\title{
Cu-Catalyzed Hydroboration of Benzylidenecyclopropanes: Reaction Optimization, (Hetero)Aryl Scope, and Origins of Pathway Selectivity
}

Jose Medina, Taeho Kang, Tuğçe Erbay, Huiling Shao, Gary Gallego, Shouliang Yang, Michelle Tran-Dubé, Paul Richardson, Joseph Derosa, Ryan Helsel, Ryan Patman, Fen Wang, Christopher Ashcroft, John Braganza, Indrawan McAlpine, Peng Liu, Keary Engle

Submitted date: 28/06/2019 - Posted date: 28/06/2019

Licence: CC BY-NC-ND 4.0

Citation information: Medina, Jose; Kang, Taeho; Erbay, Tuğçe; Shao, Huiling; Gallego, Gary; Yang, Shouliang; et al. (2019): Cu-Catalyzed Hydroboration of Benzylidenecyclopropanes: Reaction Optimization, (Hetero)Aryl Scope, and Origins of Pathway Selectivity. ChemRxiv. Preprint.

The copper-catalyzed hydroboration of benzylidenecyclopropanes, conveniently accessed in one step from readily available benzaldehydes, is reported. Under otherwise identical reaction conditions, two distinct phosphine ligands grant access to different products by either suppressing or promoting cyclopropane opening via $\beta$-carbon elimination. Computational studies provide insight into how the rigidity and steric environment of these different bis-phosphine ligands influence the relative activation energies of $\beta$-carbon elimination versus protodecupration from the key benzylcopper intermediate. The method tolerates a wide variety of heterocycles prevalent in clinical and pre-clinical drug development, giving access to valuable synthetic intermediates. The versatility of the tertiary cyclopropylboronic ester products is demonstrated through several derivatization reactions.

File list (2)

Manuscript.pdf (1.30 MiB)

view on ChemRxiv - download file

Supporting Info.pdf (25.70 MiB)

view on ChemRxiv - download file 


\title{
Cu-Catalyzed Hydroboration of Benzylidenecyclopro- panes: Reaction Optimization, (Hetero)Aryl Scope, and Origins of Pathway Selectivity
}

Jose M. Medina ${ }^{+£ \ddagger}$, Taeho Kang ${ }^{+\ddagger}$, Tuğçe G. Erbay ${ }^{\ddagger}$, Huiling Shao ${ }^{\ddagger}$, Gary M. Gallego ${ }^{\S}$, Shouliang Yang ${ }^{\S}$, Michelle Tran-Dubé ${ }^{\S}$, Paul F. Richardson ${ }^{\S}$,Joseph Derosa ${ }^{\dagger}$, Ryan T. Helsel ${ }^{\dagger}$, Ryan L. Patman ${ }^{\S}$, Fen Wang ${ }^{\S}$, Christopher P. Ashcroft $^{\S}$, John F. Braganza ${ }^{\S}$, Indrawan McAlpine ${ }^{\S^{*}}$, Peng Liu ${ }^{\ddagger^{*}}$, and Keary M. Engle ${ }^{+^{*}}$

${ }^{\dagger}$ Department of Chemistry, Scripps Research, 10550 North Torrey Pines Road, La Jolla, California 92037, United States

${ }^{\ddagger}$ Department of Chemistry, University of Pittsburgh, Pittsburgh, Pennsylvania 15260, United States

${ }^{\S}$ Pfizer Oncology Medicinal Chemistry, 10770 Science Center Drive, San Diego, California 92121, United States

Copper catalysis, hydroborations, benzylidenecyclopropanes, cyclopropylboronic esters, $\beta$-carbon elimination, heterocycles.

\begin{abstract}
The copper-catalyzed hydroboration of benzylidenecyclopropanes, conveniently accessed in one step from readily available benzaldehydes, is reported. Under otherwise identical reaction conditions, two distinct phosphine ligands grant access to different products by either suppressing or promoting cyclopropane opening via $\beta$-carbon elimination. Computational studies provide insight into how the rigidity and steric environment of these different bis-phosphine ligands influence the relative activation energies of $\beta$-carbon elimination versus protodecupration from the key benzylcopper intermediate. The method tolerates a wide variety of heterocycles prevalent in clinical and preclinical drug development, giving access to valuable synthetic intermediates. The versatility of the tertiary cyclopropylboronic ester products is demonstrated through several derivatization reactions.
\end{abstract}

Conformationally constrained small carbo- and heterocyclic ring systems are among the most important motifs in modern organic chemistry and drug discovery. ${ }^{[1]}$ Cyclopropane substructures are of special importance as they can imbue a molecule with unique properties, including increased potency, metabolic stability, and brain permeability as well as attenuated $\mathrm{p} K_{\mathrm{a}}$ and lipophilicity. ${ }^{[2]}$ Indeed, cyclopropyl groups are found in a wide range of secondary metabolites, insecticides, pharmaceutical products, for example a TRPV1 agonist, ${ }^{[3]}$ GSK1360707F, ${ }^{[4]}$ and foretinib ${ }^{[5]}$ (Figure 1A). Accessing cyclopropane motifs with diverse substitution patterns is often a challenging aspect of the synthesis of such compounds. ${ }^{[6]}$

Tertiary cyclopropylboronic esters have emerged as a versatile family of building blocks that can be further elaborated to prepare multisubstituted cyclopropane motifs. As a consequence, chemists have developed many important synthetic methods for their preparation (Figure 1B). For example, traditional cyclopropanation of vinyl boronates has been used to afford desired tertiary cyclopropylboronic esters. ${ }^{[7]}$ More recently, the Baran and Aggarwal groups independently demonstrated that decarboxylative borylation of cyclopropyl carboxylic acids produces the corresponding cyclopropylboronic esters. ${ }^{[8]}$ In addition, Harris and coworkers published a method for selective Suzuki-Miyaura coupling of gem-bis(boryl)cyclopropanes which gives tertiary cyclopropylboronic esters as products. ${ }^{[9]}$ This impressive progress notwithstanding, existing methods also have some limitations in terms of restricted scope, harsh reaction conditions, cost of reagents, and requirement of pre-functionalized precursors.

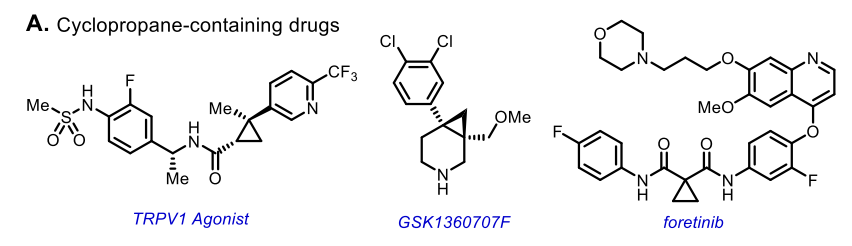

B. Precursors to tertiary cyclopropylboronic esters

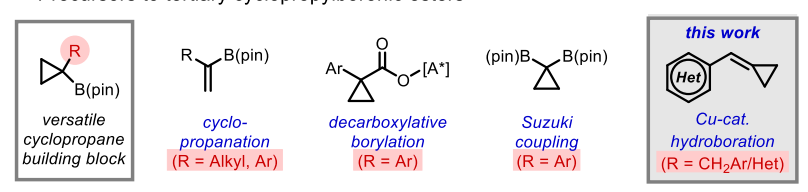

C. Ligand-controlled pathway selectivity in benzylidene cyclopropane hydroboration

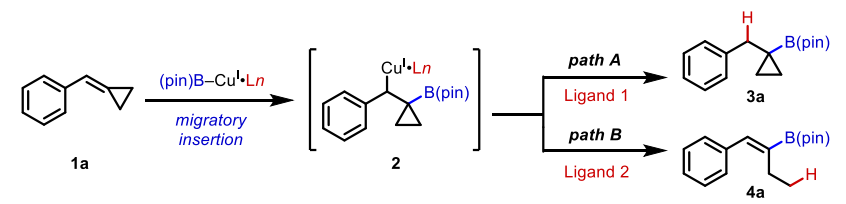

Figure 1. Overview of Proposed Approach to Preparation of Cyclopropylboronic Esters.

For ongoing programs in drug discovery at Pfizer, we sought a convenient and reliable method to access tertiary cyclopropylboronates bearing diverse $-\mathrm{CH}_{2} \mathrm{Ar} / \mathrm{Het}$ substitution at the $\alpha$-position. In this context, we were attracted to copper-boryl chemistry, which has emerged as a powerful reactivity paradigm for hydroboration and borylative difunctionalization of alkenes. ${ }^{[10]}$ This class of reactions 
often proceeds under mild conditions and exhibits broad functional group tolerance, making it well suited for our purposes. In particular, we envisioned highly strained benzylidenecyclopropanes (BCPs) 1 would be ideal tertiary cyclopropylboronic esters precursors upon $\mathrm{Cu}$ catalyzed hydroboration. ${ }^{[11]}$ The utility of the alkylidene/benzylidenecyclopropane substrate class stems largely from the relief of strain energy, which provides a thermodynamic driving force for engaging a wide array of reaction partners in complexity-generating transformations. ${ }^{[12]}$

In considering the integration of BCP substrates into copper-boryl catalysis, we envisioned two potential competitive reaction pathways (Figure 1C) from the common intermediate $\mathbf{2}$ which is generated by migratory insertion of copper-boryl complex to BCP $1 .^{[12]}$ Direct protodecupration of $\mathbf{2}$ would generate cyclopropylboronic esters $\mathbf{3}$ (path A). On the other hand, alkenylboronates 4 could also be generated if $\beta$ carbon elimination of $\mathbf{2}$ is preferred over protodecupration (path B). We envisioned that by tuning the ligand sphere around the metal we could control the pathway selectivity of this process and enable straightforward access to these coveted building blocks. At the outset, we took inspiration from the work of Shi, who described a method for 1,2-aminoboration of BCPs using BINAP as ligand. ${ }^{[11 e]}$

We began our study by preparing several representative BCPs (1a1c, Table 1). All BCP substrates were derived from the corresponding aldehydes through Wittig reactions with commercially available and inexpensive starting materials (see SI for details). ${ }^{[13]}$ The heterocycles were chosen based on their prevalence in bioactive compounds and their different electronic properties, which we thought might play a role in stabilizing intermediate $\mathbf{2}$ thereby impacting the innate tendency to undergo ring opening (Figure $1 \mathrm{C}$ ). ${ }^{[14]}$ We explored various mono- and bis-phosphine ligands with different electronic and steric properties under conditions similar to those developed for the copper-catalyzed hydroboration of styrenes. ${ }^{[15]}$ For ease of visualization, the results in Table 1 are color-coded to highlight outcomes where the ${ }^{1} \mathrm{H}$ NMR yield for one of the two isomers was higher than $70 \%$ (3 in blue and 4 in red). Entries 1-5 show results obtained with mono-phosphine ligands arranged from most electron-donating (entry 1 ) to most electronwithdrawing (entry 5). Although results varied from substrate to substrate, we observed a clear correlation between the electron-donating character of the ligand and the propensity for $\beta$-carbon elimination, which leads to formation of alkenylboronates 4. Notably, use of tris(pentafluorophenyl)phosphine (entry 5), a ligand not commonly used in copper-boryl catalysis, provided cyclopropylboronic esters 3 exclusively, albeit in variable yields, showing that $\beta$-carbon elimination could be fully suppressed with ligands that deplete electron density at the metal center.

We then turned our attention to bis-phosphine ligands containing two $-\mathrm{PPh}_{2}$ arms with varied natural bite angles $\left(\beta_{\mathrm{n}}\right)\left(\right.$ entries 6-10). ${ }^{[16]}$ Using dppm (entry $6, \beta_{\mathrm{n}}=72^{\circ}$ ), all three substrates preferentially formed cyclopropylboronic esters 3. With dppbz as the ligand (entry 7 , $\left.\beta_{\mathrm{n}}=83^{\circ}\right)$, most substrates gave 4 preferentially. In similar fashion, dppe (entry 8 ), which is known to bind copper with a larger bite-angle than dppm and dppbz $\left(\beta_{\mathrm{n}}=85^{\circ}\right)$, favored formation of alkenylboronates 4 . Larger bite angle ligands (entries 9 and 10) such as rac-BINAP $\left(\beta_{\mathrm{n}}=\right.$ $\left.92^{\circ}\right)$ and xantphos $\left(\beta_{\mathrm{n}}=111^{\circ}\right)$ switched the selectivity to favor product 3. The lack of a clear trend between bite angle and selectivity points to a complex interplay of steric and conformational factors (vide infra). With the insights gained from this study, we opted to use rac-BINAP and dppe as the ligands for selective formation of products $\mathbf{3}$ and $\mathbf{4}$, respectively.
Table 1. Ligand optimization with representative substrates

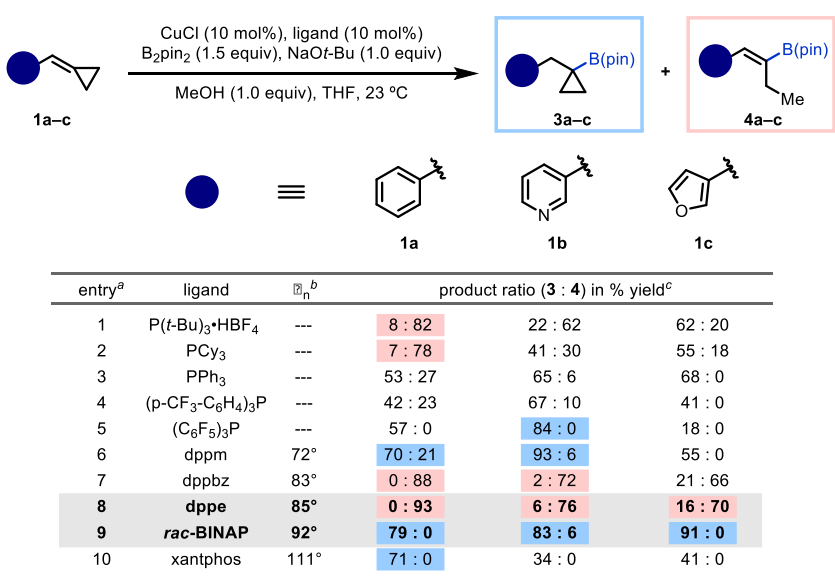

${ }^{a}$ Reaction conditions: 1 ( $\left.0.2 \mathrm{mmol}\right), \mathrm{CuCl}$ (10 mol\%), ligand (10 mol\%), $\mathrm{B}_{2} \mathrm{pin}_{2}$ (0.3 mmol), NaOt-Bu (0.2 mmol), $\mathrm{MeOH}(0.2 \mathrm{mmol})$ in THF $(0.5 \mathrm{~mL})$ at room temperature. ${ }^{b}$ Natural bite angle $\left(\beta_{\mathrm{n}}\right){ }^{c}$ Yield determined by ${ }^{1} \mathrm{H}$ NMR.

We turned our attention to exploring the scope with respect to arene substituents (Scheme 1). The protocol to prepare cyclopropylboronic esters 3 was first examined and found to tolerate a number of electronwithdrawing groups relevant to medicinal chemistry. For example, $\mathrm{BCP}$ containing fluoride at the ortho and para positions gave boronic esters $\mathbf{3} \mathbf{d}$ and $\mathbf{3 e}$ in good yields. A substrate with a bromide at the para position gave the desired cyclopropylboronic ester $3 \mathbf{f}$ in $36 \%$. As demonstrated by the synthesis of $\mathbf{3} \mathbf{g}$ and $\mathbf{3 h}$, substrates containing trifluoromethyl groups can also be utilized in the reaction. Additionally, the cyano group was well-tolerated, as shown by the synthesis of product 3i. BCPs bearing electron-donating groups also worked well under the optimized reaction conditions. With certain electron-poor substrates 1d, 1e, and 19, we found that tris(pentafluorophenyl)phosphine offered moderately higher yields (yield with this ligand in parentheses). Substrates with dimethylamine and methoxy substituents at the para position gave products $\mathbf{3} \mathbf{j}$ and $\mathbf{3 k}$, respectively. A particularly electron-rich $\mathrm{BCP}$ bearing three methoxy groups gave the desired product $\mathbf{3 1}$ in $\mathbf{8 8 \%}$. Moreover, cyclopropylboronic ester $\mathbf{3 m}$, containing the difluoro benzodioxole moiety, could be formed in excellent yield.

\section{Scheme 1. Hydroboration of BCPs with substituted arenes}

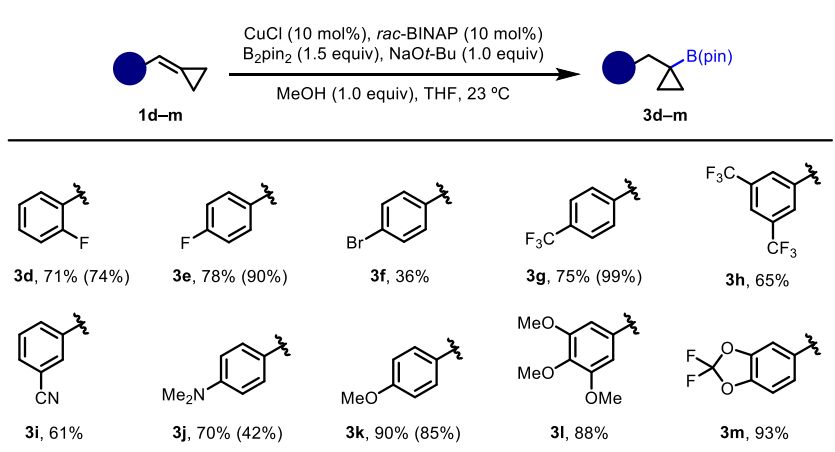

${ }^{a}$ Reaction conditions: 1 (0.2 mmol), $\mathrm{CuCl}$ (10 mol\%), rac-BINAP (10 mol\%), $\mathrm{B}_{2} \mathrm{pin}_{2}$ (0.3 mmol), NaOt-Bu (0.2 mmol), $\mathrm{MeOH}(0.2 \mathrm{mmol})$ in THF $(0.5 \mathrm{~mL})$ at room temperature. ${ }^{b}$ The values in parentheses 
correspond to NMR yields with $\left(\mathrm{C}_{6} \mathrm{~F}_{5}\right)_{3} \mathrm{P}$ as ligand in place of racBINAP

We then shifted our focus to more challenging BCPs containing heterocycles prevalent in biologically active molecules (Scheme 2). Many heterocyclic motifs are known to present problems in catalysis, as they can bind strongly to the metal center and inhibit progress. The transformation proved to be tolerant of diverse 5-membered heterocycles, including thiophene, Ts-protected pyrroles, oxazole, and thiazole to give the corresponding products $\mathbf{3 n}-\mathbf{3 s}$ in good to excellent yields. 6membered heterocycles, including various substituted pyridines, performed well in the reaction, as exemplified by formation of boronic esters $\mathbf{3 t} \mathbf{- 3 z}$. It is worth mentioning that pyridine is the most common aromatic nitrogen-containing heterocycle in drug molecules. Synthetic methods that allow access to building blocks resembling $\mathbf{3 t}-\mathbf{3 z}$ are highly sought-after. We also explored other heterocycles of interest, namely pyrimidine, quinoxaline, quinoline, pyrazolo pyrimidine, benzimidazole, and benzofuran, which gave products $\mathbf{3 z a}-\mathbf{3 z f}$ in synthetically useful to good yields. ${ }^{[17]}$

\section{Scheme 2. Hydroboration of BCPs with heterocycles}

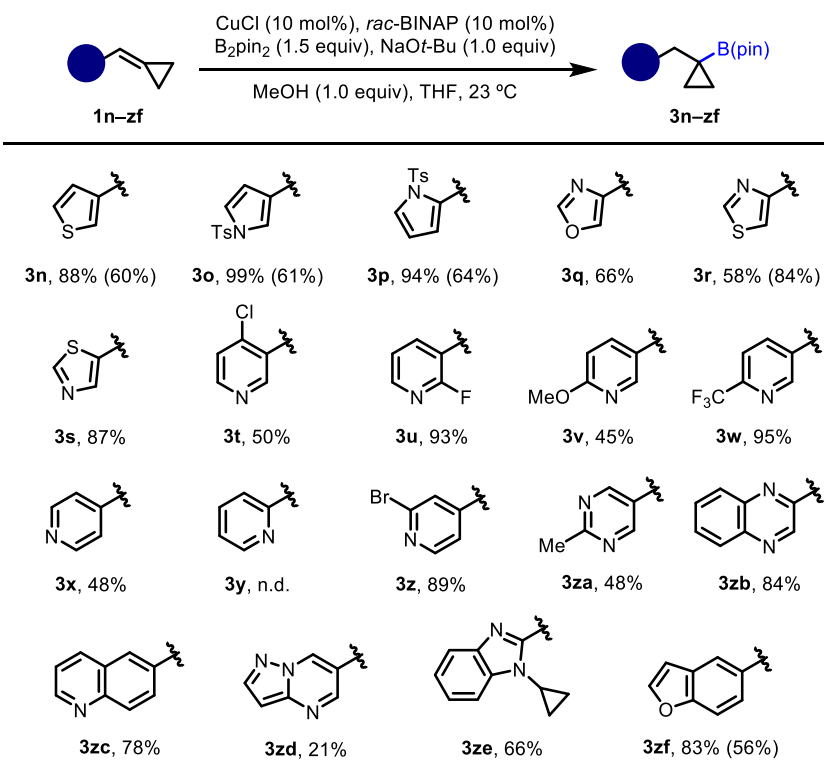

${ }^{a}$ Reaction conditions: 1 (0.2 mmol), $\mathrm{CuCl}$ (10 mol\%), rac-BINAP (10 mol\%), $\mathrm{B}_{2} \operatorname{pin}_{2}(0.3 \mathrm{mmol}), \mathrm{NaOt}-\mathrm{Bu}(0.2 \mathrm{mmol}), \mathrm{MeOH}(0.2 \mathrm{mmol})$ in THF $(0.5 \mathrm{~mL})$ at room temperature. n.d. $=$ not detected. ${ }^{b}$ The values in parentheses correspond to NMR yields with $\left(\mathrm{C}_{6} \mathrm{~F}_{5}\right)_{3} \mathrm{P}$ as ligand in place of $r a c-B I N A P$.

Next, we explored the feasibility of $\beta$-carbon elimination on a number of substrates containing various substituents and heterocyclic motifs (Scheme 3). The system developed utilizing dppe as the ligand served to give alkenylboronates in good to excellent yields.
Scheme 3. Synthesis of vinyl boronates via $\beta-C$ elimination

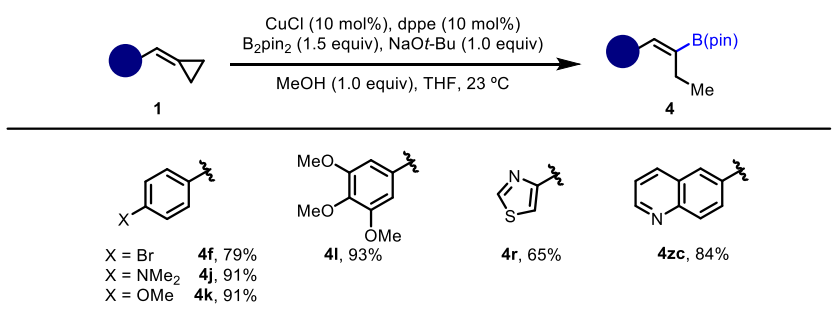

${ }^{a}$ Reaction conditions: 1 ( $\left.0.2 \mathrm{mmol}\right), \mathrm{CuCl}(10 \mathrm{~mol} \%)$, dppe (10 $\mathrm{mol} \%), \mathrm{B}_{2} \operatorname{pin}_{2}(0.3 \mathrm{mmol}), \mathrm{NaOt}$-Bu $(0.2 \mathrm{mmol}), \mathrm{MeOH}(0.2 \mathrm{mmol})$ in THF $(0.5 \mathrm{~mL})$ at room temperature.

Having established access to various tertiary cyclopropylboronic esters, we set out to demonstrate their synthetic utility through derivatization reactions (Scheme 4). We chose to focus our efforts on derivatizations of the tertiary cyclopropylboronic esters, as vinyl-boronates are widely known to be versatile building blocks. Compound 3a was chosen as the model substrate for select $\mathrm{C}-\mathrm{X}$ and $\mathrm{C}-\mathrm{C}$ bond forming transformations. Exposure of $\mathbf{3 a}$ to sodium perborate gave tertiary alcohol $\mathbf{5}$ in 99\% ${ }^{[18]}$ A modified amination protocol developed by Morken furnished primary amine 6 in modest yield. ${ }^{[19]}$ A Matteson-Aggarwal homologation delivered boronic ester $7 ;^{[20]}$ and lastly, the gram-scale conversion of $\mathbf{3 a}$ to tertiary trifluoroborate 8 occurred in $81 \%{ }^{[21]}$

Furthermore, as illustrated in Scheme 5, facile access to trifluoroborate 8 enabled several Suzuki-Miyaura couplings. Under conditions developed by Harris and coworkers, use of alkenyl triflate electrophiles gave alkenyl cyclopropanes $\mathbf{9 - 1 1}$ in good yields. ${ }^{[22]}$ Likewise, use of heteroaryl bromides efficiently delivered products 12-14 containing nitrogen-based heterocycles and substituents (e.g. fluorine, trifluoromethyl, and methyl) critical in drug development. ${ }^{[23]}$

\section{Scheme 4. Select derivatization reactions with tertiary boronic ester 3a}

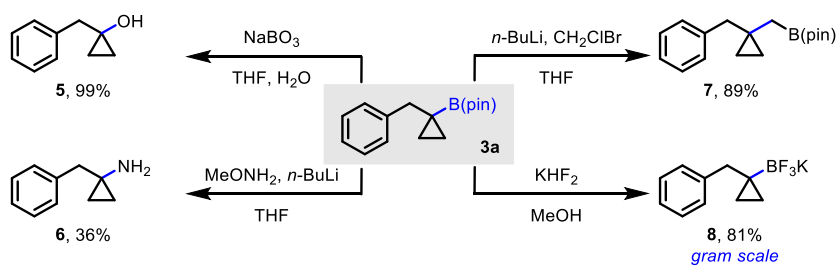

Scheme 5. Enabling Suzuki-Miyaura couplings with tertiary trifluoroborate 8

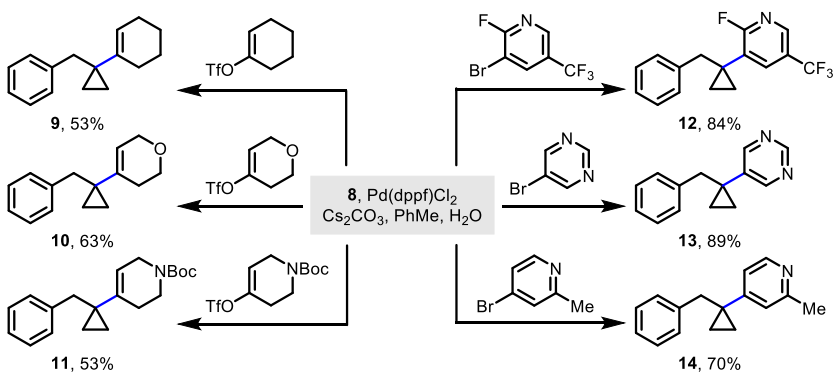


Given the wide discrepancy between outcomes obtained with seemingly related bis-phosphine ligands under otherwise identical reaction conditions, we pursued further understanding of the ligand effects with computational studies. We performed DFT calculations at the M06/6$311+\mathrm{G}(\mathrm{d}, \mathrm{p})-\mathrm{SDD} / \mathrm{SMD}(\mathrm{THF}) / / \mathrm{M} 06 \mathrm{~L} / 6-31 \mathrm{G}(\mathrm{d})-\mathrm{LANL} 2 \mathrm{DZ}$ level of theory to investigate the reaction energy profiles leading to the alkenylboronate and cyclopropylboronic ester products with dppe and BINAP ligands (see Figure S1). The calculations suggest that the pathway selectivity is determined by the activation energy difference between $\beta$-carbon elimination (TS1) and protodecupration (TS2) from the benzylic copper intermediate 2 formed via the irreversible migratory insertion of the BCP 1a (Scheme 6). ${ }^{[24]}$ Consistent with the experimental observations, with the dppe ligand the $\beta$-carbon elimination is favored over protodecupration by $3.2 \mathrm{kcal} / \mathrm{mol}$, leading to the formation of the alkenylboronate product $4 a$. The use of BINAP ligand completely reverses the pathway selectivity. $\beta$-Carbon elimination from the benzylic copper complex with BINAP as ligand requires a much higher barrier $\left(\Delta G^{\ddagger}=22.4 \mathrm{kcal} / \mathrm{mol}\right)$ than that with dppe as ligand $\left(\Delta G^{f}=13.9 \mathrm{kcal} / \mathrm{mol}\right)$. On the other hand, ligand effects have a much smaller impact on the barrier of protodecupration (TS2). Therefore, the reaction using BINAP as ligand forms the cyclopropylboronic ester 3a via favorable protodecupration.

Scheme 6. Activation energies of the selectivity-determining steps.

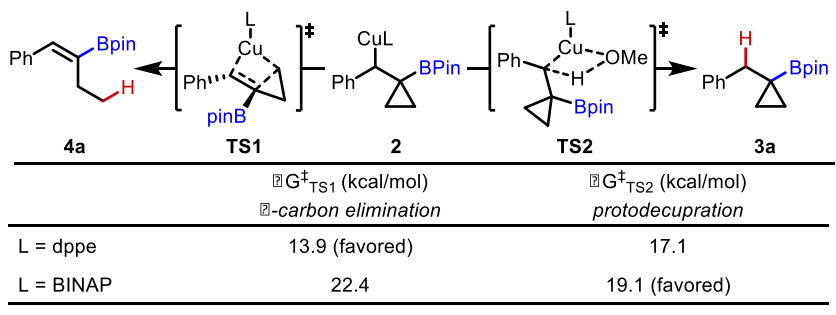

The origin of ligand effects on the $\beta$-carbon elimination barrier can be visualized in the quadrant diagrams in Figure 2 . The $\beta$-carbon elimination TS with the $\mathrm{Cu}(\mathrm{I})$ center prefers a tetrahedral geometry that places the benzylic $\left(C_{\alpha}\right)$ and $\gamma$-carbons $\left(C_{\gamma}\right)$ within the vertical region perpendicular to the $\mathrm{P}-\mathrm{Cu}-\mathrm{P}$ plane. With dppe, this vertical region is not occupied by the $-\mathrm{PPh}_{2}$ arms. Thus, no unfavorable steric repulsions are observed in TS1a. On the other hand, the more rigid BINAP is confined to a $C_{2}$-symmetric conformation, in which the vertical region is blocked by the pseudo-axial phenyl groups $\left(\mathrm{Ph}_{\mathrm{ax}}\right)$ of the $-\mathrm{PPh}_{2}$ arms. The benzylic and $\gamma$-carbons in TS1b are significantly distorted to be placed in the less occupied diagonal regions in quadrants II and IV to avoid repulsions with the BINAP ligand. Consequently, the distorted tetrahedral TS1b is energetically disfavored. An isomer of TS1b that places $\mathrm{C}_{\alpha}$ and $\mathrm{C}_{\gamma}$ in the more occupied I and III quadrants was also located and requires an even higher activation energy (26.7 $\mathrm{kcal} / \mathrm{mol})$.
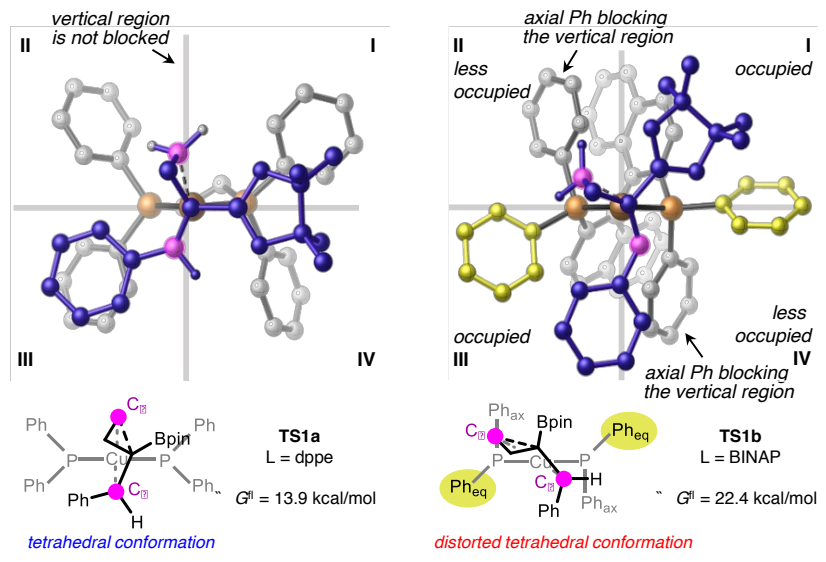

Figure 2. Origin of ligand effects on $\beta$-carbon elimination barriers.

In summary, we have developed conditions for the hydroboration of benzylidenecyclopropanes that lead to formation of two distinct products, namely cyclopropylboronic esters and alkenylboronates. Both products represent highly versatile building blocks that enable access to diverse derivatives based on downstream manipulation of boronic ester functionality. This work should be of particular interest to the pharmaceutical industry and represents an example of an emerging concept in catalysis, whereby pathway selectivity can be tuned through ligand space. ${ }^{[25]}$ Computational analysis reveals the origins of ligand effects affecting a key $\beta$-carbon elimination step, providing a conceptual framework for strategically employing this family of strained alkenes in a broader range of catalytic transformations.

\section{ASSOCIATED CONTENT}

\section{Supporting Information}

Detailed experimental and computational procedures, compound characterization, Cartesian coordinates of the calculated structures. This material is available free of charge via the Internet at http://pubs.acs.org.

\section{AUTHOR INFORMATION}

\section{Corresponding Author}

indrawan.mcalpine@pfizer.com; pengliu@pitt.edu; keary@scripps.edu

\section{Present Addresses}

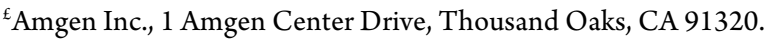

\section{Author Contributions}

${ }^{\ddagger}$ These authors contributed equally.

Notes

The authors declare no competing financial interest.

\section{ACKNOWLEDGMENT}

This work was financially supported by the National Institutes of Health (5R35GM125052-02; 1R35GM128779), The Scripps Research Institute, and Pfizer, Inc. We gratefully acknowledge the Kwanjeong Educational Foundation (Graduate Fellowship to T.K.) and the NSF for a Graduate Research Fellowship (NSF/DGE-1346837, J.D.). Calculations were performed at the Center for Research Computing at 
the University of Pittsburgh and the Extreme Science and Engineering Discovery Environment (XSEDE) supported by the NSF.

\section{REFERENCES}

(Word Style "TF_References_Section"). References are placed at the end of the manuscript. Authors are responsible for the accuracy and completeness of all references. Examples of the recommended formats for the various reference types can be found at http://pubs.acs.org/page/4authors/index.html. Detailed information on reference style can be found in The ACS Style Guide, available from Oxford Press.

(1) (a) Liebman, J. F.; Greenberg, A. A Survey of Strained Organic Molecules. Chem. Rev. 1976, 76, 311. (b) Rotstein, B. H.; Zaretsky, S.; Rai, V.; Yudin, A. K. Small Heterocycles in Multicomponent Reactions. Chem. Rev. 2014, 114, 8323. (c) de Meijere, A.; Kozhushkov, S. I.; Schill, H. Three-Membered-Ring-Based Molecular Architectures. Chem. Rev. 2006, 106, 4926.

(2) (a) Talele, T. T. The "Cyclopropyl Fragment" is a Versatile Player that Frequently Appears in Preclinical/Clinical Drug Molecules. J. Med. Chem. 2016, 59, 8712. (b) Derosa, J.; O’Duill, M. L.; Holcomb, M.; Boulous, M. N.;

Patman, R. L.; Wang, F.; Tran-Dube, M.; McAlpine, I.; Engle, K. M. Copper-Catalyzed Chan-Lam Cyclopropylation of Phenols and Azaheterocycles. J. Org. Chem. 2018, 83, 3417.

(3) Butcher, K. J.; Denton, S. M.; Field, S. E.; Gillmore, A.T.; Harbottle, G. W.; Howard, R. M.; Laity, D.A.; Ngono, C. J.; Pibworth, B. A. Convergent Asymmetric Synthesis of Two Complex TRPV1 Antagonists. Org. Process Res. Dev. 2011, 15,1192

(4) Micheli, F.; Cavanni, P.; Andreotti, D.; Arban, R.; Benedetti, R.; Bertani, B.; Bettati, M.; Bettelini, L.; Bonanomi, G.; Braggio, S.; Carletti, R.; Checchia, A.; Corsi, M.; Fazzolari, E.; Fontana, S.; Marchioro, C.; Merlo-Pich, E.; Negri, M.; Oliosi, B.; Ratti, E.; Read, K. D.; Roscic, M.; Sartori, I.; Spada, S.; Tedesco, G.; Tarsi, L.; Terreni, S.; Visentini, F.; Zocchi, A.; Zonzini, L.; Di Fabio, R. 6-(3,4Dichlorophenyl)-1-[(methyloxy)methyl]-3-azabicyclo[4.1.0]heptane: A New Potent and Selective Triple Reuptake Inhibitor. J. Med. Chem. 2010, 53, 4989.

(5) Zillhardt, M.; Park, S.-M.; Romero, I. L.; Sawada, K.; Montag, A.; Krausz, T.; Yamada, S. D.; Peter, M. E.; Lengyel, E. Foretinib (GSK1363089), an Orally Available Multikinase Inhibitor of c-Met and VEGFR-2, Blocks Proliferation, Induces Anoikis, and Impairs Ovarian Cancer Metastasis. Clin. Cancer Res. 2011, 17,4042 .

(6) Ebner, C.; Carreira, E. M. Cyclopropanation Strategies in Recent Total Syntheses. Chem. Rev. 2017, 117, 11651

(7) (a) Hussain, M. M.; Li, H.; Hussain, N.; Ureña, M.; Carroll, P. J.; Walsh, P. J. Applications of 1-Alkenyl-1,1-Heterobimetallics in the Stereoselective Synthesis of Cyclopropylboronate Esters, Trisubstituted Cyclopropanols and 2,3Disubstituted Cyclobutanones. J. Am. Chem. Soc. 2009, 131, 6516. (b) Phelan, J. P.; Lang, S. B.; Compton, J. S.; Kelly, C. B.; Dykstra, R.; Gutierrez, O.; Molander, G. A. Redox-Neutral Photocatalytic Cyclopropanation via Radical/Polar Crossover. J. Am. Chem. Soc. 2018, 140, 8037.

(8) (a) Li. C.; Wang. J.; Barton L. M.; Yu, S.; Tian, M.; Peters, D. S.; Kumar, M.; Yu, A. W.; Johnson, K. A.; Chatterjee, A. K.; Yan, M.; Baran, P. S. Decarboxylative Borylation. Science 2017, 356, eaam7355. (b) Fawcett, A.; Pradeilles, J.; Wang, Y.; Mutsuga, T.; Myers, E. L.; Aggarwal, V. K. Photoinduced Decarboxylative Borylation of Carboxylic Acids. Science 2017, 357, 283.

(9) Harris, M. R.; Wisniewska, H. M.; Jiao, W.; Wang, X.; Bradow, J. N. A Modular Approach to the Synthesis of gem-Disubstituted Cyclopropanes. Org. Lett. 2018, 20, 2867

(10) Mun, S.; Lee, J.-E.; Yun, J. Copper-Catalyzed $\beta$-Boration of $\alpha, \beta$ Unsaturated Carbonyl Compounds: Rate Acceleration by Alcohol Additives. Org. Lett. 2006, 8, 4887.

(11) (a) Lee, Y.; Hoveyda, A. H. Efficient Boron-Copper Additions to ArylSubstituted Alkenes Promoted by NHC-Based Catalysts. Enantioselective CuCatalyzed Hydroboration Reactions. J. Am. Chem. Soc. 2009, 131, 3160. (b) Lee, Y.; Jang, H.; Hoveyda, A. H. Vicinal Diboronates in High Enantiomeric Purity through Tandem Site-Selective NHC-Cu-Catalyzed Boron-Copper Additions to Terminal Alkynes. J. Am. Chem. Soc. 2009, 131, 18234. (c) Corberán, R.; Mszar, N. W.; Hoveyda, A. H. NHC-Cu-Catalyzed Enantioselective Hydroboration of Acyclic and Exocyclic 1,1-Disubstituted Aryl Alkenes. Angew. Chem. Int.
Ed. 2011, 50, 7079. (d) Matsuda, N.; Hirano, K.; Satoh, T.; Miura, M. Regioselective and Stereospecific Copper-Catalyzed Aminoboration of Styrenes with Bis(pinacolato)diboron and O-Benzoyl- $\mathrm{N}, \mathrm{N}$-dialkylhydroxylamines. J. Am. Chem. Soc. 2013, 135, 4934. (e) Jiang, H.-C.; Tang, X.-Y.; Shi, M. CopperCatalyzed Regio- and Enantioselective Aminoboration of Alkylidenecyclopropanes: The Synthesis of Cyclopropane-Containing $\beta$-Aminoalkylboranes. Chem. Commun. 2016, 52, 5273. (f) Kerchner, H.; Montgomery, J. Synthesis of Secondary and Tertiary Alkylboranes via Formal Hydroboration of Terminal and 1,1-Disubstituted Alkenes. Org. Lett. 2016, 18, 5760. (g) Han, J. T.; Han, W. J.; Kim, N.; Yun, J. Asymmetric Synthesis of Borylalkanes via Copper-Catalyzed Enantioselective Hydroallylation. J. Am. Chem. Soc. 2016, 138, 15146. (h) Jang, W. J.; Song, S. M.; Moon, J. H.; Lee, J. Y.; Yun, J. Copper-Catalyzed Enantioselective Hydroboration of Unactivated 1,1-Disubstituted Alkenes. J. Am. Chem. Soc. 2017, 139, 13660. (i) Semba, K.; Ohtagaki, Y.; Nakao, Y. Arylboration of 1Arylalkenes by Cooperative Nickel/Copper Catalysis. Org. Lett. 2016, 18, 3956. (j) Bergmann, A. M.; Dorn, S. K.; Smith, K. B.; Logan, K. M.; Brown, M. K. Catalyst-Controlled 1,2-and 1,1-Arylboration of $\alpha$-Alkyl Alkenyl Arenes. Angew. Chem. Int. Ed. 2019, 58, 1719

(12) (a) Brandi, A.; Cicchi, S.; Cordero, F. M.; Goti, A. Progress in the Synthesis and Transformations of Alkylidenecyclopropanes and Alkylidenecyclobutanes. Chem. Rev. 2014, 114, 7317. (b) Pellissier, H. Recent Developments in the Synthesis and Reactivity of Methylene- and Alkylidenecyclopropane Derivatives. Tetrahedron 2014, 70, 4991.

(13) (a) Masarwa A.; Marek, I. Selectivity in Metal-Catalyzed Carbon-Carbon Bond Cleavage of Alkylidenecyclopropanes. Chem. Eur. J. 2010, 16, 9712. (b) Yu, L.-Z.; Chen, K.; Zhu, Z.-Z.; Shi, M. Recent Advances in the Chemical Transformations of Functionalized Alkylidenecyclopropanes (FACPs). Chem. Commun. 2017, 53, 5935 .

(14) Qualitatively, the degree of aromaticity of the aryl-substituent appears to impact the inherent reactivity for ring-opening. More aromatic substituents, like phenyl in BCP 1a, appear to make the substrate more prone to rearrangement via $\beta$-carbon elimination leading to alkenylboronates $4 a$. The origin of this effect may be the decreased Brønsted basicity of the $\mathrm{C}-\mathrm{Cu}$ bond in these types of substrates, compared to those with less aromatic substituents. A thiazolecontaining substrate was found to be resistant to ring opening with all of the ligands in Table 1 (see SI).

(15) Wen, Y.; Xie, J.; Deng, C.; Li, C. Selective Synthesis of Alkylboronates by Copper(I)-Catalyzed Borylation of Allyl or Vinyl Arenes. J. Org. Chem. 2015, 80, 4142.

(16) (a) Casey, C. P.; Whiteker, G. T. The Natural Bite Angle of Chelating Diphosphines. Isr. J. Chem. 1990, 30, 299. (b) Dierkes P.; van Leeuwen P. W. N. M. The Bite Angle Makes the Difference: A Practical Ligand Parameter for Diphosphine Ligands. J. Chem. Soc., Dalton Trans., 1999, 1519. (c) van der Veen, L. A.; Keeven, P. H. Schoemaker, G. C.; Reek, J. N. H.; Kamer, P. C. J.; van Leeuwen, P. W. N. M.; Lutz, M.; Spek, A. L. Origin of the Bite Angle Effect on Rhodium Diphosphine Catalyzed Hydroformyation. Organometallics 2000, 19, 872. (d) van Leeuwen P. W. N. M.; Kamer P. C. J.; Reek, J. N. H.; Dierkes, P. Ligand Bite Angle Effects in Metal-catalyzed C-C Bond Formation. Chem. Rev. 2000, 100, 2741.

(17) For aliphatic substrate, we got low yield with significant amount of regioisomer.

(18) Farthing, C. N.; Marsden, S. P. Chiral Vinyl Dioxazaborocines in Synthesis: Asymmetric Cuprate Additions to $\beta$-Boronyl Acrylates and Vinyl Sulfones. Tetrahedron Lett. 2000, 41, 4235.

(19) Mlynarski, S. N.; Karns, A. S.; Morken, J. P. Direct Stereospecific Amination of Alkyl and Aryl Pinacol Boronates. J. Am. Chem. Soc. 2012, 134, 16449.

(20) (a) Matteson, D. S. $\alpha$-Halo Boronic Esters: Intermediates for Stereodirected Synthesis. Chem. Rev. 1989, 89, 1535. (b) Leonori, D.; Aggarwal, V. K. Lithiation-Borylation Methodology and Its Application in Synthesis. Acc. Chem. Res. 2014, 47, 3174. (c) Fujioka, Y.; Amii, H. Boron-Substituted Difluorocyclopropanes: New Building Blocks of gem-Difluorocyclopropanes. Org. Lett. 2008, 10,769 .

(21) Vedejs, E.; Chapman, R. W.; Fields, S. C.; Lin, S.; Schrimpf, M. R. Conversion of Arylboronic Acids into Potassium Aryltrifluoroborates: Convenient Precursors of Arylboron Difluoride Lewis Acids. J. Org. Chem. 1995, 60, 3020.

(22) Harris, M. R.; Li, Q.; Lian, Y.; Xiao, J.; Londregan, A. T. Construction of 1Heteroaryl-3-azabicyclo[3.1.0] hexanes by $\mathrm{sp}^{3}-\mathrm{sp}^{2}$ Suzuki-Miyaura and ChanEvans-Lam Coupling Reactions of Tertiary Trifluoroborates. Org. Lett. 2017, 19,2450 . 
(23) Fang, G.-H.; Yan, Z.-J.; Deng, M.-Z. Palladium-Catalyzed Cross-Coupling of Stereospecific Potassium Cyclopropyl Trifluoroborates with Bromides. Org. Lett. 2004, 6, 357.

(24) Dang, L.; Zhao, H.; Lin, Z.; Marder, T. B. DFT Studies of Alkene Insertions into $\mathrm{Cu}-\mathrm{B}$ Bonds in Copper(I) Boryl Complexes. Organometallics 2007, $26,2823$.
(25) (a) Mahatthananchai, J.; Dumas, A. M.; Bode, J. W. Catalytic Selective Synthesis. Angew. Chem. Int. Ed. 2012, 51, 10954. (b) Lee, Y. -C.; Kumar, K.; Waldmann, H. Ligand-Directed Divergent Synthesis of Carbo- and Heterocyclic Ring Systems. Angew. Chem. Int. Ed. 2018, 57, 5212. 


\section{Cu-Catalyzed Hydroboration of Benzylidenecyclopropanes: Reaction Optimization, (Hetero)Aryl Scope, and Origins of Pathway Selectivity}

Jose M. Medina ${ }^{+£ \ddagger}$, Taeho Kang ${ }^{\dagger}$, Tuğçe G. Erbay ${ }^{\ddagger}$, Huiling Shao ${ }^{\ddagger}$, Gary M. Gallego ${ }^{\S}$, Shouliang Yang ${ }^{\S}$, Michelle Tran-Dubé ${ }^{\S}$, Paul F. Richardson ${ }^{\S}$, Joseph Derosa ${ }^{\dagger}$, Ryan T. Helsel $^{\dagger}$, Ryan L. Patman ${ }^{\S}$, Fen Wang ${ }^{\S}$, Christopher Ashcroft $^{\S}$, John F. Braganza ${ }^{\S}$, Indrawan McAlpine ${ }^{\S^{*}}$, Peng Liu ${ }^{*^{*}}$, and Keary M. Engle ${ }^{+^{*}}$

${ }^{\dagger}$ Department of Chemistry, Scripps Research, 10550 North Torrey Pines Road, La Jolla, California 92037, United States

${ }^{\ddagger}$ Department of Chemistry, University of Pittsburgh, Pittsburgh, Pennsylvania 15260, United States

${ }^{\S}$ Pfizer Oncology Medicinal Chemistry, 10770 Science Center Drive, San Diego, California 92121, United States

\section{Supporting Information - Table of Contents}

Materials and Methods

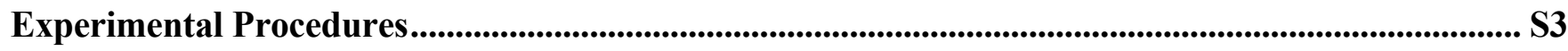

A. Synthesis of Benzylidenecyclopropanes (BCPs) ......................................................................... S3

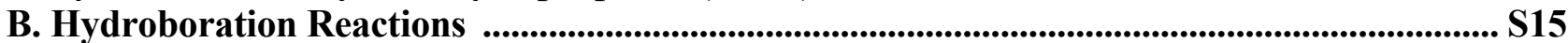

C. Exceptional Pathway Selectivity of thiazole-BCP ................................................................... S30

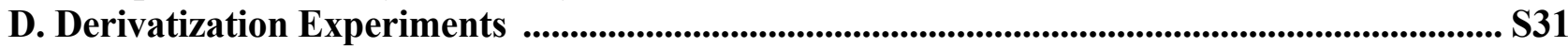

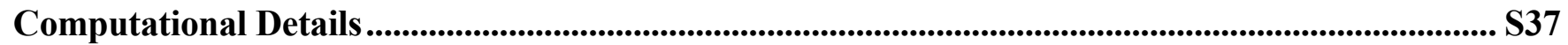

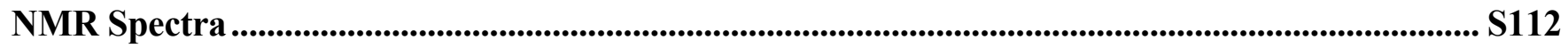

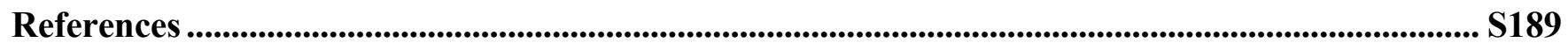




\section{Materials and Methods.}

Unless stated otherwise, reactions were conducted in flame-dried glassware under an atmosphere of nitrogen using anhydrous solvents (freshly distilled or passed through activated alumina columns). All commercially obtained reagents were used as received unless otherwise specified. Potassium tertbutoxide (KOt-Bu) and sodium tert-butoxide ( $\mathrm{NaOt}-\mathrm{Bu})$ were obtained from Oakwood Products, Inc. (3Bromopropyl) triphenylphosphonium bromide (CAS number: 3607-17-8) was obtained from Astatech. Copper chloride $(\mathrm{CuCl})$ was obtained from Sigma Aldrich. Racemic 2,2'-bis(diphenylphosphino)-1,1'binaphthyl (rac-BINAP) was obtained from Strem Chemicals. Bis(pinacolato)diboron $\left(\mathrm{B}_{2}\right.$ pin $\left.{ }_{2}\right)$ was obtained from Combi-Blocks. All aldehyde starting materials were provided by Pfizer or purchased from Alfa Aesar, Sigma Aldrich, Oakwood Products, and Combi-Blocks. Reaction temperatures were controlled using an IKAmag temperature modulator and, unless stated otherwise, reactions were performed at room temperature ( $\mathrm{rt}$, approximately $23{ }^{\circ} \mathrm{C}$ ). Thin layer chromatography (TLC) was conducted with EMD gel 60 F254 pre-coated plates $(0.25 \mathrm{~mm})$ and visualized using a combination of UV light and potassium permanganate staining. Preparative thin layer chromatography (TLC) was conducted with Analtech silica gel GF UV254 pre-coated plates (1.0 mm) and visualized using UV light. Silicycle Siliaflash P60 (particle size 0.040-0.063 mm) was used for flash column chromatography. ${ }^{1} \mathrm{H}$ and ${ }^{13} \mathrm{C}$ NMR spectra were recorded on Bruker spectrometers $(400 \mathrm{MHz}, 600 \mathrm{MHz})$ and are reported relative to deuterated solvent signals or $\mathrm{SiMe}_{4}$. The following abbreviations (or combinations thereof) were used to explain multiplicities: $\mathrm{b}=$ broad, $\mathrm{s}=$ singlet, $\mathrm{d}=$ doublet, $\mathrm{t}=$ triplet, $\mathrm{q}=$ quartet, $\mathrm{p}=$ pentet, sept $=$ septet, and $\mathrm{m}=$ multiplet. High-resolution mass spectra (HRMS) for new compounds were obtained on an Agilent LC/MSD TOF mass spectrometer. 


\section{Experimental Procedures.}

\section{A. Synthesis of Benzylidenecyclopropanes (BCPs).}

Note on BCP substrates: Some of the BCP substrates were found to decompose over time at room temperature. Some substrates were particularly unstable and showed significant decomposition within hours of being isolated. Solid substrates were generally more stable than oil substrates. We recommend that the BCPs be used soon after being made or alternatively that they be stored as frozen as stock solutions in benzene. Old BCP samples that were not appropriately stored generally gave lower product yields in hydroboration; upon re-purification by flash chromatography, the yields generally improved to reported levels.

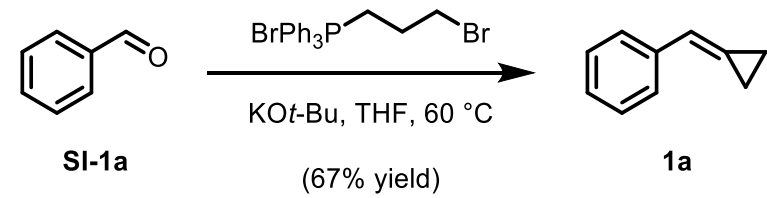

\section{Representative Procedure. ${ }^{1}$}

BCP substrate 1a. A flame-dried scintillation vial equipped with a stir bar was charged with (3bromopropyl)triphenylphosphonium bromide (1.40 g, $3.0 \mathrm{mmol}, 1.2$ equiv) and $\mathrm{KO} t$ - $\mathrm{Bu}$ (700 $\mathrm{mg}, 6.0 \mathrm{mmol}, 2.4$ equiv). The solids were suspended in THF $(10 \mathrm{~mL})$, and the mixture was allowed to stir at $60{ }^{\circ} \mathrm{C}$ for $1 \mathrm{~h}$. After the solution had cooled to room temperature, benzaldehyde (265 mg, $2.5 \mathrm{mmol}, 1.0$ equiv) was added to the suspension dropwise over $2 \mathrm{~min}$. The vial was sealed, and the suspension was allowed to stir at $60^{\circ} \mathrm{C}$ for $12 \mathrm{~h}$. The reaction mixture was cooled to room temperature and filtered through a celite pad, which was subsequently rinsed with $\mathrm{Et}_{2} \mathrm{O}$ $(3 \times 20 \mathrm{~mL})$. The filtrate was concentrated, and the resulting crude mixture was purified by flash chromatography (hexanes) to provide BCP substrate $1 \mathbf{a}(218 \mathrm{mg}, 67 \%$ yield) as a colorless oil. Spectral data match those previously reported. ${ }^{2}$ 


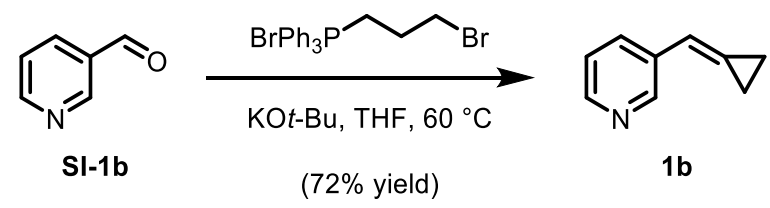

BCP substrate 1b. Purification by flash chromatography (4:1 hexanes:EtOAc) afforded BCP substrate $1 \mathbf{b}\left(235 \mathrm{mg}, 72 \%\right.$ yield) as a colorless oil. 1b: ${ }^{1} \mathbf{H}$ NMR $\left(600 \mathrm{MHz}, \mathrm{CDCl}_{3}\right): \delta 8.66(\mathrm{~s}$, $1 \mathrm{H}), 8.36(\mathrm{~d}, J=5.0 \mathrm{~Hz}, 1 \mathrm{H}), 7.76(\mathrm{~d}, J=7.8 \mathrm{~Hz}, 1 \mathrm{H}), 7.16(\mathrm{dd}, J=8.0,4.9 \mathrm{~Hz}, 1 \mathrm{H}), 6.66(\mathrm{~s}$, $1 \mathrm{H}), 1.36(\mathrm{td}, J=8.0,4.0 \mathrm{~Hz}, 2 \mathrm{H}), 1.14(\mathrm{dd}, J=9.3,6.6 \mathrm{~Hz}, 2 \mathrm{H}) ;{ }^{13} \mathbf{C} \mathbf{N M R}\left(150 \mathrm{MHz}, \mathrm{CDCl}_{3}\right)$ : $\delta 148.21,147.57,133.68,132.93,127.03,123.29,114.91,4.08,0.72$; HRMS-ESI $(\mathrm{m} / z)[\mathrm{M}+$ $\mathrm{H}]^{+}$calcd for $\mathrm{C}_{9} \mathrm{H}_{9} \mathrm{~N}, 132.0813$; found 132.0810 .

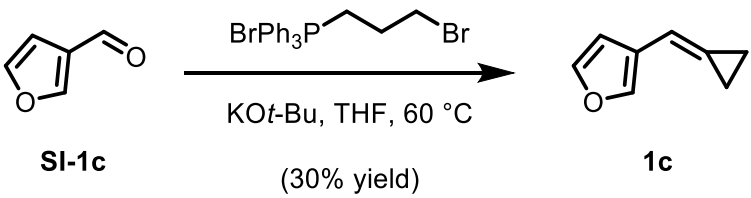

BCP substrate 1c. Purification by flash chromatography (hexanes) afforded BCP substrate 1c (88.3 mg, 30\% yield) as a colorless oil. 1c: ${ }^{1} \mathbf{H}$ NMR $\left(600 \mathrm{MHz}, \mathrm{CDCl}_{3}\right): \delta 7.47(\mathrm{~s}, 1 \mathrm{H}), 7.37(\mathrm{~s}$, $1 \mathrm{H}), 6.67(\mathrm{~s}, 1 \mathrm{H}), 6.63-6.59(\mathrm{~m}, 1 \mathrm{H}), 1.30-1.27(\mathrm{~m}, 2 \mathrm{H}), 1.21-1.18(\mathrm{~m}, 2 \mathrm{H}) ;{ }^{13} \mathrm{C}$ NMR $(150$ $\left.\mathrm{MHz}, \mathrm{CDCl}_{3}\right): \delta 143.30,139.56,124.90,123.38,108.63,108.58,3.40,1.76 ;$ HRMS-ESI $(\mathrm{m} / \mathrm{z})$ $[\mathrm{M}+\mathrm{H}]^{+}$calcd for $\mathrm{C}_{8} \mathrm{H}_{8} \mathrm{O}, 121.0653$; found 121.0657 .

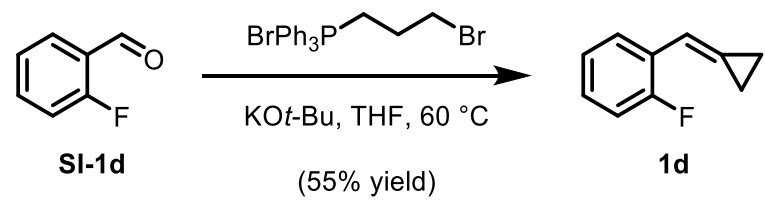

BCP substrate 1d. Purification by flash chromatography (hexanes) afforded BCP substrate 1d (204 mg, 55\% yield) as a colorless oil. 1d: ${ }^{1} \mathbf{H}$ NMR (600 MHz, $\left.\mathrm{CDCl}_{3}\right): \delta 7.74(\mathrm{td}, J=7.7,1.8$ $\mathrm{Hz}, 1 \mathrm{H}), 7.16$ (tdd, $J=7.4,5.1,1.8 \mathrm{~Hz}, 1 \mathrm{H}), 7.08$ (td, $J=7.5,1.2 \mathrm{~Hz}, 1 \mathrm{H}), 7.03$ (ddd, $J=10.6$, 8.1, $1.3 \mathrm{~Hz}, 1 \mathrm{H}), 6.98(\mathrm{p}, J=2.2 \mathrm{~Hz}, 1 \mathrm{H}), 1.43-1.38(\mathrm{~m}, 2 \mathrm{H}), 1.21-1.16(\mathrm{~m}, 2 \mathrm{H}) ;{ }^{13} \mathbf{C}$ NMR (150 MHz, $\left.\mathrm{CDCl}_{3}\right): \delta 159.89(\mathrm{~d}, J=248.3 \mathrm{~Hz}), 128.03$ (d, $\left.J=8.2 \mathrm{~Hz}\right), 127.24$ (d, $\left.J=3.9 \mathrm{~Hz}\right)$, $126.74(\mathrm{~d}, J=2.7 \mathrm{~Hz}), 125.90(\mathrm{~d}, J=12.2 \mathrm{~Hz}), 124.00(\mathrm{~d}, J=3.7 \mathrm{~Hz}), 115.61(\mathrm{~d}, J=22.0 \mathrm{~Hz})$, $110.39(\mathrm{~d}, J=5.8 \mathrm{~Hz}), 4.28,1.01$; HRMS-ESI $(\mathrm{m} / \mathrm{z})[\mathrm{M}+\mathrm{H}]^{+}$calcd for $\mathrm{C}_{10} \mathrm{H}_{9} \mathrm{~F}, 149.0767$; found 149.0761 . 


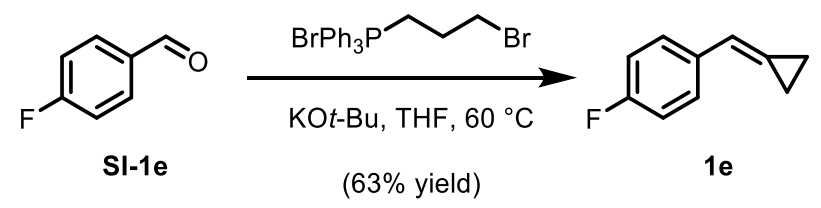

BCP substrate 1e. Purification by flash chromatography (hexanes) afforded BCP substrate 1e (233 mg, 63\% yield) as a colorless oil. 1e: ${ }^{1} \mathbf{H}$ NMR (600 MHz, $\left.\mathrm{CDCl}_{3}\right): \delta 7.46(\mathrm{dd}, J=8.8,5.6$ $\mathrm{Hz}, 2 \mathrm{H}), 7.02-6.96$ (m, 2H), 6.71-6.65 (m, 1H), 1.37 (ddd, $J=9.9,5.7,2.3 \mathrm{~Hz}, 2 \mathrm{H}), 1.18-1.11$ $(\mathrm{m}, 2 \mathrm{H}) ;{ }^{13} \mathrm{C}$ NMR $\left(150 \mathrm{MHz}, \mathrm{CDCl}_{3}\right): \delta 161.89(\mathrm{~d}, J=245.5 \mathrm{~Hz}), 134.53(\mathrm{~d}, J=3.3 \mathrm{~Hz})$, $128.06(\mathrm{~d}, J=7.8 \mathrm{~Hz}), 123.90(\mathrm{~d}, J=2.7 \mathrm{~Hz}), 117.26,115.39$ (d, $J=21.5 \mathrm{~Hz}), 4.14,0.69$; HRMS-ESI $(m / z)[\mathrm{M}+\mathrm{H}]^{+}$calcd for $\mathrm{C}_{10} \mathrm{H}_{9} \mathrm{~F}, 149.0767$; found 149.0766.

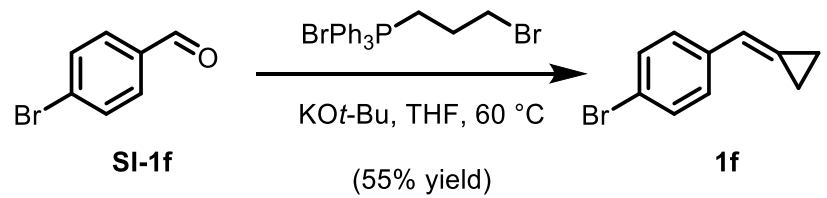

BCP substrate 1f. Purification by flash chromatography (99:1 hexanes:EtOAc) afforded BCP substrate 1f (287 mg, 55\% yield) as a colorless oil. 1f: ${ }^{1} \mathbf{H}$ NMR (600 $\left.\mathrm{MHz} \mathrm{CDCl}_{3}\right): \delta 7.46-7.40$ (m, 2H), 7.42-7.36 (m, 2H), 6.68 (p, J = 2.1 Hz, 1H), 1.39 (ddd, J=9.8, 5.7, 2.3 Hz, 2H), 1.17 $(\mathrm{ddd}, J=9.8,5.7,1.8 \mathrm{~Hz}, 2 \mathrm{H}) ;{ }^{13} \mathbf{C}$ NMR $\left(150 \mathrm{MHz}, \mathrm{CDCl}_{3}\right): \delta 137.16,131.51,128.09,125.38$, 120.34, 117.26, 4.19, 0.63.; HRMS-ESI $(\mathrm{m} / \mathrm{z})[\mathrm{M}+\mathrm{H}]^{+}$calcd for $\mathrm{C}_{10} \mathrm{H}_{9} \mathrm{Br}, 208.9966$; found 208.9958 .

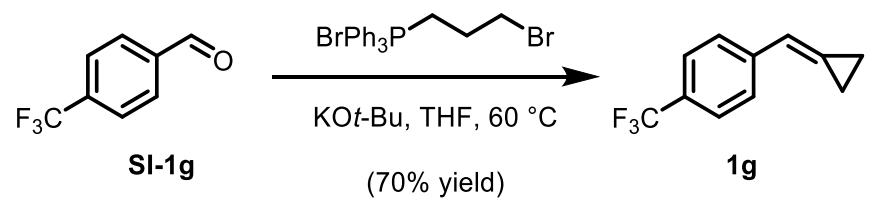

BCP substrate 1g. Purification by flash chromatography (hexanes) afforded BCP substrate $1 \mathrm{~g}$ (347 mg, 70\% yield) as a colorless oil. 1g: ${ }^{1} \mathbf{H}$ NMR $\left(600 \mathrm{MHz}, \mathrm{CDCl}_{3}\right): \delta 7.60(\mathrm{~d}, J=8.3 \mathrm{~Hz}$, 2H), $7.55(\mathrm{~d}, J=8.3 \mathrm{~Hz}, 2 \mathrm{H}), 6.79-6.75(\mathrm{~m}, 1 \mathrm{H}), 1.44$ (ddd, $J=10.1,5.9,2.2 \mathrm{~Hz}, 2 \mathrm{H}), 1.21$ (td, $J=7.8,1.8 \mathrm{~Hz}, 2 \mathrm{H}) ;{ }^{13} \mathbf{C} \mathbf{N M R}\left(150 \mathrm{MHz}, \mathrm{CDCl}_{3}\right): \delta 141.79(\mathrm{~d}, J=1.7 \mathrm{~Hz}), 128.59(\mathrm{q}, J=32.2$ $\mathrm{Hz}), 127.93,126.77,125.51$ (q, $J=3.8 \mathrm{~Hz}), 124.52$ (q, $J=271.8 \mathrm{~Hz}), 117.43,4.46,0.87$; HRMS-ESI $(m / z)[\mathrm{M}+\mathrm{H}]^{+}$calcd for $\mathrm{C}_{11} \mathrm{H}_{9} \mathrm{~F}_{3}, 199.0735$; found 199.0737. 


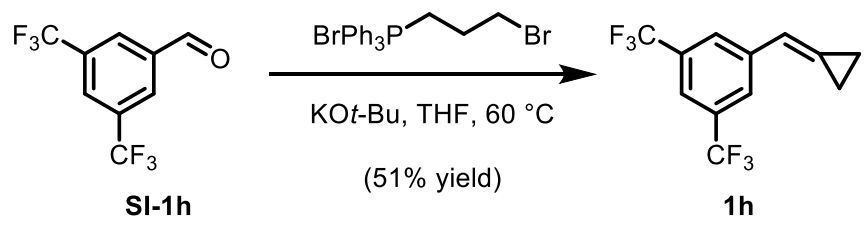

BCP substrate 1h. Purification by flash chromatography (hexanes) afforded BCP substrate $\mathbf{1 h}$ (339 mg, 51\% yield) as a colorless oil. 1h: ${ }^{1} \mathbf{H}$ NMR (600 MHz, $\left.\mathrm{CDCl}_{3}\right): \delta 7.92$ (s, 2H), 7.69 (s, $1 \mathrm{H}), 6.82(\mathrm{p}, J=2.1 \mathrm{~Hz}, 1 \mathrm{H}), 1.50(\mathrm{ddd}, J=8.2,7.0,2.3 \mathrm{~Hz}, 2 \mathrm{H}), 1.30-1.24(\mathrm{~m}, 2 \mathrm{H}) ;{ }^{13} \mathbf{C}$ NMR $\left(150 \mathrm{MHz}, \mathrm{CDCl}_{3}\right): \delta$ 140.35, 131.87 (q, $\left.J=33.0 \mathrm{~Hz}\right), 129.65,126.33$ (d, $\left.J=3.8 \mathrm{~Hz}\right), 123.62(\mathrm{q}$, $J=271.7 \mathrm{~Hz}), 120.15(\mathrm{~m}), 116.29,4.45,1.03$; HRMS-ESI $(m / z)[\mathrm{M}+\mathrm{H}]^{+}$calcd for $\mathrm{C}_{12} \mathrm{H}_{8} \mathrm{~F}_{6}$, 267.0608; found 267.0614 .

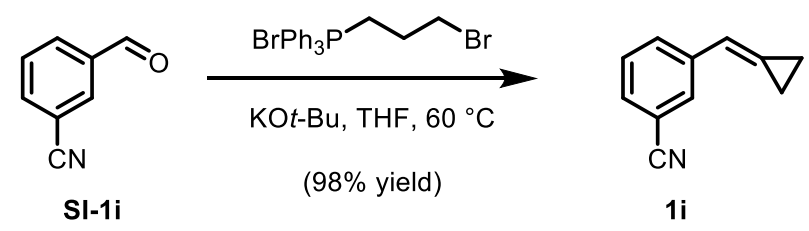

BCP substrate 1i. Purification by flash chromatography (98:2 hexanes:EtOAc) afforded BCP substrate 1i (380 mg, 98\% yield) as a colorless oil. 1i: ${ }^{1} \mathbf{H}$ NMR (600 $\left.\mathrm{MHz} \mathrm{CDCl}_{3}\right): \delta 7.81$ (s, 1H), $7.71(\mathrm{~d}, J=7.7 \mathrm{~Hz}, 1 \mathrm{H}), 7.48(\mathrm{~d}, J=7.7 \mathrm{~Hz}, 1 \mathrm{H}), 7.41(\mathrm{t}, J=7.7 \mathrm{~Hz}, 1 \mathrm{H}), 6.73$ (t, $J=2.1$ $\mathrm{Hz}, 1 \mathrm{H}), 1.46(\mathrm{ddd}, J=8.1,6.9,2.2 \mathrm{~Hz}, 2 \mathrm{H}), 1.25-1.21(\mathrm{~m}, 2 \mathrm{H}) ;{ }^{13} \mathbf{C}$ NMR $\left(150 \mathrm{MHz}, \mathrm{CDCl}_{3}\right)$ : $\delta 139.48,130.92,130.06,129.95,129.34,127.99,119.21,116.60,112.69,4.42,0.88$; HRMS$\operatorname{APCI}(\mathrm{m} / z)[\mathrm{M}+\mathrm{H}]^{+}$calcd for $\mathrm{C}_{11} \mathrm{H}_{9} \mathrm{~N}, 156.0813$; found 156.0809.

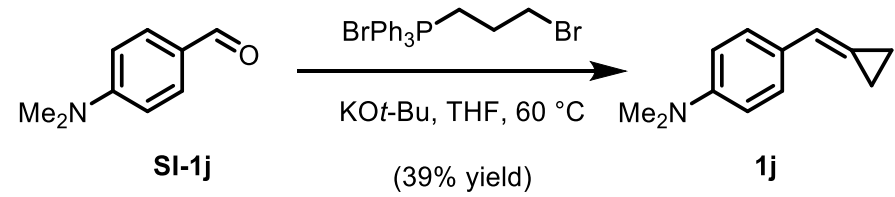

BCP substrate 1j. Purification by flash chromatography (95:5 hexanes:EtOAc) afforded BCP substrate 1j (169 mg, 39\% yield) as a colorless oil. 1j: ${ }^{\mathbf{1}} \mathbf{H}$ NMR (600 MHz, $\left.\mathrm{CDCl}_{3}\right): \delta 7.43(\mathrm{~d}, J$ $=8.7 \mathrm{~Hz}, 2 \mathrm{H}), 6.71(\mathrm{~d}, J=8.8 \mathrm{~Hz}, 2 \mathrm{H}), 6.66(\mathrm{t}, J=2.1 \mathrm{~Hz}, 1 \mathrm{H}), 2.95(\mathrm{~s}, 6 \mathrm{H}), 1.42-1.31(\mathrm{~m}, 2 \mathrm{H})$, $1.13(\mathrm{td}, J=7.2,1.8 \mathrm{~Hz}, 2 \mathrm{H}) ;{ }^{13} \mathbf{C} \mathbf{N M R}\left(150 \mathrm{MHz}, \mathrm{CDCl}_{3}\right): \delta 149.69,127.60,127.40,119.60$, 118.02, 112.77, 40.80, 4.11, 0.56; HRMS-ESI $(m / z)[\mathrm{M}+\mathrm{H}]^{+}$calcd for $\mathrm{C}_{12} \mathrm{H}_{15} \mathrm{~N}, 174.1283$; found 174.1285. 


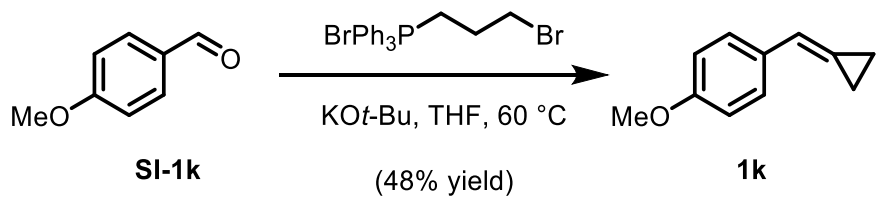

BCP substrate 1k. Purification by flash chromatography (98:2 hexanes:EtOAc) afforded BCP substrate 1k (192 mg, 48\% yield) as a colorless oil. 1k: ${ }^{1} \mathbf{H}$ NMR (600 MHz, $\left.\mathrm{CDCl}_{3}\right): \delta 7.46$ (d, $J=8.7 \mathrm{~Hz}, 2 \mathrm{H}), 6.87(\mathrm{~d}, J=8.8 \mathrm{~Hz}, 2 \mathrm{H}), 6.69$ (s, 1H), 3.80 (s, 3H), 1.38 (ddd, $J=9.7,5.5,2.3$ $\mathrm{Hz}, 2 \mathrm{H}), 1.15(\mathrm{ddd}, J=9.5,5.5,1.8 \mathrm{~Hz}, 2 \mathrm{H}) ;{ }^{13} \mathbf{C}$ NMR (150 MHz, $\left.\mathrm{CDCl}_{3}\right): \delta 158.64,131.35$, 127.80, 121.82, 117.69, 114.02, 55.41, 4.14, 0.63; HRMS-ESI $(m / z)[\mathrm{M}+\mathrm{H}]^{+}$calcd for $\mathrm{C}_{11} \mathrm{H}_{12} \mathrm{O}, 161.1966$; found 161.1965 .

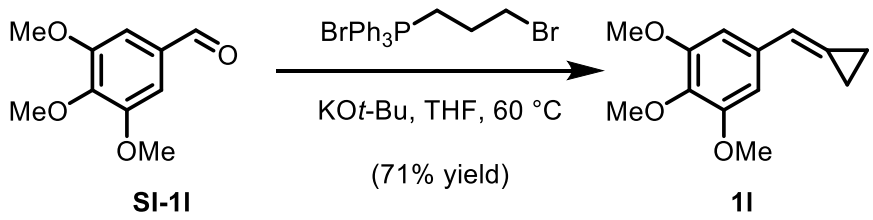

BCP substrate 11. Purification by flash chromatography (6:1 hexanes:EtOAc) afforded BCP substrate 11 (551 mg, 71\% yield) as a white solid. 1I: ${ }^{1} \mathbf{H}$ NMR $\left(600 \mathrm{MHz}, \mathrm{CDCl}_{3}\right): \delta 6.78$ (s, 2H), 6.67 (q, $J=1.9 \mathrm{~Hz}, 1 \mathrm{H}), 3.89$ (s, 6H), 3.85 (s, 3H), 1.42 (tt, $J=7.4,1.7 \mathrm{~Hz}, 2 \mathrm{H}), 1.19$ (tt, $J$ $=7.5,1.5 \mathrm{~Hz}, 2 \mathrm{H}) ;{ }^{13} \mathbf{C} \mathbf{N M R}\left(150 \mathrm{MHz}, \mathrm{CDCl}_{3}\right): \delta 153.41,137.32,134.22,123.83,118.29$, 103.79, 61.07, 56.17, 4.18, 0.73; HRMS-ESI $(\mathrm{m} / \mathrm{z})[\mathrm{M}+\mathrm{H}]^{+}$calcd for $\mathrm{C}_{13} \mathrm{H}_{16} \mathrm{O}_{3}, 221.1178$; found 221.1176 .

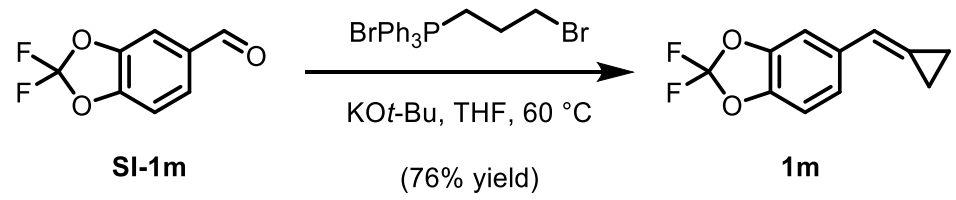

BCP substrate 1m. Purification by flash chromatography (98:2 hexanes:EtOAc) afforded BCP substrate 1m (399 mg, 76\% yield) as a white solid. 1m: ${ }^{1} \mathbf{H}$ NMR (600 MHz, $\left.\mathrm{CDCl}_{3}\right): \delta 7.32$ (d, $J=1.6 \mathrm{~Hz}, 1 \mathrm{H}), 7.13(\mathrm{dd}, J=8.3,1.6 \mathrm{~Hz}, 1 \mathrm{H}), 6.99$ (d, $J=8.3 \mathrm{~Hz}, 1 \mathrm{H}), 6.71-6.68(\mathrm{~m}, 1 \mathrm{H}), 1.40$ (ddd, $J=9.9,5.8,2.2 \mathrm{~Hz}, 2 \mathrm{H}), 1.22-1.17$ (m, 2H); ${ }^{13} \mathbf{C}$ NMR (150 MHz, $\left.\mathrm{CDCl}_{3}\right): \delta 144.31$, 142.60, 135.03, 131.77 (t, $J=254.5 \mathrm{~Hz}), 124.87,122.41,117.30,109.34,107.06,4.23,0.80$; HRMS-ESI $(\mathrm{m} / \mathrm{z})[\mathrm{M}+\mathrm{H}]^{+}$calcd for $\mathrm{C}_{11} \mathrm{H}_{8} \mathrm{~F}_{2} \mathrm{O}_{2}, 211.0571$; found 211.0566. 


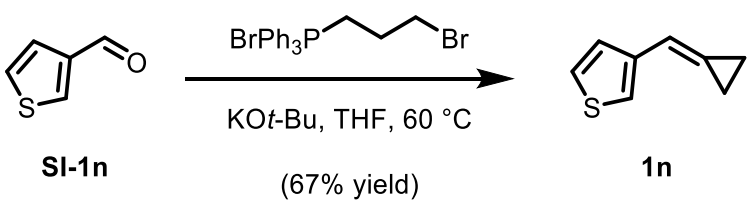

BCP substrate 1n. Purification by flash chromatography (hexanes) afforded BCP substrate 1n (227 mg, 67\% yield) as a colorless oil. 1n: ${ }^{1} \mathbf{H}$ NMR (600 MHz, $\left.\mathrm{CDCl}_{3}\right): \delta 7.43(\mathrm{~d}, J=5.1 \mathrm{~Hz}$, 1H), 7.32 (dd, $J=5.1,3.0 \mathrm{~Hz}, 1 \mathrm{H}), 7.24$ (s, 1H), 6.85 (s, 1H), 1.38 (ddd, $J=8.1,5.9,2.3 \mathrm{~Hz}$, $2 \mathrm{H}), 1.28(\mathrm{dt}, J=10.8,4.2 \mathrm{~Hz}, 2 \mathrm{H}) ;{ }^{13} \mathbf{C}$ NMR (150 MHz, $\left.\mathrm{CDCl}_{3}\right): \delta 140.78,126.01,125.64$, 123.71, 120.69, 113.16, 3.93, 1.82; HRMS-ESI $(\mathrm{m} / \mathrm{z})[\mathrm{M}+\mathrm{H}]^{+}$calcd for $\mathrm{C}_{8} \mathrm{H}_{8} \mathrm{~S}, 137.0475$; found 137.0479 .

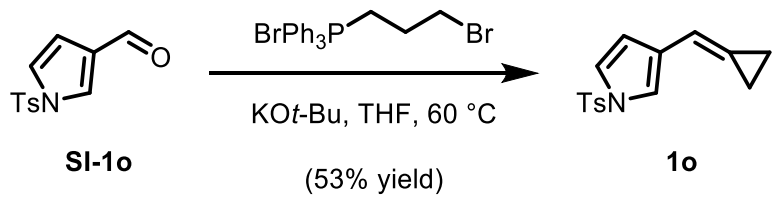

BCP substrate 10. Purification by flash chromatography (9:1 hexanes:EtOAc) afforded BCP substrate 10 (359 mg, 53\% yield) as a colorless oil. 1o: ${ }^{1} \mathbf{H}$ NMR $\left(600 \mathrm{MHz}, \mathrm{CDCl}_{3}\right): \delta 7.74(\mathrm{~d}, J$ $=8.4 \mathrm{~Hz}, 2 \mathrm{H}), 7.27(\mathrm{~d}, J=8.2 \mathrm{~Hz}, 2 \mathrm{H}), 7.19-7.06(\mathrm{~m}, 2 \mathrm{H}), 6.55$ (s, 2H), 2.39 (s, 3H), 1.27-1.20 (m, 2H), 1.20-1.13 (m, 2H); ${ }^{13} \mathbf{C}$ NMR (150 MHz, $\left.\mathrm{CDCl}_{3}\right): \delta 144.88,136.08,129.95,128.17$, $126.83,123.59,121.42,117.25,112.19,110.24,21.61,3.39,1.48 ;$ HRMS-ESI $(m / z)[M+H]^{+}$ calcd for $\mathrm{C}_{15} \mathrm{H}_{15} \mathrm{NO}_{2} \mathrm{~S}, 274.0902$; found 274.0905.

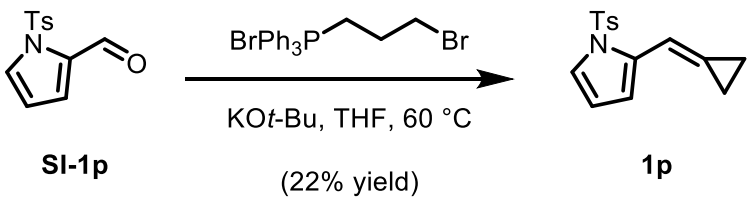

BCP substrate 1p. Purification by flash chromatography (9:1 hexanes:EtOAc) afforded BCP substrate 1p (152 mg, 22\% yield) as a colorless oil. 1p: ${ }^{1} \mathbf{H}$ NMR (600 MHz, $\left.\mathrm{CDCl}_{3}\right): \delta 7.72(\mathrm{~d}$, $J=8.1 \mathrm{~Hz}, 2 \mathrm{H}), 7.34-7.28(\mathrm{~m}, 3 \mathrm{H}), 7.19(\mathrm{~s}, 1 \mathrm{H}), 6.51(\mathrm{~s}, 1 \mathrm{H}), 6.28(\mathrm{t}, J=3.4 \mathrm{~Hz}, 1 \mathrm{H}), 2.41$ (s, $3 \mathrm{H}), 1.22(\mathrm{td}, J=7.5,7.1,3.7 \mathrm{~Hz}, 4 \mathrm{H}) ;{ }^{13} \mathbf{C} \mathbf{N M R}\left(150 \mathrm{MHz}, \mathrm{CDCl}_{3}\right): \delta 144.77,136.29,133.60$, $129.89,126.86,125.56,122.03,112.29,111.34,107.52,21.62,3.51,2.21 ;$ HRMS-ESI $(\mathrm{m} / \mathrm{z})[\mathrm{M}$ $+\mathrm{H}]^{+}$calcd for $\mathrm{C}_{15} \mathrm{H}_{15} \mathrm{NO}_{2} \mathrm{~S}, 274.0902$; found 274.0907. 


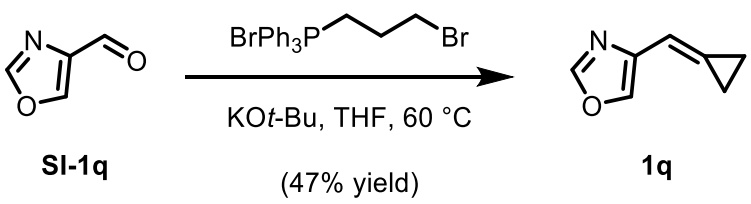

BCP substrate 1q. Purification by flash chromatography (4:1 hexanes: $\left.\mathrm{Et}_{2} \mathrm{O}\right)$ afforded $\mathrm{BCP}$ substrate 1q (143 mg, 47\% yield) as a colorless oil. 1q: ${ }^{1} \mathbf{H}$ NMR (600 MHz, $\left.\mathrm{CDCl}_{3}\right): \delta 7.86$ (s, $1 \mathrm{H}), 7.69(\mathrm{~s}, 1 \mathrm{H}), 6.65(\mathrm{~s}, 1 \mathrm{H}), 1.34(\mathrm{ddd}, J=9.4,5.7,1.8 \mathrm{~Hz}, 2 \mathrm{H}), 1.24(\mathrm{tt}, J=7.2,1.7 \mathrm{~Hz}, 2 \mathrm{H})$; ${ }^{13}$ C NMR $\left(150 \mathrm{MHz} \mathrm{CDCl}_{3}\right): \delta 151.10,139.17,134.37,127.05,108.19,3.85,2.17$; HRMS-ESI $(\mathrm{m} / \mathrm{z})[\mathrm{M}+\mathrm{H}]^{+}$calcd for $\mathrm{C}_{7} \mathrm{H}_{7} \mathrm{NO}, 122.0606$; found 122.0599 .

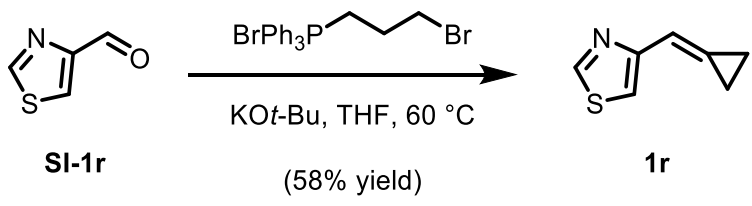

BCP substrate 1r. Purification by flash chromatography $\left(9: 1\right.$ hexanes:Et $\left.{ }_{2} \mathrm{O}\right)$ afforded $\mathrm{BCP}$ substrate 1r (199 mg, 58\% yield) as a colorless oil. 1r: ${ }^{1} \mathbf{H}$ NMR $\left(600 \mathrm{MHz}, \mathrm{CDCl}_{3}\right): \delta 8.79(\mathrm{~d}, J$ $=2.1 \mathrm{~Hz}, 1 \mathrm{H}), 7.29(\mathrm{~d}, J=2.0 \mathrm{~Hz}, 1 \mathrm{H}), 6.99(\mathrm{~s}, 1 \mathrm{H}), 1.41-1.38(\mathrm{~m}, 2 \mathrm{H}), 1.28(\mathrm{dd}, J=7.9,2.0$ $\mathrm{Hz}, 2 \mathrm{H}) .{ }^{13} \mathrm{C}$ NMR $\left(150 \mathrm{MHz}, \mathrm{CDCl}_{3}\right): \delta$ 155.61, 152.29, 127.33, 112.61, 112.57, 4.04, 2.07. HRMS-APCI $(\mathrm{m} / z)[\mathrm{M}+\mathrm{H}]^{+}$calcd for $\mathrm{C}_{7} \mathrm{H}_{7} \mathrm{NS}, 138.0377$; found 138.0378.

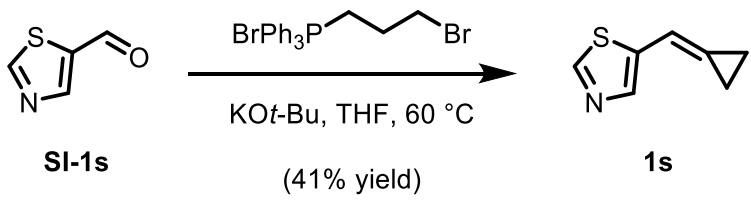

BCP substrate 1s. Purification by flash chromatography $\left(9: 1\right.$ hexanes:Et $\left.{ }_{2} \mathrm{O}\right)$ afforded $\mathrm{BCP}$ substrate 1s (141 mg, 41\% yield) as a colorless oil. 1s: ${ }^{1} \mathbf{H}$ NMR $\left(600 \mathrm{MHz}, \mathrm{CDCl}_{3}\right): \delta 8.62(\mathrm{~s}$, $1 \mathrm{H}), 7.76(\mathrm{~s}, 1 \mathrm{H}), 6.96(\mathrm{~s}, 1 \mathrm{H}), 1.36-1.33(\mathrm{~m}, 2 \mathrm{H}), 1.30(\mathrm{dt}, J=6.6,2.2 \mathrm{~Hz}, 2 \mathrm{H}) .{ }^{13} \mathbf{C}$ NMR $(150$ $\left.\mathrm{MHz}_{\mathrm{CDCl}}\right): \delta 151.37,139.87,138.61,128.00,109.29,4.15,3.62 ;$ HRMS-ESI $(\mathrm{m} / \mathrm{z})[\mathrm{M}+\mathrm{H}]^{+}$ calcd for $\mathrm{C}_{7} \mathrm{H}_{7} \mathrm{NS}, 138.0377$; found 138.0376 . 


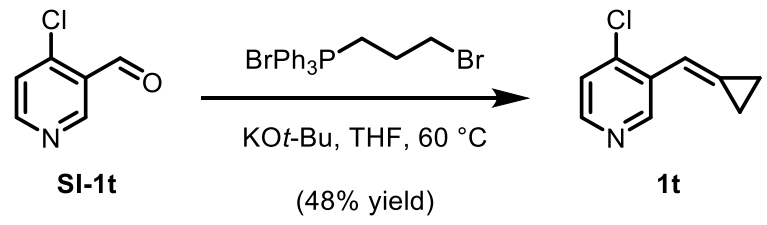

BCP substrate 1t. Purification by flash chromatography (6:1 hexanes:EtOAc) afforded BCP substrate 1t (197 mg, 48\% yield) as an orange oil. 1t: ${ }^{1} \mathbf{H}$ NMR (600 MHz, $\left.\mathrm{CDCl}_{3}\right): \delta 9.00$ (s, $1 \mathrm{H}), 8.31(\mathrm{~d}, J=5.4 \mathrm{~Hz}, 1 \mathrm{H}), 7.31(\mathrm{~d}, J=5.4 \mathrm{~Hz}, 1 \mathrm{H}), 7.08(\mathrm{~s}, 1 \mathrm{H}), 1.48$ (td, $J=8.2,2.2 \mathrm{~Hz}$, 2H), 1.31-1.26 (m, 2H); ${ }^{13} \mathbf{C}$ NMR (150 MHz, $\left.\mathrm{CDCl}_{3}\right): \delta 148.52,147.57,141.78,132.19$, 130.33, 124.72, 111.70, 4.45, 1.66; HRMS-ESI $(\mathrm{m} / \mathrm{z})[\mathrm{M}+\mathrm{H}]^{+}$calcd for $\mathrm{C}_{9} \mathrm{H}_{8} \mathrm{ClN}, 166.0424$; found 166.0423 .

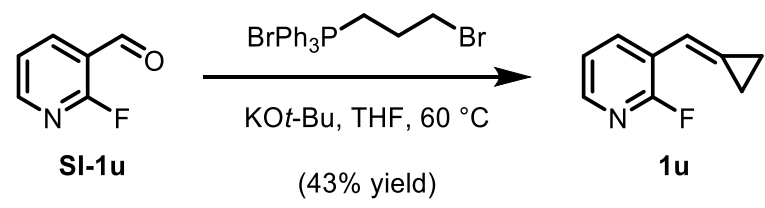

BCP substrate 1u. Purification by flash chromatography (9:1 hexanes:EtOAc) afforded BCP substrate 1u (159 mg, 43\% yield) as a colorless oil. 1u: ${ }^{1} \mathbf{H}$ NMR (600 MHz, $\left.\mathrm{CDCl}_{3}\right): \delta 8.11$ (ddd, $J=9.5,7.4,1.9 \mathrm{~Hz}, 1 \mathrm{H}), 8.04$ (d, $J=4.9 \mathrm{~Hz}, 1 \mathrm{H}), 7.15$ (ddd, $J=7.0,4.8,1.6 \mathrm{~Hz}, 1 \mathrm{H}), 6.88$ $(\mathrm{s}, 1 \mathrm{H}), 1.43(\mathrm{td}, J=8.0,2.2 \mathrm{~Hz}, 2 \mathrm{H}), 1.24(\mathrm{td}, J=7.9,1.8 \mathrm{~Hz}, 2 \mathrm{H}) ;{ }^{13} \mathbf{C}$ NMR $(150 \mathrm{MHz}$, $\left.\mathrm{CDCl}_{3}\right): \delta 160.37(\mathrm{~d}, J=240.9 \mathrm{~Hz}), 145.20(\mathrm{~d}, J=14.5 \mathrm{~Hz}), 136.94(\mathrm{~d}, J=5.0 \mathrm{~Hz}), 129.59$, $121.54(\mathrm{~d}, J=4.1 \mathrm{~Hz}), 120.83(\mathrm{~d}, J=26.9 \mathrm{~Hz}), 109.45,4.16,1.16$; HRMS-ESI $(\mathrm{m} / \mathrm{z})[\mathrm{M}+\mathrm{H}]^{+}$ calcd for $\mathrm{C}_{9} \mathrm{H}_{8} \mathrm{FN}, 150.0719$; found 150.0716.

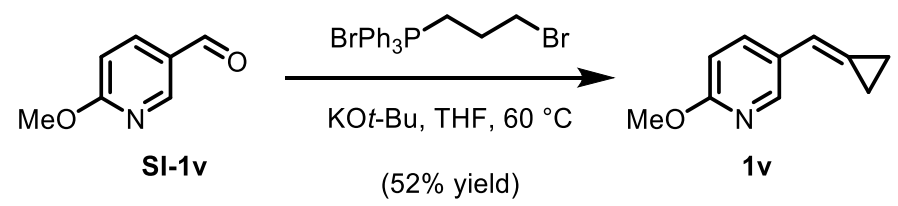

BCP substrate 1v. Purification by flash chromatography (4:1 hexanes:EtOAc) afforded BCP substrate 1v (211 mg, 52\% yield) as a colorless oil. 1v: ${ }^{1} \mathbf{H}$ NMR (600 MHz, $\left.\mathrm{CDCl}_{3}\right): \delta 8.23$ (s, 1H), 7.83 (dd, $J=8.6,2.5 \mathrm{~Hz}, 1 \mathrm{H}), 6.72$ (d, $J=8.6 \mathrm{~Hz}, 1 \mathrm{H}), 6.68$ (t, $J=2.1 \mathrm{~Hz}, 1 \mathrm{H}), 3.94$ (s, $3 \mathrm{H}), 1.38(\mathrm{ddd}, J=10.0,5.7,2.3 \mathrm{~Hz}, 2 \mathrm{H}), 1.18(\mathrm{ddd}, J=9.8,5.5,1.9 \mathrm{~Hz}, 2 \mathrm{H}) ;{ }^{13} \mathbf{C}$ NMR $(150$ $\left.\mathrm{MHz}, \mathrm{CDCl}_{3}\right): \delta 163.12,145.22,136.29,127.73,123.62,114.66,110.86,53.56,4.06,0.85$; HRMS-ESI $(\mathrm{m} / \mathrm{z})[\mathrm{M}+\mathrm{H}]^{+}$calcd for $\mathrm{C}_{10} \mathrm{H}_{11} \mathrm{NO}, 162.0919$; found 162.0921 . 


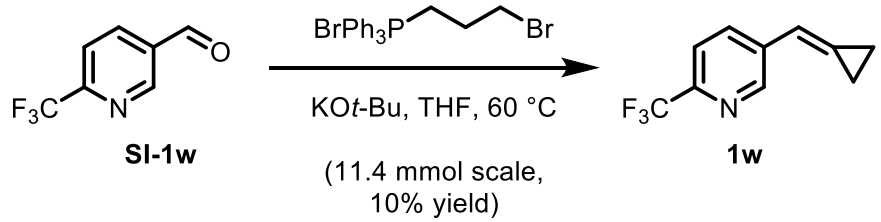

BCP substrate 1w. Purification by flash chromatography (80g of $\mathrm{SiO}_{2}$, Isco, $0-15 \%$ EtOAc/Heptanes) afforded BCP substrate $\mathbf{1 w}\left(227 \mathrm{mg}, 10 \%\right.$ yield) as a white solid. 1w: ${ }^{\mathbf{1}} \mathbf{H}$ NMR $\left(400 \mathrm{MHz}, \mathrm{CDCl}_{3}\right): \delta 8.81(\mathrm{~d}, J=2.1 \mathrm{~Hz}, 1 \mathrm{H}), 7.94(\mathrm{dd}, J=8.2,2.1 \mathrm{~Hz}, 1 \mathrm{H}), 7.60(\mathrm{~d}, J=$ $8.2 \mathrm{~Hz}, 1 \mathrm{H}), 6.78(\mathrm{~m}, 1 \mathrm{H}), 1.56-1.37(\mathrm{~m}, 2 \mathrm{H}), 1.37-1.17(\mathrm{~m}, 2 \mathrm{H}) ;{ }^{13} \mathbf{C} \mathbf{N M R}\left(100 \mathrm{MHz}, \mathrm{CDCl}_{3}\right)$ : $\delta 148.46,146.00(\mathrm{q}, J=34.6 \mathrm{~Hz}), 136.80,134.20,130.99,121.95$ (q, $J=273.6 \mathrm{~Hz}), 120.49$ (q, $J$ $=2.8 \mathrm{~Hz}), 114.36,4.54,1.34$; HRMS-ESI $(\mathrm{m} / \mathrm{z})[\mathrm{M}+\mathrm{H}]^{+}$calcd for $\mathrm{C}_{10} \mathrm{H}_{9} \mathrm{~F}_{3} \mathrm{~N}, 200.0682$; found 200.0678 .

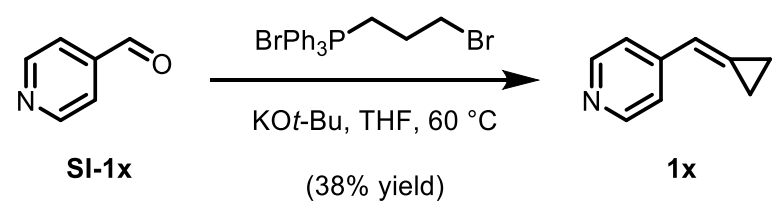

BCP substrate 1x. Purification by flash chromatography (4:1 hexanes:EtOAc) afforded BCP substrate 1x (125 mg, 38\% yield) as a colorless oil. 1x: ${ }^{1} \mathbf{H}$ NMR $\left(600 \mathrm{MHz}, \mathrm{CDCl}_{3}\right): \delta 8.53(\mathrm{~d}, J$ $=5.0 \mathrm{~Hz}, 2 \mathrm{H}), 7.38(\mathrm{~d}, J=4.6 \mathrm{~Hz}, 2 \mathrm{H}), 6.70(\mathrm{~s}, 1 \mathrm{H}), 1.48(\mathrm{td}, J=8.2,4.3 \mathrm{~Hz}, 2 \mathrm{H}), 1.25-1.21$ $(\mathrm{m}, 2 \mathrm{H}) ;{ }^{13} \mathbf{C}$ NMR $\left(150 \mathrm{MHz}, \mathrm{CDCl}_{3}\right): \delta 150.01,145.48,130.94,121.23,116.57,4.54,0.82$; HRMS-ESI $(\mathrm{m} / \mathrm{z})[\mathrm{M}+\mathrm{H}]^{+}$calcd for $\mathrm{C}_{9} \mathrm{H}_{9} \mathrm{~N}, 132.0813$; found 132.0817 .

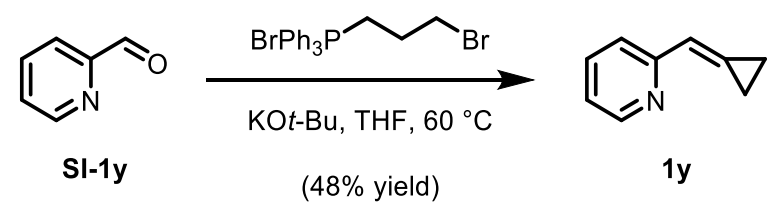

BCP substrate 1y. Purification by flash chromatography (4:1 hexanes:EtOAc) afforded BCP substrate 1y (157 mg, 48\% yield) as a colorless oil. 1y: ${ }^{\mathbf{1}} \mathbf{H} \mathbf{N M R}\left(600 \mathrm{MHz}, \mathrm{CDCl}_{3}\right): \delta 8.55$ $(\mathrm{ddd}, \mathrm{J}=4.9,1.9,1.0 \mathrm{~Hz}, 1 \mathrm{H}), 7.70$ (dt, $J=8.1,1.1 \mathrm{~Hz}, 1 \mathrm{H}), 7.63$ (td, $J=7.7,1.8 \mathrm{~Hz}, 1 \mathrm{H}), 7.10$ (ddd, $J=7.4,4.9,1.2 \mathrm{~Hz}, 1 \mathrm{H}), 6.96$ (p, $J=2.1 \mathrm{~Hz}, 1 \mathrm{H}), 1.49-1.43$ (m, 2H), 1.29-1.23 (m, 2H); ${ }^{13}$ C NMR (150 MHz, $\left.\mathrm{CDCl}_{3}\right): \delta 157.10,149.24,136.05,129.56,121.35,120.37,119.84,4.29$, 1.16; HRMS-ESI $(\mathrm{m} / \mathrm{z})[\mathrm{M}+\mathrm{H}]^{+}$calcd for $\mathrm{C}_{9} \mathrm{H}_{9} \mathrm{~N}, 132.0813$; found 132.0812. 


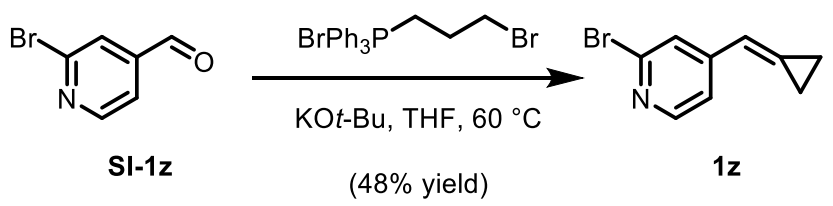

BCP substrate 1z. Purification by flash chromatography (9:1 hexanes:EtOAc) afforded BCP substrate 1z (157 mg, 48\% yield) as a colorless oil. 1z: ${ }^{1} \mathbf{H}$ NMR $\left(600 \mathrm{MHz}, \mathrm{CDCl}_{3}\right): \delta 8.27(\mathrm{~d}, J$ $=5.1 \mathrm{~Hz}, 1 \mathrm{H}), 7.55(\mathrm{~s}, 1 \mathrm{H}), 7.34(\mathrm{dd}, J=5.2,1.4 \mathrm{~Hz}, 1 \mathrm{H}), 6.66(\mathrm{p}, J=2.0 \mathrm{~Hz}, 1 \mathrm{H}), 1.50(\mathrm{ddd}, J$ $=8.5,7.3,2.2 \mathrm{~Hz}, 2 \mathrm{H}), 1.29-1.24(\mathrm{~m}, 2 \mathrm{H}) ;{ }^{13} \mathbf{C} \mathbf{N M R}\left(150 \mathrm{MHz}, \mathrm{CDCl}_{3}\right): \delta 150.19,148.42$, 142.83, 133.14, 125.21, 120.40, 115.51, 4.71, 1.12; HRMS-ESI $(m / z)[\mathrm{M}+\mathrm{H}]^{+}$calcd for $\mathrm{C}_{9} \mathrm{H}_{8} \mathrm{BrN}, 209.9918$; found 209.9918.

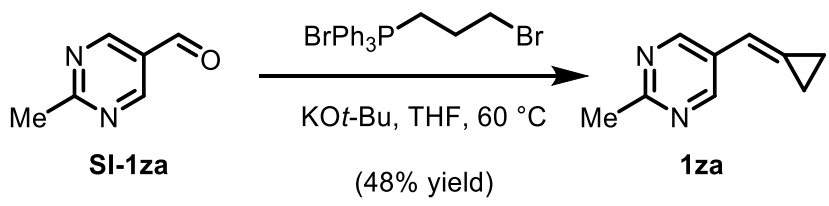

BCP substrate 1za. Reaction performed with $1.5 \mathrm{mmol}$ of aldehyde substrate due to limited material. Purification by flash chromatography (3:1 hexanes:EtOAc) afforded BCP substrate 1za (104 mg, 48\% yield) as a colorless oil. 1za: ${ }^{1} \mathbf{H}$ NMR $\left(600 \mathrm{MHz}, \mathrm{CDCl}_{3}\right): \delta 8.78(\mathrm{~s}, 2 \mathrm{H}), 6.67(\mathrm{t}$, $J=2.1 \mathrm{~Hz}, 1 \mathrm{H}), 2.74(\mathrm{~s}, 3 \mathrm{H}), 1.46(\mathrm{ddd}, J=8.2,7.0,2.3 \mathrm{~Hz}, 2 \mathrm{H}), 1.27$ (td, $J=7.8,1.7 \mathrm{~Hz}, 2 \mathrm{H})$; ${ }^{13} \mathbf{C}$ NMR $\left(150 \mathrm{MHz}, \mathrm{CDCl}_{3}\right): \delta 165.77,154.50,128.87,128.73,111.87,25.65,4.27,1.31$; HRMS-ESI $(m / z)[\mathrm{M}+\mathrm{H}]^{+}$calcd for $\mathrm{C}_{9} \mathrm{H}_{10} \mathrm{~N}_{2}, 147.0922$; found 147.0917.

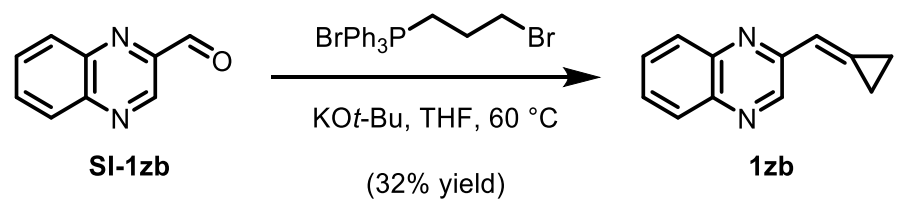

BCP substrate 1zb. Purification by flash chromatography (80g of $\mathrm{SiO}_{2}$, Isco, $0-33 \%$ EtOAc/Heptanes) afforded BCP substrate 1zb $\left(117 \mathrm{mg}, 32 \%\right.$ yield) as a brown solid. 1zb: ${ }^{1} \mathbf{H}$ NMR (400 MHz, $\left.\mathrm{CDCl}_{3}\right): \delta 9.28(\mathrm{~s}, 1 \mathrm{H}), 8.03-7.94(\mathrm{~m}, 2 \mathrm{H}), 7.71-7.58(\mathrm{~m}, 2 \mathrm{H}), 7.10(\mathrm{~m}, 1 \mathrm{H})$, $1.61-1.52$ (m, 2H), 1.38-1.29 (m, 2H); ${ }^{13} \mathbf{C}$ NMR (100 MHz, $\left.\mathrm{CDCl}_{3}\right): \delta 151.85,144.02,142.39$, 
$141.21,134.90,130.12,129.25,129.20,129.09,118.76,5.12,2.26$; HRMS-ESI $(m / z)[M+H]^{+}$ calcd for $\mathrm{C}_{12} \mathrm{H}_{10} \mathrm{~N}_{2}, 183.0917$; found 183.0915 .

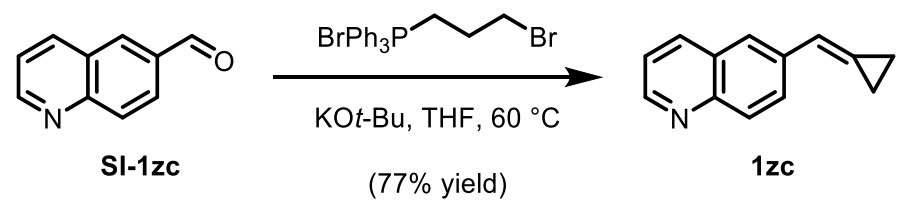

BCP substrate 1zc. Purification by flash chromatography (4:1 hexanes:EtOAc) afforded BCP substrate 1zc (284 mg, 77\% yield) as a colorless oil. 1zc: ${ }^{1} \mathbf{H}$ NMR (600 MHz, $\left.\mathrm{CDCl}_{3}\right): \delta 8.84(\mathrm{~d}$, $J=4.8 \mathrm{~Hz}, 1 \mathrm{H}), 8.12(\mathrm{~d}, J=8.3 \mathrm{~Hz}, 1 \mathrm{H}), 8.09-8.01(\mathrm{~m}, 2 \mathrm{H}), 7.77(\mathrm{~s}, 1 \mathrm{H}), 7.37$ (dd, $J=8.2,4.2$ $\mathrm{Hz}, 1 \mathrm{H}), 6.93(\mathrm{~s}, 1 \mathrm{H}), 1.54(\mathrm{td}, J=7.8,2.1 \mathrm{~Hz}, 2 \mathrm{H}), 1.27-1.23(\mathrm{~m}, 2 \mathrm{H}) ;{ }^{13} \mathbf{C} \mathbf{N M R}(150 \mathrm{MHz}$, $\left.\mathrm{CDCl}_{3}\right): \delta 149.89,147.79,136.72,136.01,129.56,128.69,128.27,126.64,125.27,121.41$, 117.96, 4.59, 0.90; HRMS-ESI $(m / z)[\mathrm{M}+\mathrm{H}]^{+}$calcd for $\mathrm{C}_{13} \mathrm{H}_{11} \mathrm{~N}, 182.0970$; found 182.0971.

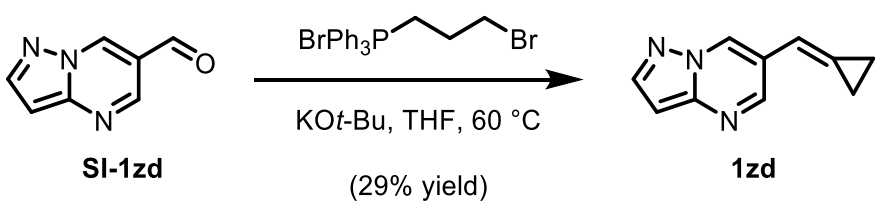

BCP substrate 1zd. Purification by flash chromatography (4:1 hexanes:EtOAc) afforded BCP substrate 1zd (108 mg, 29\% yield) as a colorless oil. 1zd: ${ }^{1} \mathbf{H}$ NMR (600 MHz, $\left.\mathrm{CDCl}_{3}\right): \delta 8.80$ $(\mathrm{d}, J=2.2 \mathrm{~Hz}, 1 \mathrm{H}), 8.71(\mathrm{~s}, 1 \mathrm{H}), 8.09(\mathrm{~d}, J=2.4 \mathrm{~Hz}, 1 \mathrm{H}), 6.72(\mathrm{~s}, 1 \mathrm{H}), 6.68(\mathrm{~d}, J=2.4 \mathrm{~Hz}, 1 \mathrm{H})$, $1.52-1.46(\mathrm{~m}, 2 \mathrm{H}), 1.29(\mathrm{td}, J=7.7,1.8 \mathrm{~Hz}, 2 \mathrm{H}) ;{ }^{13} \mathbf{C} \mathbf{N M R}\left(150 \mathrm{MHz}, \mathrm{CDCl}_{3}\right): \delta 149.17$, $147.61,145.12,131.21,127.36,120.17,111.62,97.06,4.44,1.55 ;$ HRMS-ESI $(m / z)[M+H]^{+}$ calcd for $\mathrm{C}_{10} \mathrm{H}_{9} \mathrm{~N}_{3}, 172.0875$; found 172.0876 .
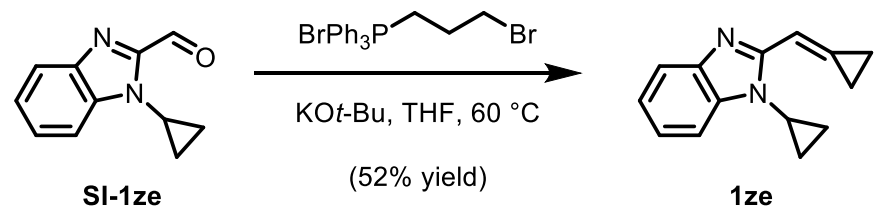

BCP substrate 1ze. Purification by flash chromatography (4:1 hexanes:EtOAc) afforded BCP substrate 1ze (272 mg, 52\% yield) as a colorless oil. 1ze: ${ }^{1} \mathbf{H}$ NMR $\left(600 \mathrm{MHz}, \mathrm{CDCl}_{3}\right): \delta 7.78-$ $7.72(\mathrm{~m}, 1 \mathrm{H}), 7.51(\mathrm{dd}, J=6.4,2.9 \mathrm{~Hz}, 1 \mathrm{H}), 7.25-7.20(\mathrm{~m}, 2 \mathrm{H}), 7.20-7.14(\mathrm{~m}, 1 \mathrm{H}), 3.30(\mathrm{tt}, J=$ $7.2,3.8 \mathrm{~Hz}, 1 \mathrm{H}), 1.67(\mathrm{td}, J=8.6,2.3 \mathrm{~Hz}, 2 \mathrm{H}), 1.33(\mathrm{td}, J=8.5,1.8 \mathrm{~Hz}, 2 \mathrm{H}), 1.25(\mathrm{dt}, J=7.0$, 
$3.4 \mathrm{~Hz}, 2 \mathrm{H}), 1.09$ (p, $J=4.9 \mathrm{~Hz}, 2 \mathrm{H}) ;{ }^{13} \mathbf{C}$ NMR (150 MHz, $\left.\mathrm{CDCl}_{3}\right): \delta 152.43,143.26,136.05$, 135.86, 122.34, 122.22, 119.78, 110.48, 107.29, 24.64, 7.63, 5.21, 2.41; HRMS-ESI $(\mathrm{m} / \mathrm{z})[\mathrm{M}+$ $\mathrm{H}]^{+}$calcd for $\mathrm{C}_{14} \mathrm{H}_{14} \mathrm{~N}_{2}, 211.1235$; found 211.1239 .

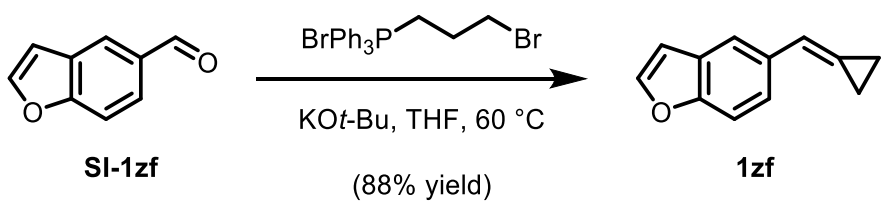

BCP substrate 1zf. Purification by flash chromatography (98:2 hexanes:EtOAc) afforded BCP substrate 1zf (374 mg, 88\% yield) as a white solid. 1zf: ${ }^{1} \mathbf{H}$ NMR (600 MHz, $\left.\mathrm{CDCl}_{3}\right): \delta 7.71$ (s, 1H), 7.59 (d, $J=2.2 \mathrm{~Hz}, 1 \mathrm{H}), 7.53(\mathrm{dd}, J=8.6,1.7 \mathrm{~Hz}, 1 \mathrm{H}), 7.45(\mathrm{~d}, J=8.5 \mathrm{~Hz}, 1 \mathrm{H}), 6.83(\mathrm{t}, J=$ $2.1 \mathrm{~Hz}, 1 \mathrm{H}), 6.75(\mathrm{~d}, J=1.3 \mathrm{~Hz}, 1 \mathrm{H}), 1.45(\mathrm{ddd}, J=9.8,5.6,2.3 \mathrm{~Hz}, 2 \mathrm{H}), 1.22-1.17$ (m, 2H); ${ }^{13}$ C NMR (150 MHz, $\left.\mathrm{CDCl}_{3}\right): \delta 154.30,145.39,133.55,127.79,123.40,122.80,119.15,118.43$, 111.38, 106.83, 4.35, 0.79; HRMS-ESI $(\mathrm{m} / \mathrm{z})[\mathrm{M}+\mathrm{H}]^{+}$calcd for $\mathrm{C}_{12} \mathrm{H}_{10} \mathrm{O}, 171.0810$; found 171.0808 . 


\section{B. Hydroboration Reactions.}

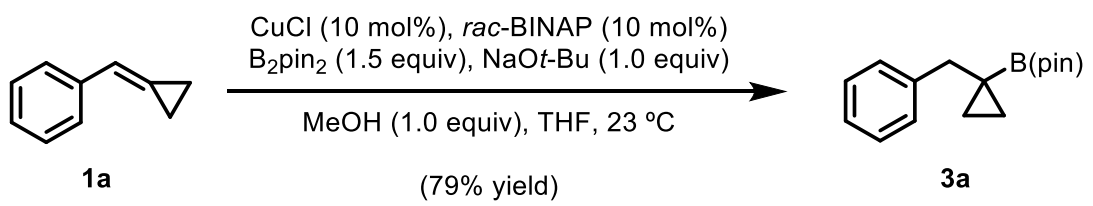

\section{Representative Procedure.}

Boronic ester 3a. A flame-dried 10-mL reaction vial equipped with a magnetic stir bar was charged with $\mathrm{CuCl}$ (2.0 mg, $0.02 \mathrm{mmol}, 0.1$ equiv), rac-BINAP (12.5 mg, $0.02 \mathrm{mmol}, 0.1$ equiv), $\mathrm{B}_{2} \mathrm{pin}_{2}$ (76.2 mg, $0.30 \mathrm{mmol}, 1.5$ equiv), and $\mathrm{NaO} t$-Bu (19.2 mg, $0.20 \mathrm{mmol}, 1.0$ equiv). The vial was capped, evacuated for $1 \mathrm{~min}$, and backfilled with $\mathrm{N}_{2}$. The solids were suspended in THF $(500 \mu \mathrm{L})$, and the reaction mixture was allowed to pre-stir for $10 \mathrm{~min}$ at room temperature. Next, $\mathrm{BCP}$ substrate 1a $(26.0 \mathrm{mg}, 0.20 \mathrm{mmol}, 1.0$ equiv) and $\mathrm{MeOH}(8.1 \mu \mathrm{L}, 0.20 \mathrm{mmol}, 1.0$ equiv) were added, and the reaction was allowed to stir at room temperature for 15-18 $\mathrm{h}$. The reaction mixture was then filtered through a pad of silica gel, which was subsequently rinsed with EtOAc $(15 \mathrm{~mL})$. The filtrate was concentrated, and the resulting crude mixture was purified by preparative thin layer chromatography (95:5 hexanes:EtOAc) to provide boronic ester 3a (40.7 mg, 79\% yield) as a colorless oil. 3a: ${ }^{1} \mathbf{H}$ NMR (600 MHz, $\left.\mathrm{CDCl}_{3}\right): \delta 7.31-7.25(\mathrm{~m}, 4 \mathrm{H}), 7.20-$ $7.15(\mathrm{~m}, 1 \mathrm{H}), 2.65(\mathrm{~s}, 2 \mathrm{H}), 1.19(\mathrm{~s}, 12 \mathrm{H}), 0.76(\mathrm{q}, J=3.6 \mathrm{~Hz}, 2 \mathrm{H}), 0.48(\mathrm{q}, J=3.6 \mathrm{~Hz}, 2 \mathrm{H}) ;{ }^{13} \mathbf{C}$ NMR (150 MHz, $\left.\mathrm{CDCl}_{3}\right): \delta 142.12,128.99,127.82,125.53,83.08,41.10,24.55,11.31$; HRMSESI $(m / z)[\mathrm{M}+\mathrm{OH}]^{-}$calcd for $\mathrm{C}_{16} \mathrm{H}_{23} \mathrm{BO}_{2}, 274.1855$; found 274.1854.

Note: We recommend using solid $\mathrm{NaO} t$ - $\mathrm{Bu}$ in this reaction. In our hands, $\mathrm{NaO} t$ - $\mathrm{Bu}$ stock solution in THF generally gave lower yields of the desired product and led to formation of unknown side products. The origin of this effect is unclear at the present time.

Any modifications from this representative procedure are specified in the following schemes. 
a)

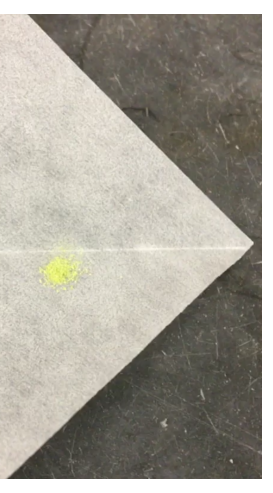

b)

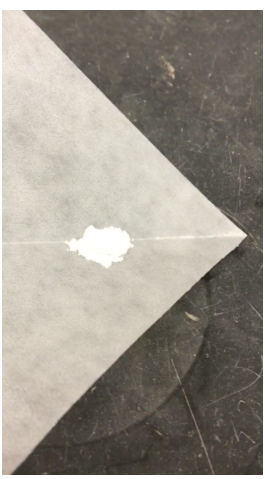

c)

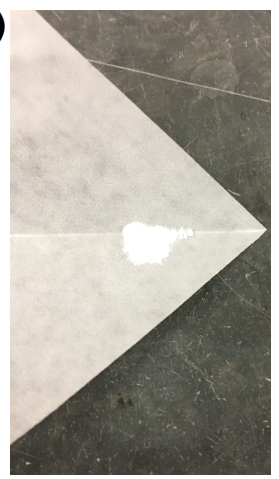

d)

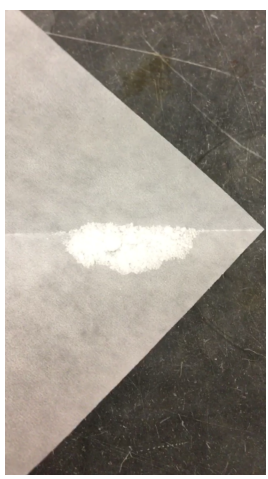

e)
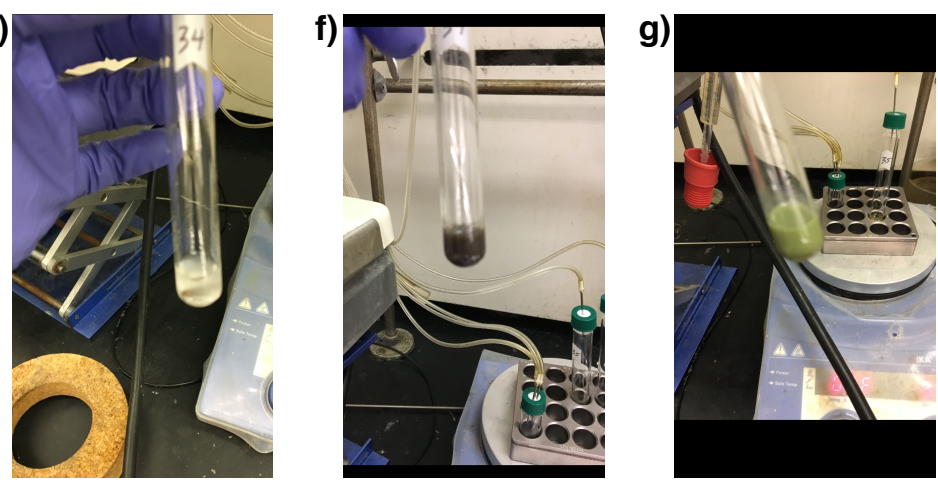

Figure S1. Picture of reagents and reaction mixture at different points of the reaction. a) $\mathrm{CuCl}, \mathrm{b}$ ) rac-BINAP, c) $\mathrm{NaO} t$-Bu, d) $\mathrm{B}_{2}$ pin 2 , e) reaction mixture after addition of THF before 10 min prestir, f) reaction mixture after 10 min pre-stir, g) reaction mixture after completion $(15-18 \mathrm{~h})$.

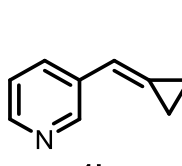

1b

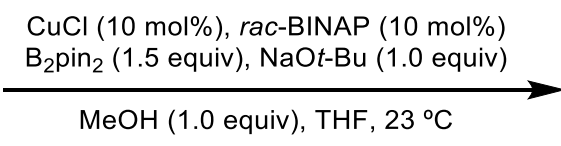

$(83 \%$ yield $)$

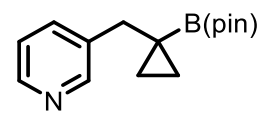

$3 \mathbf{b}$

Boronic ester 3b. Purification by preparative thin layer chromatography (4:1 hexanes:EtOAc) afforded boronic ester $3 \mathrm{~b}\left(43.3 \mathrm{mg}, 83 \%\right.$ yield) as a colorless oil. 3b: ${ }^{1} \mathbf{H}$ NMR (600 MHz, $\left.\mathrm{CDCl}_{3}\right): \delta 8.48(\mathrm{~d}, J=64.1 \mathrm{~Hz}, 2 \mathrm{H}), 7.63(\mathrm{~d}, J=7.8 \mathrm{~Hz}, 1 \mathrm{H}), 7.18(\mathrm{t}, J=6.1 \mathrm{~Hz}, 1 \mathrm{H}), 2.58(\mathrm{~s}$, $2 \mathrm{H}), 1.14(\mathrm{~d}, J=2.6 \mathrm{~Hz}, 12 \mathrm{H}), 0.78(\mathrm{t}, J=2.9 \mathrm{~Hz}, 2 \mathrm{H}), 0.47(\mathrm{t}, J=3.0 \mathrm{~Hz}, 2 \mathrm{H}) ;{ }^{13} \mathbf{C}$ NMR $(150$ $\left.\mathrm{MHz}, \mathrm{CDCl}_{3}\right): \delta 150.47,147.15,137.73,136.43,123.03,83.37,38.74,24.67,11.75$; HRMSESI $(m / z)[\mathrm{M}+\mathrm{H}]^{+}$calcd for $\mathrm{C}_{15} \mathrm{H}_{22} \mathrm{BNO}_{2}, 259.1858$; found 259.1863. 


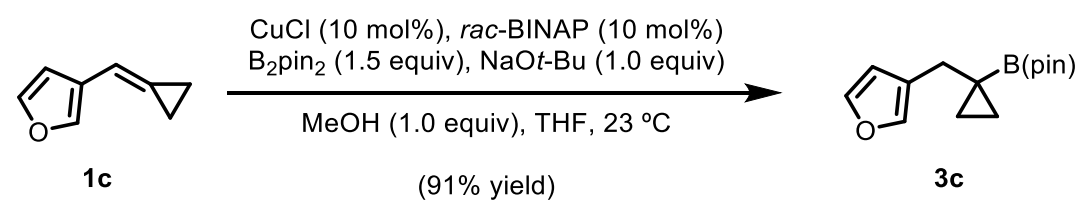

Boronic ester 3c. Purification by preparative thin layer chromatography ( $9: 1$ hexanes:EtOAc) afforded boronic ester 3c (45.1 mg, 91\% yield) as a colorless oil. 3c: ${ }^{1} \mathbf{H}$ NMR (600 MHz, $\left.\mathrm{CDCl}_{3}\right): \delta 7.30(\mathrm{t}, J=1.7 \mathrm{~Hz}, 1 \mathrm{H}), 7.23(\mathrm{t}, J=1.3 \mathrm{~Hz}, 1 \mathrm{H}), 6.35(\mathrm{~s}, 1 \mathrm{H}), 2.36(\mathrm{~s}, 2 \mathrm{H}), 1.18(\mathrm{~s}$, $12 \mathrm{H}), 0.71$ (q, $J=3.6 \mathrm{~Hz}, 2 \mathrm{H}), 0.40$ (q, $J=3.6 \mathrm{~Hz}, 2 \mathrm{H}) ;{ }^{13} \mathbf{C} \mathbf{N M R}\left(150 \mathrm{MHz}, \mathrm{CDCl}_{3}\right): \delta 142.23$, 139.43, 124.97, 111.85, 83.24, 30.90, 24.74, 11.52; HRMS-ESI $(\mathrm{m} / \mathrm{z})[\mathrm{M}+\mathrm{H}]^{+}$calcd for $\mathrm{C}_{14} \mathrm{H}_{21} \mathrm{BO}_{3}, 248.1698$; found 248.1702 .

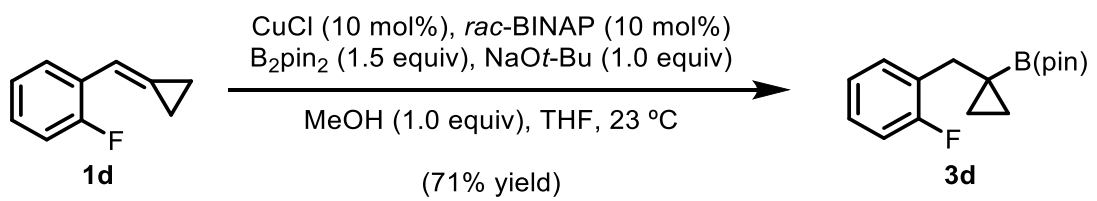

Boronic ester 3d. Purification by preparative thin layer chromatography (95:5 hexanes:EtOAc) afforded boronic ester 3d (39.2 mg, 71\% yield) as a colorless oil. 3d: ${ }^{1} \mathbf{H}$ NMR (600 MHz, $\left.\mathrm{CDCl}_{3}\right): \delta 7.41(\mathrm{td}, J=7.7,1.7 \mathrm{~Hz}, 1 \mathrm{H}), 7.13(\mathrm{tdd}, J=7.4,5.1,1.8 \mathrm{~Hz}, 1 \mathrm{H}), 7.03(\mathrm{td}, J=7.5,1.2$ Hz, 1H), 6.96 (ddd, $J=9.6,8.1,1.2 \mathrm{~Hz}, 1 \mathrm{H}), 2.68$ (s, 2H), 1.17 (s, 12H), 0.74 (q, $J=3.6 \mathrm{~Hz}$, 2H), 0.47 (q, $J=3.6 \mathrm{~Hz}, 2 \mathrm{H}) ;{ }^{13} \mathbf{C}$ NMR $\left(150 \mathrm{MHz}, \mathrm{CDCl}_{3}\right): \delta 161.29$ (d, $\left.J=244.4 \mathrm{~Hz}\right), 131.14$ (d, $J=5.0 \mathrm{~Hz}), 128.86$ (d, $J=16.0 \mathrm{~Hz}), 127.24$ (d, $J=7.8 \mathrm{~Hz}), 123.51$ (d, $J=3.4 \mathrm{~Hz}), 114.89$ (d, $J=22.7 \mathrm{~Hz}), 83.28,33.34,24.69,11.38$; HRMS-ESI $(\mathrm{m} / \mathrm{z})[\mathrm{M}+\mathrm{OH}]^{-}$calcd for $\mathrm{C}_{16} \mathrm{H}_{22} \mathrm{BFO}_{2}$, 292.1761 ; found 292.1766 .

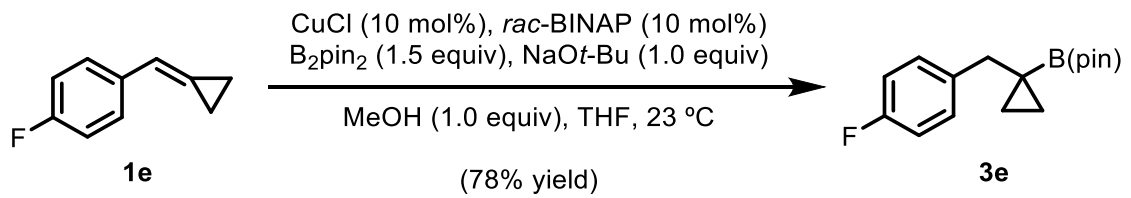

Boronic ester 3e. Purification by preparative thin layer chromatography (95:5 hexanes:EtOAc) afforded boronic ester 3e (43.1 mg, 78\% yield) as a colorless oil. 3e: ${ }^{1} \mathbf{H}$ NMR $(600 \mathrm{MHz}$, $\mathrm{CDCl}_{3}$ ): $\delta 7.25-7.20(\mathrm{~m}, 2 \mathrm{H}), 6.96-6.89(\mathrm{~m}, 2 \mathrm{H}), 2.57$ (s, 2H), 1.15 (s, 12H), 0.74 (q, $J=3.6 \mathrm{~Hz}$, 2H), 0.44 (q, $J=3.6 \mathrm{~Hz}, 2 \mathrm{H}) ;{ }^{13} \mathbf{C}$ NMR $\left(150 \mathrm{MHz}, \mathrm{CDCl}_{3}\right): \delta 161.37$ (d, $\left.J=241.7 \mathrm{~Hz}\right), 137.96$ $(\mathrm{d}, J=3.3 \mathrm{~Hz}), 130.36$ (d, $J=7.8 \mathrm{~Hz}), 114.60$ (d, $J=21.0 \mathrm{~Hz}), 83.27,40.54,24.69,11.47$; HRMS-ESI $(m / z)[\mathrm{M}+\mathrm{H}]^{+}$calcd for $\mathrm{C}_{16} \mathrm{H}_{22} \mathrm{BFO}_{2}, 277.1775$; found 277.1774. 


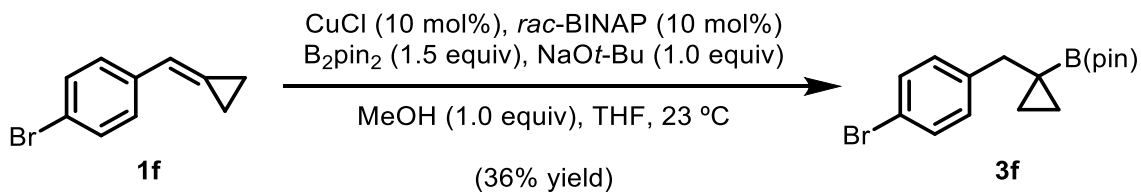

Boronic ester 3f. Purification by preparative thin layer chromatography (95:5 hexanes:EtOAc) afforded boronic ester $3 \mathbf{f f}\left(24.5 \mathrm{mg}, 36 \%\right.$ yield) as a colorless oil. 3f: ${ }^{1} \mathbf{H}$ NMR (600 MHz, $\left.\mathrm{CDCl}_{3}\right): \delta 7.38-7.33(\mathrm{~m}, 2 \mathrm{H}), 7.17-7.13(\mathrm{~m}, 2 \mathrm{H}), 2.55(\mathrm{~s}, 2 \mathrm{H}), 1.15(\mathrm{~s}, 12 \mathrm{H}), 0.76-0.72(\mathrm{~m}, 2 \mathrm{H})$, 0.46-0.42 (m, 2H).; ${ }^{13} \mathbf{C}$ NMR (150 MHz, $\left.\mathrm{CDCl}_{3}\right): \delta 141.24,130.84,130.72,119.32,83.18$, 40.64, 24.56, 11.43; HRMS-ESI $(\mathrm{m} / \mathrm{z})[\mathrm{M}+\mathrm{OH}]^{-}$calcd for $\mathrm{C}_{16} \mathrm{H}_{22} \mathrm{BBrO}_{2}, 352.0960$; found 352.0964 .

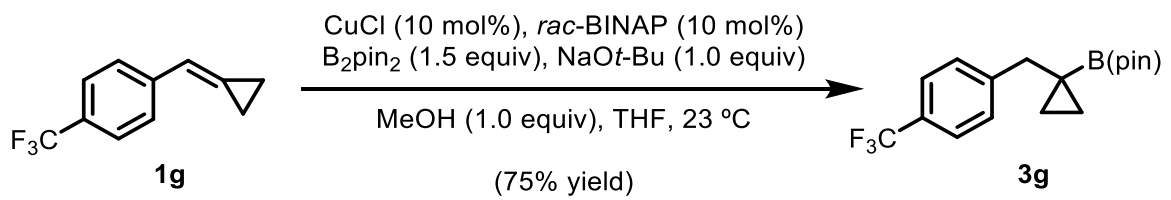

Boronic ester 3g. Purification by preparative thin layer chromatography (95:5 hexanes:EtOAc) afforded boronic ester $3 \mathrm{~g}$ (48.7 mg, 75\% yield) as a colorless oil. 3g: ${ }^{1} \mathbf{H}$ NMR (600 MHz, $\left.\mathrm{CDCl}_{3}\right): \delta 7.49(\mathrm{~d}, J=8.0 \mathrm{~Hz}, 2 \mathrm{H}), 7.39(\mathrm{~d}, J=8.0 \mathrm{~Hz}, 2 \mathrm{H}), 2.65(\mathrm{~s}, 2 \mathrm{H}), 1.15(\mathrm{~s}, 12 \mathrm{H}), 0.78(\mathrm{q}$, $J=3.6 \mathrm{~Hz}, 2 \mathrm{H}), 0.46(\mathrm{q}, J=3.6 \mathrm{~Hz}, 2 \mathrm{H}) ;{ }^{13} \mathbf{C} \mathbf{N M R}\left(150 \mathrm{MHz}, \mathrm{CDCl}_{3}\right): \delta 146.61,129.29$, $128.04(\mathrm{q}, J=31.8 \mathrm{~Hz}), 124.87,124.85,83.39,41.28,24.68,11.73$; HRMS-ESI $(\mathrm{m} / z)[\mathrm{M}+$ $\mathrm{OH}]^{-}$calcd for $\mathrm{C}_{17} \mathrm{H}_{22} \mathrm{BF}_{3} \mathrm{O}_{2}, 342.1729$; found 342.1737 .

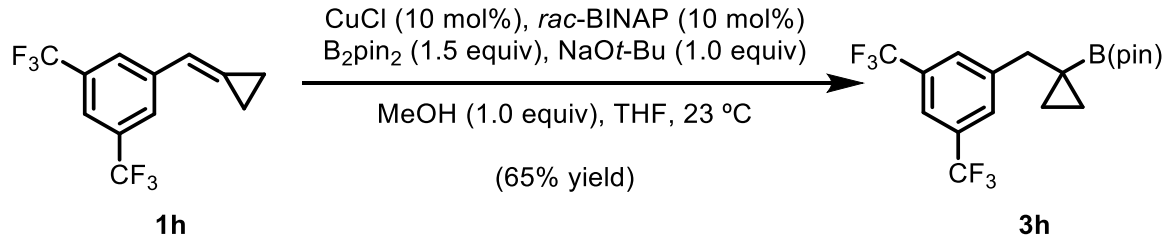

Boronic ester 3h. Purification by preparative thin layer chromatography (95:5 hexanes:EtOAc) afforded boronic ester $3 \mathbf{h}\left(51.6 \mathrm{mg}, 65 \%\right.$ yield) as a colorless oil. $3 \mathbf{h}$ : ${ }^{1} \mathbf{H}$ NMR (600 MHz, $\left.\mathrm{CDCl}_{3}\right): \delta 7.77(\mathrm{~s}, 2 \mathrm{H}), 7.69(\mathrm{~s}, 1 \mathrm{H}), 2.67(\mathrm{~s}, 2 \mathrm{H}), 1.15(\mathrm{~s}, 12 \mathrm{H}), 0.82(\mathrm{q}, J=3.9 \mathrm{~Hz}, 2 \mathrm{H}), 0.50$ $(\mathrm{q}, J=3.9 \mathrm{~Hz}, 2 \mathrm{H}) ;{ }^{13} \mathbf{C} \mathbf{N M R}\left(150 \mathrm{MHz}, \mathrm{CDCl}_{3}\right): \delta 145.11,131.09$ (q, $\left.J=32.7 \mathrm{~Hz}\right), 129.30$ (d, $J=3.9 \mathrm{~Hz}), 123.77(\mathrm{q}, J=272.6 \mathrm{~Hz}), 119.78$ (p, $J=4.0 \mathrm{~Hz}), 83.61,41.58,24.65,12.18$; HRMS-ESI $(m / z)[\mathrm{M}+\mathrm{OH}]^{-}$calcd for $\mathrm{C}_{18} \mathrm{H}_{21} \mathrm{BF}_{6} \mathrm{O}_{2}, 410.1603$; found 410.1601 . 


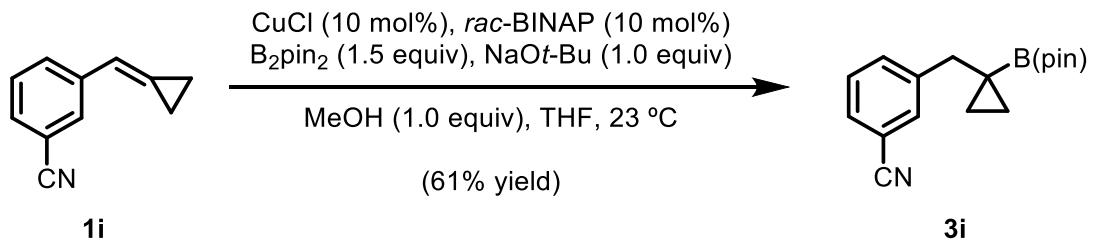

Boronic ester 3i. Purification by preparative thin layer chromatography (9:1 hexanes:EtOAc) afforded boronic ester 3i (34.5 mg, 61\% yield) as a colorless oil. 3i: ${ }^{1} \mathbf{H}$ NMR (600 MHz, $\left.\mathrm{CDCl}_{3}\right): \delta 7.65(\mathrm{~s}, 1 \mathrm{H}), 7.48(\mathrm{dd}, J=22.2,8.3 \mathrm{~Hz}, 2 \mathrm{H}), 7.33(\mathrm{t}, J=7.7 \mathrm{~Hz}, 1 \mathrm{H}), 2.60(\mathrm{~s}, 2 \mathrm{H})$, 1.15 (s, 12H), 0.79 (q, $J=3.7 \mathrm{~Hz}, 2 \mathrm{H}), 0.47$ (q, $J=3.7 \mathrm{~Hz}, 2 \mathrm{H}) ;{ }^{13} \mathbf{C}$ NMR $\left(150 \mathrm{MHz}, \mathrm{CDCl}_{3}\right): \delta$ 143.97, 133.65, 132.69, 129.52, 128.70, 119.43, 111.89, 83.46, 41.20, 24.67, 11.84; HRMS-ESI $(\mathrm{m} / \mathrm{z})[\mathrm{M}+\mathrm{H}]^{+}$calcd for $\mathrm{C}_{17} \mathrm{H}_{22} \mathrm{BNO}_{2}, 283.1858$; found 283.1858 .

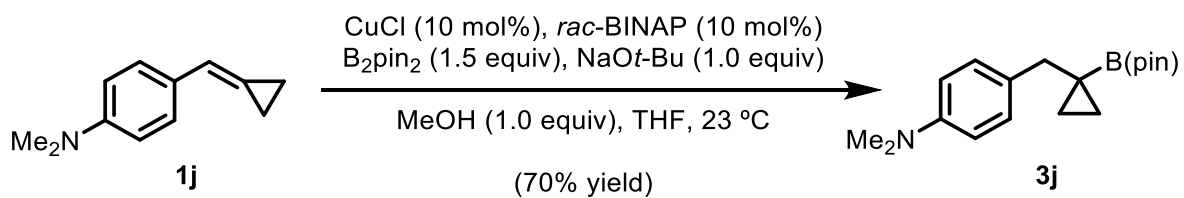

Boronic ester 3j. Purification by preparative thin layer chromatography (9:1 hexanes:EtOAc) afforded boronic ester 3j (42.2 mg, 70\% yield) as a colorless oil. 3j: ${ }^{\mathbf{1}} \mathbf{H}$ NMR (600 MHz, $\left.\mathrm{CDCl}_{3}\right): \delta 7.13(\mathrm{~d}, J=8.2 \mathrm{~Hz}, 2 \mathrm{H}), 6.66(\mathrm{~d}, J=8.3 \mathrm{~Hz}, 2 \mathrm{H}), 2.89$ (s, 6H), 2.54 (s, 2H), 1.17 (s, $12 \mathrm{H}), 0.68$ (q, $J=3.7 \mathrm{~Hz}, 2 \mathrm{H}), 0.40$ (q, $J=3.7 \mathrm{~Hz}, 2 \mathrm{H}) ;{ }^{13} \mathbf{C}$ NMR $\left(150 \mathrm{MHz}, \mathrm{CDCl}_{3}\right): \delta 149.08$, 130.55, 129.79, 112.87, 83.12, 41.16, 39.98, 24.74, 11.15; HRMS-ESI $(m / z)[\mathrm{M}+\mathrm{H}]^{+}$calcd for $\mathrm{C}_{18} \mathrm{H}_{28} \mathrm{BNO}_{2}, 301.2328$; found 301.2331.

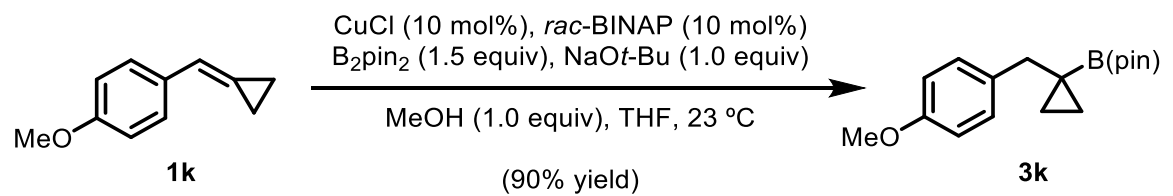

Boronic ester 3k. Purification by preparative thin layer chromatography (18:1:1 hexanes:EtOAc:PhH) afforded boronic ester 3k (51.8 mg, 90\% yield) as a colorless oil. 3k: ${ }^{\mathbf{1}} \mathbf{H}$ NMR (600 MHz, $\left.\mathrm{CDCl}_{3}\right): \delta 7.18(\mathrm{~d}, J=8.6 \mathrm{~Hz}, 2 \mathrm{H}), 6.79(\mathrm{~d}, J=8.6 \mathrm{~Hz}, 2 \mathrm{H}), 3.77(\mathrm{~s}, 3 \mathrm{H}), 2.56$ $(\mathrm{s}, 2 \mathrm{H}), 1.16(\mathrm{~s}, 12 \mathrm{H}), 0.71(\mathrm{q}, J=3.6 \mathrm{~Hz}, 2 \mathrm{H}), 0.42$ (q, $J=3.6 \mathrm{~Hz}, 2 \mathrm{H}) ;{ }^{13} \mathrm{C}$ NMR $(150 \mathrm{MHz}$, $\left.\mathrm{CDCl}_{3}\right): \delta 157.74,134.37,130.01,113.36,83.17,55.34,40.27,24.70,11.29 ;$ HRMS-ESI $(\mathrm{m} / z)$ $[\mathrm{M}+\mathrm{H}]^{+}$calcd for $\mathrm{C}_{17} \mathrm{H}_{25} \mathrm{BO}_{3}, 288.2011$; found 288.2008. 


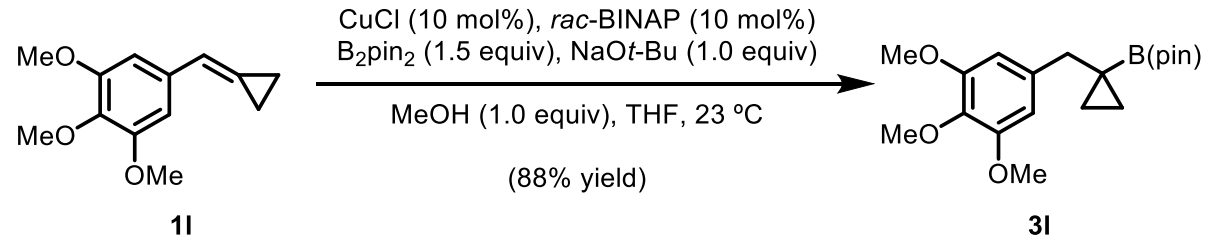

Boronic ester 31. Purification by preparative thin layer chromatography (3:1 hexanes:EtOAc) afforded boronic ester $\mathbf{3 l}$ (61.3 mg, 88\% yield) as a white solid. 3I: ${ }^{1} \mathbf{H}$ NMR (600 MHz, $\left.\mathrm{CDCl}_{3}\right)$ : $\delta 6.51(\mathrm{~s}, 2 \mathrm{H}), 3.85(\mathrm{~s}, 6 \mathrm{H}), 3.82(\mathrm{~s}, 3 \mathrm{H}), 2.56(\mathrm{~s}, 2 \mathrm{H}), 1.17(\mathrm{~s}, 12 \mathrm{H}), 0.76(\mathrm{q}, J=3.5 \mathrm{~Hz}, 2 \mathrm{H})$, 0.46 (q, $J=3.6 \mathrm{~Hz}, 2 \mathrm{H}) ;{ }^{13} \mathbf{C}$ NMR $\left(150 \mathrm{MHz}, \mathrm{CDCl}_{3}\right): \delta 152.76,138.15,136.04,106.04,83.18$, 60.94, 56.09, 41.65, 24.76, 11.71; HRMS-ESI $(\mathrm{m} / \mathrm{z})[\mathrm{M}+\mathrm{H}]^{+}$calcd for $\mathrm{C}_{19} \mathrm{H}_{29} \mathrm{BO}_{5}, 348.2223$; found 348.2215 .

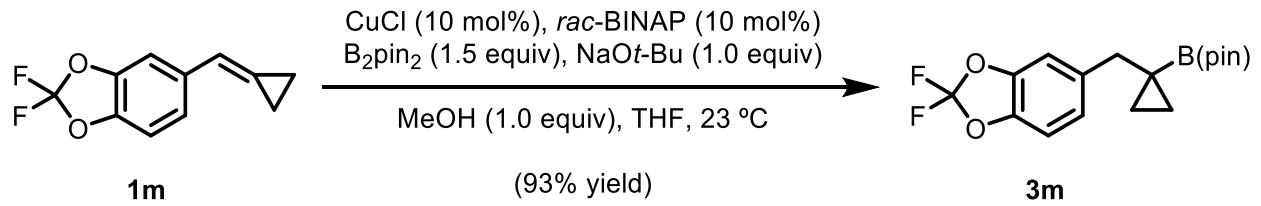

Boronic ester 3m. Purification by preparative thin layer chromatography (9:1 hexanes:EtOAc) afforded boronic ester $\mathbf{3 m}$ (62.9 mg, 93\% yield) as a white solid. 3m: ${ }^{1} \mathbf{H}$ NMR (600 MHz, $\left.\mathrm{CDCl}_{3}\right): \delta 7.07(\mathrm{~d}, J=1.6 \mathrm{~Hz}, 1 \mathrm{H}), 6.94(\mathrm{dd}, J=8.2,1.6 \mathrm{~Hz}, 1 \mathrm{H}), 6.90(\mathrm{~d}, J=8.2 \mathrm{~Hz}, 1 \mathrm{H}), 2.57$ $(\mathrm{s}, 2 \mathrm{H}), 1.16(\mathrm{~s}, 12 \mathrm{H}), 0.76(\mathrm{q}, J=3.6 \mathrm{~Hz}, 2 \mathrm{H}), 0.44(\mathrm{q}, J=3.6 \mathrm{~Hz}, 2 \mathrm{H}) ;{ }^{13} \mathbf{C} \mathbf{N M R}(150 \mathrm{MHz}$, $\left.\mathrm{CDCl}_{3}\right): \delta 143.64,141.94,138.70,131.78(\mathrm{t}, J=253.7 \mathrm{~Hz}), 123.77,110.25,108.67,83.40$, 41.31, 24.70, 11.69; HRMS-ESI $(m / z)[\mathrm{M}+\mathrm{OH}]^{-}$calcd for $\mathrm{C}_{17} \mathrm{H}_{21} \mathrm{BF}_{2} \mathrm{O}_{4}, 354.1565$; found 354.1566 .

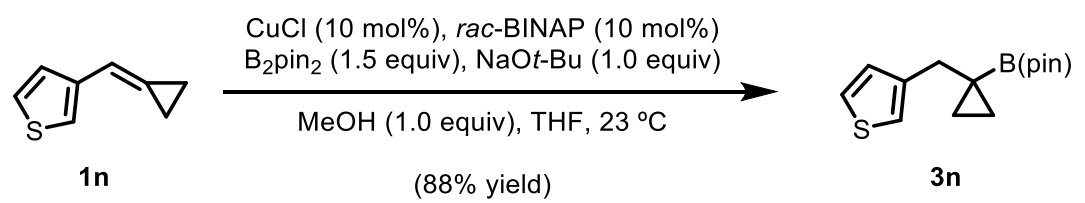

Boronic ester 3n. Purification by preparative thin layer chromatography (95:5 hexanes:EtOAc) afforded boronic ester 3n (46.4 mg, 88\% yield) as a colorless oil. 3n: ${ }^{1} \mathbf{H}$ NMR (600 MHz, $\left.\mathrm{CDCl}_{3}\right): \delta 7.18(\mathrm{dd}, J=4.9,3.0 \mathrm{~Hz}, 1 \mathrm{H}), 7.04-6.96(\mathrm{~m}, 2 \mathrm{H}), 2.59$ (s, 2H), $1.16(\mathrm{~s}, 12 \mathrm{H}), 0.73(\mathrm{q}$, $J=3.5 \mathrm{~Hz}, 2 \mathrm{H}), 0.43(\mathrm{q}, J=3.6 \mathrm{~Hz}, 2 \mathrm{H}) ;{ }^{13} \mathbf{C} \mathbf{N M R}\left(150 \mathrm{MHz}, \mathrm{CDCl}_{3}\right) \delta 142.83,129.06$, 
124.51, 120.61, 83.22, 36.17, 24.70, 11.58; HRMS-ESI $(m / z)[\mathrm{M}+\mathrm{H}]^{+}$calcd for $\mathrm{C}_{14} \mathrm{H}_{21} \mathrm{BO}_{2} \mathrm{~S}$, 264.1470; found 264.1468.

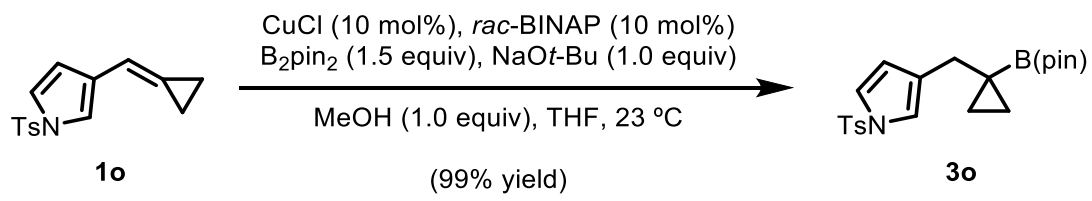

Boronic ester 3o. Purification by preparative thin layer chromatography (4:1 hexanes:EtOAc) afforded boronic ester $30\left(83.3 \mathrm{mg}, 99 \%\right.$ yield) as a white amorphous solid. 3o: ${ }^{1} \mathbf{H}$ NMR (600 $\left.\mathrm{MHz}, \mathrm{CDCl}_{3}\right): \delta 7.70(\mathrm{~d}, J=8.3 \mathrm{~Hz}, 2 \mathrm{H}), 7.25(\mathrm{~d}, J=8.4 \mathrm{~Hz}, 2 \mathrm{H}), 7.03(\mathrm{t}, J=2.7 \mathrm{~Hz}, 1 \mathrm{H}), 6.94$ $(\mathrm{s}, 1 \mathrm{H}), 6.21(\mathrm{~d}, J=1.5 \mathrm{~Hz}, 1 \mathrm{H}), 2.38(\mathrm{~s}, 3 \mathrm{H}), 2.31(\mathrm{~s}, 2 \mathrm{H}), 1.11(\mathrm{~s}, 12 \mathrm{H}), 0.68(\mathrm{q}, J=3.6 \mathrm{~Hz}$, 2H), 0.35 (q, $J=3.6 \mathrm{~Hz}, 2 \mathrm{H}) ;{ }^{13} \mathbf{C}$ NMR $\left(150 \mathrm{MHz}, \mathrm{CDCl}_{3}\right) \delta 144.63,136.60,129.92,129.79$, 126.82, 120.44, 117.86, 115.47, 83.16, 32.95, 24.63, 21.68, 11.50; HRMS-ESI $(\mathrm{m} / z)[\mathrm{M}+\mathrm{H}]^{+}$ calcd for $\mathrm{C}_{21} \mathrm{H}_{28} \mathrm{BNO}_{4} \mathrm{~S}, 401.1947$; found 401.1948 .

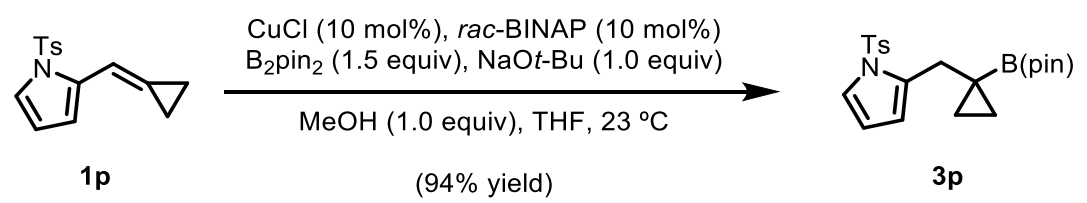

Boronic ester 3p. Purification by preparative thin layer chromatography (4:1 hexanes:EtOAc) afforded boronic ester $\mathbf{3 p}$ (75.7 $\mathrm{mg}, 94 \%$ yield) as a yellow amorphous solid. $\mathbf{3 p}$ : ${ }^{1}$ H NMR (600 $\left.\mathrm{MHz}, \mathrm{CDCl}_{3}\right): \delta 7.62(\mathrm{~d}, J=8.2 \mathrm{~Hz}, 2 \mathrm{H}), 7.27(\mathrm{~d}, J=7.7 \mathrm{~Hz}, 2 \mathrm{H}), 7.23(\mathrm{t}, J=2.4 \mathrm{~Hz}, 1 \mathrm{H})$, 6.19-6.14 (m, 2H), $2.66(\mathrm{~s}, 2 \mathrm{H}), 2.40(\mathrm{~s}, 3 \mathrm{H}), 1.07(\mathrm{~s}, 12 \mathrm{H}), 0.74(\mathrm{q}, J=3.7 \mathrm{~Hz}, 2 \mathrm{H}), 0.36(\mathrm{q}, J=$ $3.7 \mathrm{~Hz}, 2 \mathrm{H}) ;{ }^{13} \mathbf{C}$ NMR (150 MHz, $\left.\mathrm{CDCl}_{3}\right): \delta 144.38,136.89,136.41,129.91,126.81,122.04$, 112.21, 111.45, 83.10, 33.69, 24.58, 21.72, 11.38; HRMS-ESI $(\mathrm{m} / \mathrm{z})[\mathrm{M}+\mathrm{H}]^{+}$calcd for $\mathrm{C}_{21} \mathrm{H}_{28} \mathrm{BNO}_{4} \mathrm{~S}, 401.1947$; found 401.1944 .

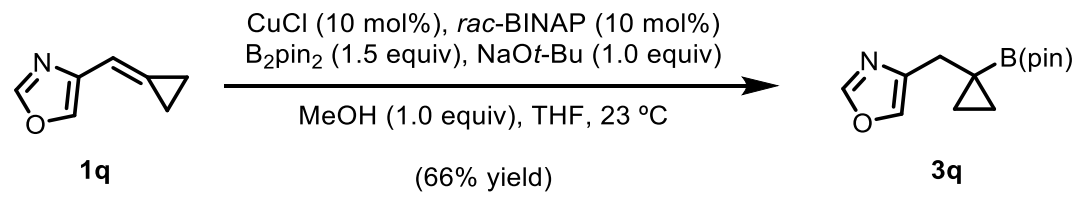

Boronic ester 3q. Purification by preparative thin layer chromatography (4:1 hexanes:EtOAc) afforded boronic ester 3q (32.8 mg, 66\% yield) as a white solid. 3q: ${ }^{1} \mathbf{H}$ NMR (600 MHz, $\left.\mathrm{CDCl}_{3}\right): \delta 7.77(\mathrm{~s}, 1 \mathrm{H}), 7.47(\mathrm{~s}, 1 \mathrm{H}), 2.53(\mathrm{~s}, 2 \mathrm{H}), 1.18(\mathrm{~s}, 12 \mathrm{H}), 0.75(\mathrm{q}, J=3.6 \mathrm{~Hz}, 2 \mathrm{H}), 0.49$ 
$(\mathrm{q}, J=3.6 \mathrm{~Hz}, 2 \mathrm{H}) ;{ }^{13} \mathbf{C}$ NMR $\left(150 \mathrm{MHz}, \mathrm{CDCl}_{3}\right): \delta 150.41,140.79,134.88,83.31,32.30,24.72$, 11.33; HRMS-ESI $(m / z)[\mathrm{M}+\mathrm{H}]^{+}$calcd for $\mathrm{C}_{13} \mathrm{H}_{20} \mathrm{BNO}_{3}, 249.1651$; found 249.1654.

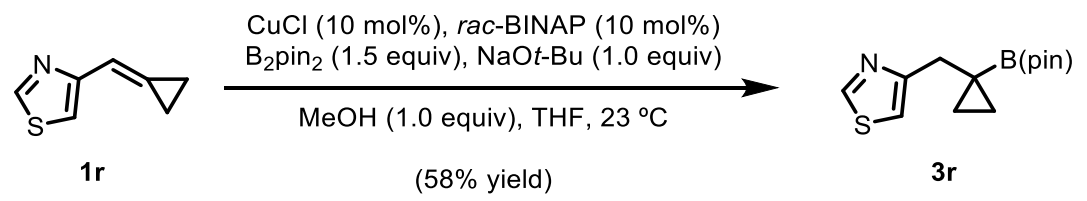

Boronic ester 3r. Purification by preparative thin layer chromatography (4:1 hexanes:EtOAc) afforded boronic ester $\mathbf{3 r}$ (30.6 mg, 58\% yield) as a colorless oil. 3r: ${ }^{1} \mathbf{H}$ NMR (600 MHz, $\left.\mathrm{CDCl}_{3}\right): \delta 8.70(\mathrm{~d}, J=2.0 \mathrm{~Hz}, 1 \mathrm{H}), 7.08(\mathrm{~d}, J=2.0 \mathrm{~Hz}, 1 \mathrm{H}), 2.83(\mathrm{~s}, 2 \mathrm{H}), 1.17(\mathrm{~s}, 12 \mathrm{H}), 0.78(\mathrm{q}$, $J=3.6 \mathrm{~Hz}, 2 \mathrm{H}), 0.52(\mathrm{q}, J=3.6 \mathrm{~Hz}, 2 \mathrm{H}) ;{ }^{13} \mathbf{C} \mathbf{N M R}\left(150 \mathrm{MHz}, \mathrm{CDCl}_{3}\right): \delta 158.55,151.64$, 113.05, 83.27, 37.15, 24.68, 11.48; HRMS-ESI $(\mathrm{m} / \mathrm{z})[\mathrm{M}+\mathrm{H}]^{+}$calcd for $\mathrm{C}_{13} \mathrm{H}_{20} \mathrm{BNO}_{2} \mathrm{~S}$, 265.1422 ; found 265.1428 .

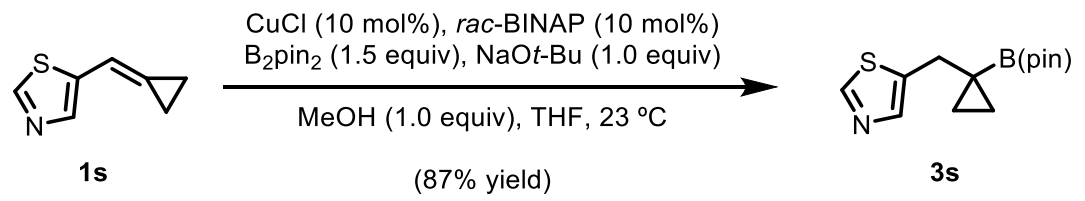

Boronic ester 3s. Purification by preparative thin layer chromatography (4:1 hexanes:EtOAc) afforded boronic ester 3s (46.0 mg, 87\% yield) as a colorless oil. 3s: ${ }^{1} \mathbf{H}$ NMR (600 MHz, $\left.\mathrm{CDCl}_{3}\right): \delta 8.61(\mathrm{~s}, 1 \mathrm{H}), 7.63(\mathrm{~s}, 1 \mathrm{H}), 2.77(\mathrm{~s}, 2 \mathrm{H}), 1.17(\mathrm{~s}, 12 \mathrm{H}), 0.80(\mathrm{q}, J=3.7 \mathrm{~Hz}, 2 \mathrm{H}), 0.50$ $(\mathrm{q}, J=3.7 \mathrm{~Hz}, 2 \mathrm{H}) .{ }^{13} \mathbf{C}$ NMR $\left(150 \mathrm{MHz}, \mathrm{CDCl}_{3}\right): \delta 151.37,140.71,139.40,83.51,33.03,24.67$, 12.15; HRMS-ESI $(\mathrm{m} / \mathrm{z})[\mathrm{M}+\mathrm{H}]^{+}$calcd for $\mathrm{C}_{13} \mathrm{H}_{20} \mathrm{BNO}_{2} \mathrm{~S}, 265.1422$; found 265.1428 .

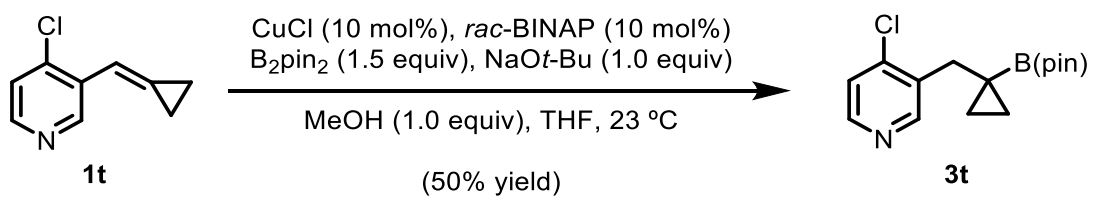

Boronic ester 3t. Purification by preparative thin layer chromatography (4:1 hexanes:EtOAc) afforded boronic ester 3t (29.5 mg, 50\% yield) as a colorless oil. 3t: ${ }^{1} \mathbf{H}$ NMR (600 MHz, $\left.\mathrm{CDCl}_{3}\right): \delta 8.70(\mathrm{~s}, 1 \mathrm{H}), 8.32(\mathrm{~d}, J=5.3 \mathrm{~Hz}, 1 \mathrm{H}), 7.26(\mathrm{~d}, J=5.3 \mathrm{~Hz}, 1 \mathrm{H}), 2.78(\mathrm{~s}, 2 \mathrm{H}), 1.19(\mathrm{~s}$, $12 \mathrm{H}), 0.86(\mathrm{q}, J=3.7 \mathrm{~Hz}, 2 \mathrm{H}), 0.51(\mathrm{t}, J=3.1 \mathrm{~Hz}, 2 \mathrm{H}) ;{ }^{13} \mathbf{C} \mathbf{N M R}\left(150 \mathrm{MHz}, \mathrm{CDCl}_{3}\right): \delta 151.33$, 147.81, 144.15, 135.52, 124.14, 83.52, 35.54, 24.69, 11.87; HRMS-ESI $(m / z)[\mathrm{M}+\mathrm{H}]^{+}$calcd for $\mathrm{C}_{15} \mathrm{H}_{21} \mathrm{BCINO}_{2}$, 293.1468; found 293.1472. 


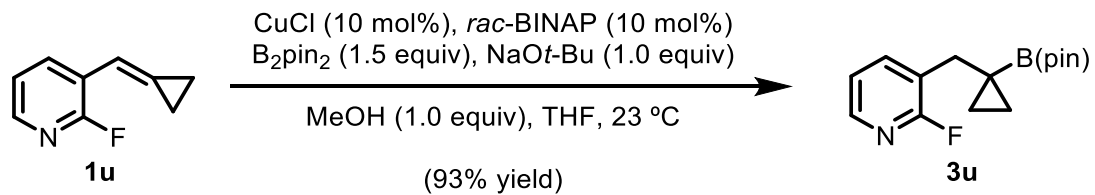

Boronic ester 3u. Purification by preparative thin layer chromatography (6:1 hexanes:EtOAc) afforded boronic ester 3u (51.8 mg, 93\% yield) as a colorless oil. 3u: ${ }^{1} \mathbf{H}$ NMR (600 MHz, $\left.\mathrm{CDCl}_{3}\right): \delta 8.02(\mathrm{~d}, J=4.0 \mathrm{~Hz}, 1 \mathrm{H}), 7.86(\mathrm{ddd}, J=9.5,7.3,1.9 \mathrm{~Hz}, 1 \mathrm{H}), 7.09(\mathrm{ddd}, J=7.0,4.8$, $1.8 \mathrm{~Hz}, 1 \mathrm{H}), 2.62(\mathrm{~s}, 2 \mathrm{H}), 1.15(\mathrm{~s}, 12 \mathrm{H}), 0.80(\mathrm{q}, J=3.7 \mathrm{~Hz}, 2 \mathrm{H}), 0.52(\mathrm{q}, J=3.7 \mathrm{~Hz}, 2 \mathrm{H}) ;{ }^{13} \mathbf{C}$ NMR (150 MHz, $\left.\mathrm{CDCl}_{3}\right): \delta 162.23(\mathrm{~d}, J=238.0 \mathrm{~Hz}), 144.69(\mathrm{~d}, J=14.5 \mathrm{~Hz}), 141.12(\mathrm{~d}, J=5.9$ Hz), 124.09 (d, $J=31.0 \mathrm{~Hz}), 121.01$ (d, $J=4.1 \mathrm{~Hz}), 83.39,33.70,24.65,11.68$; HRMS-ESI $(m / z)[\mathrm{M}+\mathrm{H}]^{+}$calcd for $\mathrm{C}_{15} \mathrm{H}_{21} \mathrm{BFNO}_{2}, 277.1764$; found 277.1770.

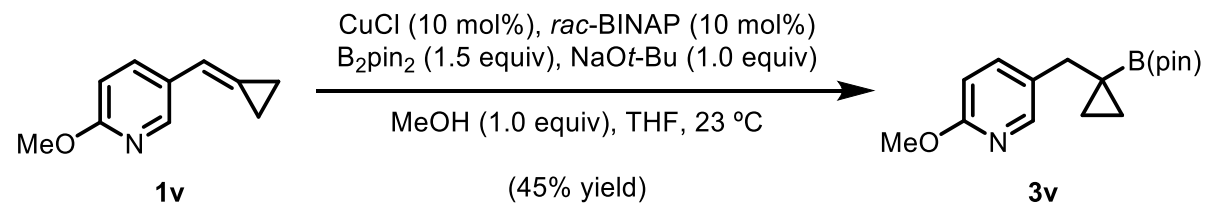

Boronic ester 3v. Purification by preparative thin layer chromatography (9:1 hexanes:EtOAc) afforded boronic ester 3v (26.0 mg, 45\% yield) as a colorless oil. 3v: ${ }^{1} \mathbf{H}$ NMR (600 MHz, $\left.\mathrm{CDCl}_{3}\right): \delta 8.01(\mathrm{~s}, 1 \mathrm{H}), 7.56(\mathrm{dd}, J=8.5,2.4 \mathrm{~Hz}, 1 \mathrm{H}), 6.64(\mathrm{~d}, J=8.4 \mathrm{~Hz}, 1 \mathrm{H}), 3.91(\mathrm{~s}, 3 \mathrm{H}), 2.50$ $(\mathrm{s}, 2 \mathrm{H}), 1.16(\mathrm{~s}, 12 \mathrm{H}), 0.74(\mathrm{q}, J=3.6 \mathrm{~Hz}, 2 \mathrm{H}), 0.44(\mathrm{q}, J=3.6 \mathrm{~Hz}, 2 \mathrm{H}) ;{ }^{13} \mathbf{C ~ N M R}(150 \mathrm{MHz}$, $\left.\mathrm{CDCl}_{3}\right): \delta 162.68,146.64,139.77,130.28,109.92,83.32,53.39,37.69,24.72,11.56$; HRMSESI $(m / z)[\mathrm{M}+\mathrm{H}]^{+}$calcd for $\mathrm{C}_{16} \mathrm{H}_{24} \mathrm{BNO}_{3}, 289.1964$; found 289.1969.

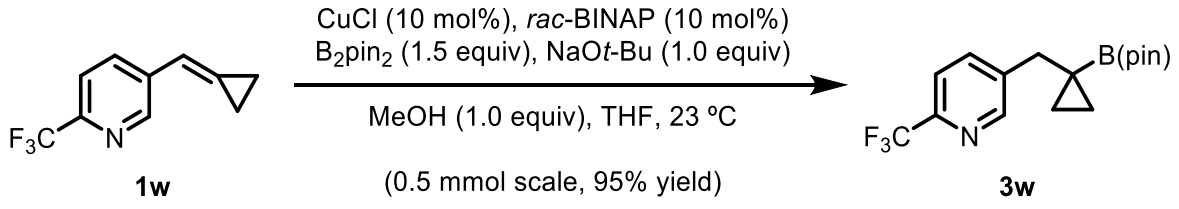

Boronic ester 3w. Purification by preparative thin layer chromatography $\left(4 \mathrm{~g}\right.$ of $\mathrm{SiO}_{2}, \mathrm{Isco}_{0} 0_{-}$ 20\% EtOAc/Heptanes) afforded boronic ester 3w (156.0 mg, 95\% yield) as a colorless oil. 3w: ${ }^{1}$ H NMR $\left(400 \mathrm{MHz}, \mathrm{CDCl}_{3}\right): \delta 8.64-8.52(\mathrm{~m}, 1 \mathrm{H}), 7.78(\mathrm{dd}, J=8.1,2.1 \mathrm{~Hz}, 1 \mathrm{H}), 7.53(\mathrm{~d}, J=$ $8.1 \mathrm{~Hz}, 1 \mathrm{H}), 2.61(\mathrm{~s}, 2 \mathrm{H}), 1.11(\mathrm{~s}, 12 \mathrm{H}), 0.83-0.71(\mathrm{~m}, 2 \mathrm{H}), 0.51-0.42(\mathrm{~m}, 2 \mathrm{H}) ;{ }^{13} \mathrm{C}$ NMR $(100$ $\left.\mathrm{MHz}, \mathrm{CDCl}_{3}\right): \delta 150.83,145.89(\mathrm{q}, J=34.4 \mathrm{~Hz}), 141.39,137.56,122.05(\mathrm{q}, J=273.7 \mathrm{~Hz})$, 
$119.84(\mathrm{q}, J=2.9 \mathrm{~Hz}), 83.63,38.82,24.74,12.11$; HRMS-ESI $(m / z)[\mathrm{M}+\mathrm{H}]^{+}$calcd for $\mathrm{C}_{16} \mathrm{H}_{22} \mathrm{BF}_{3} \mathrm{NO}_{2}, 322.1727$; found 322.1727 .

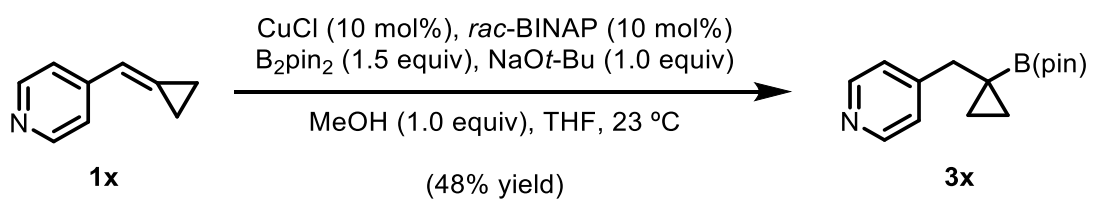

Boronic ester 3x. Purification by preparative thin layer chromatography (4:1 hexanes:EtOAc) afforded boronic ester 3x (24.7 mg, 48\% yield) as a colorless oil. 3x: ${ }^{1} \mathbf{H}$ NMR (600 MHz, $\left.\mathrm{CDCl}_{3}\right): \delta 8.45(\mathrm{~d}, J=5.1 \mathrm{~Hz}, 2 \mathrm{H}), 7.22(\mathrm{~d}, J=6.1 \mathrm{~Hz}, 2 \mathrm{H}), 2.58(\mathrm{~s}, 2 \mathrm{H}), 1.15(\mathrm{~s}, 12 \mathrm{H}), 0.81$ (q, $J=3.7 \mathrm{~Hz}, 2 \mathrm{H}), 0.47$ (q, $J=3.7 \mathrm{~Hz}, 2 \mathrm{H}) ;{ }^{13} \mathbf{C}$ NMR (150 MHz, $\left.\mathrm{CDCl}_{3}\right): \delta 151.57,149.31$, 124.46, 83.45, 40.90, 24.66, 11.84; HRMS-ESI $(\mathrm{m} / \mathrm{z})[\mathrm{M}+\mathrm{H}]^{+}$calcd for $\mathrm{C}_{15} \mathrm{H}_{22} \mathrm{BNO}_{2}$, 259.1858; found 259.1860 .

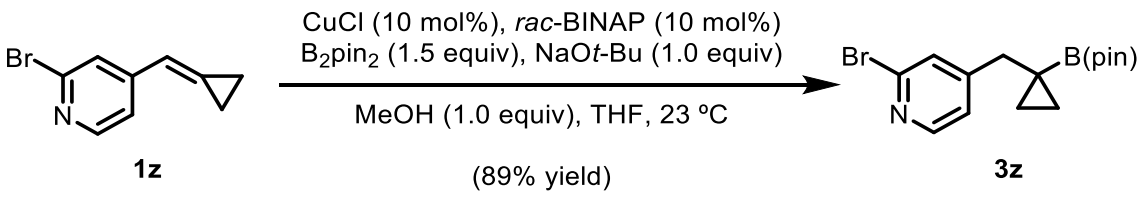

Boronic ester 3z. Purification by preparative thin layer chromatography (4:1 hexanes:EtOAc) afforded boronic ester 3z (60.4 mg, 89\% yield) as a colorless oil. 3z: ${ }^{1} \mathbf{H}$ NMR (600 MHz, $\left.\mathrm{CDCl}_{3}\right): \delta 8.21(\mathrm{~d}, J=5.1 \mathrm{~Hz}, 1 \mathrm{H}), 7.48(\mathrm{~s}, 1 \mathrm{H}), 7.15(\mathrm{~d}, J=5.0 \mathrm{~Hz}, 1 \mathrm{H}), 2.53(\mathrm{~s}, 2 \mathrm{H}), 1.17$ (s, $12 \mathrm{H}), 0.82(\mathrm{q}, J=3.7 \mathrm{~Hz}, 2 \mathrm{H}), 0.47$ (q, $J=3.7 \mathrm{~Hz}, 2 \mathrm{H}) ;{ }^{13} \mathbf{C}$ NMR $\left(150 \mathrm{MHz}, \mathrm{CDCl}_{3}\right): \delta 154.89$, 149.53, 142.12, 128.49, 123.63, 83.58, 40.75, 24.68, 12.01; HRMS-ESI $(m / z)[\mathrm{M}+\mathrm{H}]^{+}$calcd for $\mathrm{C}_{15} \mathrm{H}_{21} \mathrm{BBrNO}_{2}, 337.0963$; found 337.0962.

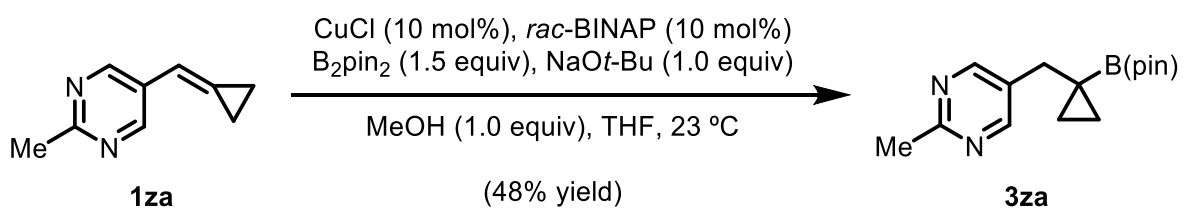

Boronic ester 3za. Purification by preparative thin layer chromatography (3:1 hexanes:EtOAc) afforded boronic ester 3za (26.5 mg, 48\% yield) as a colorless oil. 3za: ${ }^{1} \mathbf{H}$ NMR (600 MHz, $\left.\mathrm{CDCl}_{3}\right): \delta 8.57(\mathrm{~s}, 2 \mathrm{H}), 2.70(\mathrm{~s}, 3 \mathrm{H}), 2.50(\mathrm{~s}, 2 \mathrm{H}), 1.16(\mathrm{~s}, 12 \mathrm{H}), 0.80(\mathrm{t}, J=3.0 \mathrm{~Hz}, 2 \mathrm{H}), 0.48(\mathrm{q}$, $J=3.8 \mathrm{~Hz}, 2 \mathrm{H}) ;{ }^{13} \mathbf{C}$ NMR $\left(150 \mathrm{MHz}, \mathrm{CDCl}_{3}\right): \delta 165.55,157.31,131.86,83.55,36.09,25.62$, 24.72, 12.03; HRMS-ESI $(\mathrm{m} / \mathrm{z})[\mathrm{M}+\mathrm{H}]^{+}$calcd for $\mathrm{C}_{15} \mathrm{H}_{23} \mathrm{BN}_{2} \mathrm{O}_{2}, 274.1967$; found 274.1970. 


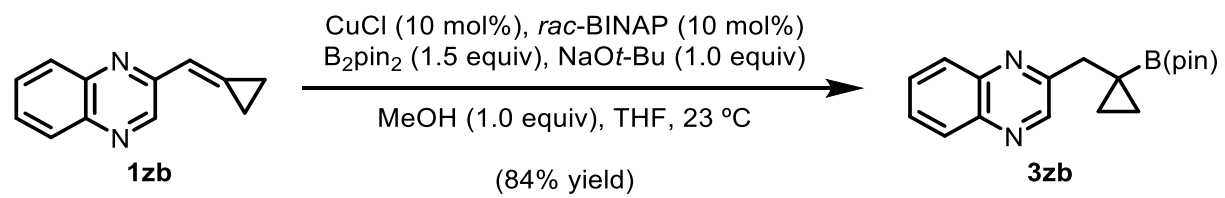

Boronic ester 3zb. Purification by preparative thin layer chromatography (4:1 hexanes:EtOAc) afforded boronic ester 3zb (48.0 mg, 84\% yield) as a white solid. 3zb: ${ }^{1} \mathbf{H}$ NMR (400 MHz, $\left.\mathrm{CDCl}_{3}\right): \delta 8.96(\mathrm{~s}, 1 \mathrm{H}), 8.11-7.94(\mathrm{~m}, 2 \mathrm{H}), 7.75-7.62(\mathrm{~m}, 2 \mathrm{H}), 2.99(\mathrm{~s}, 2 \mathrm{H}), 1.16(\mathrm{~s}, 12 \mathrm{H}), 0.86-$ $0.79(\mathrm{~m}, 2 \mathrm{H}), 0.64-0.58(\mathrm{~m}, 2 \mathrm{H}) ;{ }^{13} \mathbf{C} \mathbf{N M R}\left(101 \mathrm{MHz}, \mathrm{CDCl}_{3}\right): \delta 157.62,146.52,145.88$, 141.35, 129.88, 129.28, 129.12, 128.99, 83.63, 42.37, 41.39, 24.84, 12.18; HRMS-ESI $(\mathrm{m} / \mathrm{z})$ [M $+\mathrm{H}]^{+}$calcd for $\mathrm{C}_{18} \mathrm{H}_{23} \mathrm{BN}_{2} \mathrm{O}_{2}, 310.1962$; found 310.1967 .

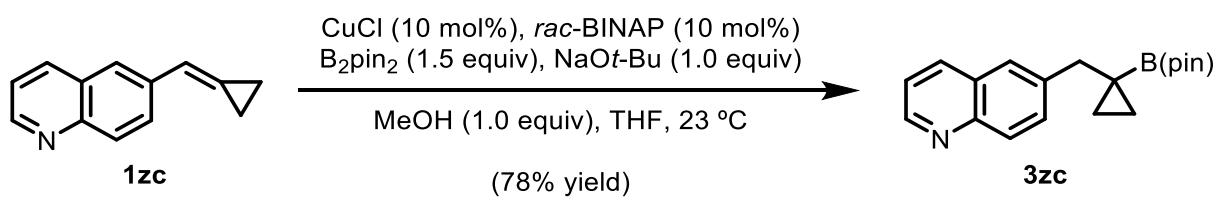

Boronic ester 3zc. Purification by preparative thin layer chromatography (4:1 hexanes:EtOAc) afforded boronic ester 3zc (48.0 mg, 78\% yield) as a white solid. 3zc: ${ }^{1} \mathbf{H}$ NMR (600 MHz, $\left.\mathrm{CDCl}_{3}\right): \delta 8.84(\mathrm{dd}, J=4.2,1.8 \mathrm{~Hz}, 1 \mathrm{H}), 8.08(\mathrm{~d}, J=8.3 \mathrm{~Hz}, 1 \mathrm{H}), 7.99(\mathrm{~d}, J=8.2 \mathrm{~Hz}, 1 \mathrm{H}), 7.69$ $(\mathrm{d}, J=9.9 \mathrm{~Hz}, 2 \mathrm{H}), 7.35(\mathrm{dd}, J=8.2,4.1 \mathrm{~Hz}, 1 \mathrm{H}), 2.80(\mathrm{~s}, 2 \mathrm{H}), 1.14(\mathrm{~s}, 12 \mathrm{H}), 0.81(\mathrm{q}, J=3.5$ $\mathrm{Hz}, 2 \mathrm{H}), 0.53(\mathrm{t}, J=2.9 \mathrm{~Hz}, 2 \mathrm{H}) ;{ }^{13} \mathbf{C} \mathbf{N M R}\left(150 \mathrm{MHz}, \mathrm{CDCl}_{3}\right): \delta 149.58,147.26,140.98$, 135.73, 131.90, 128.76, 128.26, 126.63, 120.99, 83.32, 41.39, 24.68, 11.74; HRMS-ESI $(\mathrm{m} / \mathrm{z})$ $[\mathrm{M}+\mathrm{H}]^{+}$calcd for $\mathrm{C}_{19} \mathrm{H}_{24} \mathrm{BNO}_{2}, 309.2015$; found 309.2020.

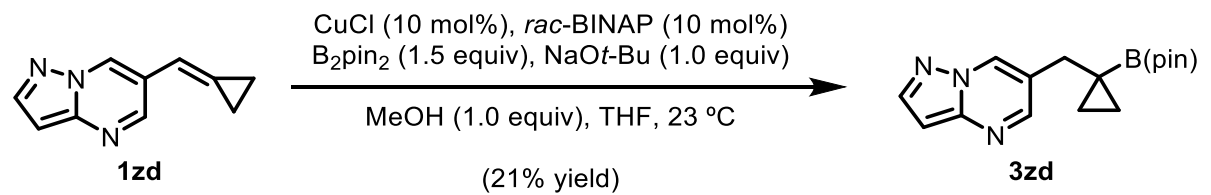

Boronic ester 3zd. Purification by preparative thin layer chromatography (4:1 hexanes:EtOAc) afforded boronic ester 3zd (12.6 mg, 21\% yield) as a colorless oil. 3zd: ${ }^{\mathbf{1}} \mathbf{H}$ NMR (600 MHz, $\left.\mathrm{CDCl}_{3}\right): \delta 8.64(\mathrm{~s}, 1 \mathrm{H}), 8.48(\mathrm{~s}, 1 \mathrm{H}), 8.06(\mathrm{~s}, 1 \mathrm{H}), 6.64(\mathrm{~s}, 1 \mathrm{H}), 2.60(\mathrm{~s}, 2 \mathrm{H}), 1.16(\mathrm{~s}, 12 \mathrm{H}), 0.85$ $(\mathrm{q}, J=3.7 \mathrm{~Hz}, 2 \mathrm{H}), 0.54(\mathrm{q}, J=3.7 \mathrm{~Hz}, 2 \mathrm{H}) ;{ }^{13} \mathbf{C}$ NMR $\left(150 \mathrm{MHz}, \mathrm{CDCl}_{3}\right): \delta 152.18,147.62$, 144.46, 133.52, 122.49, 96.29, 83.69, 36.08, 24.76, 12.13; HRMS-ESI $(m / z)[\mathrm{M}+\mathrm{H}]^{+}$calcd for $\mathrm{C}_{16} \mathrm{H}_{22} \mathrm{BN}_{3} \mathrm{O}_{2}, 299.1920$; found 299.1925. 

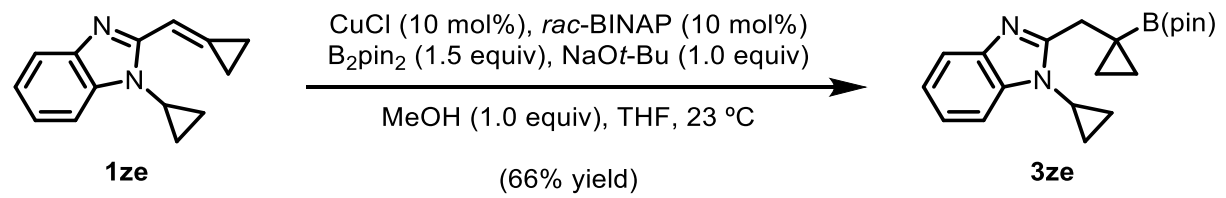

Boronic ester 3ze. Purification by preparative thin layer chromatography (2:1 hexanes:EtOAc) afforded boronic ester 3ze (44.7 mg, 66\% yield) as a colorless oil. 3ze: ${ }^{1} \mathbf{H}$ NMR (600 MHz, $\left.\mathrm{CDCl}_{3}\right): \delta 7.71(\mathrm{~d}, J=7.0 \mathrm{~Hz}, 1 \mathrm{H}), 7.50(\mathrm{~d}, J=7.5 \mathrm{~Hz}, 1 \mathrm{H}), 7.20(\mathrm{p}, J=7.2 \mathrm{~Hz}, 2 \mathrm{H}), 3.24(\mathrm{tt}, J$ $=7.2,3.9 \mathrm{~Hz}, 1 \mathrm{H}), 3.04(\mathrm{~s}, 2 \mathrm{H}), 1.21(\mathrm{t}, J=6.5 \mathrm{~Hz}, 2 \mathrm{H}), 1.15(\mathrm{~s}, 12 \mathrm{H}), 1.10(\mathrm{q}, J=5.4,4.4 \mathrm{~Hz}$, 2H), 0.90 (q, $J=3.8 \mathrm{~Hz}, 2 \mathrm{H}), 0.58$ (q, $J=3.8 \mathrm{~Hz}, 2 \mathrm{H}) ;{ }^{13} \mathbf{C ~ N M R}\left(150 \mathrm{MHz}, \mathrm{CDCl}_{3}\right) \delta 156.84$, $142.58,136.27,121.66,121.42,119.42,110.16$, 83.26, 33.38, 24.99, 24.64, 11.31, 7.08; HRMSESI $(m / z)[\mathrm{M}+\mathrm{H}]^{+}$calcd for $\mathrm{C}_{20} \mathrm{H}_{27} \mathrm{BN}_{2} \mathrm{O}_{2}, 338.2280$; found 338.2285.

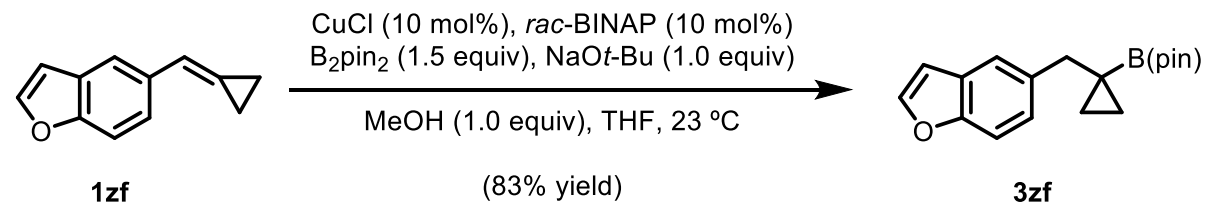

Boronic ester 3zf. Purification by preparative thin layer chromatography (95:5 hexanes:EtOAc) afforded boronic ester 3zf (49.4 mg, 83\% yield) as a white solid. 3zf: ${ }^{1} \mathbf{H}$ NMR (600 MHz, $\left.\mathrm{CDCl}_{3}\right): \delta 7.56(\mathrm{~d}, J=2.3 \mathrm{~Hz}, 1 \mathrm{H}), 7.48(\mathrm{~s}, 1 \mathrm{H}), 7.36(\mathrm{~d}, J=8.5 \mathrm{~Hz}, 1 \mathrm{H}), 7.22(\mathrm{dd}, J=8.5,1.8$ $\mathrm{Hz}, 1 \mathrm{H}), 6.69(\mathrm{dd}, J=2.3,1.0 \mathrm{~Hz}, 1 \mathrm{H}), 2.71(\mathrm{~s}, 2 \mathrm{H}), 1.15(\mathrm{~s}, 12 \mathrm{H}), 0.74(\mathrm{q}, J=3.6 \mathrm{~Hz}, 2 \mathrm{H}), 0.47$ $(\mathrm{q}, J=3.6 \mathrm{~Hz}, 2 \mathrm{H}) ;{ }^{13} \mathbf{C}$ NMR $\left(150 \mathrm{MHz}, \mathrm{CDCl}_{3}\right) \delta 153.56,144.76,136.57,127.10,125.65$, 121.01, 110.44, 106.44, 83.09, 40.96, 24.58, 11.31; HRMS-ESI $(m / z)[\mathrm{M}+\mathrm{H}]^{+}$calcd for $\mathrm{C}_{18} \mathrm{H}_{23} \mathrm{BO}_{3}, 298.1855$; found 298.1854.

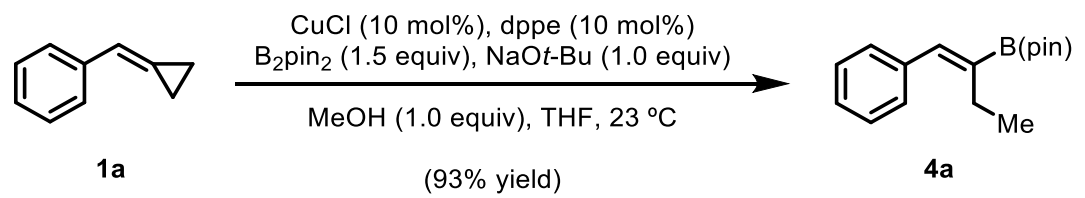

Boronic ester 4a. Purification by preparative thin layer chromatography (95:5 hexanes:EtOAc) afforded boronic ester 4a (48.0 mg, 93\% yield) as a colorless oil. 4a: ${ }^{1} \mathbf{H}$ NMR (600 MHz, $\left.\mathrm{CDCl}_{3}\right): \delta 7.32(\mathrm{~m}, 4 \mathrm{H}), 7.26-7.21(\mathrm{~m}, 1 \mathrm{H}), 7.20(\mathrm{~s}, 1 \mathrm{H}), 2.39(\mathrm{q}, J=7.5,2 \mathrm{H}), 1.31(\mathrm{~s}, 12 \mathrm{H})$, $1.10(\mathrm{t}, J=7.5 \mathrm{~Hz}, 3 \mathrm{H}) ;{ }^{13} \mathbf{C} \mathbf{N M R}\left(150 \mathrm{MHz}, \mathrm{CDCl}_{3}\right): \delta 141.37,137.91,128.95,128.08$, 
127.01, 83.38, 24.80, 22.68, 14.66.; Note: The signal corresponding to the carbon atom bonded to boron was not observed, likely due to quadrupolar relaxation. HRMS-ESI $(\mathrm{m} / \mathrm{z})[\mathrm{M}+\mathrm{OH}]^{-}$ calcd for $\mathrm{C}_{16} \mathrm{H}_{23} \mathrm{BO}_{2}, 274.1855$; found 274.1852.

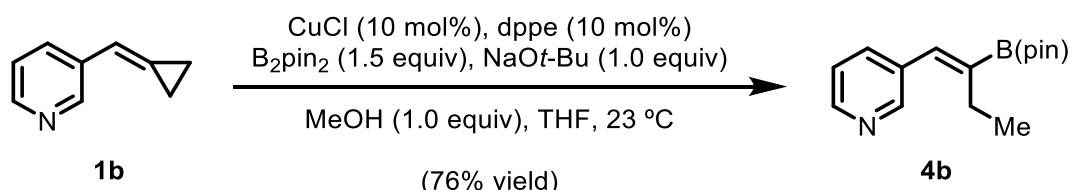

Boronic ester 4b. Purification by preparative thin layer chromatography (7:1 hexanes:EtOAc) afforded boronic ester $\mathbf{4 b}\left(39.4 \mathrm{mg}, 76 \%\right.$ yield) as a colorless oil. 4b: ${ }^{1} \mathbf{H}$ NMR (600 MHz, $\left.\mathrm{CDCl}_{3}\right): \delta 8.55(\mathrm{~s}, 1 \mathrm{H}), 8.45(\mathrm{~d}, J=4.2 \mathrm{~Hz}, 1 \mathrm{H}), 7.59(\mathrm{~d}, J=7.9 \mathrm{~Hz}, 1 \mathrm{H}), 7.24(\mathrm{dd}, J=7.9 \mathrm{~Hz}$, $4.2 \mathrm{~Hz}, 1 \mathrm{H}), 2.34(\mathrm{q}, J=7.5 \mathrm{~Hz}, 2 \mathrm{H}), 1.29(\mathrm{~s}, 12 \mathrm{H}), 1.08(\mathrm{t}, J=7.5 \mathrm{~Hz}, 3 \mathrm{H}) ;{ }^{13} \mathbf{C}$ NMR $(150$ $\left.\mathrm{MHz}, \mathrm{CDCl}_{3}\right): \delta 151.18,148.12,137.57,136.12,133.72,123.30,83.84,25.00,22.99,14.74 ;$ Note: The carbon signal bound to the boron atom was not observed, likely due to quadrupolar relaxation. HRMS-ESI $(\mathrm{m} / \mathrm{z})[\mathrm{M}+\mathrm{H}]^{+}$calcd for $\mathrm{C}_{15} \mathrm{H}_{22} \mathrm{BNO}_{2}, 259.1858$; found 259.1859.

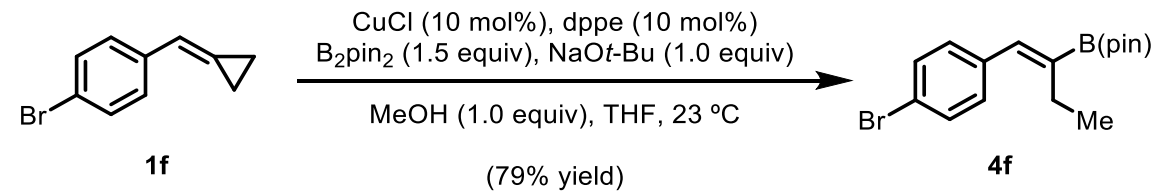

Boronic ester 4f. Purification by preparative thin layer chromatography (95:5 hexanes:EtOAc) afforded boronic ester $4 \mathbf{f}\left(53.0 \mathrm{mg}, 79 \%\right.$ yield) as a colorless oil. 4f: ${ }^{1} \mathbf{H}$ NMR (600 MHz, $\left.\mathrm{CDCl}_{3}\right): \delta 7.45(\mathrm{~d}, J=8.4 \mathrm{~Hz}, 2 \mathrm{H}), 7.18(\mathrm{~d}, J=8.4 \mathrm{~Hz}, 2 \mathrm{H}), 7.11(\mathrm{~s}, 1 \mathrm{H}), 2.35$ (q, $J=7.5 \mathrm{~Hz}$, $3 \mathrm{H}), 1.31(\mathrm{~s}, 12 \mathrm{H}), 1.08(\mathrm{t}, J=7.5 \mathrm{~Hz}, 3 \mathrm{H}) ;{ }^{13} \mathbf{C} \mathbf{N M R}\left(150 \mathrm{MHz}, \mathrm{CDCl}_{3}\right): \delta 139.99,136.73$, 131.24, 130.53, 120.97, 83.49, 24.78. 22.66, 14.51; Note: The carbon signal bound to the boron atom was not observed, likely due to quadrupolar relaxation. HRMS-ESI $(\mathrm{m} / \mathrm{z})[\mathrm{M}+\mathrm{OH}]^{-}$calcd for $\mathrm{C}_{16} \mathrm{H}_{22} \mathrm{BBrO}_{2}, 352.0960$; found 352.0968.

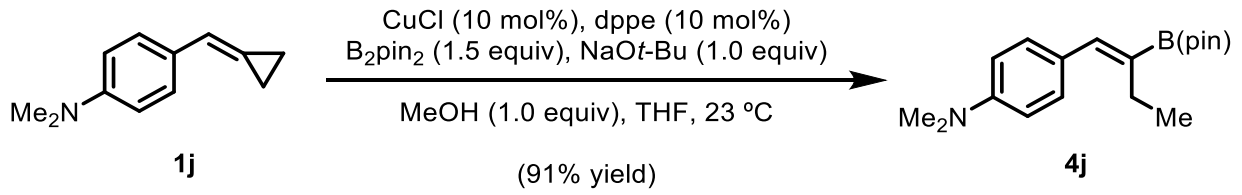

Boronic ester $\mathbf{4 j}$. Purification by preparative thin layer chromatography (9:1 hexanes:EtOAc) afforded boronic ester $\mathbf{4 j}$ (55.1 mg, 91\% yield) as a colorless oil. 4j: ${ }^{1} \mathbf{H} \mathbf{~ N M R}(600 \mathrm{MHz}$, 
$\left.\mathrm{CDCl}_{3}\right): \delta 7.28(\mathrm{~d}, J=8.6 \mathrm{~Hz}, 2 \mathrm{H}), 7.09(\mathrm{~s}, 1 \mathrm{H}), 6.67(\mathrm{~d}, J=8.6 \mathrm{~Hz}, 2 \mathrm{H}), 2.95(\mathrm{~s}, 6 \mathrm{H}), 2.43(\mathrm{q}, J$ $=7.5 \mathrm{~Hz}, 2 \mathrm{H}), 1.28(\mathrm{~s}, 12 \mathrm{H}), 1.10(\mathrm{t}, J=7.5 \mathrm{~Hz}, 3 \mathrm{H}) ;{ }^{13} \mathbf{C} \mathbf{N M R}\left(150 \mathrm{MHz}, \mathrm{CDCl}_{3}\right): \delta 149.75$, $141.81,130.72,126.45,112.04,83.31,40.58,25.01,22.91,14.81$; Note: The carbon signal bound to the boron atom was not observed, likely due to quadrupolar relaxation. HRMS-ESI $(m / z)[\mathrm{M}+\mathrm{H}]^{+}$calcd for $\mathrm{C}_{18} \mathrm{H}_{28} \mathrm{BNO}_{2}, 301.2328$; found 301.2327.

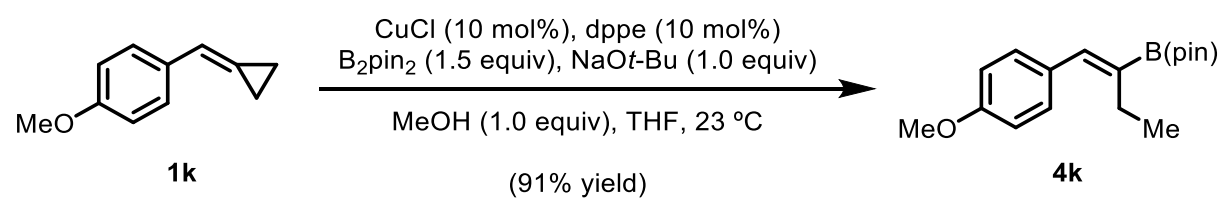

Boronic ester 4k. Purification by preparative thin layer chromatography (10:1 hexanes:EtOAc) afforded boronic ester $4 \mathbf{k}\left(52.3 \mathrm{mg}, 91 \%\right.$ yield) as a colorless oil. 4k: ${ }^{1} \mathbf{H}$ NMR (600 MHz, $\mathrm{CDCl}_{3}$ ): $\delta 7.29$ (d, $\left.J=8.6 \mathrm{~Hz}, 2 \mathrm{H}\right), 7.14(\mathrm{~s}, 1 \mathrm{H}), 6.87$ (d, $\left.J=8.6 \mathrm{~Hz}, 2 \mathrm{H}\right), 3.81$ (s, 3H), 2.40 (q, $J$ $=7.4 \mathrm{~Hz} 2 \mathrm{H}), 1.30(\mathrm{~s}, 12 \mathrm{H}), 1.11(\mathrm{q}, J=7.4 \mathrm{~Hz}, 3 \mathrm{H}) ;{ }^{13} \mathbf{C} \mathbf{~ N M R}\left(150 \mathrm{MHz}, \mathrm{CDCl}_{3}\right): \delta 158.66$, $140.95,130.55,130.44,113.52,83.27,55.20,24.79,22.62,14.58$; Note: The carbon signal bound to the boron atom was not observed, likely due to quadrupolar relaxation. HRMS-ESI $(\mathrm{m} / \mathrm{z})[\mathrm{M}+\mathrm{H}]^{+}$calcd for $\mathrm{C}_{17} \mathrm{H}_{25} \mathrm{BO}_{3}, 288.2011$; found 288.2010.

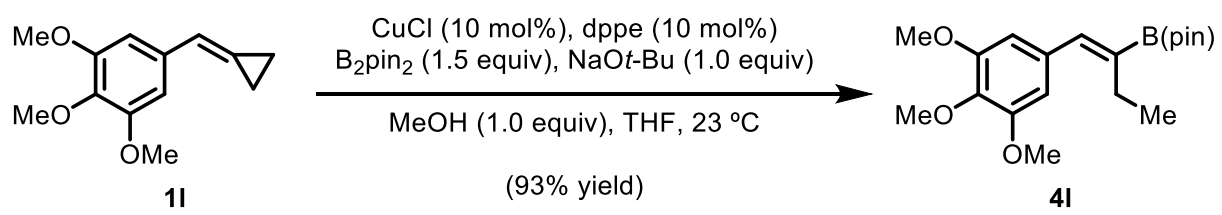

Boronic ester 4l. Purification by preparative thin layer chromatography (5:1 hexanes:EtOAc) afforded boronic ester $\mathbf{4 l}$ ( $65.0 \mathrm{mg}, 93 \%$ yield) as a white solid. $4 \mathbf{l}$ : ${ }^{1} \mathbf{H}$ NMR (600 MHz, $\left.\mathrm{CDCl}_{3}\right)$ : $\delta 7.26(\mathrm{~s}, 1 \mathrm{H}), 6.58(\mathrm{~s}, 2 \mathrm{H}), 3.86(\mathrm{~s}, 3 \mathrm{H}), 3.84(\mathrm{~s}, 6 \mathrm{H}), 2.42(\mathrm{q}, J=7.5 \mathrm{~Hz}, 2 \mathrm{H}), 1.31(\mathrm{~s}, 12 \mathrm{H})$, $1.13(\mathrm{t}, J=7.5 \mathrm{~Hz}, 3 \mathrm{H}) ;{ }^{13} \mathbf{C}$ NMR $\left(150 \mathrm{MHz}, \mathrm{CDCl}_{3}\right): \delta 152.82,141.34,137.20,133.43$, $106.14,83.42,60.91,56.00,24.80,22.88,14.61$; Note: The carbon signal bound to the boron atom was not observed, likely due to quadrupolar relaxation. HRMS-ESI $(\mathrm{m} / \mathrm{z})[\mathrm{M}+\mathrm{H}]^{+}$calcd for $\mathrm{C}_{19} \mathrm{H}_{29} \mathrm{BO}_{5}, 348.2223$; found 348.2225 . 


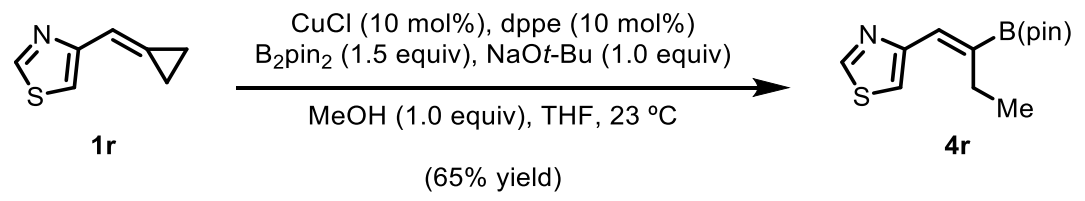

Boronic ester 4r. Purification by preparative thin layer chromatography (10:1 hexanes:EtOAc) afforded boronic ester 4r (34.7 mg, 65\% yield) as a colorless oil. 4r: ${ }^{1} \mathbf{H}$ NMR (600 MHz, $\left.\mathrm{CDCl}_{3}\right): \delta 8.80(\mathrm{~d}, J=1.9 \mathrm{~Hz}, 1 \mathrm{H}), 7.29$ (d, $\left.J=1.9 \mathrm{~Hz}, 1 \mathrm{H}\right), 7.23$ (s, 1H) 2.70 (q, $J=7.5 \mathrm{~Hz}$, 2H), 1.30 (s, 12H), 1.10 (q, $J=7.5 \mathrm{~Hz}, 3 \mathrm{H}) ;{ }^{13} \mathbf{C}$ NMR (150 MHz, $\left.\mathrm{CDCl}_{3}\right): \delta 154.66,151.75$, 132.95, 117.98, 83.49, 24.78, 23.15, 14.17; Note: The carbon signal bound to the boron atom was not observed, likely due to quadrupolar relaxation. HRMS-ESI $(m / z)[\mathrm{M}+\mathrm{H}]^{+}$calcd for $\mathrm{C}_{13} \mathrm{H}_{20} \mathrm{BNO}_{2} \mathrm{~S}, 265.1422$; found 265.1422.

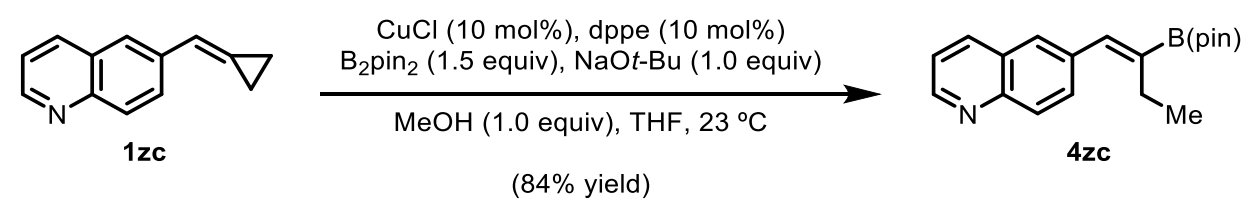

Boronic ester 4zc. Purification by preparative thin layer chromatography (10:1 hexanes:EtOAc) afforded boronic ester 4zc (52.0 mg, 84\% yield) as a white solid. 4zc: ${ }^{1} \mathbf{H}$ NMR (600 MHz, $\left.\mathrm{CDCl}_{3}\right): \delta 8.86(\mathrm{~d}, J=3.0 \mathrm{~Hz}, 1 \mathrm{H}), 8.10(\mathrm{~d}, J=8.1 \mathrm{~Hz}, 1 \mathrm{H}), 8.03(\mathrm{~d}, J=8.7 \mathrm{~Hz}, 1 \mathrm{H}), 7.71(\mathrm{~s}$, $1 \mathrm{H}), 7.67(\mathrm{dd}, J=8.7,1.9 \mathrm{~Hz}, 1 \mathrm{H}), 7.36(\mathrm{dd}, \mathrm{J}=8.1 \mathrm{~Hz}, 3.0 \mathrm{~Hz}, 1 \mathrm{H}) 7.33(\mathrm{~s}, 1 \mathrm{H}), 2.44(\mathrm{q}, J=$ $7.5 \mathrm{~Hz}, 2 \mathrm{H}) 2.80(\mathrm{~s}, 2 \mathrm{H}), 1.31(\mathrm{~s}, 12 \mathrm{H}), 1.13(\mathrm{t}, J=7.5 \mathrm{~Hz}, 3 \mathrm{H}) ;{ }^{13} \mathbf{C} \mathbf{N M R}\left(150 \mathrm{MHz}, \mathrm{CDCl}_{3}\right): \delta$ $150.51,147.61,140.65,136.48,136.40,131.10,129.26,128.32,127.75,121.45,83.76,25.02$, 23.01, 14.85; Note: The carbon signal bound to the boron atom was not observed, likely due to quadrupolar relaxation. HRMS-ESI $(m / z)[\mathrm{M}+\mathrm{H}]^{+}$calcd for $\mathrm{C}_{19} \mathrm{H}_{24} \mathrm{BNO}_{2}, 309.2015$; found 309.2015. 


\section{Exceptional pathway selectivity of thiazole-BCP.}

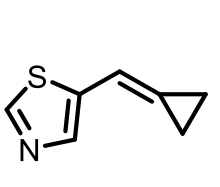

$1 \mathrm{~s}$

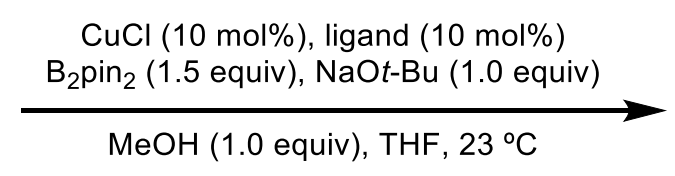

$\mathrm{MeOH}$ (1.0 equiv), THF, $23^{\circ} \mathrm{C}$

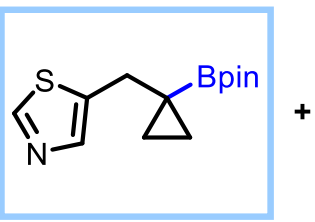

$3 s$

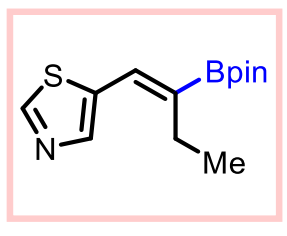

4s

$\begin{array}{ccc}\text { entry } & \text { ligand } & \text { product ratio }(\mathbf{3}: \mathbf{4}) \text { in } \% \text { yield } \\ 1 & \mathrm{P}(t-\mathrm{Bu})_{3} \cdot \mathrm{HBF}_{4} & 60: 16 \\ 2 & \mathrm{PCy}_{3} & 77: 6 \\ 3 & \mathrm{PPh}_{3} & 79: 0 \\ 4 & \left(\mathrm{p}-\mathrm{CF}_{3}-\mathrm{C}_{6} \mathrm{H}_{4}\right)_{3} \mathrm{P} & 97: 0 \\ 5 & \left(\mathrm{C}_{6} \mathrm{~F}_{5}\right)_{3} \mathrm{P} & 78: 0 \\ \ldots & \text { dppm } & 77: 0 \\ 6 & \text { dppbz } & 49: 15 \\ 7 & \text { dppe } & 70: 6 \\ 8 & \text { rac-BINAP } & 87: 0 \\ 9 & \text { xantphos } & 45: 0 \\ 10 & & \end{array}$




\section{Derivatization Experiments.}

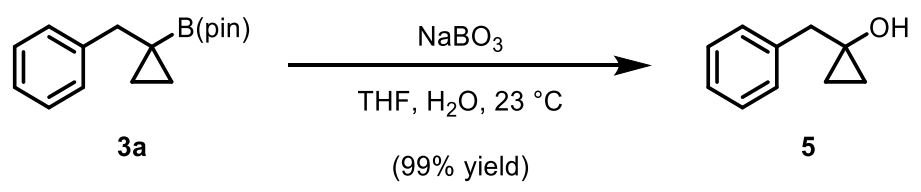

Alcohol 5. Alcohol 5 was prepared according to a modified procedure. ${ }^{3}$ A $10-\mathrm{mL}$ reaction vial with a magnetic stir bar was charged with $\mathrm{NaBO}_{3}(29.9 \mathrm{mg}, 0.30 \mathrm{mmol}, 3.0$ equiv), THF (1.0 $\mathrm{mL}), \mathrm{H}_{2} \mathrm{O}(1.0 \mathrm{~mL})$, and boronic ester $3 \mathrm{a}(25.8 \mathrm{mg}, 0.10 \mathrm{mmol}, 1.0$ equiv). The mixture was allowed to stir at room temperature for $2 \mathrm{~h}$. The reaction was then diluted with $\mathrm{H}_{2} \mathrm{O}(2.0 \mathrm{~mL})$ and extracted with EtOAc $(4 \times 3 \mathrm{~mL})$. The combined organic layers were combined, dried over $\mathrm{Na}_{2} \mathrm{SO}_{4}$, concentrated, and purified by preparative thin layer chromatography (4:1 hexanes:EtOAc) to give alcohol 5 as a colorless oil (16.0 mg, 99\% yield). Spectral data match those previously reported. ${ }^{4}$

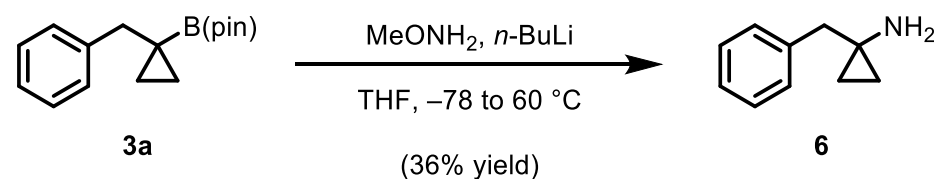

Amine 6. Amine 6 was prepared according to a modified procedure. ${ }^{5}$ A flame-dried 10-mL reaction vial with a magnetic stir bar was charged with $\mathrm{MeONH}_{2}(500 \mu \mathrm{L}, 1.0 \mathrm{M}$ solution in THF, $0.50 \mathrm{mmol}, 5.0$ equiv) and THF (1.0 mL). The vial was cooled to $-78{ }^{\circ} \mathrm{C}$, and $n$ - $\mathrm{BuLi}(200$ $\mu \mathrm{L}, 2.5 \mathrm{M}$ solution in hexane, $0.50 \mathrm{mmol}, 5.0$ equiv) was added dropwise over $1 \mathrm{~min}$. The reaction was allowed to stir at $-78{ }^{\circ} \mathrm{C}$ for $30 \mathrm{~min}$. Then, a solution of boronic ester $\mathbf{3 a}(25.8 \mathrm{mg}$, $0.10 \mathrm{mmol}, 1.0$ equiv) in $200 \mu \mathrm{L}$ of THF was added dropwise over $1 \mathrm{~min}$. The reaction mixture was allowed to warm to room temperature and was then heated to $60{ }^{\circ} \mathrm{C}$ for $15 \mathrm{~h}$. The reaction mixture was cooled to room temperature and quenched with $\mathrm{H}_{2} \mathrm{O}(3.0 \mathrm{~mL})$. The mixture were extracted with EtOAc $(4 \times 3 \mathrm{~mL})$, and the combined organic layers were dried over $\mathrm{Na}_{2} \mathrm{SO}_{4}$, concentrated, and purified by preparative thin layer chromatography (9:1 EtOAc:MeOH) to give amine 6 (5.3 mg, 36\% yield) as a colorless oil. Spectral data match those previously reported. ${ }^{6}$ 


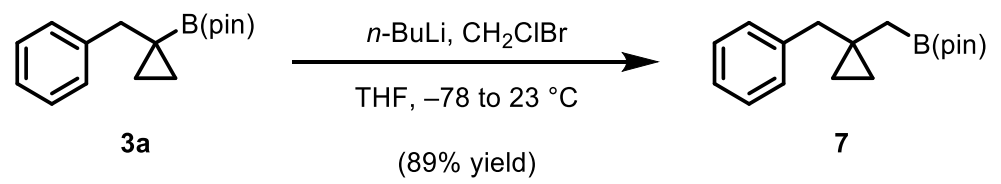

Boronic ester 7. Boronic ester 7 was prepared according to a modified procedure. ${ }^{7}$ A flamedried $10-\mathrm{mL}$ reaction vial with a magnetic stir bar was charged with boronic ester $\mathbf{3 a}$ (25.8 $\mathrm{mg}$, $0.10 \mathrm{mmol}, 1.0$ equiv), THF (1.0 mL), and $\mathrm{CH}_{2} \mathrm{BrCl}(7.8 \mu \mathrm{L}, 0.12 \mathrm{mmol}, 1.2$ equiv). The vial was cooled to $-78^{\circ} \mathrm{C}$, and $n$-BuLi $(48 \mu \mathrm{L}, 2.5 \mathrm{M}$ solution in hexane, $0.12 \mathrm{mmol}, 1.2$ equiv) was added dropwise over $1 \mathrm{~min}$. The reaction mixture was allowed to stir at $-78{ }^{\circ} \mathrm{C}$ for $15 \mathrm{~min}$ and then at room temperature for $16 \mathrm{~h}$. The reaction was quenched with saturated $\mathrm{NH}_{4} \mathrm{Cl}(1.0 \mathrm{~mL})$ and diluted with $\mathrm{H}_{2} \mathrm{O}(2.0 \mathrm{~mL})$. The mixture was extracted with EtOAc $(4 \times 2 \mathrm{~mL})$, and the combined organic layers were dried over $\mathrm{Na}_{2} \mathrm{SO}_{4}$, concentrated, and purified by preparative thin layer chromatography (95:5 hexanes:EtOAc) to give boronic ester 7 (24.2 $\mathrm{mg}, 89 \%$ yield) as a colorless oil. 7: ${ }^{1} \mathbf{H}$ NMR (600 MHz, $\left.\mathrm{CDCl}_{3}\right): \delta 7.29(\mathrm{t}, J=7.4 \mathrm{~Hz}, 2 \mathrm{H}), 7.25(\mathrm{~d}, J=7.4 \mathrm{~Hz}, 2 \mathrm{H})$, $7.21(\mathrm{t}, J=7.3 \mathrm{~Hz}, 1 \mathrm{H}), 2.65(\mathrm{~s}, 2 \mathrm{H}), 1.27(\mathrm{~s}, 12 \mathrm{H}), 0.87(\mathrm{~s}, 2 \mathrm{H}), 0.47$ (q, $J=3.5,3.1 \mathrm{~Hz}, 2 \mathrm{H})$, $0.44(\mathrm{~d}, J=4.1 \mathrm{~Hz}, 2 \mathrm{H}) ;{ }^{13} \mathrm{C}$ NMR $\left(150 \mathrm{MHz}, \mathrm{CDCl}_{3}\right): \delta 140.83,129.69,128.11,125.95,83.01$, 45.18, 25.01, 24.69, 17.69, 12.36; HRMS-ESI $(\mathrm{m} / \mathrm{z})[\mathrm{M}+\mathrm{H}]^{+}$calcd for $\mathrm{C}_{17} \mathrm{H}_{25} \mathrm{BO}_{2}, 272.2062$; found 272.2065 .

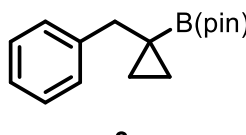

$3 a$

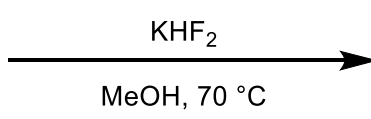

$(81 \%$ yield $)$

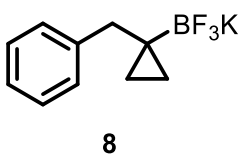

Trifluoroborate 8. Trifluoroborate 8 was prepared according to a modified procedure. ${ }^{8}$ A flamedried 100-mL round-bottom flask with a stir bar was charged with $\mathrm{KHF}_{2}(2.79 \mathrm{~g}, 35.8$ mmol, 7.0 equiv), $\mathrm{MeOH}(40 \mathrm{~mL})$, and boronic ester 3a (1.31 g, $5.11 \mathrm{mmol}, 1.0$ equiv). The mixture was heated to $70{ }^{\circ} \mathrm{C}$ and allowed to stir vigorously for $4 \mathrm{~h}$. The reaction mixture was then cooled to room temperature and concentrated. The remaining solids were triturated with a heptane: $\mathrm{Et}_{2} \mathrm{O}$ (4:1) solvent mixture $(60 \mathrm{~mL})$ for $15 \mathrm{~min}$. The solids were collected by filtration, and further washed with cold $\mathrm{Et}_{2} \mathrm{O}(30 \mathrm{~mL})$. The heptane $/ \mathrm{Et}_{2} \mathrm{O}$ filtrate was then discarded. The precipitate was then washed with hot MeCN $(300 \mathrm{~mL})$, and the filtrate was collected in a clean roundbottom flask. Upon concentration trifluoroborate 8 (988 $\mathrm{mg}, 81 \%$ yield) was obtained as a white solid. 8: ${ }^{1} \mathbf{H}$ NMR (600 MHz, DMSO- $\left.d_{6}\right) \delta 7.15$ (t, $\left.J=7.5 \mathrm{~Hz}, 2 \mathrm{H}\right), 7.10-7.07$ (m, 2H), 7.07- 
$7.03(\mathrm{~m}, 1 \mathrm{H}), 2.53(\mathrm{~s}, 2 \mathrm{H}), 0.01(\mathrm{q}, J=2.6 \mathrm{~Hz}, 2 \mathrm{H}),-0.42(\mathrm{~s}, 2 \mathrm{H}) ;{ }^{13} \mathbf{C}$ NMR $(150 \mathrm{MHz}, \mathrm{DMSO}-$ $\left.d_{6}\right) \delta 142.54,129.89,127.00,124.44,40.73,6.70$. HRMS-ESI $(m / z)[\mathrm{M}-\mathrm{K}]^{+}$calcd for $\mathrm{C}_{10} \mathrm{H}_{11} \mathrm{BF}_{3}, 198.0942$; found 198.0942 .

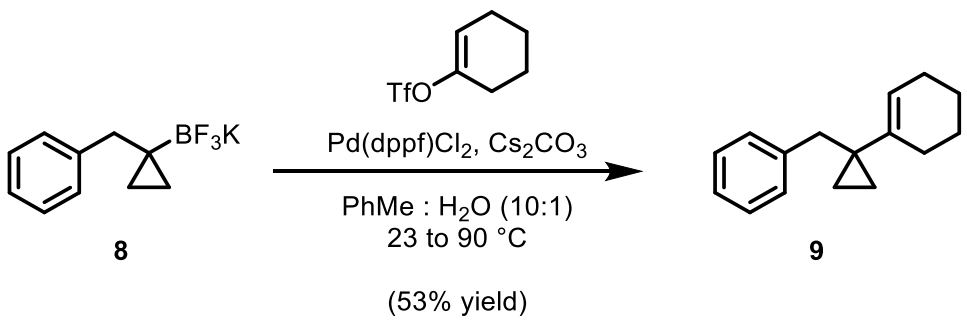

Representative Procedure for Suzuki Couplings.

Suzuki product 9. Suzuki product 9 was prepared according to a modified procedure. ${ }^{9}$ A $10-\mathrm{mL}$ reaction vial equipped with a magnetic stir bar was charged with trifluoroborate $\mathbf{8}(23.8 \mathrm{mg}, 0.10$ mmol, 1.0 equiv), $\mathrm{Pd}(\mathrm{dppf}) \mathrm{Cl}_{2}\left(7.3 \mathrm{mg}, 0.01 \mathrm{mmol}, 0.1\right.$ equiv), $\mathrm{Cs}_{2} \mathrm{CO}_{3}$ (97.8 mg, $0.30 \mathrm{mmol}$, 3.0 equiv), alkenyl triflate (46.0 mg, $0.20 \mathrm{mmol}, 2.0$ equiv), toluene (2.5 mL), and $\mathrm{H}_{2} \mathrm{O}(250 \mu \mathrm{L})$. The vial was capped and purged with $\mathrm{N}_{2}$ for $10 \mathrm{~min}$ at room temperature. The reaction vial was then placed in an aluminum heating block, which was kept at $90{ }^{\circ} \mathrm{C}$ for $15 \mathrm{~h}$. The reaction vial was then cooled to room temperature, and the crude mixture filtered through a pad of silica gel that was subsequently rinsed with 1:1 hexanes:EtOAc $(15 \mathrm{~mL})$. The filtrate was concentrated, and the resulting crude mixture was purified by preparative thin layer chromatography (hexanes) to provide Suzuki product $9\left(11.3 \mathrm{mg}, 53 \%\right.$ yield) as a colorless oil. 9: ${ }^{1} \mathbf{H}$ NMR (600 MHz, $\left.\mathrm{CDCl}_{3}\right): \delta 7.23(\mathrm{t}, J=7.5 \mathrm{~Hz}, 2 \mathrm{H}), 7.16(\mathrm{t}, J=7.3 \mathrm{~Hz}, 1 \mathrm{H}), 7.13(\mathrm{~d}, J=7.5 \mathrm{~Hz}, 2 \mathrm{H}), 5.29$ (s, 1H), 2.67 (s, 2H), 2.02-1.99 (m, 2H), 1.89-1.85 (m, 2H), 1.57 (dd, J= 7.6, 4.3 Hz, 2H), 1.49$1.45(\mathrm{~m}, 2 \mathrm{H}), 0.54(\mathrm{~d}, J=4.1 \mathrm{~Hz}, 2 \mathrm{H}), 0.49(\mathrm{~d}, J=3.7 \mathrm{~Hz}, 2 \mathrm{H}) ;{ }^{13} \mathbf{C} \mathbf{N M R}\left(150 \mathrm{MHz}, \mathrm{CDCl}_{3}\right): \delta$ $140.68,139.08,129.45,127.84,125.83,123.58,42.68,27.70,27.05,25.36,23.27,22.80,11.41$; HRMS-ESI $(m / z)[\mathrm{M}+\mathrm{H}]^{+}$calcd for $\mathrm{C}_{16} \mathrm{H}_{20}, 213.1643$; found 213.1639. 


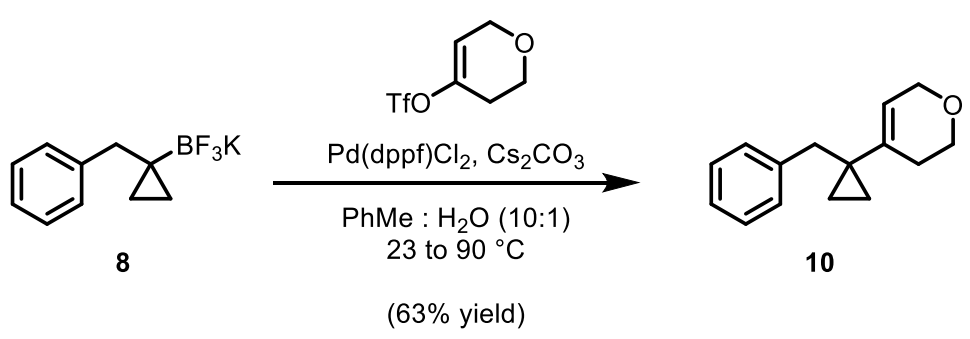

Suzuki product 10. Purification by preparative thin layer chromatography (98:2 hexanes:EtOAc) afforded Suzuki product 10 (13.4 mg, 63\% yield) as a colorless oil. 10: ${ }^{1} \mathbf{H}$ NMR $\left(600 \mathrm{MHz}, \mathrm{CDCl}_{3}\right): \delta 7.26-7.23(\mathrm{~m}, 2 \mathrm{H}), 7.17(\mathrm{dd}, J=20.4,7.4 \mathrm{~Hz}, 3 \mathrm{H}), 5.30(\mathrm{~s}, 1 \mathrm{H})$, $4.02(\mathrm{~d}, J=2.7 \mathrm{~Hz}, 2 \mathrm{H}), 3.72(\mathrm{t}, J=5.5 \mathrm{~Hz}, 2 \mathrm{H}), 2.72(\mathrm{~s}, 2 \mathrm{H}), 2.10$ (s, 2H), 0.63 (q, $J=4.2 \mathrm{~Hz}$, $2 \mathrm{H}), 0.57$ (q, $J=4.1 \mathrm{~Hz}, 2 \mathrm{H}) ;{ }^{13} \mathbf{C}$ NMR $\left(150 \mathrm{MHz}, \mathrm{CDCl}_{3}\right): \delta 140.11,137.37,129.31,128.05$, 126.07, 121.84, 65.65, 64.58, 42.22, 27.06, 26.65, 11.38; HRMS-ESI $(\mathrm{m} / \mathrm{z})[\mathrm{M}+\mathrm{H}]^{+}$calcd for $\mathrm{C}_{15} \mathrm{H}_{18} \mathrm{O}, 215.1436$; found 215.1436.

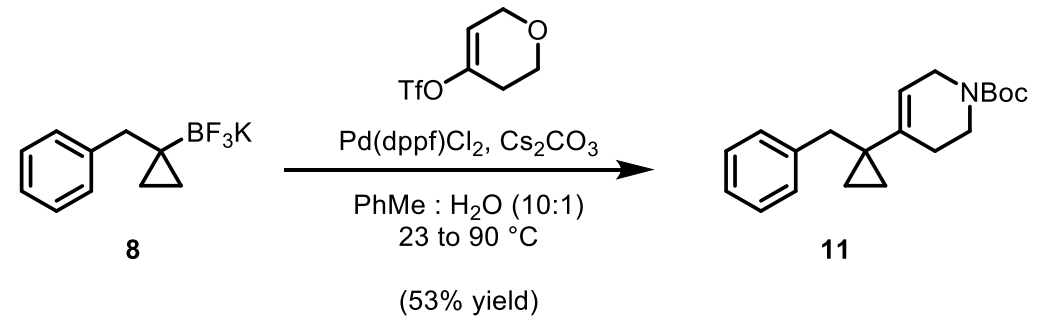

Suzuki product 11. Purification by preparative thin layer chromatography (95:5 hexanes:EtOAc) afforded Suzuki product 11 (16.4 mg, 53\% yield) as a colorless oil. 11: ${ }^{1} \mathbf{H}$ $\operatorname{NMR}\left(600 \mathrm{MHz}, \mathrm{CDCl}_{3}\right): \delta 7.24(\mathrm{t}, J=7.5 \mathrm{~Hz}, 2 \mathrm{H}), 7.17(\mathrm{t}, J=7.5 \mathrm{~Hz}, 1 \mathrm{H}), 7.12(\mathrm{~d}, J=7.2 \mathrm{~Hz}$, $2 \mathrm{H}), 5.22(\mathrm{~s}, 1 \mathrm{H}), 3.74(\mathrm{~s}, 2 \mathrm{H}), 3.41(\mathrm{~s}, 2 \mathrm{H}), 2.70(\mathrm{~s}, 2 \mathrm{H}), 2.09(\mathrm{~s}, 2 \mathrm{H}), 1.45(\mathrm{~s}, 9 \mathrm{H}), 0.57(\mathrm{~d}, J=$ $4.1 \mathrm{~Hz}, 2 \mathrm{H}) ;{ }^{13} \mathbf{C}$ NMR $\left(150 \mathrm{MHz}, \mathrm{CDCl}_{3}\right): \delta 155.10,140.08,138.38,129.30,128.05,126.09$, 120.08, 79.52, 43.63, 42.43, 41.26, 40.04, 28.62, 26.96, 11.44; HRMS-ESI $(\mathrm{m} / \mathrm{z})[\mathrm{M}+\mathrm{Na}]^{+}$ calcd for $\mathrm{C}_{20} \mathrm{H}_{27} \mathrm{NO}_{2}, 336.1939$; found 336.1941 . 


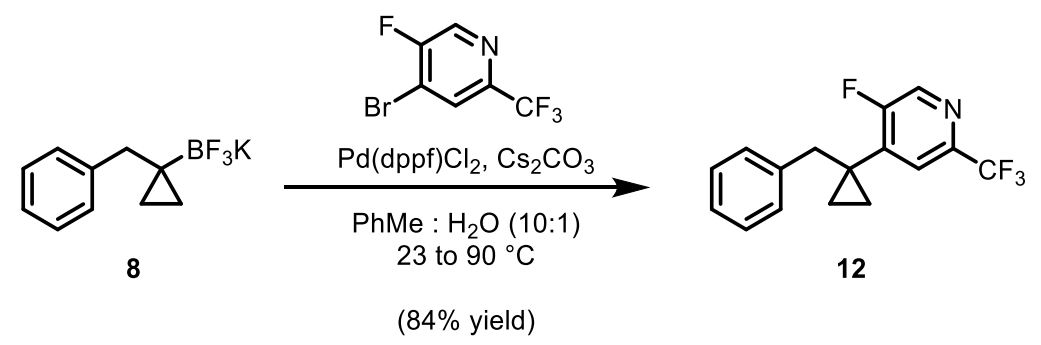

Suzuki product 12. Purification by preparative thin layer chromatography ( $4: 1$ hexanes:EtOAc) afforded Suzuki product $12\left(24.9 \mathrm{mg}, 84 \%\right.$ yield) as a colorless oil. 12: ${ }^{1} \mathbf{H}$ NMR (600 MHz, $\left.\mathrm{CDCl}_{3}\right): \delta 8.31(\mathrm{~s}, 1 \mathrm{H}), 7.46(\mathrm{dd}, J=8.5,2.5 \mathrm{~Hz}, 1 \mathrm{H}), 7.19(\mathrm{q}, J=7.4,6.8 \mathrm{~Hz}, 3 \mathrm{H}), 6.95-6.90$ (m, 2H), $2.90(\mathrm{~s}, 2 \mathrm{H}), 1.05-1.00(\mathrm{~m}, 2 \mathrm{H}), 0.89-0.86(\mathrm{~m}, 2 \mathrm{H}) ;{ }^{13} \mathbf{C}$ NMR $\left(150 \mathrm{MHz}, \mathrm{CDCl}_{3}\right): \delta$ $164.70(\mathrm{~d}, J=247.4 \mathrm{~Hz}), 143.30(\mathrm{dd}, J=17.3,4.6 \mathrm{~Hz}), 139.96(\mathrm{dd}, J=7.1,3.5 \mathrm{~Hz}), 138.41$, 129.30, 128.39, 127.65 (d, $J=28.7 \mathrm{~Hz}), 126.80,124.67$ (qd, $J=33.4,4.6 \mathrm{~Hz}), 123.10$ (q, $J=$ $271.7 \mathrm{~Hz}), 44.01,22.82(\mathrm{~d}, J=3.6 \mathrm{~Hz}), 11.98$; HRMS-ESI $(\mathrm{m} / \mathrm{z})[\mathrm{M}+\mathrm{H}]^{+}$calcd for $\mathrm{C}_{16} \mathrm{H}_{13} \mathrm{~F}_{4} \mathrm{~N}$, 296.1062; found 296.1064 .

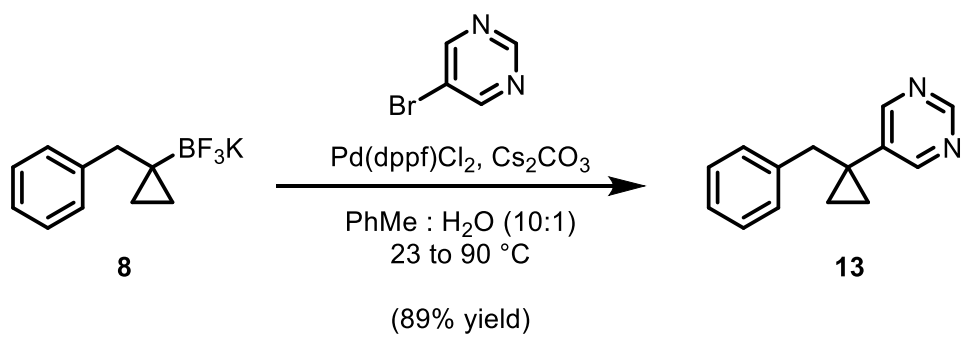

Suzuki product 13. Purification by preparative thin layer chromatography (4:1 hexanes:EtOAc) afforded Suzuki product 13 (18.7 mg, 89\% yield) as a colorless oil. 13: ${ }^{1} \mathbf{H}$ NMR (600 MHz, $\mathrm{CDCl}_{3}$ ): $\delta 8.99$ (s, 1H), 8.43 (s, 2H), $7.20(\mathrm{dq}, J=14.1,7.0 \mathrm{~Hz}, 3 \mathrm{H}), 6.99(\mathrm{~d}, J=7.1 \mathrm{~Hz}, 2 \mathrm{H})$, $2.92(\mathrm{~s}, 2 \mathrm{H}), 1.06-1.00(\mathrm{~m}, 2 \mathrm{H}), 0.92(\mathrm{~d}, J=5.7 \mathrm{~Hz}, 2 \mathrm{H}) ;{ }^{13} \mathbf{C}$ NMR $\left(150 \mathrm{MHz}, \mathrm{CDCl}_{3}\right): \delta$ $157.82,156.58,138.35,138.04,129.32,128.52,126.80,45.16,22.69,12.26$; HRMS-ESI $(\mathrm{m} / \mathrm{z})$ $[\mathrm{M}+\mathrm{H}]^{+}$calcd for $\mathrm{C}_{14} \mathrm{H}_{14} \mathrm{~N}_{2}, 211.1235$; found 211.1239 . 


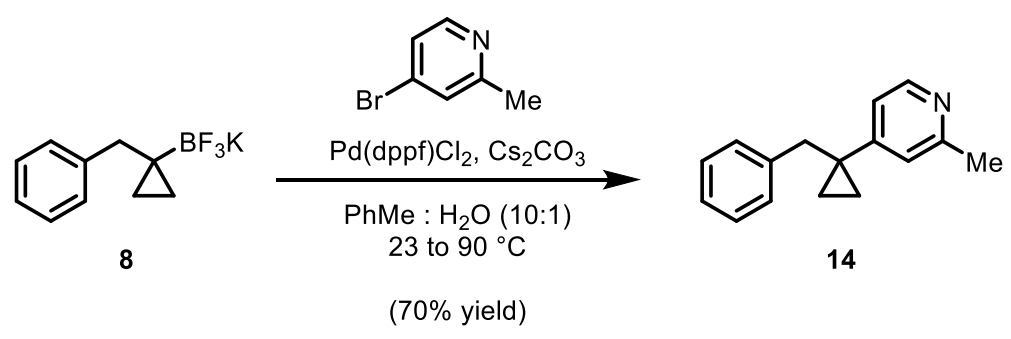

Suzuki product 14. Purification by preparative thin layer chromatography $4: 1$ hexanes:EtOAc) afforded Suzuki product 14 (15.7 mg, 70\% yield) as a colorless oil. 14: ${ }^{1} \mathbf{H}$ NMR $\left(600 \mathrm{MHz}, \mathrm{CDCl}_{3}\right): \delta 8.28(\mathrm{~d}, J=5.3 \mathrm{~Hz}, 1 \mathrm{H}), 7.23-7.12(\mathrm{~m}, 3 \mathrm{H}), 7.05(\mathrm{~d}, J=6.8 \mathrm{~Hz}, 2 \mathrm{H})$, 6.95-6.78 (m, 2H), $3.00(\mathrm{~s}, 2 \mathrm{H}), 2.46(\mathrm{~s}, 3 \mathrm{H}), 0.94(\mathrm{~s}, 4 \mathrm{H}) ;{ }^{13} \mathbf{C}$ NMR $\left(150 \mathrm{MHz}, \mathrm{CDCl}_{3}\right): \delta$ 158.06, 154.69, 148.84, 139.02, 129.30, 128.22, 126.35, 122.67, 120.45, 43.66, 24.62, 24.51, 14.25; HRMS-ESI $(\mathrm{m} / \mathrm{z})[\mathrm{M}+\mathrm{H}]^{+}$calcd for $\mathrm{C}_{16} \mathrm{H}_{17} \mathrm{~N}, 224.1439$; found 224.1444. 


\section{Computational Methods}

All DFT calculations were performed with Gaussian $16 .{ }^{10}$ Geometry optimizations were performed in the gas phase with the M06L functional ${ }^{11}$ with a mixed basis set of LANL2DZ for $\mathrm{Cu}$ and 6-31G(d) for other atoms. Single point calculations were performed at the M06 level of theory with a mixed basis set of SDD for $\mathrm{Cu}$ and $6-311+\mathrm{G}(\mathrm{d}, \mathrm{p})$ for other atoms in THF using the SMD solvation model. ${ }^{12}$ Gibbs free energies were calculated at the standard conditions (298K, 1 $\mathrm{mol} / \mathrm{L}$ ) using Cramer and Truhlar's quasiharmonic approximation ${ }^{13}$ using Goodvibes v2.0.1. ${ }^{14}$ Images of structures were generated using CYLview. ${ }^{15}$

\section{Additional Computational Results}

The proposed mechanisms of the Cu-catalyzed hydroboration of benzylidenecyclopropane $(\mathrm{BCP})$ are presented in Scheme S1. The $[\mathrm{L}] \mathrm{Cu}(\mathrm{I})$ - OR complex first undergoes borylation with $\mathrm{B}_{2}$ (pin) $)_{2}$ to form the $[\mathrm{L}] \mathrm{Cu}(\mathrm{I})-\mathrm{B}(\mathrm{pin})$ intermediate IM2. Subsequent migratory insertion of the BCP substrate 1a into Cu-Bpin forms benzylic copper(I) complex 2, a key common intermediate to form both alkenyl boronates (4a) and boronic esters (3a). The two different products are generated through distinct transition states. Protodecupration (TS2) of 2 releases the boronic ester product 3a and regenerates IM1. Conversely, $\beta$-carbon elimination (TS1) may take place to cleave the cyclopropane ring. A subsequent protodecupration (TS3) would then form the alkenyl boronate product $4 \mathbf{a}$. 
Scheme S1. Proposed reaction mechanisms.

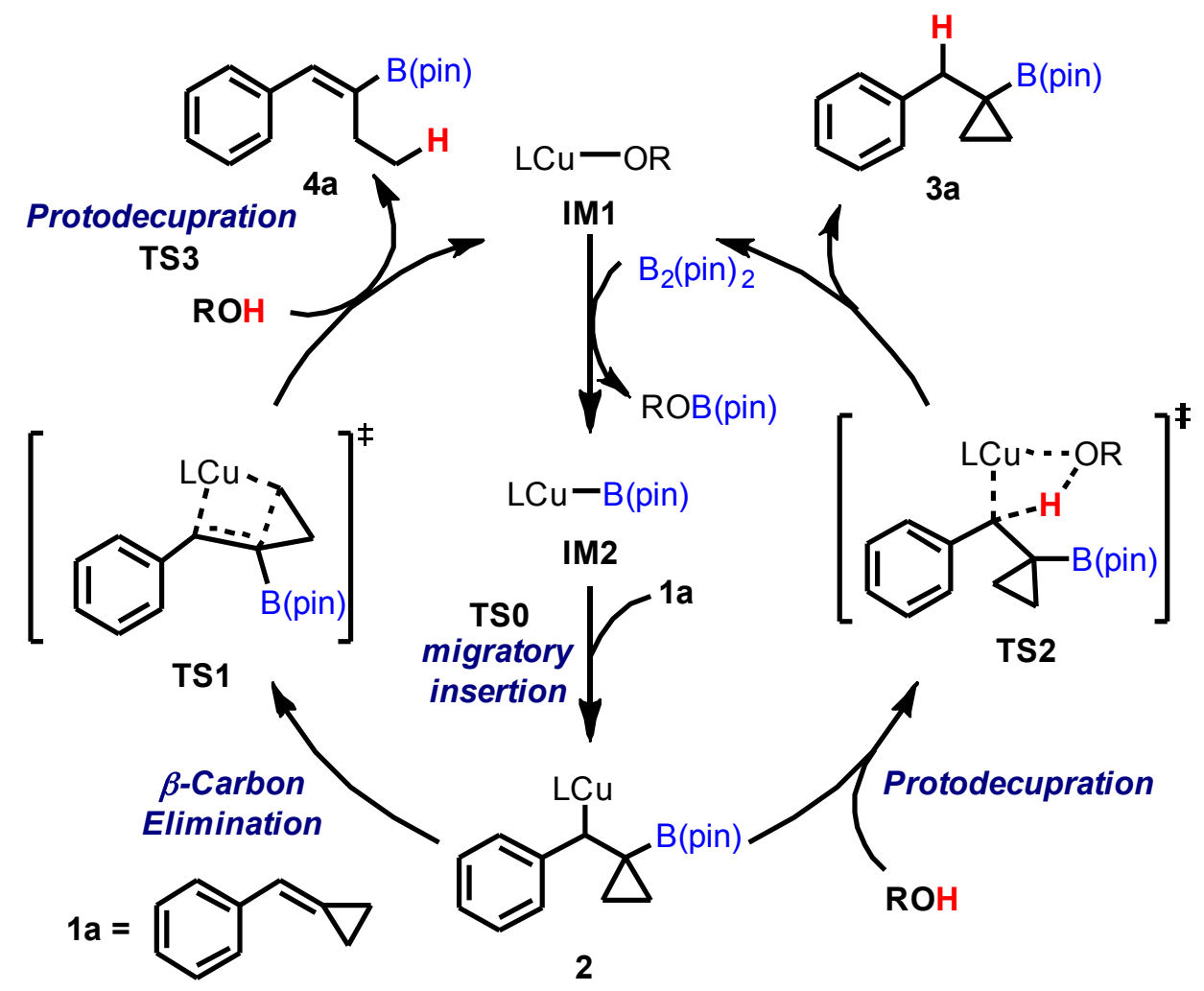

The computed energy profiles for the two competing pathways with the (S)-BINAP and dppe-supported $\mathrm{Cu}(\mathrm{I})-\mathrm{B}($ pin) complexes are shown in Figure $\mathrm{S} 1$. In reactions using both $(S)$ BINAP and dppe ligands, the key intermediates $\mathbf{1 6}$ and $\mathbf{2 0}$ are generated via an irreversible BCP migratory insertion (TS4 and TS6). In reaction with the (S)-BINAP ligand, the Cu-Bpin complex preferentially adds to the $R e$-face of the $\mathrm{BCP}$ double bond, leading to the $(R)$-benzylic $\mathrm{Cu}(\mathrm{I})$ complex 16. Addition to the opposite face of $\mathrm{BCP}$ (vide infra) requires higher barrier. Nonetheless, this newly formed benzylic chiral center is ablated in subsequent steps, eventually leading to achiral products in both pathways depicted in Scheme S1. The benzylic $\mathrm{Cu}(\mathrm{I})$ intermediates $\mathbf{1 6}$ and $\mathbf{2 0}$ adopt a distorted tetrahedral conformation with one of the oxygen atoms on Bpin datively bonded onto the $\mathrm{Cu}$ center, where the distortion from the tetrahedral geometry is more substantial in the $(S)$-BINAP-bound species 16. From the dppe-supported complex 20, the $\beta$-carbon elimination transition state (TS1a) is more favorable over protodecupration (TS2a) by $3.2 \mathrm{kcal} / \mathrm{mol}$, leading to the formation of the alkenyl boronate product $4 \mathrm{a}$. The most favorable $\beta$-carbon elimination transition state (TS1a) places the Ph and Bpin groups on the opposite sides 
of the double bond, leading to the experimentally observed $Z$ stereoisomer of the alkenyl boronate. Another $\beta$-carbon elimination transition state (TS8), which would eventually lead to the $E$-stereoisomer of the alkenyl boronate, is $3.5 \mathrm{kcal} / \mathrm{mol}$ less stable than TS1a.

In contrast, the (S)-BINAP-supported complex 16 favors the protodecupration (TS2b) over $\beta$-carbon elimination (TS1b) by $3.2 \mathrm{kcal} / \mathrm{mol}$, leading to the formation of the boronic ester. This less favorable $\beta$-carbon elimination transition state, TS1b, would eventually lead to the $Z$-alkenyl boronate. The $\beta$-carbon elimination to form the $E$-double bond is expected to be even less favorable, because of the steric repulsions between Ph and BPin groups. However, after multiple attempts, this $\beta$-carbon elimination transition state still cannot be located, because of the unfavorable steric interactions in this transition state.

Overall, the computational studies indicated the hydroboration reaction with the dppesupport $\mathrm{Cu}$ catalyst favors the $Z$-alkenyl boronate $\mathbf{4 a}$, while the use of BINAP-supported $\mathrm{Cu}$ catalyst leads to the cyclopropylboronic ester 3a. This reversal of product selectivity is in good agreement with experiment. 

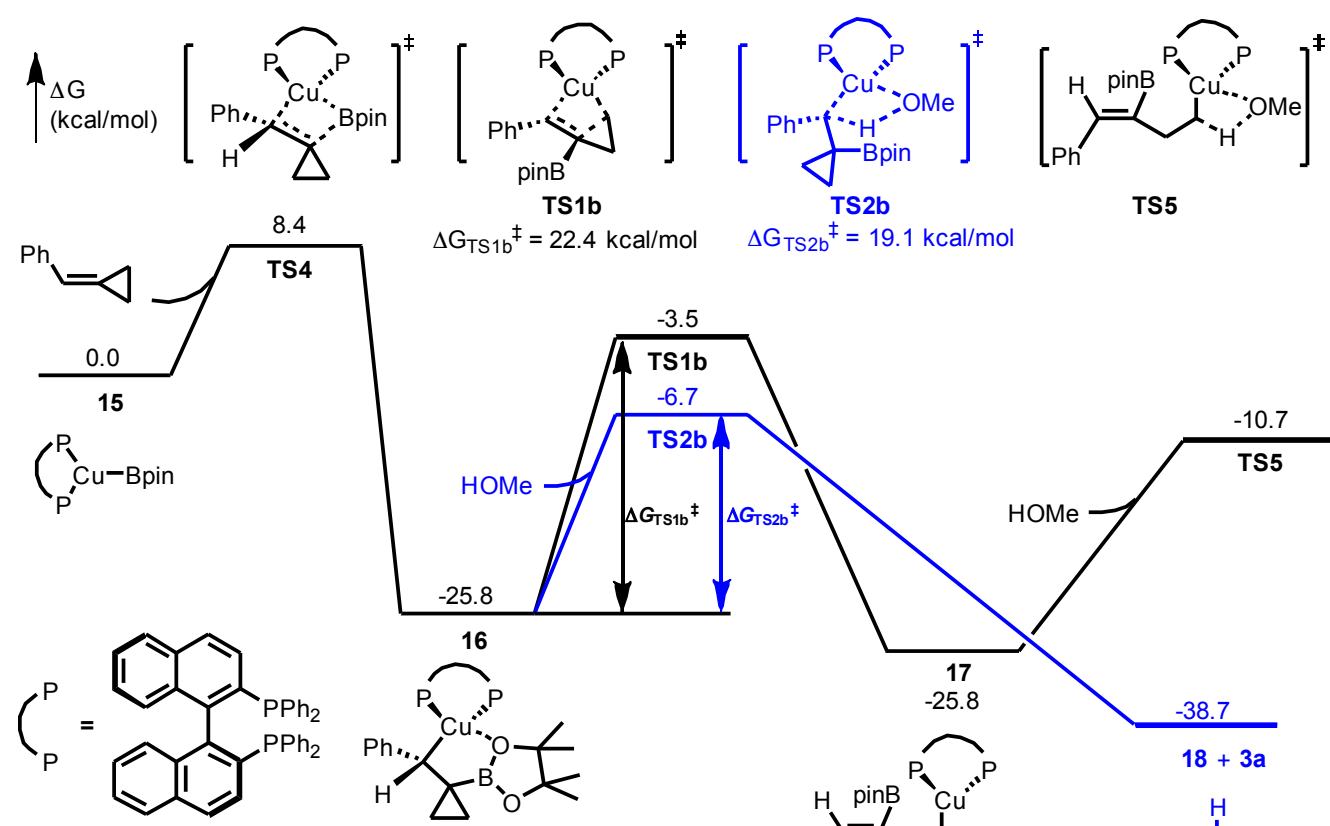

(S)-BINAP

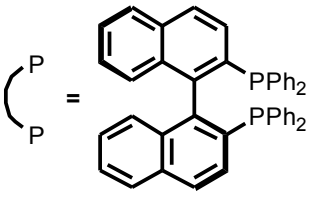

Bpin

$\int_{P^{\prime}}^{P^{\prime}}$ Cu-Bpin

$\zeta_{\mathrm{P}}^{\mathrm{P}}=\underbrace{\mathrm{PPh}_{2}}_{\mathrm{DPPE}}$

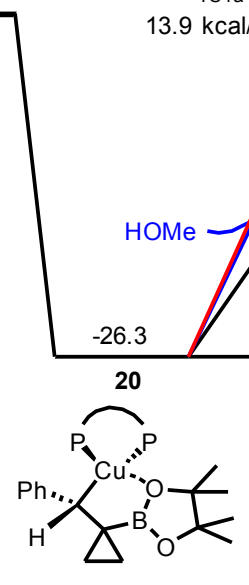

$\Delta \mathrm{G}_{\mathrm{TS} 2 \mathrm{~b}}{ }^{\ddagger}=19.1 \mathrm{kcal} / \mathrm{mol}$

TS5
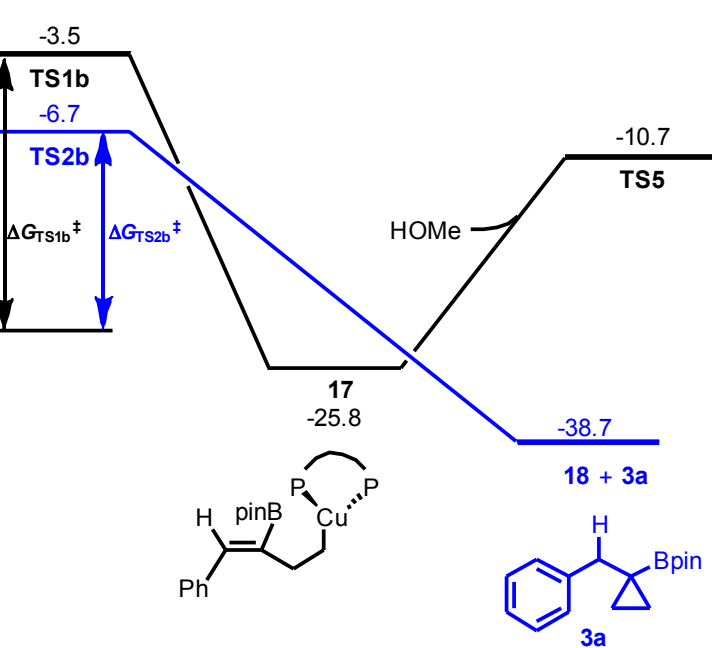

$\left(_{P^{\prime}}^{\mathrm{P}} \mathrm{Cu}-\mathrm{OMe}\right.$

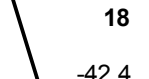

$-42.4$

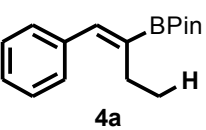

-8.9
TS8

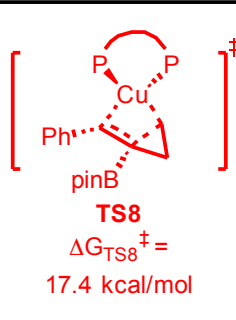

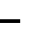

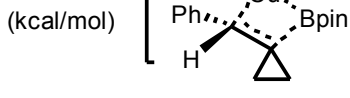

$\stackrel{P h}{\longrightarrow}$

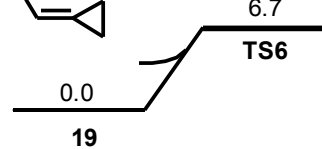

$\Delta \mathrm{G}_{\mathrm{TS} 1 \mathrm{a}}{ }^{\ddagger}=$

$13.9 \mathrm{kcal} / \mathrm{mol}$
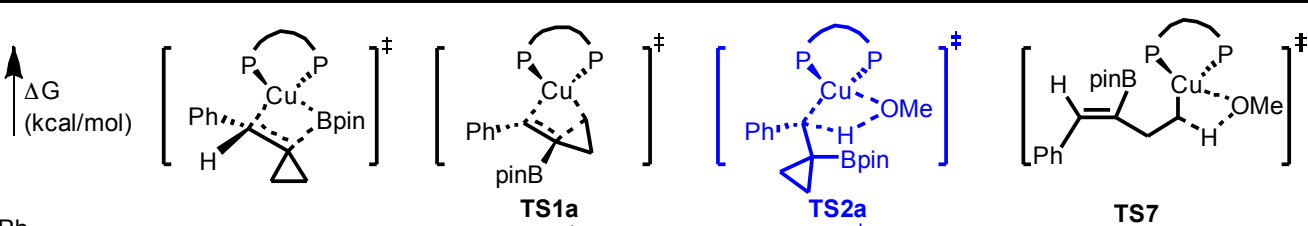

$\Delta \mathrm{G}_{\mathrm{TS} 2 \mathrm{a}}^{\ddagger}=$

$17.1 \mathrm{kcal} / \mathrm{mol}$

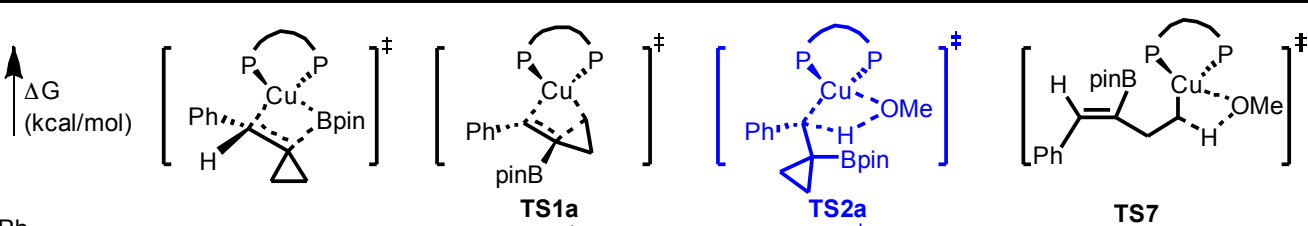

TS7$$
\text { (7.1 }
$$

$-8.9$

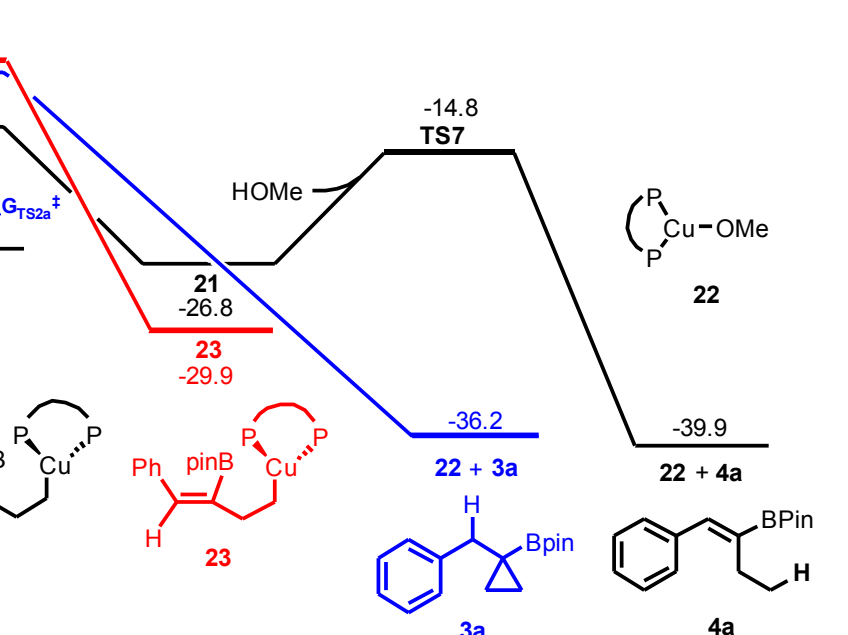

$3 a$

Figure S1. Computed reaction energy profiles of the hydroboration of benzylidenecycloprane 1 with $(S)$-BINAP (top) and dppe (bottom)-supported $\mathrm{Cu}$ catalysts. 
We also considered the alternative pathway with the (S)-BINAP ligand that initiates via the $\mathrm{Cu}$ Bpin complex adding to the opposite face of the BCP $\pi$ bond. The computed energy profile in this pathway is shown in Figure S2. The calculations suggest this pathway is much less favorable because the initial migratory insertion (TS1BINAP2) is much less favorable than the competing pathway via TS4.

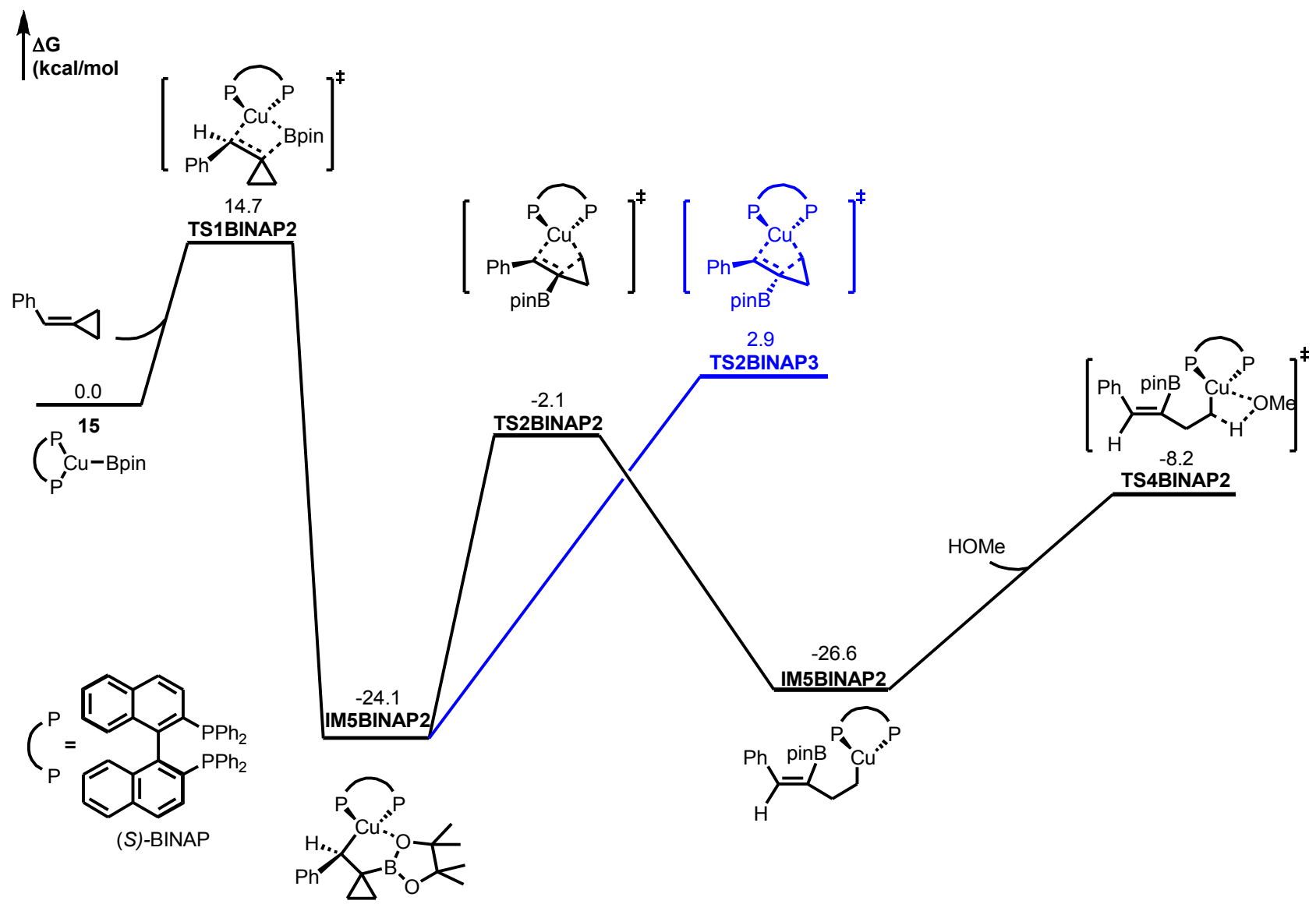

Figure S2. Alternative diastereomeric TS with the $(S)$-BINAP-supported Cu catalyst. 
Two $\beta$-carbon elimination transition states with (S)-BINAP as ligand were located (Figure S3). In the more favorable transition state TS1b, the $\gamma$ - and benzylic carbons (highlighted in pink) are placed in the less occupied quadrants II and IV. In TS1b', the $\gamma$ - and benzylic carbons are placed in the more occupied quadrants I and III. Therefore, greater steric repulsions with the ligand are observed in TS1b'.
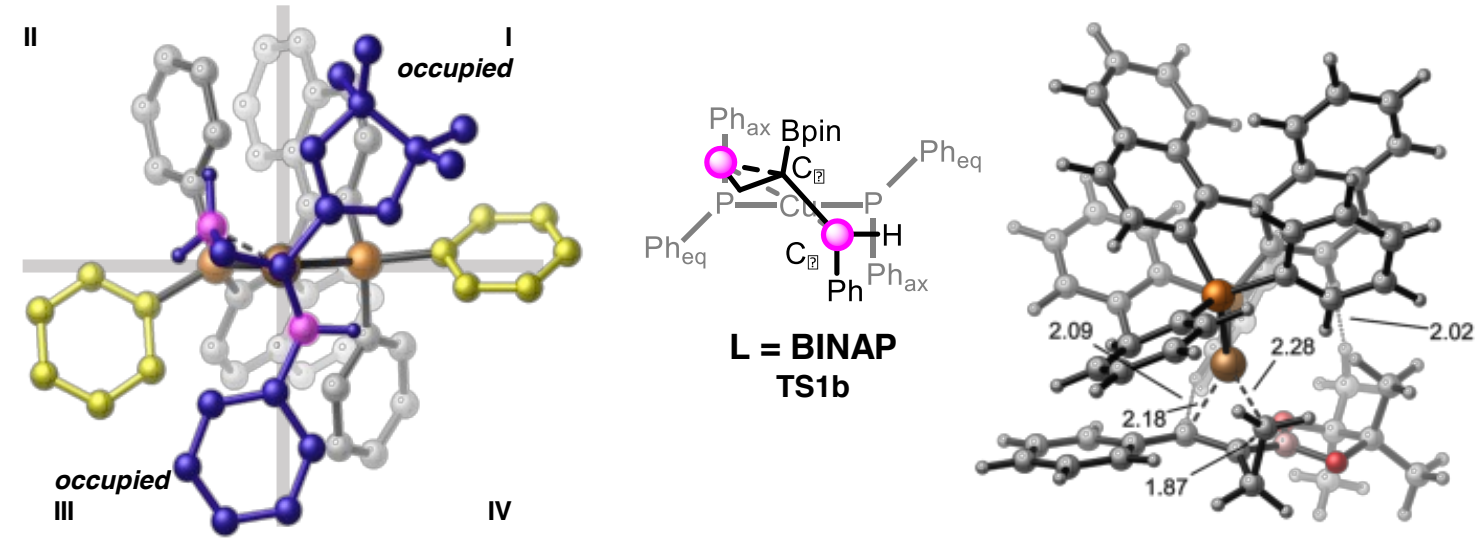

" $\mathrm{G}^{\mathrm{fl}}=22.4 \mathrm{kcal} / \mathrm{mol}$
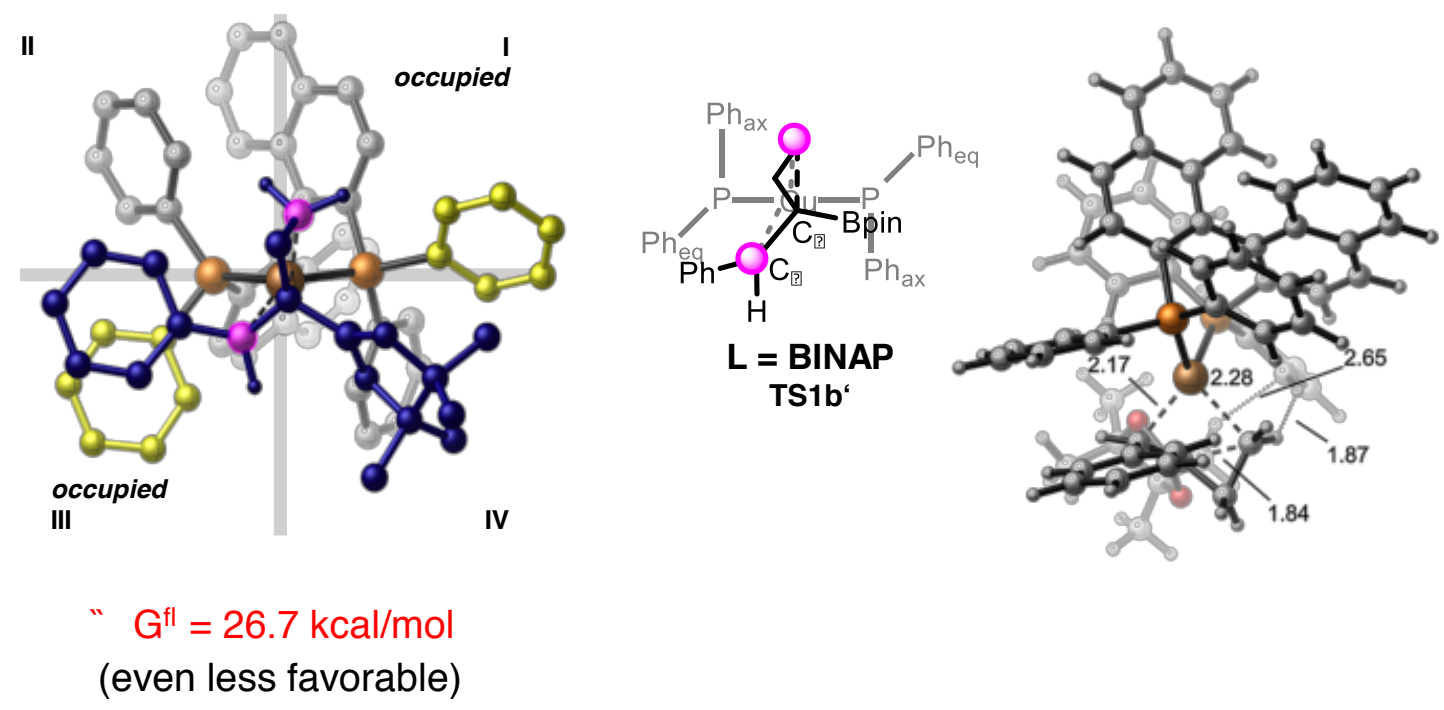

(even less favorable)

Figure S3. $\beta$-Carbon elimination transition states for $(S)$-BINAP.

The optimized geometries of the protodecupration transition states with both dppe and BINAP ligands (TS2a and TS2b, respectively) are shown in Figure S4. These transition states are less crowded than the $\beta$-carbon elimination transition states due to a smaller substrate bite 
angle. Therefore, both TS2a and TS2b experience smaller ligand-substrate steric repulsions and are less distorted from the tetrahedral geometry.
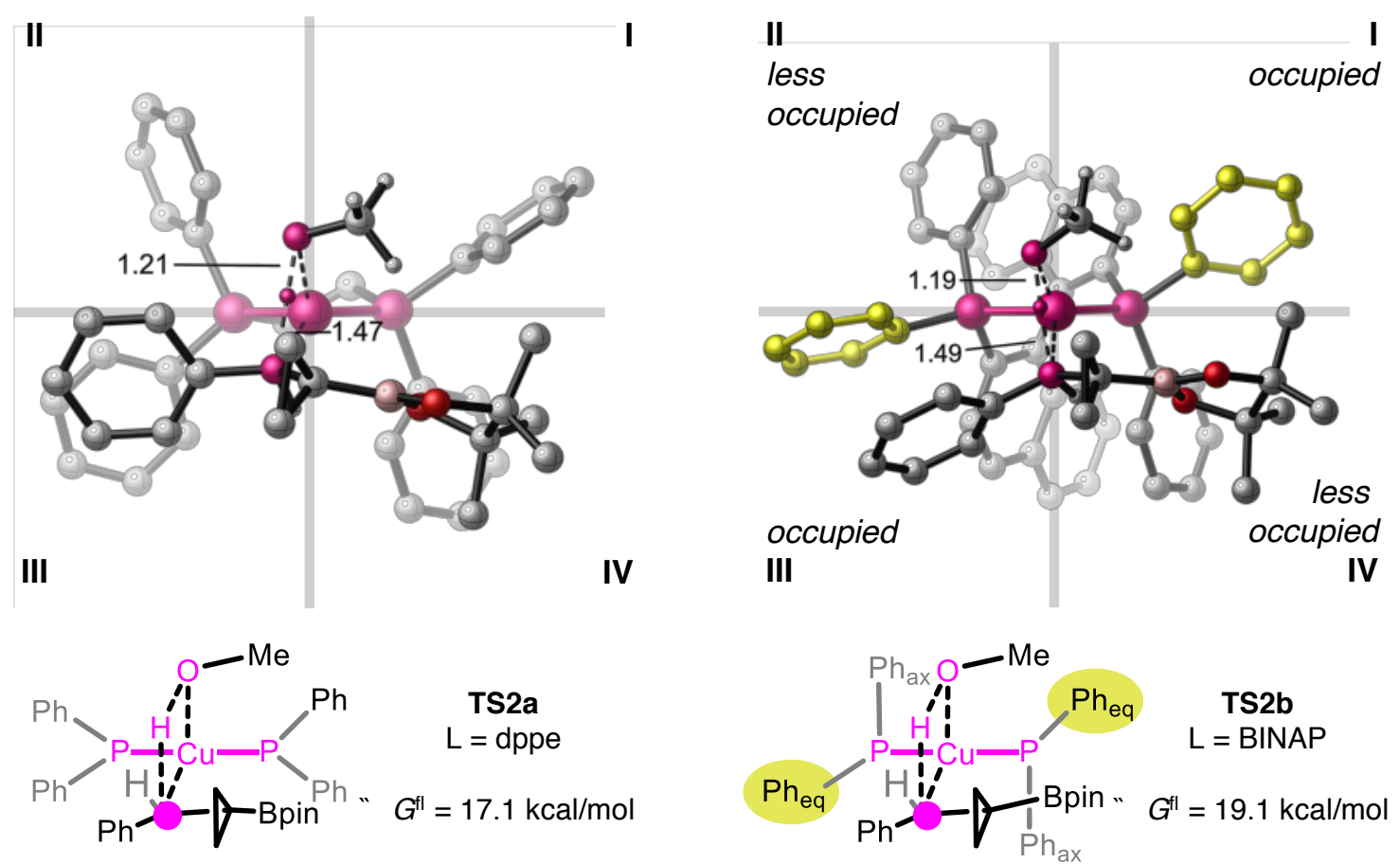

Figure S4. Protodecupration transition states. 


\section{Cartesian Coordinates}

Cartesian coordinates of the structures shown in Figure S1:

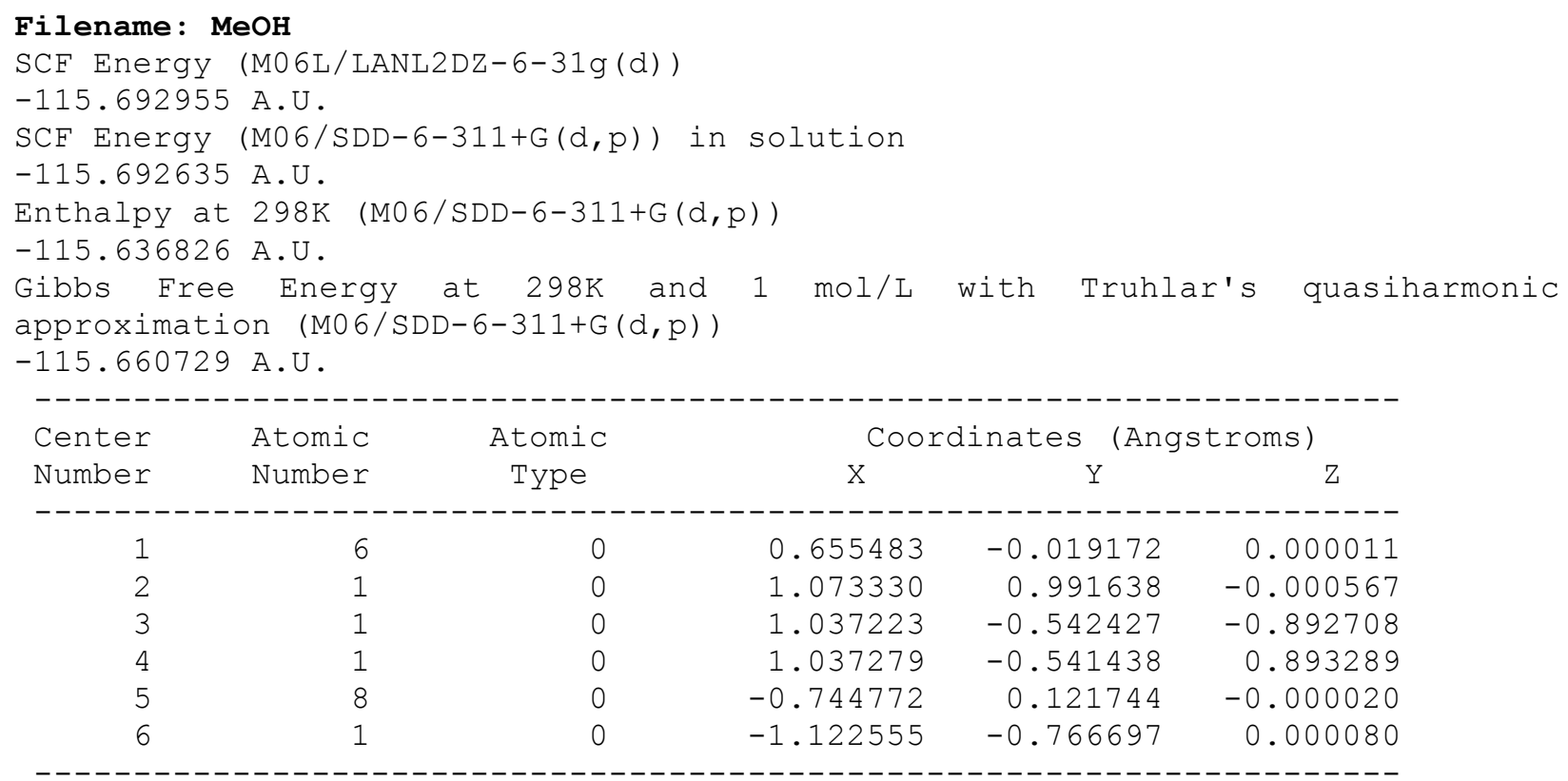




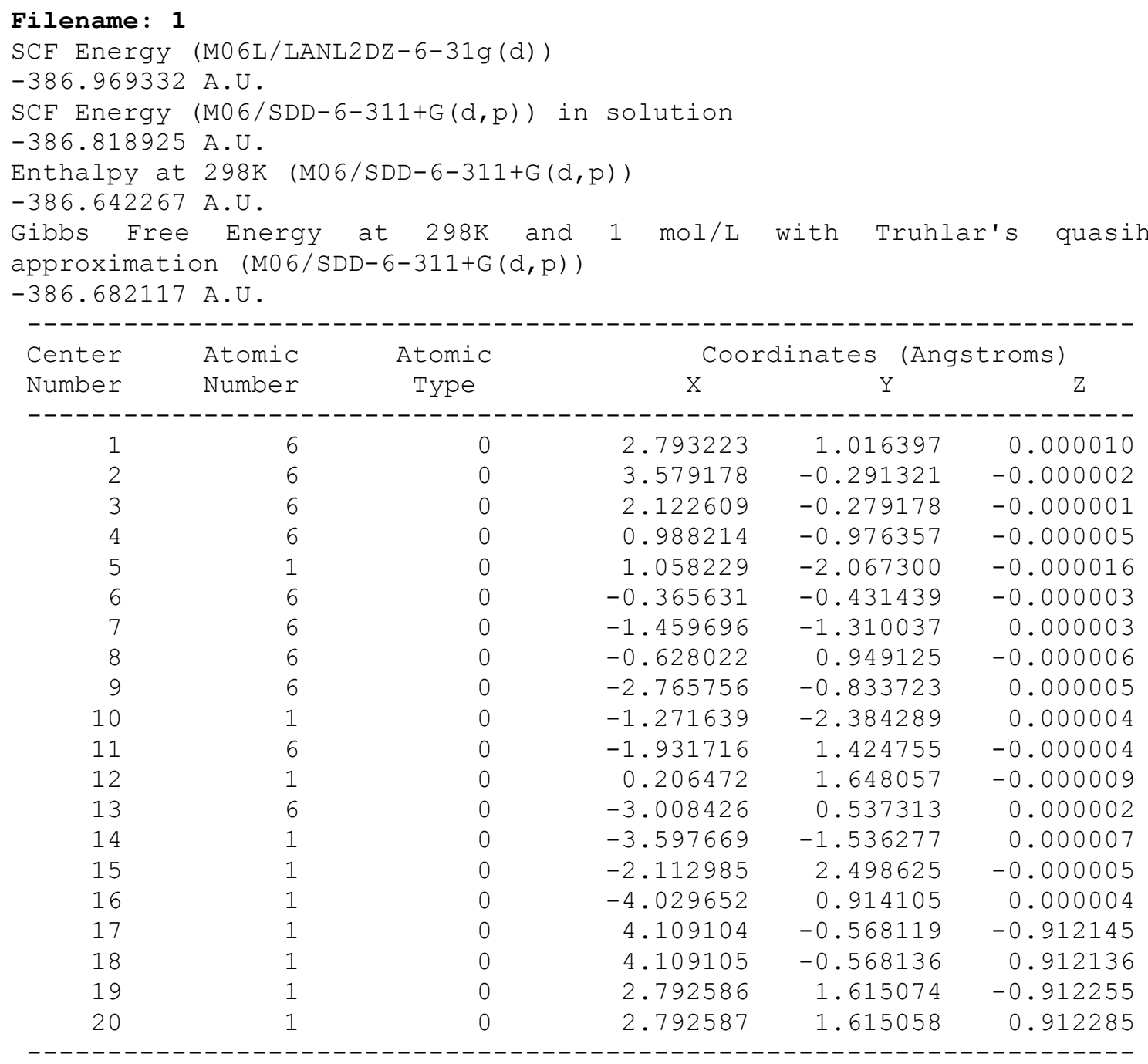


Filename: 2

SCF Energy (M06L/LANL2DZ-6-31g (d))

-798.849867 A.U.

$\mathrm{SCF}$ Energy (M06/SDD-6-311+G(d,p)) in solution

-798.605633 A.U.

Enthalpy at $298 \mathrm{~K}(\mathrm{M} 06 / \mathrm{SDD}-6-311+\mathrm{G}(\mathrm{d}, \mathrm{p}))$

-798.221136 A.U.

Gibbs Free Energy at $298 \mathrm{~K}$ and 1 mol/L with Truhlar's quasiharmonic approximation (M06/SDD-6-311+G(d,p))

-798.282519 A.U.

\begin{tabular}{|c|c|c|c|c|c|}
\hline \multirow{2}{*}{$\begin{array}{l}\text { Center } \\
\text { Number }\end{array}$} & \multirow{2}{*}{$\begin{array}{l}\text { Atomic } \\
\text { Number }\end{array}$} & \multirow{2}{*}{$\begin{array}{c}\text { Atomic } \\
\text { Type }\end{array}$} & \multicolumn{3}{|c|}{ Coordinates (Angstroms) } \\
\hline & & & $\mathrm{X}$ & $\mathrm{Y}$ & $\mathrm{Z}$ \\
\hline & & & 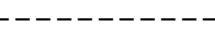 & ---------- & --------- \\
\hline 1 & 6 & 0 & -1.394919 & -0.468984 & 0.031782 \\
\hline 2 & 1 & 0 & -0.983311 & -1.462711 & 0.232762 \\
\hline 3 & 6 & 0 & -2.855867 & -0.436269 & 0.016079 \\
\hline 4 & 6 & 0 & -3.543861 & -1.623182 & -0.293149 \\
\hline 5 & 6 & 0 & -3.623789 & 0.699530 & 0.327924 \\
\hline 6 & 6 & 0 & -4.931053 & -1.666651 & -0.336003 \\
\hline 7 & 1 & 0 & -2.964806 & -2.520098 & -0.514446 \\
\hline 8 & 6 & 0 & -5.012906 & 0.653897 & 0.296628 \\
\hline 9 & 1 & 0 & -3.128245 & 1.616226 & 0.638497 \\
\hline 10 & 6 & 0 & -5.673719 & -0.523846 & -0.045299 \\
\hline 11 & 1 & 0 & -5.436317 & -2.597138 & -0.589831 \\
\hline 12 & 1 & 0 & -5.584731 & 1.544859 & 0.551328 \\
\hline 13 & 1 & 0 & -6.761558 & -0.555021 & -0.069972 \\
\hline 14 & 6 & 0 & -0.489064 & 0.509501 & -0.211365 \\
\hline 15 & 5 & 0 & 1.009154 & 0.135216 & -0.116035 \\
\hline 16 & 8 & 0 & 1.480666 & -1.126841 & 0.172152 \\
\hline 17 & 8 & 0 & 2.017496 & 1.052839 & -0.316204 \\
\hline 18 & 6 & 0 & 2.903000 & -0.992282 & 0.417223 \\
\hline 19 & 6 & 0 & 3.257993 & 0.303659 & -0.376987 \\
\hline 20 & 6 & 0 & -0.824599 & 1.929223 & -0.587772 \\
\hline 21 & 1 & 0 & -0.129721 & 2.254298 & -1.375198 \\
\hline 22 & 1 & 0 & -1.832899 & 1.997328 & -1.017596 \\
\hline 23 & 6 & 0 & -0.686928 & 2.896201 & 0.585934 \\
\hline 24 & 1 & 0 & -1.359113 & 2.628352 & 1.411765 \\
\hline 25 & 1 & 0 & 0.335782 & 2.881203 & 0.981244 \\
\hline 26 & 1 & 0 & -0.914825 & 3.925955 & 0.286120 \\
\hline 27 & 6 & 0 & 3.078889 & -0.829687 & 1.921167 \\
\hline 28 & 1 & 0 & 2.618223 & -1.680752 & 2.433348 \\
\hline 29 & 1 & 0 & 4.137318 & -0.789793 & 2.204750 \\
\hline 30 & 1 & 0 & 2.591745 & 0.084023 & 2.281305 \\
\hline 31 & 6 & 0 & 3.602442 & -2.246483 & -0.066905 \\
\hline 32 & 1 & 0 & 4.691273 & -2.147717 & 0.029392 \\
\hline 33 & 1 & 0 & 3.289453 & -3.106773 & 0.534634 \\
\hline 34 & 1 & 0 & 3.366334 & -2.463762 & -1.112542 \\
\hline 35 & 6 & 0 & 4.364663 & 1.141070 & 0.231770 \\
\hline 36 & 1 & 0 & 5.303624 & 0.574484 & 0.274971 \\
\hline 37 & 1 & 0 & 4.539539 & 2.033458 & -0.378980 \\
\hline 38 & 1 & 0 & 4.113560 & 1.470712 & 1.244031 \\
\hline 39 & 6 & 0 & 3.538977 & 0.036557 & -1.849486 \\
\hline 40 & 1 & 0 & 2.754359 & -0.584522 & -2.296927 \\
\hline 41 & 1 & 0 & 3.566108 & 0.987862 & -2.391051 \\
\hline 42 & 1 & 0 & 4.501594 & -0.468070 & -1.994278 \\
\hline
\end{tabular}


Filename: 3

SCF Energy (M06L/LANL2DZ-6-31g (d))

-798.840914 A.U.

$\mathrm{SCF}$ Energy (M06/SDD-6-311+G(d,p)) in solution

-798.601016 A.U.

Enthalpy at $298 \mathrm{~K}(\mathrm{M} 06 / \mathrm{SDD}-6-311+\mathrm{G}(\mathrm{d}, \mathrm{p}))$

-798.217012 A.U.

Gibbs Free Energy at $298 \mathrm{~K}$ and 1 mol/L with Truhlar's quasiharmonic approximation (M06/SDD-6-311+G(d,p))

-798.276646 A.U.

\begin{tabular}{|c|c|c|c|c|c|}
\hline \multirow{2}{*}{$\begin{array}{l}\text { Center } \\
\text { Number }\end{array}$} & \multirow{2}{*}{$\begin{array}{l}\text { Atomic } \\
\text { Number }\end{array}$} & \multirow{2}{*}{$\begin{array}{c}\text { Atomic } \\
\text { Type }\end{array}$} & \multicolumn{3}{|c|}{ Coordinates (Angstroms) } \\
\hline & & & $\mathrm{X}$ & Y & Z \\
\hline & & & 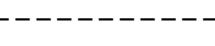 & ---------- & ---------- \\
\hline 1 & 6 & 0 & -1.596563 & 1.647217 & -0.930343 \\
\hline 2 & 6 & 0 & -0.288519 & 1.960468 & -0.228475 \\
\hline 3 & 6 & 0 & 0.273973 & 3.363027 & -0.385880 \\
\hline 4 & 6 & 0 & -0.317233 & 2.956512 & 0.914288 \\
\hline 5 & 1 & 0 & -0.287953 & 4.050699 & -1.016024 \\
\hline 6 & 1 & 0 & 0.350037 & 2.787518 & 1.756658 \\
\hline 7 & 5 & 0 & 0.735032 & 0.804192 & -0.144256 \\
\hline 8 & 8 & 0 & 1.784969 & 0.777591 & 0.745283 \\
\hline 9 & 8 & 0 & 0.686793 & -0.297459 & -0.963697 \\
\hline 10 & 6 & 0 & 2.622586 & -0.343524 & 0.355925 \\
\hline 11 & 6 & 0 & 1.614565 & -1.261307 & -0.403517 \\
\hline 12 & 6 & 0 & 3.224770 & -0.957720 & 1.602897 \\
\hline 13 & 1 & 0 & 3.798988 & -1.859352 & 1.354124 \\
\hline 14 & 1 & 0 & 3.907074 & -0.247042 & 2.081996 \\
\hline 15 & 1 & 0 & 2.455039 & -1.229006 & 2.331051 \\
\hline 16 & 6 & 0 & 3.711802 & 0.214045 & -0.549732 \\
\hline 17 & 1 & 0 & 3.284926 & 0.643285 & -1.464000 \\
\hline 18 & 1 & 0 & 4.249925 & 1.008841 & -0.022329 \\
\hline 19 & 1 & 0 & 4.435850 & -0.557169 & -0.838214 \\
\hline 20 & 6 & 0 & 2.217666 & -2.073498 & -1.531191 \\
\hline 21 & 1 & 0 & 2.985842 & -2.758859 & -1.150586 \\
\hline 22 & 1 & 0 & 1.441479 & -2.675464 & -2.015873 \\
\hline 23 & 1 & 0 & 2.672372 & -1.433637 & -2.293141 \\
\hline 24 & 6 & 0 & 0.802736 & -2.150958 & 0.528536 \\
\hline 25 & 1 & 0 & -0.041041 & -2.576624 & -0.025380 \\
\hline 26 & 1 & 0 & 1.404165 & -2.972744 & 0.935607 \\
\hline 27 & 1 & 0 & 0.389134 & -1.575252 & 1.365185 \\
\hline 28 & 1 & 0 & 1.354037 & 3.480136 & -0.448602 \\
\hline 29 & 1 & 0 & -1.288625 & 3.370491 & 1.181786 \\
\hline 30 & 1 & 0 & -1.409981 & 1.465753 & -1.999159 \\
\hline 31 & 6 & 0 & -2.324759 & 0.453166 & -0.360907 \\
\hline 32 & 6 & 0 & -2.991415 & -0.437968 & -1.206451 \\
\hline 33 & 6 & 0 & -2.348315 & 0.198578 & 1.015001 \\
\hline 34 & 6 & 0 & -3.670158 & -1.542157 & -0.699543 \\
\hline 35 & 1 & 0 & -2.966993 & -0.263514 & -2.282850 \\
\hline 36 & 6 & 0 & -3.023501 & -0.906390 & 1.527255 \\
\hline 37 & 1 & 0 & -1.822387 & 0.871605 & 1.692038 \\
\hline 38 & 6 & 0 & -3.689057 & -1.781428 & 0.672527 \\
\hline 39 & 1 & 0 & -4.179404 & -2.223385 & -1.379935 \\
\hline 40 & 1 & 0 & -3.027138 & -1.085558 & 2.601787 \\
\hline 41 & 1 & 0 & -4.215436 & -2.646530 & 1.072463 \\
\hline 42 & 1 & 0 & -2.250625 & 2.535396 & -0.890336 \\
\hline
\end{tabular}


Filename: 4

SCF Energy (M06L/LANL2DZ-6-31g(d))

-2985.837834 A.U.

$\mathrm{SCF}$ Energy (M06/SDD-6-311+G(d,p)) in solution

-2986.317682 A.U.

Enthalpy at $298 \mathrm{~K}(\mathrm{M} 06 / \mathrm{SDD}-6-311+\mathrm{G}(\mathrm{d}, \mathrm{p}))$

-2985.462819 A.U.

Gibbs Free Energy at $298 \mathrm{~K}$ and 1 mol/L with Truhlar's quasiharmonic approximation (M06/SDD-6-311+G(d,p))

-2985.582906 A.U.

\begin{tabular}{|c|c|c|c|c|c|}
\hline \multirow{2}{*}{$\begin{array}{l}\text { Center } \\
\text { Number }\end{array}$} & \multirow{2}{*}{$\begin{array}{l}\text { Atomic } \\
\text { Number }\end{array}$} & \multirow{2}{*}{$\begin{array}{c}\text { Atomic } \\
\text { Type }\end{array}$} & \multicolumn{3}{|c|}{ Coordinates (Angstroms) } \\
\hline & & & $\mathrm{X}$ & Y & Z \\
\hline & & & & & \\
\hline 1 & 29 & 0 & 1.675047 & 0.018982 & -0.028453 \\
\hline 2 & 15 & 0 & 0.001472 & 1.700649 & 0.147385 \\
\hline 3 & 15 & 0 & 0.030479 & -1.689812 & -0.182273 \\
\hline 4 & 6 & 0 & -1.429178 & 1.175167 & 1.168017 \\
\hline 5 & 6 & 0 & -0.676999 & 2.229345 & -1.464803 \\
\hline 6 & 6 & 0 & 0.591409 & 3.250713 & 0.920575 \\
\hline 7 & 6 & 0 & 0.617382 & -3.237558 & -0.960963 \\
\hline 8 & 6 & 0 & -0.597876 & -2.219373 & 1.450436 \\
\hline 9 & 6 & 0 & -1.430426 & -1.183894 & -1.167568 \\
\hline 10 & 6 & 0 & -2.357005 & 0.227309 & 0.720559 \\
\hline 11 & 6 & 0 & -1.435935 & 1.559472 & 2.536355 \\
\hline 12 & 6 & 0 & -0.040313 & 1.749306 & -2.615373 \\
\hline 13 & 6 & 0 & -1.785021 & 3.073881 & -1.603118 \\
\hline 14 & 6 & 0 & -0.244814 & 4.324625 & 1.257672 \\
\hline 15 & 6 & 0 & 1.968816 & 3.359570 & 1.147694 \\
\hline 16 & 6 & 0 & 1.998037 & -3.357645 & -1.162073 \\
\hline 17 & 6 & 0 & -0.221958 & -4.301074 & -1.322773 \\
\hline 18 & 6 & 0 & 0.024220 & -1.678962 & 2.581972 \\
\hline 19 & 6 & 0 & -1.654406 & -3.121776 & 1.620809 \\
\hline 20 & 6 & 0 & -1.452817 & -1.550775 & -2.540763 \\
\hline 21 & 6 & 0 & -2.369631 & -0.261726 & -0.692093 \\
\hline 22 & 6 & 0 & -3.332670 & -0.294404 & 1.628186 \\
\hline 23 & 6 & 0 & -2.344353 & 1.042478 & 3.422471 \\
\hline 24 & 1 & 0 & -0.694534 & 2.274286 & 2.887893 \\
\hline 25 & 1 & 0 & 0.817017 & 1.080355 & -2.508426 \\
\hline 26 & 6 & 0 & -0.499451 & 2.108633 & -3.879797 \\
\hline 27 & 6 & 0 & -2.245072 & 3.429880 & -2.865645 \\
\hline 28 & 1 & 0 & -2.313100 & 3.427653 & -0.717509 \\
\hline 29 & 1 & 0 & -1.321716 & 4.243429 & 1.108668 \\
\hline 30 & 6 & 0 & 0.287757 & 5.486431 & 1.803872 \\
\hline 31 & 6 & 0 & 2.499145 & 4.528176 & 1.691977 \\
\hline 32 & 1 & 0 & 2.623705 & 2.521478 & 0.897057 \\
\hline 33 & 1 & 0 & 2.654993 & -2.525526 & -0.897432 \\
\hline 34 & 6 & 0 & 2.528260 & -4.528096 & -1.702159 \\
\hline 35 & 6 & 0 & 0.310754 & -5.464445 & -1.865738 \\
\hline 36 & 1 & 0 & -1.301283 & -4.208719 & -1.198855 \\
\hline 37 & 1 & 0 & 0.842912 & -0.967251 & 2.449401 \\
\hline 38 & 6 & 0 & -0.401356 & -2.032410 & 3.859591 \\
\hline 39 & 6 & 0 & -2.081522 & -3.471589 & 2.896626 \\
\hline 40 & 1 & 0 & -2.165416 & -3.532148 & 0.749880 \\
\hline 41 & 1 & 0 & -0.700142 & -2.241744 & -2.915270 \\
\hline 42 & 6 & 0 & -2.389207 & -1.044726 & -3.403420 \\
\hline 43 & 6 & 0 & -3.378164 & 0.242776 & -1.573571 \\
\hline
\end{tabular}




\begin{tabular}{|c|c|c|c|c|c|}
\hline 44 & 6 & 0 & -4.315716 & -1.234599 & 1.223867 \\
\hline 45 & 6 & 0 & -3.316680 & 0.109019 & 3.000466 \\
\hline 46 & 1 & 0 & -2.325031 & 1.346545 & 4.469310 \\
\hline 47 & 1 & 0 & 0.000321 & 1.722696 & -4.766698 \\
\hline 48 & 6 & 0 & -1.602654 & 2.948545 & -4.005379 \\
\hline 49 & 1 & 0 & -3.120871 & 4.069795 & -2.961342 \\
\hline 50 & 1 & 0 & -0.370146 & 6.314035 & 2.06452 \\
\hline 51 & 6 & 0 & 1.662643 & 5.590807 & 2.01816 \\
\hline 52 & 1 & 0 & 3.572026 & 4.599917 & 1.86266 \\
\hline 53 & 1 & 0 & 3.603470 & -4.608648 & -1.853105 \\
\hline 54 & 6 & 0 & 1.688653 & -5.580934 & -2.051763 \\
\hline 55 & 1 & 0 & -0.349608 & -6.283543 & -2.14630 \\
\hline 56 & 1 & 0 & 0.085609 & -1.598537 & 4.73132 \\
\hline 57 & 6 & 0 & -1.455834 & -2.927646 & 4.0173 \\
\hline 58 & 1 & 0 & -2.918204 & -4.157924 & 3.01753 \\
\hline 59 & 1 & 0 & -2.380455 & -1.333028 & -4.45482 \\
\hline 60 & 6 & 0 & -3.378077 & -0.143407 & -2.95085 \\
\hline 61 & 6 & 0 & -4.378132 & 1.151473 & -1.13938 \\
\hline 62 & 1 & 0 & -4.341066 & -1.557593 & 0.18497 \\
\hline 63 & 6 & 0 & -5.225002 & -1.745672 & 2.12073 \\
\hline 64 & 6 & 0 & -4.258723 & -0.441952 & 3.90031 \\
\hline 65 & 1 & 0 & -1.971205 & 3.222374 & -4.9927 \\
\hline 66 & 1 & 0 & 2.077457 & 6.502673 & 2.44505 \\
\hline 67 & 1 & 0 & 2.103532 & -6.493797 & -2.47642 \\
\hline 68 & 1 & 0 & -1.798849 & -3.197330 & 5.0150 \\
\hline 69 & 6 & 0 & -4.351087 & 0.393313 & -3.82601 \\
\hline 70 & 6 & 0 & -5.318060 & 1.648237 & -2.01248 \\
\hline 71 & 1 & 0 & -4.393066 & 1.461554 & -0.09637 \\
\hline 72 & 1 & 0 & -5.969244 & -2.465099 & 1.78300 \\
\hline 73 & 6 & 0 & -5.197509 & -1.351460 & 3.4739 \\
\hline 74 & 1 & 0 & -4.223514 & -0.127480 & 4.9435 \\
\hline 75 & 1 & 0 & -4.327082 & 0.092674 & $-4.8736 \varepsilon$ \\
\hline 76 & 6 & 0 & -5.305824 & 1.271722 & -3.37092 \\
\hline 77 & 1 & 0 & -6.075003 & 2.342739 & -1.65152 \\
\hline 78 & 1 & 0 & -5.918488 & -1.766475 & $4.1760^{\circ}$ \\
\hline 79 & 1 & 0 & -6.050783 & 1.675716 & -4.05412 \\
\hline 80 & 6 & 0 & 5.870553 & 0.464374 & 0.6264 \\
\hline 81 & 6 & 0 & 5.876731 & -0.433328 & -0.6414 \\
\hline 82 & 8 & 0 & 4.503345 & 0.936044 & 0.66252 \\
\hline 83 & 8 & 0 & 4.507620 & -0.897544 & -0.69620 \\
\hline 84 & 5 & 0 & 3.695954 & 0.021211 & -0.0220 \\
\hline 85 & 6 & 0 & 6.105195 & -0.323508 & 1.9097 \\
\hline 86 & 1 & 0 & 7.144670 & -0.664236 & 1.99943 \\
\hline 87 & 1 & 0 & 5.877479 & 0.313479 & 2.7713 \\
\hline 88 & 1 & 0 & 5.447733 & -1.199242 & 1.95647 \\
\hline 89 & 6 & 0 & 6.800784 & 1.660705 & 0.56736 \\
\hline 90 & 1 & 0 & 6.722664 & 2.242987 & 1.49292 \\
\hline 91 & 1 & 0 & 7.846976 & 1.343871 & 0.4588 \\
\hline 92 & 1 & 0 & 6.553283 & 2.323610 & -0.26712 \\
\hline 93 & 6 & 0 & 6.799270 & -1.634931 & -0.5696 \\
\hline 94 & 1 & 0 & 6.730600 & -2.216660 & -1.49632 \\
\hline 95 & 1 & 0 & 7.845662 & -1.324135 & $-0.4465 \varepsilon$ \\
\hline 96 & 1 & 0 & 6.536468 & -2.296503 & 0.26121 \\
\hline 97 & 6 & 0 & 6.133245 & 0.353087 & -1.92142 \\
\hline 98 & 1 & 0 & 7.175680 & 0.688183 & -1.9968 \\
\hline 99 & 1 & 0 & 5.913924 & -0.282792 & -2.78598 \\
\hline 100 & 1 & 0 & 5.481234 & 1.232339 & -1.9771 \\
\hline
\end{tabular}


Medina et al.: BCP Hydroboration-Supporting Information - S50 
Filename: 5

SCF Energy (M06L/LANL2DZ-6-31g(d))

-3372.880431 A.U.

$\mathrm{SCF}$ Energy (M06/SDD-6-311+G(d,p)) in solution

-3373.204726 A.U.

Enthalpy at $298 \mathrm{~K}(\mathrm{M} 06 / \mathrm{SDD}-6-311+\mathrm{G}(\mathrm{d}, \mathrm{p}))$

-3372.170267 A.U.

Gibbs Free Energy at $298 \mathrm{~K}$ and 1 mol/L with Truhlar's quasiharmonic approximation (M06/SDD-6-311+G(d,p))

-3372.306074 A. U.

\begin{tabular}{|c|c|c|c|c|c|}
\hline \multirow{2}{*}{$\begin{array}{l}\text { Center } \\
\text { Number }\end{array}$} & \multirow{2}{*}{$\begin{array}{l}\text { Atomic } \\
\text { Number }\end{array}$} & \multirow{2}{*}{$\begin{array}{c}\text { Atomic } \\
\text { Type }\end{array}$} & \multicolumn{3}{|c|}{ Coordinates (Angstroms) } \\
\hline & & & $\mathrm{X}$ & Y & Z \\
\hline------- & & & ------- & -------- & -------- \\
\hline 1 & 6 & 0 & 2.846739 & 1.732619 & 0.659259 \\
\hline 2 & 1 & 0 & 3.076922 & 1.383800 & 1.678641 \\
\hline 3 & 6 & 0 & 3.856632 & 1.112014 & -0.287666 \\
\hline 4 & 6 & 0 & 2.531545 & 3.168747 & 0.736375 \\
\hline 5 & 5 & 0 & 4.105612 & -0.382824 & -0.012677 \\
\hline 6 & 6 & 0 & 2.782820 & 4.151576 & -0.247474 \\
\hline 7 & 6 & 0 & 1.848072 & 3.627563 & 1.891617 \\
\hline 8 & 8 & 0 & 5.192889 & -1.093217 & -0.477445 \\
\hline 9 & 8 & 0 & 3.225030 & -1.182728 & 0.701709 \\
\hline 10 & 6 & 0 & 2.357122 & 5.470636 & -0.094197 \\
\hline 11 & 1 & 0 & 3.303412 & 3.888382 & -1.164647 \\
\hline 12 & 6 & 0 & 1.418839 & 4.935742 & 2.040101 \\
\hline 13 & 1 & 0 & 1.636085 & 2.899272 & 2.679949 \\
\hline 14 & 6 & 0 & 4.910250 & -2.495640 & -0.265514 \\
\hline 15 & 6 & 0 & 3.891011 & -2.453442 & 0.915223 \\
\hline 16 & 6 & 0 & 1.663946 & 5.881932 & 1.039478 \\
\hline 17 & 1 & 0 & 2.583855 & 6.191061 & -0.882117 \\
\hline 18 & 1 & 0 & 0.890481 & 5.224602 & 2.949606 \\
\hline 19 & 6 & 0 & 6.208069 & -3.215702 & 0.042208 \\
\hline 20 & 6 & 0 & 4.302861 & -3.025122 & -1.556828 \\
\hline 21 & 6 & 0 & 2.847555 & -3.552402 & 0.913458 \\
\hline 22 & 6 & 0 & 4.574138 & -2.373786 & 2.273704 \\
\hline 23 & 1 & 0 & 1.335693 & 6.913488 & 1.152223 \\
\hline 24 & 1 & 0 & 6.021975 & -4.268371 & 0.291844 \\
\hline 25 & 1 & 0 & 6.867463 & -3.189567 & -0.832123 \\
\hline 26 & 1 & 0 & 6.740518 & -2.750983 & 0.876801 \\
\hline 27 & 1 & 0 & 5.002400 & -2.856735 & -2.382508 \\
\hline 28 & 1 & 0 & 4.091540 & -4.100478 & -1.499253 \\
\hline 29 & 1 & 0 & 3.371396 & -2.500442 & -1.800312 \\
\hline 30 & 1 & 0 & 3.318147 & -4.542953 & 0.965674 \\
\hline 31 & 1 & 0 & 2.196737 & -3.443773 & 1.790519 \\
\hline 32 & 1 & 0 & 2.214335 & -3.514477 & 0.019224 \\
\hline 33 & 1 & 0 & 3.825296 & -2.149393 & 3.040957 \\
\hline 34 & 1 & 0 & 5.062427 & -3.319388 & 2.538539 \\
\hline 35 & 1 & 0 & 5.329466 & -1.579299 & 2.290183 \\
\hline 36 & 29 & 0 & 1.090270 & 0.775138 & 0.321693 \\
\hline 37 & 15 & 0 & -0.449784 & -0.295387 & 1.705198 \\
\hline 38 & 15 & 0 & -0.262773 & 0.619426 & -1.596666 \\
\hline 39 & 6 & 0 & -1.353300 & -1.624784 & 0.827373 \\
\hline 40 & 6 & 0 & -1.692565 & 0.913084 & 2.285444 \\
\hline 41 & 6 & 0 & 0.148956 & -1.077424 & 3.246387 \\
\hline 42 & 6 & 0 & 0.189343 & 1.874802 & -2.842060 \\
\hline 43 & 6 & 0 & -0.229762 & -0.976642 & -2.469803 \\
\hline
\end{tabular}


Medina et al.: BCP Hydroboration-Supporting Information - S52

\begin{tabular}{|c|c|c|c|c|c|}
\hline 44 & 6 & 0 & -2.032796 & 0.935472 & -1.199686 \\
\hline 45 & 6 & 0 & -2.298293 & -1.363234 & -0.170549 \\
\hline 46 & 6 & 0 & -0.875693 & -2.953373 & 0.995644 \\
\hline 47 & 6 & 0 & -1.482905 & 2.259738 & 1.964267 \\
\hline 48 & 6 & 0 & -2.824280 & 0.558332 & 3.02916 \\
\hline 49 & 6 & 0 & -0.614654 & -1.944717 & 4.04040 \\
\hline 50 & 6 & 0 & 1.439610 & -0.732876 & 3.6642 \\
\hline 51 & 6 & 0 & 0.496444 & 3.154641 & -2.35521 \\
\hline 52 & 6 & 0 & 0.363970 & 1.609785 & -4.20452 \\
\hline 53 & 6 & 0 & 0.711770 & -1.923921 & -2.05066 \\
\hline 54 & 6 & 0 & -1.122224 & -1.308033 & -3.4974 \\
\hline 55 & 6 & 0 & -2.568295 & 2.223361 & -1.4626 \\
\hline 56 & 6 & 0 & -2.791109 & 0.016355 & -0.46492 \\
\hline 57 & 6 & 0 & -2.828219 & -2.444066 & -0.94704 \\
\hline 58 & 6 & 0 & -1.340662 & -3.989068 & 0.229647 \\
\hline 59 & 1 & 0 & -0.116635 & -3.148325 & 1.74985 \\
\hline 60 & 1 & 0 & -0.598816 & 2.546076 & 1.3879 \\
\hline 61 & 6 & 0 & -2.389523 & 3.233304 & $2.3747 C$ \\
\hline 62 & 6 & 0 & -3.732900 & 1.530127 & 3.4323 \\
\hline 63 & 1 & 0 & -3.009254 & -0.487765 & 3.27368 \\
\hline 64 & 1 & 0 & -1.610322 & -2.244772 & 3.71248 \\
\hline 65 & 6 & 0 & -0.100481 & -2.447496 & $5.2304]$ \\
\hline 66 & 6 & 0 & 1.948373 & -1.228158 & 4.86375 \\
\hline 67 & 1 & 0 & 2.044859 & -0.084362 & 3.02748 \\
\hline 68 & 1 & 0 & 0.425153 & 3.359842 & $-1.2845 \xi$ \\
\hline 69 & 6 & 0 & 0.932778 & 4.155210 & -3.2157 \\
\hline 70 & 6 & 0 & 0.819699 & 2.609294 & -5.0613 \\
\hline 71 & 1 & 0 & 0.162404 & 0.614765 & -4.5975 \\
\hline 72 & 1 & 0 & 1.387049 & -1.672088 & -1.2263 \\
\hline 73 & 6 & 0 & 0.764691 & -3.177986 & -2.6538 \\
\hline 74 & 6 & 0 & -1.067728 & -2.561228 & -4.0981 \\
\hline 75 & 1 & 0 & -1.878848 & -0.586352 & $-3.8084 \varepsilon$ \\
\hline 76 & 1 & 0 & -1.983553 & 2.932913 & -2.0436 \\
\hline 77 & 6 & 0 & -3.803148 & 2.594931 & -0.99792 \\
\hline 78 & 6 & 0 & -4.094913 & 0.380888 & -0.0026 \\
\hline 79 & 6 & 0 & -3.823916 & -2.247482 & -1.9391 \\
\hline 80 & 6 & 0 & -2.333266 & -3.772296 & -0.7523 \\
\hline 81 & 1 & 0 & -0.949527 & -4.997037 & 0.37302 \\
\hline 82 & 1 & 0 & -2.212353 & 4.275671 & 2.11431 \\
\hline 83 & 6 & 0 & -3.517281 & 2.868213 & 3.10502 \\
\hline 84 & 1 & 0 & -4.621422 & 1.240747 & 3.9913 \\
\hline 85 & 1 & 0 & -0.700864 & -3.123439 & 5.8371 \\
\hline 86 & 6 & 0 & 1.180121 & -2.084428 & 5.64712 \\
\hline 87 & 1 & 0 & 2.951805 & -0.947963 & 5.18102 \\
\hline 88 & 1 & 0 & 1.171604 & 5.137729 & -2.81182 \\
\hline 89 & 6 & 0 & 1.097287 & 3.883310 & -4.5728 \\
\hline 90 & 1 & 0 & 0.961468 & 2.387347 & -6.1178 \\
\hline 91 & 1 & 0 & 1.494378 & -3.912832 & -2.3136 \\
\hline 92 & 6 & 0 & -0.124795 & -3.497146 & -3.67698 \\
\hline 93 & 1 & 0 & -1.778274 & -2.816973 & $-4.8824 \varepsilon$ \\
\hline 94 & 1 & 0 & -4.189252 & 3.593082 & -1.2052 \\
\hline 95 & 6 & 0 & -4.596049 & 1.697299 & -0.2502 \\
\hline 96 & 6 & 0 & -4.913674 & -0.514816 & 0.73322 \\
\hline 97 & 1 & 0 & -4.211157 & -1.244783 & -2.10792 \\
\hline 98 & 6 & 0 & -4.300899 & -3.297631 & -2.6895 \\
\hline 99 & 6 & 0 & -2.834405 & -4.828296 & -1.5479 \\
\hline 100 & 1 & 0 & -4.234627 & 3.625483 & 3.41772 \\
\hline
\end{tabular}


Medina et al.: BCP Hydroboration-Supporting Information - S53

\begin{tabular}{|c|c|c|c|c|c|}
\hline 101 & 1 & 0 & 1.579057 & -2.475058 & 6.581720 \\
\hline 102 & 1 & 0 & 1.457346 & 4.659391 & -5.245947 \\
\hline 103 & 1 & 0 & -0.093336 & -4.482231 & -4.139680 \\
\hline 104 & 6 & 0 & -5.864799 & 2.066685 & 0.254863 \\
\hline 105 & 6 & 0 & -6.147400 & -0.129258 & 1.203533 \\
\hline 106 & 1 & 0 & -4.548383 & -1.520242 & 0.933829 \\
\hline 107 & 1 & 0 & -5.067547 & -3.117412 & -3.441463 \\
\hline 108 & 6 & 0 & -3.801736 & -4.601740 & -2.498976 \\
\hline 109 & 1 & 0 & -2.437140 & -5.830854 & -1.387544 \\
\hline 110 & 1 & 0 & -6.224375 & 3.077646 & 0.062479 \\
\hline 111 & 6 & 0 & -6.629580 & 1.174792 & 0.968664 \\
\hline 112 & 1 & 0 & -6.754826 & -0.835945 & 1.766716 \\
\hline 113 & 1 & 0 & -4.182694 & -5.423989 & -3.102019 \\
\hline 114 & 1 & 0 & -7.605275 & 1.470371 & 1.350212 \\
\hline 115 & 6 & 0 & 3.881528 & 1.416940 & -1.785224 \\
\hline 116 & 6 & 0 & 5.017864 & 1.858899 & -0.928787 \\
\hline 117 & 1 & 0 & 5.108432 & 2.917209 & -0.696391 \\
\hline 118 & 1 & 0 & 5.969288 & 1.339829 & -1.033635 \\
\hline 119 & 1 & 0 & 3.168960 & 2.153897 & -2.153785 \\
\hline 120 & 1 & 0 & 4.052960 & 0.593173 & -2.47996 \\
\hline
\end{tabular}


Filename: 6

SCF Energy (M06L/LANL2DZ-6-31g(d))

-3372.887308 A. U.

$\mathrm{SCF}$ Energy (M06/SDD-6-311+G(d,p)) in solution

-3373.205401 A.U.

Enthalpy at $298 \mathrm{~K}(\mathrm{M} 06 / \mathrm{SDD}-6-311+\mathrm{G}(\mathrm{d}, \mathrm{p}))$

-3372.170309 A.U.

Gibbs Free Energy at $298 \mathrm{~K}$ and 1 mol/L with Truhlar's quasiharmonic approximation (M06/SDD-6-311+G(d,p))

-3372.306164 A.U.

\begin{tabular}{|c|c|c|c|c|c|}
\hline \multirow{2}{*}{$\begin{array}{l}\text { Center } \\
\text { Number }\end{array}$} & \multirow{2}{*}{$\begin{array}{l}\text { Atomic } \\
\text { Number }\end{array}$} & \multirow{2}{*}{$\begin{array}{c}\text { Atomic } \\
\text { Type }\end{array}$} & \multicolumn{3}{|c|}{ Coordinates (Angstroms) } \\
\hline & & & $\mathrm{X}$ & Y & $\mathrm{Z}$ \\
\hline & & & & & \\
\hline 1 & 6 & 0 & -4.295149 & 0.344999 & -0.074633 \\
\hline 2 & 1 & 0 & -4.441025 & 0.623305 & 0.974965 \\
\hline 3 & 6 & 0 & -3.480160 & 1.137298 & -0.813124 \\
\hline 4 & 6 & 0 & -4.987481 & -0.884407 & -0.458400 \\
\hline 5 & 6 & 0 & -1.533080 & 1.297308 & -2.369874 \\
\hline 6 & 6 & 0 & -2.998186 & 0.860990 & -2.219376 \\
\hline 7 & 5 & 0 & -2.880409 & 2.430046 & -0.184121 \\
\hline 8 & 6 & 0 & -5.357748 & -1.194635 & -1.780074 \\
\hline 9 & 6 & 0 & -5.333045 & -1.811906 & 0.541306 \\
\hline 10 & 1 & 0 & -1.073063 & 0.778992 & -3.227959 \\
\hline 11 & 1 & 0 & -1.502883 & 2.373092 & -2.607282 \\
\hline 12 & 1 & 0 & -3.662810 & 1.359975 & -2.951679 \\
\hline 13 & 1 & 0 & -3.094195 & -0.213107 & -2.435195 \\
\hline 14 & 8 & 0 & -2.976663 & 3.663680 & -0.788119 \\
\hline 15 & 8 & 0 & -2.221165 & 2.483231 & 1.029965 \\
\hline 16 & 6 & 0 & -5.984813 & -2.397740 & -2.086150 \\
\hline 17 & 1 & 0 & -5.182923 & -0.466533 & -2.567994 \\
\hline 18 & 6 & 0 & -5.950698 & -3.017591 & 0.236532 \\
\hline 19 & 1 & 0 & -5.106715 & -1.570150 & 1.580548 \\
\hline 20 & 6 & 0 & -2.095542 & 4.560206 & -0.070153 \\
\hline 21 & 6 & 0 & -1.991560 & 3.882274 & 1.334554 \\
\hline 22 & 6 & 0 & -6.269567 & -3.324170 & -1.085090 \\
\hline 23 & 1 & 0 & -6.264296 & -2.608427 & -3.117813 \\
\hline 24 & 1 & 0 & -6.187309 & -3.720824 & 1.034107 \\
\hline 25 & 6 & 0 & -2.710872 & 5.945306 & -0.058833 \\
\hline 26 & 6 & 0 & -0.771619 & 4.568331 & -0.821186 \\
\hline 27 & 6 & 0 & -0.631101 & 4.022748 & 1.989986 \\
\hline 28 & 6 & 0 & -3.097646 & 4.300979 & 2.292031 \\
\hline 29 & 1 & 0 & -6.757224 & -4.266636 & -1.329338 \\
\hline 30 & 1 & 0 & -2.104897 & 6.636444 & 0.541417 \\
\hline 31 & 1 & 0 & -2.759003 & 6.341174 & -1.079107 \\
\hline 32 & 1 & 0 & -3.727171 & 5.935394 & 0.346061 \\
\hline 33 & 1 & 0 & -0.946301 & 4.867426 & -1.861065 \\
\hline 34 & 1 & 0 & -0.047808 & 5.264276 & -0.379153 \\
\hline 35 & 1 & 0 & -0.324584 & 3.564437 & -0.838887 \\
\hline 36 & 1 & 0 & -0.358359 & 5.080288 & 2.102387 \\
\hline 37 & 1 & 0 & -0.642181 & 3.568540 & 2.987049 \\
\hline 38 & 1 & 0 & 0.145304 & 3.524853 & 1.395936 \\
\hline 39 & 1 & 0 & -3.082044 & 3.638784 & 3.165402 \\
\hline 40 & 1 & 0 & -2.971066 & 5.334679 & 2.636551 \\
\hline 41 & 1 & 0 & -4.083273 & 4.213186 & 1.819891 \\
\hline 42 & 29 & 0 & -0.447666 & 0.920142 & -0.709924 \\
\hline 43 & 15 & 0 & 1.937253 & 1.324822 & -0.692048 \\
\hline
\end{tabular}


Medina et al.: BCP Hydroboration-Supporting Information - S55

\begin{tabular}{|c|c|c|c|c|c|}
\hline 44 & 15 & 0 & -0.498523 & -0.667656 & 1.008268 \\
\hline 45 & 6 & 0 & 1.907550 & -0.297944 & -1.580193 \\
\hline 46 & 6 & 0 & 3.358065 & 1.200312 & 0.457997 \\
\hline 47 & 6 & 0 & 2.496346 & 2.565991 & -1.921711 \\
\hline 48 & 6 & 0 & -1.329339 & -0.163122 & 2.54962 \\
\hline 49 & 6 & 0 & -1.356450 & -2.195295 & 0.49200 \\
\hline 50 & 6 & 0 & 1.137029 & -1.300024 & 1.56321 \\
\hline 51 & 6 & 0 & 1.789980 & -1.502242 & -0.87413 \\
\hline 52 & 6 & 0 & 1.733979 & -0.302872 & -2.98903 \\
\hline 53 & 6 & 0 & 3.184141 & 1.598113 & 1.787643 \\
\hline 54 & 6 & 0 & 4.605756 & 0.701641 & 0.05946 \\
\hline 55 & 6 & 0 & 1.558083 & 2.995639 & -2.87451 \\
\hline 56 & 6 & 0 & 3.753817 & 3.182623 & -1.8970 \\
\hline 57 & 6 & 0 & -0.728344 & 0.797838 & 3.3743 \\
\hline 58 & 6 & 0 & -2.628524 & -0.581229 & 2.85008 \\
\hline 59 & 6 & 0 & -1.834742 & -2.263820 & -0.81912 \\
\hline 60 & 6 & 0 & -1.515602 & -3.298892 & 1.34067 \\
\hline 61 & 6 & 0 & 1.462071 & -1.465394 & 2.9336 \\
\hline 62 & 6 & 0 & 2.081295 & -1.615376 & 0.5873 \\
\hline 63 & 6 & 0 & 1.459366 & -2.707601 & -1.5735 \\
\hline 64 & 6 & 0 & 1.435519 & -1.452879 & -3.6719 \\
\hline 65 & 1 & 0 & 1.832753 & 0.631284 & -3.5374 \\
\hline 66 & 1 & 0 & 2.212099 & 1.978932 & 2.10620 \\
\hline 67 & 6 & 0 & 4.229771 & 1.498394 & 2.7017 \\
\hline 68 & 6 & 0 & 5.652192 & 0.604949 & 0.9702 \\
\hline 69 & 1 & 0 & 4.748535 & 0.371555 & -0.97049 \\
\hline 70 & 1 & 0 & 0.561728 & 2.551458 & -2.87412 \\
\hline 71 & 6 & 0 & 1.881876 & 3.984142 & -3.79711 \\
\hline 72 & 6 & 0 & 4.070025 & 4.181893 & $-2.8145 \varepsilon$ \\
\hline 73 & 1 & 0 & 4.491522 & 2.891316 & -1.15238 \\
\hline 74 & 1 & 0 & 0.277701 & 1.151878 & 3.1419 \\
\hline 75 & 6 & 0 & -1.398511 & 1.306434 & 4.48021 \\
\hline 76 & 6 & 0 & -3.309250 & -0.056133 & $3.9458^{\circ}$ \\
\hline 77 & 1 & 0 & -3.114917 & -1.315580 & $2.2110 \varepsilon$ \\
\hline 78 & 1 & 0 & -1.683980 & -1.405376 & -1.47742 \\
\hline 79 & 6 & 0 & -2.478053 & -3.410757 & -1.2757 \\
\hline 80 & 6 & 0 & -2.162302 & -4.442589 & 0.8848 \\
\hline 81 & 1 & 0 & -1.137023 & -3.255097 & 2.36331 \\
\hline 82 & 1 & 0 & 0.713203 & -1.239453 & 3.68965 \\
\hline 83 & 6 & 0 & 2.703117 & -1.901045 & 3.32179 \\
\hline 84 & 6 & 0 & 3.360635 & -2.125839 & 0.9747 \\
\hline 85 & 6 & 0 & 1.275146 & -3.942057 & -0.8989 \\
\hline 86 & 6 & 0 & 1.264611 & -2.678529 & -2.98982 \\
\hline 87 & 1 & 0 & 1.309930 & -1.429210 & $-4.7544]$ \\
\hline 88 & 1 & 0 & 4.076299 & 1.800955 & 3.73636 \\
\hline 89 & 6 & 0 & 5.464472 & 1.000186 & 2.2935 \\
\hline 90 & 1 & 0 & 6.610978 & 0.198754 & 0.65193 \\
\hline 91 & 1 & 0 & 1.139714 & 4.298343 & -4.5293 \\
\hline 92 & 6 & 0 & 3.140602 & 4.581100 & -3.7706 \\
\hline 93 & 1 & 0 & 5.052298 & 4.650564 & -2.7786 \\
\hline 94 & 1 & 0 & -0.909764 & 2.044303 & 5.1158 \\
\hline 95 & 6 & 0 & -2.696617 & 0.884826 & 4.7676 \\
\hline 96 & 1 & 0 & -4.324421 & -0.389867 & 4.1580 \\
\hline 97 & 1 & 0 & -2.856814 & -3.448504 & -2.2958 \\
\hline 98 & 6 & 0 & -2.646447 & -4.497016 & -0.4226 \\
\hline 99 & 1 & 0 & -2.290351 & -5.294975 & 1.55065 \\
\hline 100 & 1 & 0 & 2.941859 & -2.010652 & 4.38003 \\
\hline
\end{tabular}


Medina et al.: BCP Hydroboration-Supporting Information - S56

$\begin{array}{rrrrrr}101 & 6 & 0 & 3.685280 & -2.236535 & 2.363263 \\ 102 & 6 & 0 & 4.344163 & -2.507993 & 0.026768 \\ 103 & 1 & 0 & 1.396174 & -3.978615 & 0.182169 \\ 104 & 6 & 0 & 0.910094 & -5.077557 & -1.583409 \\ 105 & 6 & 0 & 0.903087 & -3.866904 & -3.666808 \\ 106 & 1 & 0 & 6.280338 & 0.909452 & 3.008865 \\ 107 & 1 & 0 & 3.391879 & 5.362869 & -4.485505 \\ 108 & 1 & 0 & -3.226290 & 1.291254 & 5.627539 \\ 109 & 1 & 0 & -3.160703 & -5.390236 & -0.775004 \\ 110 & 6 & 0 & 4.971392 & -2.688330 & 2.742521 \\ 111 & 6 & 0 & 5.581411 & -2.956868 & 0.426892 \\ 112 & 1 & 0 & 4.110872 & -2.436535 & -1.033968 \\ 113 & 1 & 0.752116 & -6.006747 & -1.038393 \\ 114 & 6 & 0 & 0.725829 & -5.045096 & -2.980679 \\ 115 & 1 & 0.760550 & -3.825546 & -4.746691 \\ 116 & 1 & 0 & 5.204362 & -2.757064 & 3.805322 \\ 117 & 6 & 0 & 6.904607 & -3.041656 & 1.796667 \\ 118 & 1 & 0 & 0.439273 & -3.246242 & -0.320557 \\ 119 & 1 & 0 & 6.888682 & -5.951022 & -3.511805 \\ 120 & 1 & 0.393366 & 2.101709 \\ -------------\end{array}$


Filename: 7

SCF Energy (M06L/LANL2DZ-6-31g(d))

-2689.759104 A.U.

$\mathrm{SCF}$ Energy (M06/SDD-6-311+G(d,p)) in solution

-2690.310960 A.U.

Enthalpy at $298 \mathrm{~K}(\mathrm{M} 06 / \mathrm{SDD}-6-311+\mathrm{G}(\mathrm{d}, \mathrm{p}))$

-2689.603710 A.U.

Gibbs Free Energy at $298 \mathrm{~K}$ and 1 mol/L with Truhlar's quasiharmonic approximation (M06/SDD-6-311+G(d,p))

-2689.710851 A.U.

\begin{tabular}{|c|c|c|c|c|c|}
\hline \multirow{2}{*}{$\begin{array}{l}\text { Center } \\
\text { Number }\end{array}$} & \multirow{2}{*}{$\begin{array}{l}\text { Atomic } \\
\text { Number }\end{array}$} & \multirow{2}{*}{$\begin{array}{c}\text { Atomic } \\
\text { Type }\end{array}$} & \multicolumn{3}{|c|}{ Coordinates (Angstroms) } \\
\hline & & & $\mathrm{X}$ & Y & $\mathrm{Z}$ \\
\hline & & & & & \\
\hline 1 & 29 & 0 & -0.367398 & -2.042628 & -1.198566 \\
\hline 2 & 15 & 0 & -1.784744 & -0.297183 & -0.813291 \\
\hline 3 & 15 & 0 & 1.626758 & -1.091125 & -0.578622 \\
\hline 4 & 6 & 0 & -0.894971 & 1.303717 & -0.841906 \\
\hline 5 & 6 & 0 & -2.382729 & -0.553467 & 0.897008 \\
\hline 6 & 6 & 0 & -3.329065 & -0.013805 & -1.748585 \\
\hline 7 & 6 & 0 & 2.891566 & -2.368114 & -0.241618 \\
\hline 8 & 6 & 0 & 2.502764 & 0.272592 & -1.412621 \\
\hline 9 & 6 & 0 & 1.162709 & -0.440631 & 1.083756 \\
\hline 10 & 6 & 0 & 0.094566 & 1.628042 & 0.093339 \\
\hline 11 & 6 & 0 & -1.065989 & 2.129749 & -1.986489 \\
\hline 12 & 6 & 0 & -2.121877 & -1.789602 & 1.502857 \\
\hline 13 & 6 & 0 & -3.095677 & 0.422088 & 1.602957 \\
\hline 14 & 6 & 0 & -4.092644 & 1.158943 & -1.665097 \\
\hline 15 & 6 & 0 & -3.801543 & -1.076902 & -2.527334 \\
\hline 16 & 6 & 0 & 2.431341 & -3.692145 & -0.160525 \\
\hline 17 & 6 & 0 & 4.254407 & -2.100260 & -0.066347 \\
\hline 18 & 6 & 0 & 2.204749 & 0.521258 & -2.757619 \\
\hline 19 & 6 & 0 & 3.436446 & 1.090173 & -0.762427 \\
\hline 20 & 6 & 0 & 1.411491 & -1.269434 & 2.209175 \\
\hline 21 & 6 & 0 & 0.419015 & 0.734829 & 1.246831 \\
\hline 22 & 6 & 0 & 0.833292 & 2.847083 & -0.052825 \\
\hline 23 & 6 & 0 & -0.322279 & 3.265626 & -2.168455 \\
\hline 24 & 1 & 0 & -1.799629 & 1.846209 & -2.738718 \\
\hline 25 & 1 & 0 & -1.570357 & -2.558580 & 0.951321 \\
\hline 26 & 6 & 0 & -2.557416 & -2.033109 & 2.803519 \\
\hline 27 & 6 & 0 & -3.524585 & 0.175417 & 2.901750 \\
\hline 28 & 1 & 0 & -3.293931 & 1.390991 & 1.143387 \\
\hline 29 & 1 & 0 & -3.729989 & 2.001444 & -1.075844 \\
\hline 30 & 6 & 0 & -5.299926 & 1.263250 & -2.346803 \\
\hline 31 & 6 & 0 & -5.015603 & -0.973946 & -3.202409 \\
\hline 32 & 1 & 0 & -3.209628 & -1.991038 & -2.594698 \\
\hline 33 & 1 & 0 & 1.372133 & -3.917080 & -0.325923 \\
\hline 34 & 6 & 0 & 3.328167 & -4.720811 & 0.119564 \\
\hline 35 & 6 & 0 & 5.144119 & -3.136107 & 0.200988 \\
\hline 36 & 1 & 0 & 4.627611 & -1.081033 & -0.153407 \\
\hline 37 & 1 & 0 & 1.458394 & -0.095381 & -3.260042 \\
\hline 38 & 6 & 0 & 2.834437 & 1.557071 & -3.441901 \\
\hline 39 & 6 & 0 & 4.067137 & 2.123100 & -1.447339 \\
\hline 40 & 1 & 0 & 3.641900 & 0.937639 & 0.297961 \\
\hline 41 & 1 & 0 & 1.972582 & -2.191770 & 2.073008 \\
\hline 42 & 6 & 0 & 0.951862 & -0.937676 & 3.457030 \\
\hline 43 & 6 & 0 & -0.028209 & 1.113502 & 2.553360 \\
\hline
\end{tabular}




\begin{tabular}{|c|c|c|c|c|c|}
\hline 44 & 6 & 0 & 1.791904 & 3.272472 & 0.903017 \\
\hline 45 & 6 & 0 & 0.629716 & 3.668910 & -1.205257 \\
\hline 46 & 1 & 0 & -0.463999 & 3.878875 & -3.058556 \\
\hline 47 & 1 & 0 & -2.344845 & -2.993670 & 3.269789 \\
\hline 48 & 6 & 0 & -3.252833 & -1.051920 & 3.504584 \\
\hline 49 & 1 & 0 & -4.058099 & 0.948931 & 3.451971 \\
\hline 50 & 1 & 0 & -5.882714 & 2.180316 & -2.277920 \\
\hline 51 & 6 & 0 & -5.764680 & 0.195482 & -3.114002 \\
\hline 52 & 1 & 0 & -5.372478 & -1.809254 & -3.801941 \\
\hline 53 & 1 & 0 & 2.963984 & -5.744770 & 0.182118 \\
\hline 54 & 6 & 0 & 4.681337 & -4.446741 & 0.300840 \\
\hline 55 & 1 & 0 & 6.203208 & -2.918560 & 0.330452 \\
\hline 56 & 1 & 0 & 2.586677 & 1.745561 & -4.485122 \\
\hline 57 & 6 & 0 & 3.767348 & 2.357651 & -2.787703 \\
\hline 58 & 1 & 0 & 4.776046 & 2.763862 & -0.925506 \\
\hline 59 & 1 & 0 & 1.147240 & -1.593246 & 4.305743 \\
\hline 60 & 6 & 0 & 0.228540 & 0.256776 & 3.669106 \\
\hline 61 & 6 & 0 & -0.748900 & 2.312808 & 2.788097 \\
\hline 62 & 1 & 0 & 1.967480 & 2.659535 & 1.784585 \\
\hline 63 & 6 & 0 & 2.499018 & 4.440621 & 0.733926 \\
\hline 64 & 6 & 0 & 1.385906 & 4.853966 & -1.360486 \\
\hline 65 & 1 & 0 & -3.582511 & -1.239438 & 4.525457 \\
\hline 66 & 1 & 0 & -6.711210 & 0.278927 & -3.645203 \\
\hline 67 & 1 & 0 & 5.379941 & -5.255539 & 0.509837 \\
\hline 68 & 1 & 0 & 4.252376 & 3.175738 & -3.317795 \\
\hline 69 & 6 & 0 & -0.247199 & 0.620779 & 4.950857 \\
\hline 70 & 6 & 0 & -1.185195 & 2.647444 & 4.048992 \\
\hline 71 & 1 & 0 & -0.960774 & 2.973592 & 1.949682 \\
\hline 72 & 1 & 0 & 3.222727 & 4.745938 & 1.487963 \\
\hline 73 & 6 & 0 & 2.302125 & 5.239505 & -0.410238 \\
\hline 74 & 1 & 0 & 1.221131 & 5.460185 & -2.251472 \\
\hline 75 & 1 & 0 & -0.048866 & -0.050543 & 5.786490 \\
\hline 76 & 6 & 0 & -0.938386 & 1.793507 & 5.143001 \\
\hline 77 & 1 & 0 & -1.733363 & 3.575799 & 4.201396 \\
\hline 78 & 1 & 0 & 2.872823 & 6.157587 & -0.538080 \\
\hline 79 & 1 & 0 & -1.294851 & 2.064335 & 6.135260 \\
\hline 80 & 8 & 0 & -0.680677 & -3.881004 & -0.975049 \\
\hline 81 & 6 & 0 & -1.940884 & -4.409348 & -1.153760 \\
\hline 82 & 1 & 0 & -2.243321 & -4.486619 & -2.221348 \\
\hline 83 & 1 & 0 & -2.006407 & -5.436876 & -0.744380 \\
\hline 84 & 1 & 0 & -2.749753 & -3.827267 & -0.649814 \\
\hline
\end{tabular}


Filename: 8

SCF Energy (M06L/LANL2DZ-6-31g (d))

-2295.142921 A.U.

SCF Energy (M06/SDD-6-311+G(d,p)) in solution

-2295.918339 A.U.

Enthalpy at $298 \mathrm{~K}(\mathrm{M} 06 / \mathrm{SDD}-6-311+\mathrm{G}(\mathrm{d}, \mathrm{p}))$

-2295.273692 A.U.

Gibbs Free Energy at $298 \mathrm{~K}$ and 1 mol/L with Truhlar's quasiharmonic approximation (M06/SDD-6-311+G $(\mathrm{d}, \mathrm{p}))$

-2295.371443 A.U.

\begin{tabular}{|c|c|c|c|c|c|}
\hline \multirow{2}{*}{$\begin{array}{l}\text { Center } \\
\text { Number }\end{array}$} & \multirow{2}{*}{$\begin{array}{l}\text { Atomic } \\
\text { Number }\end{array}$} & \multirow{2}{*}{$\begin{array}{c}\text { Atomic } \\
\text { Type }\end{array}$} & \multicolumn{3}{|c|}{ Coordinates (Angstroms) } \\
\hline & & & $\mathrm{X}$ & Y & $\mathrm{Z}$ \\
\hline & & & & & \\
\hline 1 & 15 & 0 & 0.369694 & 1.895746 & -0.121231 \\
\hline 2 & 15 & 0 & -2.061206 & -0.114350 & -0.024363 \\
\hline 3 & 6 & 0 & -1.359094 & 2.591457 & -0.197275 \\
\hline 4 & 6 & 0 & -2.343663 & 1.654687 & 0.488989 \\
\hline 5 & 6 & 0 & -3.138862 & -0.994014 & 1.153696 \\
\hline 6 & 6 & 0 & -4.196588 & -1.821528 & 0.762103 \\
\hline 7 & 6 & 0 & -4.944131 & -2.507541 & 1.716541 \\
\hline 8 & 6 & 0 & -4.645134 & -2.378472 & 3.069551 \\
\hline 9 & 6 & 0 & -3.583906 & -1.567016 & 3.468137 \\
\hline 10 & 6 & 0 & -2.831200 & -0.886658 & 2.518603 \\
\hline 11 & 6 & 0 & -2.896822 & -0.191268 & -1.643940 \\
\hline 12 & 6 & 0 & -4.198018 & 0.285069 & -1.855949 \\
\hline 13 & 6 & 0 & -4.765844 & 0.240712 & -3.124754 \\
\hline 14 & 6 & 0 & -4.037850 & -0.274325 & -4.197281 \\
\hline 15 & 6 & 0 & -2.744270 & -0.748551 & -3.998461 \\
\hline 16 & 6 & 0 & -2.176274 & -0.709691 & -2.727183 \\
\hline 17 & 6 & 0 & 0.665295 & 1.815256 & 1.684614 \\
\hline 18 & 6 & 0 & 1.404550 & 0.733958 & 2.187600 \\
\hline 19 & 6 & 0 & 1.608113 & 0.600973 & 3.560496 \\
\hline 20 & 6 & 0 & 1.081632 & 1.540316 & 4.441947 \\
\hline 21 & 6 & 0 & 0.353729 & 2.624438 & 3.950483 \\
\hline 22 & 6 & 0 & 0.149057 & 2.761461 & 2.581532 \\
\hline 23 & 6 & 0 & 1.356936 & 3.328138 & -0.677521 \\
\hline 24 & 6 & 0 & 1.065552 & 3.921980 & -1.914563 \\
\hline 25 & 6 & 0 & 1.831077 & 4.979827 & -2.391769 \\
\hline 26 & 6 & 0 & 2.911489 & 5.453345 & -1.649784 \\
\hline 27 & 6 & 0 & 3.219578 & 4.860187 & -0.428567 \\
\hline 28 & 6 & 0 & 2.449808 & 3.806421 & 0.056170 \\
\hline 29 & 1 & 0 & -1.408706 & 3.601216 & 0.232207 \\
\hline 30 & 1 & 0 & -1.612573 & 2.691892 & -1.262030 \\
\hline 31 & 1 & 0 & -3.382466 & 1.953803 & 0.294919 \\
\hline 32 & 1 & 0 & -2.200265 & 1.690677 & 1.577570 \\
\hline 33 & 1 & 0 & -4.437689 & -1.930680 & -0.294424 \\
\hline 34 & 1 & 0 & -5.765789 & -3.146704 & 1.397588 \\
\hline 35 & 1 & 0 & -5.231261 & -2.915528 & 3.812958 \\
\hline 36 & 1 & 0 & -3.335341 & -1.470449 & 4.523553 \\
\hline 37 & 1 & 0 & -1.985501 & -0.274426 & 2.839447 \\
\hline 38 & 1 & 0 & -4.774716 & 0.679986 & -1.018327 \\
\hline 39 & 1 & 0 & -5.778852 & 0.608551 & -3.279137 \\
\hline 40 & 1 & 0 & -4.483593 & -0.306297 & -5.190019 \\
\hline 41 & 1 & 0 & -2.174126 & -1.152403 & -4.832802 \\
\hline 42 & 1 & 0 & -1.164316 & -1.088252 & -2.559668 \\
\hline 43 & 1 & 0 & 1.818476 & -0.012122 & 1.504722 \\
\hline
\end{tabular}


Medina et al.: BCP Hydroboration-Supporting Information - S60

\begin{tabular}{|c|c|c|c|c|c|}
\hline 44 & 1 & 0 & 2.179001 & -0.246904 & 3.934653 \\
\hline 45 & 1 & 0 & 1.238629 & 1.432894 & 5.514094 \\
\hline 46 & 1 & 0 & -0.053370 & 3.365511 & 4.63663 \\
\hline 47 & 1 & 0 & -0.415364 & 3.615869 & 2.20569 \\
\hline 48 & 1 & 0 & 0.233432 & 3.551076 & -2.51389 \\
\hline 49 & 1 & 0 & 1.585202 & 5.433638 & -3.3504 \\
\hline 50 & 1 & 0 & 3.513246 & 6.278786 & -2.0254 \\
\hline 51 & 1 & 0 & 4.063612 & 5.221973 & 0.1564 \\
\hline 52 & 1 & 0 & 2.694940 & 3.354836 & 1.0167 \\
\hline 53 & 29 & 0 & 0.228991 & -0.533010 & $-0.4041 \varepsilon$ \\
\hline 54 & 5 & 0 & 1.716309 & -1.881129 & -0.5234 \\
\hline 55 & 8 & 0 & 2.141549 & -2.659856 & -1.5982 \\
\hline 56 & 8 & 0 & 2.553502 & -2.111849 & 0.58058 \\
\hline 57 & 6 & 0 & 3.139684 & -3.592056 & -1.1259 \\
\hline 58 & 6 & 0 & 3.698370 & -2.873034 & 0.1323 \\
\hline 59 & 6 & 0 & 4.154168 & -3.826475 & -2.2289 \\
\hline 60 & 1 & 0 & 4.974747 & -4.468831 & -1.8810 \\
\hline 61 & 1 & 0 & 3.675820 & -4.325634 & -3.0793 \\
\hline 62 & 1 & 0 & 4.581353 & -2.886297 & -2.5903 \\
\hline 63 & 6 & 0 & 2.416684 & -4.891290 & -0.7904 \\
\hline 64 & 1 & 0 & 1.868434 & -5.236599 & -1.6736 \\
\hline 65 & 1 & 0 & 3.112741 & -5.683807 & -0.4865 \\
\hline 66 & 1 & 0 & 1.690914 & -4.737582 & 0.0162 \\
\hline 67 & 6 & 0 & 4.798780 & -1.871390 & -0.1986 \\
\hline 68 & 1 & 0 & 4.995817 & -1.248132 & 0.6810 \\
\hline 69 & 1 & 0 & 5.735724 & -2.366601 & -0.4842 \\
\hline 70 & 1 & 0 & 4.492097 & -1.210153 & -1.0172 \\
\hline 71 & 6 & 0 & 4.146526 & -3.794773 & 1.2501 \\
\hline 72 & 1 & 0 & 4.965342 & -4.448229 & 0.9193 \\
\hline 73 & 1 & 0 & 4.512150 & -3.206105 & 2.0997 \\
\hline 74 & 1 & 0 & 3.326014 & -4.424202 & $1.6073=$ \\
\hline
\end{tabular}


Filename: 9

SCF Energy (M06L/LANL2DZ-6-31g(d))

-2682.184444 A.U.

$\mathrm{SCF}$ Energy (M06/SDD-6-311+G(d,p)) in solution

-2682.805762 A.U.

Enthalpy at $298 \mathrm{~K}(\mathrm{M} 06 / \mathrm{SDD}-6-311+\mathrm{G}(\mathrm{d}, \mathrm{p}))$

-2681.981657 A.U.

Gibbs Free Energy at $298 \mathrm{~K}$ and 1 mol/L with Truhlar's quasiharmonic approximation (M06/SDD-6-311+G(d,p))

-2682.095468 A.U.

\begin{tabular}{|c|c|c|c|c|c|}
\hline \multirow{2}{*}{$\begin{array}{l}\text { Center } \\
\text { Number }\end{array}$} & \multirow{2}{*}{$\begin{array}{l}\text { Atomic } \\
\text { Number }\end{array}$} & \multirow{2}{*}{$\begin{array}{c}\text { Atomic } \\
\text { Type }\end{array}$} & \multicolumn{3}{|c|}{ Coordinates (Angstroms) } \\
\hline & & & $\mathrm{X}$ & Y & $\mathrm{Z}$ \\
\hline & & & & & \\
\hline 1 & 6 & 0 & -0.211471 & -1.187942 & -2.194710 \\
\hline 2 & 1 & 0 & -0.415818 & -0.358630 & -2.891137 \\
\hline 3 & 6 & 0 & 1.205403 & -1.665508 & -2.425530 \\
\hline 4 & 6 & 0 & -1.408577 & -2.044663 & -2.137187 \\
\hline 5 & 6 & 0 & 1.790709 & -2.907633 & -1.743863 \\
\hline 6 & 6 & 0 & 1.565991 & -2.902479 & -3.219989 \\
\hline 7 & 5 & 0 & 2.264839 & -0.550272 & -2.328965 \\
\hline 8 & 6 & 0 & -1.452883 & -3.442048 & -1.927139 \\
\hline 9 & 6 & 0 & -2.674249 & -1.404412 & -2.209679 \\
\hline 10 & 1 & 0 & 1.140833 & -3.485526 & -1.090048 \\
\hline 11 & 1 & 0 & 2.824782 & -2.866078 & -1.396857 \\
\hline 12 & 1 & 0 & 2.441086 & -2.880492 & -3.867849 \\
\hline 13 & 1 & 0 & 0.733340 & -3.485659 & -3.608151 \\
\hline 14 & 8 & 0 & 3.502634 & -0.586212 & -2.935020 \\
\hline 15 & 8 & 0 & 2.124840 & 0.551230 & -1.495945 \\
\hline 16 & 6 & 0 & -2.659284 & -4.122409 & -1.755990 \\
\hline 17 & 1 & 0 & -0.534438 & -4.021811 & -1.892156 \\
\hline 18 & 6 & 0 & -3.869863 & -2.078133 & -2.033920 \\
\hline 19 & 1 & 0 & -2.688427 & -0.325437 & -2.382666 \\
\hline 20 & 6 & 0 & 4.340004 & 0.376387 & -2.254834 \\
\hline 21 & 6 & 0 & 3.297582 & 1.384571 & -1.670549 \\
\hline 22 & 6 & 0 & -3.879578 & -3.456370 & -1.790405 \\
\hline 23 & 1 & 0 & -2.634487 & -5.201887 & -1.597723 \\
\hline 24 & 1 & 0 & -4.806364 & -1.520763 & -2.085679 \\
\hline 25 & 6 & 0 & 5.310333 & 0.975377 & -3.253080 \\
\hline 26 & 6 & 0 & 5.089926 & -0.390947 & -1.173806 \\
\hline 27 & 6 & 0 & 3.676885 & 1.970708 & -0.324601 \\
\hline 28 & 6 & 0 & 2.915101 & 2.483685 & -2.651748 \\
\hline 29 & 1 & 0 & -4.815733 & -3.993891 & -1.650907 \\
\hline 30 & 1 & 0 & 5.917547 & 1.761187 & -2.785334 \\
\hline 31 & 1 & 0 & 5.990832 & 0.201414 & -3.624446 \\
\hline 32 & 1 & 0 & 4.790968 & 1.405881 & -4.114111 \\
\hline 33 & 1 & 0 & 5.647359 & -1.211976 & -1.637822 \\
\hline 34 & 1 & 0 & 5.797935 & 0.245148 & -0.627923 \\
\hline 35 & 1 & 0 & 4.392446 & -0.821588 & -0.445328 \\
\hline 36 & 1 & 0 & 4.597199 & 2.564958 & -0.400424 \\
\hline 37 & 1 & 0 & 2.880781 & 2.632593 & 0.039739 \\
\hline 38 & 1 & 0 & 3.826804 & 1.192697 & 0.430702 \\
\hline 39 & 1 & 0 & 2.041778 & 3.025447 & -2.269485 \\
\hline 40 & 1 & 0 & 3.729426 & 3.204018 & -2.796980 \\
\hline 41 & 1 & 0 & 2.645368 & 2.066192 & -3.628624 \\
\hline 42 & 29 & 0 & -0.322235 & -0.167235 & -0.458613 \\
\hline 43 & 15 & 0 & 0.435993 & -0.334304 & 1.725466 \\
\hline
\end{tabular}


Medina et al.: BCP Hydroboration-Supporting Information - S62

\begin{tabular}{|c|c|c|c|c|c|}
\hline 44 & 15 & 0 & -2.009642 & 1.233967 & 0.401672 \\
\hline 45 & 6 & 0 & -0.606994 & 0.803231 & 2.764901 \\
\hline 46 & 6 & 0 & -0.106090 & -1.974014 & 2.321727 \\
\hline 47 & 6 & 0 & 2.119087 & -0.138594 & 2.380818 \\
\hline 48 & 6 & 0 & -2.031947 & 0.827788 & 2.220061 \\
\hline 49 & 6 & 0 & -3.768524 & 1.419994 & -0.018912 \\
\hline 50 & 6 & 0 & -1.365107 & 2.945080 & $0.4337^{\circ}$ \\
\hline 51 & 1 & 0 & -0.589655 & 0.501486 & 3.82121 \\
\hline 52 & 1 & 0 & -0.178444 & 1.813075 & 2.70078 \\
\hline 53 & 6 & 0 & -0.961683 & -2.724000 & 1.50415 \\
\hline 54 & 6 & 0 & 0.271022 & -2.485964 & 3.57034 \\
\hline 55 & 6 & 0 & 2.545229 & 1.005869 & 3.06684 \\
\hline 56 & 6 & 0 & 3.066934 & -1.125487 & 2.06491 \\
\hline 57 & 1 & 0 & -2.654810 & 1.544469 & 2.77326 \\
\hline 58 & 1 & 0 & -2.490135 & -0.165338 & 2.33376 \\
\hline 59 & 6 & 0 & -4.777463 & 0.739873 & 0.674730 \\
\hline 60 & 6 & 0 & -4.117973 & 2.163275 & -1.15712 \\
\hline 61 & 6 & 0 & -2.118856 & 4.035034 & 0.88658 \\
\hline 62 & 6 & 0 & -0.036350 & 3.151382 & 0.03727 \\
\hline 63 & 1 & 0 & -1.241223 & -2.349782 & 0.51497 \\
\hline 64 & 6 & 0 & -1.437714 & -3.961798 & 1.93026 \\
\hline 65 & 6 & 0 & -0.198515 & -3.726467 & 3.98933 \\
\hline 66 & 1 & 0 & 0.946461 & -1.914297 & 4.20827 \\
\hline 67 & 1 & 0 & 1.836094 & 1.793579 & 3.31698 \\
\hline 68 & 6 & 0 & 3.880991 & 1.155844 & 3.4328 \\
\hline 69 & 6 & 0 & 4.397419 & -0.976060 & 2.43814 \\
\hline 70 & 1 & 0 & 2.752151 & -2.016719 & 1.51932 \\
\hline 71 & 1 & 0 & -4.534263 & 0.142287 & 1.55182 \\
\hline 72 & 6 & 0 & -6.101286 & 0.812648 & 0.24941 \\
\hline 73 & 6 & 0 & -5.440506 & 2.236829 & -1.5768 \\
\hline 74 & 1 & 0 & -3.342946 & 2.688965 & -1.7160 \\
\hline 75 & 1 & 0 & -3.157487 & 3.885022 & 1.18352 \\
\hline 76 & 6 & 0 & -1.554099 & 5.305528 & 0.94469 \\
\hline 77 & 6 & 0 & 0.528896 & 4.423025 & 0.10408 \\
\hline 78 & 1 & 0 & 0.548894 & 2.303598 & $-0.3357]$ \\
\hline 79 & 1 & 0 & -2.098136 & -4.530359 & 1.2776 \\
\hline 80 & 6 & 0 & -1.054895 & -4.463752 & 3.1713 \\
\hline 81 & 1 & 0 & 0.105388 & -4.121514 & 4.9574 \\
\hline 82 & 1 & 0 & 4.194172 & 2.051311 & 3.9671 \\
\hline 83 & 6 & 0 & 4.810854 & 0.167911 & 3.12062 \\
\hline 84 & 1 & 0 & 5.117959 & -1.752502 & 2.1854 \\
\hline 85 & 1 & 0 & -6.872796 & 0.279043 & 0.8018 \\
\hline 86 & 6 & 0 & -6.437835 & 1.561221 & $-0.8746 \varepsilon$ \\
\hline 87 & 1 & 0 & -5.693837 & 2.821584 & -2.45938 \\
\hline 88 & 1 & 0 & -2.149153 & 6.147758 & 1.29391 \\
\hline 89 & 6 & 0 & -0.229111 & 5.500210 & 0.55720 \\
\hline 90 & 1 & 0 & 1.561408 & 4.575595 & -0.20982 \\
\hline 91 & 1 & 0 & -1.417847 & -5.435481 & 3.50225 \\
\hline 92 & 1 & 0 & 5.854060 & 0.287413 & 3.40687 \\
\hline 93 & 1 & 0 & -7.472867 & 1.615162 & -1.20662 \\
\hline 94 & 1 & 0 & 0.210278 & 6.495187 & 0.60250 \\
\hline
\end{tabular}


Filename: 10

SCF Energy (M06L/LANL2DZ-6-31g(d))

-2682.191660 A.U.

$\mathrm{SCF}$ Energy (M06/SDD-6-311+G(d,p)) in solution

-2682.806587 A.U.

Enthalpy at $298 \mathrm{~K}(\mathrm{M} 06 / \mathrm{SDD}-6-311+\mathrm{G}(\mathrm{d}, \mathrm{p}))$

-2681.982093 A.U.

Gibbs Free Energy at $298 \mathrm{~K}$ and 1 mol/L with Truhlar's quasiharmonic approximation (M06/SDD-6-311+G(d,p))

-2681.982093 A.U.

\begin{tabular}{|c|c|c|c|c|c|}
\hline \multirow{2}{*}{$\begin{array}{l}\text { Center } \\
\text { Number }\end{array}$} & \multirow{2}{*}{$\begin{array}{l}\text { Atomic } \\
\text { Number }\end{array}$} & \multirow{2}{*}{$\begin{array}{c}\text { Atomic } \\
\text { Type }\end{array}$} & \multicolumn{3}{|c|}{ Coordinates (Angstroms) } \\
\hline & & & $\mathrm{X}$ & Y & $\mathrm{Z}$ \\
\hline & & & & & \\
\hline 1 & 6 & 0 & 3.097722 & -1.132579 & -1.588281 \\
\hline 2 & 1 & 0 & 3.243598 & -1.924809 & -0.844796 \\
\hline 3 & 6 & 0 & 1.877500 & -1.074123 & -2.177242 \\
\hline 4 & 6 & 0 & 4.300184 & -0.329541 & -1.800720 \\
\hline 5 & 6 & 0 & 0.655420 & 1.053444 & -2.458427 \\
\hline 6 & 6 & 0 & 1.401863 & -0.072269 & -3.196447 \\
\hline 7 & 5 & 0 & 0.755042 & -2.049987 & -1.730093 \\
\hline 8 & 6 & 0 & 4.310399 & 0.960588 & -2.366482 \\
\hline 9 & 6 & 0 & 5.533200 & -0.850759 & -1.367555 \\
\hline 10 & 1 & 0 & 1.385728 & 1.814801 & -2.126889 \\
\hline 11 & 1 & 0 & -0.029746 & 1.567528 & -3.149842 \\
\hline 12 & 1 & 0 & 0.690459 & -0.584452 & -3.859974 \\
\hline 13 & 1 & 0 & 2.219933 & 0.280856 & -3.845000 \\
\hline 14 & 8 & 0 & -0.071438 & -2.711724 & -2.607490 \\
\hline 15 & 8 & 0 & 0.439522 & -2.305367 & -0.409656 \\
\hline 16 & 6 & 0 & 5.498462 & 1.666360 & -2.520728 \\
\hline 17 & 1 & 0 & 3.373039 & 1.432151 & -2.651553 \\
\hline 18 & 6 & 0 & 6.719987 & -0.144691 & -1.518083 \\
\hline 19 & 1 & 0 & 5.547467 & -1.839898 & -0.908189 \\
\hline 20 & 6 & 0 & -1.183738 & -3.235795 & -1.841875 \\
\hline 21 & 6 & 0 & -0.596754 & -3.320120 & -0.391854 \\
\hline 22 & 6 & 0 & 6.711524 & 1.119170 & -2.104332 \\
\hline 23 & 1 & 0 & 5.473409 & 2.662517 & -2.961110 \\
\hline 24 & 1 & 0 & 7.656498 & -0.582183 & -1.174854 \\
\hline 25 & 6 & 0 & -1.599477 & -4.568819 & -2.431582 \\
\hline 26 & 6 & 0 & -2.307735 & -2.219078 & -1.974393 \\
\hline 27 & 6 & 0 & -1.579779 & -2.967740 & 0.708044 \\
\hline 28 & 6 & 0 & 0.094043 & -4.642574 & -0.094893 \\
\hline 29 & 1 & 0 & 7.638306 & 1.677524 & -2.224893 \\
\hline 30 & 1 & 0 & -2.404357 & -5.022368 & -1.838825 \\
\hline 31 & 1 & 0 & -1.973598 & -4.425022 & -3.450862 \\
\hline 32 & 1 & 0 & -0.764043 & -5.273641 & -2.476626 \\
\hline 33 & 1 & 0 & -2.562114 & -2.094837 & -3.032660 \\
\hline 34 & 1 & 0 & -3.213062 & -2.519450 & -1.432148 \\
\hline 35 & 1 & 0 & -1.988913 & -1.236690 & -1.597547 \\
\hline 36 & 1 & 0 & -2.464454 & -3.617964 & 0.674280 \\
\hline 37 & 1 & 0 & -1.107956 & -3.078517 & 1.691904 \\
\hline 38 & 1 & 0 & -1.915881 & -1.926994 & 0.606211 \\
\hline 39 & 1 & 0 & 0.632254 & -4.558502 & 0.856153 \\
\hline 40 & 1 & 0 & -0.624406 & -5.466956 & -0.011995 \\
\hline 41 & 1 & 0 & 0.819657 & -4.895785 & -0.876745 \\
\hline 42 & 29 & 0 & -0.218383 & 0.484699 & -0.734770 \\
\hline 43 & 15 & 0 & -2.388263 & 1.039347 & 0.150720 \\
\hline
\end{tabular}




\begin{tabular}{|c|c|c|c|c|c|}
\hline 44 & 15 & 0 & 0.491642 & 0.505921 & 1.548803 \\
\hline 45 & 6 & 0 & -2.193480 & 1.108124 & 1.998825 \\
\hline 46 & 6 & 0 & -2.677448 & 2.805141 & -0.236997 \\
\hline 47 & 6 & 0 & -4.025501 & 0.278336 & -0.093302 \\
\hline 48 & 6 & 0 & -0.784687 & 1.582738 & 2.358465 \\
\hline 49 & 6 & 0 & 2.070117 & 1.361055 & 1.879018 \\
\hline 50 & 6 & 0 & 0.605247 & -0.934839 & 2.670005 \\
\hline 51 & 1 & 0 & -2.960911 & 1.751903 & 2.451326 \\
\hline 52 & 1 & 0 & -2.351113 & 0.090370 & 2.382881 \\
\hline 53 & 6 & 0 & -1.672208 & 3.519095 & -0.899504 \\
\hline 54 & 6 & 0 & -3.848554 & 3.471522 & 0.149439 \\
\hline 55 & 6 & 0 & -4.550051 & -0.690175 & 0.773613 \\
\hline 56 & 6 & 0 & -4.713380 & 0.547975 & -1.287638 \\
\hline 57 & 1 & 0 & -0.649924 & 1.634945 & 3.448022 \\
\hline 58 & 1 & 0 & -0.619627 & 2.599933 & 1.975092 \\
\hline 59 & 6 & 0 & 2.911873 & 1.618083 & 0.792414 \\
\hline 60 & 6 & 0 & 2.485491 & 1.714158 & 3.169850 \\
\hline 61 & 6 & 0 & -0.246800 & -1.159989 & 3.757406 \\
\hline 62 & 6 & 0 & 1.583947 & -1.896318 & 2.375442 \\
\hline 63 & 1 & 0 & -0.770590 & 2.991455 & -1.220384 \\
\hline 64 & 6 & 0 & -1.831922 & 4.878285 & -1.162914 \\
\hline 65 & 6 & 0 & -4.009086 & 4.825474 & -0.122842 \\
\hline 66 & 1 & 0 & -4.642750 & 2.919298 & 0.653658 \\
\hline 67 & 1 & 0 & -4.037347 & -0.933135 & 1.703519 \\
\hline 68 & 6 & 0 & -5.730656 & -1.359580 & 0.460579 \\
\hline 69 & 6 & 0 & -5.892378 & -0.119288 & -1.596180 \\
\hline 70 & 1 & 0 & -4.316106 & 1.291802 & -1.978815 \\
\hline 71 & 1 & 0 & 2.586949 & 1.325668 & -0.207942 \\
\hline 72 & 6 & 0 & 4.154832 & 2.216657 & 0.989712 \\
\hline 73 & 6 & 0 & 3.717740 & 2.327744 & 3.363489 \\
\hline 74 & 1 & 0 & 1.847454 & 1.491784 & 4.026461 \\
\hline 75 & 1 & 0 & -1.008287 & -0.427829 & 4.024060 \\
\hline 76 & 6 & 0 & -0.132083 & -2.319419 & 4.523495 \\
\hline 77 & 6 & 0 & 1.705741 & -3.044828 & 3.147131 \\
\hline 78 & 1 & 0 & 2.246086 & -1.739377 & 1.524628 \\
\hline 79 & 1 & 0 & -1.044735 & 5.424065 & -1.679815 \\
\hline 80 & 6 & 0 & -2.998981 & 5.530959 & -0.777013 \\
\hline 81 & 1 & 0 & -4.924602 & 5.333801 & 0.175453 \\
\hline 82 & 1 & 0 & -6.125174 & -2.103130 & 1.151406 \\
\hline 83 & 6 & 0 & -6.406058 & -1.077806 & -0.723672 \\
\hline 84 & 1 & 0 & -6.412455 & 0.108518 & -2.525053 \\
\hline 85 & 1 & 0 & 4.810314 & 2.386278 & 0.136242 \\
\hline 86 & 6 & 0 & 4.553619 & 2.575931 & 2.273723 \\
\hline 87 & 1 & 0 & 4.034968 & 2.603267 & 4.368151 \\
\hline 88 & 1 & 0 & -0.804628 & -2.476913 & 5.365341 \\
\hline 89 & 6 & 0 & 0.841332 & -3.266134 & 4.219729 \\
\hline 90 & 1 & 0 & 2.476278 & -3.776095 & 2.906895 \\
\hline 91 & 1 & 0 & -3.126914 & 6.591096 & -0.989474 \\
\hline 92 & 1 & 0 & -7.327991 & -1.602586 & -0.966698 \\
\hline 93 & 1 & 0 & 5.524249 & 3.043632 & 2.431336 \\
\hline 94 & 1 & 0 & 0.930693 & -4.170916 & 4.818246 \\
\hline
\end{tabular}


Filename: 11

SCF Energy (M06L/LANL2DZ-6-31g (d))

-1999.061407 A.U.

$\mathrm{SCF}$ Energy (M06/SDD-6-311+G(d,p)) in solution

-1999.907344 A.U.

Enthalpy at $298 \mathrm{~K}(\mathrm{M} 06 / \mathrm{SDD}-6-311+\mathrm{G}(\mathrm{d}, \mathrm{p}))$

-1999.410310 A.U.

Gibbs Free Energy at $298 \mathrm{~K}$ and 1 mol/L with Truhlar's quasiharmonic approximation (M06/SDD-6-311+G(d,p))

-1999.495345 A.U.

\begin{tabular}{|c|c|c|c|c|c|}
\hline \multirow{2}{*}{$\begin{array}{l}\text { Center } \\
\text { Number }\end{array}$} & \multirow{2}{*}{$\begin{array}{l}\text { Atomic } \\
\text { Number }\end{array}$} & \multirow{2}{*}{$\begin{array}{c}\text { Atomic } \\
\text { Type }\end{array}$} & \multicolumn{3}{|c|}{ Coordinates (Angstroms) } \\
\hline & & & $\mathrm{X}$ & Y & Z \\
\hline & & & & & \\
\hline 1 & 29 & 0 & 0.112109 & -1.544294 & 1.022128 \\
\hline 2 & 15 & 0 & -1.465370 & -0.163129 & -0.274888 \\
\hline 3 & 15 & 0 & 1.663555 & -0.406074 & -0.137819 \\
\hline 4 & 6 & 0 & -0.585632 & -0.463671 & -1.885487 \\
\hline 5 & 6 & 0 & -3.075129 & -0.976024 & -0.531567 \\
\hline 6 & 6 & 0 & -1.856165 & 1.614495 & -0.282540 \\
\hline 7 & 6 & 0 & 0.927301 & -0.204624 & -1.846755 \\
\hline 8 & 6 & 0 & 3.405453 & -0.806797 & -0.517552 \\
\hline 9 & 6 & 0 & 1.696772 & 1.327922 & 0.444149 \\
\hline 10 & 1 & 0 & -0.765292 & -1.530226 & -2.073722 \\
\hline 11 & 1 & 0 & -1.060916 & 0.083988 & -2.711528 \\
\hline 12 & 6 & 0 & -3.304754 & -2.189192 & 0.132718 \\
\hline 13 & 6 & 0 & -4.061429 & -0.440352 & -1.371567 \\
\hline 14 & 6 & 0 & -1.162323 & 2.537154 & -1.075638 \\
\hline 15 & 6 & 0 & -2.789977 & 2.099775 & 0.647033 \\
\hline 16 & 1 & 0 & 1.169461 & 0.807630 & -2.196422 \\
\hline 17 & 1 & 0 & 1.445378 & -0.893154 & -2.525314 \\
\hline 18 & 6 & 0 & 3.684322 & -1.905184 & -1.343779 \\
\hline 19 & 6 & 0 & 4.474831 & -0.125033 & 0.076017 \\
\hline 20 & 6 & 0 & 2.336383 & 2.343867 & -0.279813 \\
\hline 21 & 6 & 0 & 0.983141 & 1.664666 & 1.598948 \\
\hline 22 & 1 & 0 & -2.540425 & -2.591359 & 0.809492 \\
\hline 23 & 6 & 0 & -4.511762 & -2.859255 & -0.063107 \\
\hline 24 & 6 & 0 & -5.262663 & -1.115732 & -1.555885 \\
\hline 25 & 1 & 0 & -3.887890 & 0.513145 & -1.872207 \\
\hline 26 & 1 & 0 & -0.427153 & 2.191617 & -1.801612 \\
\hline 27 & 6 & 0 & -1.393994 & 3.903904 & -0.944765 \\
\hline 28 & 6 & 0 & -3.027891 & 3.463350 & 0.768126 \\
\hline 29 & 1 & 0 & -3.338147 & 1.396202 & 1.274354 \\
\hline 30 & 1 & 0 & 2.866560 & -2.475420 & -1.786356 \\
\hline 31 & 6 & 0 & 4.995939 & -2.290518 & -1.594697 \\
\hline 32 & 6 & 0 & 5.787794 & -0.516360 & -0.172810 \\
\hline 33 & 1 & 0 & 4.280237 & 0.720414 & 0.734188 \\
\hline 34 & 1 & 0 & 2.907043 & 2.093089 & -1.175611 \\
\hline 35 & 6 & 0 & 2.256611 & 3.666760 & 0.141335 \\
\hline 36 & 6 & 0 & 0.894753 & 2.990000 & 2.015534 \\
\hline 37 & 1 & 0 & 0.469112 & 0.876137 & 2.153463 \\
\hline 38 & 1 & 0 & -4.692445 & -3.799537 & 0.455210 \\
\hline 39 & 6 & 0 & -5.486458 & -2.328117 & -0.903277 \\
\hline 40 & 1 & 0 & -6.026752 & -0.696708 & -2.208924 \\
\hline 41 & 1 & 0 & -0.839038 & 4.604606 & -1.567171 \\
\hline 42 & 6 & 0 & -2.326580 & 4.372129 & -0.024100 \\
\hline 43 & 1 & 0 & -3.763479 & 3.820026 & 1.487287 \\
\hline
\end{tabular}


Medina et al.: BCP Hydroboration-Supporting Information - S66

\begin{tabular}{|c|c|c|c|c|c|}
\hline 44 & 1 & 0 & 5.192839 & -3.142113 & -2.243461 \\
\hline 45 & 6 & 0 & 6.053442 & -1.594365 & -1.012079 \\
\hline 46 & 1 & 0 & 6.607338 & 0.027896 & 0.293365 \\
\hline 47 & 1 & 0 & 2.757732 & 4.448428 & -0.427438 \\
\hline 48 & 6 & 0 & 1.529339 & 3.991314 & 1.286166 \\
\hline 49 & 1 & 0 & 0.319309 & 3.240108 & 2.904754 \\
\hline 50 & 1 & 0 & -6.428788 & -2.854805 & -1.047377 \\
\hline 51 & 1 & 0 & -2.508116 & 5.440621 & 0.076862 \\
\hline 52 & 1 & 0 & 7.080472 & -1.897404 & -1.206308 \\
\hline 53 & 1 & 0 & 1.454449 & 5.029101 & 1.606587 \\
\hline 54 & 8 & 0 & -0.791250 & -2.808925 & 2.014988 \\
\hline 55 & 6 & 0 & -0.196221 & -3.945812 & 2.528255 \\
\hline 56 & 1 & 0 & 0.745610 & -3.751373 & 3.081996 \\
\hline 57 & 1 & 0 & -0.875132 & -4.449774 & 3.243314 \\
\hline 58 & 1 & 0 & 0.053484 & -4.702973 & 1.754394 \\
\hline
\end{tabular}


Filename: 12

SCF Energy (M06L/LANL2DZ-6-31g (d))

-2682.198806 A.U.

$\mathrm{SCF}$ Energy (M06/SDD-6-311+G(d,p)) in solution

-2682.810894 A.U.

Enthalpy at $298 \mathrm{~K}(\mathrm{M} 06 / \mathrm{SDD}-6-311+\mathrm{G}(\mathrm{d}, \mathrm{p}))$

-2681.987005 A.U.

Gibbs Free Energy at 298K and 1 mol/L with Truhlar's quasiharmonic approximation (M06/SDD-6-311+G(d,p))

-2682.101211 A.U.

\begin{tabular}{|c|c|c|c|c|c|}
\hline \multirow{2}{*}{$\begin{array}{l}\text { Center } \\
\text { Number }\end{array}$} & \multirow{2}{*}{$\begin{array}{l}\text { Atomic } \\
\text { Number }\end{array}$} & \multirow{2}{*}{$\begin{array}{c}\text { Atomic } \\
\text { Type }\end{array}$} & \multicolumn{3}{|c|}{ Coordinates (Angstroms) } \\
\hline & & & $\mathrm{X}$ & Y & Z \\
\hline 1 & 6 & 0 & 2808487 & 0.405026 & -2.534060 \\
\hline $\begin{array}{l}1 \\
2\end{array}$ & 1 & 0 & 2.979639 & 1.366560 & -3.033273 \\
\hline 3 & 6 & 0 & 1.654600 & -0.248752 & -2.793726 \\
\hline 4 & 6 & 0 & 3.852139 & -0.005753 & -1.597652 \\
\hline 5 & 6 & 0 & -0.496175 & 1.000566 & -2.922150 \\
\hline 6 & 6 & 0 & 0.626042 & 0.326632 & -3.733858 \\
\hline 7 & 5 & 0 & 1.139088 & -1.511613 & -2.040375 \\
\hline 8 & 6 & 0 & 4.167411 & -1.357056 & -1.377187 \\
\hline 9 & 6 & 0 & 4.575761 & 0.959466 & -0.876780 \\
\hline 10 & 1 & 0 & -0.268368 & 2.080029 & -2.856691 \\
\hline 11 & 1 & 0 & -1.453810 & 0.931694 & -3.461045 \\
\hline 12 & 1 & 0 & 0.200453 & -0.494312 & -4.329745 \\
\hline 13 & 1 & 0 & 1.107536 & 1.013678 & -4.450639 \\
\hline 14 & 8 & 0 & 0.552078 & -2.583086 & -2.673651 \\
\hline 15 & 8 & 0 & 1.118910 & -1.643123 & -0.663170 \\
\hline 16 & 6 & 0 & 5.127481 & -1.730355 & -0.444060 \\
\hline 17 & 1 & 0 & 3.660157 & -2.118160 & -1.970905 \\
\hline 18 & 6 & 0 & 5.535269 & 0.588851 & 0.058769 \\
\hline 19 & 1 & 0 & 4.350806 & 2.014869 & -1.035972 \\
\hline 20 & 6 & 0 & -0.138287 & -3.357371 & -1.658900 \\
\hline 21 & 6 & 0 & 0.634948 & -2.975153 & -0.355122 \\
\hline 22 & 6 & 0 & 5.814134 & -0.758464 & 0.284024 \\
\hline 23 & 1 & 0 & 5.351566 & -2.786589 & -0.293812 \\
\hline 24 & 1 & 0 & 6.065497 & 1.358181 & 0.619124 \\
\hline 25 & 6 & 0 & -0.064063 & -4.824576 & -2.030840 \\
\hline 26 & 6 & 0 & -1.584148 & -2.880347 & -1.653412 \\
\hline 27 & 6 & 0 & -0.224227 & -2.881456 & 0.889886 \\
\hline 28 & 6 & 0 & 1.858111 & -3.844035 & -0.102085 \\
\hline 29 & 1 & 0 & 6.569916 & -1.049344 & 1.012201 \\
\hline 30 & 1 & 0 & -0.518801 & -5.447110 & -1.249517 \\
\hline 31 & 1 & 0 & -0.611729 & -5.002341 & -2.962575 \\
\hline 32 & 1 & 0 & 0.967999 & -5.155507 & -2.179492 \\
\hline 33 & 1 & 0 & -2.015092 & -3.016485 & -2.651165 \\
\hline 34 & 1 & 0 & -2.198247 & -3.430989 & -0.929251 \\
\hline 35 & 1 & 0 & -1.646951 & -1.808229 & -1.413277 \\
\hline 36 & 1 & 0 & -0.746939 & -3.826850 & 1.088187 \\
\hline 37 & 1 & 0 & 0.395675 & -2.640170 & 1.762004 \\
\hline 38 & 1 & 0 & -0.976970 & -2.090981 & 0.777629 \\
\hline 39 & 1 & 0 & 2.466740 & -3.384715 & 0.685751 \\
\hline 40 & 1 & 0 & 1.575637 & -4.853357 & 0.221120 \\
\hline 41 & 1 & 0 & 2.479313 & -3.933794 & -1.001344 \\
\hline 42 & 29 & 0 & -0.641310 & 0.450298 & -0.989742 \\
\hline 43 & 15 & 0 & -2.589739 & 0.433653 & 0.394899 \\
\hline
\end{tabular}


Medina et al.: BCP Hydroboration-Supporting Information - S68

\begin{tabular}{|c|c|c|c|c|c|}
\hline 44 & 15 & 0 & 0.553760 & 1.143046 & 0.971713 \\
\hline 45 & 6 & 0 & -2.024512 & 1.046389 & 2.058185 \\
\hline 46 & 6 & 0 & -3.664235 & 1.816917 & -0.139519 \\
\hline 47 & 6 & 0 & -3.765534 & -0.900791 & 0.797227 \\
\hline 48 & 6 & 0 & -0.819956 & 1.977039 & 1.907065 \\
\hline 49 & 6 & 0 & 1.747880 & 2.499461 & 0.699078 \\
\hline 50 & 6 & 0 & 1.369237 & 0.110606 & 2.238237 \\
\hline 51 & 1 & 0 & -2.848892 & 1.545445 & 2.586414 \\
\hline 52 & 1 & 0 & -1.744042 & 0.166663 & 2.652362 \\
\hline 53 & 6 & 0 & -3.225743 & 2.635522 & -1.186298 \\
\hline 54 & 6 & 0 & -4.875966 & 2.114985 & 0.499707 \\
\hline 55 & 6 & 0 & -3.535814 & -1.797444 & 1.850830 \\
\hline 56 & 6 & 0 & -4.837822 & -1.157301 & -0.072473 \\
\hline 57 & 1 & 0 & -0.479045 & 2.351745 & 2.882696 \\
\hline 58 & 1 & 0 & -1.098184 & 2.860620 & 1.314132 \\
\hline 59 & 6 & 0 & 1.670783 & 3.181953 & -0.521758 \\
\hline 60 & 6 & 0 & 2.728851 & 2.869202 & 1.626826 \\
\hline 61 & 6 & 0 & 0.871791 & -0.070904 & 3.535348 \\
\hline 62 & 6 & 0 & 2.530129 & -0.585007 & 1.863332 \\
\hline 63 & 1 & 0 & -2.290556 & 2.389357 & -1.693871 \\
\hline 64 & 6 & 0 & -3.982009 & 3.736913 & -1.582925 \\
\hline 65 & 6 & 0 & -5.632848 & 3.209288 & 0.096072 \\
\hline 66 & 1 & 0 & -5.231534 & 1.475251 & 1.308641 \\
\hline 67 & 1 & 0 & -2.700776 & -1.643235 & 2.533685 \\
\hline 68 & 6 & 0 & -4.358090 & -2.904710 & 2.036906 \\
\hline 69 & 6 & 0 & -5.658868 & -2.263223 & 0.116676 \\
\hline 70 & 1 & 0 & -5.030050 & -0.478045 & -0.902996 \\
\hline 71 & 1 & 0 & 0.934383 & 2.858624 & -1.260857 \\
\hline 72 & 6 & 0 & 2.542646 & 4.230604 & -0.800654 \\
\hline 73 & 6 & 0 & 3.608753 & 3.908909 & 1.340229 \\
\hline 74 & 1 & 0 & 2.811844 & 2.330079 & 2.570704 \\
\hline 75 & 1 & 0 & -0.011697 & 0.470785 & 3.869955 \\
\hline 76 & 6 & 0 & 1.504800 & -0.934204 & 4.428152 \\
\hline 77 & 6 & 0 & 3.168986 & -1.431772 & 2.761944 \\
\hline 78 & 1 & 0 & 2.931791 & -0.466511 & 0.858108 \\
\hline 79 & 1 & 0 & -3.632292 & 4.365991 & -2.399506 \\
\hline 80 & 6 & 0 & -5.184769 & 4.023404 & -0.944423 \\
\hline 81 & 1 & 0 & -6.575465 & 3.429846 & 0.594515 \\
\hline 82 & 1 & 0 & -4.164365 & -3.583685 & 2.865821 \\
\hline 83 & 6 & 0 & -5.423122 & -3.142451 & 1.172042 \\
\hline 84 & 1 & 0 & -6.487950 & -2.439557 & -0.566563 \\
\hline 85 & 1 & 0 & 2.475024 & 4.751775 & -1.754286 \\
\hline 86 & 6 & 0 & 3.515052 & 4.593664 & 0.129496 \\
\hline 87 & 1 & 0 & 4.372104 & 4.186261 & 2.065818 \\
\hline 88 & 1 & 0 & 1.100259 & -1.061824 & 5.431133 \\
\hline 89 & 6 & 0 & 2.653615 & -1.619275 & 4.044246 \\
\hline 90 & 1 & 0 & 4.074446 & -1.950708 & 2.448564 \\
\hline 91 & 1 & 0 & -5.779149 & 4.879949 & -1.258225 \\
\hline 92 & 1 & 0 & -6.065193 & -4.009009 & 1.317795 \\
\hline 93 & 1 & 0 & 4.206613 & 5.404906 & -0.092203 \\
\hline 94 & 1 & 0 & 3.148728 & -2.292181 & 4.742277 \\
\hline
\end{tabular}


Filename: TS5

SCF Energy (M06L/LANL2DZ-6-31g (d))

-3372.828200 A.U.

SCF Energy (M06/SDD-6-311+G(d,p)) in solution

-3373.148312 A.U.

Enthalpy at $298 \mathrm{~K}(\mathrm{M} 06 / \mathrm{SDD}-6-311+\mathrm{G}(\mathrm{d}, \mathrm{p}))$

-3372.116458 A.U.

Gibbs Free Energy at $298 \mathrm{~K}$ and 1 mol/L with Truhlar's quasiharmonic approximation ( $06 / \mathrm{SDD}-6-311+\mathrm{G}(\mathrm{d}, \mathrm{p}))$

-3372.251669 A.U.

\begin{tabular}{|c|c|c|c|c|c|}
\hline \multirow{2}{*}{$\begin{array}{l}\text { Center } \\
\text { Number }\end{array}$} & \multirow{2}{*}{$\begin{array}{l}\text { Atomic } \\
\text { Number }\end{array}$} & \multirow{2}{*}{$\begin{array}{c}\text { Atomic } \\
\text { Type }\end{array}$} & \multicolumn{3}{|c|}{ Coordinates (Angstroms) } \\
\hline & & & $\mathrm{X}$ & Y & $\mathrm{Z}$ \\
\hline & & & & & \\
\hline 1 & 6 & 0 & -2.393632 & 0.675657 & 2.376422 \\
\hline 2 & 6 & 0 & -2.668782 & -0.684418 & 2.005328 \\
\hline 3 & 6 & 0 & -2.582197 & -1.855416 & 2.917950 \\
\hline 4 & 6 & 0 & -3.907949 & -1.476573 & 2.309204 \\
\hline 5 & 1 & 0 & -2.403039 & -1.667093 & 3.976534 \\
\hline 6 & 1 & 0 & -2.124888 & -2.767513 & 2.534537 \\
\hline 7 & 1 & 0 & -4.333263 & -2.172154 & 1.589386 \\
\hline 8 & 1 & 0 & -4.637863 & -0.956305 & 2.928806 \\
\hline 9 & 29 & 0 & -1.149104 & -0.125122 & 0.817017 \\
\hline 10 & 15 & 0 & -0.315683 & 1.197380 & -0.853973 \\
\hline 11 & 15 & 0 & 0.976130 & -0.948756 & 1.552491 \\
\hline 12 & 6 & 0 & 0.858220 & 0.038763 & -1.656338 \\
\hline 13 & 6 & 0 & 0.598836 & 2.699347 & -0.344984 \\
\hline 14 & 6 & 0 & -1.339602 & 1.832937 & -2.227637 \\
\hline 15 & 6 & 0 & 0.931395 & -1.220853 & 3.358385 \\
\hline 16 & 6 & 0 & 1.575980 & -2.531452 & 0.864457 \\
\hline 17 & 6 & 0 & 2.393868 & 0.194770 & 1.256948 \\
\hline 18 & 6 & 0 & 2.159993 & -0.199465 & -1.217276 \\
\hline 19 & 6 & 0 & 0.293036 & -0.806450 & -2.652695 \\
\hline 20 & 6 & 0 & 0.597395 & 3.028671 & 1.013941 \\
\hline 21 & 6 & 0 & 1.247817 & 3.542669 & -1.253677 \\
\hline 22 & 6 & 0 & -0.893262 & 1.956939 & -3.549202 \\
\hline 23 & 6 & 0 & -2.624016 & 2.277082 & -1.896134 \\
\hline 24 & 6 & 0 & 0.565838 & -0.145896 & 4.185010 \\
\hline 25 & 6 & 0 & 1.082621 & -2.486579 & 3.936112 \\
\hline 26 & 6 & 0 & 0.728181 & -3.264687 & 0.028738 \\
\hline 27 & 6 & 0 & 2.876103 & -2.994856 & 1.105453 \\
\hline 28 & 6 & 0 & 3.038333 & 0.849495 & 2.336708 \\
\hline 29 & 6 & 0 & 2.755342 & 0.522335 & -0.054689 \\
\hline 30 & 6 & 0 & 2.956402 & -1.201817 & -1.869737 \\
\hline 31 & 6 & 0 & 1.027137 & -1.791542 & -3.256608 \\
\hline 32 & 1 & 0 & -0.751111 & -0.659581 & -2.928612 \\
\hline 33 & 1 & 0 & 0.074041 & 2.365585 & 1.709287 \\
\hline 34 & 6 & 0 & 1.254143 & 4.170570 & 1.463103 \\
\hline 35 & 6 & 0 & 1.910917 & 4.678827 & -0.803395 \\
\hline 36 & 1 & 0 & 1.242125 & 3.302871 & -2.317577 \\
\hline 37 & 1 & 0 & 0.104079 & 1.610918 & -3.822781 \\
\hline 38 & 6 & 0 & -1.725697 & 2.502774 & -4.522718 \\
\hline 39 & 6 & 0 & -3.448133 & 2.835358 & -2.867770 \\
\hline 40 & 1 & 0 & -2.983930 & 2.169011 & -0.873477 \\
\hline 41 & 1 & 0 & 0.419042 & 0.841504 & 3.742943 \\
\hline 42 & 6 & 0 & 0.384401 & -0.325133 & 5.551151 \\
\hline 43 & 6 & 0 & 0.883781 & -2.668188 & 5.303256 \\
\hline
\end{tabular}




\begin{tabular}{|c|c|c|c|c|c|}
\hline 44 & 1 & 0 & 1.348554 & -3.338207 & 3.311985 \\
\hline 45 & 1 & 0 & -0.283134 & -2.897495 & -0.157095 \\
\hline 46 & 6 & 0 & 1.182010 & -4.437464 & -0.570339 \\
\hline 47 & 6 & 0 & 3.323110 & -4.168659 & 0.509844 \\
\hline 48 & 1 & 0 & 3.544308 & -2.418428 & 1.74760 \\
\hline 49 & 1 & 0 & 2.784338 & 0.570674 & 3.3566 \\
\hline 50 & 6 & 0 & 3.980101 & 1.823996 & 2.1233 \\
\hline 51 & 6 & 0 & 3.743419 & 1.526680 & -0.2914 \\
\hline 52 & 6 & 0 & 4.307189 & -1.447109 & -1.51651 \\
\hline 53 & 6 & 0 & 2.383349 & -1.998407 & -2.90998 \\
\hline 54 & 1 & 0 & 0.573378 & -2.426817 & -4.01860 \\
\hline 55 & 1 & 0 & 1.251404 & 4.415086 & 2.52460 \\
\hline 56 & 6 & 0 & 1.916934 & 4.992586 & $0.5550^{\circ}$ \\
\hline 57 & 1 & 0 & 2.433567 & 5.317834 & -1.5135 \\
\hline 58 & 1 & 0 & -1.372318 & 2.587443 & -5.54925 \\
\hline 59 & 6 & 0 & -3.003533 & 2.943669 & -4.1837 \\
\hline 60 & 1 & 0 & -4.444147 & 3.178960 & -2.59112 \\
\hline 61 & 1 & 0 & 0.107276 & 0.522751 & 6.17521 \\
\hline 62 & 6 & 0 & 0.538833 & -1.591314 & 6.1144 \\
\hline 63 & 1 & 0 & 1.000932 & -3.660798 & 5.7350 \\
\hline 64 & 1 & 0 & 0.524401 & -4.992645 & -1.23830 \\
\hline 65 & 6 & 0 & 2.478157 & -4.887260 & -0.3353 \\
\hline 66 & 1 & 0 & 4.340254 & -4.514555 & 0.6882 \\
\hline 67 & 1 & 0 & 4.460810 & 2.316586 & 2.9689 \\
\hline 68 & 6 & 0 & 4.345554 & 2.205313 & 0.8136 \\
\hline 69 & 6 & 0 & 4.130774 & 1.907046 & -1.6018 \\
\hline 70 & 1 & 0 & 4.758310 & -0.867874 & -0.71362 \\
\hline 71 & 6 & 0 & 5.054426 & -2.400975 & -2.17038 \\
\hline 72 & 6 & 0 & 3.170946 & -2.980410 & -3.5523 \\
\hline 73 & 1 & 0 & 2.439084 & 5.882199 & 0.9042 \\
\hline 74 & 1 & 0 & -3.651318 & 3.371852 & -4.9472 \\
\hline 75 & 1 & 0 & 0.383488 & -1.737244 & 7.18182 \\
\hline 76 & 1 & 0 & 2.837161 & -5.795277 & -0.8176 \\
\hline 77 & 6 & 0 & 5.287518 & 3.232244 & 0.56848 \\
\hline 78 & 6 & 0 & 5.053068 & 2.906164 & -1.8082 \\
\hline 79 & 1 & 0 & 3.675680 & 1.401450 & -2.4520 \\
\hline 80 & 1 & 0 & 6.092151 & -2.561733 & -1.8826 \\
\hline 81 & 6 & 0 & 4.485624 & -3.177217 & -3.1980 \\
\hline 82 & 1 & 0 & 2.711907 & -3.578515 & -4.3398 \\
\hline 83 & 1 & 0 & 5.731705 & 3.743711 & 1.4225 \\
\hline 84 & 6 & 0 & 5.636374 & 3.579781 & -0.7151 \\
\hline 85 & 1 & 0 & 5.331558 & 3.183073 & -2.8236 \\
\hline 86 & 1 & 0 & 5.083934 & -3.932188 & -3.7050 \\
\hline 87 & 1 & 0 & 6.362462 & 4.371598 & -0.8906 \\
\hline 88 & 5 & 0 & -2.477082 & -1.397677 & -0.0869 \\
\hline 89 & 8 & 0 & -3.097122 & -0.742322 & -1.13838 \\
\hline 90 & 8 & 0 & -2.760327 & -2.753494 & -0.0963 \\
\hline 91 & 6 & 0 & -3.995916 & -1.669776 & -1.7961 \\
\hline 92 & 6 & 0 & -3.439719 & -3.059734 & -1.3440 \\
\hline 93 & 6 & 0 & -3.930461 & -1.407611 & -3.2889 \\
\hline 94 & 6 & 0 & -5.391840 & -1.365185 & -1.2715 \\
\hline 95 & 6 & 0 & -4.502851 & -4.105391 & -1.0695 \\
\hline 96 & 6 & 0 & -2.379145 & -3.619316 & -2.2823 \\
\hline 97 & 1 & 0 & -4.562687 & -2.112917 & -3.8447 \\
\hline 98 & 1 & 0 & -4.283976 & -0.392603 & -3.5030 \\
\hline 99 & 1 & 0 & -2.907548 & -1.488344 & -3.6715 \\
\hline 100 & 1 & 0 & -5.624480 & -0.312983 & -1.4767 \\
\hline
\end{tabular}


Medina et al.: BCP Hydroboration-Supporting Information - S71

$\begin{array}{rrrrrr}101 & 1 & 0 & -6.159497 & -1.988318 & -1.747070 \\ 102 & 1 & 0 & -5.451336 & -1.511457 & -0.186002 \\ 103 & 1 & 0 & -5.074200 & -4.331049 & -1.979719 \\ 104 & 1 & 0 & -4.036096 & -5.035115 & -0.726134 \\ 105 & 1 & 0 & -5.205535 & -3.777992 & -0.297010 \\ 106 & 1 & 0 & -1.934429 & -4.511083 & -1.826019 \\ 107 & 1 & 0 & -2.797437 & -3.904766 & -3.255425 \\ 108 & 1 & 0 & -1.574307 & -2.891361 & -2.445365 \\ 109 & 6 & 0 & -3.216289 & 1.784584 & 1.941406 \\ 110 & 6 & 0 & -4.344127 & 1.599423 & 1.110141 \\ 111 & 6 & 0 & -2.921460 & 3.109121 & 2.331357 \\ 112 & 6 & 0 & -5.123143 & 2.674961 & 0.700051 \\ 113 & 1 & 0 & -4.591265 & 0.592966 & 0.777128 \\ 114 & 6 & 0 & -3.693913 & 4.180533 & 1.908409 \\ 115 & 1 & 0 & -2.057081 & 3.284143 & 2.975673 \\ 116 & 6 & 0 & -4.804909 & 3.976403 & 1.086081 \\ 117 & 1 & 0 & -5.989261 & 2.492466 & 0.061847 \\ 118 & 1 & 0 & -3.429504 & 5.189702 & 2.223530 \\ 119 & 1 & 0 & -5.412388 & 4.817906 & 0.758014 \\ 120 & 1 & 0 & -1.716901 & 0.848931 & 3.214913\end{array}$


Filename: TS6

SCF Energy (M06L/LANL2DZ-6-31g(d))

-3372.854343 A.U.

$\mathrm{SCF}$ Energy (M06/SDD-6-311+G(d,p)) in solution

-3373.168245 A.U.

Enthalpy at $298 \mathrm{~K}(\mathrm{M} 06 / \mathrm{SDD}-6-311+\mathrm{G}(\mathrm{d}, \mathrm{p}))$

-3372.135793 A.U.

Gibbs Free Energy at $298 \mathrm{~K}$ and 1 mol/L with Truhlar's quasiharmonic approximation (M06/SDD-6-311+G(d,p))

-3372.270537 A.U.

\begin{tabular}{|c|c|c|c|c|c|}
\hline \multirow{2}{*}{$\begin{array}{l}\text { Center } \\
\text { Number }\end{array}$} & \multirow{2}{*}{$\begin{array}{l}\text { Atomic } \\
\text { Number }\end{array}$} & \multirow{2}{*}{$\begin{array}{c}\text { Atomic } \\
\text { Type }\end{array}$} & \multicolumn{3}{|c|}{ Coordinates (Angstroms) } \\
\hline & & & $\mathrm{X}$ & Y & Z \\
\hline & & & & & \\
\hline 1 & 6 & 0 & 3.429547 & 1.004396 & -0.843564 \\
\hline 2 & 6 & 0 & 3.019726 & 1.681529 & 0.376672 \\
\hline 3 & 29 & 0 & 1.294565 & 0.636231 & -0.461505 \\
\hline 4 & 15 & 0 & -0.880472 & 1.140731 & -1.281921 \\
\hline 5 & 15 & 0 & 0.373632 & -0.742463 & 1.221908 \\
\hline 6 & 6 & 0 & -2.356094 & 1.113516 & -0.188008 \\
\hline 7 & 6 & 0 & -1.323177 & -0.022155 & -2.620324 \\
\hline 8 & 6 & 0 & -0.893833 & 2.795572 & -2.055211 \\
\hline 9 & 6 & 0 & 1.193905 & -1.836537 & 2.447945 \\
\hline 10 & 6 & 0 & -0.460550 & 0.387652 & 2.403750 \\
\hline 11 & 6 & 0 & -0.884056 & -1.793612 & 0.415672 \\
\hline 12 & 6 & 0 & -2.766870 & -0.085160 & 0.406550 \\
\hline 13 & 6 & 0 & -3.003581 & 2.325815 & 0.162585 \\
\hline 14 & 6 & 0 & -0.414208 & -1.028871 & -2.960379 \\
\hline 15 & 6 & 0 & -2.566817 & 0.021038 & -3.263712 \\
\hline 16 & 6 & 0 & -1.173778 & 3.018610 & -3.409211 \\
\hline 17 & 6 & 0 & -0.504786 & 3.883530 & -1.257932 \\
\hline 18 & 6 & 0 & 2.358820 & -1.331811 & 3.042339 \\
\hline 19 & 6 & 0 & 0.691130 & -3.064910 & 2.892825 \\
\hline 20 & 6 & 0 & -0.138275 & 1.747512 & 2.369999 \\
\hline 21 & 6 & 0 & -1.331642 & -0.085932 & 3.391976 \\
\hline 22 & 6 & 0 & -0.372275 & -3.033040 & -0.066371 \\
\hline 23 & 6 & 0 & -2.170085 & -1.397176 & 0.029499 \\
\hline 24 & 6 & 0 & -3.822412 & -0.067104 & 1.373672 \\
\hline 25 & 6 & 0 & -4.022961 & 2.352555 & 1.077571 \\
\hline 26 & 1 & 0 & -2.691912 & 3.252935 & -0.311908 \\
\hline 27 & 1 & 0 & 0.541094 & -1.071940 & -2.431671 \\
\hline 28 & 6 & 0 & -0.741166 & -1.979795 & -3.922294 \\
\hline 29 & 6 & 0 & -2.888658 & -0.923624 & -4.232227 \\
\hline 30 & 1 & 0 & -3.292948 & 0.785845 & -2.983530 \\
\hline 31 & 1 & 0 & -1.444180 & 2.184179 & -4.053562 \\
\hline 32 & 6 & 0 & -1.102428 & 4.303596 & -3.941352 \\
\hline 33 & 6 & 0 & -0.458457 & 5.168530 & -1.787083 \\
\hline 34 & 1 & 0 & -0.224261 & 3.722193 & -0.214387 \\
\hline 35 & 1 & 0 & 2.757144 & -0.374548 & 2.708219 \\
\hline 36 & 6 & 0 & 3.011327 & -2.040919 & 4.043886 \\
\hline 37 & 6 & 0 & 1.356009 & -3.783201 & 3.884624 \\
\hline 38 & 1 & 0 & -0.227516 & -3.464974 & 2.464943 \\
\hline 39 & 1 & 0 & 0.567665 & 2.110655 & 1.619415 \\
\hline 40 & 6 & 0 & -0.699722 & 2.627570 & 3.293054 \\
\hline 41 & 6 & 0 & -1.899863 & 0.793932 & 4.305432 \\
\hline 42 & 1 & 0 & -1.576443 & -1.148227 & 3.435821 \\
\hline 43 & 1 & 0 & 0.642876 & -3.318483 & 0.207337 \\
\hline
\end{tabular}




\begin{tabular}{|c|c|c|c|c|c|}
\hline 44 & 6 & 0 & -1.110997 & -3.871099 & -0.853619 \\
\hline 45 & 6 & 0 & -2.968466 & -2.278208 & -0.782567 \\
\hline 46 & 6 & 0 & -4.242248 & -1.239321 & 2.052973 \\
\hline 47 & 6 & 0 & -4.450470 & 1.169331 & 1.720591 \\
\hline 48 & 1 & 0 & -4.511990 & 3.294428 & 1.32621 \\
\hline 49 & 1 & 0 & -0.034746 & -2.774496 & -4.15970 \\
\hline 50 & 6 & 0 & -1.977746 & -1.926516 & -4.5605 \\
\hline 51 & 1 & 0 & -3.863700 & -0.892843 & -4.7158 \\
\hline 52 & 1 & 0 & -1.320182 & 4.460403 & -4.99652 \\
\hline 53 & 6 & 0 & -0.758382 & 5.382264 & -3.13067 \\
\hline 54 & 1 & 0 & -0.161520 & 5.997090 & -1.1469 \\
\hline 55 & 1 & 0 & 3.913829 & -1.630432 & 4.4942 \\
\hline 56 & 6 & 0 & 2.516690 & -3.275387 & 4.4624 \\
\hline 57 & 1 & 0 & 0.954078 & -4.739695 & 4.2154 \\
\hline 58 & 1 & 0 & -0.434445 & 3.683511 & 3.2551 \\
\hline 59 & 6 & 0 & -1.587011 & 2.152913 & 4.25475 \\
\hline 60 & 1 & 0 & -2.594847 & 0.419106 & 5.05553 \\
\hline 61 & 1 & 0 & -0.688845 & -4.813992 & -1.2032 \\
\hline 62 & 6 & 0 & -2.432463 & -3.526758 & -1.2281 \\
\hline 63 & 6 & 0 & -4.283605 & -1.945250 & -1.19543 \\
\hline 64 & 1 & 0 & -3.762311 & -2.186832 & 1.8143 \\
\hline 65 & 6 & 0 & -5.228060 & -1.193521 & 3.01221 \\
\hline 66 & 6 & 0 & -5.465287 & 1.183659 & 2.70542 \\
\hline 67 & 1 & 0 & -2.240838 & -2.676471 & -5.3047 \\
\hline 68 & 1 & 0 & -0.708757 & 6.386265 & -3.5484 \\
\hline 69 & 1 & 0 & 3.030141 & -3.836158 & 5.24171 \\
\hline 70 & 1 & 0 & -2.031892 & 2.839175 & 4.97392 \\
\hline 71 & 6 & 0 & -3.215020 & -4.377870 & -2.03889 \\
\hline 72 & 6 & 0 & -5.029335 & -2.800705 & -1.9766 \\
\hline 73 & 1 & 0 & -4.708567 & -0.991330 & -0.8916 \\
\hline 74 & 1 & 0 & -5.527476 & -2.107703 & 3.5219 \\
\hline 75 & 6 & 0 & -5.850882 & 0.027225 & 3.34223 \\
\hline 76 & 1 & 0 & -5.932220 & 2.136707 & 2.95435 \\
\hline 77 & 1 & 0 & -2.780904 & -5.322733 & -2.36700 \\
\hline 78 & 6 & 0 & -4.495569 & -4.029216 & -2.40603 \\
\hline 79 & 1 & 0 & -6.038167 & -2.516309 & -2.2716 \\
\hline 80 & 1 & 0 & -6.630310 & 0.051952 & 4.1017 \\
\hline 81 & 1 & 0 & -5.090698 & -4.696535 & -3.0268 \\
\hline 82 & 5 & 0 & 3.813347 & -0.500960 & -0.76188 \\
\hline 83 & 8 & 0 & 4.286837 & -1.185840 & -1.86220 \\
\hline 84 & 8 & 0 & 3.747054 & -1.282344 & 0.3680 \\
\hline 85 & 6 & 0 & 4.224167 & -2.589606 & -1.5133 \\
\hline 86 & 6 & 0 & 4.379155 & -2.548061 & 0.0366 \\
\hline 87 & 6 & 0 & 5.323126 & -3.332187 & -2.2430 \\
\hline 88 & 6 & 0 & 2.847346 & -3.074954 & -1.9516 \\
\hline 89 & 6 & 0 & 3.676535 & -3.672568 & 0.76802 \\
\hline 90 & 6 & 0 & 5.828967 & -2.444598 & 0.4875 \\
\hline 91 & 1 & 0 & 5.351529 & -4.385362 & -1.9354 \\
\hline 92 & 1 & 0 & 5.146639 & -3.302482 & -3.3237 \\
\hline 93 & 1 & 0 & 6.304665 & -2.889377 & -2.0502 \\
\hline 94 & 1 & 0 & 2.724075 & -2.888059 & -3.0249 \\
\hline 95 & 1 & 0 & 2.703035 & -4.147007 & -1.7675 \\
\hline 96 & 1 & 0 & 2.056781 & -2.524235 & -1.4209 \\
\hline 97 & 1 & 0 & 4.073425 & -4.644507 & 0.4464 \\
\hline 98 & 1 & 0 & 3.826103 & -3.584324 & 1.8495 \\
\hline 99 & 1 & 0 & 2.597658 & -3.662833 & 0.58611 \\
\hline 100 & 1 & 0 & 5.853480 & -2.194023 & 1.5538 \\
\hline
\end{tabular}


Medina et al.: BCP Hydroboration-Supporting Information - S74

\begin{tabular}{|c|c|c|c|c|c|}
\hline 101 & 1 & 0 & 6.367023 & -3.389325 & 0.344313 \\
\hline 102 & 1 & 0 & 6.361026 & -1.657160 & -0.058629 \\
\hline 103 & 6 & 0 & 3.786002 & 1.613519 & -2.154777 \\
\hline 104 & 6 & 0 & 2.395039 & 1.111859 & -2.396851 \\
\hline 105 & 1 & 0 & 1.649826 & 1.900082 & -2.531692 \\
\hline 106 & 1 & 0 & 2.295326 & 0.251427 & -3.058264 \\
\hline 107 & 1 & 0 & 3.928004 & 2.692369 & -2.168093 \\
\hline 108 & 1 & 0 & 4.576190 & 1.099088 & -2.702536 \\
\hline 109 & 1 & 0 & 3.196184 & 1.075045 & 1.266423 \\
\hline 110 & 6 & 0 & 2.850367 & 3.089216 & 0.639856 \\
\hline 111 & 6 & 0 & 2.618300 & 4.090534 & -0.335012 \\
\hline 112 & 6 & 0 & 2.847662 & 3.529768 & 1.989321 \\
\hline 113 & 6 & 0 & 2.450934 & 5.424897 & 0.016684 \\
\hline 114 & 1 & 0 & 2.524610 & 3.818544 & -1.384676 \\
\hline 115 & 6 & 0 & 2.655395 & 4.857296 & 2.334861 \\
\hline 116 & 1 & 0 & 3.000997 & 2.785087 & 2.773384 \\
\hline 117 & 6 & 0 & 2.466048 & 5.830895 & 1.350047 \\
\hline 118 & 1 & 0 & 2.292775 & 6.159635 & -0.773804 \\
\hline 119 & 1 & 0 & 2.663719 & 5.140603 & 3.387768 \\
\hline 120 & 1 & 0 & 2.332325 & 6.877272 & 1.617300 \\
\hline
\end{tabular}


Filename: TS7

SCF Energy (M06L/LANL2DZ-6-31g(d))

-3488.570200 A.U.

$\mathrm{SCF}$ Energy (M06/SDD-6-311+G(d,p)) in solution

-3488.881408 A.U.

Enthalpy at $298 \mathrm{~K}(\mathrm{M} 06 / \mathrm{SDD}-6-311+\mathrm{G}(\mathrm{d}, \mathrm{p}))$

$-3487.794350 \mathrm{~A} . \mathrm{U}$.

Gibbs Free Energy at $298 \mathrm{~K}$ and 1 mol/L with Truhlar's quasiharmonic approximation (M06/SDD-6-311+G(d,p))

-3487.936427 A.U.

\begin{tabular}{|c|c|c|c|c|c|}
\hline \multirow{2}{*}{$\begin{array}{l}\text { Center } \\
\text { Number }\end{array}$} & \multirow{2}{*}{$\begin{array}{l}\text { Atomic } \\
\text { Number }\end{array}$} & \multirow{2}{*}{$\begin{array}{c}\text { Atomic } \\
\text { Type }\end{array}$} & \multicolumn{3}{|c|}{ Coordinates (Angstroms) } \\
\hline & & & $\mathrm{X}$ & $\mathrm{Y}$ & $\mathrm{Z}$ \\
\hline & & & & & \\
\hline 1 & 6 & 0 & 3.844670 & 1.942601 & -0.279417 \\
\hline 2 & 6 & 0 & 4.548068 & 2.800906 & -1.308811 \\
\hline 3 & 6 & 0 & 4.883674 & 2.995177 & 0.126195 \\
\hline 4 & 5 & 0 & 4.399727 & 0.516601 & -0.089547 \\
\hline 5 & 1 & 0 & 3.952957 & 3.567071 & -1.804774 \\
\hline 6 & 1 & 0 & 5.287550 & 2.328099 & -1.954058 \\
\hline 7 & 1 & 0 & 5.857629 & 2.658177 & 0.478424 \\
\hline 8 & 1 & 0 & 4.504351 & 3.881619 & 0.631177 \\
\hline 9 & 8 & 0 & 5.644989 & 0.123713 & -0.535821 \\
\hline 10 & 8 & 0 & 3.766969 & -0.472260 & 0.645482 \\
\hline 11 & 6 & 0 & 5.780550 & -1.281572 & -0.213552 \\
\hline 12 & 6 & 0 & 4.801092 & -1.434092 & 0.985865 \\
\hline 13 & 6 & 0 & 7.232278 & -1.570325 & 0.108683 \\
\hline 14 & 6 & 0 & 5.324084 & -2.068219 & -1.434185 \\
\hline 15 & 6 & 0 & 4.193264 & -2.811581 & 1.140007 \\
\hline 16 & 6 & 0 & 5.408932 & -0.972535 & 2.304485 \\
\hline 17 & 1 & 0 & 7.358423 & -2.606539 & 0.448444 \\
\hline 18 & 1 & 0 & 7.851538 & -1.432381 & -0.784546 \\
\hline 19 & 1 & 0 & 7.612571 & -0.901940 & 0.887022 \\
\hline 20 & 1 & 0 & 5.893682 & -1.742631 & -2.311653 \\
\hline 21 & 1 & 0 & 5.474924 & -3.147427 & -1.305538 \\
\hline 22 & 1 & 0 & 4.259678 & -1.895151 & -1.637036 \\
\hline 23 & 1 & 0 & 4.979885 & -3.558957 & 1.309620 \\
\hline 24 & 1 & 0 & 3.512670 & -2.837545 & 1.999290 \\
\hline 25 & 1 & 0 & 3.627878 & -3.102194 & 0.250700 \\
\hline 26 & 1 & 0 & 4.615714 & -0.890241 & 3.055939 \\
\hline 27 & 1 & 0 & 6.160639 & -1.679878 & 2.675148 \\
\hline 28 & 1 & 0 & 5.882023 & 0.011875 & 2.202592 \\
\hline 29 & 8 & 0 & 1.378745 & 1.960365 & -2.436464 \\
\hline 30 & 6 & 0 & 2.324063 & 1.469900 & -3.340508 \\
\hline 31 & 1 & 0 & 2.934819 & 2.279998 & -3.774598 \\
\hline 32 & 1 & 0 & 3.024227 & 0.742280 & -2.880218 \\
\hline 33 & 1 & 0 & 1.822848 & 0.957016 & -4.180407 \\
\hline 34 & 1 & 0 & 1.875274 & 2.111643 & -1.367468 \\
\hline 35 & 6 & 0 & 1.842190 & 3.333088 & 0.678405 \\
\hline 36 & 6 & 0 & 0.983944 & 3.230706 & 1.792066 \\
\hline 37 & 6 & 0 & 2.103056 & 4.637675 & 0.212357 \\
\hline 38 & 6 & 0 & 0.422736 & 4.345092 & 2.403270 \\
\hline 39 & 1 & 0 & 0.763382 & 2.232226 & 2.181721 \\
\hline 40 & 6 & 0 & 1.560247 & 5.757051 & 0.835918 \\
\hline 41 & 1 & 0 & 2.736541 & 4.776887 & -0.663627 \\
\hline 42 & 6 & 0 & 0.713556 & 5.625448 & 1.935157 \\
\hline 43 & 1 & 0 & -0.238365 & 4.214961 & 3.262205 \\
\hline
\end{tabular}




\begin{tabular}{|c|c|c|c|c|c|}
\hline 44 & 1 & 0 & 1.793232 & 6.748639 & 0.446598 \\
\hline 45 & 1 & 0 & 0.278552 & 6.503079 & 2.410114 \\
\hline 46 & 6 & 0 & 2.358396 & 2.106013 & 0.038854 \\
\hline 47 & 1 & 0 & 2.116573 & 1.272090 & 0.724215 \\
\hline 48 & 29 & 0 & 0.587085 & 0.796108 & -0.763469 \\
\hline 49 & 15 & 0 & 0.294952 & -1.455475 & -0.42659 \\
\hline 50 & 15 & 0 & -1.565746 & 1.543917 & -0.3216 \\
\hline 51 & 6 & 0 & -1.424421 & -1.975672 & -0.8531 \\
\hline 52 & 6 & 0 & 0.547497 & -2.095756 & 1.26021 \\
\hline 53 & 6 & 0 & 1.316057 & -2.468919 & -1.554725 \\
\hline 54 & 6 & 0 & -2.010329 & 3.283998 & -0.01402 \\
\hline 55 & 6 & 0 & -2.723316 & 1.115643 & -1.6731 \\
\hline 56 & 6 & 0 & -2.060548 & 0.607152 & 1.1727 \\
\hline 57 & 6 & 0 & -2.522557 & -1.526730 & -0.1130 \\
\hline 58 & 6 & 0 & -1.644327 & -2.716926 & -2.04344 \\
\hline 59 & 6 & 0 & 1.102369 & -1.235481 & 2.2149 \\
\hline 60 & 6 & 0 & 0.147762 & -3.380720 & 1.64919 \\
\hline 61 & 6 & 0 & 1.766786 & -3.768628 & -1.30262 \\
\hline 62 & 6 & 0 & 1.625989 & -1.872028 & -2.7836 \\
\hline 63 & 6 & 0 & -3.108912 & 3.701311 & 0.74774 \\
\hline 64 & 6 & 0 & -1.235406 & 4.236868 & -0.68522 \\
\hline 65 & 6 & 0 & -2.183665 & 0.672486 & -2.88558 \\
\hline 66 & 6 & 0 & -4.109628 & 1.270561 & -1.55700 \\
\hline 67 & 6 & 0 & -1.876808 & 1.264658 & 2.4212 \\
\hline 68 & 6 & 0 & -2.368167 & -0.756019 & 1.15772 \\
\hline 69 & 6 & 0 & -3.848766 & -1.824989 & -0.56034 \\
\hline 70 & 6 & 0 & -2.910149 & -2.992501 & -2.49185 \\
\hline 71 & 1 & 0 & -0.791447 & -3.069765 & -2.61841 \\
\hline 72 & 1 & 0 & 1.408625 & -0.233434 & 1.9159 \\
\hline 73 & 6 & 0 & 1.262227 & -1.654665 & 3.5320 \\
\hline 74 & 6 & 0 & 0.322535 & -3.803461 & 2.9629 \\
\hline 75 & 1 & 0 & -0.336270 & -4.039494 & 0.9265 \\
\hline 76 & 1 & 0 & 1.548798 & -4.246461 & -0.34830 \\
\hline 77 & 6 & 0 & 2.515415 & -4.450351 & -2.2596 \\
\hline 78 & 6 & 0 & 2.359705 & -2.558276 & -3.74611 \\
\hline 79 & 1 & 0 & 1.283759 & -0.853478 & -2.9748 \\
\hline 80 & 1 & 0 & -3.713751 & 2.966711 & 1.28072 \\
\hline 81 & 6 & 0 & -3.421223 & 5.053946 & 0.8406 \\
\hline 82 & 6 & 0 & -1.562672 & 5.586581 & -0.6025 \\
\hline 83 & 1 & 0 & -0.366568 & 3.911648 & -1.26026 \\
\hline 84 & 1 & 0 & -1.098935 & 0.576814 & -2.9813 \\
\hline 85 & 6 & 0 & -3.019577 & 0.368185 & -3.9573 \\
\hline 86 & 6 & 0 & -4.942915 & 0.960473 & -2.6252 \\
\hline 87 & 1 & 0 & -4.538971 & 1.623964 & -0.6190 \\
\hline 88 & 1 & 0 & -1.601610 & 2.317879 & 2.42198 \\
\hline 89 & 6 & 0 & -2.030401 & 0.601666 & 3.6099 \\
\hline 90 & 6 & 0 & -2.585748 & -1.448369 & 2.3944 \\
\hline 91 & 6 & 0 & -4.999382 & -1.390724 & 0.1474 \\
\hline 92 & 6 & 0 & -4.043920 & -2.550055 & $-1.7770 \varepsilon$ \\
\hline 93 & 1 & 0 & -3.054795 & -3.556738 & -3.4133 \\
\hline 94 & 1 & 0 & 1.681032 & -0.970412 & 4.2684 \\
\hline 95 & 6 & 0 & 0.876862 & -2.940214 & 3.90618 \\
\hline 96 & 1 & 0 & -0.002803 & -4.799549 & 3.2587 \\
\hline 97 & 1 & 0 & 2.871798 & -5.457047 & -2.0467 \\
\hline 98 & 6 & 0 & 2.809038 & -3.850474 & -3.4827 \\
\hline 99 & 1 & 0 & 2.594789 & -2.074092 & -4.6927 \\
\hline 100 & 1 & 0 & -4.271616 & 5.372553 & 1.44168 \\
\hline
\end{tabular}


Medina et al.: BCP Hydroboration-Supporting Information - S77

$\begin{array}{rrrrrr}101 & 6 & 0 & -2.652462 & 5.996790 & 0.160560 \\ 102 & 1 & 0 & -0.947060 & 6.320348 & -1.119642 \\ 103 & 1 & 0 & -2.590607 & 0.020245 & -4.895733 \\ 104 & 6 & 0 & -4.398661 & 0.505851 & -3.825817 \\ 105 & 1 & 0 & -6.021585 & 1.064502 & -2.518499 \\ 106 & 1 & 0 & -1.880704 & 1.126475 & 4.553755 \\ 107 & 6 & 0 & -2.403285 & -0.761756 & 3.635699 \\ 108 & 6 & 0 & -2.978047 & -2.810860 & 2.442001 \\ 109 & 1 & 0 & -4.871131 & -0.831546 & 1.072203 \\ 110 & 6 & 0 & -6.265143 & -1.651955 & -0.323074 \\ 111 & 6 & 0 & -5.358424 & -2.798330 & -2.237902 \\ 112 & 1 & 0 & 0.992254 & -3.266200 & 4.938624 \\ 113 & 1 & 0 & 3.396451 & -4.387212 & -4.225626 \\ 114 & 1 & 0 & -2.899772 & 7.054628 & 0.234290 \\ 115 & 1 & 0 & -5.053633 & 0.260613 & -4.660569 \\ 116 & 6 & 0 & -2.605358 & -1.453722 & 4.852423 \\ 117 & 6 & 0 & -3.184765 & -3.452550 & 3.641495 \\ 118 & 1 & 0 & -3.116615 & -3.353752 & 1.509109 \\ 119 & 1 & 0 & -7.131271 & -1.305024 & 0.237934 \\ 120 & 6 & 0 & -6.450877 & -2.359740 & -1.528388 \\ 121 & 1 & 0 & -5.485473 & -3.345606 & -3.172049 \\ 122 & 1 & 0 & -2.454322 & -0.912977 & 5.786792 \\ 123 & 6 & 0 & -2.992693 & -2.773214 & 4.860751 \\ 124 & 1 & 0 & -3.491939 & -4.497205 & 3.647626 \\ 125 & 1 & 0 & -7.457421 & -2.556678 & -1.893056 \\ 126 & 1 & 0 & -3.153146 & -3.292310 & 5.804028 \\ --------------19\end{array}$


Filename: TS8

SCF Energy (M06L/LANL2DZ-6-31g(d))

-3488.579879 A.U.

$\mathrm{SCF}$ Energy (M06/SDD-6-311+G(d,p)) in solution

$-3488.888207 \mathrm{~A}$. U.

Enthalpy at $298 \mathrm{~K}(\mathrm{M} 06 / \mathrm{SDD}-6-311+\mathrm{G}(\mathrm{d}, \mathrm{p}))$

-3487.800112 A.U.

Gibbs Free Energy at $298 \mathrm{~K}$ and 1 mol/L with Truhlar's quasiharmonic approximation (M06/SDD-6-311+G(d,p))

-3487.942743 A.U.

\begin{tabular}{|c|c|c|c|c|c|}
\hline \multirow{2}{*}{$\begin{array}{l}\text { Center } \\
\text { Number }\end{array}$} & \multirow{2}{*}{$\begin{array}{l}\text { Atomic } \\
\text { Number }\end{array}$} & \multirow{2}{*}{$\begin{array}{c}\text { Atomic } \\
\text { Type }\end{array}$} & \multicolumn{3}{|c|}{ Coordinates (Angstroms) } \\
\hline & & & $\mathrm{X}$ & Y & $\mathrm{Z}$ \\
\hline & & & & & \\
\hline 1 & 6 & 0 & -3.710500 & 2.079021 & 0.579688 \\
\hline 2 & 6 & 0 & -4.060713 & 0.912909 & 1.176461 \\
\hline 3 & 6 & 0 & -2.092814 & 0.065860 & 2.515802 \\
\hline 4 & 6 & 0 & -3.587683 & 0.438753 & 2.529142 \\
\hline 5 & 5 & 0 & -4.892496 & -0.088502 & 0.335437 \\
\hline 6 & 1 & 0 & -1.499293 & 0.997075 & 2.596295 \\
\hline 7 & 1 & 0 & -1.847634 & -0.531141 & 3.406614 \\
\hline 8 & 1 & 0 & -4.159540 & -0.461524 & 2.792532 \\
\hline 9 & 1 & 0 & -3.831256 & 1.172659 & 3.315211 \\
\hline 10 & 8 & 0 & -5.472020 & -1.223587 & 0.859364 \\
\hline 11 & 8 & 0 & -5.192214 & 0.089901 & -1.004301 \\
\hline 12 & 6 & 0 & -6.347843 & -1.766943 & -0.152078 \\
\hline 13 & 6 & 0 & -5.779885 & -1.148305 & -1.470799 \\
\hline 14 & 6 & 0 & -7.753081 & -1.277630 & 0.175553 \\
\hline 15 & 6 & 0 & -6.286556 & -3.280528 & -0.087803 \\
\hline 16 & 6 & 0 & -4.651486 & -1.971475 & -2.078500 \\
\hline 17 & 6 & 0 & -6.828659 & -0.826687 & -2.518382 \\
\hline 18 & 1 & 0 & -8.502201 & -1.695654 & -0.507870 \\
\hline 19 & 1 & 0 & -8.011149 & -1.581484 & 1.195547 \\
\hline 20 & 1 & 0 & -7.812246 & -0.184179 & 0.125821 \\
\hline 21 & 1 & 0 & -6.674954 & -3.629789 & 0.875527 \\
\hline 22 & 1 & 0 & -6.897318 & -3.731540 & -0.880954 \\
\hline 23 & 1 & 0 & -5.260160 & -3.645264 & -0.189784 \\
\hline 24 & 1 & 0 & -5.012647 & -2.931760 & -2.467007 \\
\hline 25 & 1 & 0 & -4.208865 & -1.414970 & -2.913881 \\
\hline 26 & 1 & 0 & -3.861297 & -2.146915 & -1.338965 \\
\hline 27 & 1 & 0 & -6.354063 & -0.393182 & -3.406510 \\
\hline 28 & 1 & 0 & -7.354796 & -1.736573 & -2.835352 \\
\hline 29 & 1 & 0 & -7.568963 & -0.110827 & -2.149090 \\
\hline 30 & 1 & 0 & -2.281334 & -0.858688 & 1.398184 \\
\hline 31 & 8 & 0 & -2.436069 & -1.577977 & 0.443668 \\
\hline 32 & 6 & 0 & -2.500189 & -2.876154 & 0.955941 \\
\hline 33 & 1 & 0 & -2.320463 & -3.623185 & 0.163564 \\
\hline 34 & 1 & 0 & -1.739932 & -3.053574 & 1.745135 \\
\hline 35 & 1 & 0 & -3.491835 & -3.088431 & 1.395838 \\
\hline 36 & 29 & 0 & -0.624407 & -0.217459 & 0.871669 \\
\hline 37 & 15 & 0 & 0.235775 & 1.105132 & -0.777395 \\
\hline 38 & 15 & 0 & 1.241284 & -1.523065 & 1.324175 \\
\hline 39 & 6 & 0 & 1.386545 & 0.084411 & -1.793942 \\
\hline 40 & 6 & 0 & 1.220654 & 2.511800 & -0.170178 \\
\hline 41 & 6 & 0 & -0.939346 & 1.783233 & -1.999942 \\
\hline 42 & 6 & 0 & 1.272727 & -2.264515 & 2.992387 \\
\hline 43 & 6 & 0 & 1.499858 & -2.891491 & 0.153568 \\
\hline
\end{tabular}




\begin{tabular}{|c|c|c|c|c|c|}
\hline 44 & 6 & 0 & 2.741582 & -0.462891 & 1.223406 \\
\hline 45 & 6 & 0 & 2.613889 & -0.380502 & -1.309604 \\
\hline 46 & 6 & 0 & 0.905212 & -0.394497 & -3.041555 \\
\hline 47 & 6 & 0 & 1.070918 & 2.869635 & 1.174214 \\
\hline 48 & 6 & 0 & 2.127569 & 3.223666 & -0.966236 \\
\hline 49 & 6 & 0 & -0.675497 & 2.896768 & -2.80724 \\
\hline 50 & 6 & 0 & -2.180781 & 1.142261 & -2.0999 \\
\hline 51 & 6 & 0 & 0.797191 & -1.467267 & 4.04547 \\
\hline 52 & 6 & 0 & 1.641381 & -3.586651 & 3.26240 \\
\hline 53 & 6 & 0 & 0.465422 & -3.154821 & -0.75355 \\
\hline 54 & 6 & 0 & 2.680299 & -3.643258 & 0.08867 \\
\hline 55 & 6 & 0 & 3.338844 & -0.011725 & 2.42948 \\
\hline 56 & 6 & 0 & 3.186659 & 0.054064 & 0.0017 \\
\hline 57 & 6 & 0 & 3.386300 & -1.294449 & -2.0993 \\
\hline 58 & 6 & 0 & 1.618676 & -1.294838 & -3.78862 \\
\hline 59 & 1 & 0 & -0.055877 & -0.040473 & -3.40857 \\
\hline 60 & 1 & 0 & 0.362471 & 2.318103 & 1.79732 \\
\hline 61 & 6 & 0 & 1.808002 & 3.920690 & 1.7115 \\
\hline 62 & 6 & 0 & 2.856203 & 4.279931 & -0.4309 \\
\hline 63 & 1 & 0 & 2.293473 & 2.921743 & -2.00097 \\
\hline 64 & 1 & 0 & 0.272708 & 3.424193 & -2.7180 \\
\hline 65 & 6 & 0 & -1.635135 & 3.358785 & -3.70153 \\
\hline 66 & 6 & 0 & -3.138793 & 1.606090 & -3.00014 \\
\hline 67 & 1 & 0 & -2.412230 & 0.298021 & -1.4435 \\
\hline 68 & 1 & 0 & 0.464915 & -0.448879 & 3.8345 \\
\hline 69 & 6 & 0 & 0.730664 & -1.966302 & 5.34108 \\
\hline 70 & 6 & 0 & 1.557652 & -4.090034 & 4.55862 \\
\hline 71 & 1 & 0 & 1.980991 & -4.233022 & 2.45514 \\
\hline 72 & 1 & 0 & -0.443476 & -2.548453 & -0.71430 \\
\hline 73 & 6 & 0 & 0.606747 & -4.164406 & -1.70158 \\
\hline 74 & 6 & 0 & 2.817599 & -4.650024 & -0.8610 \\
\hline 75 & 1 & 0 & 3.503873 & -3.417923 & 0.7679 \\
\hline 76 & 1 & 0 & 2.994401 & -0.419031 & 3.37743 \\
\hline 77 & 6 & 0 & 4.341988 & 0.921791 & 2.4269 \\
\hline 78 & 6 & 0 & 4.270769 & 0.990526 & -0.0163 \\
\hline 79 & 6 & 0 & 4.652638 & -1.777365 & -1.67778 \\
\hline 80 & 6 & 0 & 2.873333 & -1.768668 & -3.3467 \\
\hline 81 & 1 & 0 & 1.225088 & -1.652244 & -4.74038 \\
\hline 82 & 1 & 0 & 1.678242 & 4.189143 & 2.75893 \\
\hline 83 & 6 & 0 & 2.698469 & 4.628611 & 0.90936 \\
\hline 84 & 1 & 0 & 3.571292 & 4.813982 & -1.0546 \\
\hline 85 & 1 & 0 & -1.423901 & 4.231181 & -4.3180 \\
\hline 86 & 6 & 0 & -2.867561 & 2.712609 & -3.80000 \\
\hline 87 & 1 & 0 & -4.111918 & 1.120385 & -3.0300 \\
\hline 88 & 1 & 0 & 0.361807 & -1.332827 & 6.14572 \\
\hline 89 & 6 & 0 & 1.110608 & -3.282079 & 5.60025 \\
\hline 90 & 1 & 0 & 1.842756 & -5.122656 & 4.7530 \\
\hline 91 & 1 & 0 & -0.199118 & -4.356815 & -2.4082 \\
\hline 92 & 6 & 0 & 1.781028 & -4.911592 & -1.7556 \\
\hline 93 & 1 & 0 & 3.745158 & -5.217415 & -0.9184 \\
\hline 94 & 1 & 0 & 4.782101 & 1.257041 & 3.36622 \\
\hline 95 & 6 & 0 & 4.836650 & 1.449316 & 1.2139 \\
\hline 96 & 6 & 0 & 4.807086 & 1.506040 & -1.22512 \\
\hline 97 & 1 & 0 & 5.059710 & -1.437006 & -0.7280 \\
\hline 98 & 6 & 0 & 5.366395 & -2.671585 & -2.4415 \\
\hline 99 & 6 & 0 & 3.625288 & -2.697735 & -4.1038 \\
\hline 100 & 1 & 0 & 3.280630 & 5.447359 & 1.3295 \\
\hline
\end{tabular}


Medina et al.: BCP Hydroboration-Supporting Information - S80

$\begin{array}{rrrrrr}101 & 1 & 0 & -3.621848 & 3.083280 & -4.492421 \\ 102 & 1 & 0 & 1.044587 & -3.680524 & 6.611085 \\ 103 & 1 & 0 & 1.897394 & -5.692454 & -2.505691 \\ 104 & 6 & 0 & 5.879664 & 2.404995 & 1.189893 \\ 105 & 6 & 0 & 5.832501 & 2.422853 & -1.217399 \\ 106 & 1 & 0 & 4.392239 & 1.169534 & -2.173086 \\ 107 & 1 & 0 & 6.335998 & -3.023664 & -2.093084 \\ 108 & 6 & 0 & 4.849965 & -3.142489 & -3.665430 \\ 109 & 1 & 0 & 3.210839 & -3.051336 & -5.048150 \\ 110 & 1 & 0 & 6.290253 & 2.747159 & 2.139962 \\ 111 & 6 & 0 & 6.372504 & 2.885371 & -0.000087 \\ 112 & 1 & 0 & 6.226153 & 2.798811 & -2.160398 \\ 113 & 1 & 0 & 5.420696 & -3.854304 & -4.259235 \\ 114 & 1 & 0 & 7.179306 & 3.616108 & -0.005526 \\ 115 & 6 & 0 & -2.869704 & 3.194337 & 1.006897 \\ 116 & 6 & 0 & -2.468015 & 3.463169 & 2.329558 \\ 117 & 6 & 0 & -2.434978 & 4.086212 & 0.007264 \\ 118 & 6 & 0 & -1.678568 & 4.568577 & 2.625686 \\ 119 & 1 & 0 & -2.793324 & 2.813428 & 3.136851 \\ 120 & 6 & 0 & -1.620694 & 5.172890 & 0.298378 \\ 121 & 1 & 0 & -2.744688 & 3.899543 & -1.022405 \\ 122 & 6 & 0 & -1.243677 & 5.425369 & 1.615216 \\ 123 & 1 & 0 & -1.394860 & 4.760764 & 3.660320 \\ 124 & 1 & 0 & -1.282264 & 5.825718 & -0.506090 \\ 125 & 1 & 0 & -0.608187 & 6.277458 & 1.852558 \\ 126 & 1 & 0 & -4.082542 & 2.214314 & -0.439623 \\ -------------1\end{array}$


Filename: TS9

SCF Energy (M06L/LANL2DZ-6-31g(d))

-2682.135243 A.U.

$\mathrm{SCF}$ Energy (M06/SDD-6-311+G(d,p)) in solution

-2682.752177 A.U.

Enthalpy at $298 \mathrm{~K}(\mathrm{M} 06 / \mathrm{SDD}-6-311+\mathrm{G}(\mathrm{d}, \mathrm{p}))$

-2681.930660 A.U.

Gibbs Free Energy at $298 \mathrm{~K}$ and 1 mol/L with Truhlar's quasiharmonic approximation (M06/SDD-6-311+G(d,p))

-2682.042938 A.U.

\begin{tabular}{|c|c|c|c|c|c|}
\hline \multirow{2}{*}{$\begin{array}{l}\text { Center } \\
\text { Number }\end{array}$} & \multirow{2}{*}{$\begin{array}{l}\text { Atomic } \\
\text { Number }\end{array}$} & \multirow{2}{*}{$\begin{array}{c}\text { Atomic } \\
\text { Type }\end{array}$} & \multicolumn{3}{|c|}{ Coordinates (Angstroms) } \\
\hline & & & $\mathrm{X}$ & Y & Z \\
\hline 1 & 6 & 0 & 10 & 0600335 & 2513597 \\
\hline $\begin{array}{l}1 \\
2\end{array}$ & $\begin{array}{l}6 \\
6\end{array}$ & $\begin{array}{l}0 \\
0\end{array}$ & $\begin{array}{r}-1.001 / 16 \\
0.075784\end{array}$ & $\begin{array}{r}0.600335 \\
-0.354778\end{array}$ & $\begin{array}{l}2.513592 \\
2.634080\end{array}$ \\
\hline 3 & 6 & 0 & 1.079309 & -0.371876 & 3.735475 \\
\hline 4 & 6 & 0 & 0.095015 & -1.509381 & 3.575035 \\
\hline 5 & 5 & 0 & 1.294540 & -1.292957 & 1.014276 \\
\hline 6 & 1 & 0 & 0.930037 & 0.329748 & 4.556081 \\
\hline 7 & 1 & 0 & 2.124013 & -0.577141 & 3.505390 \\
\hline 8 & 1 & 0 & 0.475456 & -2.476555 & 3.252553 \\
\hline 9 & 1 & 0 & -0.743992 & -1.573193 & 4.267891 \\
\hline 10 & 8 & 0 & 2.650411 & -1.022514 & 1.123002 \\
\hline 11 & 8 & 0 & 1.062295 & -2.629477 & 0.757850 \\
\hline 12 & 6 & 0 & 3.342503 & -2.143072 & 0.518987 \\
\hline 13 & 6 & 0 & 2.347626 & -3.313452 & 0.771379 \\
\hline 14 & 6 & 0 & 4.697794 & -2.290746 & 1.179615 \\
\hline 15 & 6 & 0 & 3.501842 & -1.810711 & -0.960838 \\
\hline 16 & 6 & 0 & 2.333331 & -4.379419 & -0.305162 \\
\hline 17 & 6 & 0 & 2.517454 & -3.940239 & 2.147090 \\
\hline 18 & 1 & 0 & 5.225346 & -3.176777 & 0.802452 \\
\hline 19 & 1 & 0 & 5.316188 & -1.412667 & 0.960357 \\
\hline 20 & 1 & 0 & 4.610289 & -2.378033 & 2.266979 \\
\hline 21 & 1 & 0 & 4.014224 & -0.845949 & -1.059309 \\
\hline 22 & 1 & 0 & 4.086988 & -2.571347 & -1.492689 \\
\hline 23 & 1 & 0 & 2.520709 & -1.728843 & -1.448182 \\
\hline 24 & 1 & 0 & 3.319604 & -4.853698 & -0.398930 \\
\hline 25 & 1 & 0 & 1.606893 & -5.160685 & -0.053179 \\
\hline 26 & 1 & 0 & 2.048492 & -3.965759 & -1.277784 \\
\hline 27 & 1 & 0 & 1.656828 & -4.582761 & 2.363921 \\
\hline 28 & 1 & 0 & 3.424817 & -4.554805 & 2.202003 \\
\hline 29 & 1 & 0 & 2.575174 & -3.174548 & 2.930020 \\
\hline 30 & 29 & 0 & -0.050406 & 0.204019 & 0.704899 \\
\hline 31 & 15 & 0 & 1.117449 & 1.845080 & -0.476658 \\
\hline 32 & 15 & 0 & -1.215302 & -0.322981 & -1.229369 \\
\hline 33 & 6 & 0 & 0.818284 & 1.350542 & -2.242115 \\
\hline 34 & 6 & 0 & 0.415497 & 3.540249 & -0.453346 \\
\hline 35 & 6 & 0 & 2.924634 & 2.072129 & -0.409845 \\
\hline 36 & 6 & 0 & -0.643783 & 0.960453 & -2.443050 \\
\hline 37 & 6 & 0 & -3.030400 & -0.182487 & -1.362170 \\
\hline 38 & 6 & 0 & -0.828778 & -1.921435 & -2.015360 \\
\hline 39 & 1 & 0 & 1.088061 & 2.162543 & -2.932440 \\
\hline 40 & 1 & 0 & 1.488858 & 0.503591 & -2.450957 \\
\hline 41 & 6 & 0 & -0.806899 & 3.718838 & 0.205023 \\
\hline 42 & 6 & 0 & 1.015206 & 4.634370 & -1.088124 \\
\hline 43 & 6 & 0 & 3.717668 & 2.344117 & -1.532374 \\
\hline
\end{tabular}


Medina et al.: BCP Hydroboration-Supporting Information - S82

\begin{tabular}{|c|c|c|c|c|c|}
\hline 44 & 6 & 0 & 3.546867 & 1.911404 & 0.834887 \\
\hline 45 & 1 & 0 & -0.841475 & 0.643183 & -3.476502 \\
\hline 46 & 1 & 0 & -1.286595 & 1.833774 & -2.259750 \\
\hline 47 & 6 & 0 & -3.628378 & 0.860959 & -0.645003 \\
\hline 48 & 6 & 0 & -3.835083 & -1.039265 & -2.120293 \\
\hline 49 & 6 & 0 & -1.316397 & -3.086606 & -1.40485 \\
\hline 50 & 6 & 0 & -0.019158 & -2.047269 & -3.1490 \\
\hline 51 & 1 & 0 & -1.252087 & 2.863352 & 0.7214 \\
\hline 52 & 6 & 0 & -1.429683 & 4.964602 & 0.21200 \\
\hline 53 & 6 & 0 & 0.396442 & 5.880387 & -1.07299 \\
\hline 54 & 1 & 0 & 1.978533 & 4.512024 & -1.58562 \\
\hline 55 & 1 & 0 & 3.257260 & 2.460548 & -2.5135 \\
\hline 56 & 6 & 0 & 5.100447 & 2.458482 & -1.4111 \\
\hline 57 & 6 & 0 & 4.926515 & 2.036496 & 0.9569 \\
\hline 58 & 1 & 0 & 2.940923 & 1.651207 & 1.70213 \\
\hline 59 & 1 & 0 & -3.006461 & 1.501739 & -0.01719 \\
\hline 60 & 6 & 0 & -5.002965 & 1.056592 & -0.69924 \\
\hline 61 & 6 & 0 & -5.213779 & -0.847473 & -2.1629 \\
\hline 62 & 1 & 0 & -3.384534 & -1.859991 & -2.67698 \\
\hline 63 & 1 & 0 & -1.928478 & -3.003286 & -0.5054 \\
\hline 64 & 6 & 0 & -1.031229 & -4.337667 & -1.93563 \\
\hline 65 & 6 & 0 & 0.283711 & -3.304920 & -3.66980 \\
\hline 66 & 1 & 0 & 0.381217 & -1.160744 & -3.64037 \\
\hline 67 & 1 & 0 & -2.381394 & 5.091357 & 0.72575 \\
\hline 68 & 6 & 0 & -0.828536 & 6.045642 & -0.42713 \\
\hline 69 & 1 & 0 & 0.871654 & 6.727733 & -1.56479 \\
\hline 70 & 1 & 0 & 5.705605 & 2.665564 & -2.29230 \\
\hline 71 & 6 & 0 & 5.706112 & 2.307052 & -0.16604 \\
\hline 72 & 1 & 0 & 5.396654 & 1.904196 & 1.92992 \\
\hline 73 & 1 & 0 & -5.455326 & 1.860495 & -0.1213 \\
\hline 74 & 6 & 0 & -5.799054 & 0.200137 & -1.4564 \\
\hline 75 & 1 & 0 & -5.832853 & -1.520225 & -2.7545 \\
\hline 76 & 1 & 0 & -1.426913 & -5.230634 & -1.45454 \\
\hline 77 & 6 & 0 & -0.228682 & -4.451519 & -3.0708 \\
\hline 78 & 1 & 0 & 0.915239 & -3.384246 & $-4.5533]$ \\
\hline 79 & 1 & 0 & -1.309892 & 7.022133 & -0.4171 \\
\hline 80 & 1 & 0 & 6.787313 & 2.394076 & -0.0723 \\
\hline 81 & 1 & 0 & -6.878038 & 0.342643 & -1.4871 \\
\hline 82 & 1 & 0 & 0.003321 & -5.433033 & -3.4806 \\
\hline 83 & 6 & 0 & -2.394077 & 0.175917 & 2.45011 \\
\hline 84 & 6 & 0 & -2.756322 & -1.135523 & 2.0656 \\
\hline 85 & 6 & 0 & -3.444384 & 1.063085 & 2.7638 \\
\hline 86 & 6 & 0 & -4.086749 & -1.524701 & 1.9931 \\
\hline 87 & 1 & 0 & -1.961634 & -1.840578 & 1.8113 \\
\hline 88 & 6 & 0 & -4.774070 & 0.664663 & 2.70092 \\
\hline 89 & 1 & 0 & -3.195073 & 2.081638 & 3.07003 \\
\hline 90 & 6 & 0 & -5.110997 & -0.632165 & 2.3131 \\
\hline 91 & 1 & 0 & -4.330657 & -2.540222 & 1.6782 \\
\hline 92 & 1 & 0 & -5.559476 & 1.376226 & 2.9584 \\
\hline 93 & 1 & 0 & -6.153310 & -0.940273 & 2.25333 \\
\hline 94 & 1 & 0 & -0.815474 & 1.620390 & 2.8560 \\
\hline
\end{tabular}


Filename: TS10

SCF Energy (M06L/LANL2DZ-6-31g (d))

-2682.169540 A.U.

$\mathrm{SCF}$ Energy (M06/SDD-6-311+G(d,p)) in solution

-2682.783287 A.U.

Enthalpy at $298 \mathrm{~K}(\mathrm{M} 06 / \mathrm{SDD}-6-311+\mathrm{G}(\mathrm{d}, \mathrm{p}))$

-2681.961006 A.U.

Gibbs Free Energy at $298 \mathrm{~K}$ and 1 mol/L with Truhlar's quasiharmonic approximation (M06/SDD-6-311+G(d,p))

-2682.073331 A.U.

\begin{tabular}{|c|c|c|c|c|c|}
\hline \multirow{2}{*}{$\begin{array}{l}\text { Center } \\
\text { Number }\end{array}$} & \multirow{2}{*}{$\begin{array}{l}\text { Atomic } \\
\text { Number }\end{array}$} & \multirow{2}{*}{$\begin{array}{c}\text { Atomic } \\
\text { Type }\end{array}$} & \multicolumn{3}{|c|}{ Coordinates (Angstroms) } \\
\hline & & & $\mathrm{X}$ & Y & $\mathrm{Z}$ \\
\hline & & & & & \\
\hline 1 & 6 & 0 & -0.446497 & -2.262539 & -0.494413 \\
\hline 2 & 6 & 0 & 0.404229 & -1.837455 & -1.605589 \\
\hline 3 & 6 & 0 & 0.029259 & -0.312057 & -2.628333 \\
\hline 4 & 6 & 0 & 0.047127 & -1.753288 & -3.047945 \\
\hline 5 & 5 & 0 & 1.931396 & -1.813536 & -1.300358 \\
\hline 6 & 1 & 0 & 0.886282 & 0.283123 & -2.937638 \\
\hline 7 & 1 & 0 & -0.939651 & 0.186139 & -2.715839 \\
\hline 8 & 1 & 0 & -0.915741 & -2.185487 & -3.324732 \\
\hline 9 & 1 & 0 & 0.830380 & -2.043979 & -3.752360 \\
\hline 10 & 8 & 0 & 2.850343 & -1.094469 & -2.029438 \\
\hline 11 & 8 & 0 & 2.508134 & -2.602019 & -0.331730 \\
\hline 12 & 6 & 0 & 4.159430 & -1.610891 & -1.688043 \\
\hline 13 & 6 & 0 & 3.918120 & -2.268602 & -0.287363 \\
\hline 14 & 6 & 0 & 5.146219 & -0.460962 & -1.684015 \\
\hline 15 & 6 & 0 & 4.517819 & -2.624167 & -2.767118 \\
\hline 16 & 6 & 0 & 4.707397 & -3.540078 & -0.042086 \\
\hline 17 & 6 & 0 & 4.106222 & -1.301237 & 0.870334 \\
\hline 18 & 1 & 0 & 6.144436 & -0.803819 & -1.381515 \\
\hline 19 & 1 & 0 & 5.227143 & -0.033366 & -2.689916 \\
\hline 20 & 1 & 0 & 4.832841 & 0.339302 & -1.006199 \\
\hline 21 & 1 & 0 & 4.483861 & -2.134508 & -3.746192 \\
\hline 22 & 1 & 0 & 5.523463 & -3.037032 & -2.623987 \\
\hline 23 & 1 & 0 & 3.803641 & -3.456233 & -2.783173 \\
\hline 24 & 1 & 0 & 5.786246 & -3.340021 & -0.071378 \\
\hline 25 & 1 & 0 & 4.467692 & -3.943682 & 0.947652 \\
\hline 26 & 1 & 0 & 4.478668 & -4.311394 & -0.782933 \\
\hline 27 & 1 & 0 & 3.768574 & -1.772072 & 1.800198 \\
\hline 28 & 1 & 0 & 5.155804 & -1.006287 & 0.992613 \\
\hline 29 & 1 & 0 & 3.514818 & -0.390747 & 0.720829 \\
\hline 30 & 29 & 0 & -0.180272 & -0.148903 & -0.417861 \\
\hline 31 & 15 & 0 & -2.074332 & 1.082210 & 0.153412 \\
\hline 32 & 15 & 0 & 1.125098 & 1.296104 & 0.788279 \\
\hline 33 & 6 & 0 & -1.408072 & 2.347896 & 1.339744 \\
\hline 34 & 6 & 0 & -2.944146 & 2.062120 & -1.113991 \\
\hline 35 & 6 & 0 & -3.410998 & 0.301990 & 1.118475 \\
\hline 36 & 6 & 0 & 0.011410 & 2.775717 & 0.981722 \\
\hline 37 & 6 & 0 & 2.644441 & 2.107300 & 0.166754 \\
\hline 38 & 6 & 0 & 1.461719 & 0.838539 & 2.527575 \\
\hline 39 & 1 & 0 & -2.084331 & 3.211042 & 1.415495 \\
\hline 40 & 1 & 0 & -1.410435 & 1.860951 & 2.325266 \\
\hline 41 & 6 & 0 & -2.655256 & 3.410371 & -1.359944 \\
\hline 42 & 6 & 0 & -3.848418 & 1.405407 & -1.964558 \\
\hline 43 & 6 & 0 & -4.724568 & 0.784145 & 1.154881 \\
\hline
\end{tabular}




\begin{tabular}{|c|c|c|c|c|c|}
\hline 44 & 6 & 0 & -3.071918 & -0.823057 & 1.878662 \\
\hline 45 & 1 & 0 & 0.416031 & 3.467478 & 1.733949 \\
\hline 46 & 1 & 0 & 0.036933 & 3.305013 & 0.018646 \\
\hline 47 & 6 & 0 & 2.623074 & 2.528313 & -1.17155 \\
\hline 48 & 6 & 0 & 3.816323 & 2.287931 & 0.91047 \\
\hline 49 & 6 & 0 & 1.802105 & 1.762969 & 3.52450 \\
\hline 50 & 6 & 0 & 1.298267 & -0.507583 & 2.87771 \\
\hline 51 & 1 & 0 & -1.957219 & 3.947797 & -0.71832 \\
\hline 52 & 6 & 0 & -3.260951 & 4.086370 & -2.41641 \\
\hline 53 & 6 & 0 & -4.456831 & 2.084055 & -3.01389 \\
\hline 54 & 1 & 0 & -4.087728 & 0.356977 & -1.77937 \\
\hline 55 & 1 & 0 & -4.999911 & 1.656243 & 0.56223 \\
\hline 56 & 6 & 0 & -5.681867 & 0.146608 & 1.93819 \\
\hline 57 & 6 & 0 & -4.030218 & -1.453256 & 2.66556 \\
\hline 58 & 1 & 0 & -2.053551 & -1.214198 & 1.81952 \\
\hline 59 & 1 & 0 & 1.728002 & 2.353592 & -1.77207 \\
\hline 60 & 6 & 0 & 3.731720 & 3.144970 & -1.73980 \\
\hline 61 & 6 & 0 & 4.931604 & 2.893764 & 0.33466 \\
\hline 62 & 1 & 0 & 3.863653 & 1.940926 & 1.94230 \\
\hline 63 & 1 & 0 & 1.934071 & 2.815417 & 3.26939 \\
\hline 64 & 6 & 0 & 1.981867 & 1.348183 & 4.84069 \\
\hline 65 & 6 & 0 & 1.484317 & -0.923387 & 4.19359 \\
\hline 66 & 1 & 0 & 1.018967 & -1.221495 & 2.09751 \\
\hline 67 & 1 & 0 & -3.028713 & 5.136484 & -2.58684 \\
\hline 68 & 6 & 0 & -4.163644 & 3.427152 & -3.24602 \\
\hline 69 & 1 & 0 & -5.164670 & 1.560582 & -3.65458 \\
\hline 70 & 1 & 0 & -6.702570 & 0.525526 & 1.95858 \\
\hline 71 & 6 & 0 & -5.336220 & -0.971451 & 2.69451 \\
\hline 72 & 1 & 0 & -3.759763 & -2.340331 & 3.23595 \\
\hline 73 & 1 & 0 & 3.696745 & 3.467877 & -2.77885 \\
\hline 74 & 6 & 0 & 4.890413 & 3.330321 & -0.98684 \\
\hline 75 & 1 & 0 & 5.837210 & 3.024626 & 0.92504 \\
\hline 76 & 1 & 0 & 2.243617 & 2.074893 & 5.60803 \\
\hline 77 & 6 & 0 & 1.823796 & 0.004204 & 5.17560 \\
\hline 78 & 1 & 0 & 1.357465 & -1.972965 & 4.45310 \\
\hline 79 & 1 & 0 & -4.636363 & 3.956755 & -4.07101 \\
\hline 80 & 1 & 0 & -6.089380 & -1.472641 & 3.30049 \\
\hline 81 & 1 & 0 & 5.762975 & 3.803305 & -1.43380 \\
\hline 82 & 1 & 0 & 1.961439 & -0.318236 & 6.20631 \\
\hline 83 & 1 & 0 & 0.121407 & -2.737271 & 0.30735 \\
\hline 84 & 6 & 0 & -1.835906 & -2.665031 & -0.58050 \\
\hline 85 & 6 & 0 & -2.775786 & -2.056590 & -1.44526 \\
\hline 86 & 6 & 0 & -2.341424 & -3.658036 & 0.28827 \\
\hline 87 & 6 & 0 & -4.120159 & -2.410281 & -1.42886 \\
\hline 88 & 1 & 0 & -2.447907 & -1.258036 & -2.11196 \\
\hline 89 & 6 & 0 & -3.684610 & -4.005462 & 0.30596 \\
\hline 90 & 1 & 0 & -1.645484 & -4.152399 & 0.96884 \\
\hline 91 & 6 & 0 & -4.593901 & -3.384646 & -0.55188 \\
\hline 92 & 1 & 0 & -4.811776 & -1.909276 & -2.10862 \\
\hline 93 & 1 & 0 & -4.029860 & -4.773110 & 0.99945 \\
\hline 94 & 1 & 0 & -5.648623 & -3.652021 & -0.53439 \\
\hline
\end{tabular}


Filename: TS11

SCF Energy (M06L/LANL2DZ-6-31g(d))

-2797.876979 A.U.

$\mathrm{SCF}$ Energy (M06/SDD-6-311+G(d,p)) in solution

-2798.484900 A.U.

Enthalpy at $298 \mathrm{~K}(\mathrm{M} 06 / \mathrm{SDD}-6-311+\mathrm{G}(\mathrm{d}, \mathrm{p}))$

-2797.608252 A.U.

Gibbs Free Energy at $298 \mathrm{~K}$ and 1 mol/L with Truhlar's quasiharmonic approximation (M06/SDD-6-311+G(d,p))

-2797.728914 A.U.

\begin{tabular}{|c|c|c|c|c|c|}
\hline \multirow{2}{*}{$\begin{array}{l}\text { Center } \\
\text { Number }\end{array}$} & \multirow{2}{*}{$\begin{array}{l}\text { Atomic } \\
\text { Number }\end{array}$} & \multirow{2}{*}{$\begin{array}{c}\text { Atomic } \\
\text { Type }\end{array}$} & \multicolumn{3}{|c|}{ Coordinates (Angstroms) } \\
\hline & & & $\mathrm{X}$ & Y & $\mathrm{Z}$ \\
\hline & & & & & \\
\hline 1 & 6 & 0 & -0.881922 & -2.996141 & -0.995168 \\
\hline 2 & 6 & 0 & -0.736598 & -3.800178 & -2.266325 \\
\hline 3 & 6 & 0 & -0.720337 & -4.518284 & -0.964762 \\
\hline 4 & 5 & 0 & -2.294431 & -2.506399 & -0.622472 \\
\hline 5 & 1 & 0 & 0.210740 & -3.706137 & -2.797373 \\
\hline 6 & 1 & 0 & -1.607014 & -3.910118 & -2.911375 \\
\hline 7 & 1 & 0 & -1.579279 & -5.132942 & -0.699468 \\
\hline 8 & 1 & 0 & 0.238196 & -4.889547 & -0.603733 \\
\hline 9 & 8 & 0 & -3.455999 & -3.118863 & -1.050098 \\
\hline 10 & 8 & 0 & -2.545388 & -1.453185 & 0.244764 \\
\hline 11 & 6 & 0 & -4.557419 & -2.307834 & -0.578661 \\
\hline 12 & 6 & 0 & -3.931375 & -1.592216 & 0.653696 \\
\hline 13 & 6 & 0 & -5.730707 & -3.212537 & -0.261006 \\
\hline 14 & 6 & 0 & -4.913372 & -1.338749 & -1.698512 \\
\hline 15 & 6 & 0 & -4.514264 & -0.230944 & 0.971840 \\
\hline 16 & 6 & 0 & -3.934379 & -2.466615 & 1.901637 \\
\hline 17 & 1 & 0 & -6.555633 & -2.641855 & 0.184977 \\
\hline 18 & 1 & 0 & -6.104063 & -3.681703 & -1.177890 \\
\hline 19 & 1 & 0 & -5.447233 & -4.010370 & 0.431627 \\
\hline 20 & 1 & 0 & -5.147795 & -1.902883 & -2.607401 \\
\hline 21 & 1 & 0 & -5.780344 & -0.716926 & -1.442595 \\
\hline 22 & 1 & 0 & -4.067150 & -0.677035 & -1.925132 \\
\hline 23 & 1 & 0 & -5.589202 & -0.314345 & 1.180171 \\
\hline 24 & 1 & 0 & -4.032283 & 0.189861 & 1.862221 \\
\hline 25 & 1 & 0 & -4.379497 & 0.479585 & 0.150269 \\
\hline 26 & 1 & 0 & -3.295956 & -2.003611 & 2.662654 \\
\hline 27 & 1 & 0 & -4.942403 & -2.574833 & 2.320079 \\
\hline 28 & 1 & 0 & -3.540105 & -3.467330 & 1.687176 \\
\hline 29 & 8 & 0 & 0.288985 & -0.441864 & -2.641880 \\
\hline 30 & 6 & 0 & -0.998742 & -0.334851 & -3.166696 \\
\hline 31 & 1 & 0 & -1.152810 & -1.012120 & -4.025608 \\
\hline 32 & 1 & 0 & -1.772975 & -0.588305 & -2.408701 \\
\hline 33 & 1 & 0 & -1.208572 & 0.693961 & -3.504917 \\
\hline 34 & 1 & 0 & 0.248177 & -1.235479 & -1.734175 \\
\hline 35 & 29 & 0 & 0.313073 & 0.155644 & -0.504375 \\
\hline 36 & 15 & 0 & 2.300940 & 1.009293 & 0.351513 \\
\hline 37 & 15 & 0 & -0.827641 & 1.933360 & 0.298640 \\
\hline 38 & 6 & 0 & 1.706540 & 2.470930 & 1.344116 \\
\hline 39 & 6 & 0 & 3.484345 & 0.209564 & 1.480216 \\
\hline 40 & 6 & 0 & 3.312527 & 1.786598 & -0.949348 \\
\hline 41 & 6 & 0 & 0.519519 & 3.160174 & 0.672719 \\
\hline 42 & 6 & 0 & -1.524505 & 1.631629 & 1.964290 \\
\hline 43 & 6 & 0 & -2.100170 & 2.908476 & -0.564741 \\
\hline
\end{tabular}




\begin{tabular}{|c|c|c|c|c|c|}
\hline 44 & 1 & 0 & 1.405329 & 2.094562 & 2.332339 \\
\hline 45 & 1 & 0 & 2.524134 & 3.184702 & 1.513217 \\
\hline 46 & 6 & 0 & 4.749704 & -0.201887 & 1.045562 \\
\hline 47 & 6 & 0 & 3.073552 & -0.129967 & 2.778020 \\
\hline 48 & 6 & 0 & 4.379528 & 2.649215 & -0.661341 \\
\hline 49 & 6 & 0 & 2.978355 & 1.520630 & -2.28333 \\
\hline 50 & 1 & 0 & 0.826609 & 3.604073 & -0.2856 \\
\hline 51 & 1 & 0 & 0.149575 & 3.976034 & 1.30973 \\
\hline 52 & 6 & 0 & -1.196577 & 0.430452 & 2.60465 \\
\hline 53 & 6 & 0 & -2.360235 & 2.545615 & 2.616270 \\
\hline 54 & 6 & 0 & -2.223970 & 4.300910 & -0.486416 \\
\hline 55 & 6 & 0 & -2.999572 & 2.192537 & -1.36575 \\
\hline 56 & 1 & 0 & 5.072818 & 0.021833 & 0.0297 \\
\hline 57 & 6 & 0 & 5.592372 & -0.909724 & 1.8974 \\
\hline 58 & 6 & 0 & 3.920554 & -0.830910 & 3.62842 \\
\hline 59 & 1 & 0 & 2.076474 & 0.145723 & 3.12617 \\
\hline 60 & 1 & 0 & 4.655753 & 2.843424 & 0.37631 \\
\hline 61 & 6 & 0 & 5.100701 & 3.240535 & -1.6922 \\
\hline 62 & 6 & 0 & 3.704831 & 2.118664 & -3.3125 \\
\hline 63 & 1 & 0 & 2.147335 & 0.845806 & -2.50960 \\
\hline 64 & 1 & 0 & -0.579966 & -0.301494 & 2.07753 \\
\hline 65 & 6 & 0 & -1.679906 & 0.157031 & 3.88184 \\
\hline 66 & 6 & 0 & -2.855919 & 2.264501 & 3.88608 \\
\hline 67 & 1 & 0 & -2.640638 & 3.474490 & 2.1176 \\
\hline 68 & 1 & 0 & -1.527468 & 4.880461 & 0.11932 \\
\hline 69 & 6 & 0 & -3.234924 & 4.957482 & -1.18441 \\
\hline 70 & 6 & 0 & -4.016916 & 2.846573 & -2.0517 \\
\hline 71 & 1 & 0 & -2.879081 & 1.110876 & -1.4490 \\
\hline 72 & 1 & 0 & 6.571081 & -1.226803 & 1.5407 \\
\hline 73 & 6 & 0 & 5.185874 & -1.219832 & 3.1919 \\
\hline 74 & 1 & 0 & 3.589105 & -1.078928 & 4.63550 \\
\hline 75 & 1 & 0 & 5.930442 & 3.907233 & -1.46275 \\
\hline 76 & 6 & 0 & 4.761371 & 2.975776 & -3.0195 \\
\hline 77 & 1 & 0 & 3.441681 & 1.908967 & -4.34773 \\
\hline 78 & 1 & 0 & -1.417886 & -0.780472 & 4.3700 \\
\hline 79 & 6 & 0 & -2.513863 & 1.071626 & 4.5216 \\
\hline 80 & 1 & 0 & -3.513780 & 2.976980 & 4.38102 \\
\hline 81 & 1 & 0 & -3.319816 & 6.040950 & -1.1199 \\
\hline 82 & 6 & 0 & -4.134992 & 4.232304 & -1.96192 \\
\hline 83 & 1 & 0 & -4.709253 & 2.274263 & -2.6676 \\
\hline 84 & 1 & 0 & 5.847312 & -1.772726 & 3.8565 \\
\hline 85 & 1 & 0 & 5.327301 & 3.439586 & -3.8260 \\
\hline 86 & 1 & 0 & -2.903620 & 0.852555 & 5.51425 \\
\hline 87 & 1 & 0 & -4.923487 & 4.749333 & -2.5057 \\
\hline 88 & 6 & 0 & 1.643034 & -2.640142 & -0.5328 \\
\hline 89 & 6 & 0 & 2.228888 & -3.127762 & 0.64752 \\
\hline 90 & 6 & 0 & 2.435316 & -2.656078 & -1.6950 \\
\hline 91 & 6 & 0 & 3.534909 & -3.606368 & 0.67402 \\
\hline 92 & 1 & 0 & 1.637612 & -3.116908 & 1.56613 \\
\hline 93 & 6 & 0 & 3.739054 & -3.145028 & -1.67583 \\
\hline 94 & 1 & 0 & 2.022014 & -2.241082 & -2.6150 \\
\hline 95 & 6 & 0 & 4.297667 & -3.622781 & -0.4919 \\
\hline 96 & 1 & 0 & 3.961536 & -3.963584 & 1.6113 \\
\hline 97 & 1 & 0 & 4.328331 & -3.140472 & -2.5927 \\
\hline 98 & 1 & 0 & 5.321486 & -3.994931 & -0.4752 \\
\hline 99 & 6 & 0 & 0.256175 & -2.082175 & -0.53672 \\
\hline 100 & 1 & 0 & 0.012116 & -1.823811 & 0.51389 \\
\hline
\end{tabular}


Medina et al.: BCP Hydroboration-Supporting Information - S87 


\section{Filename: TS12}

SCF Energy (M06L/LANL2DZ-6-31g (d))

-2797.890458 A.U.

SCF Energy (M06/SDD-6-311+G(d,p)) in solution

-2798.493603 A.U.

Enthalpy at $298 \mathrm{~K}(\mathrm{M} 06 / \mathrm{SDD}-6-311+\mathrm{G}(\mathrm{d}, \mathrm{p}))$

-2797.616797 A.U.

Gibbs Free Energy at $298 \mathrm{~K}$ and 1 mol/L with Truhlar's quasiharmonic approximation ( $06 / \mathrm{SDD}-6-311+\mathrm{G}(\mathrm{d}, \mathrm{p}))$

-2797.737829 A.U.

\begin{tabular}{|c|c|c|c|c|c|}
\hline \multirow{2}{*}{$\begin{array}{l}\text { Center } \\
\text { Number }\end{array}$} & \multirow{2}{*}{$\begin{array}{l}\text { Atomic } \\
\text { Number }\end{array}$} & \multirow{2}{*}{$\begin{array}{c}\text { Atomic } \\
\text { Type }\end{array}$} & \multicolumn{3}{|c|}{ Coordinates (Angstroms) } \\
\hline & & & $\mathrm{X}$ & Y & $\mathrm{Z}$ \\
\hline & & & & & \\
\hline 1 & 6 & 0 & -0.246346 & 0.834158 & 2.675103 \\
\hline 2 & 6 & 0 & -1.690099 & 0.402784 & 2.975738 \\
\hline 3 & 1 & 0 & -0.283240 & 1.689804 & 1.972461 \\
\hline 4 & 1 & 0 & 0.216384 & 1.277530 & 3.572664 \\
\hline 5 & 1 & 0 & 0.649002 & -0.310036 & 2.753212 \\
\hline 6 & 8 & 0 & 1.399593 & -1.268196 & 2.692489 \\
\hline 7 & 6 & 0 & 2.684926 & -0.826221 & 2.998472 \\
\hline 8 & 1 & 0 & 3.439495 & -1.322867 & 2.361356 \\
\hline 9 & 1 & 0 & 2.801730 & 0.267334 & 2.828220 \\
\hline 10 & 1 & 0 & 2.956766 & -1.026601 & 4.050000 \\
\hline 11 & 29 & 0 & 0.624126 & -0.139493 & 0.886792 \\
\hline 12 & 15 & 0 & -0.043028 & -1.236204 & -1.023697 \\
\hline 13 & 15 & 0 & 2.355107 & 0.834844 & -0.351631 \\
\hline 14 & 6 & 0 & 0.714393 & -0.195960 & -2.375334 \\
\hline 15 & 6 & 0 & 0.991728 & -2.740136 & -1.132745 \\
\hline 16 & 6 & 0 & -1.632636 & -1.717805 & -1.771433 \\
\hline 17 & 6 & 0 & 2.169423 & 0.121698 & -2.052455 \\
\hline 18 & 6 & 0 & 4.159978 & 0.730978 & -0.101579 \\
\hline 19 & 6 & 0 & 2.009415 & 2.616988 & -0.529194 \\
\hline 20 & 1 & 0 & 0.633819 & -0.710059 & -3.343008 \\
\hline 21 & 1 & 0 & 0.127217 & 0.731003 & -2.451747 \\
\hline 22 & 6 & 0 & 1.727658 & -3.114369 & -0.001104 \\
\hline 23 & 6 & 0 & 1.127207 & -3.476368 & -2.317562 \\
\hline 24 & 6 & 0 & -2.123808 & -3.022907 & -1.643376 \\
\hline 25 & 6 & 0 & -2.438329 & -0.748470 & -2.383502 \\
\hline 26 & 1 & 0 & 2.624944 & 0.763917 & -2.817957 \\
\hline 27 & 1 & 0 & 2.757163 & -0.807481 & -2.047038 \\
\hline 28 & 6 & 0 & 4.970078 & 1.849093 & 0.121673 \\
\hline 29 & 6 & 0 & 4.740321 & -0.546166 & -0.073090 \\
\hline 30 & 6 & 0 & 1.741900 & 3.323575 & 0.653713 \\
\hline 31 & 6 & 0 & 1.957197 & 3.299602 & -1.749514 \\
\hline 32 & 1 & 0 & 1.607712 & -2.552568 & 0.932180 \\
\hline 33 & 6 & 0 & 2.595959 & -4.204141 & -0.063261 \\
\hline 34 & 6 & 0 & 1.993174 & -4.563342 & -2.373332 \\
\hline 35 & 1 & 0 & 0.542070 & -3.201790 & -3.196686 \\
\hline 36 & 1 & 0 & -1.524127 & -3.782514 & -1.142067 \\
\hline 37 & 6 & 0 & -3.384441 & -3.351245 & -2.132683 \\
\hline 38 & 6 & 0 & -3.698493 & -1.078553 & -2.868062 \\
\hline 39 & 1 & 0 & -2.095212 & 0.282737 & -2.450144 \\
\hline 40 & 1 & 0 & 4.534509 & 2.847534 & 0.107103 \\
\hline 41 & 6 & 0 & 6.334482 & 1.692667 & 0.355852 \\
\hline 42 & 6 & 0 & 6.103430 & -0.696720 & 0.150446 \\
\hline 43 & 1 & 0 & 4.114717 & -1.430554 & -0.215262 \\
\hline
\end{tabular}




\begin{tabular}{|c|c|c|c|c|c|}
\hline 44 & 1 & 0 & 1.757896 & 2.791426 & 1.606789 \\
\hline 45 & 6 & 0 & 1.446295 & 4.682036 & 0.616478 \\
\hline 46 & 6 & 0 & 1.642877 & 4.656213 & -1.787076 \\
\hline 47 & 1 & 0 & 2.157539 & 2.774731 & -2.682857 \\
\hline 48 & 1 & 0 & 3.163476 & -4.488900 & 0.821769 \\
\hline 49 & 6 & 0 & 2.732103 & -4.925116 & -1.24639 \\
\hline 50 & 1 & 0 & 2.092924 & -5.131488 & -3.2968 \\
\hline 51 & 1 & 0 & -3.754843 & -4.368619 & -2.01557 \\
\hline 52 & 6 & 0 & -4.174812 & -2.382805 & -2.74653 \\
\hline 53 & 1 & 0 & -4.315298 & -0.309847 & -3.33121 \\
\hline 54 & 1 & 0 & 6.954343 & 2.571422 & 0.52593 \\
\hline 55 & 6 & 0 & 6.904946 & 0.423339 & 0.36732 \\
\hline 56 & 1 & 0 & 6.540206 & -1.693744 & 0.1654 \\
\hline 57 & 1 & 0 & 1.241912 & 5.216293 & 1.54271 \\
\hline 58 & 6 & 0 & 1.388538 & 5.349587 & -0.6064 \\
\hline 59 & 1 & 0 & 1.597064 & 5.173116 & -2.74434 \\
\hline 60 & 1 & 0 & 3.410293 & -5.775803 & -1.29319 \\
\hline 61 & 1 & 0 & -5.163496 & -2.640854 & -3.1221 \\
\hline 62 & 1 & 0 & 7.971337 & 0.304642 & 0.54992 \\
\hline 63 & 1 & 0 & 1.137907 & 6.408701 & -0.63841 \\
\hline 64 & 6 & 0 & -2.506043 & 0.276554 & 1.71669 \\
\hline 65 & 6 & 0 & -2.964316 & -0.877521 & 1.17359 \\
\hline 66 & 1 & 0 & -3.602561 & -0.786016 & 0.28802 \\
\hline 67 & 6 & 0 & -2.726093 & -2.262024 & 1.58088 \\
\hline 68 & 6 & 0 & -1.492080 & -2.691034 & $2.0967^{\circ}$ \\
\hline 69 & 6 & 0 & -3.717227 & -3.231750 & 1.35274 \\
\hline 70 & 6 & 0 & -1.264170 & -4.034516 & 2.37867 \\
\hline 71 & 1 & 0 & -0.684784 & -1.976988 & 2.24371 \\
\hline 72 & 6 & 0 & -3.496609 & -4.570493 & 1.6504 \\
\hline 73 & 1 & 0 & -4.669619 & -2.918467 & 0.9229 \\
\hline 74 & 6 & 0 & -2.265059 & -4.979432 & 2.1634 \\
\hline 75 & 1 & 0 & -0.289951 & -4.336136 & 2.76205 \\
\hline 76 & 1 & 0 & -4.283585 & -5.302352 & 1.4712 \\
\hline 77 & 1 & 0 & -2.085925 & -6.030551 & 2.3849 \\
\hline 78 & 5 & 0 & -2.819820 & 1.591950 & 0.96793 \\
\hline 79 & 8 & 0 & -2.681961 & 2.841320 & 1.53508 \\
\hline 80 & 8 & 0 & -3.216738 & 1.655992 & -0.3569 \\
\hline 81 & 6 & 0 & -3.205418 & 3.798549 & 0.5848 \\
\hline 82 & 6 & 0 & -3.052570 & 3.033843 & -0.76442 \\
\hline 83 & 6 & 0 & -2.397304 & 5.076538 & 0.6882 \\
\hline 84 & 1 & 0 & -2.561770 & 5.546477 & 1.6643 \\
\hline 85 & 1 & 0 & -1.325385 & 4.881022 & 0.5835 \\
\hline 86 & 1 & 0 & -2.698120 & 5.794434 & -0.08630 \\
\hline 87 & 6 & 0 & -4.660347 & 4.050455 & $0.9560^{\circ}$ \\
\hline 88 & 1 & 0 & -4.715665 & 4.378635 & 1.9991 \\
\hline 89 & 1 & 0 & -5.111310 & 4.827446 & 0.3268 \\
\hline 90 & 1 & 0 & -5.257969 & 3.136820 & 0.8576 \\
\hline 91 & 6 & 0 & -1.648192 & 3.160278 & -1.3433 \\
\hline 92 & 1 & 0 & -1.535524 & 2.467077 & -2.1868 \\
\hline 93 & 1 & 0 & -1.442386 & 4.172524 & -1.71221 \\
\hline 94 & 1 & 0 & -0.884793 & 2.909239 & -0.5934 \\
\hline 95 & 6 & 0 & -4.096245 & 3.360826 & -1.8127 \\
\hline 96 & 1 & 0 & -4.051477 & 4.420491 & -2.0954 \\
\hline 97 & 1 & 0 & -3.918828 & 2.765808 & -2.7167 \\
\hline 98 & 1 & 0 & -5.107207 & 3.139045 & -1.4586 \\
\hline 99 & 1 & 0 & -2.187134 & 1.156808 & 3.6098 \\
\hline 100 & 1 & 0 & -1.701451 & -0.534729 & 3.55031 \\
\hline
\end{tabular}


Medina et al.: BCP Hydroboration-Supporting Information - S90 


\section{Filename: TS13}

SCF Energy (M06L/LANL2DZ-6-31g (d))

-2682.163645 A.U. $\mathrm{SCF}$ Energy (M06/SDD-6-311+G(d,p)) in solution

-2682.777106 A.U.

Enthalpy at $298 \mathrm{~K}$ (M06/SDD-6-311+G(d,p))

$-2681.955265 \mathrm{~A} . \mathrm{U}$.

Gibbs Free Energy at $298 \mathrm{~K}$ and 1 mol/L with Truhlar's quasiharmonic approximation (M06/SDD-6-311+G(d,p))

-2682.067739 A.U.

\begin{tabular}{|c|c|c|c|c|c|}
\hline \multirow{2}{*}{$\begin{array}{l}\text { Center } \\
\text { Number }\end{array}$} & \multirow{2}{*}{$\begin{array}{l}\text { Atomic } \\
\text { Number }\end{array}$} & \multirow{2}{*}{$\begin{array}{c}\text { Atomic } \\
\text { Type }\end{array}$} & \multicolumn{3}{|c|}{ Coordinates (Angstroms) } \\
\hline & & & $\mathrm{X}$ & Y & Z \\
\hline------1 & & & ---------- & ---------- & --------- \\
\hline 1 & 6 & 0 & -0.218883 & -0.375962 & -2.425945 \\
\hline 2 & 1 & 0 & 0.494917 & -0.655928 & -3.207120 \\
\hline 3 & 6 & 0 & -1.044832 & -1.464911 & -1.918227 \\
\hline 4 & 6 & 0 & -0.561803 & 1.027547 & -2.389972 \\
\hline 5 & 6 & 0 & -0.300385 & -2.867841 & -0.949826 \\
\hline 6 & 6 & 0 & -0.805305 & -2.872352 & -2.358355 \\
\hline 7 & 5 & 0 & -2.516260 & -1.304367 & -1.418255 \\
\hline 8 & 6 & 0 & 0.146034 & 1.940722 & -3.211412 \\
\hline 9 & 6 & 0 & -1.548817 & 1.599843 & -1.548829 \\
\hline 10 & 1 & 0 & -0.952602 & -3.293689 & -0.190523 \\
\hline 11 & 1 & 0 & 0.758251 & -3.119547 & -0.851281 \\
\hline 12 & 1 & 0 & -0.039060 & -2.980449 & -3.130778 \\
\hline 13 & 1 & 0 & -1.678198 & -3.491437 & -2.585297 \\
\hline 14 & 8 & 0 & -2.986760 & -1.751302 & -0.204906 \\
\hline 15 & 8 & 0 & -3.513135 & -0.864852 & -2.253292 \\
\hline 16 & 6 & 0 & -0.096606 & 3.307058 & -3.176478 \\
\hline 17 & 1 & 0 & 0.901172 & 1.543462 & -3.893555 \\
\hline 18 & 6 & 0 & -1.803575 & 2.962819 & -1.534163 \\
\hline 19 & 1 & 0 & -2.103040 & 0.956732 & -0.863549 \\
\hline 20 & 6 & 0 & -4.428889 & -1.862189 & -0.340437 \\
\hline 21 & 6 & 0 & -4.736443 & -0.834783 & -1.473749 \\
\hline 22 & 6 & 0 & -1.077344 & 3.842329 & -2.339428 \\
\hline 23 & 1 & 0 & 0.483355 & 3.965795 & -3.824552 \\
\hline 24 & 1 & 0 & -2.581293 & 3.348592 & -0.871439 \\
\hline 25 & 6 & 0 & -5.071580 & -1.553167 & 0.994653 \\
\hline 26 & 6 & 0 & -4.711904 & -3.299858 & -0.755601 \\
\hline 27 & 6 & 0 & -5.891001 & -1.213114 & -2.379949 \\
\hline 28 & 6 & 0 & -4.916024 & 0.584352 & -0.952426 \\
\hline 29 & 1 & 0 & -1.272202 & 4.912286 & -2.316390 \\
\hline 30 & 1 & 0 & -6.166050 & -1.555092 & 0.907732 \\
\hline 31 & 1 & 0 & -4.790860 & -2.313024 & 1.733022 \\
\hline 32 & 1 & 0 & -4.753646 & -0.580936 & 1.382608 \\
\hline 33 & 1 & 0 & -4.274385 & -3.982557 & -0.019040 \\
\hline 34 & 1 & 0 & -5.787897 & -3.502470 & -0.815764 \\
\hline 35 & 1 & 0 & -4.267071 & -3.526074 & -1.732383 \\
\hline 36 & 1 & 0 & -6.824641 & -1.294418 & -1.808467 \\
\hline 37 & 1 & 0 & -6.031861 & -0.443596 & -3.146579 \\
\hline 38 & 1 & 0 & -5.712200 & -2.164697 & -2.888976 \\
\hline
\end{tabular}


Medina et al.: BCP Hydroboration-Supporting Information - S92

\begin{tabular}{|c|c|c|c|c|c|}
\hline 39 & 1 & 0 & -4.851314 & 1.288989 & -1.788260 \\
\hline 40 & 1 & 0 & -5.889378 & 0.708670 & -0.461411 \\
\hline 41 & 1 & 0 & -4.137245 & 0.849019 & -0.227744 \\
\hline 42 & 29 & 0 & 0.411734 & -0.851863 & -0.437351 \\
\hline 43 & 15 & 0 & 2.572301 & -0.812148 & 0.354604 \\
\hline 44 & 15 & 0 & -0.174055 & 0.446634 & 1.438636 \\
\hline 45 & 6 & 0 & 2.451918 & -0.158425 & 2.105887 \\
\hline 46 & 6 & 0 & 3.601358 & -2.308258 & 0.555502 \\
\hline 47 & 6 & 0 & 3.675088 & 0.383520 & -0.475753 \\
\hline 48 & 6 & 0 & 1.033792 & -0.259729 & 2.665856 \\
\hline 49 & 6 & 0 & -1.722557 & 0.574676 & 2.392914 \\
\hline 50 & 6 & 0 & 0.419336 & 2.177203 & 1.397110 \\
\hline 51 & 1 & 0 & 3.166158 & -0.688087 & 2.749559 \\
\hline 52 & 1 & 0 & 2.758793 & 0.896149 & 2.077248 \\
\hline 53 & 6 & 0 & 3.203987 & -3.275522 & 1.490667 \\
\hline 54 & 6 & 0 & 4.704505 & -2.578872 & -0.263220 \\
\hline 55 & 6 & 0 & 4.877253 & 0.828550 & 0.089795 \\
\hline 56 & 6 & 0 & 3.254771 & 0.919656 & -1.697704 \\
\hline 57 & 1 & 0 & 0.962866 & 0.265485 & 3.629342 \\
\hline 58 & 1 & 0 & 0.762704 & -1.310683 & 2.840333 \\
\hline 59 & 6 & 0 & -2.073001 & -0.355050 & 3.379536 \\
\hline 60 & 6 & 0 & -2.630040 & 1.597975 & 2.080495 \\
\hline 61 & 6 & 0 & 0.360667 & 3.013921 & 2.520421 \\
\hline 62 & 6 & 0 & 1.056502 & 2.631858 & 0.239975 \\
\hline 63 & 1 & 0 & 2.328877 & -3.094236 & 2.118246 \\
\hline 64 & 6 & 0 & 3.909021 & -4.466035 & 1.625325 \\
\hline 65 & 6 & 0 & 5.404579 & -3.775507 & -0.131672 \\
\hline 66 & 1 & 0 & 5.024334 & -1.842505 & -0.999624 \\
\hline 67 & 1 & 0 & 5.220010 & 0.409627 & 1.036993 \\
\hline 68 & 6 & 0 & 5.636021 & 1.801461 & -0.553001 \\
\hline 69 & 6 & 0 & 4.016358 & 1.891381 & -2.341791 \\
\hline 70 & 1 & 0 & 2.300386 & 0.588054 & -2.115042 \\
\hline 71 & 1 & 0 & -1.393097 & -1.169120 & 3.629008 \\
\hline 72 & 6 & 0 & -3.283824 & -0.245830 & 4.058113 \\
\hline 73 & 6 & 0 & -3.841456 & 1.702574 & 2.756716 \\
\hline 74 & 1 & 0 & -2.368499 & 2.328273 & 1.312590 \\
\hline 75 & 1 & 0 & -0.146427 & 2.673192 & 3.424460 \\
\hline 76 & 6 & 0 & 0.936939 & 4.279500 & 2.481703 \\
\hline 77 & 6 & 0 & 1.636775 & 3.897216 & 0.202886 \\
\hline 78 & 1 & 0 & 1.078149 & 1.991211 & -0.641454 \\
\hline 79 & 1 & 0 & 3.592004 & -5.201351 & 2.363022 \\
\hline 80 & 6 & 0 & 5.013758 & -4.719639 & 0.813836 \\
\hline 81 & 1 & 0 & 6.264800 & -3.967539 & -0.770957 \\
\hline 82 & 1 & 0 & 6.566099 & 2.147495 & -0.104996 \\
\hline 83 & 6 & 0 & 5.205106 & 2.334853 & -1.767872 \\
\hline 84 & 1 & 0 & 3.670218 & 2.311672 & -3.285082 \\
\hline 85 & 1 & 0 & -3.534647 & -0.970413 & 4.831353 \\
\hline 86 & 6 & 0 & -4.169625 & 0.784962 & 3.753670 \\
\hline 87 & 1 & 0 & -4.530299 & 2.507801 & 2.504879 \\
\hline 88 & 1 & 0 & 0.886982 & 4.924833 & 3.357515 \\
\hline 89 & 6 & 0 & 1.579388 & 4.720202 & 1.324133 \\
\hline 90 & 1 & 0 & 2.120297 & 4.236543 & -0.711996 \\
\hline
\end{tabular}


Medina et al.: BCP Hydroboration-Supporting Information - S93

\begin{tabular}{|c|c|c|c|c|c|}
\hline 91 & 1 & 0 & 5.563593 & -5.653392 & 0.915157 \\
\hline 92 & 1 & 0 & 5.798346 & 3.101200 & -2.264204 \\
\hline 93 & 1 & 0 & -5.114919 & 0.869676 & 4.286673 \\
\hline 94 & 1 & 0 & 2.029451 & 5.711473 & 1.297857 \\
\hline
\end{tabular}


Cartesian coordinates of the structures shown in Figure S2:

\section{Filename: TS1BINAP2}

SCF Energy (M06L/LANL2DZ-6-31g (d))

-3372.822273 A.U.

$\mathrm{SCF}$ Energy (M06/SDD-6-311+G(d,p)) in solution

-3373.141072 A.U.

Enthalpy at $298 \mathrm{~K}(\mathrm{M} 06 / \mathrm{SDD}-6-311+\mathrm{G}(\mathrm{d}, \mathrm{p}))$

-3372.108131 A.U.

Gibbs Free Energy at $298 \mathrm{~K}$ and 1 mol/L with Truhlar's quasiharmonic approximation (M06/SDD-6-311+G(d,p))

-3372.241524 A.U.

\begin{tabular}{|c|c|c|c|c|c|}
\hline \multirow{2}{*}{$\begin{array}{l}\text { Center } \\
\text { Number }\end{array}$} & \multirow{2}{*}{$\begin{array}{l}\text { Atomic } \\
\text { Number }\end{array}$} & \multirow{2}{*}{$\begin{array}{l}\text { Atomic } \\
\text { Type }\end{array}$} & \multicolumn{3}{|c|}{ Coordinates (Angstroms) } \\
\hline & & & $\mathrm{x}$ & Y & Z \\
\hline & & & & 17180118 & $-1-------$ \\
\hline 1 & 6 & 0 & -2.690506 & 1.189118 & -1.867301 \\
\hline 2 & 6 & 0 & -3.228223 & -0.042693 & -1.303745 \\
\hline 3 & 6 & 0 & -4.695097 & -0.251209 & -1.109839 \\
\hline 4 & 6 & 0 & -3.948535 & -1.025912 & -2.169023 \\
\hline 5 & 1 & 0 & -5.338003 & 0.575275 & -1.412889 \\
\hline 6 & 1 & 0 & -5.062145 & -0.797584 & -0.242311 \\
\hline 7 & 1 & 0 & -3.809681 & -2.093106 & -2.012978 \\
\hline 8 & 1 & 0 & -4.091938 & -0.743267 & -3.212694 \\
\hline 9 & 29 & 0 & -1.367796 & 0.256968 & -0.516678 \\
\hline 10 & 15 & 0 & 0.373792 & -1.080131 & -1.455165 \\
\hline 11 & 15 & 0 & 0.041685 & 1.531895 & 0.855574 \\
\hline 12 & 6 & 0 & 1.289083 & -1.550775 & 0.063945 \\
\hline 13 & 6 & 0 & 1.574053 & -0.344962 & -2.631402 \\
\hline 14 & 6 & 0 & 0.003247 & -2.673253 & -2.273809 \\
\hline 15 & 6 & 0 & -0.514129 & 3.262690 & 1.058700 \\
\hline 16 & 6 & 0 & 0.376936 & 0.990358 & 2.567326 \\
\hline 17 & 6 & 0 & 1.759218 & 1.692373 & 0.158716 \\
\hline 18 & 6 & 0 & 2.238449 & -0.728793 & 0.669448 \\
\hline 19 & 6 & 0 & 0.841064 & -2.715101 & 0.749096 \\
\hline 20 & 6 & 0 & 1.328186 & 0.957850 & -3.078366 \\
\hline 21 & 6 & 0 & 2.685083 & -1.038064 & -3.125248 \\
\hline 22 & 6 & 0 & 0.796037 & -3.823301 & -2.181072 \\
\hline 23 & 6 & 0 & -1.143098 & -2.705656 & -3.074264 \\
\hline 24 & 6 & 0 & -0.797152 & 4.009388 & -0.095030 \\
\hline 25 & 6 & 0 & -0.730170 & 3.848851 & 2.310295 \\
\hline 26 & 6 & 0 & -0.409402 & -0.019631 & 3.124732 \\
\hline 27 & 6 & 0 & 1.414114 & 1.552315 & 3.322705 \\
\hline 28 & 6 & 0 & 2.193590 & 2.933085 & -0.373887 \\
\hline 29 & 6 & 0 & 2.616189 & 0.588118 & 0.082990 \\
\hline 30 & 6 & 0 & 2.861470 & -1.140742 & 1.895032 \\
\hline 31 & 6 & 0 & 1.386821 & -3.092290 & 1.947489 \\
\hline 32 & 1 & 0 & 0.041458 & -3.304171 & 0.305813 \\
\hline 33 & 1 & 0 & 0.456650 & 1.491839 & -2.689190 \\
\hline 34 & 6 & 0 & 2.191610 & 1.566885 & -3.984832 \\
\hline 35 & 6 & 0 & 3.548368 & -0.428159 & -4.028550 \\
\hline 36 & 1 & 0 & 2.882064 & -2.057206 & -2.790887 \\
\hline
\end{tabular}


Medina et al.: BCP Hydroboration-Supporting Information - S95

\begin{tabular}{|c|c|c|c|c|c|}
\hline 37 & 1 & 0 & 1.690297 & -3.819428 & -1.557454 \\
\hline 38 & 6 & 0 & 0.436284 & -4.983813 & -2.861520 \\
\hline 39 & 6 & 0 & -1.500050 & -3.862611 & -3.758502 \\
\hline 40 & 1 & 0 & -1.768542 & -1.815453 & -3.134431 \\
\hline 41 & 1 & 0 & -0.689817 & 3.543514 & -1.075747 \\
\hline 42 & 6 & 0 & -1.219839 & 5.328736 & 0.001447 \\
\hline 43 & 6 & 0 & -1.185853 & 5.162443 & 2.403555 \\
\hline 44 & 1 & 0 & -0.543618 & 3.280564 & 3.219148 \\
\hline 45 & 1 & 0 & -1.223176 & -0.440084 & 2.532322 \\
\hline 46 & 6 & 0 & -0.147388 & -0.479071 & 4.413441 \\
\hline 47 & 6 & 0 & 1.666814 & 1.098552 & 4.611915 \\
\hline 48 & 1 & 0 & 2.033388 & 2.338581 & 2.887803 \\
\hline 49 & 1 & 0 & 1.552099 & 3.805084 & -0.286441 \\
\hline 50 & 6 & 0 & 3.412059 & 3.066783 & -0.989476 \\
\hline 51 & 6 & 0 & 3.895109 & 0.715873 & -0.546816 \\
\hline 52 & 6 & 0 & 3.900835 & -0.396457 & 2.508218 \\
\hline 53 & 6 & 0 & 2.431788 & -2.343268 & 2.538497 \\
\hline 54 & 1 & 0 & 1.030645 & -3.990800 & 2.454476 \\
\hline 55 & 1 & 0 & 1.997908 & 2.586128 & -4.315896 \\
\hline 56 & 6 & 0 & 3.304453 & 0.875748 & -4.457146 \\
\hline 57 & 1 & 0 & 4.422378 & -0.967287 & -4.391079 \\
\hline 58 & 1 & 0 & 1.057848 & -5.873846 & -2.775959 \\
\hline 59 & 6 & 0 & -0.713190 & -5.007089 & -3.648730 \\
\hline 60 & 1 & 0 & -2.402126 & -3.872496 & -4.369000 \\
\hline 61 & 1 & 0 & -1.432530 & 5.890965 & -0.904950 \\
\hline 62 & 6 & 0 & -1.417536 & 5.908538 & 1.252993 \\
\hline 63 & 1 & 0 & -1.352945 & 5.603359 & 3.385047 \\
\hline 64 & 1 & 0 & -0.749719 & -1.282559 & 4.835649 \\
\hline 65 & 6 & 0 & 0.892317 & 0.074470 & 5.155042 \\
\hline 66 & 1 & 0 & 2.485401 & 1.528468 & 5.187226 \\
\hline 67 & 1 & 0 & 3.717575 & 4.033063 & -1.391623 \\
\hline 68 & 6 & 0 & 4.290118 & 1.968601 & -1.108895 \\
\hline 69 & 6 & 0 & 4.789946 & -0.378835 & -0.663608 \\
\hline 70 & 1 & 0 & 4.230882 & 0.531868 & 2.046867 \\
\hline 71 & 6 & 0 & 4.498032 & -0.828177 & 3.670797 \\
\hline 72 & 6 & 0 & 3.049337 & -2.749357 & 3.743438 \\
\hline 73 & 1 & 0 & 3.986213 & 1.353160 & -5.159394 \\
\hline 74 & 1 & 0 & -0.994246 & -5.917236 & -4.176051 \\
\hline 75 & 1 & 0 & -1.770060 & 6.936040 & 1.327985 \\
\hline 76 & 1 & 0 & 1.106398 & -0.295553 & 6.156568 \\
\hline 77 & 6 & 0 & 5.540960 & 2.077019 & -1.761446 \\
\hline 78 & 6 & 0 & 6.002679 & -0.241858 & -1.297810 \\
\hline 79 & 1 & 0 & 4.500326 & -1.343149 & -0.250189 \\
\hline 80 & 1 & 0 & 5.300959 & -0.240935 & 4.113605 \\
\hline 81 & 6 & 0 & 4.069962 & -2.013225 & 4.299442 \\
\hline 82 & 1 & 0 & 2.702333 & -3.666496 & 4.219910 \\
\hline 83 & 1 & 0 & 5.821451 & 3.039966 & -2.188625 \\
\hline 84 & 6 & 0 & 6.384205 & 0.995669 & -1.855715 \\
\hline 85 & 1 & 0 & 6.670701 & -1.098014 & -1.375844 \\
\hline 86 & 1 & 0 & 4.544088 & -2.341941 & 5.222592 \\
\hline 87 & 1 & 0 & 7.344791 & 1.091253 & -2.358907 \\
\hline 88 & 5 & 0 & -2.540528 & -1.276785 & 0.279035 \\
\hline
\end{tabular}


Medina et al.: BCP Hydroboration-Supporting Information - S96

\begin{tabular}{|c|c|c|c|c|c|}
\hline 89 & 8 & 0 & -3.311548 & -1.234580 & 1.430366 \\
\hline 90 & 8 & 0 & -2.332514 & -2.586221 & -0.129097 \\
\hline 91 & 6 & 0 & -3.470022 & -2.588673 & 1.932479 \\
\hline 92 & 6 & 0 & -3.140218 & -3.473274 & 0.685007 \\
\hline 93 & 6 & 0 & -4.885236 & -2.734979 & 2.45948 \\
\hline 94 & 6 & 0 & -2.475257 & -2.767958 & 3.06979 \\
\hline 95 & 6 & 0 & -2.326553 & -4.721991 & 0.97476 \\
\hline 96 & 6 & 0 & -4.366479 & -3.856756 & -0.133087 \\
\hline 97 & 1 & 0 & -5.072892 & -3.761639 & 2.80058 \\
\hline 98 & 1 & 0 & -5.036561 & -2.064203 & 3.31211 \\
\hline 99 & 1 & 0 & -5.632222 & -2.483709 & 1.70047 \\
\hline 100 & 1 & 0 & -2.662227 & -2.000119 & 3.82916 \\
\hline 101 & 1 & 0 & -2.577318 & -3.751516 & 3.54528 \\
\hline 102 & 1 & 0 & -1.440172 & -2.656946 & 2.72195 \\
\hline 103 & 1 & 0 & -2.904061 & -5.439588 & 1.57208 \\
\hline 104 & 1 & 0 & -2.055471 & -5.208189 & 0.02943 \\
\hline 105 & 1 & 0 & -1.401490 & -4.498001 & 1.51432 \\
\hline 106 & 1 & 0 & -4.041442 & -4.307333 & -1.07848 \\
\hline 107 & 1 & 0 & -4.990168 & -4.587911 & 0.39608 \\
\hline 108 & 1 & 0 & -4.988710 & -2.987561 & -0.37177 \\
\hline 109 & 6 & 0 & -3.235735 & 2.469982 & -1.46591 \\
\hline 110 & 6 & 0 & -3.827241 & 2.662018 & -0.19637 \\
\hline 111 & 6 & 0 & -3.241222 & 3.569641 & -2.34903 \\
\hline 112 & 6 & 0 & -4.403587 & 3.874910 & 0.15324 \\
\hline 113 & 1 & 0 & -3.821390 & 1.833241 & 0.51459 \\
\hline 114 & 6 & 0 & -3.831148 & 4.777536 & -1.99929 \\
\hline 115 & 1 & 0 & -2.792803 & 3.448819 & -3.33728 \\
\hline 116 & 6 & 0 & -4.421110 & 4.942567 & -0.74546 \\
\hline 117 & 1 & 0 & -4.843466 & 3.991908 & 1.14374 \\
\hline 118 & 1 & 0 & -3.835272 & 5.600065 & -2.71543 \\
\hline 119 & 1 & 0 & -4.880467 & 5.890557 & -0.46991 \\
\hline 120 & 1 & 0 & -2.308745 & 1.122281 & -2.89016 \\
\hline
\end{tabular}




\section{Filename: TS2BINAP2}

SCF Energy (M06L/LANL2DZ-6-31g (d))

-3372.846433 A.U.

$\mathrm{SCF}$ Energy (M06/SDD-6-311+G(d,p)) in solution

-3373.166489 A.U.

Enthalpy at $298 \mathrm{~K}(\mathrm{M} 06 / \mathrm{SDD}-6-311+\mathrm{G}(\mathrm{d}, \mathrm{p}))$

-3372.133817 A.U.

Gibbs Free Energy at $298 \mathrm{~K}$ and 1 mol/L with Truhlar's quasiharmonic approximation (M06/SDD-6-311+G(d,p))

-3372.268374 A.U.

$\begin{array}{lcccc}\text { Center } & \text { Atomic } & \text { Atomic } & \text { Coordinates (Angstroms) } \\ \text { Number } & \text { Number } & \text { Type } & \mathrm{X} & \mathrm{Y}\end{array}$

\begin{tabular}{|c|c|c|c|c|c|}
\hline 1 & 6 & 0 & -2.184337 & -2.244830 & 0.649655 \\
\hline 2 & 1 & 0 & -1.798473 & -3.086207 & 1.234457 \\
\hline 3 & 6 & 0 & -2.877368 & -1.224842 & 1.419340 \\
\hline 4 & 6 & 0 & -2.400191 & -2.522815 & -0.751062 \\
\hline 5 & 6 & 0 & -1.981014 & -0.203440 & 2.697882 \\
\hline 6 & 6 & 0 & -2.955926 & -1.318548 & 2.907559 \\
\hline 7 & 5 & 0 & -4.017452 & -0.309834 & 0.863038 \\
\hline 8 & 6 & 0 & -2.049785 & -3.784344 & -1.287501 \\
\hline 9 & 6 & 0 & -2.918513 & -1.583838 & -1.671383 \\
\hline 10 & 1 & 0 & -2.354241 & 0.813032 & 2.808661 \\
\hline 11 & 1 & 0 & -0.998703 & -0.392238 & 3.134849 \\
\hline 12 & 1 & 0 & -2.528081 & -2.236850 & 3.322196 \\
\hline 13 & 1 & 0 & -3.911973 & -1.081342 & 3.384195 \\
\hline 14 & 8 & 0 & -3.984392 & 1.065390 & 0.868794 \\
\hline 15 & 8 & 0 & -5.233906 & -0.817819 & 0.483059 \\
\hline 16 & 6 & 0 & -2.194498 & -4.071834 & -2.638070 \\
\hline 17 & 1 & 0 & -1.639285 & -4.538766 & -0.613585 \\
\hline 18 & 6 & 0 & -3.064900 & -1.872498 & -3.019178 \\
\hline 19 & 1 & 0 & -3.172487 & -0.586120 & -1.312574 \\
\hline 20 & 6 & 0 & -5.360615 & 1.510547 & 0.735026 \\
\hline 21 & 6 & 0 & -6.031364 & 0.300877 & 0.012712 \\
\hline 22 & 6 & 0 & -2.701827 & -3.123135 & -3.527280 \\
\hline 23 & 1 & 0 & -1.902699 & -5.056777 & -3.004210 \\
\hline 24 & 1 & 0 & -3.470228 & -1.107133 & -3.685282 \\
\hline 25 & 6 & 0 & -5.380468 & 2.811441 & -0.039060 \\
\hline 26 & 6 & 0 & -5.883895 & 1.717528 & 2.150149 \\
\hline 27 & 6 & 0 & -7.476433 & 0.051879 & 0.395530 \\
\hline 28 & 6 & 0 & -5.886853 & 0.364065 & -1.501198 \\
\hline 29 & 1 & 0 & -2.817786 & -3.351595 & -4.584655 \\
\hline 30 & 1 & 0 & -6.412388 & 3.137392 & -0.224218 \\
\hline 31 & 1 & 0 & -4.876071 & 3.597629 & 0.534287 \\
\hline 32 & 1 & 0 & -4.864646 & 2.720018 & -0.999574 \\
\hline 33 & 1 & 0 & -5.233550 & 2.424089 & 2.677170 \\
\hline 34 & 1 & 0 & -6.903410 & 2.121156 & 2.152649 \\
\hline 35 & 1 & 0 & -5.887966 & 0.776475 & 2.713801 \\
\hline 36 & 1 & 0 & -8.101672 & 0.917283 & 0.140662 \\
\hline 37 & 1 & 0 & -7.863648 & -0.815344 & -0.150096 \\
\hline 38 & 1 & 0 & -7.583476 & -0.149799 & 1.465328 \\
\hline
\end{tabular}


Medina et al.: BCP Hydroboration-Supporting Information - S98

\begin{tabular}{|c|c|c|c|c|c|}
\hline 39 & 1 & 0 & -6.117746 & -0.616729 & -1.930093 \\
\hline 40 & 1 & 0 & -6.566884 & 1.107732 & -1.935135 \\
\hline 41 & 1 & 0 & -4.864891 & 0.628277 & -1.796637 \\
\hline 42 & 29 & 0 & -0.924025 & -0.485652 & 0.762494 \\
\hline 43 & 15 & 0 & -0.196880 & 1.383026 & -0.408955 \\
\hline 44 & 15 & 0 & 1.198279 & -1.422805 & 1.085664 \\
\hline 45 & 6 & 0 & 1.183639 & 0.898859 & -1.539173 \\
\hline 46 & 6 & 0 & 0.574971 & 2.614174 & 0.704302 \\
\hline 47 & 6 & 0 & -1.352811 & 2.386452 & -1.431334 \\
\hline 48 & 6 & 0 & 1.115840 & -2.543784 & 2.522493 \\
\hline 49 & 6 & 0 & 1.780612 & -2.459471 & -0.295385 \\
\hline 50 & 6 & 0 & 2.570783 & -0.241754 & 1.368787 \\
\hline 51 & 6 & 0 & 2.440362 & 0.494615 & -1.063847 \\
\hline 52 & 6 & 0 & 0.897131 & 0.761144 & -2.923615 \\
\hline 53 & 6 & 0 & 0.473098 & 2.436332 & 2.087675 \\
\hline 54 & 6 & 0 & 1.303492 & 3.704356 & 0.211261 \\
\hline 55 & 6 & 0 & -1.574681 & 3.753404 & -1.221797 \\
\hline 56 & 6 & 0 & -2.134556 & 1.731203 & -2.397415 \\
\hline 57 & 6 & 0 & 0.871699 & -2.028836 & 3.807422 \\
\hline 58 & 6 & 0 & 1.046742 & -3.932811 & 2.343027 \\
\hline 59 & 6 & 0 & 0.999620 & -2.538650 & -1.452198 \\
\hline 60 & 6 & 0 & 3.010286 & -3.130187 & -0.244963 \\
\hline 61 & 6 & 0 & 3.182278 & -0.111712 & 2.642253 \\
\hline 62 & 6 & 0 & 2.914003 & 0.652270 & 0.346611 \\
\hline 63 & 6 & 0 & 3.400746 & -0.055837 & -1.983995 \\
\hline 64 & 6 & 0 & 1.806021 & 0.245567 & -3.807995 \\
\hline 65 & 1 & 0 & -0.062524 & 1.095022 & -3.304902 \\
\hline 66 & 1 & 0 & -0.077577 & 1.575315 & 2.468650 \\
\hline 67 & 6 & 0 & 1.090583 & 3.326367 & 2.962009 \\
\hline 68 & 6 & 0 & 1.910139 & 4.600451 & 1.084823 \\
\hline 69 & 1 & 0 & 1.409998 & 3.837753 & -0.866372 \\
\hline 70 & 1 & 0 & -1.018116 & 4.285330 & -0.453963 \\
\hline 71 & 6 & 0 & -2.508150 & 4.449570 & -1.987292 \\
\hline 72 & 6 & 0 & -3.054990 & 2.430357 & -3.171390 \\
\hline 73 & 1 & 0 & -2.017902 & 0.658762 & -2.548505 \\
\hline 74 & 1 & 0 & 0.879346 & -0.950675 & 3.970069 \\
\hline 75 & 6 & 0 & 0.608710 & -2.873297 & 4.880188 \\
\hline 76 & 6 & 0 & 0.771174 & -4.775727 & 3.416322 \\
\hline 77 & 1 & 0 & 1.198940 & -4.356733 & 1.351667 \\
\hline 78 & 1 & 0 & 0.048963 & -2.005390 & -1.493595 \\
\hline 79 & 6 & 0 & 1.442656 & -3.274969 & -2.547726 \\
\hline 80 & 6 & 0 & 3.448563 & -3.866834 & -1.339358 \\
\hline 81 & 1 & 0 & 3.627096 & -3.062037 & 0.652721 \\
\hline 82 & 1 & 0 & 2.960964 & -0.842684 & 3.415418 \\
\hline 83 & 6 & 0 & 4.071647 & 0.895344 & 2.912071 \\
\hline 84 & 6 & 0 & 3.864163 & 1.692428 & 0.608841 \\
\hline 85 & 6 & 0 & 4.680499 & -0.505715 & -1.565914 \\
\hline 86 & 6 & 0 & 3.077036 & -0.185563 & -3.370010 \\
\hline 87 & 1 & 0 & 1.556123 & 0.167976 & -4.866173 \\
\hline 88 & 1 & 0 & 1.017845 & 3.167630 & 4.036757 \\
\hline 89 & 6 & 0 & 1.808465 & 4.409902 & 2.461353 \\
\hline 90 & 1 & 0 & 2.489307 & 5.433303 & 0.689518 \\
\hline
\end{tabular}


Medina et al.: BCP Hydroboration-Supporting Information - S99

\begin{tabular}{|c|c|c|c|c|c|}
\hline 91 & 1 & 0 & -2.659029 & 5.513030 & -1.808145 \\
\hline 92 & 6 & 0 & -3.241449 & 3.797336 & -2.973611 \\
\hline 93 & 1 & 0 & -3.636194 & 1.898964 & -3.924443 \\
\hline 94 & 1 & 0 & 0.430857 & -2.449631 & 5.867153 \\
\hline 95 & 6 & 0 & 0.557376 & -4.252505 & 4.688457 \\
\hline 96 & 1 & 0 & 0.722386 & -5.851081 & 3.253309 \\
\hline 97 & 1 & 0 & 0.824744 & -3.322674 & -3.443148 \\
\hline 98 & 6 & 0 & 2.666572 & -3.936238 & -2.492499 \\
\hline 99 & 1 & 0 & 4.410920 & -4.375256 & -1.300848 \\
\hline 100 & 1 & 0 & 4.535060 & 0.970320 & 3.896133 \\
\hline 101 & 6 & 0 & 4.421750 & 1.836889 & 1.918161 \\
\hline 102 & 6 & 0 & 4.269923 & 2.618107 & -0.387606 \\
\hline 103 & 1 & 0 & 4.946959 & -0.446535 & -0.513119 \\
\hline 104 & 6 & 0 & 5.584249 & -1.029470 & -2.462398 \\
\hline 105 & 6 & 0 & 4.023625 & -0.730817 & -4.267377 \\
\hline 106 & 1 & 0 & 2.300349 & 5.101663 & 3.143338 \\
\hline 107 & 1 & 0 & -3.965557 & 4.345717 & -3.573706 \\
\hline 108 & 1 & 0 & 0.341949 & -4.914273 & 5.525219 \\
\hline 109 & 1 & 0 & 3.019118 & -4.503740 & -3.35258 \\
\hline 110 & 6 & 0 & 5.322404 & 2.894900 & 2.181989 \\
\hline 111 & 6 & 0 & 5.157932 & 3.630170 & -0.104353 \\
\hline 112 & 1 & 0 & 3.858770 & 2.527047 & -1.391005 \\
\hline 113 & 1 & 0 & 6.556464 & -1.368085 & -2.107917 \\
\hline 114 & 6 & 0 & 5.259697 & -1.142830 & -3.827780 \\
\hline 115 & 1 & 0 & 3.749445 & -0.816386 & -5.318993 \\
\hline 116 & 1 & 0 & 5.730173 & 2.988769 & 3.18878 \\
\hline 117 & 6 & 0 & 5.686797 & 3.779041 & 1.19335 \\
\hline 118 & 1 & 0 & 5.449472 & 4.325977 & -0.889596 \\
\hline 119 & 1 & 0 & 5.982167 & -1.559086 & -4.52765 \\
\hline 120 & 1 & 0 & 6.385200 & 4.586053 & 1.40782 \\
\hline
\end{tabular}




\section{Filename: TS2BINAP3}

SCF Energy (M06L/LANL2DZ-6-31g (d))

-3372.843230 A.U.

$\mathrm{SCF}$ Energy (M06/SDD-6-311+G(d,p)) in solution

-3373.157696 A.U.

Enthalpy at $298 \mathrm{~K}(\mathrm{M} 06 / \mathrm{SDD}-6-311+\mathrm{G}(\mathrm{d}, \mathrm{p}))$

-3372.125433 A.U.

Gibbs Free Energy at $298 \mathrm{~K}$ and 1 mol/L with Truhlar's quasiharmonic approximation (M06/SDD-6-311+G(d,p))

-3372.260482 A.U.

\begin{tabular}{|c|c|c|c|c|c|}
\hline \multirow{2}{*}{$\begin{array}{l}\text { Center } \\
\text { Number }\end{array}$} & \multirow{2}{*}{$\begin{array}{l}\text { Atomic } \\
\text { Number }\end{array}$} & \multirow{2}{*}{$\begin{array}{l}\text { Atomic } \\
\text { Type }\end{array}$} & \multicolumn{3}{|c|}{ Coordinates (Angstroms) } \\
\hline & & & $\mathrm{X}$ & Y & Z \\
\hline 1 & 6 & 0 & -3.243925 & 1.308510 & -0.073606 \\
\hline 2 & 6 & 0 & -3.490722 & 0.142534 & -0.917736 \\
\hline 3 & 6 & 0 & -2.604467 & 0.010338 & -2.509986 \\
\hline 4 & 6 & 0 & -4.082737 & 0.117565 & -2.282554 \\
\hline 5 & 5 & 0 & -3.627762 & -1.187899 & -0.116367 \\
\hline 6 & 1 & 0 & -2.225222 & -0.910750 & -2.953687 \\
\hline 7 & 1 & 0 & -2.137963 & 0.926794 & -2.882628 \\
\hline 8 & 1 & 0 & -4.594361 & 1.011678 & -2.633109 \\
\hline 9 & 1 & 0 & -4.646769 & -0.786896 & -2.512958 \\
\hline 10 & 8 & 0 & -4.010871 & -2.389712 & -0.668124 \\
\hline 11 & 8 & 0 & -3.514834 & -1.239438 & 1.255200 \\
\hline 12 & 6 & 0 & -4.443790 & -3.230473 & 0.437925 \\
\hline 13 & 6 & 0 & -3.652084 & -2.630486 & 1.641158 \\
\hline 14 & 6 & 0 & -4.118257 & -4.675050 & 0.122345 \\
\hline 15 & 6 & 0 & -5.948344 & -3.036060 & 0.565728 \\
\hline 16 & 6 & 0 & -4.371249 & -2.693945 & 2.973007 \\
\hline 17 & 6 & 0 & -2.245082 & -3.190351 & 1.757690 \\
\hline 18 & 1 & 0 & -4.390654 & -5.323668 & 0.964849 \\
\hline 19 & 1 & 0 & -4.690122 & -5.006014 & -0.752220 \\
\hline 20 & 1 & 0 & -3.055904 & -4.817217 & -0.096627 \\
\hline 21 & 1 & 0 & -6.428029 & -3.282617 & -0.387215 \\
\hline 22 & 1 & 0 & -6.375045 & -3.682616 & 1.341564 \\
\hline 23 & 1 & 0 & -6.196957 & -1.996586 & 0.809367 \\
\hline 24 & 1 & 0 & -4.567818 & -3.734793 & 3.260064 \\
\hline 25 & 1 & 0 & -3.750976 & -2.242495 & 3.755343 \\
\hline 26 & 1 & 0 & -5.322825 & -2.155470 & 2.946957 \\
\hline 27 & 1 & 0 & -1.659758 & -2.572008 & 2.448863 \\
\hline 28 & 1 & 0 & -2.241275 & -4.223294 & 2.127295 \\
\hline 29 & 1 & 0 & -1.743532 & -3.173010 & 0.781536 \\
\hline 30 & 1 & 0 & -3.244407 & 1.073439 & 0.990170 \\
\hline 31 & 6 & 0 & -3.523917 & 2.690278 & -0.372451 \\
\hline 32 & 6 & 0 & -3.673476 & 3.244123 & -1.668729 \\
\hline 33 & 6 & 0 & -3.654740 & 3.603294 & 0.704582 \\
\hline 34 & 6 & 0 & -4.015297 & 4.578256 & -1.859105 \\
\hline 35 & 1 & 0 & -3.502822 & 2.627913 & -2.548827 \\
\hline 36 & 6 & 0 & -3.972189 & 4.935984 & 0.509642 \\
\hline 37 & 1 & 0 & -3.503777 & 3.225746 & 1.718100 \\
\hline 38 & 6 & 0 & -4.181246 & 5.440105 & -0.777805 \\
\hline
\end{tabular}


Medina et al.: BCP Hydroboration-Supporting Information - S101

\begin{tabular}{|c|c|c|c|c|c|}
\hline 39 & 1 & 0 & -4.138919 & 4.952117 & -2.876398 \\
\hline 40 & 1 & 0 & -4.063329 & 5.594734 & 1.373663 \\
\hline 41 & 1 & 0 & -4.444561 & 6.485172 & -0.931550 \\
\hline 42 & 29 & 0 & -1.318893 & 0.291040 & -0.661438 \\
\hline 43 & 15 & 0 & 0.296546 & 1.699023 & 0.320218 \\
\hline 44 & 15 & 0 & 0.336951 & -1.493943 & -0.850552 \\
\hline 45 & 6 & 0 & 2.043478 & 1.608077 & -0.238399 \\
\hline 46 & 6 & 0 & 0.327171 & 1.291349 & 2.101170 \\
\hline 47 & 6 & 0 & -0.097258 & 3.479117 & 0.246574 \\
\hline 48 & 6 & 0 & 0.027958 & -3.253639 & -1.279938 \\
\hline 49 & 6 & 0 & 1.355800 & -1.000983 & -2.305210 \\
\hline 50 & 6 & 0 & 1.472547 & -1.588247 & 0.578019 \\
\hline 51 & 6 & 0 & 2.834425 & 0.463336 & -0.057251 \\
\hline 52 & 6 & 0 & 2.510563 & 2.639614 & -1.098685 \\
\hline 53 & 6 & 0 & -0.695156 & 0.493952 & 2.629588 \\
\hline 54 & 6 & 0 & 1.342960 & 1.746406 & 2.952295 \\
\hline 55 & 6 & 0 & 0.087706 & 4.359995 & 1.317044 \\
\hline 56 & 6 & 0 & -0.591893 & 3.980241 & -0.964509 \\
\hline 57 & 6 & 0 & -1.185235 & -3.564846 & -1.905873 \\
\hline 58 & 6 & 0 & 0.964848 & -4.280012 & -1.085025 \\
\hline 59 & 6 & 0 & 0.981005 & 0.117529 & -3.055731 \\
\hline 60 & 6 & 0 & 2.477901 & -1.736194 & -2.706421 \\
\hline 61 & 6 & 0 & 1.164692 & -2.587119 & 1.544747 \\
\hline 62 & 6 & 0 & 2.455961 & -0.636086 & 0.871037 \\
\hline 63 & 6 & 0 & 4.086776 & 0.350512 & -0.745283 \\
\hline 64 & 6 & 0 & 3.708910 & 2.546001 & -1.753567 \\
\hline 65 & 1 & 0 & 1.901965 & 3.531000 & -1.233685 \\
\hline 66 & 1 & 0 & -1.486544 & 0.124410 & 1.972710 \\
\hline 67 & 6 & 0 & -0.700079 & 0.160885 & 3.981556 \\
\hline 68 & 6 & 0 & 1.334388 & 1.415249 & 4.303076 \\
\hline 69 & 1 & 0 & 2.161759 & 2.339256 & 2.542182 \\
\hline 70 & 1 & 0 & 0.443367 & 3.986449 & 2.275470 \\
\hline 71 & 6 & 0 & -0.195716 & 5.715003 & 1.169766 \\
\hline 72 & 6 & 0 & -0.859722 & 5.335342 & -1.115113 \\
\hline 73 & 1 & 0 & -0.777583 & 3.292482 & -1.791894 \\
\hline 74 & 1 & 0 & -1.944138 & -2.793223 & -2.024473 \\
\hline 75 & 6 & 0 & -1.449021 & -4.860728 & -2.343474 \\
\hline 76 & 6 & 0 & 0.694265 & -5.576255 & -1.510186 \\
\hline 77 & 1 & 0 & 1.906990 & -4.063640 & -0.581592 \\
\hline 78 & 1 & 0 & 0.114313 & 0.702090 & -2.740530 \\
\hline 79 & 6 & 0 & 1.718668 & 0.506114 & -4.170794 \\
\hline 80 & 6 & 0 & 3.215541 & -1.349890 & -3.819346 \\
\hline 81 & 1 & 0 & 2.789020 & -2.608789 & -2.132655 \\
\hline 82 & 1 & 0 & 0.396684 & -3.321658 & 1.316381 \\
\hline 83 & 6 & 0 & 1.815148 & -2.659223 & 2.745249 \\
\hline 84 & 6 & 0 & 3.164452 & -0.716578 & 2.119832 \\
\hline 85 & 6 & 0 & 4.907933 & -0.801396 & -0.635756 \\
\hline 86 & 6 & 0 & 4.522545 & 1.397419 & -1.615993 \\
\hline 87 & 1 & 0 & 4.048067 & 3.358396 & -2.396736 \\
\hline 88 & 1 & 0 & -1.499724 & -0.464202 & 4.378125 \\
\hline 89 & 6 & 0 & 0.312275 & 0.621391 & 4.820114 \\
\hline 90 & 1 & 0 & 2.141167 & 1.759286 & 4.94832 \\
\hline
\end{tabular}


Medina et al.: BCP Hydroboration-Supporting Information - S102

\begin{tabular}{|c|c|c|c|c|c|}
\hline 91 & 1 & 0 & -0.054446 & 6.389034 & 2.013234 \\
\hline 92 & 6 & 0 & -0.660899 & 6.205862 & -0.046884 \\
\hline 93 & 1 & 0 & -1.259506 & 5.704747 & -2.056949 \\
\hline 94 & 1 & 0 & -2.400850 & -5.080091 & -2.825037 \\
\hline 95 & 6 & 0 & -0.510601 & -5.869643 & -2.147467 \\
\hline 96 & 1 & 0 & 1.430875 & -6.361268 & -1.346539 \\
\hline 97 & 1 & 0 & 1.419855 & 1.387374 & -4.736112 \\
\hline 98 & 6 & 0 & 2.838007 & -0.226806 & -4.553497 \\
\hline 99 & 1 & 0 & 4.098509 & -1.919239 & -4.105130 \\
\hline 100 & 1 & 0 & 1.558237 & -3.436819 & 3.464942 \\
\hline 101 & 6 & 0 & 2.828155 & -1.727543 & 3.072481 \\
\hline 102 & 6 & 0 & 4.171019 & 0.215833 & 2.479171 \\
\hline 103 & 1 & 0 & 4.588051 & -1.618633 & 0.008141 \\
\hline 104 & 6 & 0 & 6.088607 & -0.908302 & -1.336334 \\
\hline 105 & 6 & 0 & 5.738152 & 1.258830 & -2.322912 \\
\hline 106 & 1 & 0 & 0.311286 & 0.356150 & 5.876283 \\
\hline 107 & 1 & 0 & -0.889595 & 7.264613 & -0.158198 \\
\hline 108 & 1 & 0 & -0.717675 & -6.884033 & -2.483812 \\
\hline 109 & 1 & 0 & 3.420334 & 0.077176 & -5.421861 \\
\hline 110 & 6 & 0 & 3.496092 & -1.769492 & 4.316659 \\
\hline 111 & 6 & 0 & 4.810770 & 0.147475 & 3.697242 \\
\hline 112 & 1 & 0 & 4.435796 & 1.004679 & 1.778512 \\
\hline 113 & 1 & 0 & 6.696729 & -1.805676 & -1.232788 \\
\hline 114 & 6 & 0 & 6.513073 & 0.128979 & -2.189266 \\
\hline 115 & 1 & 0 & 6.049374 & 2.070533 & -2.980983 \\
\hline 116 & 1 & 0 & 3.218924 & -2.549066 & 5.026636 \\
\hline 117 & 6 & 0 & 4.472897 & -0.851600 & 4.629549 \\
\hline 118 & 1 & 0 & 5.580278 & 0.877957 & 3.941753 \\
\hline 119 & 1 & 0 & 7.447226 & 0.034273 & -2.74001 \\
\hline 120 & 1 & 0 & 4.980654 & -0.894706 & 5.59149 \\
\hline
\end{tabular}




\section{Filename: TS4BINAP2}

SCF Energy (M06L/LANL2DZ-6-31g (d))

-3488.574565 A. U.

$\mathrm{SCF}$ Energy (M06/SDD-6-311+G(d,p)) in solution

-3488.882789 A.U.

Enthalpy at $298 \mathrm{~K}(\mathrm{M} 06 / \mathrm{SDD}-6-311+\mathrm{G}(\mathrm{d}, \mathrm{p}))$

-3487.795626 A.U.

Gibbs Free Energy at $298 \mathrm{~K}$ and 1 mol/L with Truhlar's quasiharmonic approximation (M06/SDD-6-311+G(d,p))

-3487.938792 A.U.

\begin{tabular}{|c|c|c|c|c|c|}
\hline \multirow{2}{*}{$\begin{array}{l}\text { Center } \\
\text { Number }\end{array}$} & \multirow{2}{*}{$\begin{array}{l}\text { Atomic } \\
\text { Number }\end{array}$} & \multirow{2}{*}{$\begin{array}{l}\text { Atomic } \\
\text { Type }\end{array}$} & \multicolumn{3}{|c|}{ Coordinates (Angstroms) } \\
\hline & & & $\mathrm{X}$ & Y & Z \\
\hline 1 & 6 & 0 & -3.432804 & -1.291943 & -1.682112 \\
\hline 2 & 1 & 0 & -2.680621 & -1.703031 & -2.367956 \\
\hline 3 & 6 & 0 & -3.525375 & 0.059551 & -1.649678 \\
\hline 4 & 6 & 0 & -4.155846 & -2.338760 & -0.961010 \\
\hline 5 & 6 & 0 & -1.702686 & 1.854687 & -1.984170 \\
\hline 6 & 6 & 0 & -2.674361 & 0.848314 & -2.621739 \\
\hline 7 & 5 & 0 & -4.420153 & 0.927799 & -0.718727 \\
\hline 8 & 6 & 0 & -5.489187 & -2.225382 & -0.537225 \\
\hline 9 & 6 & 0 & -3.495639 & -3.558266 & -0.730800 \\
\hline 10 & 1 & 0 & -0.770252 & 1.878047 & -2.578611 \\
\hline 11 & 1 & 0 & -2.087114 & 2.881590 & -2.103445 \\
\hline 12 & 1 & 0 & -3.354607 & 1.365624 & -3.320155 \\
\hline 13 & 1 & 0 & -2.114522 & 0.130625 & -3.242921 \\
\hline 14 & 8 & 0 & -4.663621 & 2.247161 & -1.029818 \\
\hline 15 & 8 & 0 & -5.061187 & 0.528708 & 0.432487 \\
\hline 16 & 6 & 0 & -6.117884 & -3.270834 & 0.127689 \\
\hline 17 & 1 & 0 & -6.036445 & -1.309321 & -0.742159 \\
\hline 18 & 6 & 0 & -4.120942 & -4.601358 & -0.059657 \\
\hline 19 & 1 & 0 & -2.464615 & -3.669821 & -1.073907 \\
\hline 20 & 6 & 0 & -5.709371 & 2.716312 & -0.149199 \\
\hline 21 & 6 & 0 & -5.649302 & 1.704200 & 1.045587 \\
\hline 22 & 6 & 0 & -5.436521 & -4.461256 & 0.377695 \\
\hline 23 & 1 & 0 & -7.154854 & -3.159973 & 0.443062 \\
\hline 24 & 1 & 0 & -3.578400 & -5.528253 & 0.123424 \\
\hline 25 & 6 & 0 & -7.008103 & 2.630545 & -0.941422 \\
\hline 26 & 6 & 0 & -5.412985 & 4.154559 & 0.226059 \\
\hline 27 & 6 & 0 & -4.714773 & 2.139547 & 2.164217 \\
\hline 28 & 6 & 0 & -7.002057 & 1.310010 & 1.610387 \\
\hline 29 & 1 & 0 & -5.933102 & -5.279722 & 0.897166 \\
\hline 30 & 1 & 0 & -7.862400 & 3.013412 & -0.370227 \\
\hline 31 & 1 & 0 & -6.911943 & 3.224011 & -1.856749 \\
\hline 32 & 1 & 0 & -7.226674 & 1.596764 & -1.235547 \\
\hline 33 & 1 & 0 & -5.452754 & 4.789364 & -0.666148 \\
\hline 34 & 1 & 0 & -6.152137 & 4.533524 & 0.943812 \\
\hline 35 & 1 & 0 & -4.416834 & 4.253177 & 0.667715 \\
\hline 36 & 1 & 0 & -5.088013 & 3.036892 & 2.673163 \\
\hline 37 & 1 & 0 & -4.644590 & 1.337777 & 2.909221 \\
\hline 38 & 1 & 0 & -3.705092 & 2.324510 & 1.779428 \\
\hline
\end{tabular}




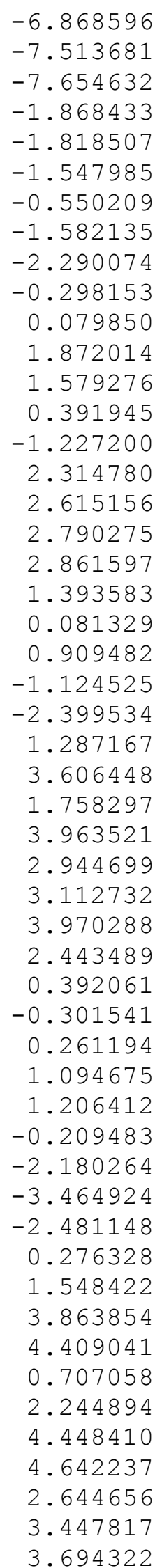

0.569647

2.179405

0.868840

2.054441

2.266386

3.625902

3.811641

4.164325

4.108358

1.061966

$-1.055776$

1.814446

$-1.092116$

$-2.262104$

$-1.803318$

3.246148

2. 221942

0.404478

$-0.860507$

$-1.148520$

$-1.856357$

$-3.546976$

$-3.080024$

$-1.062772$

4.150056

3.504200

2. 222927

2. 553961

0.408590

$-0.749288$

$-0.727709$

$-0.997175$

$-1.303768$

$-0.845252$

$-2.721033$

$-4.409465$

$-3.862858$

$-3.659813$

$-3.607527$

$-1.604821$

$-0.064232$

3. 934316

5.300821

4.649280

2.791549

1.956252

2.543551

2. 866641

2. 550456

1.290869

$-0.676661$

$-1.868819$
2.407689

2.043311

0.850459

$-0.518143$

0.663348

0.822083

1. 262285

$-0.146270$

1. 485149

$-0.442093$

0.380268

$-0.513208$

1. 449676

$-0.957076$

1. 410430

$-1.558721$

1.105574

$-1.244564$

0.941358

2. 856589

$-2.260268$

$-0.753367$

1.983800

1.594587

$-1.852465$

$-2.038461$

2. 213026

1.282434

$-2.658517$

$-0.526086$

1.834960

3. 725126

3. 251768

$-2.419392$

$-3.336350$

$-1.828466$

0.246650

1.865593

2.715405

2. 312754

1. 161050

$-1.499969$

$-2.592477$

$-2.784098$

$-1.845765$

2. 072974

3. 477888

2. 547051

0.429586

$-3.221560$

$-3.326377$

$-1.204310$ 
Medina et al.: BCP Hydroboration-Supporting Information - S105

\begin{tabular}{|c|c|c|c|c|c|}
\hline 91 & 6 & 0 & 5.292027 & -0.503983 & 1.370314 \\
\hline 92 & 6 & 0 & 3.755183 & -0.784312 & 3.247470 \\
\hline 93 & 1 & 0 & 2.274440 & -1.034549 & 4.801584 \\
\hline 94 & 1 & 0 & 0.012729 & -2.389662 & -4.343490 \\
\hline 95 & 6 & 0 & 0.766141 & -4.000405 & -3.120167 \\
\hline 96 & 1 & 0 & 1.515886 & -5.399406 & -1.660333 \\
\hline 97 & 1 & 0 & -2.093416 & -4.600957 & 3.153488 \\
\hline 98 & 6 & 0 & -3.357086 & -2.873446 & 2.869874 \\
\hline 99 & 1 & 0 & -4.389071 & -1.037177 & 2.398501 \\
\hline 100 & 1 & 0 & 0.740914 & 5.996315 & -2.81353 \\
\hline 101 & 6 & 0 & 2.836048 & 5.551689 & -3.05715 \\
\hline 102 & 1 & 0 & 4.869608 & 4.839093 & -3.15538 \\
\hline 103 & 1 & 0 & 1.571524 & 2.534385 & 4.33335 \\
\hline 104 & 6 & 0 & 3.589567 & 2.862452 & 3.64558 \\
\hline 105 & 1 & 0 & 5.503099 & 3.103004 & 2.679177 \\
\hline 106 & 1 & 0 & 3.547609 & -0.655583 & -4.411700 \\
\hline 107 & 6 & 0 & 3.851842 & -1.835216 & -2.625068 \\
\hline 108 & 6 & 0 & 4.107670 & -3.038635 & -0.516910 \\
\hline 109 & 1 & 0 & 5.472758 & -0.452283 & 0.29813 \\
\hline 110 & 6 & 0 & 6.337841 & -0.343674 & 2.24946 \\
\hline 111 & 6 & 0 & 4.850205 & -0.609229 & 4.12591 \\
\hline 112 & 1 & 0 & 0.917247 & -4.677610 & -3.95927 \\
\hline 113 & 1 & 0 & -4.195996 & -3.301530 & 3.41667 \\
\hline 114 & 1 & 0 & 3.040555 & 6.447516 & -3.640983 \\
\hline 115 & 1 & 0 & 3.973976 & 3.103817 & 4.635455 \\
\hline 116 & 6 & 0 & 4.395326 & -2.957289 & -3.292778 \\
\hline 117 & 6 & 0 & 4.648337 & -4.108446 & -1.19254 \\
\hline 118 & 1 & 0 & 3.992052 & -3.084739 & 0.56418 \\
\hline 119 & 1 & 0 & 7.342872 & -0.172869 & 1.86719 \\
\hline 120 & 6 & 0 & 6.118591 & -0.393293 & 3.64140 \\
\hline 121 & 1 & 0 & 4.664619 & -0.647859 & 5.19949 \\
\hline 122 & 1 & 0 & 4.497866 & -2.915334 & -4.377260 \\
\hline 123 & 6 & 0 & 4.790491 & -4.073832 & -2.594243 \\
\hline 124 & 1 & 0 & 4.962911 & -4.992034 & -0.639537 \\
\hline 125 & 1 & 0 & 6.953078 & -0.260701 & 4.32772 \\
\hline 126 & 1 & 0 & 5.212178 & -4.929020 & -3.11936 \\
\hline
\end{tabular}




\section{Filename: IM4BINAP2}

SCF Energy (M06L/LANL2DZ-6-31g (d))

-3372.876452 A.U.

$\mathrm{SCF}$ Energy (M06/SDD-6-311+G(d,p)) in solution

-3373.20249 A.U.

Enthalpy at $298 \mathrm{~K}(\mathrm{M} 06 / \mathrm{SDD}-6-311+\mathrm{G}(\mathrm{d}, \mathrm{p}))$

-3372.167916 A.U.

Gibbs Free Energy at $298 \mathrm{~K}$ and 1 mol/L with Truhlar's quasiharmonic approximation (M06/SDD-6-311+G(d,p))

-3372.303433 A.U.

\begin{tabular}{|c|c|c|c|c|c|}
\hline \multirow{2}{*}{$\begin{array}{l}\text { Center } \\
\text { Number }\end{array}$} & \multirow{2}{*}{$\begin{array}{l}\text { Atomic } \\
\text { Number }\end{array}$} & \multirow{2}{*}{$\begin{array}{c}\text { Atomic } \\
\text { Type }\end{array}$} & \multicolumn{3}{|c|}{ Coordinates (Angstroms) } \\
\hline & & & $x$ & $\mathrm{Y}$ & Z \\
\hline 1 & 6 & 0 & & 200066 & 0350617 \\
\hline $\begin{array}{l}1 \\
2\end{array}$ & $\begin{array}{l}0 \\
1\end{array}$ & 0 & -2.261841 & $2.4 \angle 0600$ & 0.350612 \\
\hline 2 & 1 & 0 & -2.630929 & 2.147420 & 1.355025 \\
\hline 3 & 6 & 0 & -1.663385 & 3.764474 & 0.447096 \\
\hline 4 & 6 & 0 & -1.506844 & 4.694346 & -0.607690 \\
\hline 5 & 6 & 0 & -1.119148 & 4.166890 & 1.693486 \\
\hline 6 & 6 & 0 & -0.893045 & 5.929439 & -0.416466 \\
\hline 7 & 1 & 0 & -1.856752 & 4.450053 & -1.607140 \\
\hline 8 & 6 & 0 & -0.484187 & 5.385404 & 1.877389 \\
\hline 9 & 1 & 0 & -1.211216 & 3.478261 & 2.538346 \\
\hline 10 & 6 & 0 & -0.369654 & 6.294183 & 0.821224 \\
\hline 11 & 1 & 0 & -0.811228 & 6.611851 & -1.263582 \\
\hline 12 & 1 & 0 & -0.086030 & 5.638182 & 2.860299 \\
\hline 13 & 1 & 0 & 0.113144 & 7.259317 & 0.964132 \\
\hline 14 & 29 & 0 & -0.602959 & 1.278098 & 0.259829 \\
\hline 15 & 15 & 0 & 1.635567 & 1.418005 & -0.391219 \\
\hline 16 & 15 & 0 & -0.419813 & -1.013757 & 0.885864 \\
\hline 17 & 6 & 0 & 1.787807 & -0.031635 & -1.501061 \\
\hline 18 & 6 & 0 & 2.858500 & 1.272059 & 0.963251 \\
\hline 19 & 6 & 0 & 2.346735 & 2.807799 & -1.339967 \\
\hline 20 & 6 & 0 & -1.418953 & -1.417473 & 2.363529 \\
\hline 21 & 6 & 0 & -1.002427 & -2.055052 & -0.493946 \\
\hline 22 & 6 & 0 & 1.236501 & -1.698321 & 1.302171 \\
\hline 23 & 6 & 0 & 2.005568 & -1.342692 & -1.072999 \\
\hline 24 & 6 & 0 & 1.421956 & 0.207779 & -2.856152 \\
\hline 25 & 6 & 0 & 2.388281 & 1.274058 & 2.280416 \\
\hline 26 & 6 & 0 & 4.239100 & 1.233590 & $0.73227 \varepsilon$ \\
\hline 27 & 6 & 0 & 3.339170 & 2.677768 & -2.320725 \\
\hline 28 & 6 & 0 & 1.905570 & 4.085691 & -0.978087 \\
\hline 29 & 6 & 0 & -1.819962 & -0.341762 & 3.163663 \\
\hline 30 & 6 & 0 & -1.751084 & -2.717326 & 2.763390 \\
\hline 31 & 6 & 0 & -1.569217 & -1.401952 & -1.596210 \\
\hline 32 & 6 & 0 & -0.834778 & -3.443772 & -0.538761 \\
\hline 33 & 6 & 0 & 1.541638 & -2.024995 & 2.650335 \\
\hline 34 & 6 & 0 & 2.269413 & -1.681552 & 0.356625 \\
\hline 35 & 6 & 0 & 1.966916 & -2.413790 & -2.026080 \\
\hline 36 & 6 & 0 & 1.339379 & -0.811497 & -3.766849 \\
\hline 37 & 1 & 0 & 1.202420 & 1.227571 & -3.170275 \\
\hline 38 & 1 & 0 & 1.310416 & 1.306016 & 2.461100 \\
\hline
\end{tabular}




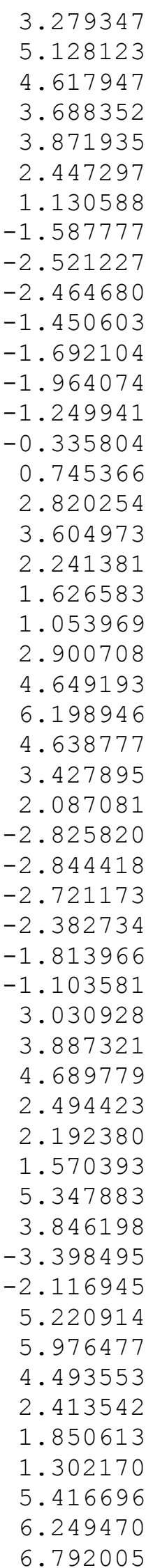

3.279347

1.220472

1.171316

1.245553

1.687009

3.808588

5.214645

4.196369

0.674339

$-0.558106$

$-2.934845$

$-3.567340$

$-0.316043$

$-2.124587$

$-4.168212$

$-3.957536$

$-2.026607$

$-2.324231$

$-1.996958$

$-3.757747$

$-2.143014$

$-0.605540$

1.215623

1.164790

1.120914

3.698130

5.078263

6.199709

0.289145

$-1.855750$

$-3.950398$

$-1.601346$

$-3.510170$

$-5.246807$

$-2.566528$

$-2.307236$

$-1.966815$

$-3.986134$

$-4.770497$

$-3.206962$

1.116358

5.960546

$-2.028196$

$-4.076451$

$-2.564507$

$-2.221307$

$-1.724511$

$-5.792442$

$-4.497707$

$-2.979490$

$-2.792289$

$-2.522184$

$-2.187336$
3.349136

1. 798742

$-0.290003$

$-2.613884$

$-2.932055$

$-1.585723$

$-0.218687$

2.837006

4.347388

3.938471

2.150865

$-1.565667$

$-2.717683$

$-1.652155$

0.283890

3.391508

3.043861

0.751321

$-1.665267$

$-3.388500$

$-4.798544$

4.369909

3.108367

1.608079

$-3.697278$

$-2.564672$

$-1.292725$

4.959088

4.736024

4.235683

$-3.576844$

$-2.743280$

$-1.681177$

4.085902

2. 119050

$-0.163113$

$-0.631899$

$-2.595854$

$-4.318253$

3.942186

$-3.046439$

5.657131

$-3.622577$

2. 513470

0.251322

$-1.206229$

$-2.292139$

$-3.935213$

$-5.350120$

3.561492

1. 601775

$-0.469197$ 
Medina et al.: BCP Hydroboration-Supporting Information - S108

\begin{tabular}{|c|c|c|c|c|c|}
\hline 91 & 1 & 0 & 1.809325 & -5.307141 & -4.661917 \\
\hline 92 & 1 & 0 & 7.272142 & -2.719323 & 1.918219 \\
\hline 93 & 6 & 0 & -3.363467 & 2.073468 & -0.643994 \\
\hline 94 & 6 & 0 & -4.147605 & 3.128768 & -1.408880 \\
\hline 95 & 6 & 0 & -3.185959 & 2.265340 & -2.150859 \\
\hline 96 & 1 & 0 & -3.885863 & 4.168543 & -1.234854 \\
\hline 97 & 1 & 0 & -5.209274 & 2.955096 & -1.569936 \\
\hline 98 & 1 & 0 & -3.594368 & 1.513803 & -2.829502 \\
\hline 99 & 1 & 0 & -2.235937 & 2.683073 & -2.484991 \\
\hline 100 & 5 & 0 & -4.189603 & 0.798512 & -0.352817 \\
\hline 101 & 8 & 0 & -3.742012 & -0.324236 & 0.313473 \\
\hline 102 & 8 & 0 & -5.480394 & 0.651431 & -0.821313 \\
\hline 103 & 6 & 0 & -5.833825 & -0.740249 & -0.680970 \\
\hline 104 & 6 & 0 & -4.875247 & -1.222656 & 0.454091 \\
\hline 105 & 6 & 0 & -5.545495 & -1.400172 & -2.022930 \\
\hline 106 & 1 & 0 & -6.093195 & -0.869465 & -2.809442 \\
\hline 107 & 1 & 0 & -4.475623 & -1.352230 & -2.259310 \\
\hline 108 & 1 & 0 & -5.849517 & -2.453865 & -2.038954 \\
\hline 109 & 6 & 0 & -7.309658 & -0.840043 & -0.348537 \\
\hline 110 & 1 & 0 & -7.909051 & -0.473601 & -1.189280 \\
\hline 111 & 1 & 0 & -7.598391 & -1.881913 & -0.158386 \\
\hline 112 & 1 & 0 & -7.566605 & -0.243806 & 0.531804 \\
\hline 113 & 6 & 0 & -5.437665 & -0.996484 & 1.849805 \\
\hline 114 & 1 & 0 & -5.780713 & 0.037468 & 1.975837 \\
\hline 115 & 1 & 0 & -6.278572 & -1.667762 & 2.063248 \\
\hline 116 & 1 & 0 & -4.651083 & -1.185003 & 2.589127 \\
\hline 117 & 6 & 0 & -4.408748 & -2.656828 & 0.297920 \\
\hline 118 & 1 & 0 & -3.864025 & -2.813172 & -0.638583 \\
\hline 119 & 1 & 0 & -3.744264 & -2.930676 & 1.12470 \\
\hline 120 & 1 & 0 & -5.268336 & -3.340312 & 0.317398 \\
\hline
\end{tabular}




\section{Filename: IM5BINAP2}

SCF Energy (M06L/LANL2DZ-6-31g (d))

-3372.885487 A.U.

$\mathrm{SCF}$ Energy (M06/SDD-6-311+G(d,p)) in solution

-3373.206187 A.U.

Enthalpy at $298 \mathrm{~K}(\mathrm{M} 06 / \mathrm{SDD}-6-311+\mathrm{G}(\mathrm{d}, \mathrm{p}))$

-3372.171421 A.U.

Gibbs Free Energy at $298 \mathrm{~K}$ and 1 mol/L with Truhlar's quasiharmonic approximation (M06/SDD-6-311+G(d,p))

-3372.307409 A.U.

\begin{tabular}{|c|c|c|c|c|c|}
\hline \multirow{2}{*}{$\begin{array}{l}\text { Center } \\
\text { Number }\end{array}$} & \multirow{2}{*}{$\begin{array}{l}\text { Atomic } \\
\text { Number }\end{array}$} & \multirow{2}{*}{$\begin{array}{l}\text { Atomic } \\
\text { Type }\end{array}$} & \multicolumn{3}{|c|}{ Coordinates (Angstroms) } \\
\hline & & & $\mathrm{X}$ & Y & Z \\
\hline 1 & 6 & 0 & -4.001190 & -1.505603 & -1.582254 \\
\hline 2 & 1 & 0 & -3.710107 & -2.323285 & -2.253763 \\
\hline 3 & 6 & 0 & -3.501554 & -0.278844 & -1.848844 \\
\hline 4 & 6 & 0 & -4.916459 & -1.904202 & -0.519044 \\
\hline 5 & 6 & 0 & -1.462316 & 0.874131 & -2.803527 \\
\hline 6 & 6 & 0 & -2.646062 & -0.061189 & -3.078519 \\
\hline 7 & 5 & 0 & -3.635792 & 0.982891 & -0.938721 \\
\hline 8 & 6 & 0 & -5.727880 & -0.988118 & 0.171147 \\
\hline 9 & 6 & 0 & -5.049684 & -3.262505 & -0.189624 \\
\hline 10 & 1 & 0 & -0.702433 & 0.757517 & -3.595879 \\
\hline 11 & 1 & 0 & -1.811422 & 1.917063 & -2.889433 \\
\hline 12 & 1 & 0 & -3.295590 & 0.325576 & -3.888389 \\
\hline 13 & 1 & 0 & -2.298419 & -1.045163 & -3.434662 \\
\hline 14 & 8 & 0 & -4.227891 & 2.161476 & -1.326040 \\
\hline 15 & 8 & 0 & -3.095383 & 1.063536 & 0.335028 \\
\hline 16 & 6 & 0 & -6.586576 & -1.401456 & 1.180643 \\
\hline 17 & 1 & 0 & -5.713587 & 0.059575 & -0.127226 \\
\hline 18 & 6 & 0 & -5.920842 & -3.682771 & 0.808112 \\
\hline 19 & 1 & 0 & -4.441215 & -3.992573 & -0.724534 \\
\hline 20 & 6 & 0 & -3.837548 & 3.178564 & -0.363655 \\
\hline 21 & 6 & 0 & -3.506207 & 2.333836 & 0.906547 \\
\hline 22 & 6 & 0 & -6.686420 & -2.753587 & 1.509055 \\
\hline 23 & 1 & 0 & -7.202925 & -0.666508 & 1.698317 \\
\hline 24 & 1 & 0 & -5.996465 & -4.743583 & 1.046363 \\
\hline 25 & 6 & 0 & -4.982883 & 4.156288 & -0.198857 \\
\hline 26 & 6 & 0 & -2.608756 & 3.869156 & -0.930942 \\
\hline 27 & 6 & 0 & -2.365191 & 2.871645 & 1.747755 \\
\hline 28 & 6 & 0 & -4.719911 & 2.061638 & 1.784399 \\
\hline 29 & 1 & 0 & -7.371425 & -3.080597 & 2.289807 \\
\hline 30 & 1 & 0 & -4.752383 & 4.897720 & 0.577043 \\
\hline 31 & 1 & 0 & -5.151032 & 4.695048 & -1.137543 \\
\hline 32 & 1 & 0 & -5.916099 & 3.652550 & 0.070342 \\
\hline 33 & 1 & 0 & -2.834078 & 4.258175 & -1.930214 \\
\hline 34 & 1 & 0 & -2.272605 & 4.703757 & -0.302191 \\
\hline 35 & 1 & 0 & -1.779829 & 3.157273 & -1.030643 \\
\hline 36 & 1 & 0 & -2.587255 & 3.883052 & 2.111705 \\
\hline 37 & 1 & 0 & -2.209212 & 2.229998 & 2.623965 \\
\hline 38 & 1 & 0 & -1.427354 & 2.905159 & 1.181119 \\
\hline
\end{tabular}




\begin{tabular}{|c|c|c|c|c|c|}
\hline 39 & 1 & 0 & -4.482727 & 1.261321 & 2.495499 \\
\hline 40 & 1 & 0 & -5.011637 & 2.953999 & 2.351475 \\
\hline 41 & 1 & 0 & -5.584216 & 1.736862 & 1.195468 \\
\hline 42 & 29 & 0 & -0.645664 & 0.543937 & -0.993156 \\
\hline 43 & 15 & 0 & 1.097537 & 1.869341 & -0.130802 \\
\hline 44 & 15 & 0 & -0.057341 & -1.474867 & 0.051176 \\
\hline 45 & 6 & 0 & 2.324275 & 0.871267 & -1.090135 \\
\hline 46 & 6 & 0 & 1.876488 & 2.099116 & 1.510164 \\
\hline 47 & 6 & 0 & 1.108890 & 3.555791 & -0.851702 \\
\hline 48 & 6 & 0 & -1.330426 & -2.328238 & 1.046626 \\
\hline 49 & 6 & 0 & 0.525074 & -2.699262 & -1.173264 \\
\hline 50 & 6 & 0 & 1.364424 & -1.372477 & 1.221830 \\
\hline 51 & 6 & 0 & 2.798151 & -0.354677 & -0.606094 \\
\hline 52 & 6 & 0 & 2.644621 & 1.262111 & -2.416500 \\
\hline 53 & 6 & 0 & 1.152237 & 1.737355 & 2.650763 \\
\hline 54 & 6 & 0 & 3.180362 & 2.589932 & 1.661353 \\
\hline 55 & 6 & 0 & 0.546225 & 3.727596 & -2.128607 \\
\hline 56 & 6 & 0 & 1.484687 & 4.698364 & -0.133072 \\
\hline 57 & 6 & 0 & -2.129809 & -1.518564 & 1.865548 \\
\hline 58 & 6 & 0 & -1.514523 & -3.715019 & 1.079844 \\
\hline 59 & 6 & 0 & 0.327621 & -2.413670 & -2.529040 \\
\hline 60 & 6 & 0 & 1.206101 & -3.869597 & -0.813138 \\
\hline 61 & 6 & 0 & 1.217199 & -1.775745 & 2.574413 \\
\hline 62 & 6 & 0 & 2.571369 & -0.807747 & 0.800769 \\
\hline 63 & 6 & 0 & 3.611989 & -1.182242 & -1.448692 \\
\hline 64 & 6 & 0 & 3.405014 & 0.470802 & -3.236783 \\
\hline 65 & 1 & 0 & 2.291680 & 2.220116 & -2.788028 \\
\hline 66 & 1 & 0 & 0.150454 & 1.320712 & 2.533580 \\
\hline 67 & 6 & 0 & 1.715676 & 1.864519 & 3.917458 \\
\hline 68 & 6 & 0 & 3.742030 & 2.720742 & 2.926744 \\
\hline 69 & 1 & 0 & 3.761030 & 2.856762 & 0.777101 \\
\hline 70 & 1 & 0 & 0.173619 & 2.855331 & -2.666726 \\
\hline 71 & 6 & 0 & 0.415042 & 4.993641 & -2.686645 \\
\hline 72 & 6 & 0 & 1.331820 & 5.967835 & -0.687265 \\
\hline 73 & 1 & 0 & 1.891236 & 4.602925 & 0.871495 \\
\hline 74 & 1 & 0 & -2.027606 & -0.435862 & 1.805306 \\
\hline 75 & 6 & 0 & -3.067681 & -2.084876 & 2.721918 \\
\hline 76 & 6 & 0 & -2.453427 & -4.278806 & 1.939788 \\
\hline 77 & 1 & 0 & -0.924310 & -4.359857 & 0.431028 \\
\hline 78 & 1 & 0 & -0.169850 & -1.480816 & -2.803047 \\
\hline 79 & 6 & 0 & 0.791467 & -3.290207 & -3.506643 \\
\hline 80 & 6 & 0 & 1.658465 & -4.748916 & -1.791141 \\
\hline 81 & 1 & 0 & 1.400092 & -4.079093 & 0.240183 \\
\hline 82 & 1 & 0 & 0.274961 & -2.211186 & 2.900300 \\
\hline 83 & 6 & 0 & 2.238531 & -1.629989 & 3.478005 \\
\hline 84 & 6 & 0 & 3.658158 & -0.682460 & 1.725278 \\
\hline 85 & 6 & 0 & 4.148234 & -2.417897 & -1.004051 \\
\hline 86 & 6 & 0 & 3.907559 & -0.769263 & -2.785393 \\
\hline 87 & 1 & 0 & 3.642342 & 0.800210 & -4.248422 \\
\hline 88 & 1 & 0 & 1.147180 & 1.562769 & 4.795825 \\
\hline 89 & 6 & 0 & 3.010579 & 2.357520 & 4.056203 \\
\hline 90 & 1 & 0 & 4.762220 & 3.086738 & 3.031484 \\
\hline
\end{tabular}


Medina et al.: BCP Hydroboration-Supporting Information - S111

\begin{tabular}{|c|c|c|c|c|c|}
\hline 91 & 1 & 0 & -0.020588 & 5.099406 & -3.679008 \\
\hline 92 & 6 & 0 & 0.807991 & 6.120790 & -1.967315 \\
\hline 93 & 1 & 0 & 1.627385 & 6.842522 & -0.109816 \\
\hline 94 & 1 & 0 & -3.694463 & -1.443240 & 3.339737 \\
\hline 95 & 6 & 0 & -3.223483 & -3.467756 & 2.768938 \\
\hline 96 & 1 & 0 & -2.589684 & -5.359497 & 1.954308 \\
\hline 97 & 1 & 0 & 0.643754 & -3.052920 & -4.558886 \\
\hline 98 & 6 & 0 & 1.453106 & -4.458976 & -3.138830 \\
\hline 99 & 1 & 0 & 2.193636 & -5.652535 & -1.502266 \\
\hline 100 & 1 & 0 & 2.105095 & -1.945366 & 4.51335 \\
\hline 101 & 6 & 0 & 3.481706 & -1.086469 & 3.08615 \\
\hline 102 & 6 & 0 & 4.919060 & -0.150797 & 1.34892 \\
\hline 103 & 1 & 0 & 3.927428 & -2.756028 & 0.006368 \\
\hline 104 & 6 & 0 & 4.927838 & -3.195485 & -1.828541 \\
\hline 105 & 6 & 0 & 4.705039 & -1.594274 & -3.612348 \\
\hline 106 & 1 & 0 & 3.458269 & 2.445801 & 5.044861 \\
\hline 107 & 1 & 0 & 0.692050 & 7.114210 & -2.397277 \\
\hline 108 & 1 & 0 & -3.967565 & -3.910684 & 3.428992 \\
\hline 109 & 1 & 0 & 1.824039 & -5.139726 & -3.903490 \\
\hline 110 & 6 & 0 & 4.549109 & -0.935945 & 4.00263 \\
\hline 111 & 6 & 0 & 5.941930 & -0.028128 & 2.26042 \\
\hline 112 & 1 & 0 & 5.072237 & 0.167905 & 0.31982 \\
\hline 113 & 1 & 0 & 5.321599 & -4.142000 & -1.461318 \\
\hline 114 & 6 & 0 & 5.209818 & -2.785105 & -3.146598 \\
\hline 115 & 1 & 0 & 4.915098 & -1.260556 & -4.628552 \\
\hline 116 & 1 & 0 & 4.391271 & -1.244469 & 5.03642 \\
\hline 117 & 6 & 0 & 5.758603 & -0.419504 & 3.602246 \\
\hline 118 & 1 & 0 & 6.900136 & 0.380271 & 1.94325 \\
\hline 119 & 1 & 0 & 5.825636 & -3.410031 & -3.79097 \\
\hline 120 & 1 & 0 & 6.574130 & -0.314221 & 4.31567 \\
\hline
\end{tabular}




\section{NMR Spectra}

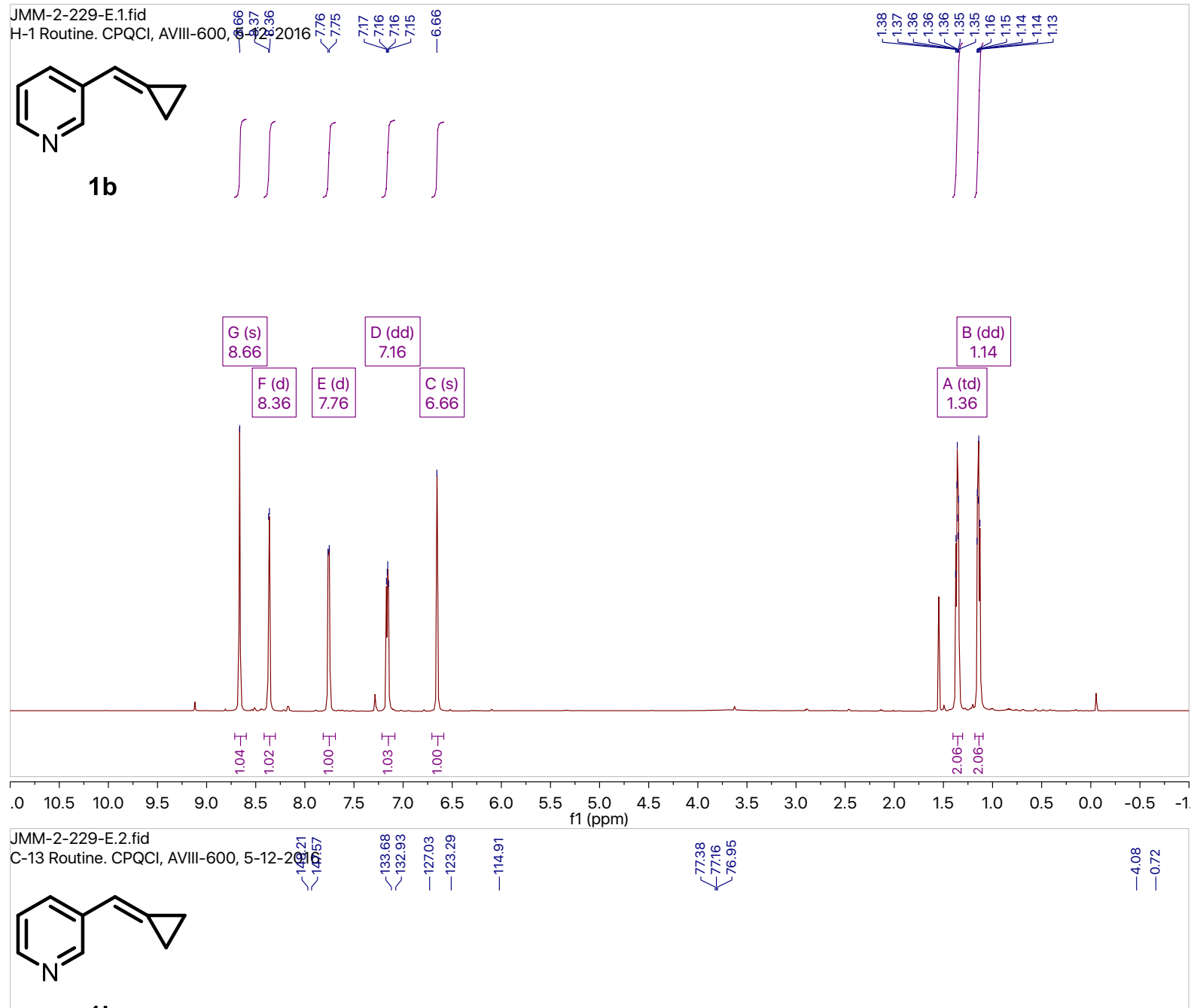

$1 b$

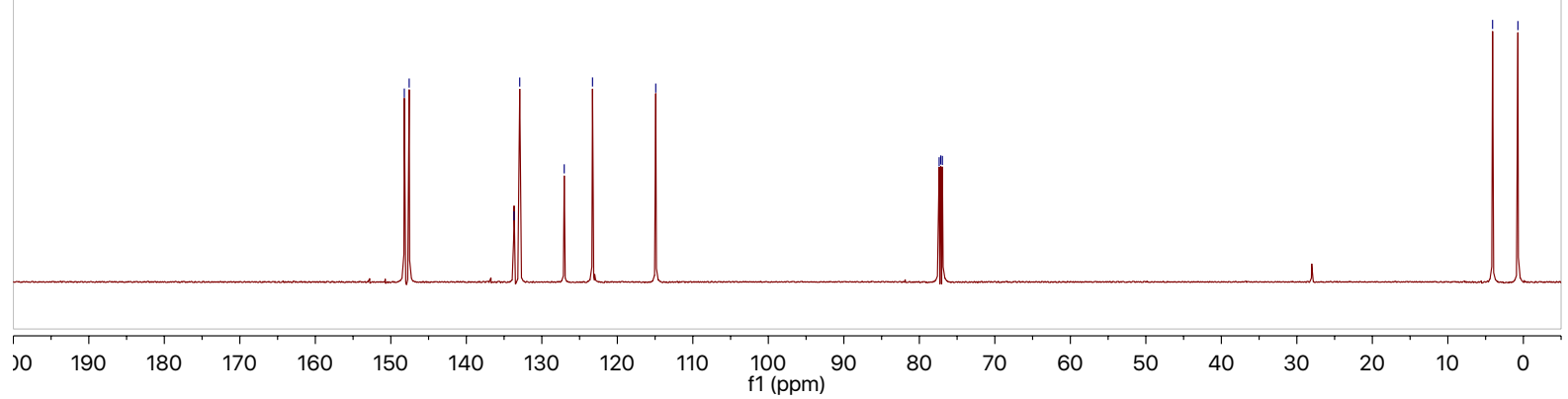




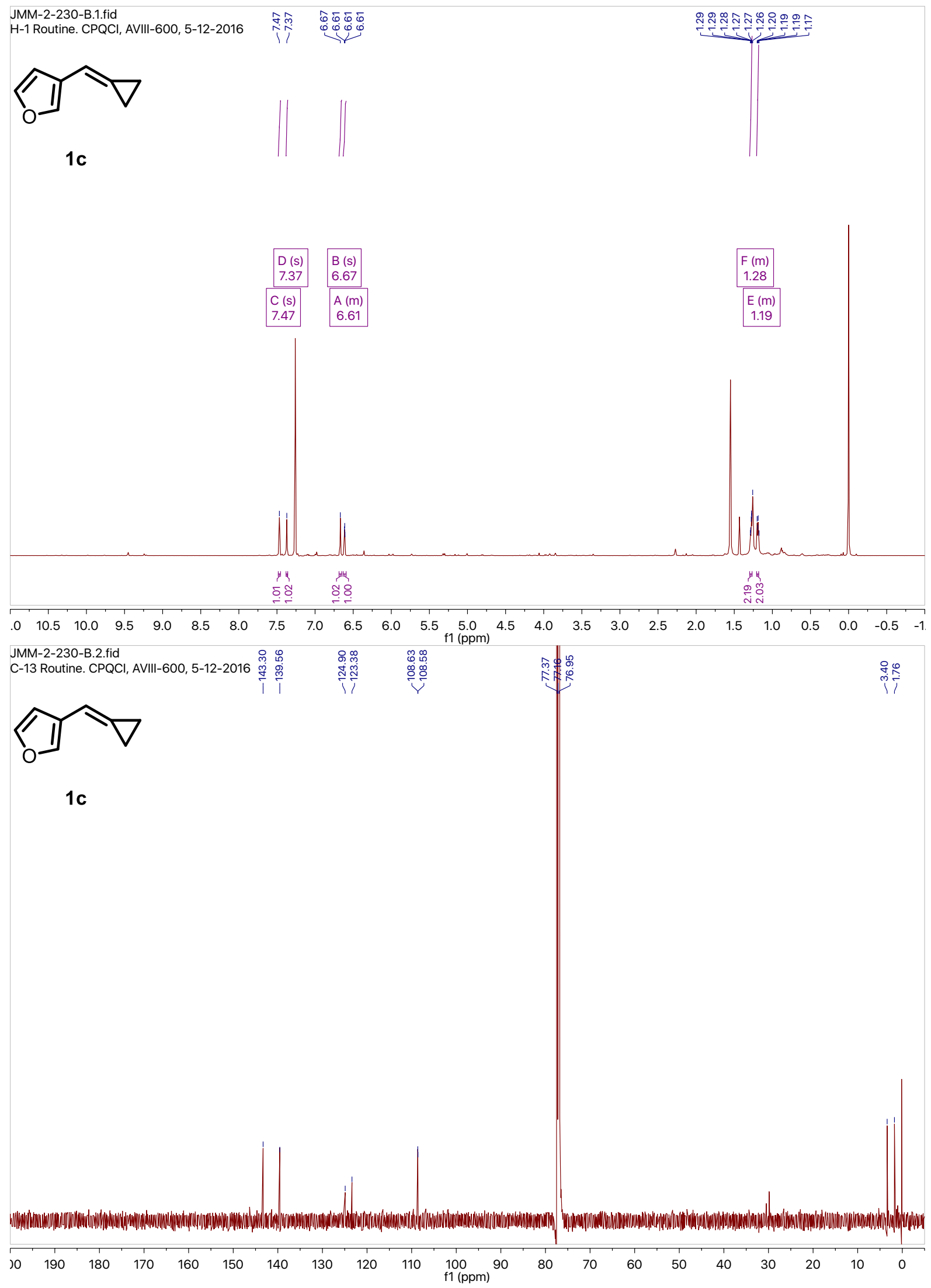



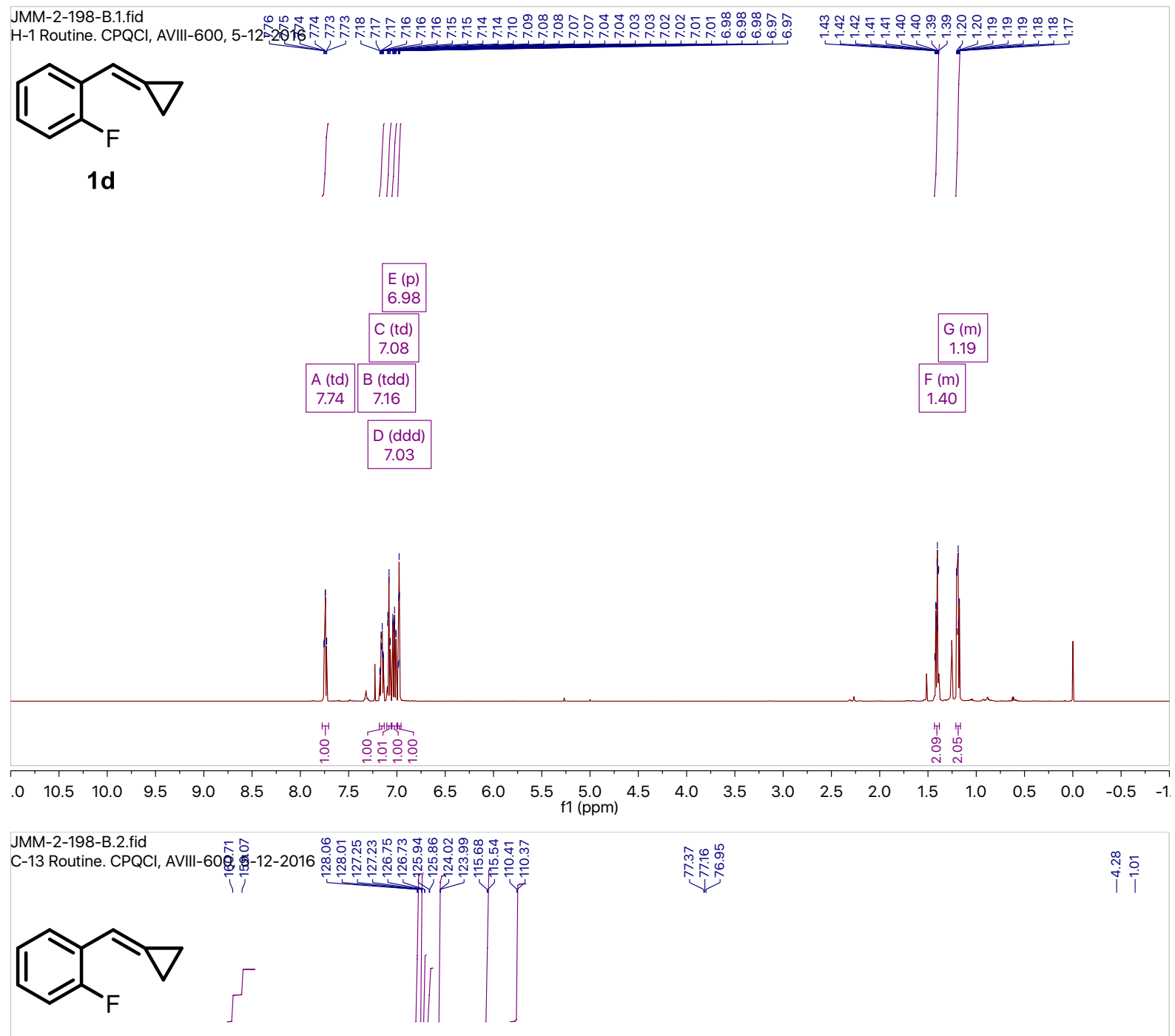

1d

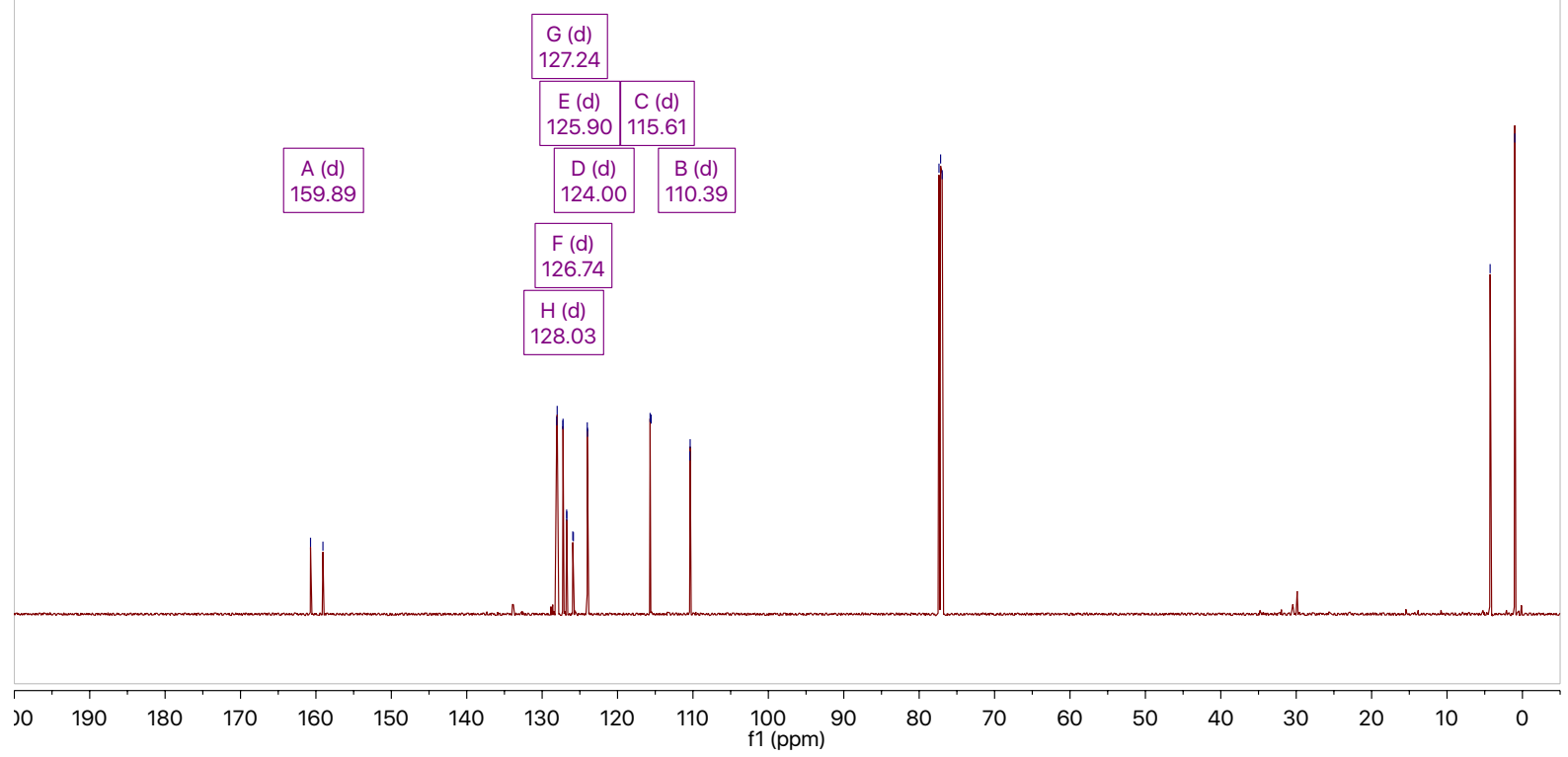




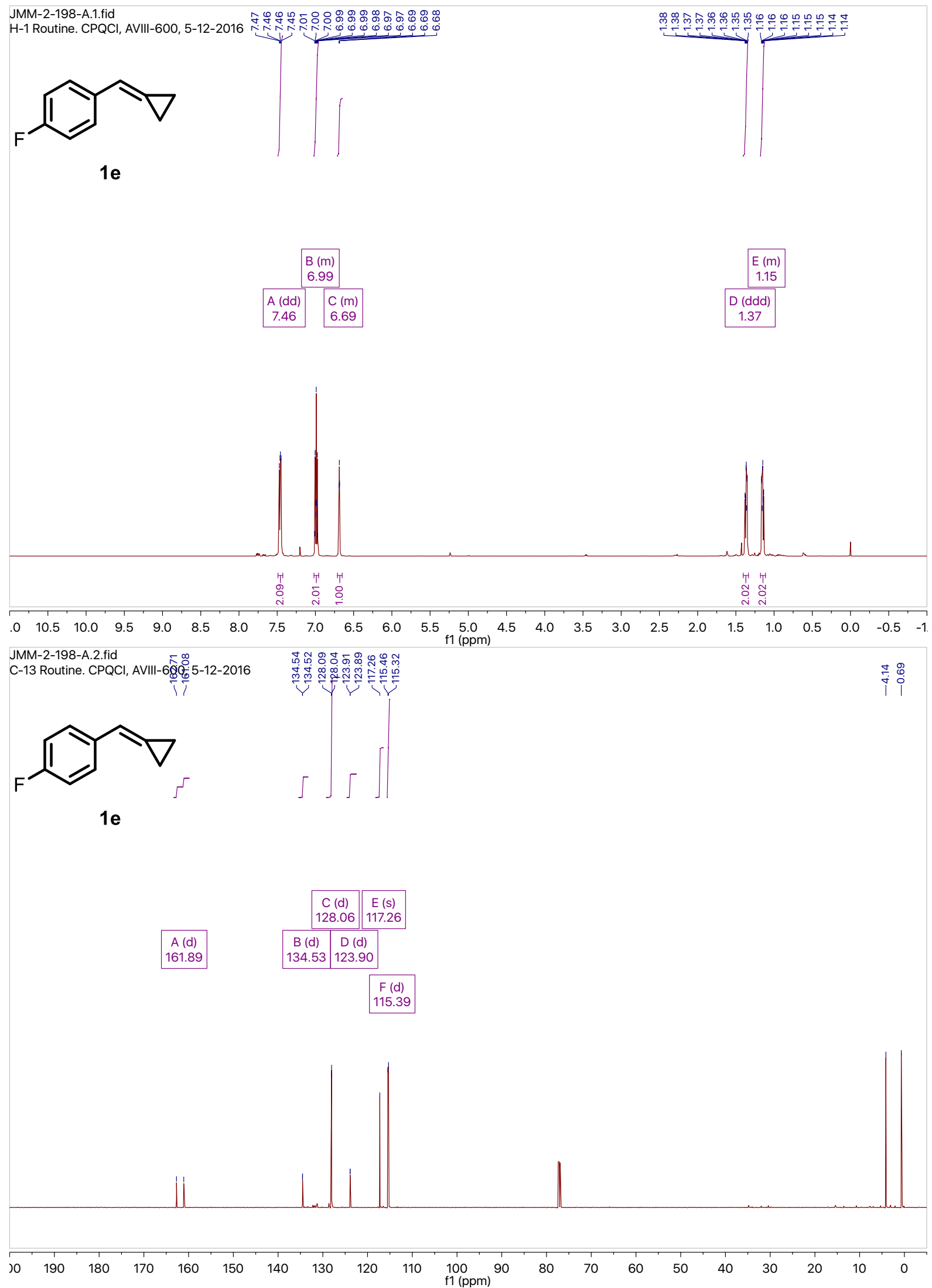


Medina et al.: BCP Hydroboration-Supporting Information - S116
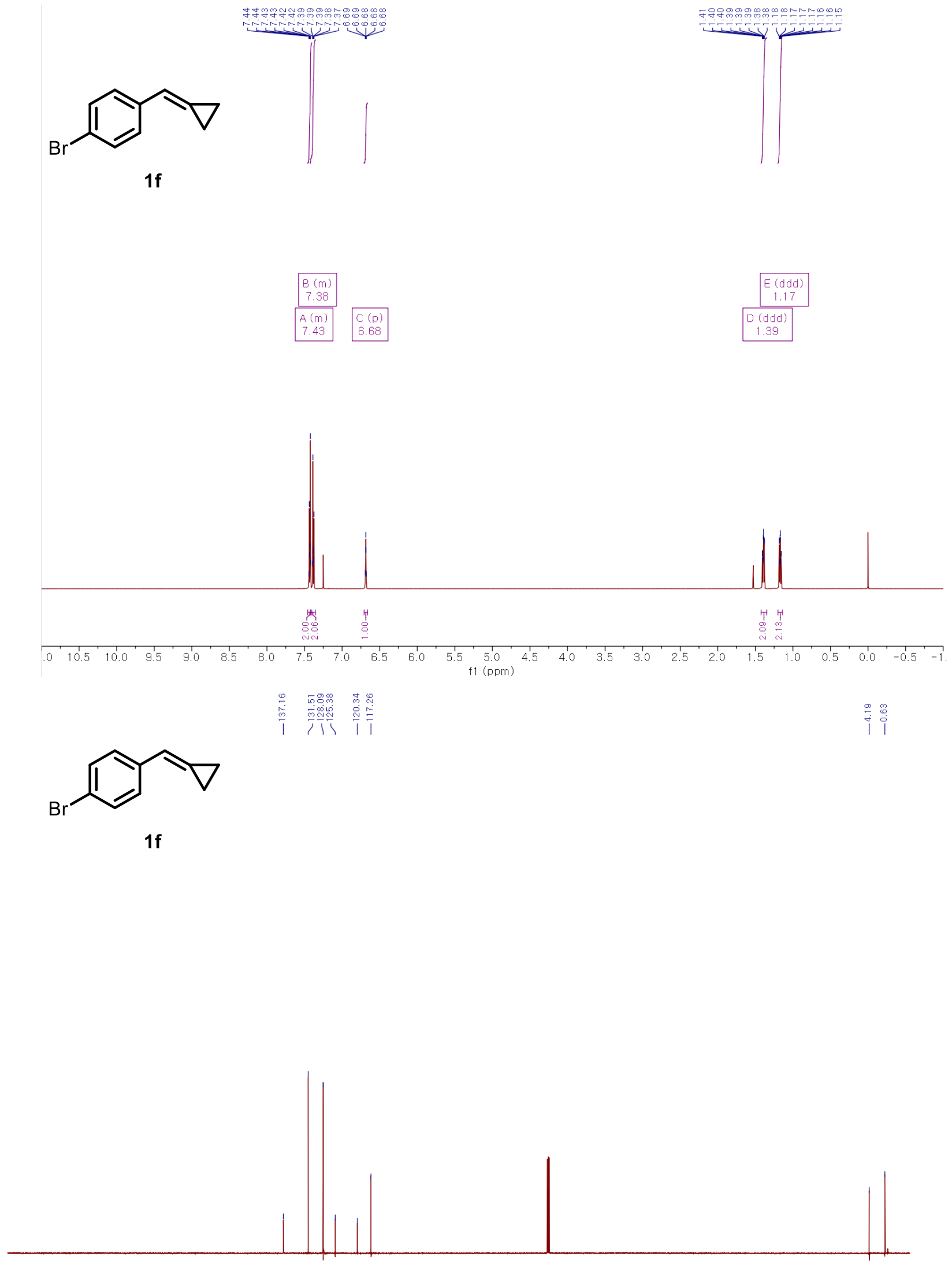

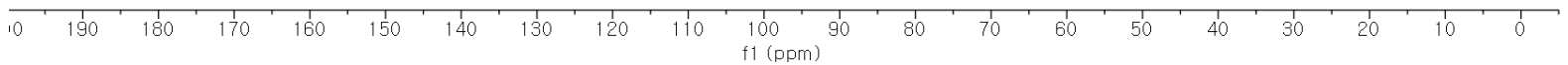




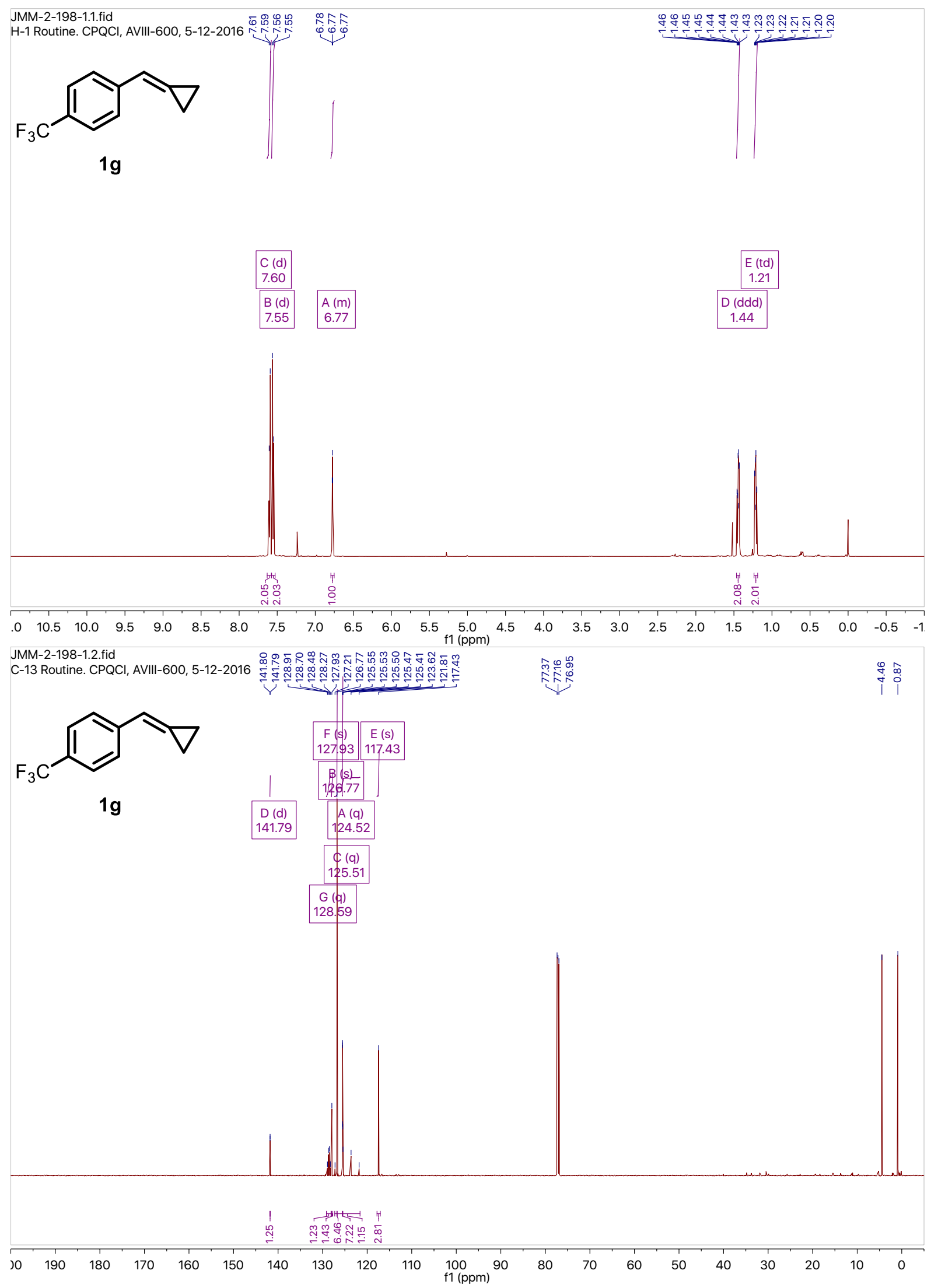




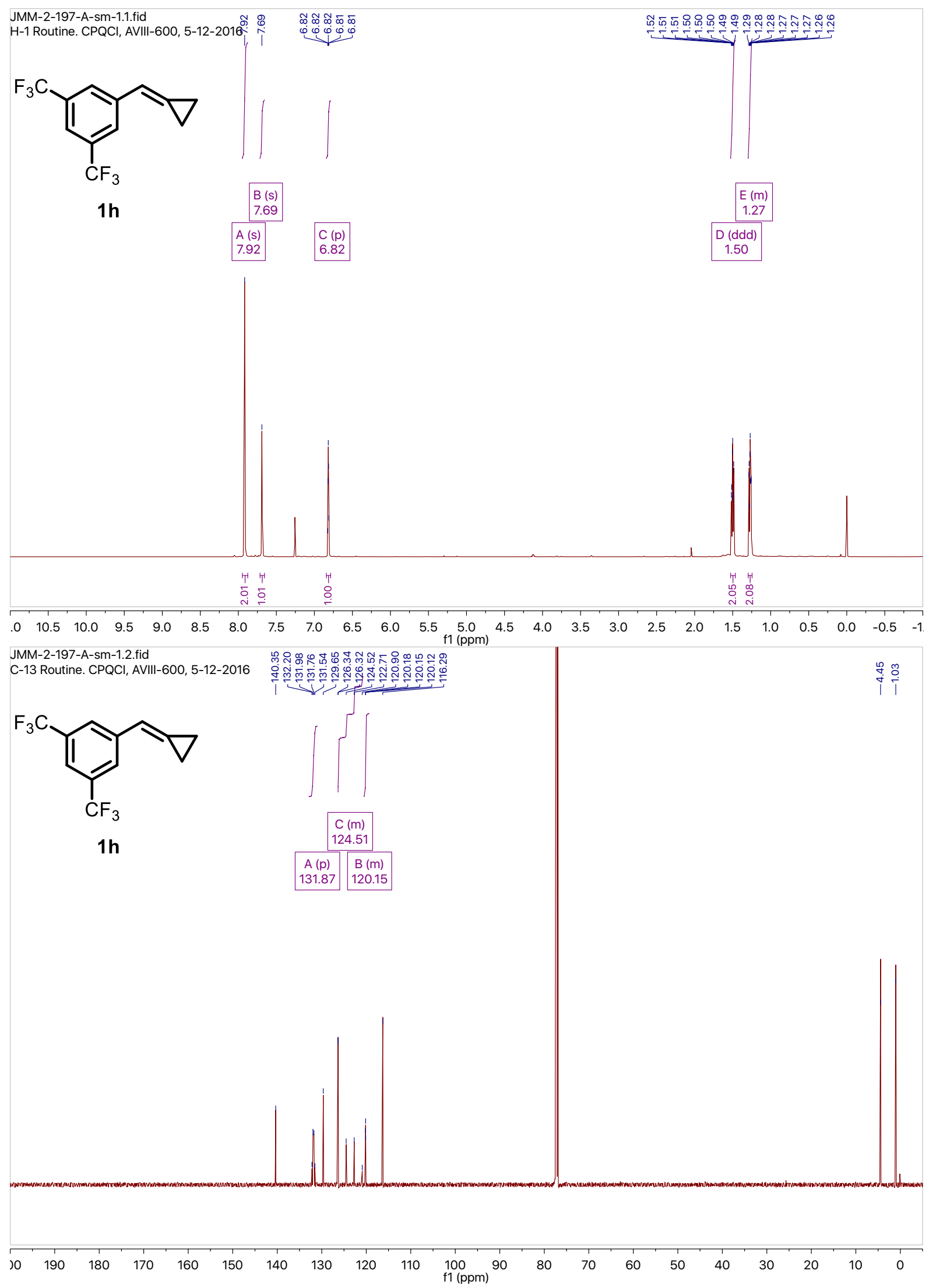




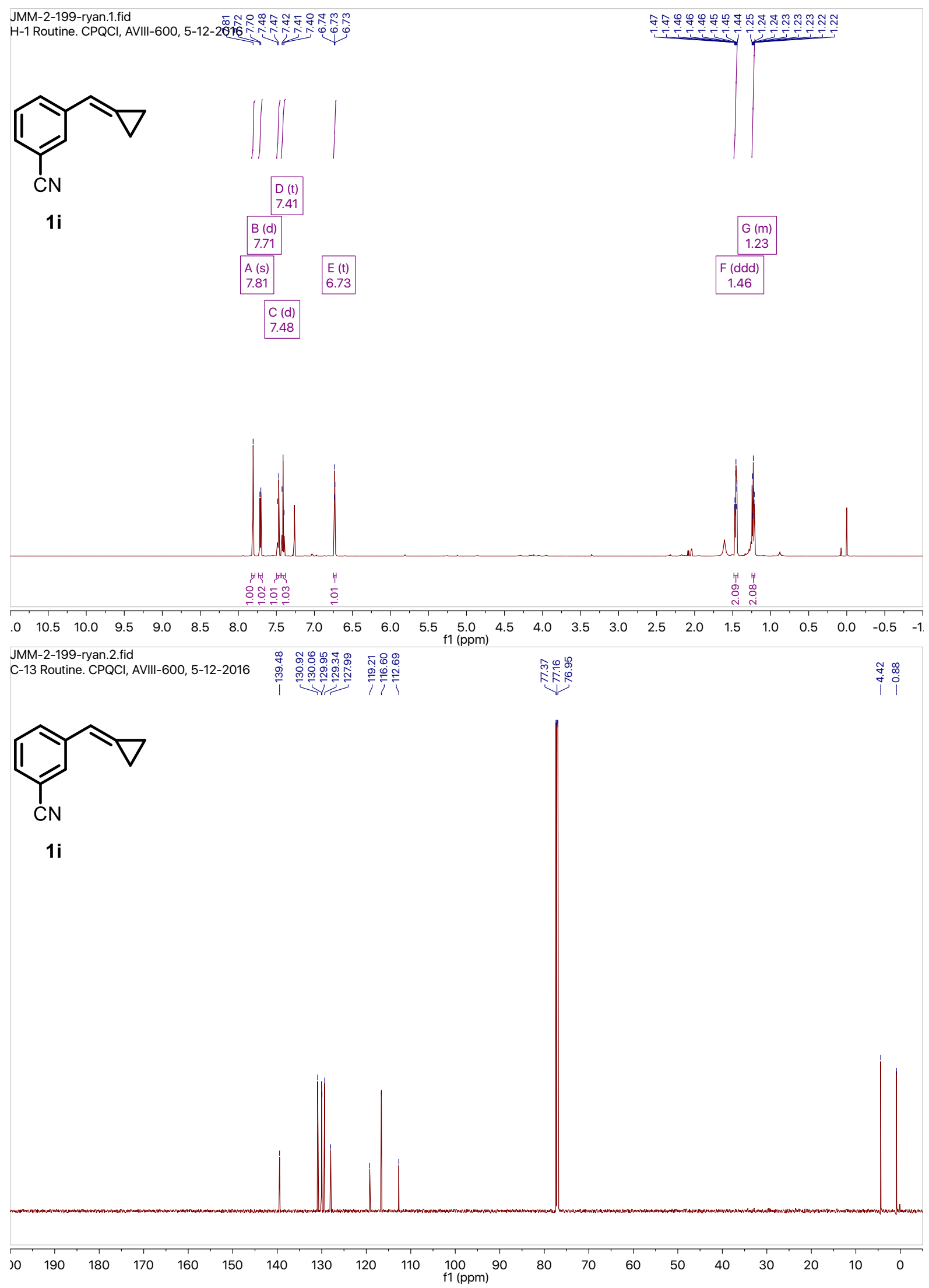



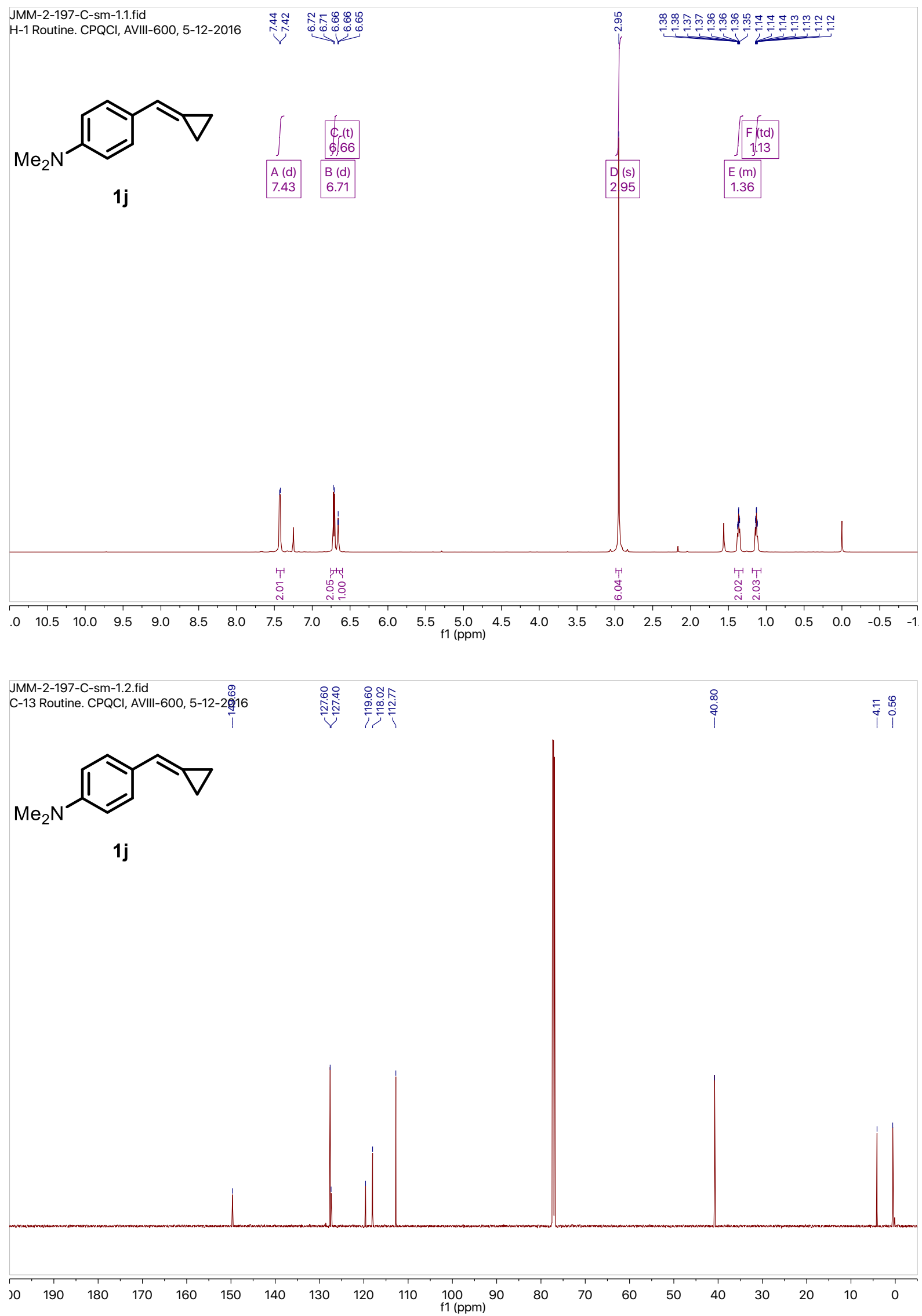


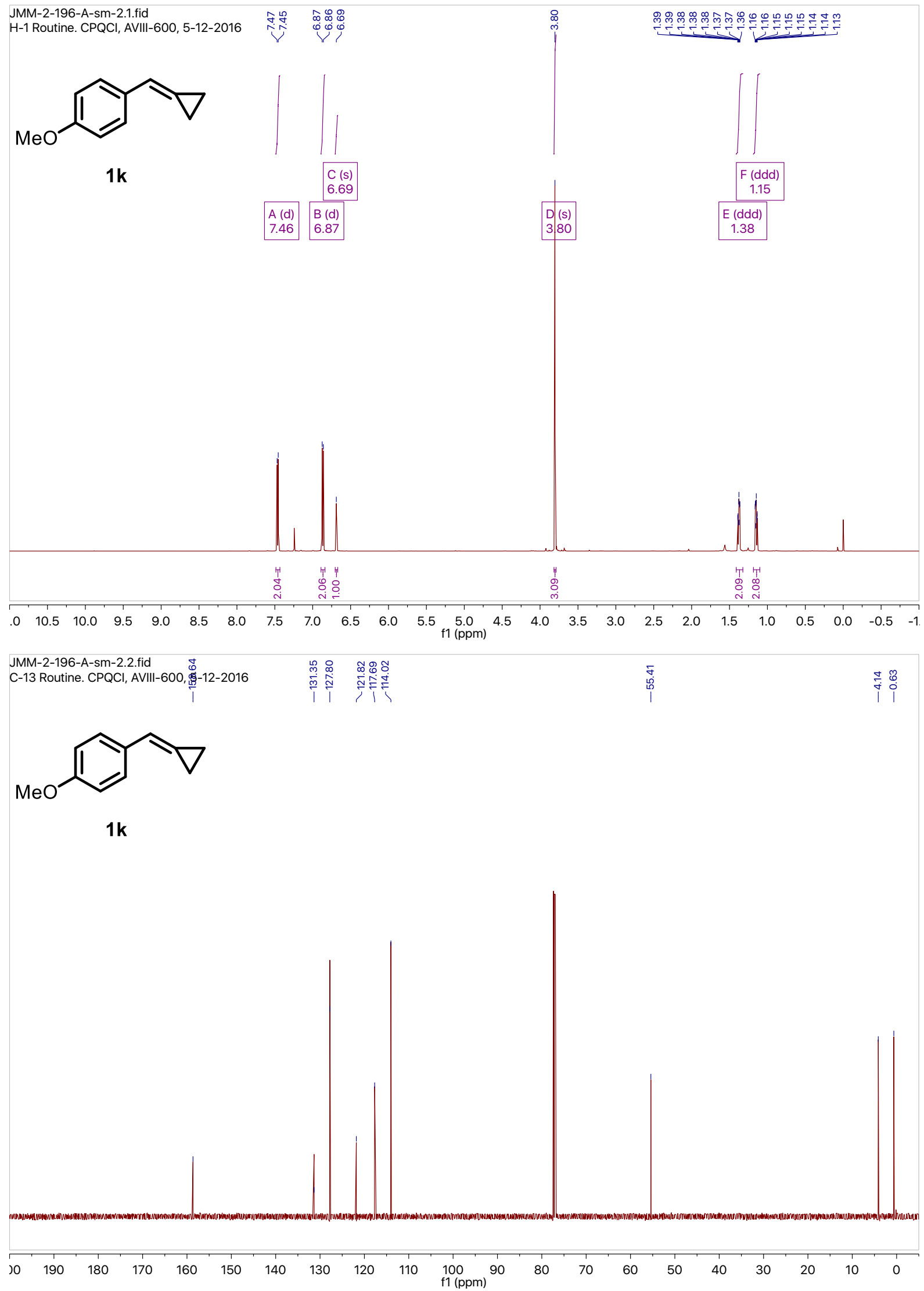




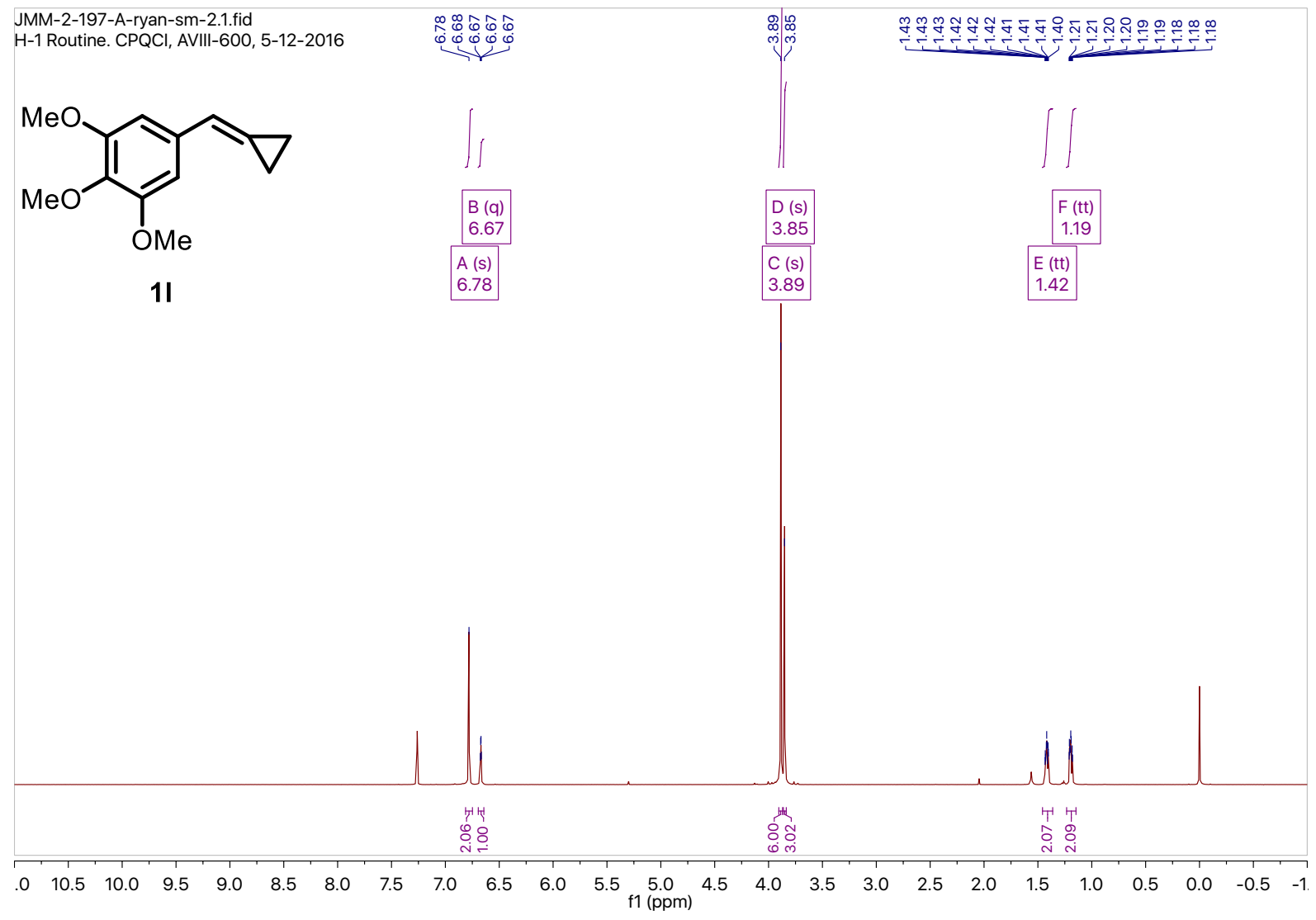

JMM-2-197-A-ryan-sm-2.2.fid
C-13 Routine. CPQCI, AVIII-60, 5-12

11

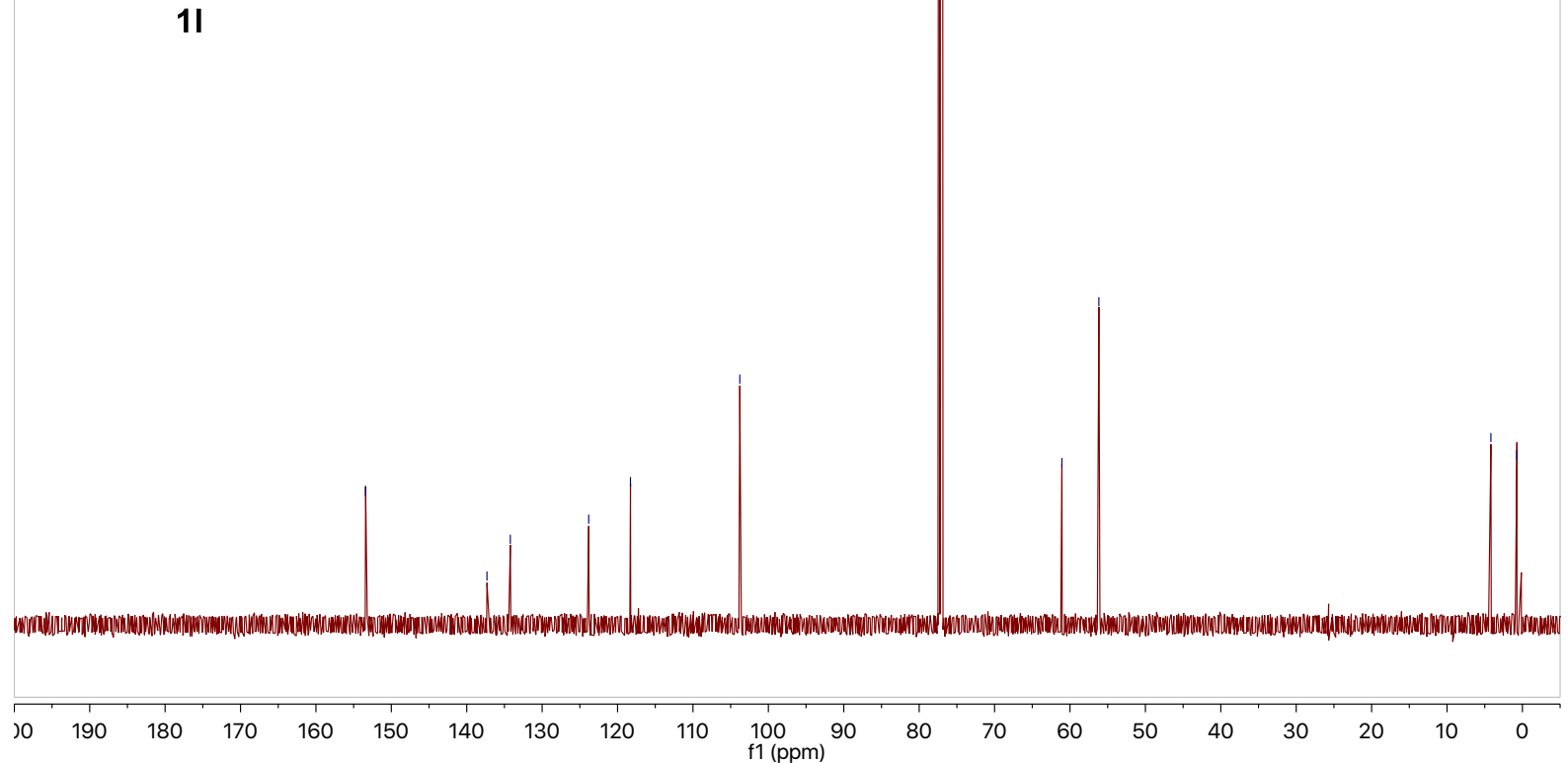



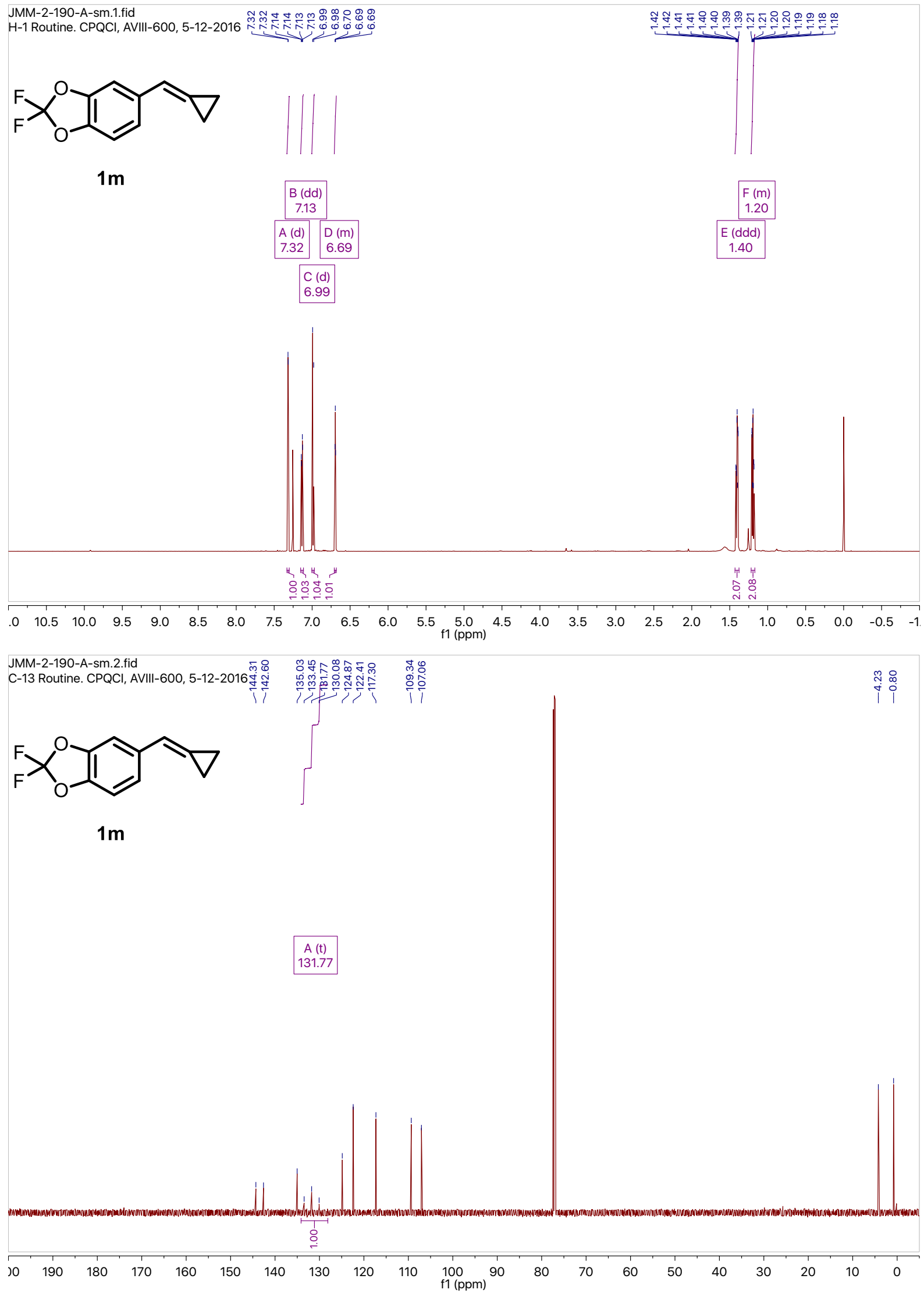

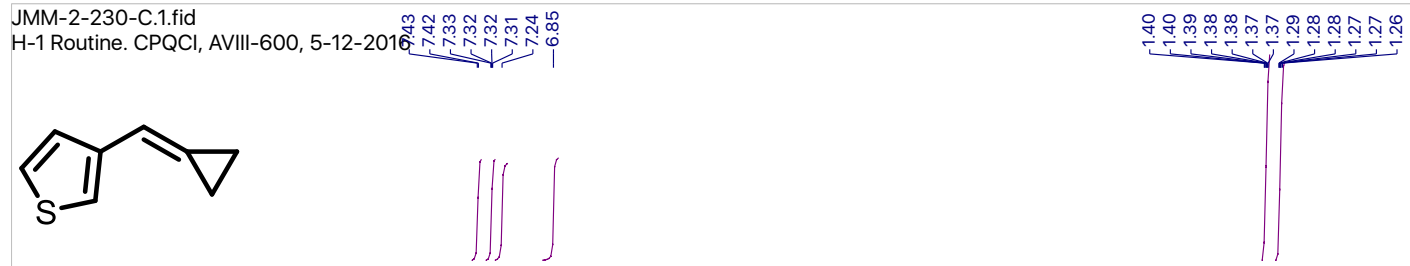

$1 \mathrm{n}$

\begin{tabular}{|l|}
\hline \begin{tabular}{|l|}
\hline B (s) \\
7.24
\end{tabular} \\
\begin{tabular}{|l|l|}
\hline D (d) & A (s) \\
7.43 & 6.85 \\
\hline \multicolumn{2}{|c|}{ (dd) } \\
7.32
\end{tabular} \\
\hline
\end{tabular}
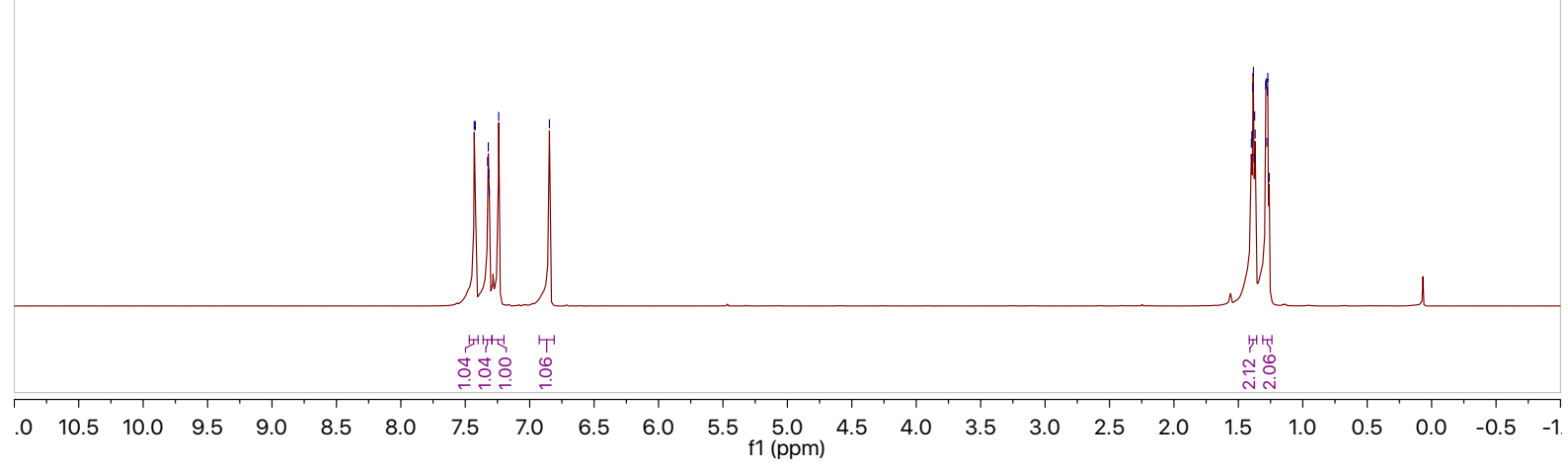

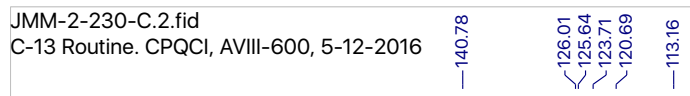

诲尔

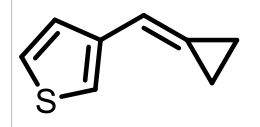

$1 n$

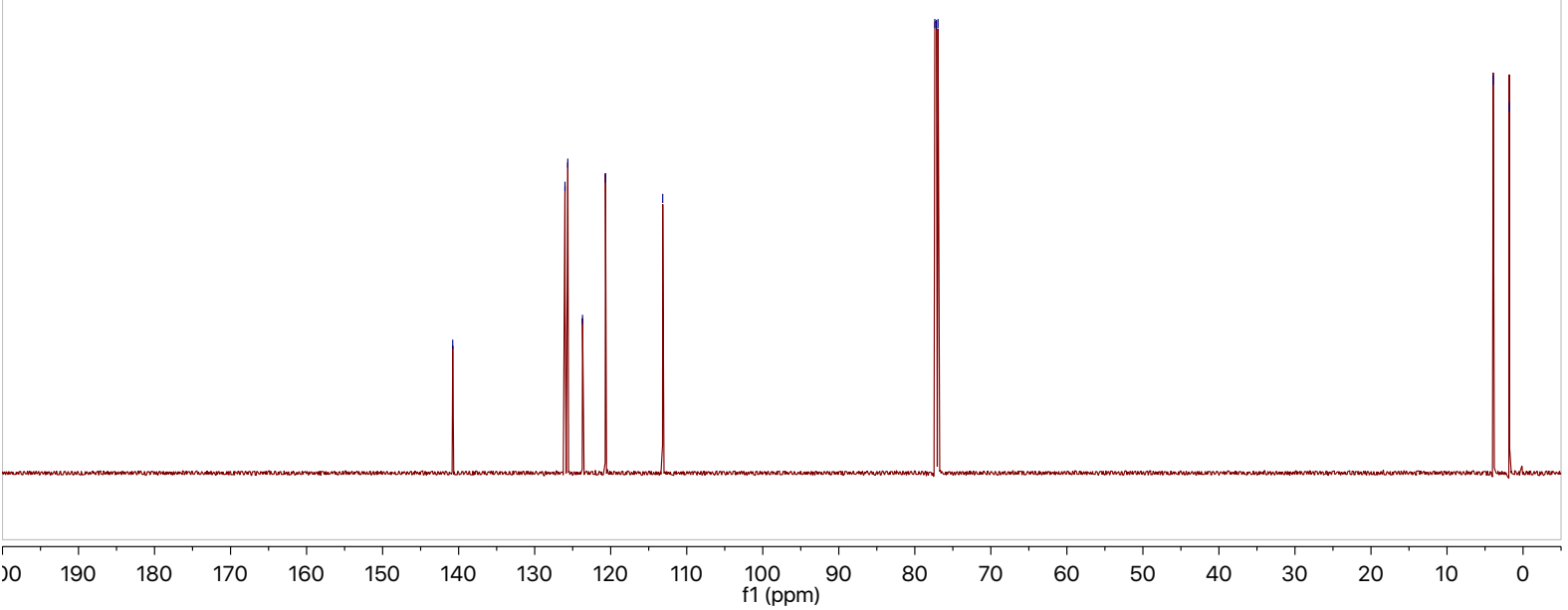



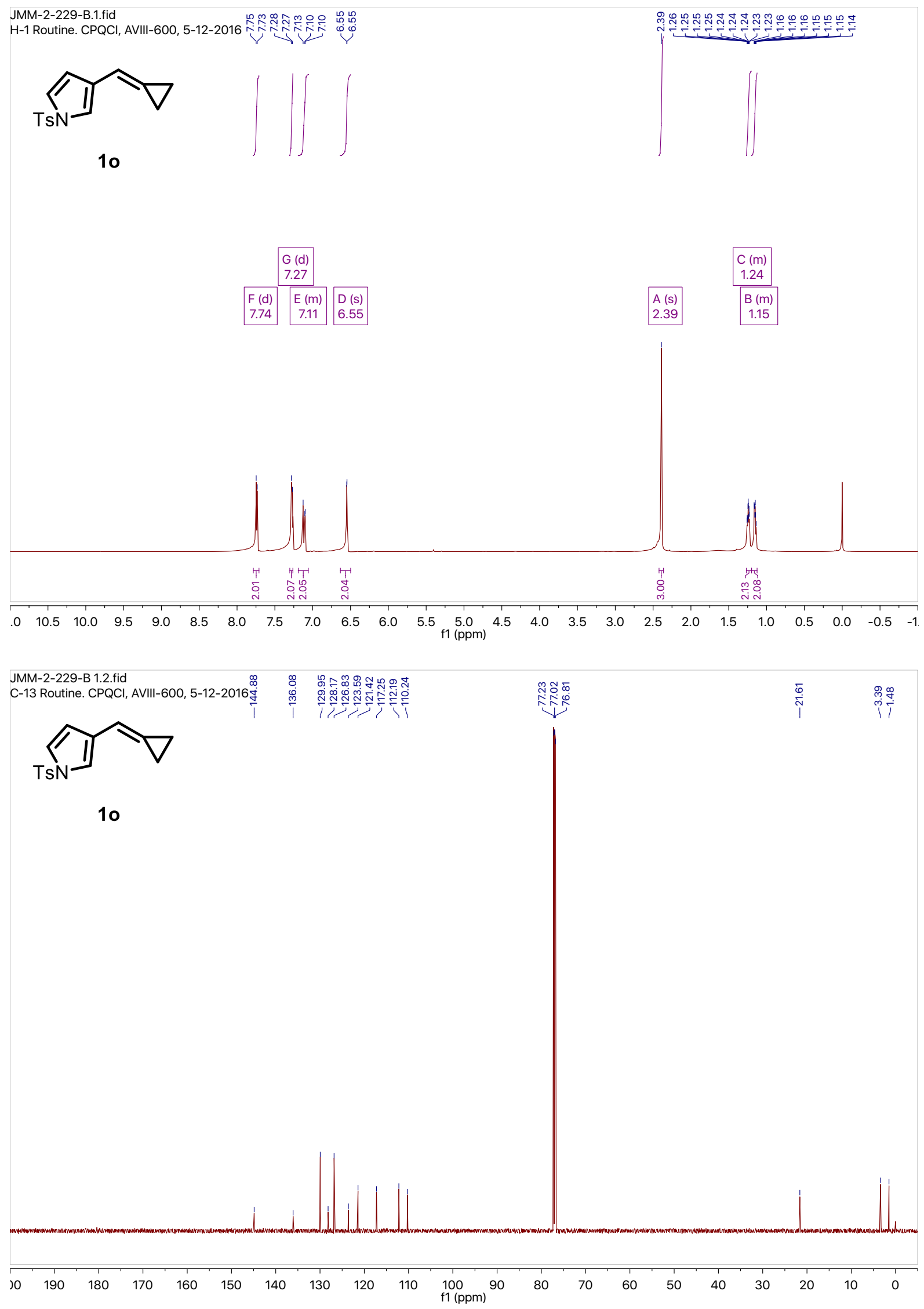


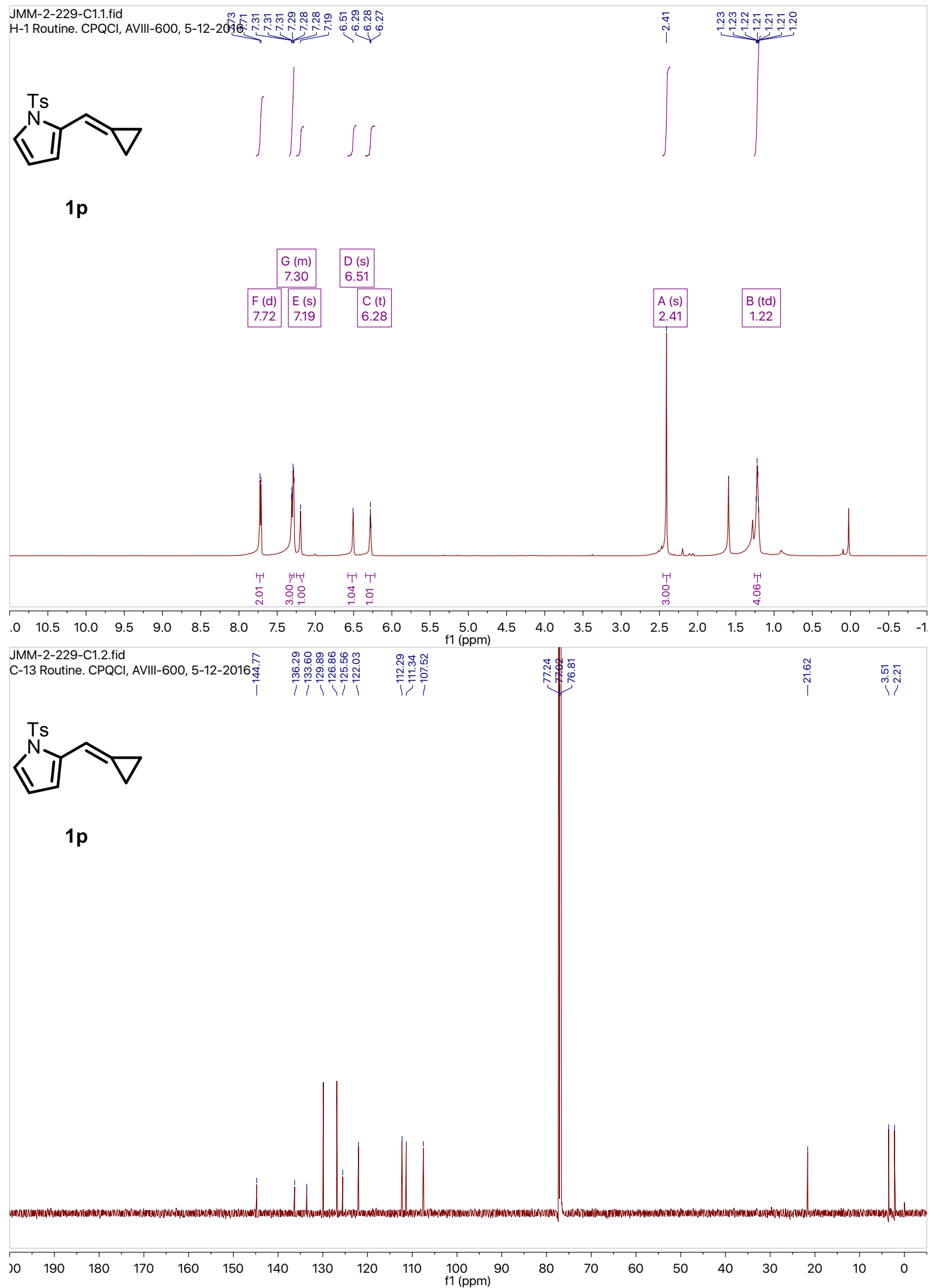




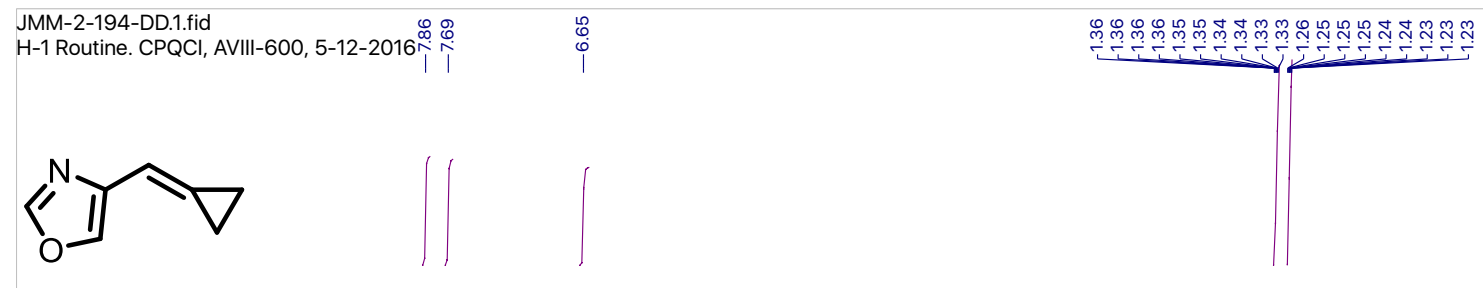

$1 q$
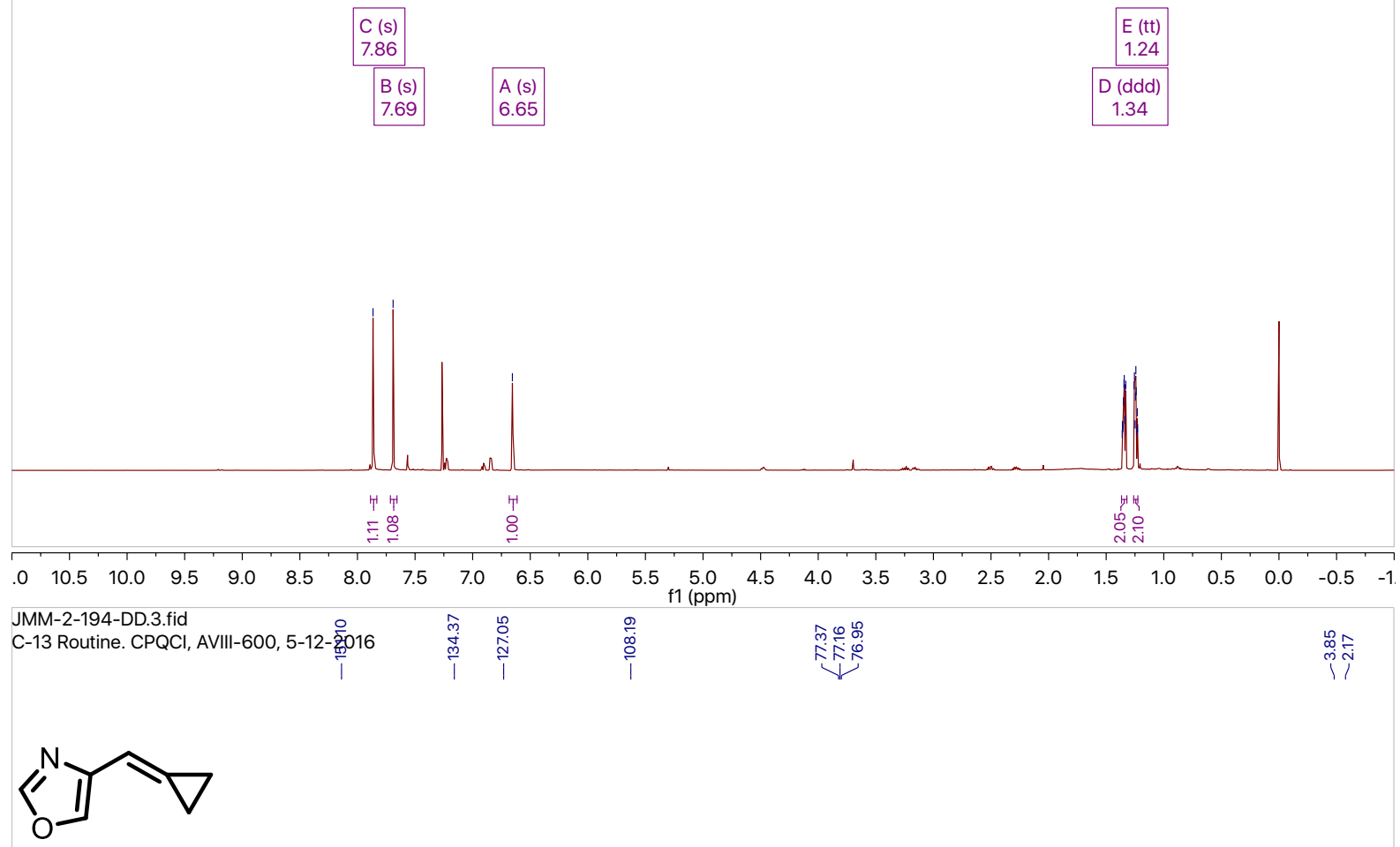

$1 q$
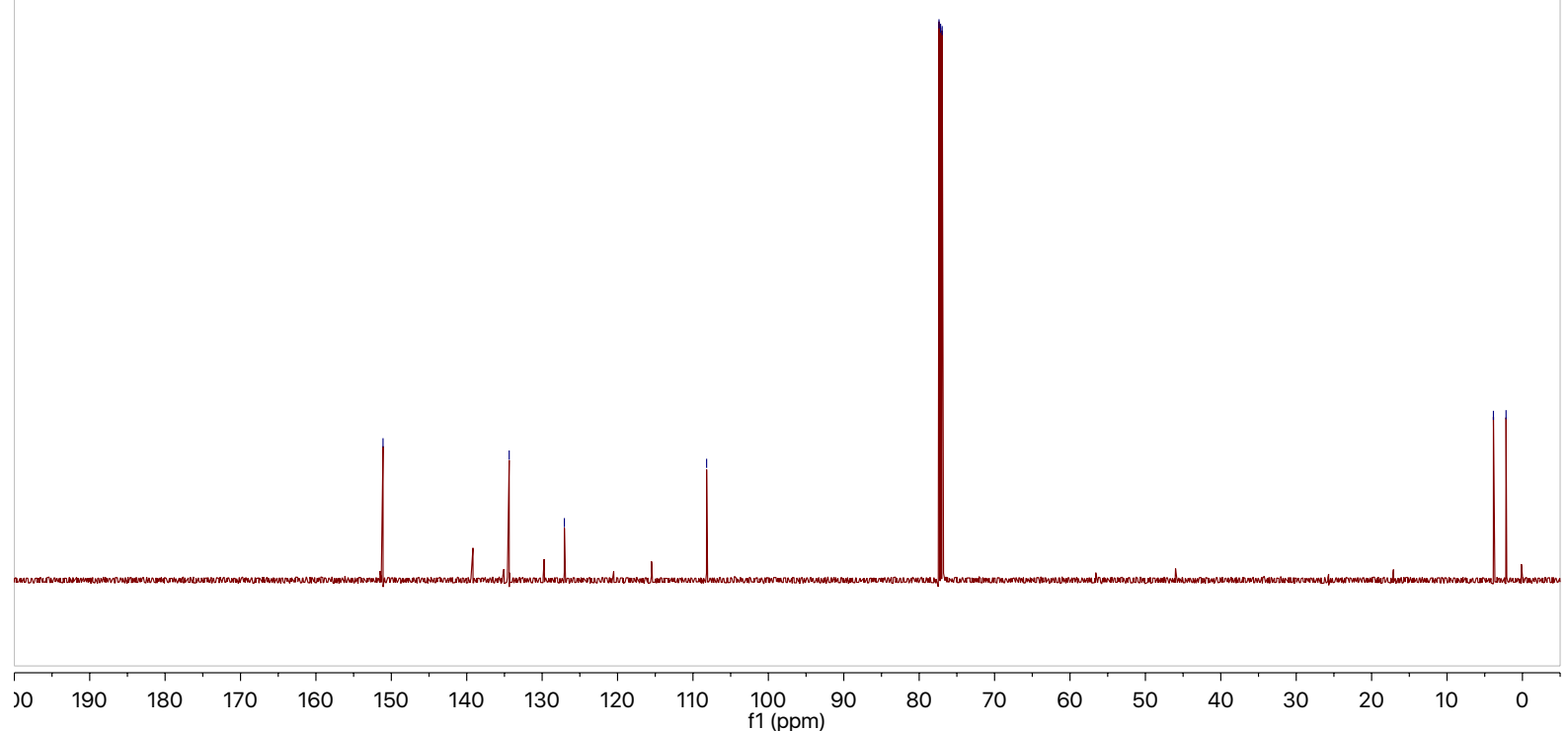


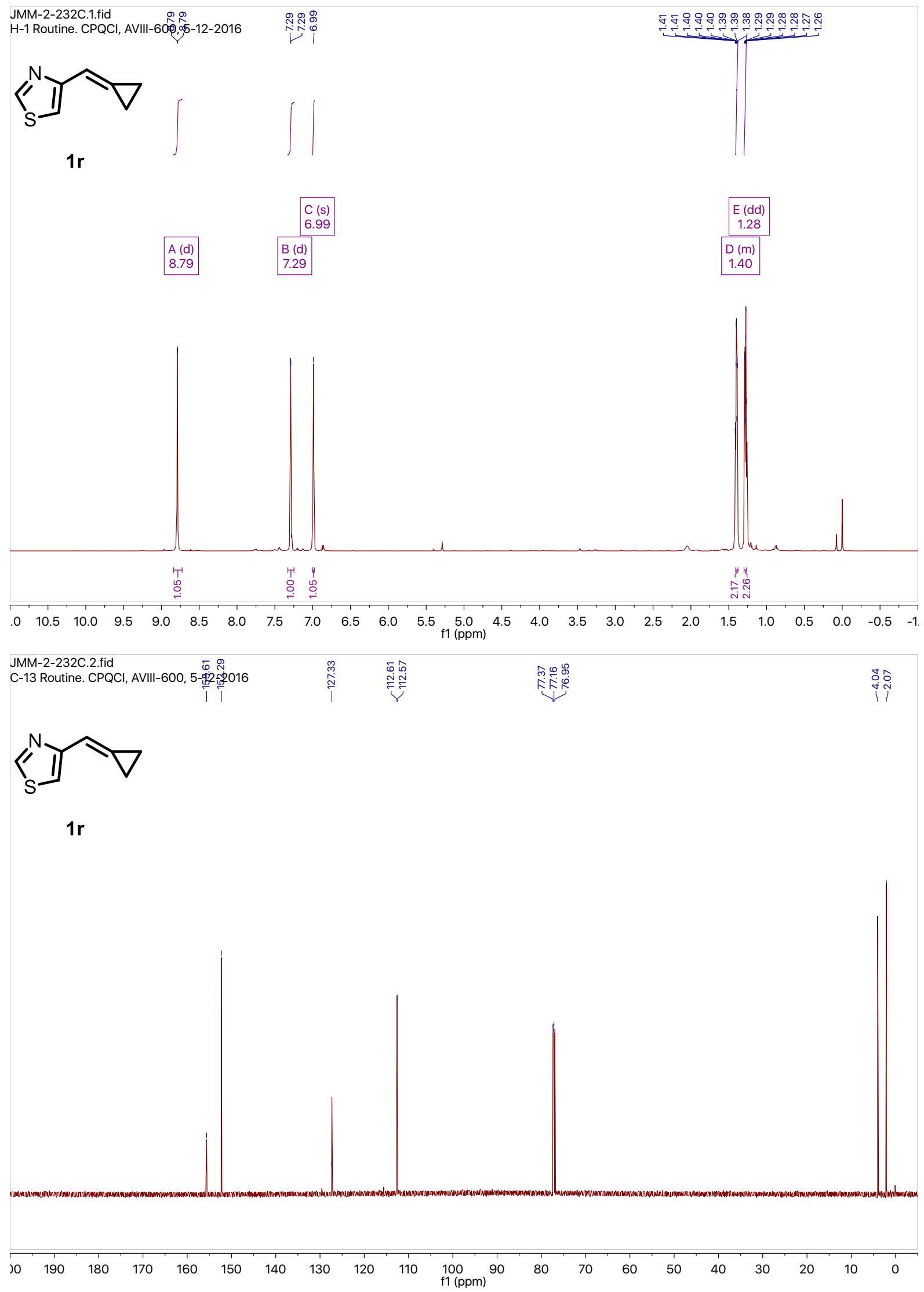




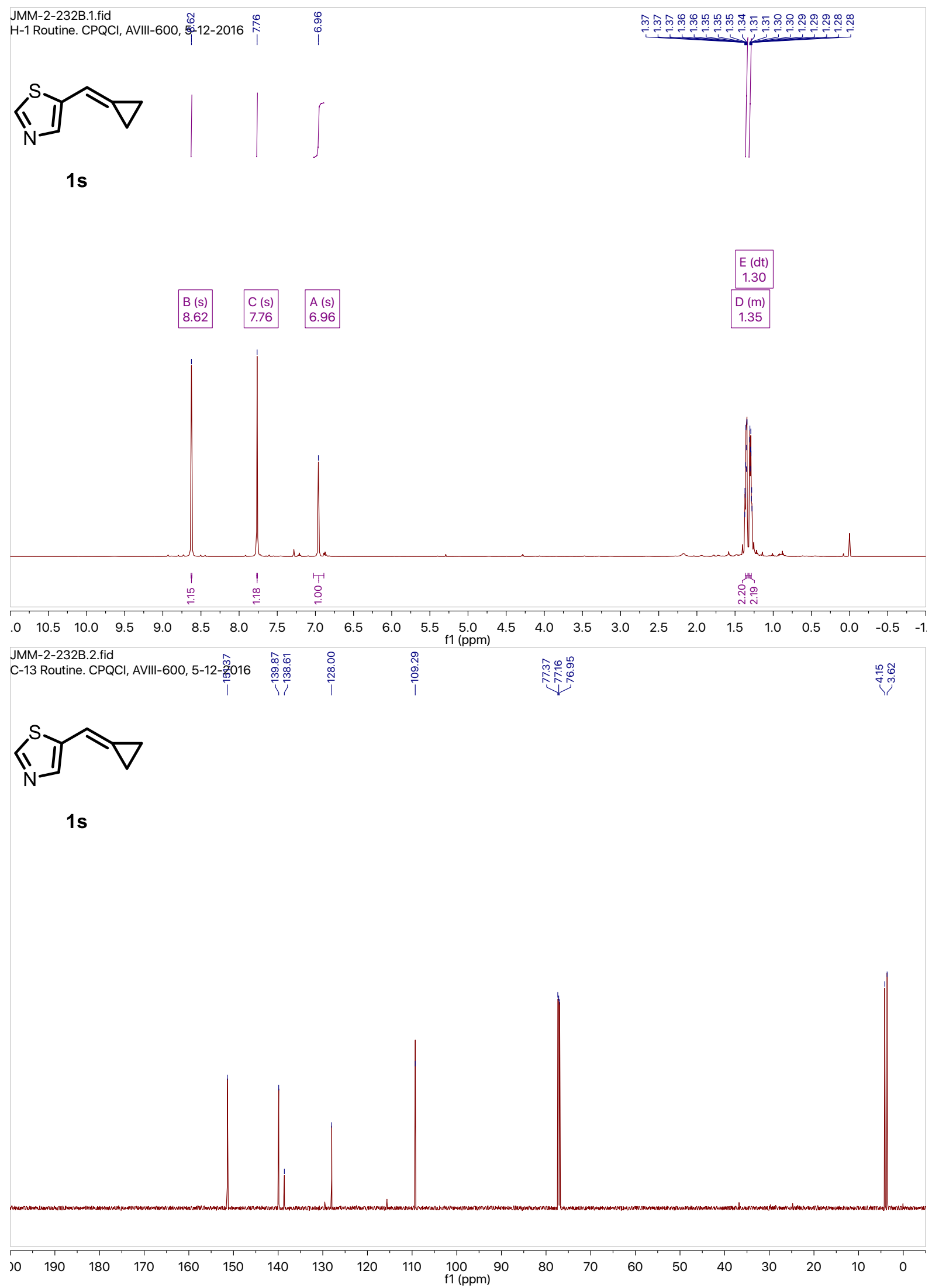




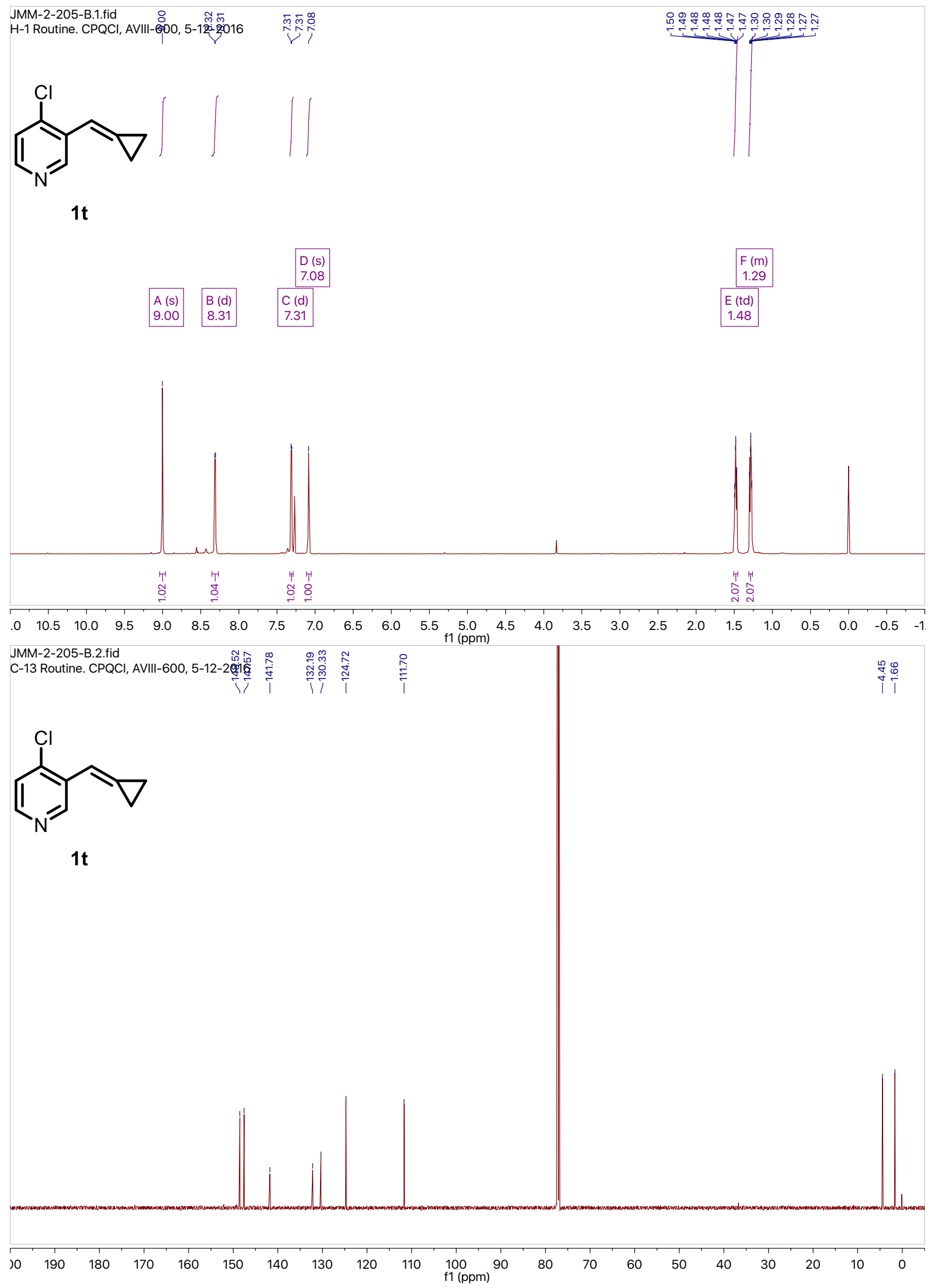




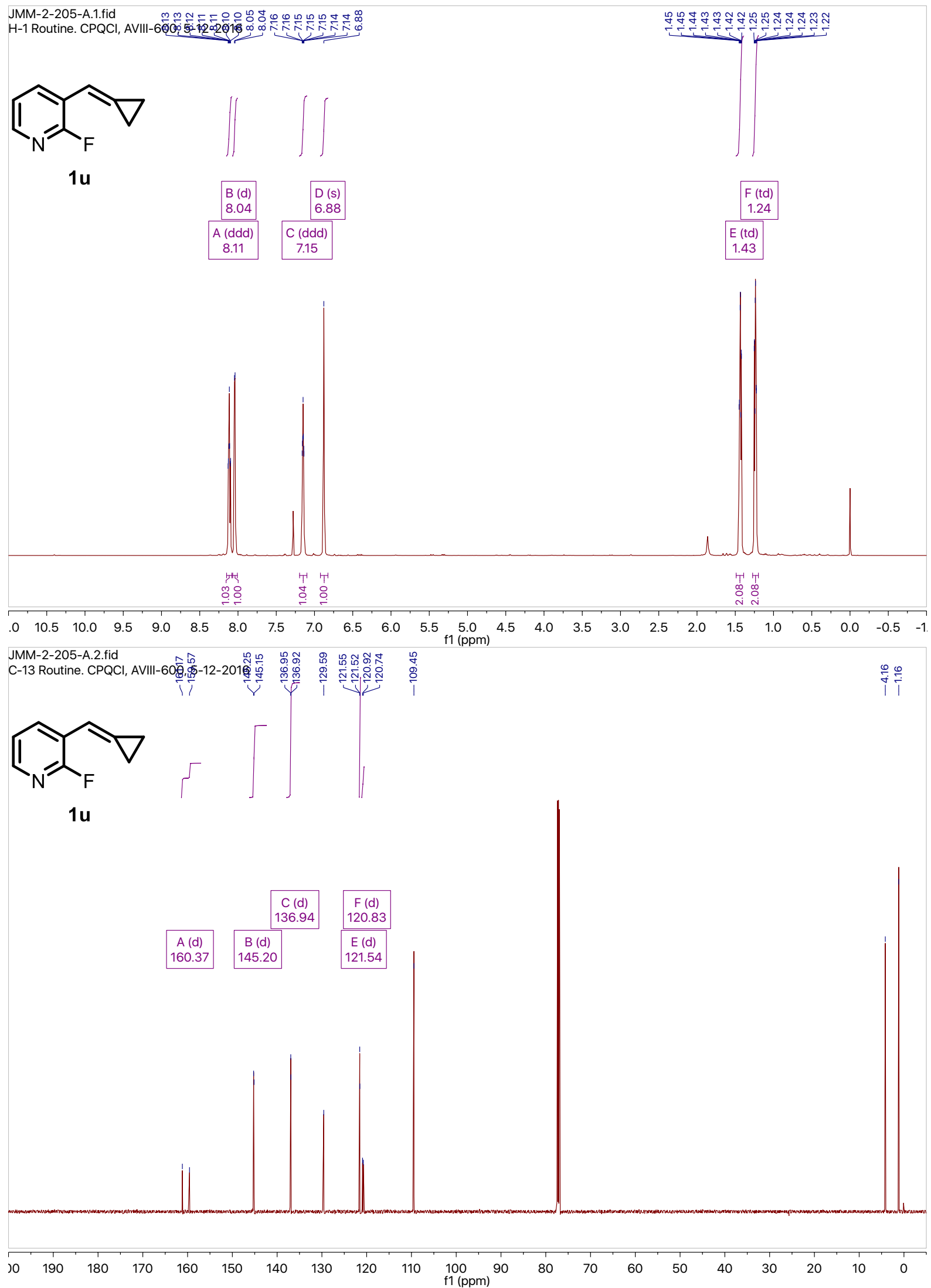



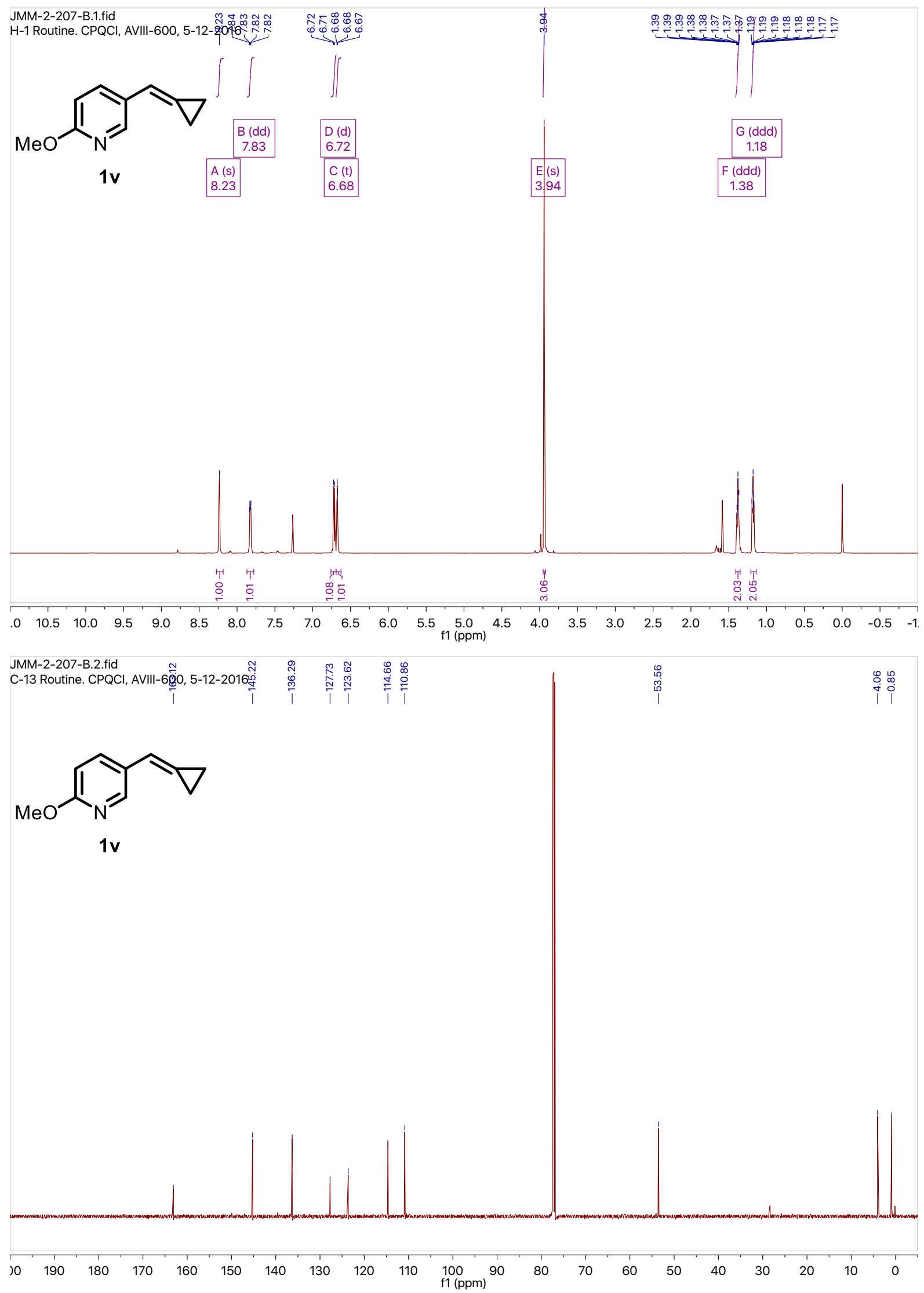
Medina et al.: BCP Hydroboration-Supporting Information - S133
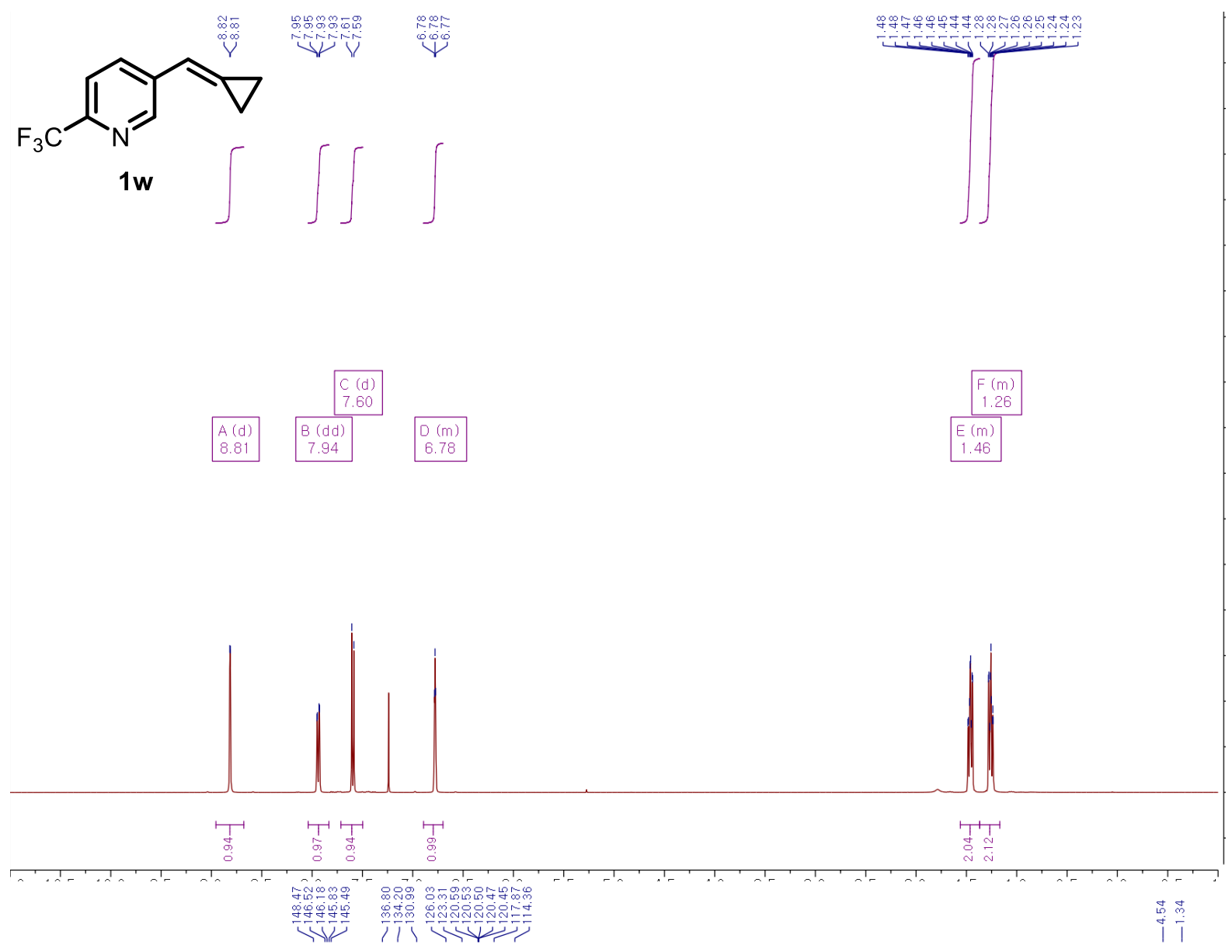<smiles>FC(F)(F)c1ccc(C=C2CC2)cn1</smiles>

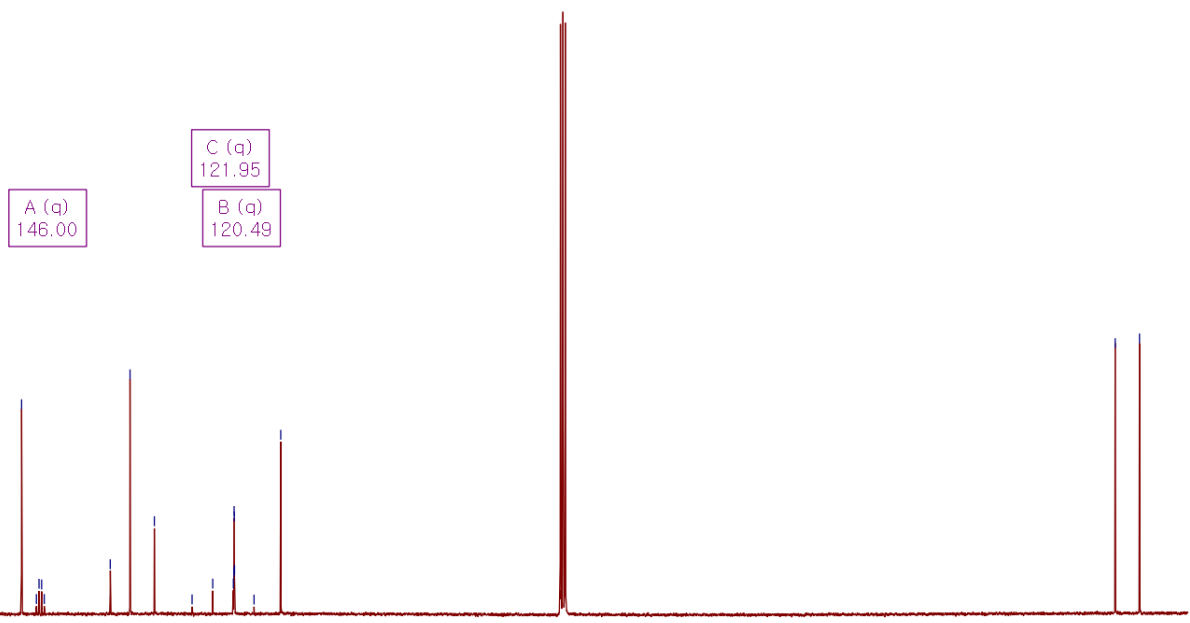

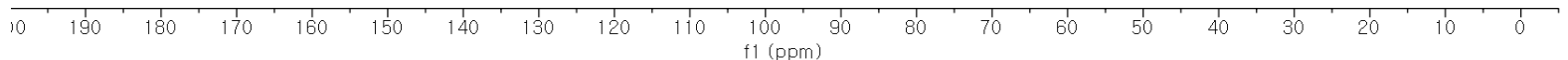



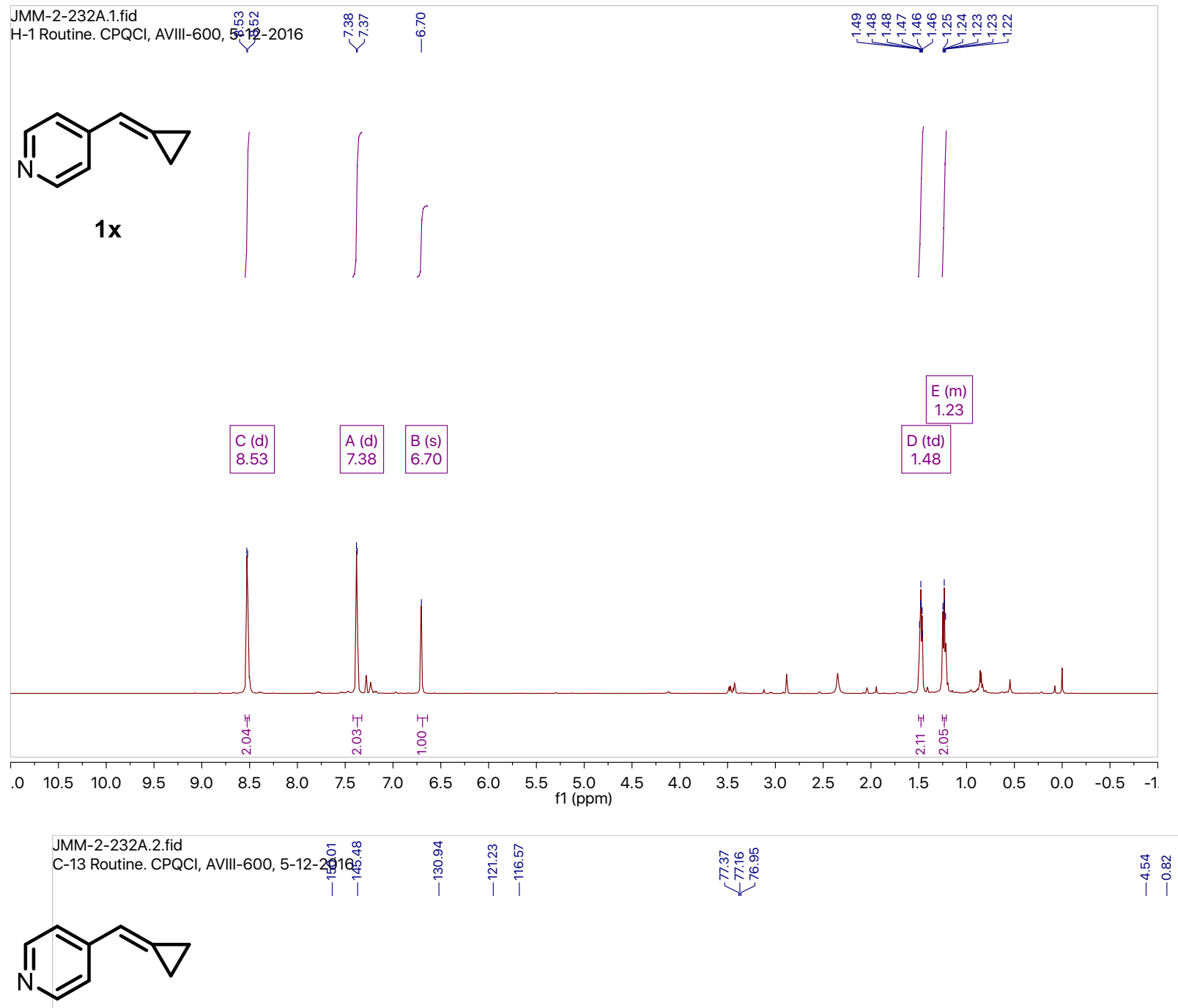

$1 x$

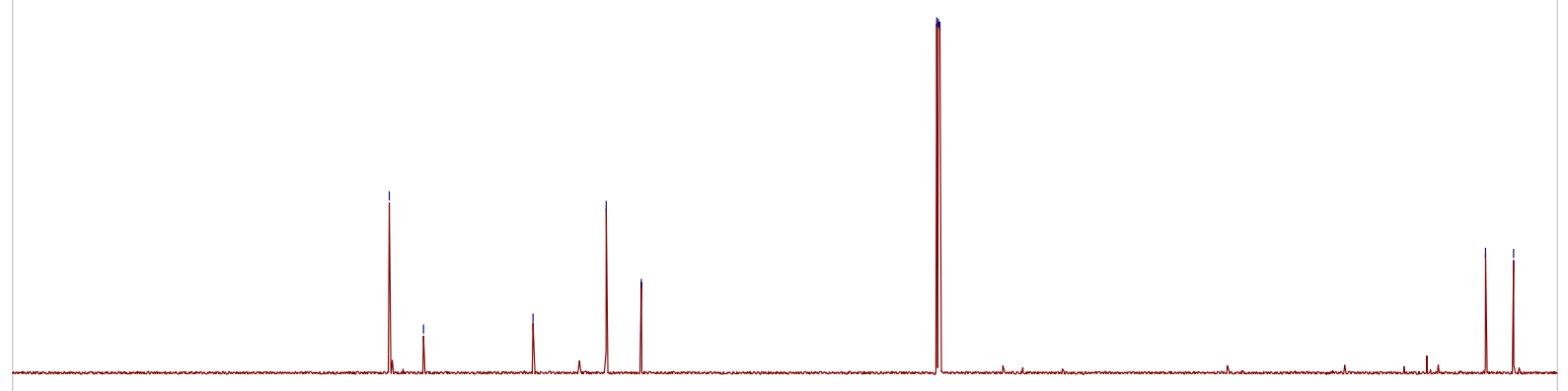

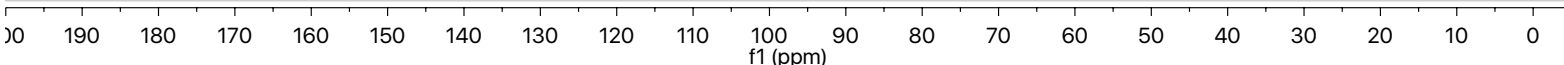


Medina et al.: BCP Hydroboration-Supporting Information - S135

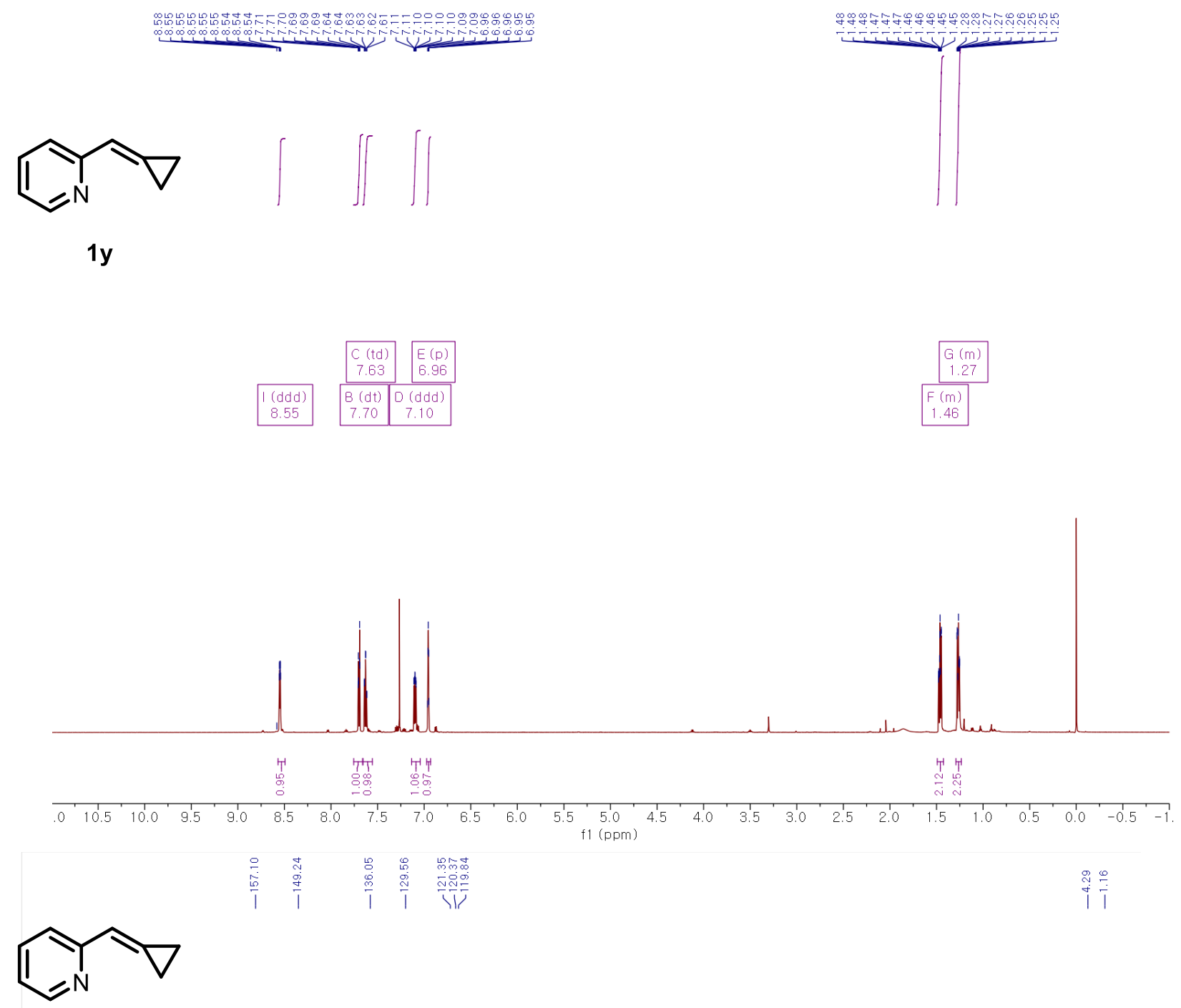

$1 y$
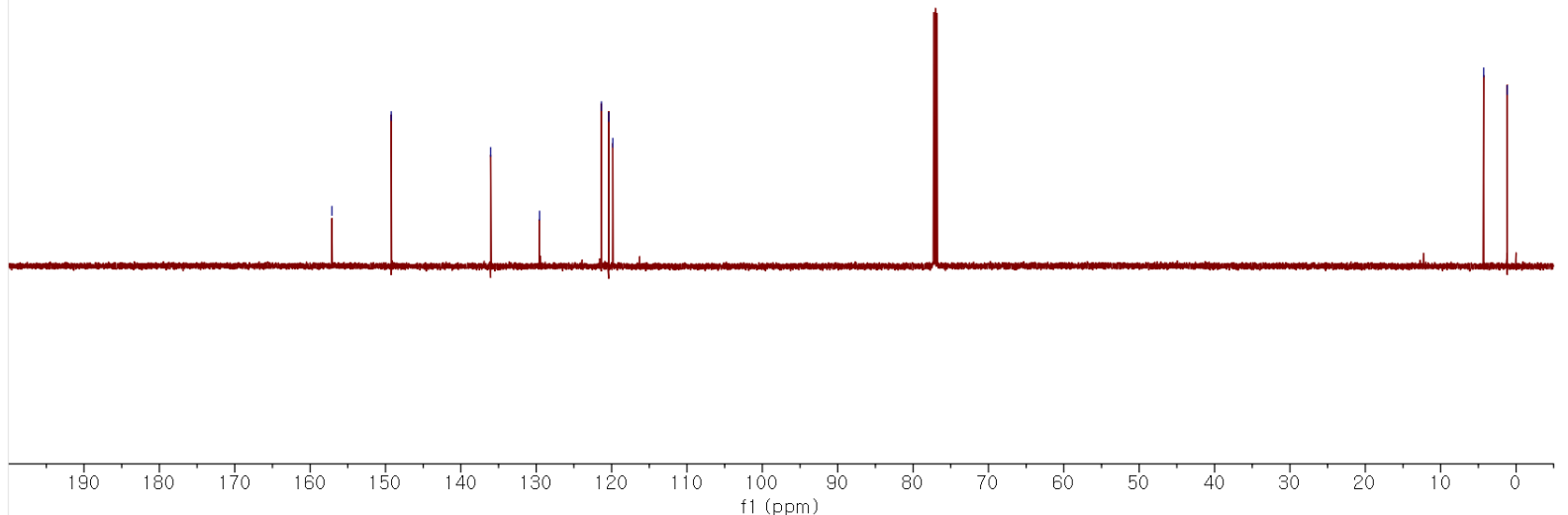

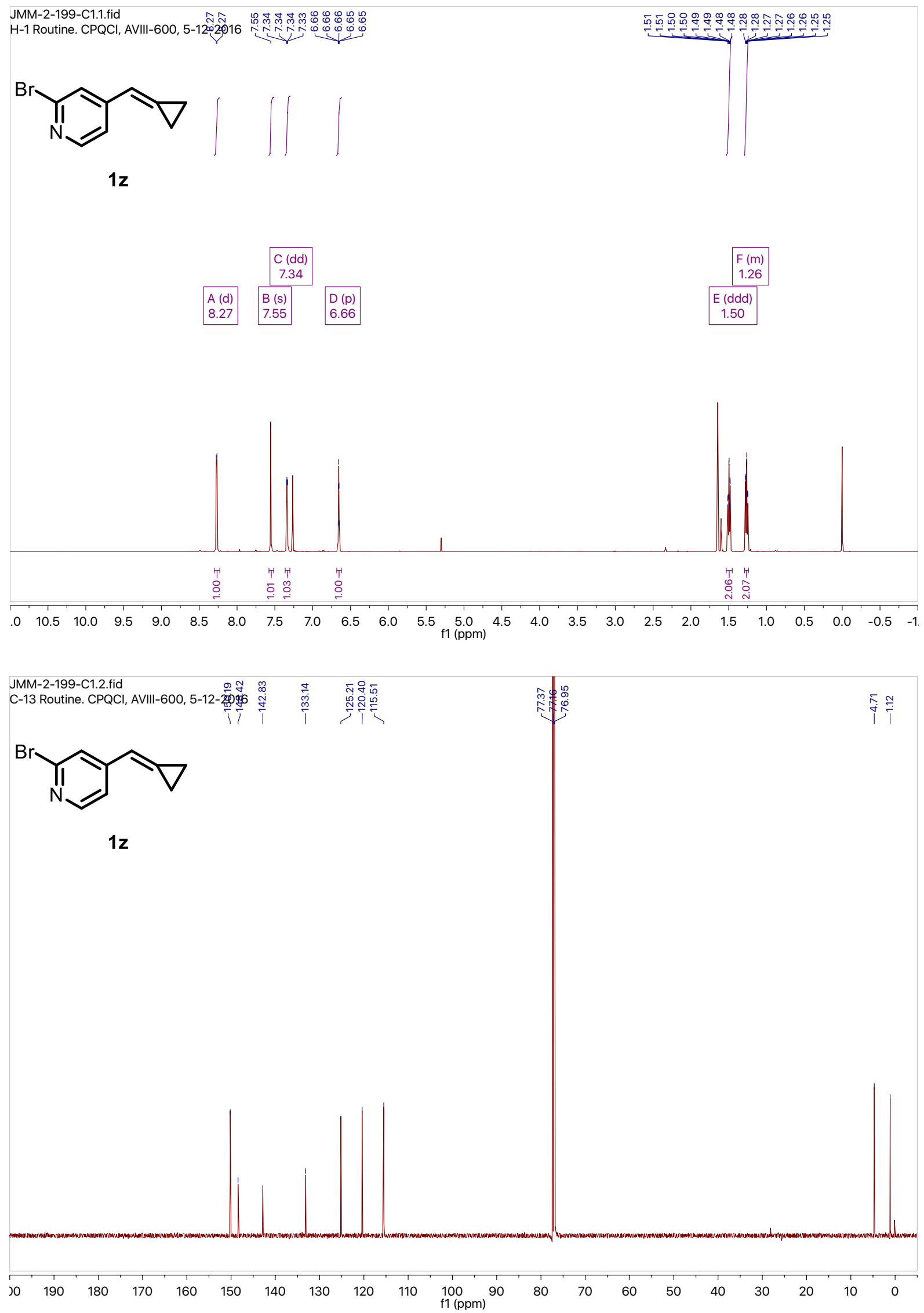
Medina et al.: BCP Hydroboration-Supporting Information - S137
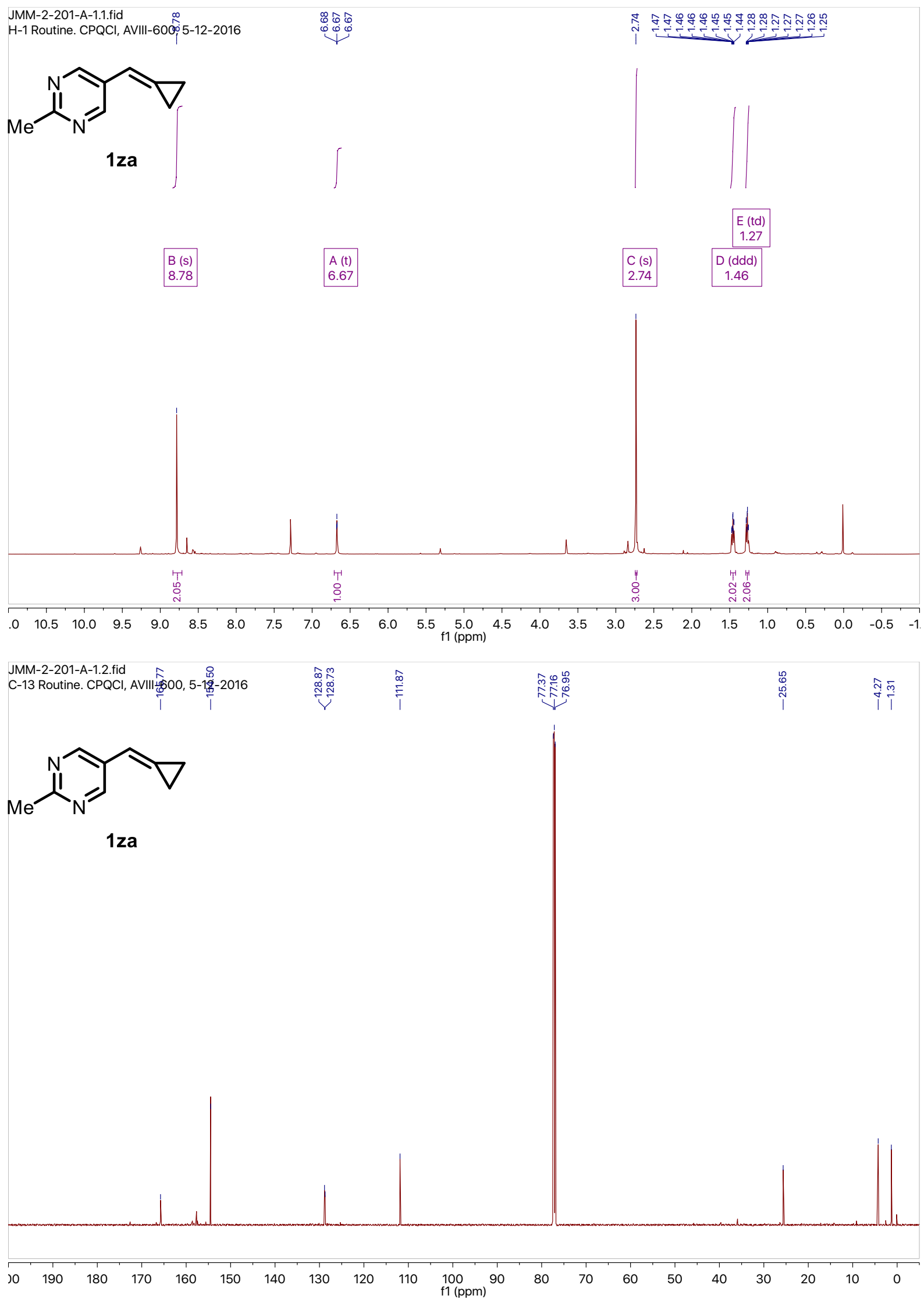

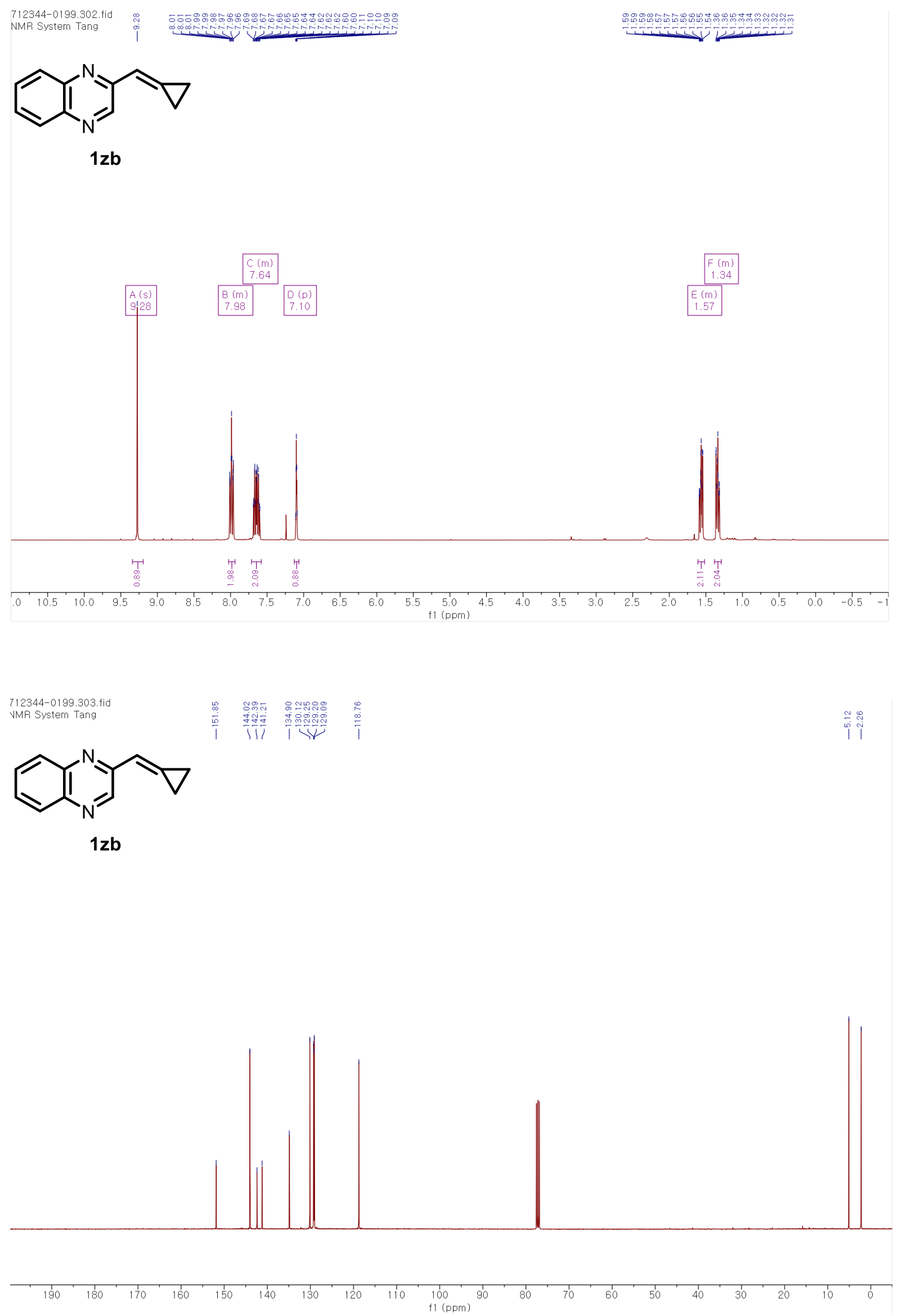


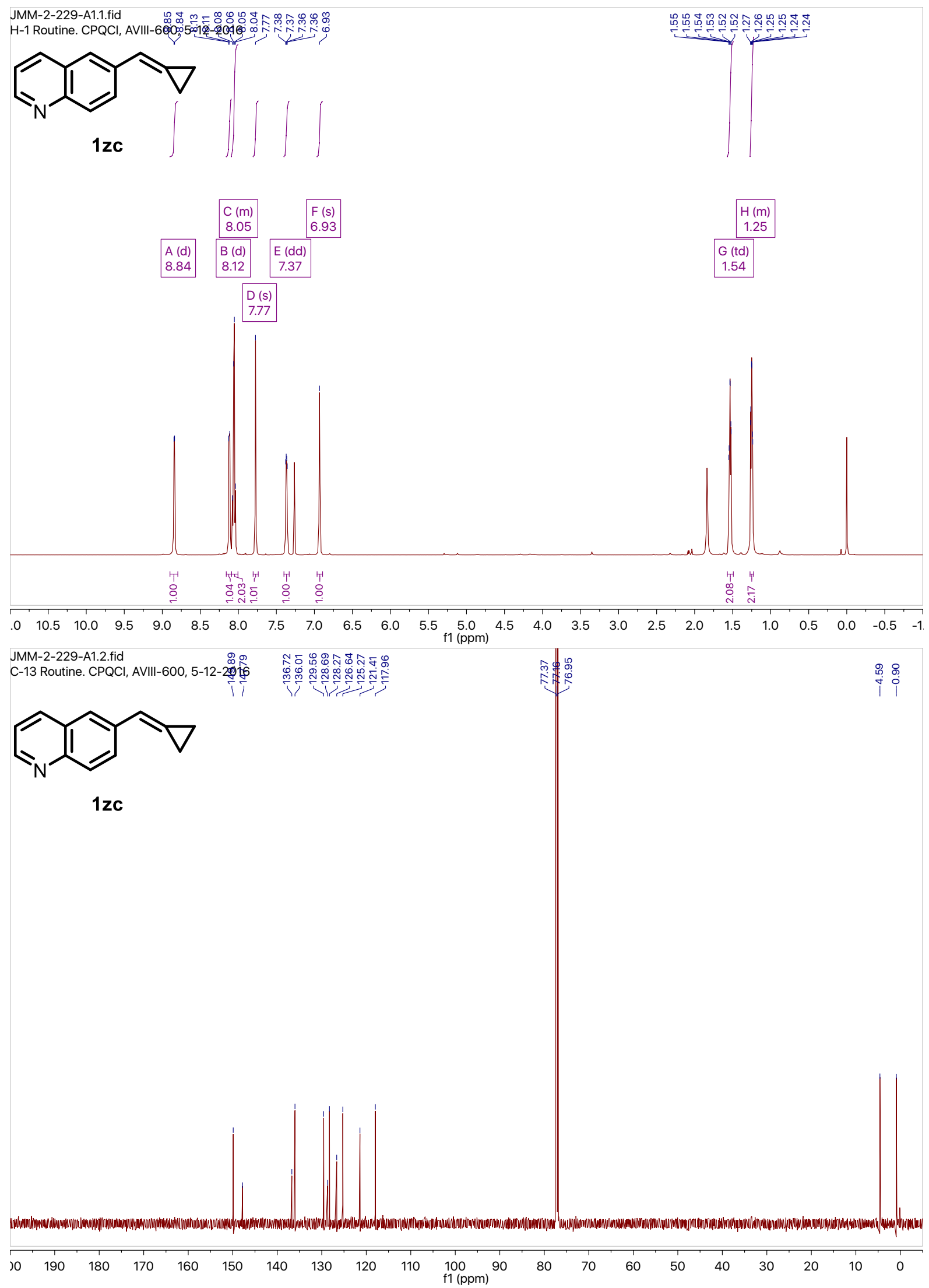




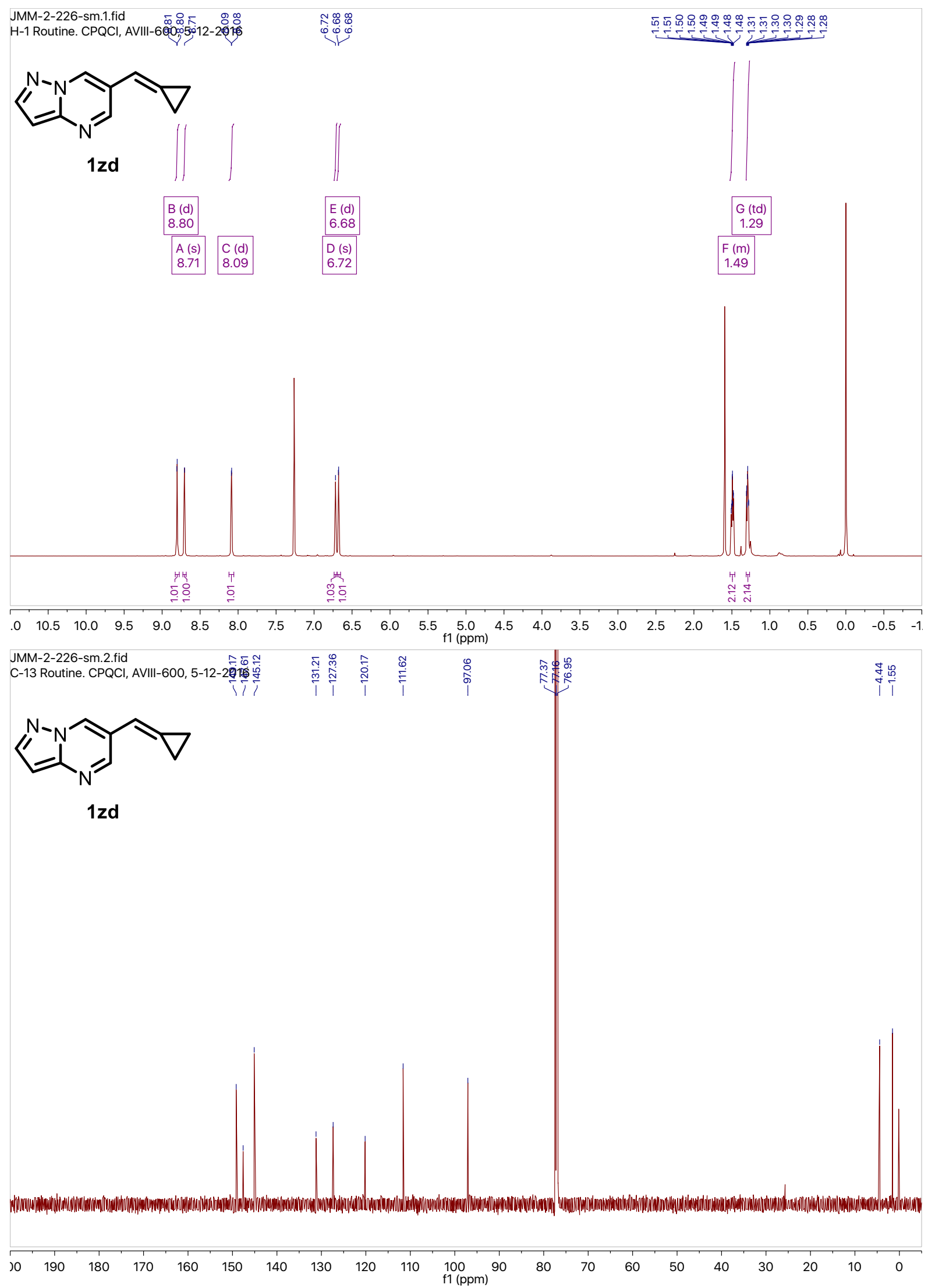



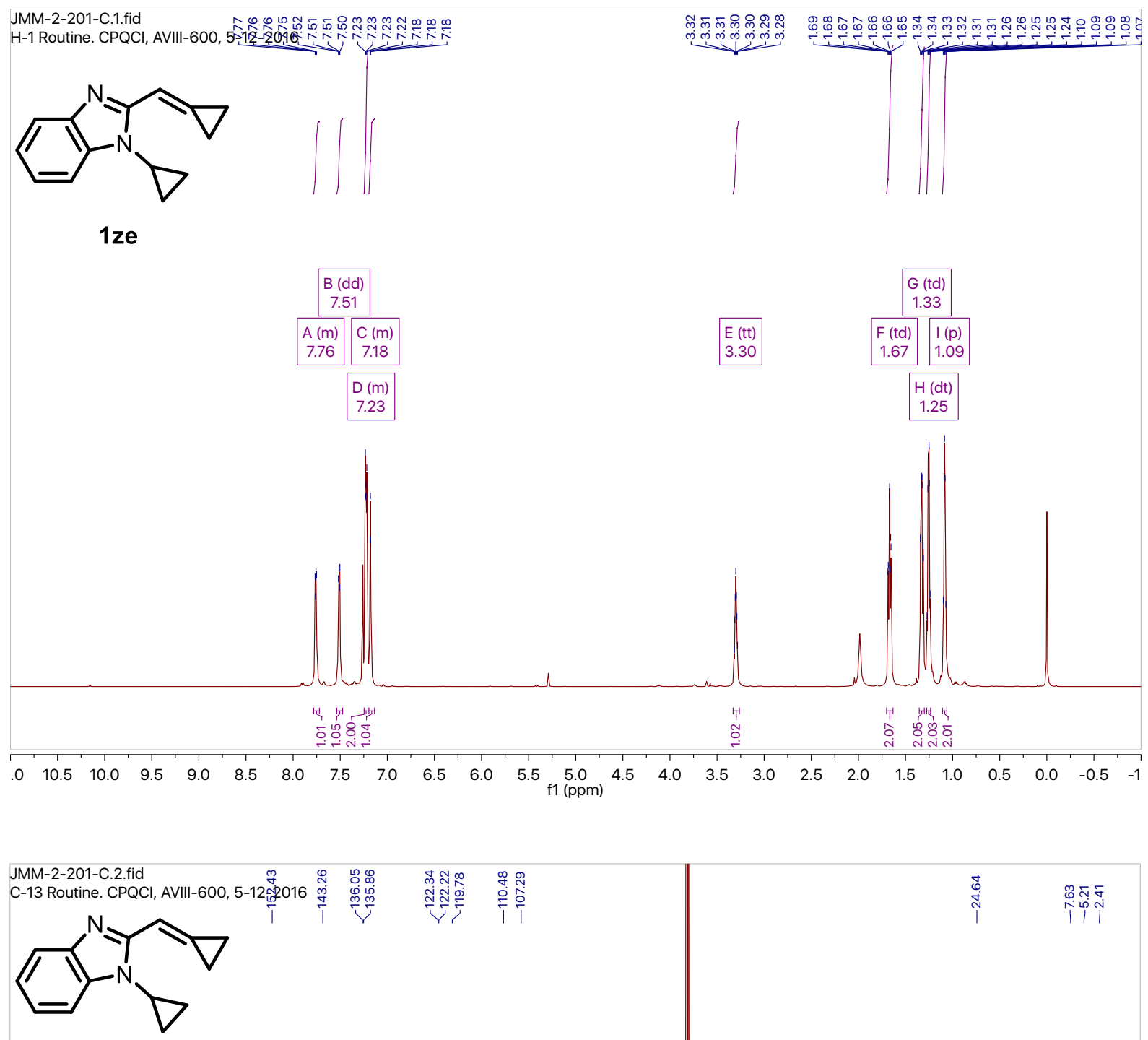

$1 z e$

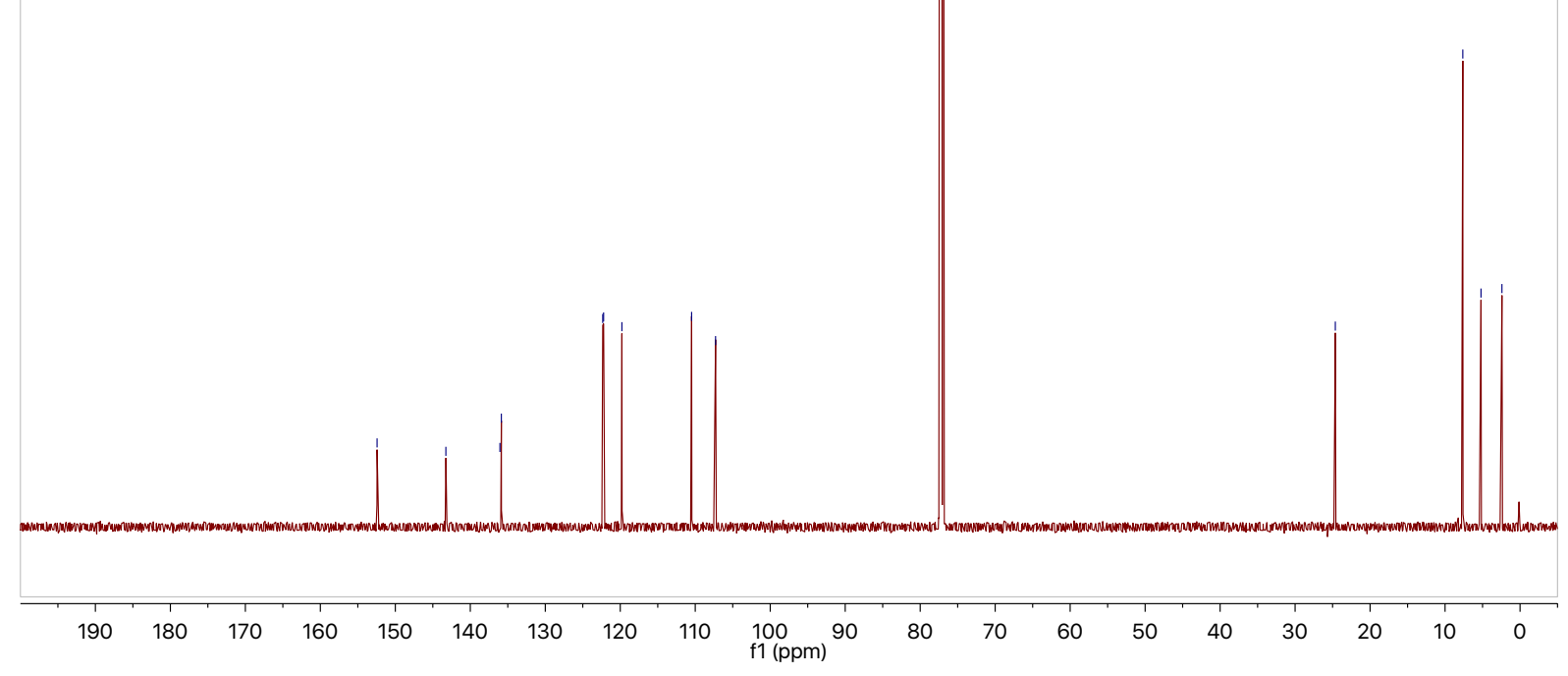



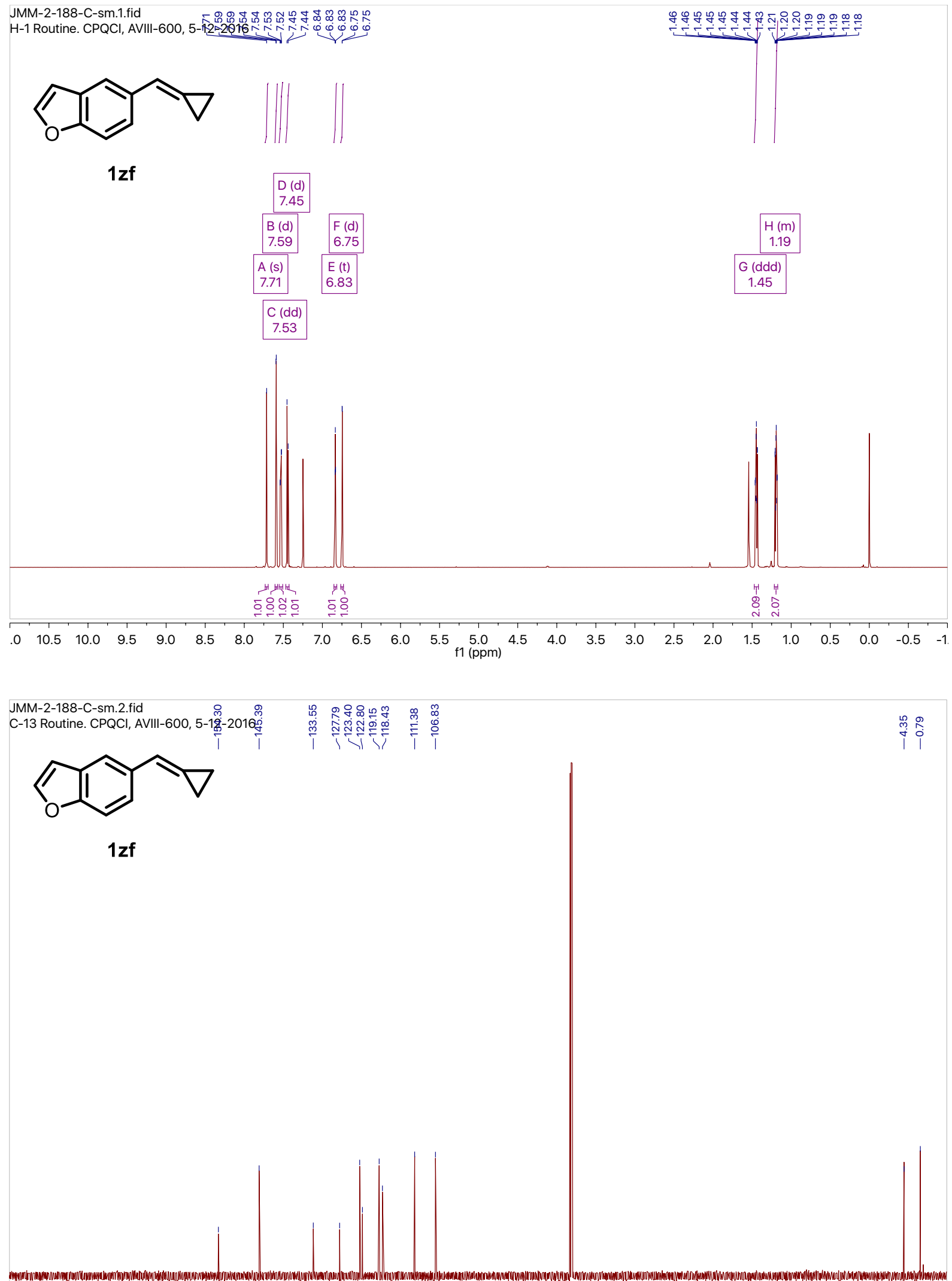


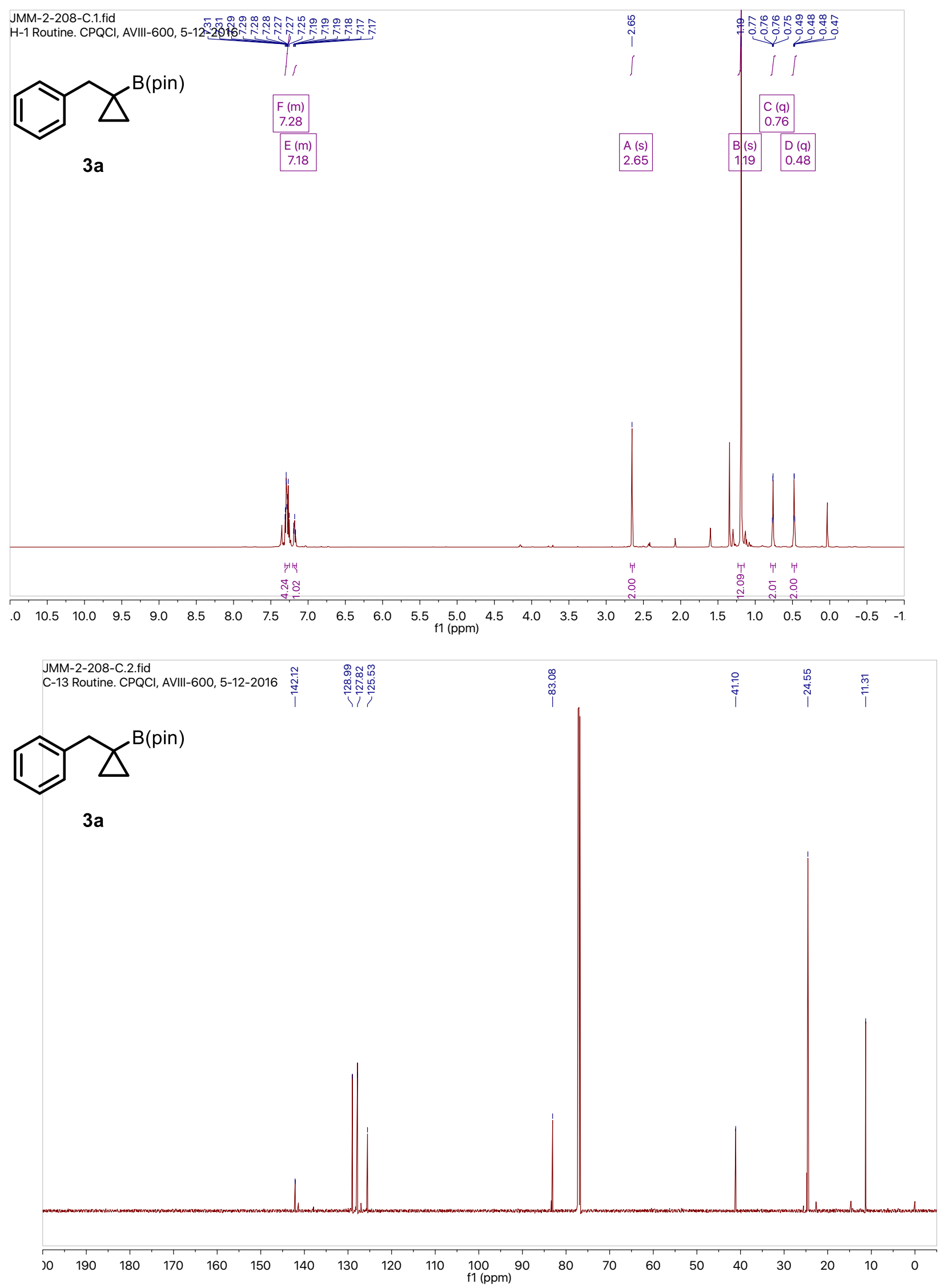




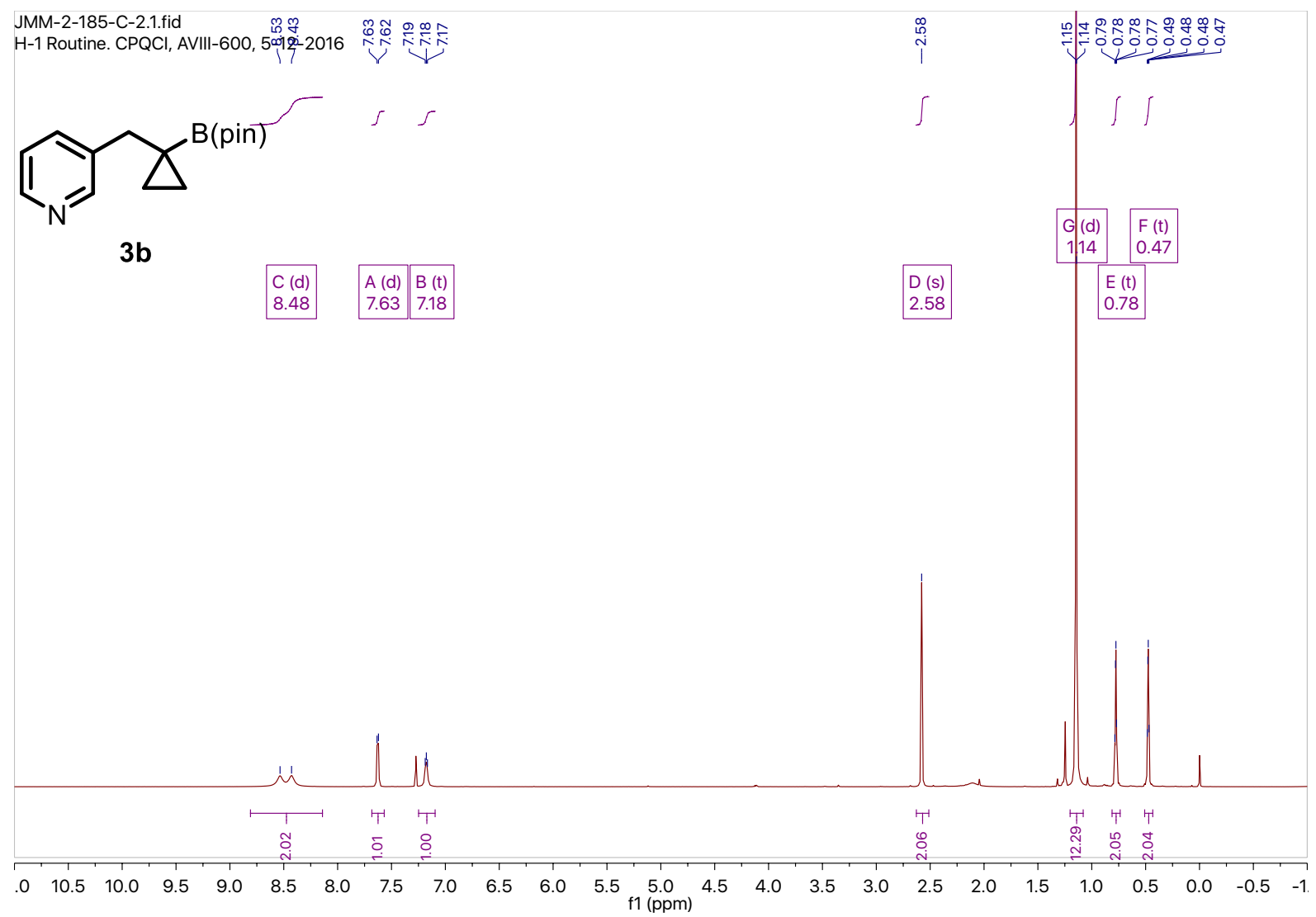

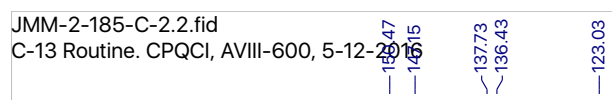

$\overbrace{N}^{B(\text { pin })}$

$3 b$
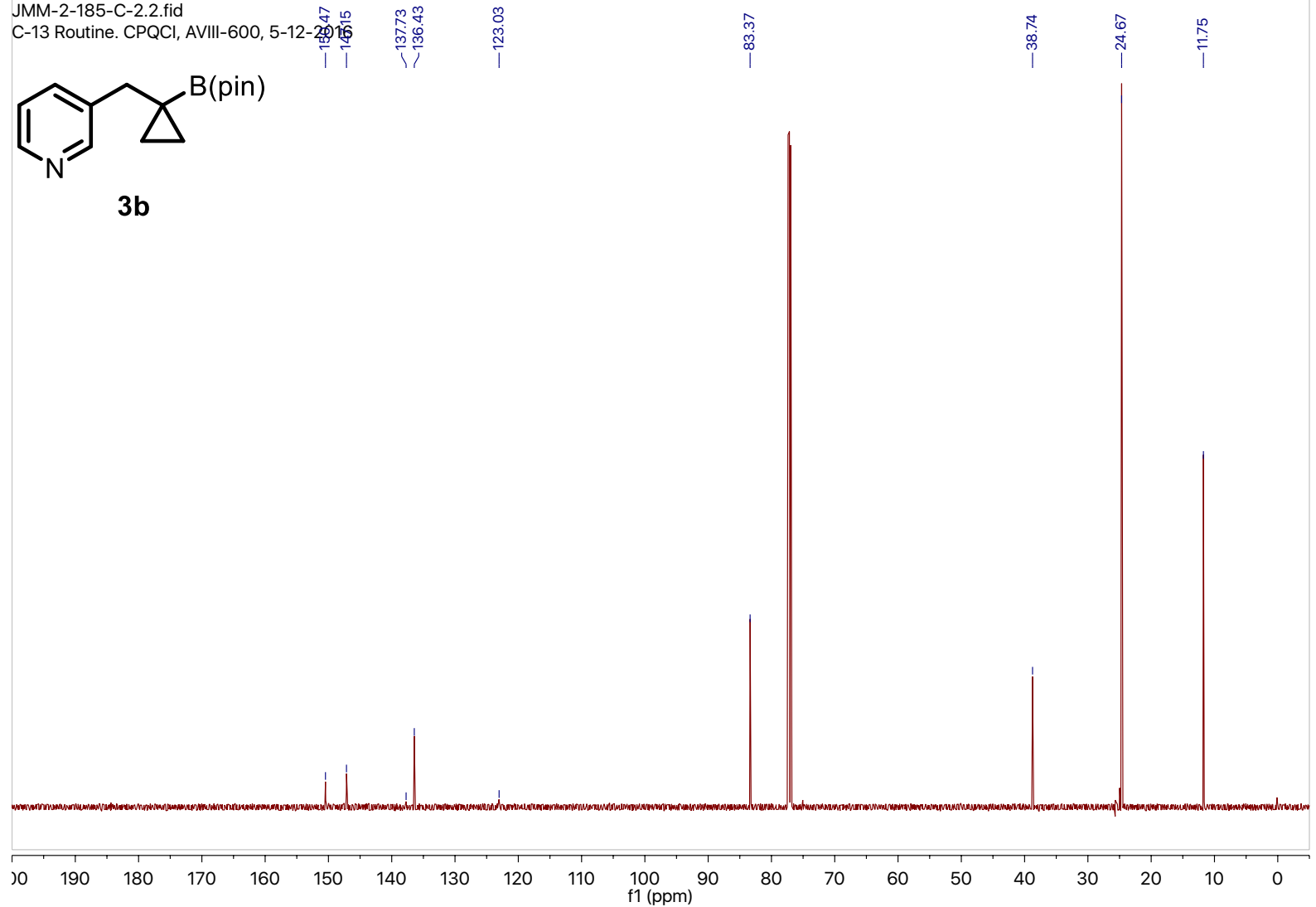

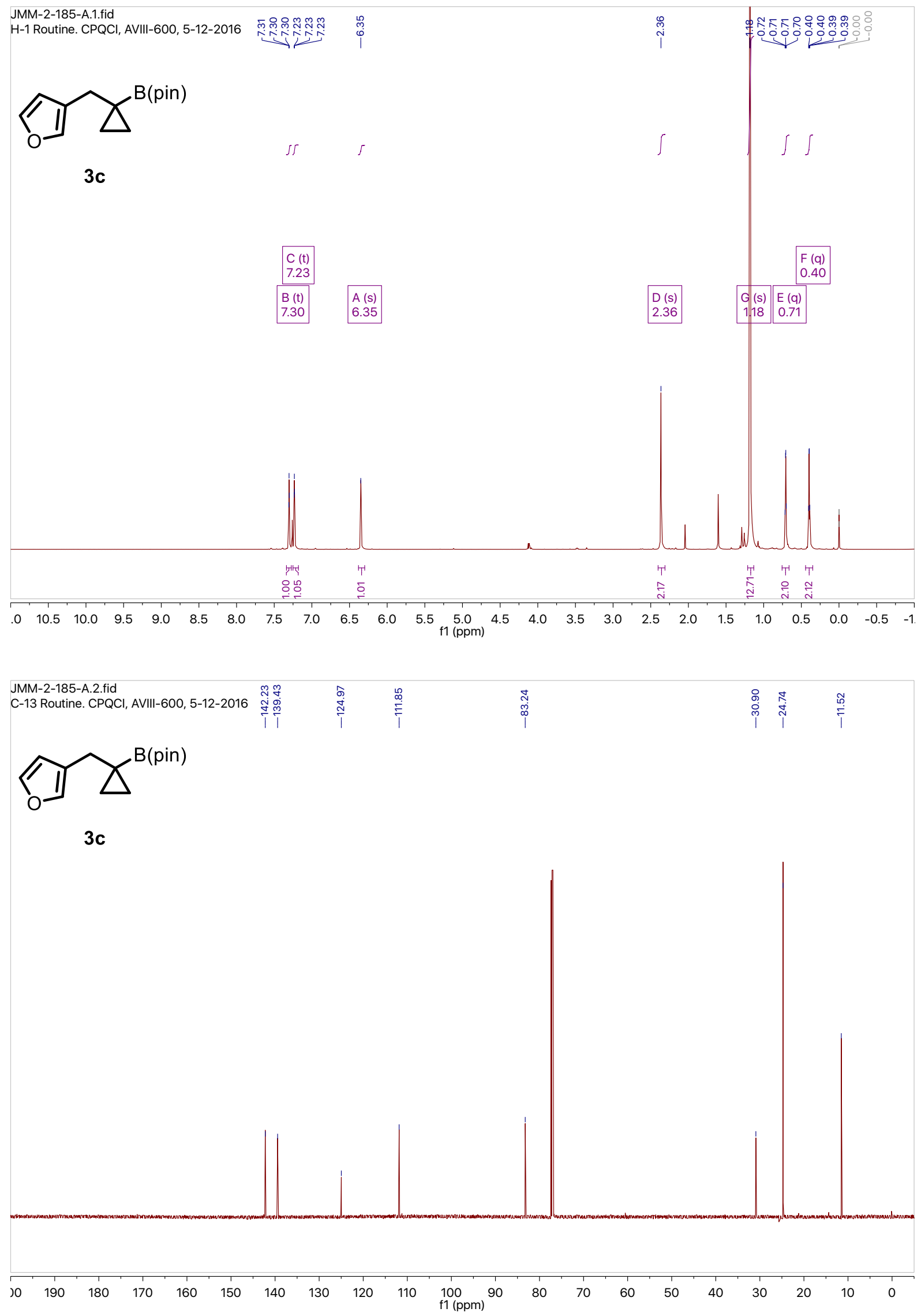

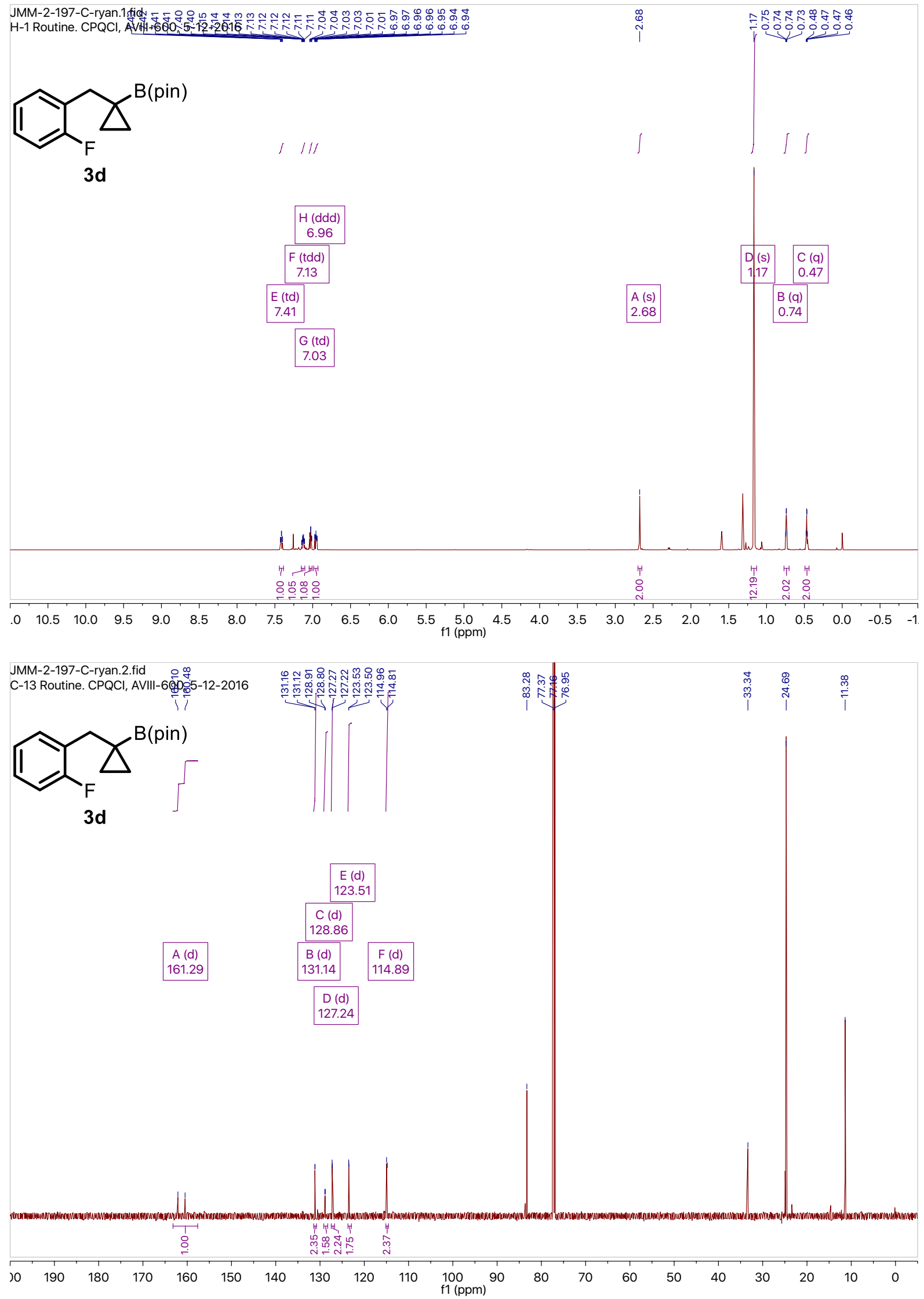

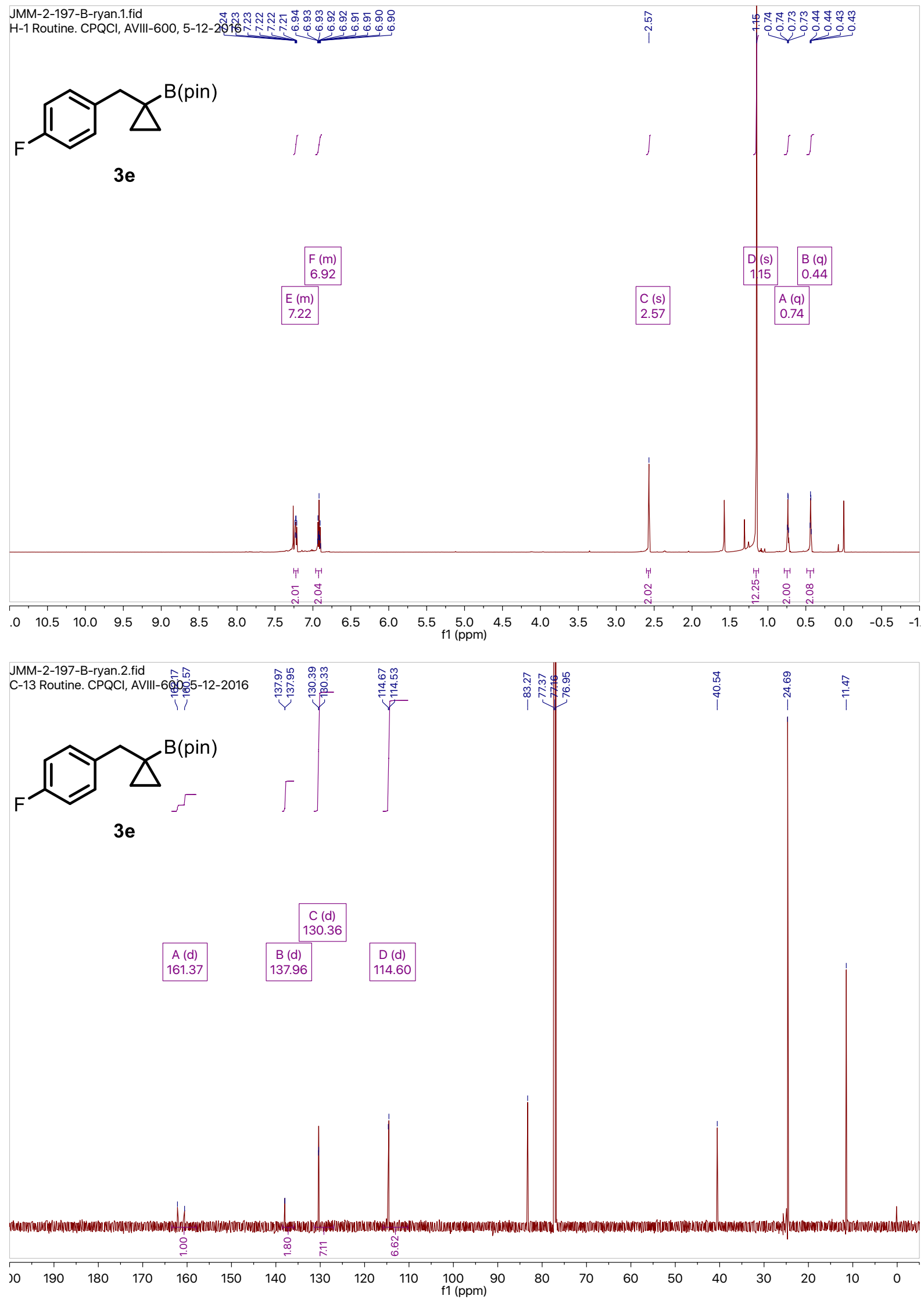
<smiles>Brc1ccc(CC2(Br)CC2)cc1</smiles>

$3 f$

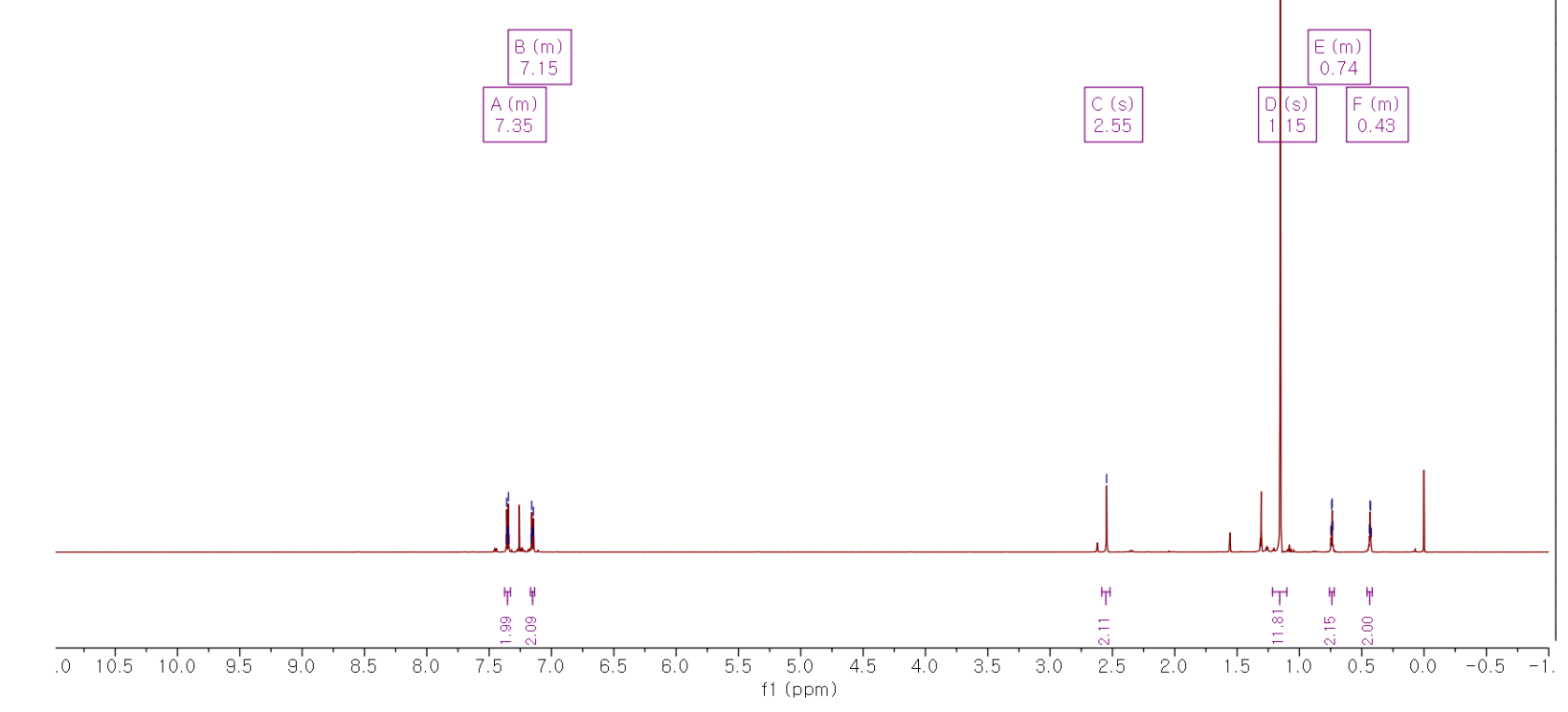<smiles>Brc1ccc(CC2(Br)CC2)cc1</smiles>

$3 f$

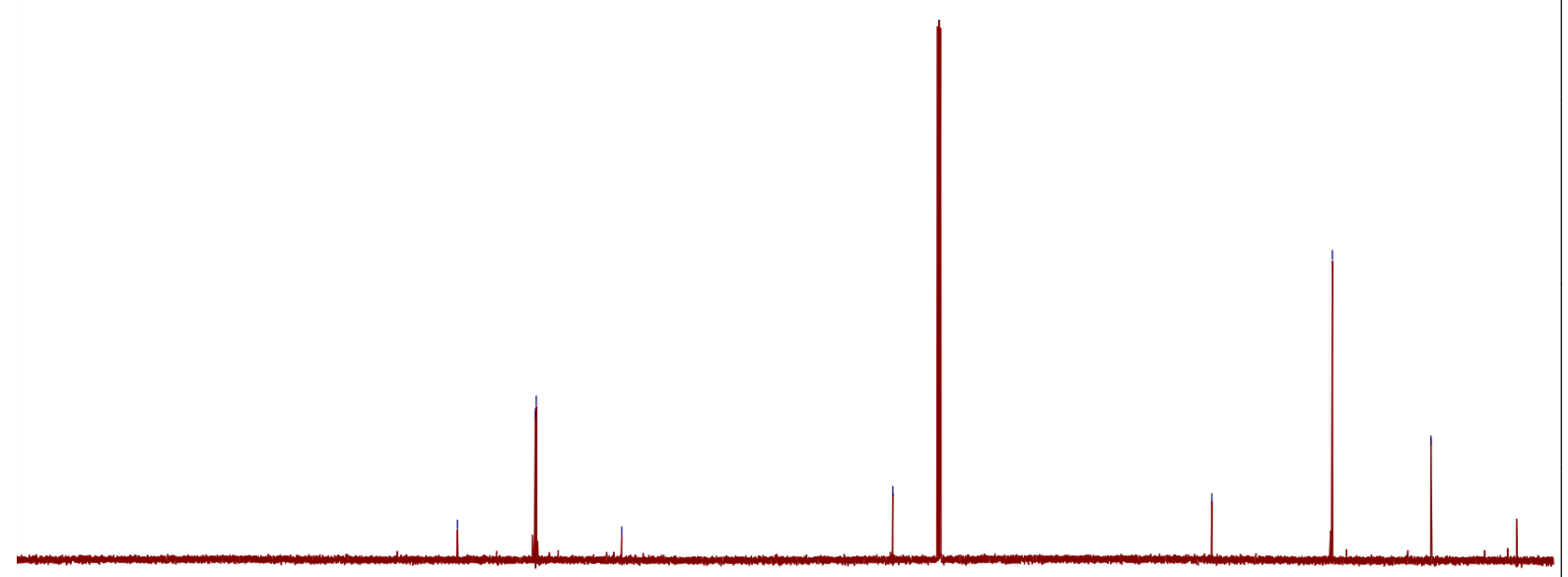



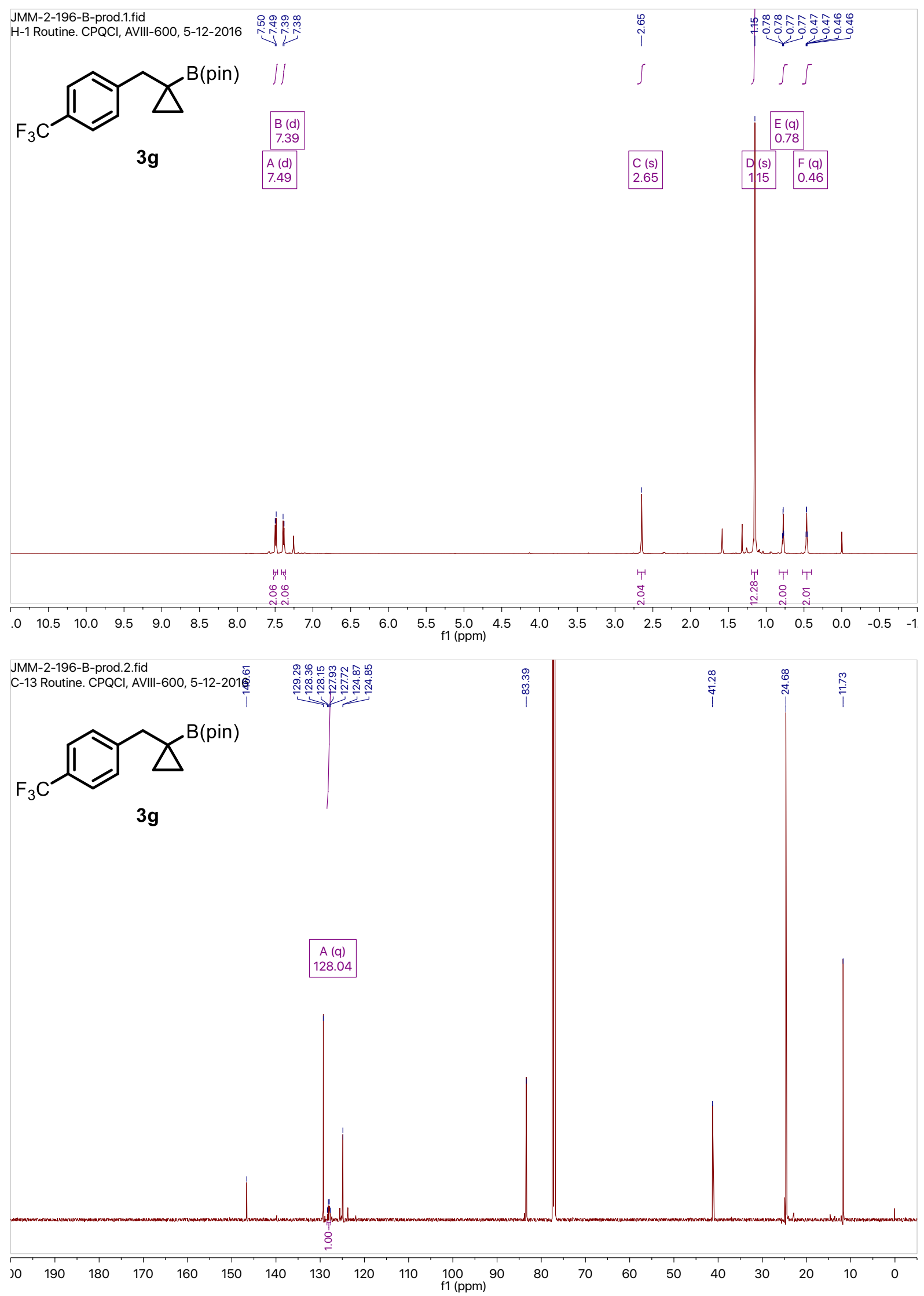

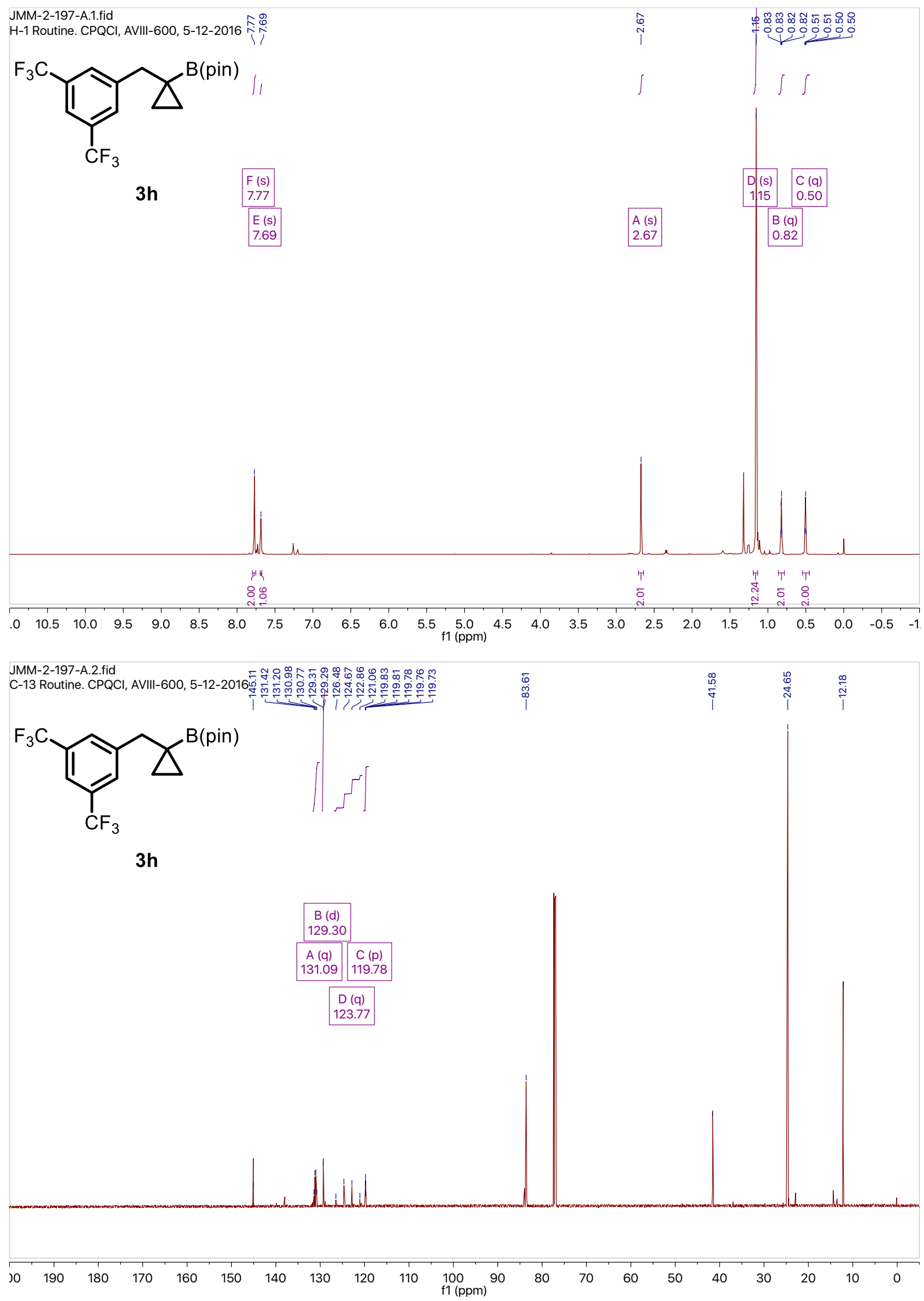

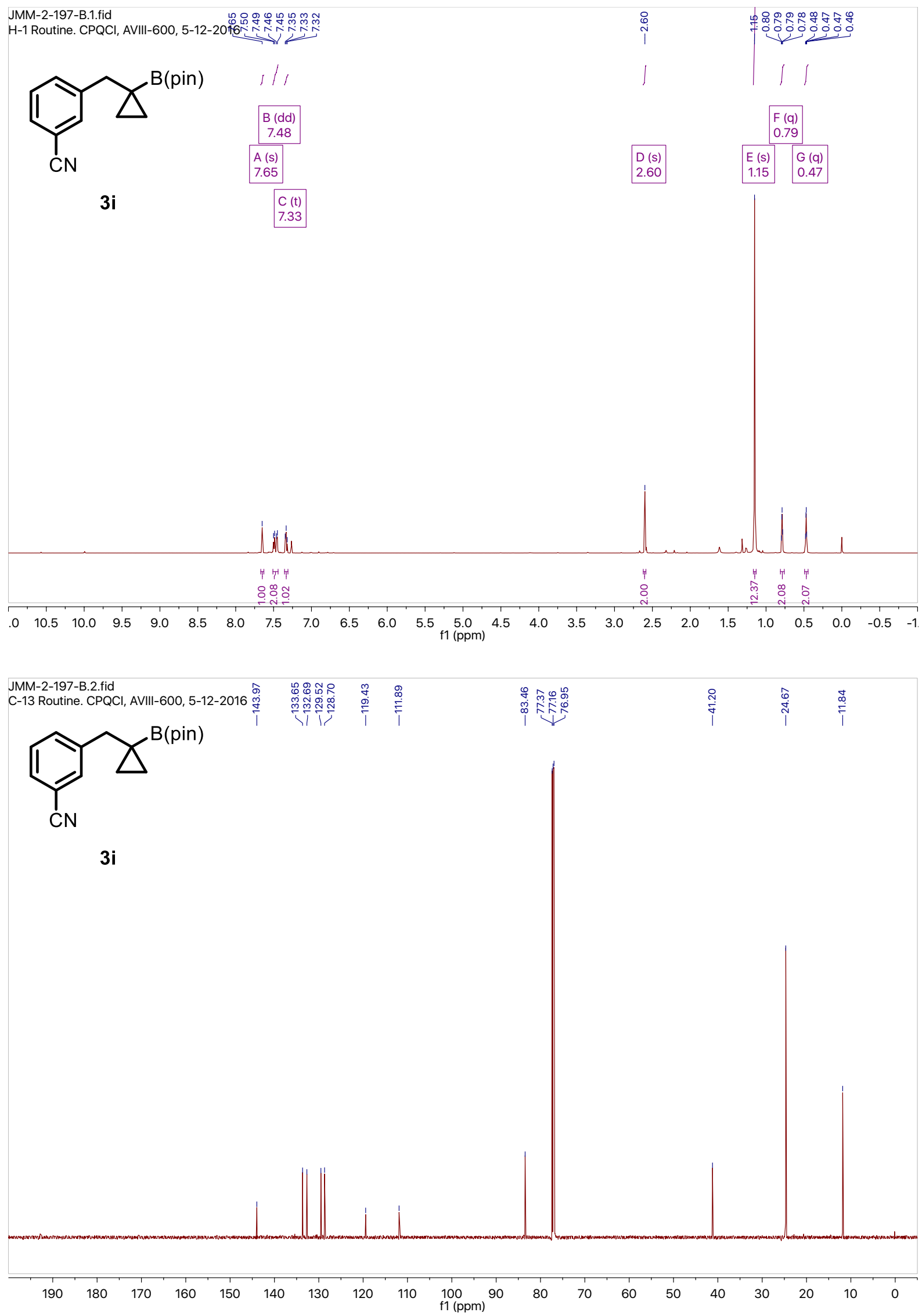


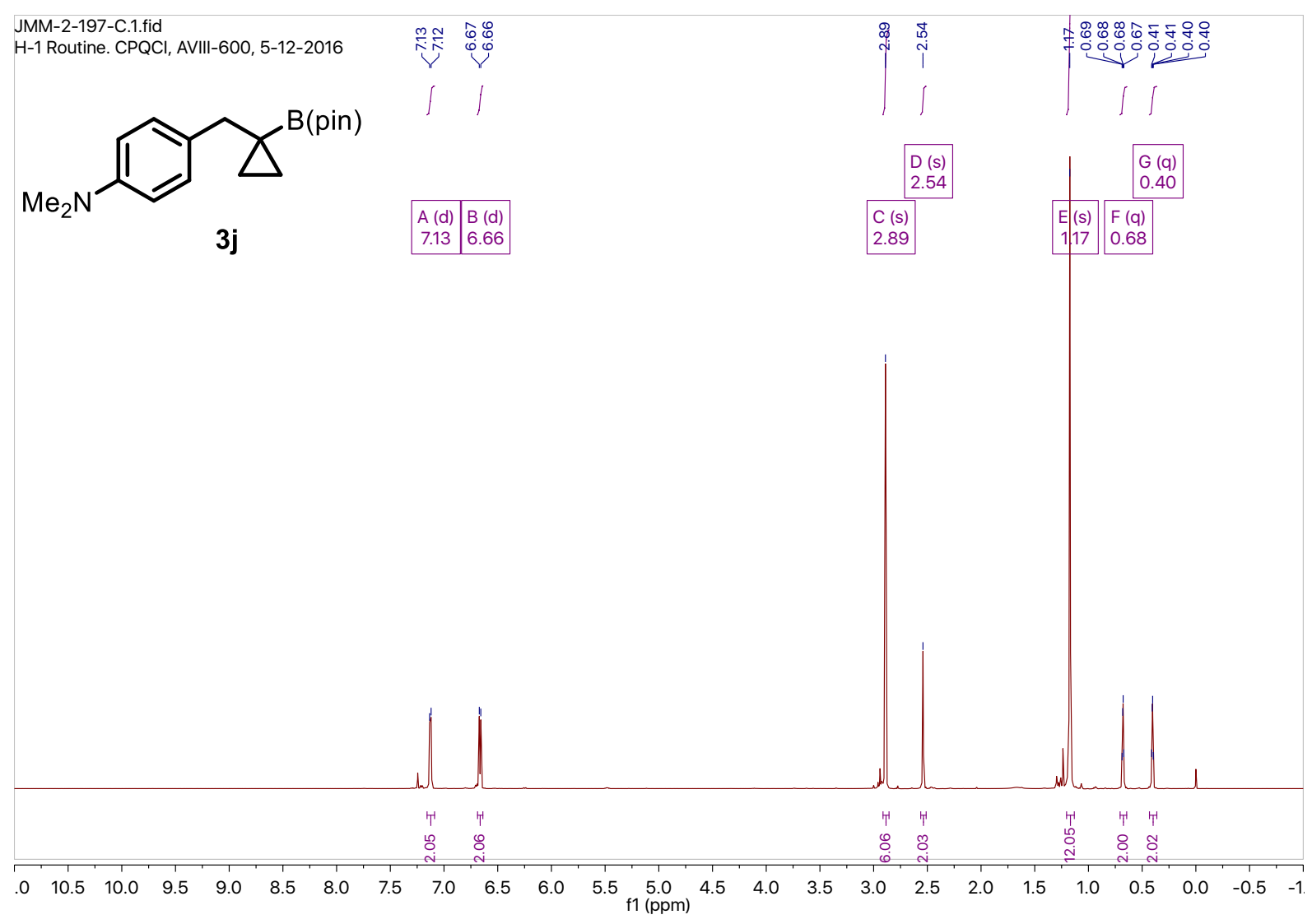

\section{JMM-2-197-C.2.fid}

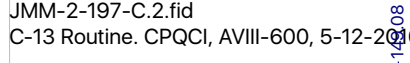

\begin{tabular}{|c|c|c|}
\hline 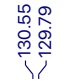 & 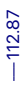 & 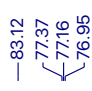 \\
\hline
\end{tabular}

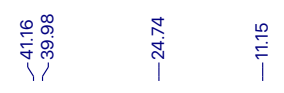

$\triangle_{\mathrm{Me}_{2} \mathrm{~N}}^{\mathrm{B}(\mathrm{pin})}$

3j
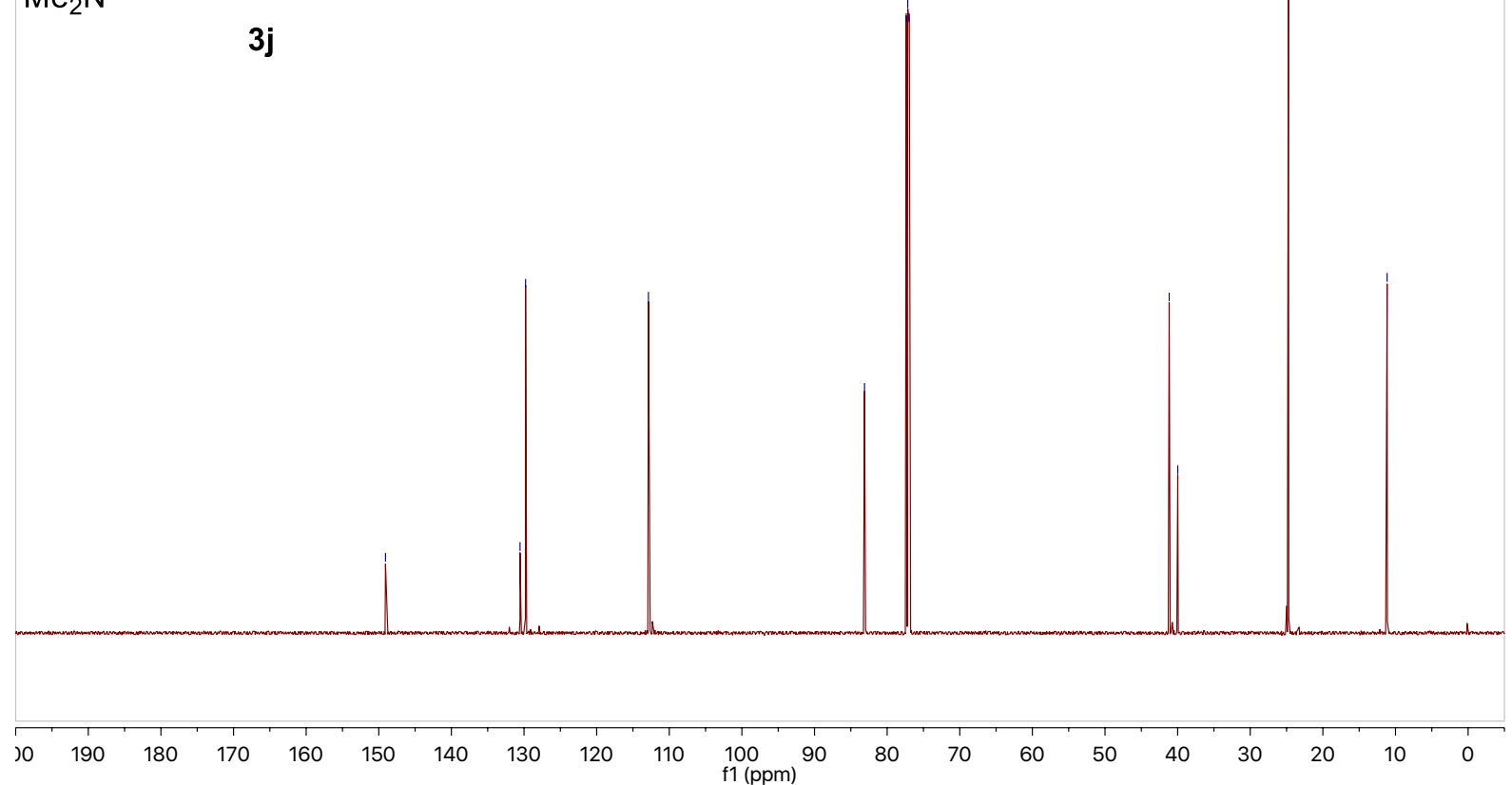


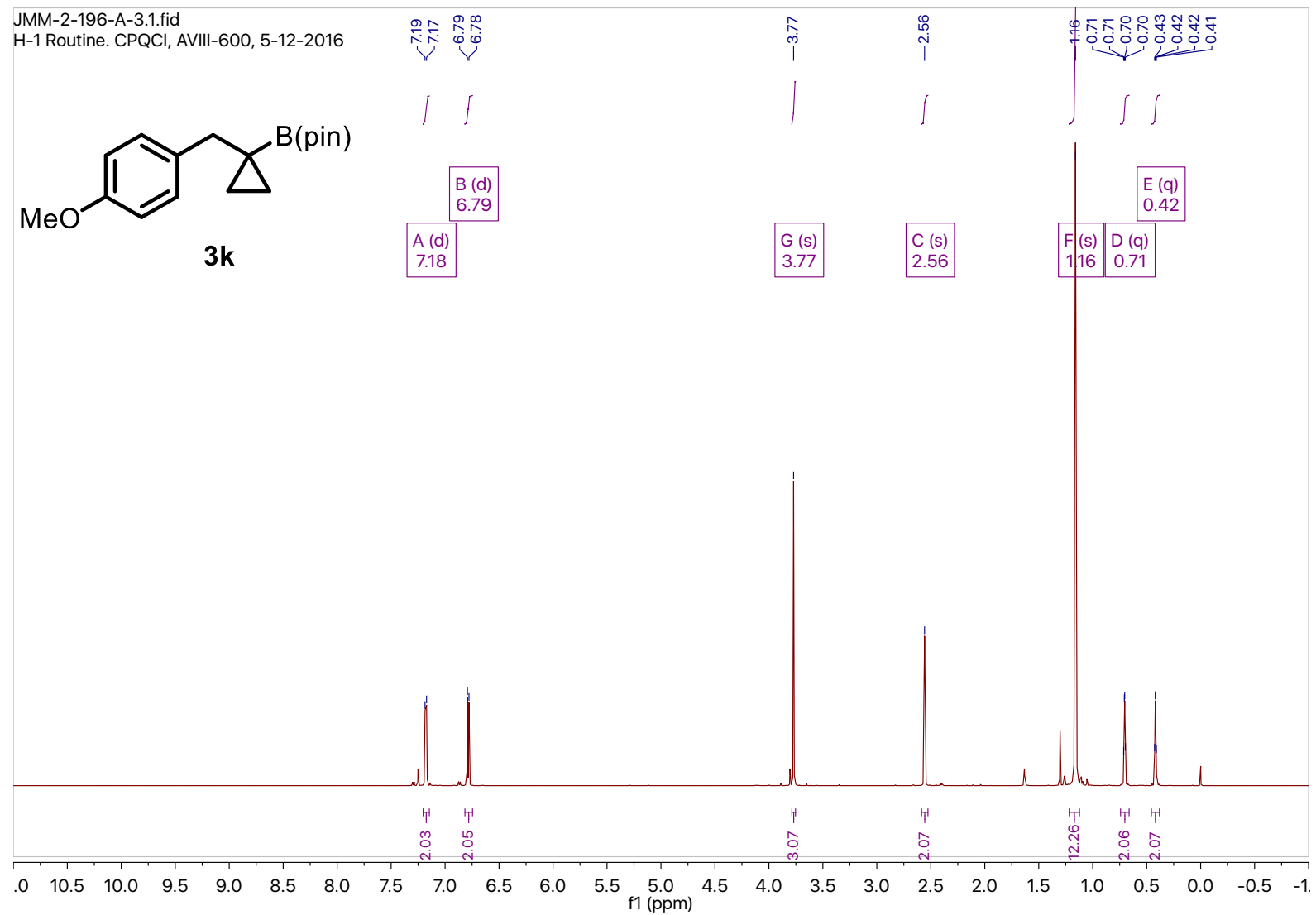

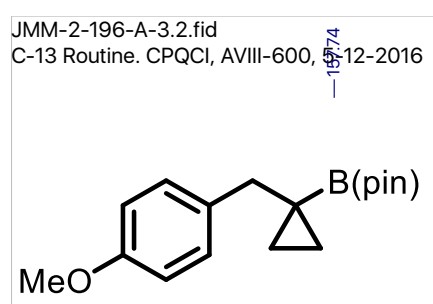

$3 \mathbf{k}$
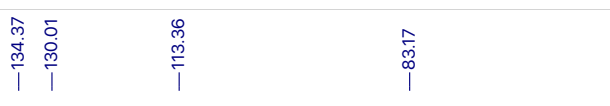

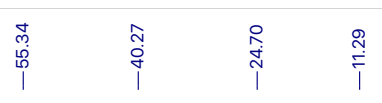

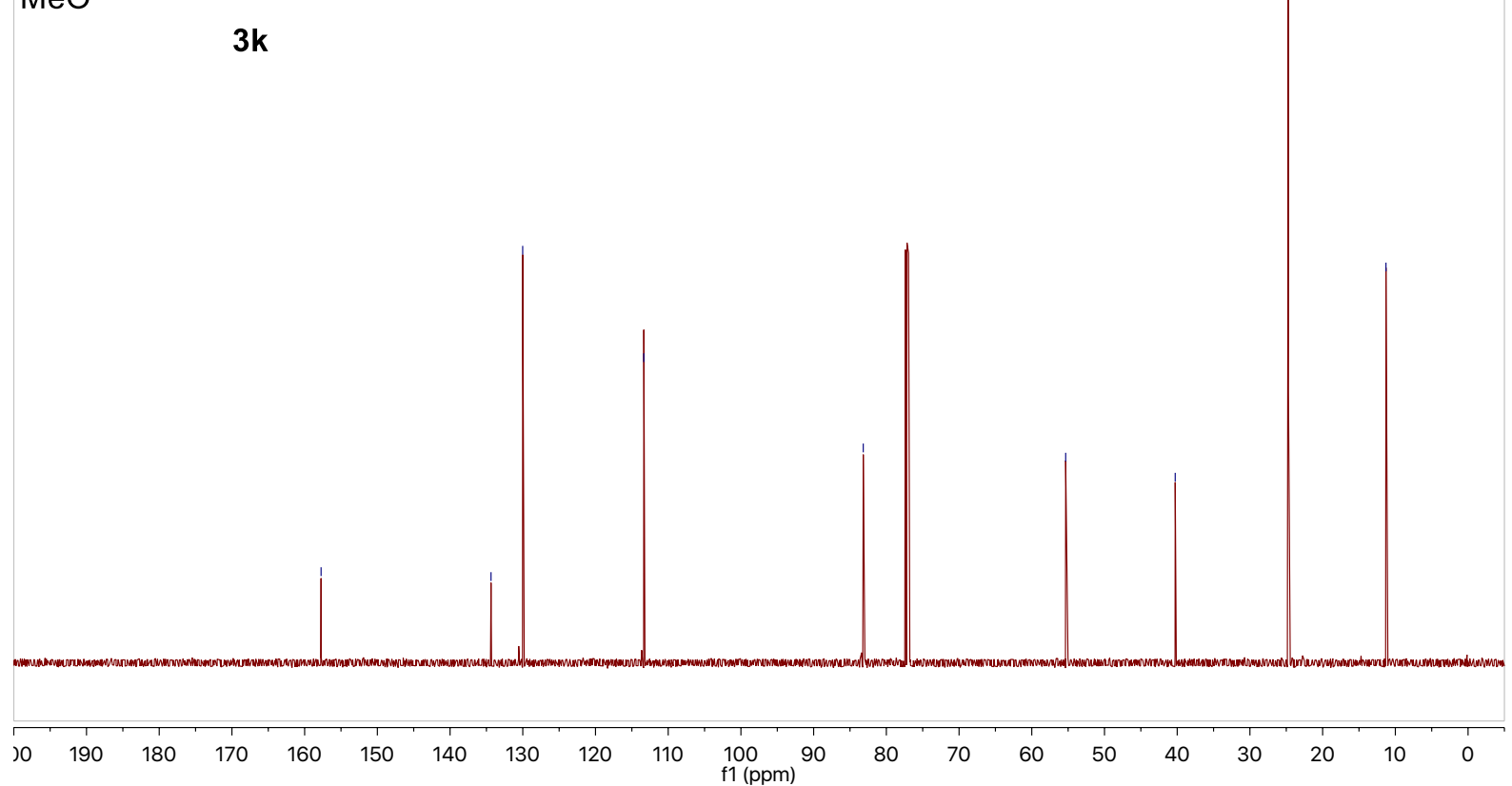



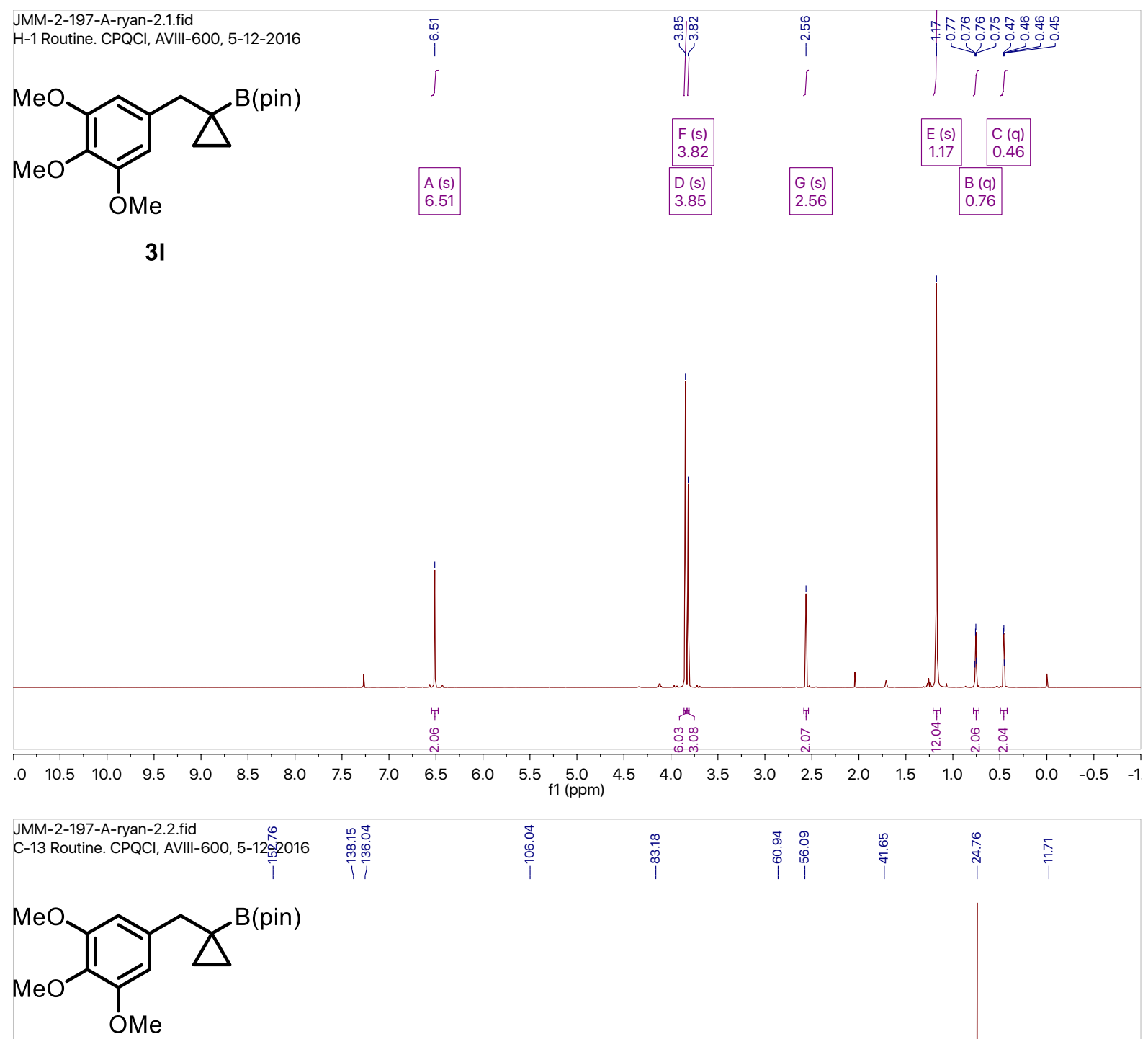

31
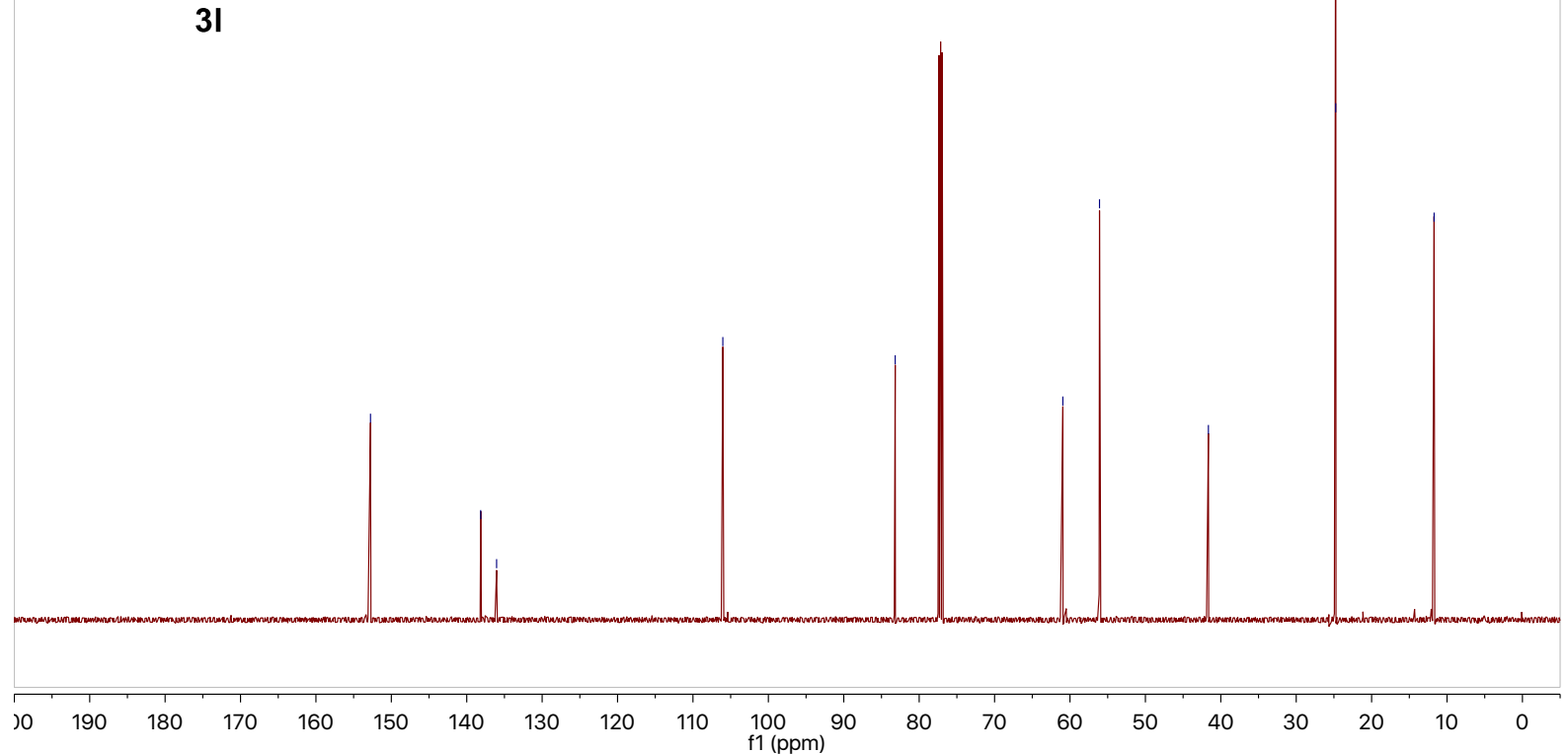

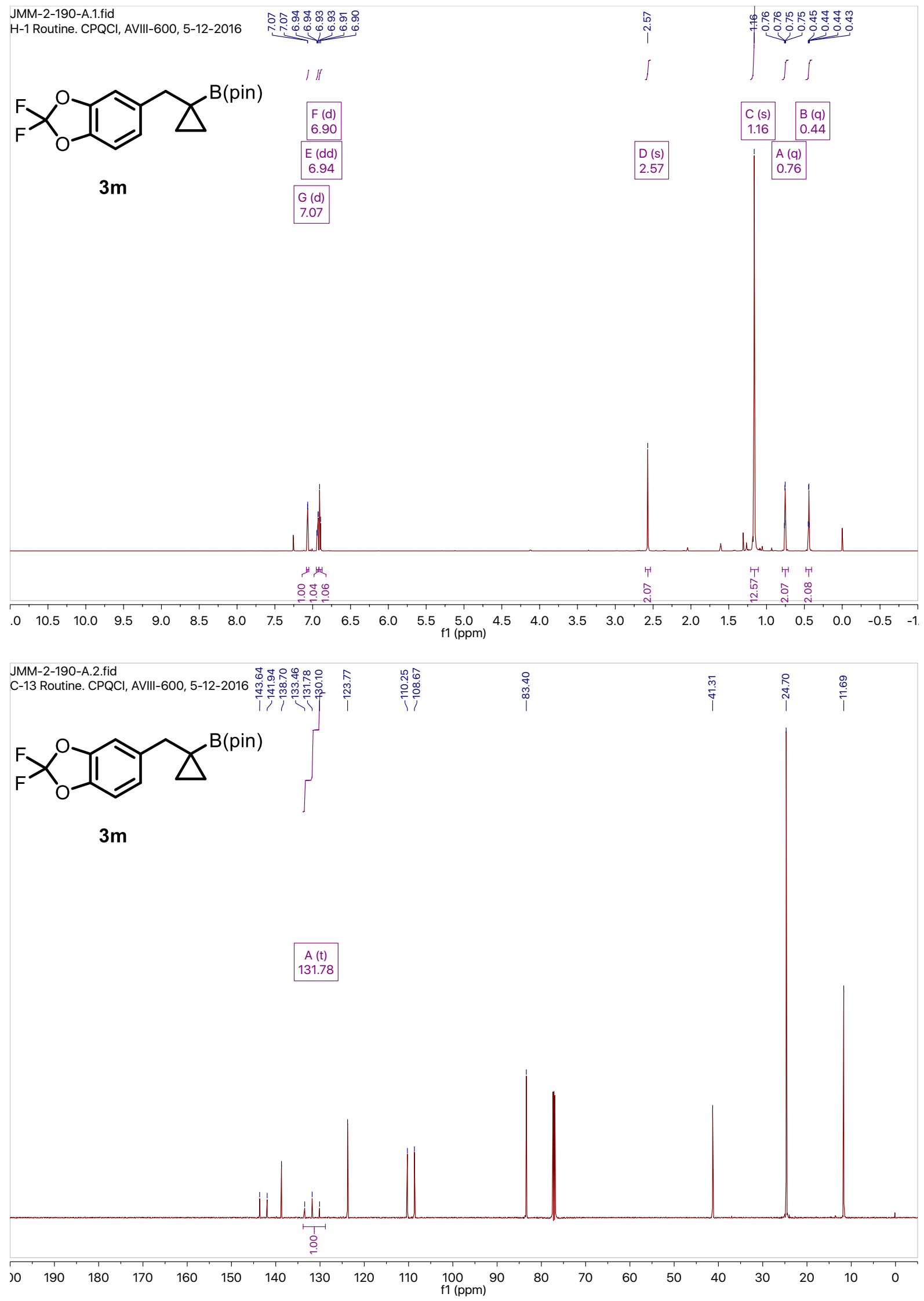

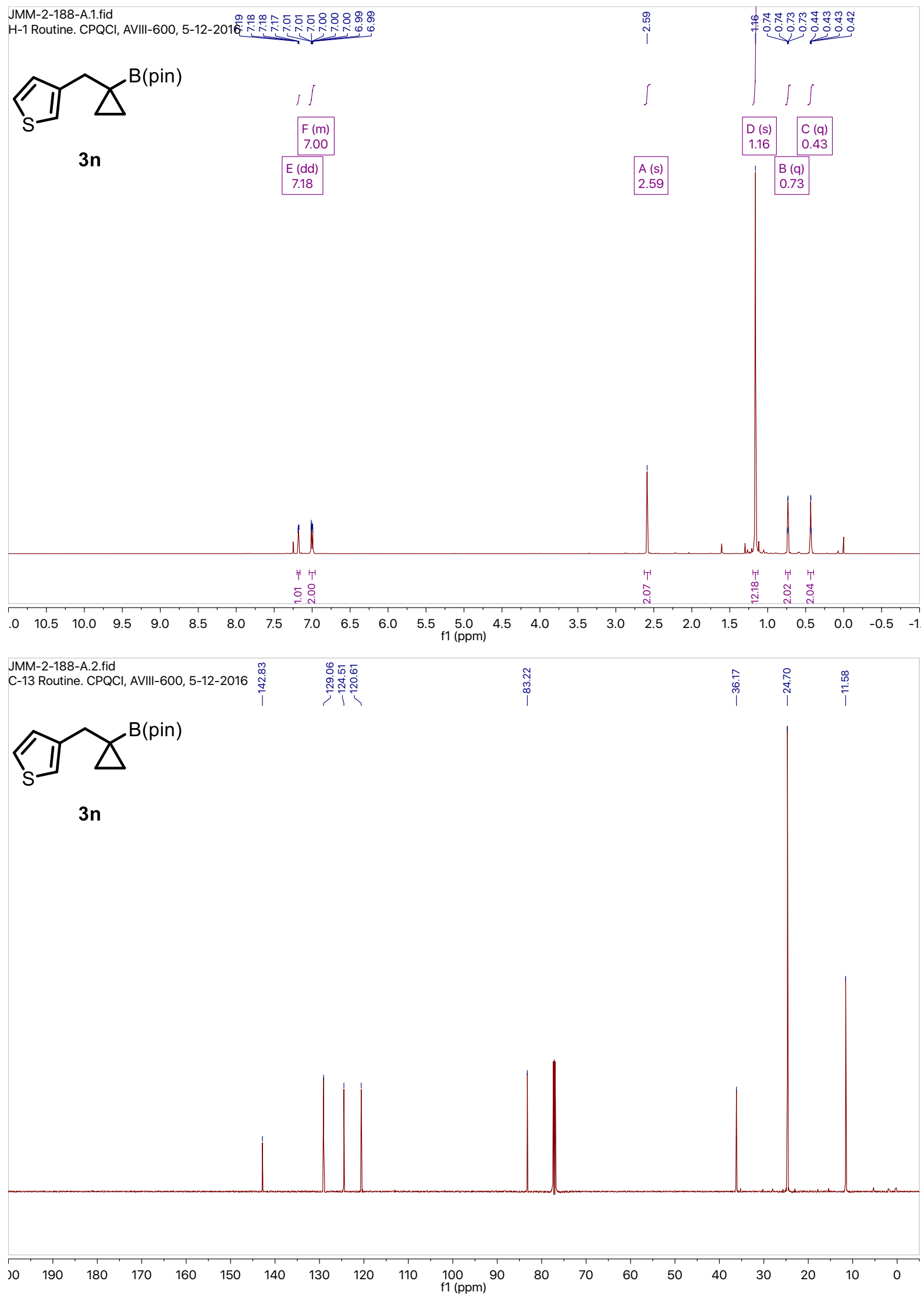

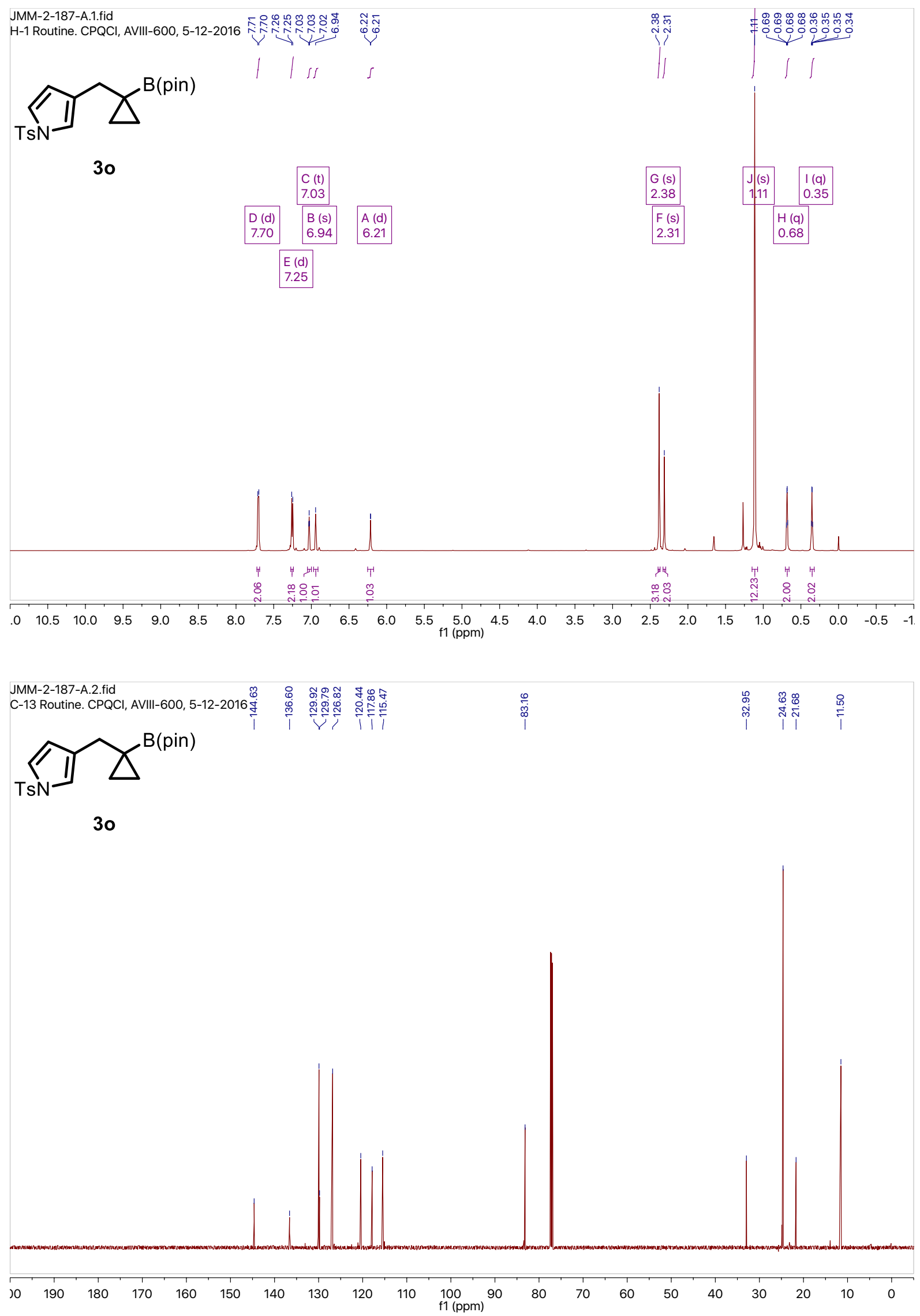


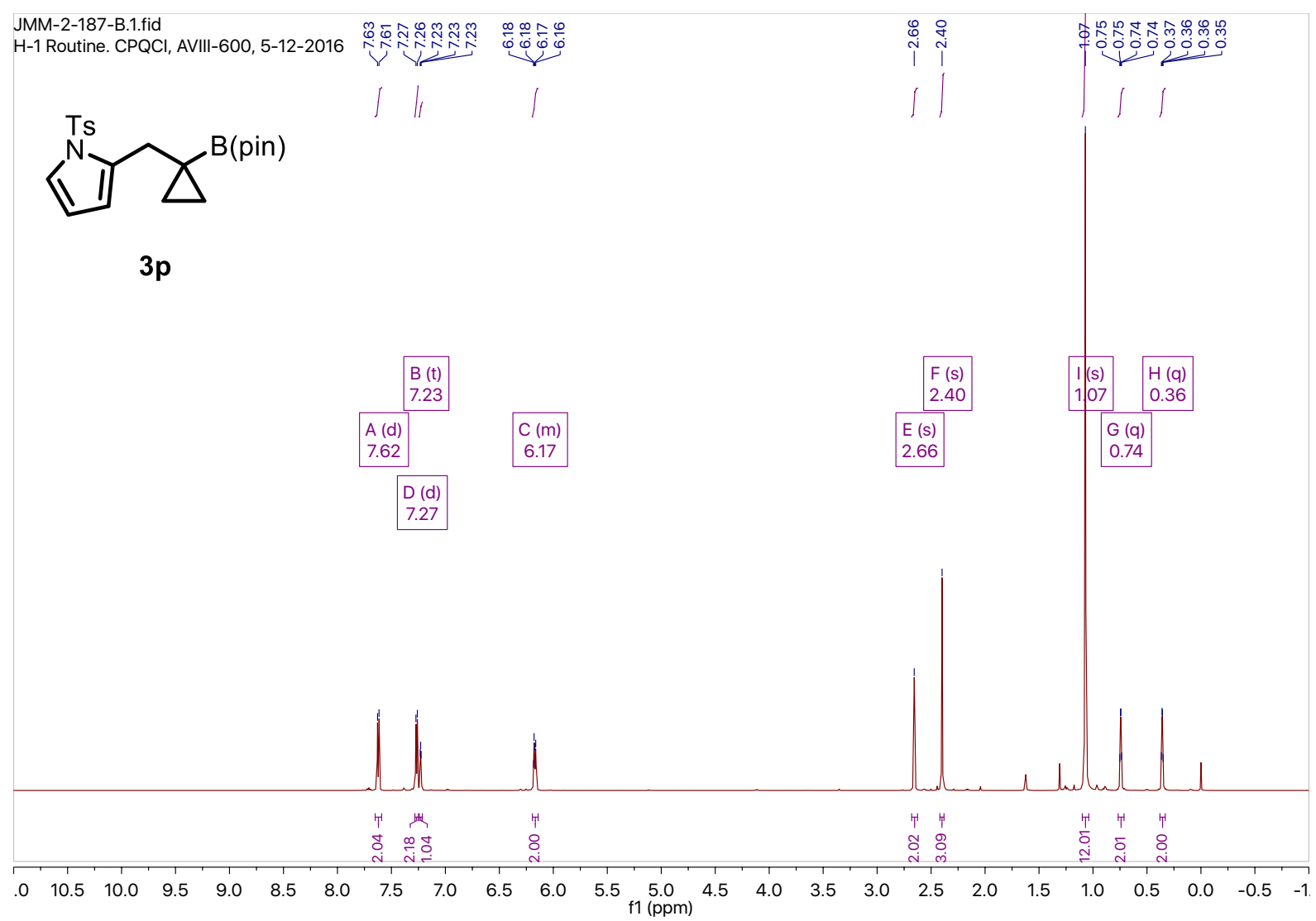

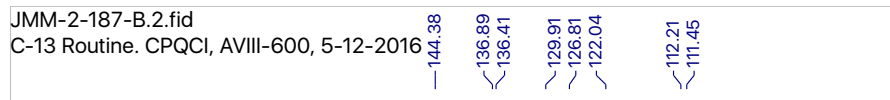

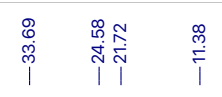

$\mathbb{1}^{\mathrm{T}} \mathrm{\triangle}^{\mathrm{B}(\mathrm{pin})}$

$3 p$

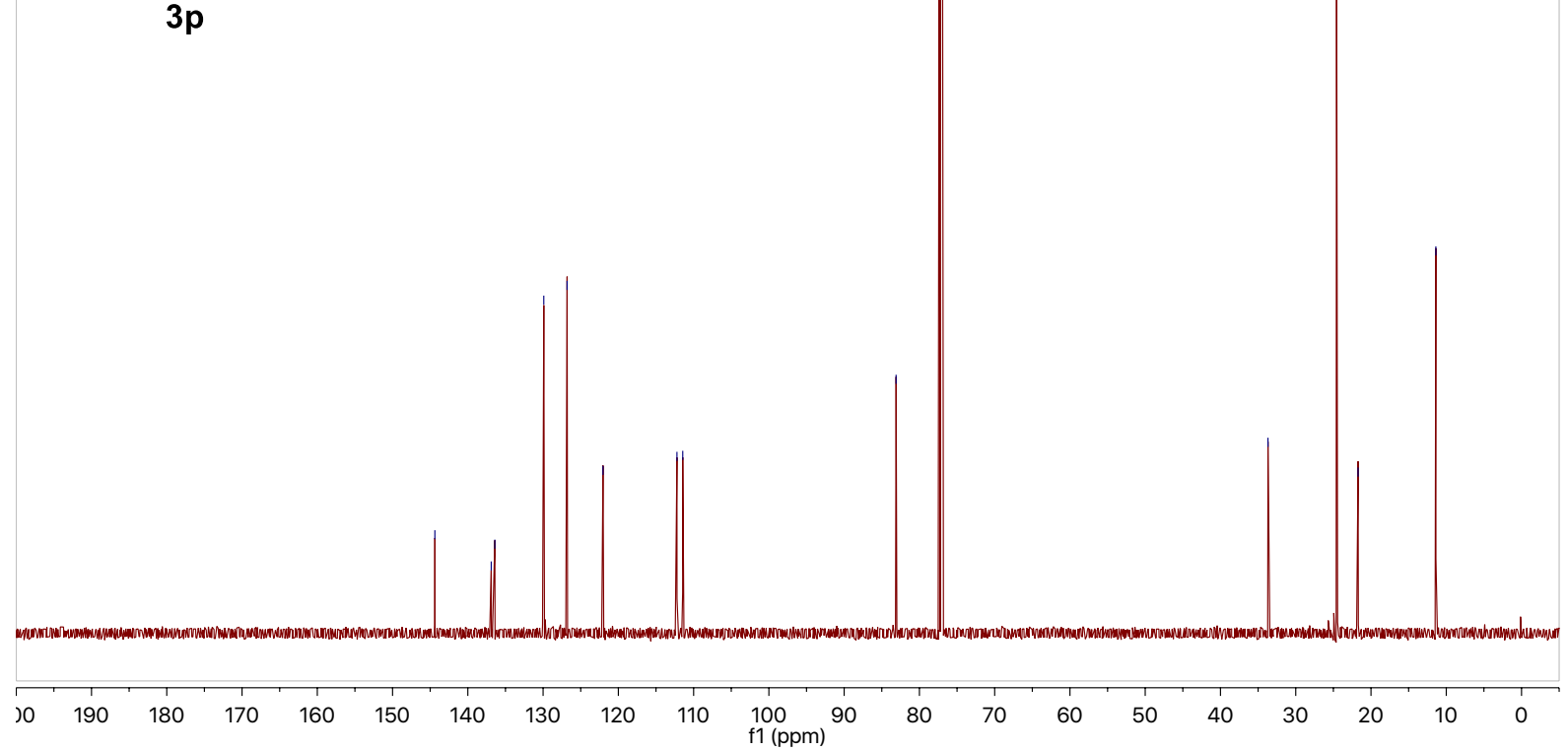



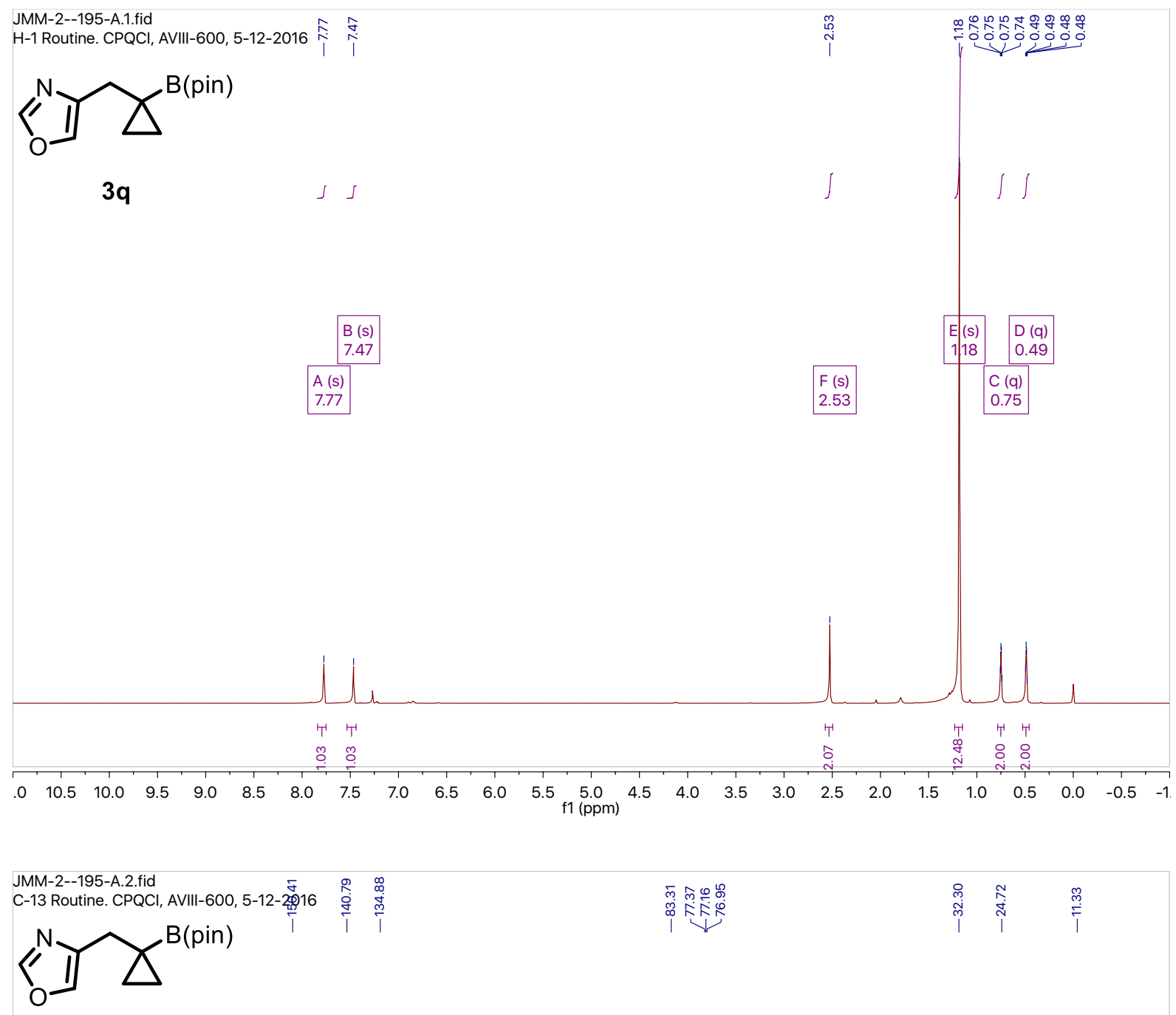

$3 q$

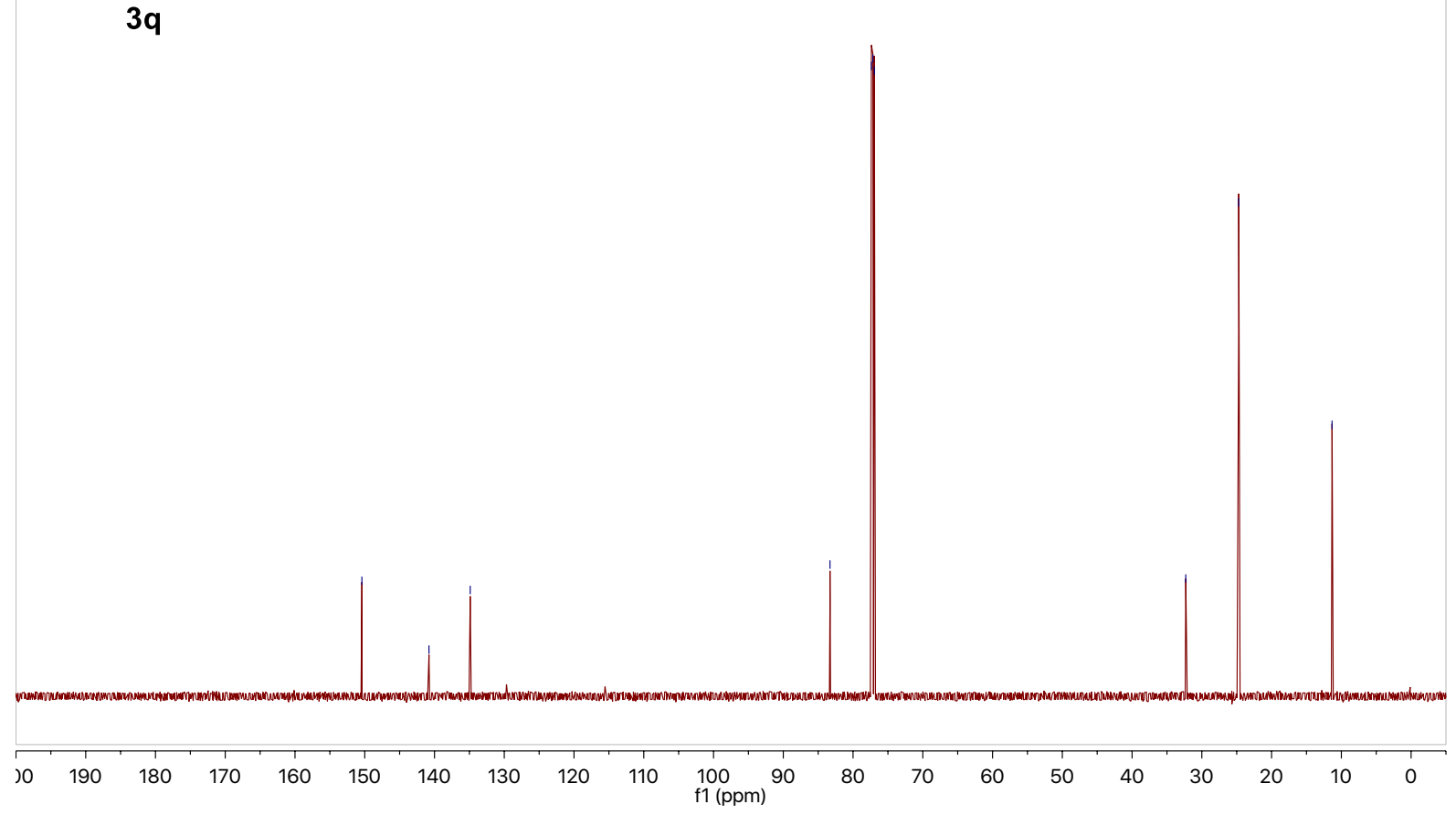




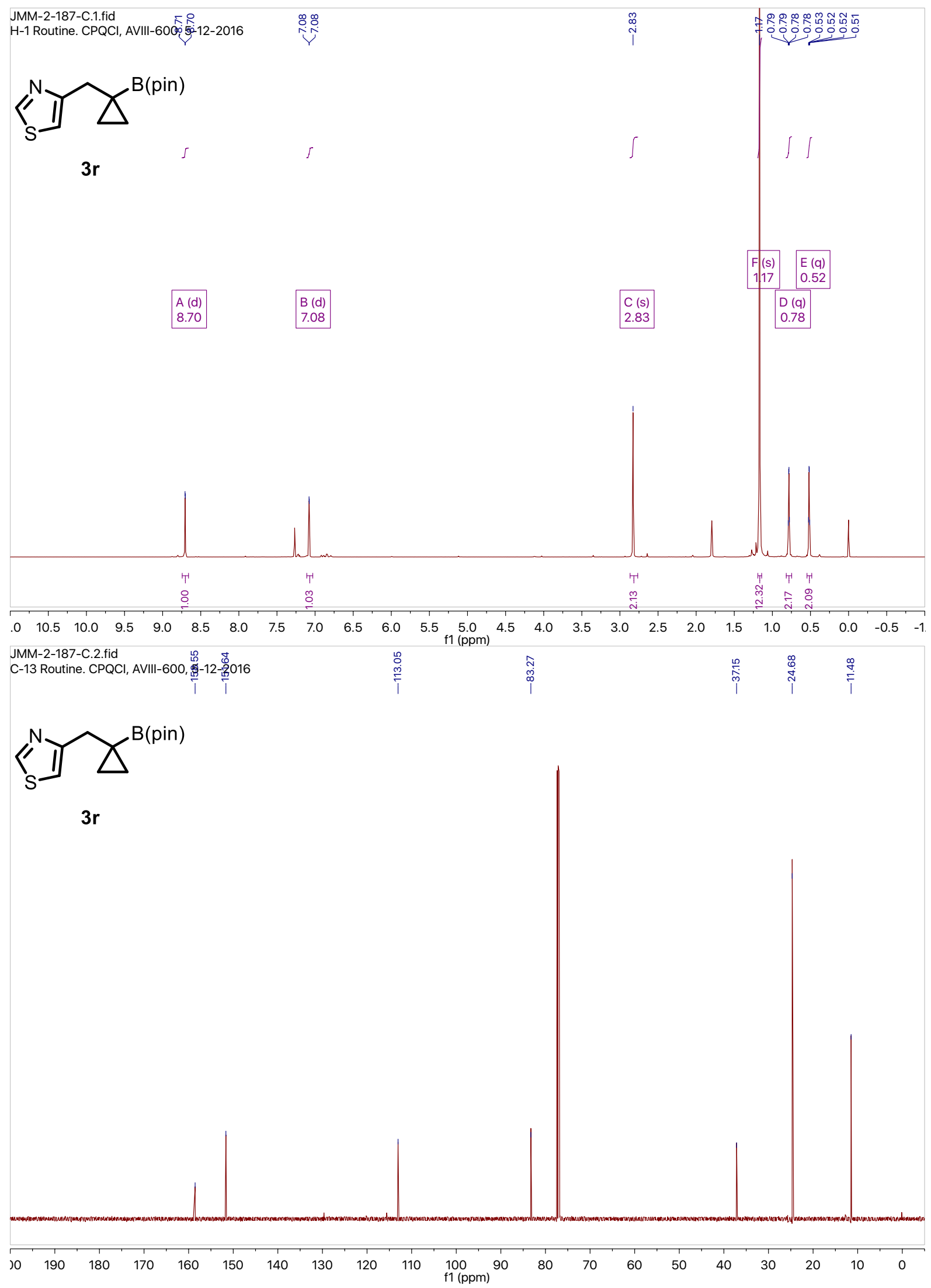




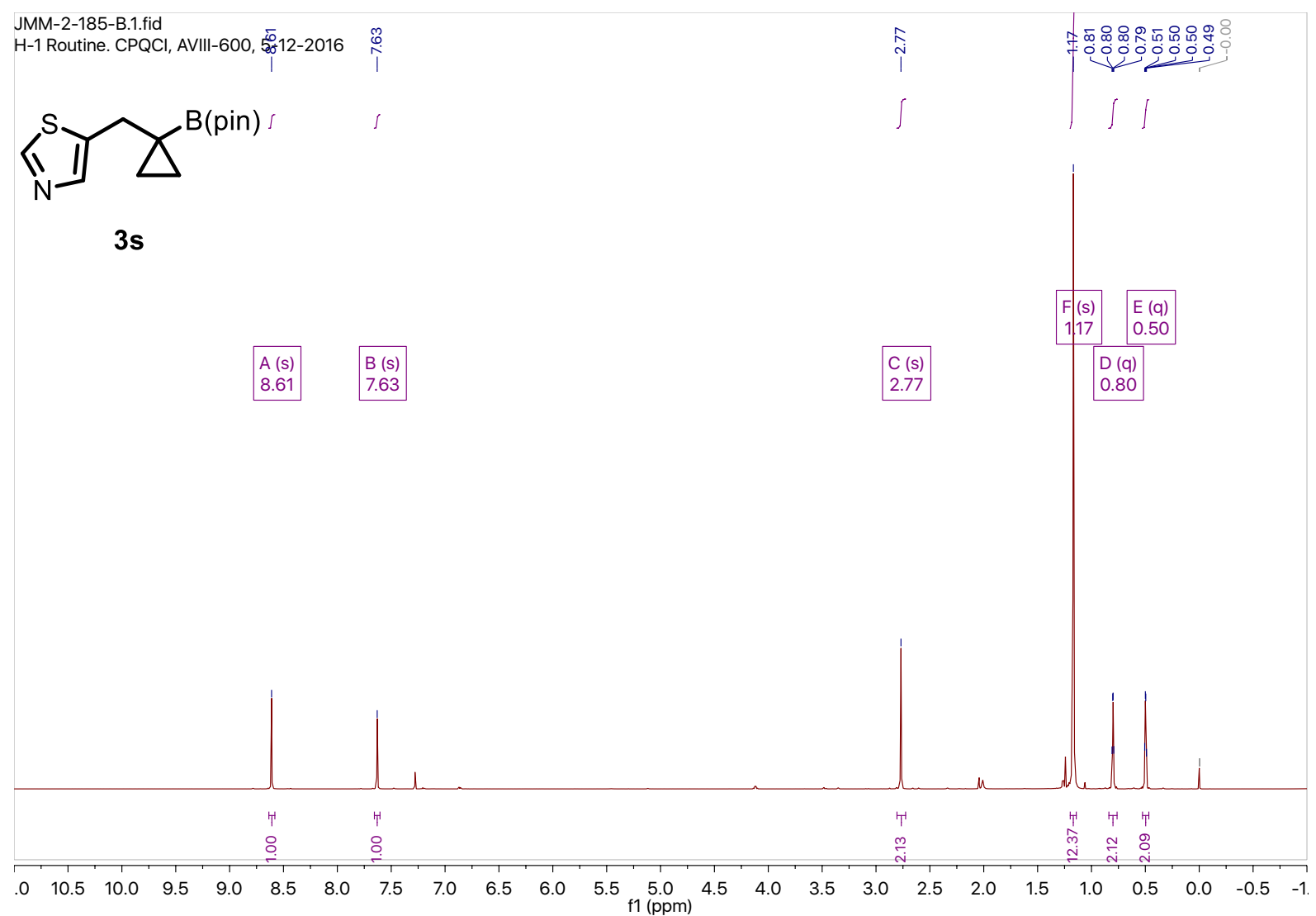

JMM-2-185-B.2.fid
C-13 Routine. CPQCl, AVIII-600, 5-12-忽016

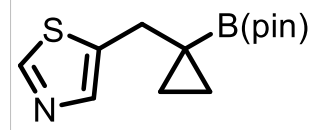

3s

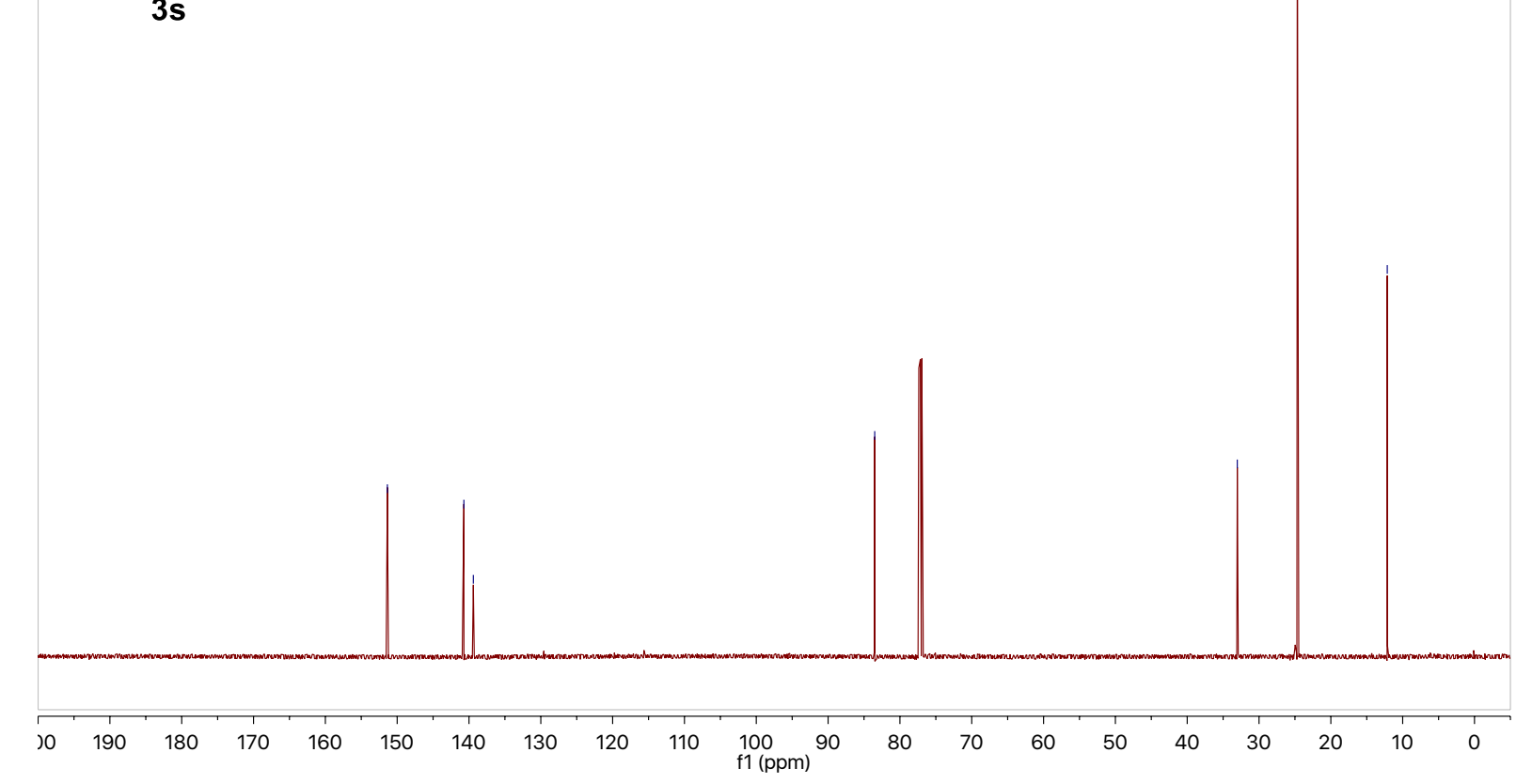



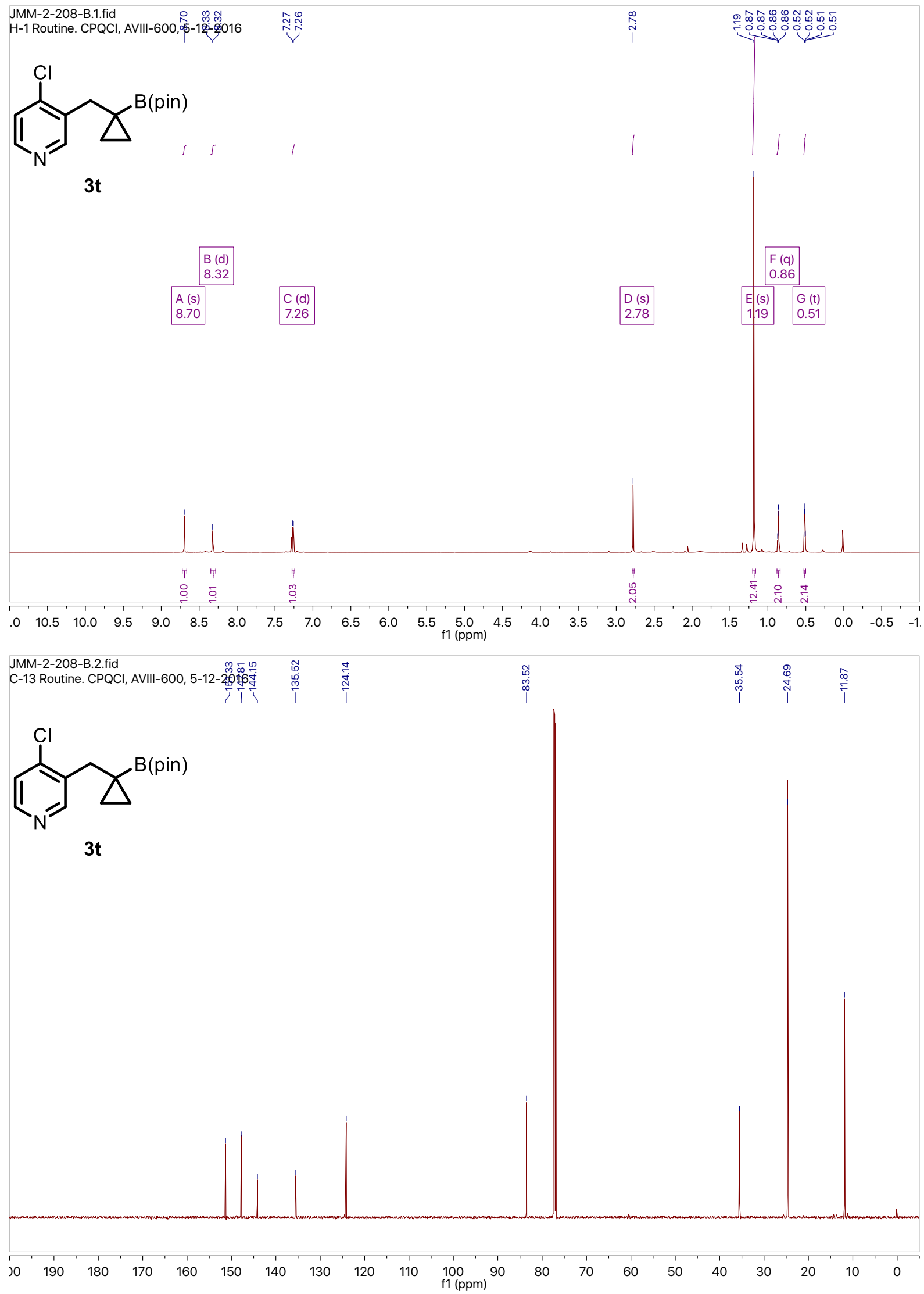

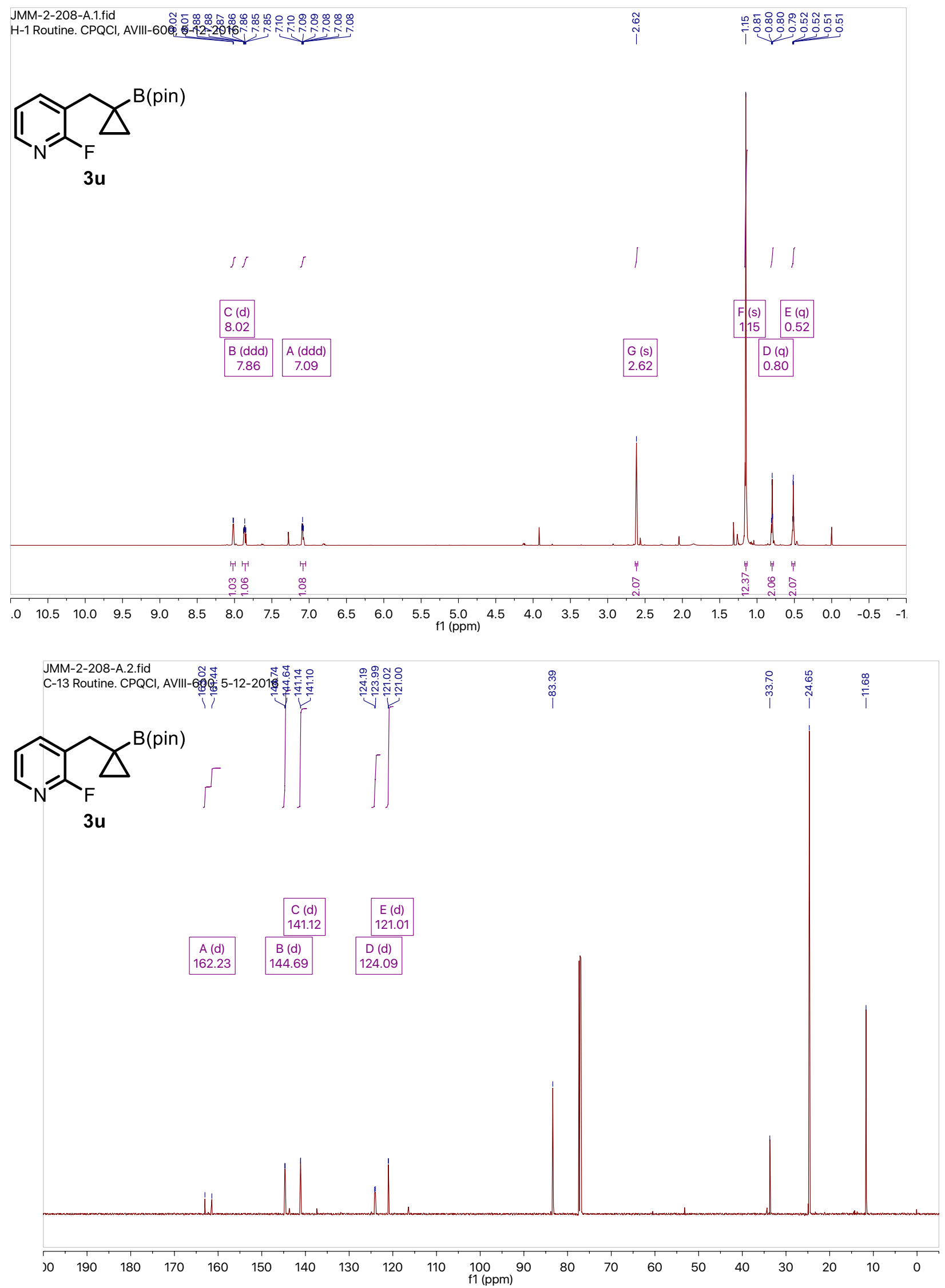

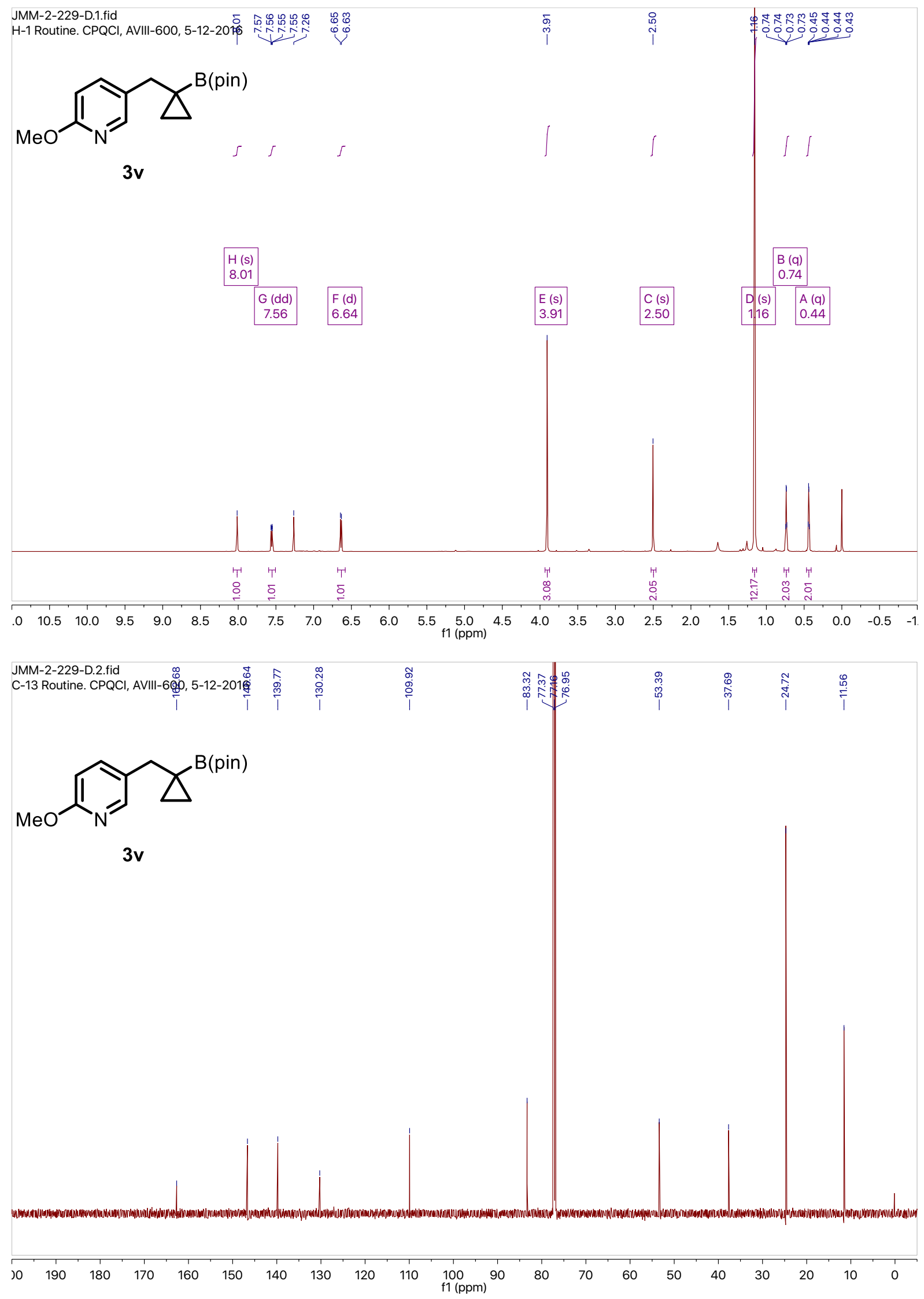
Medina et al.: BCP Hydroboration-Supporting Information - S165

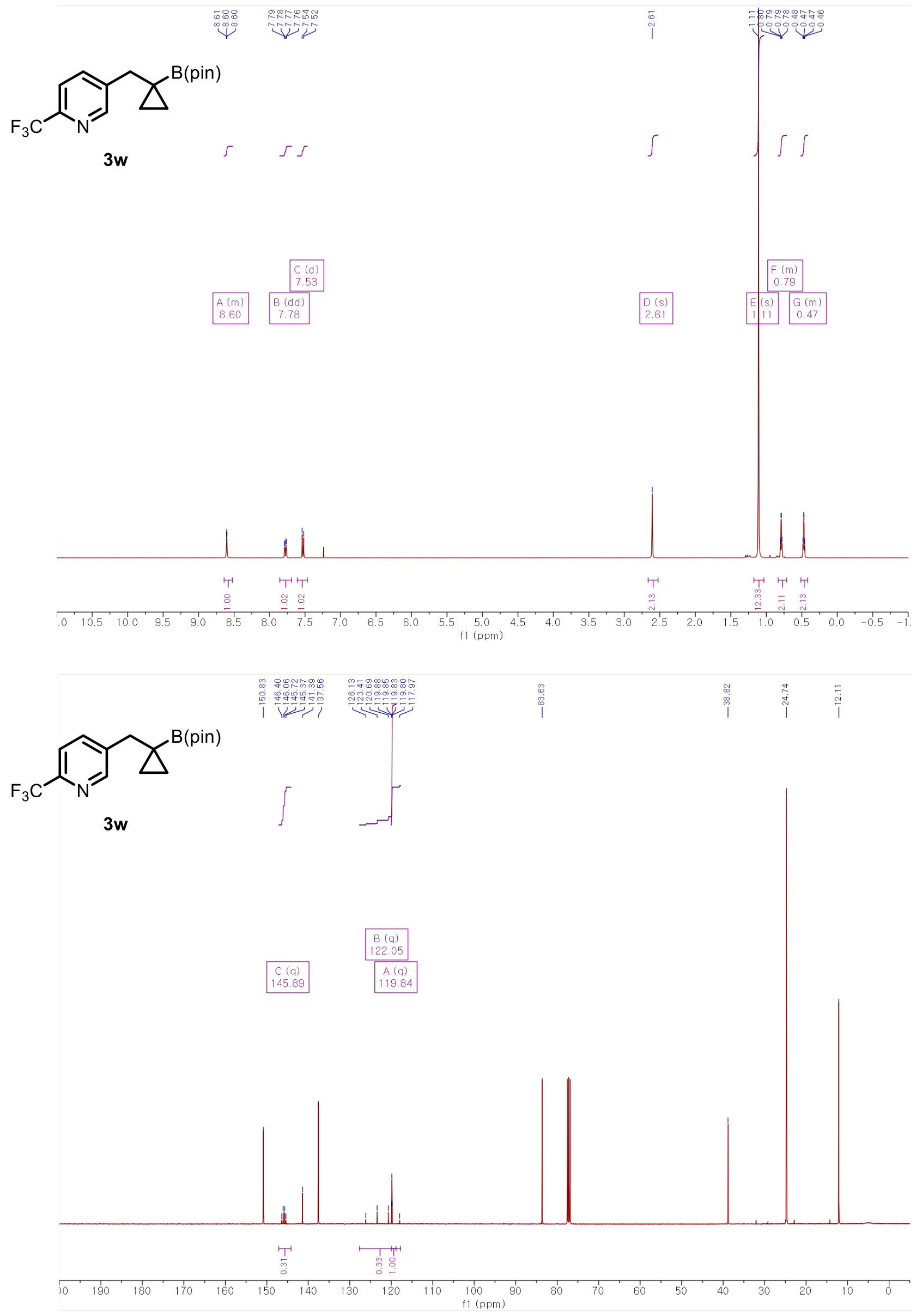




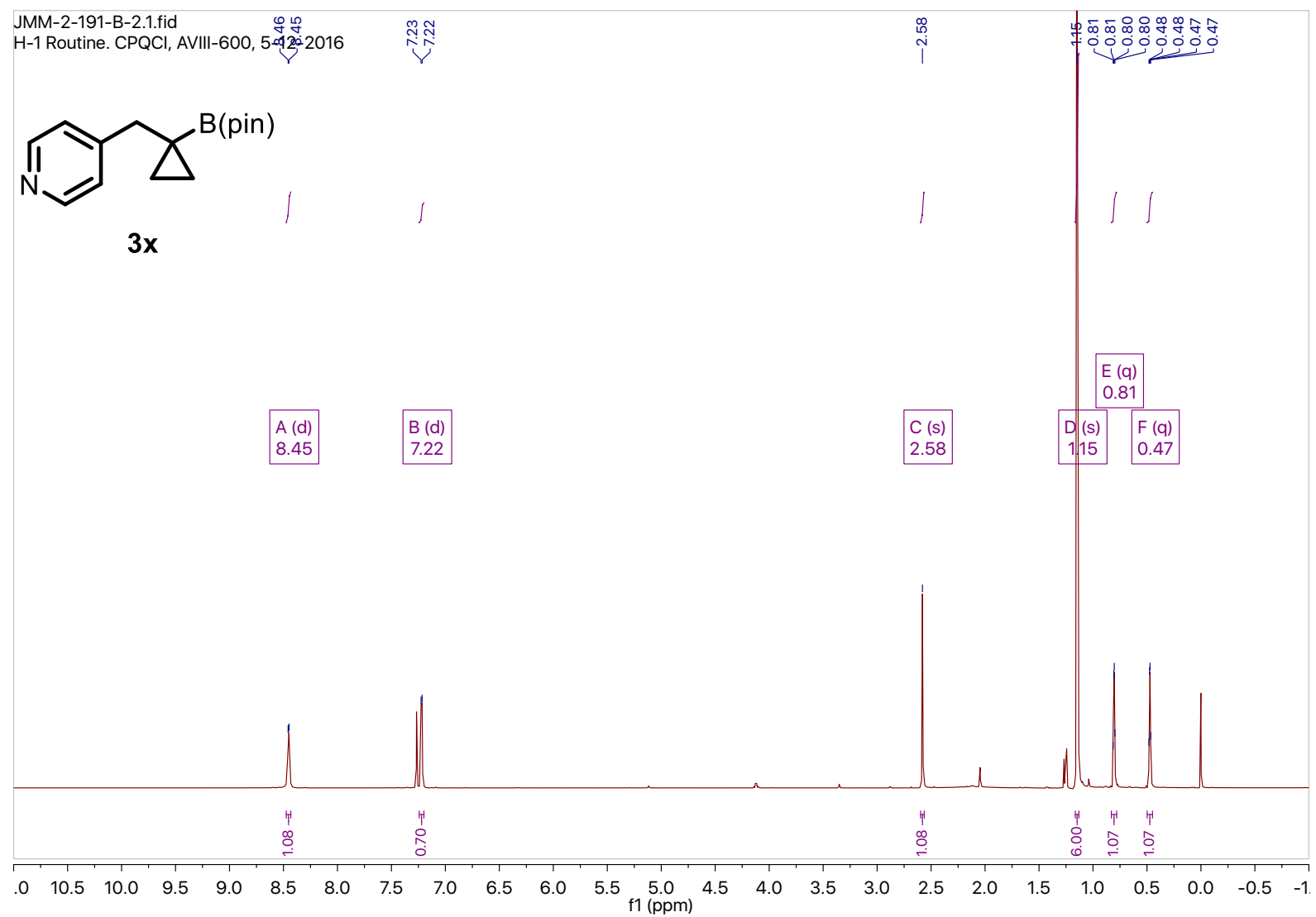

\begin{tabular}{|c|c|c|}
\hline 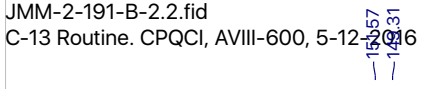 & $\frac{i}{\stackrel{i}{+}}$ & 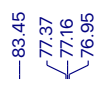 \\
\hline
\end{tabular}

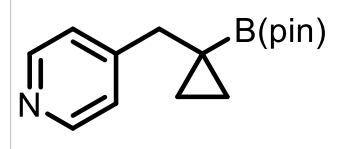

$3 \mathbf{x}$
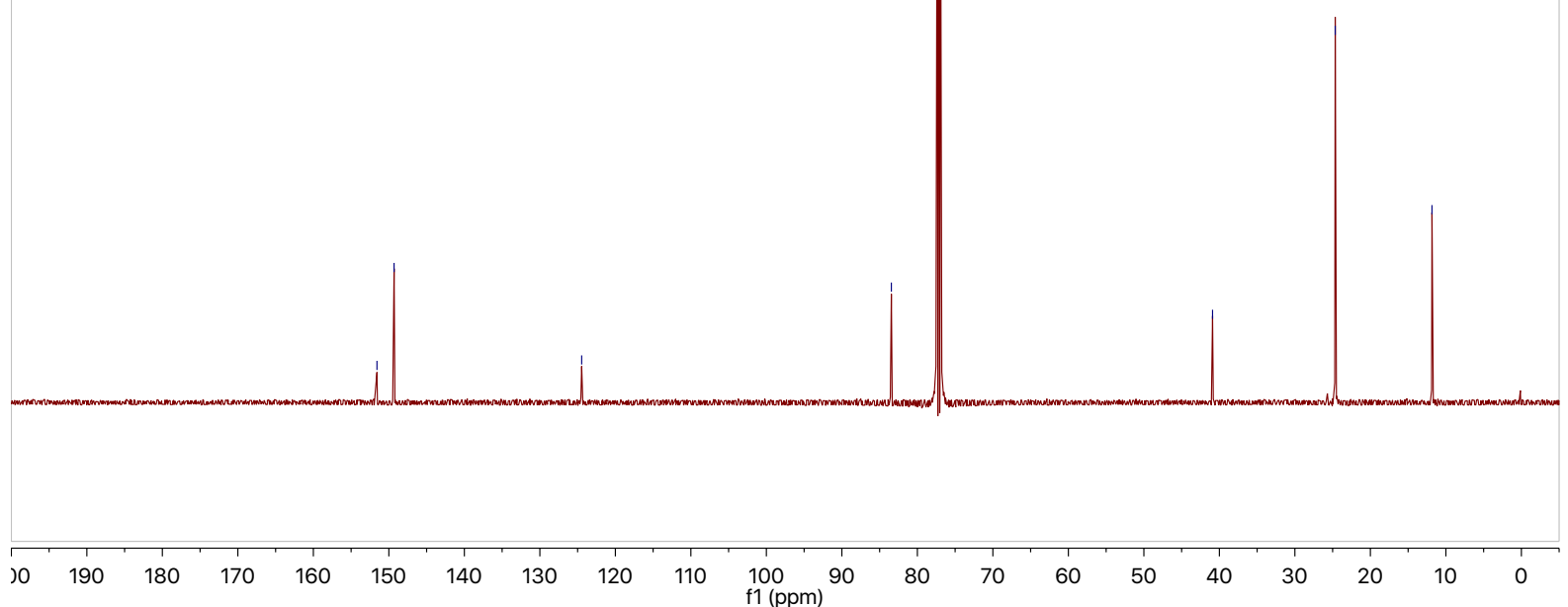

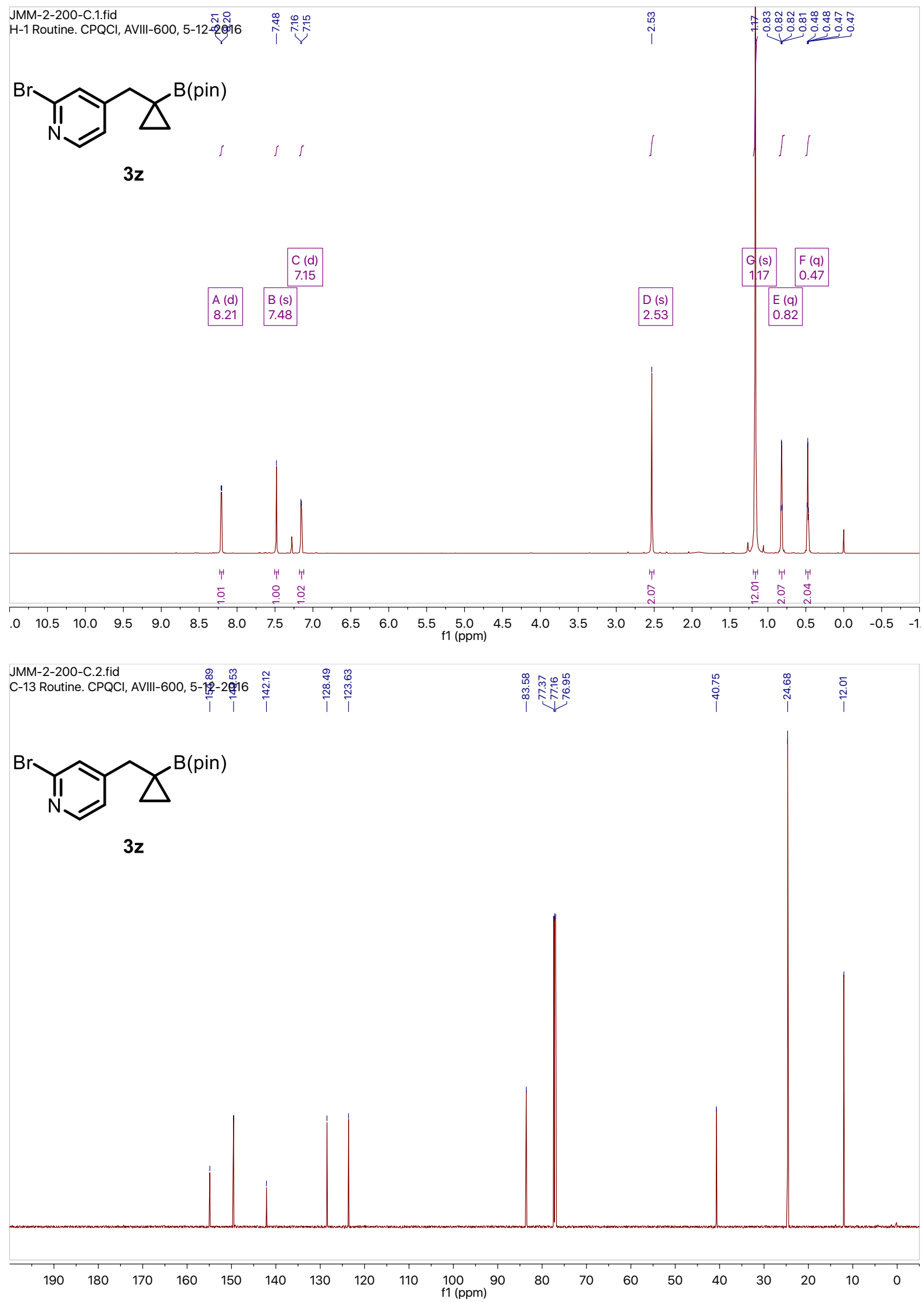


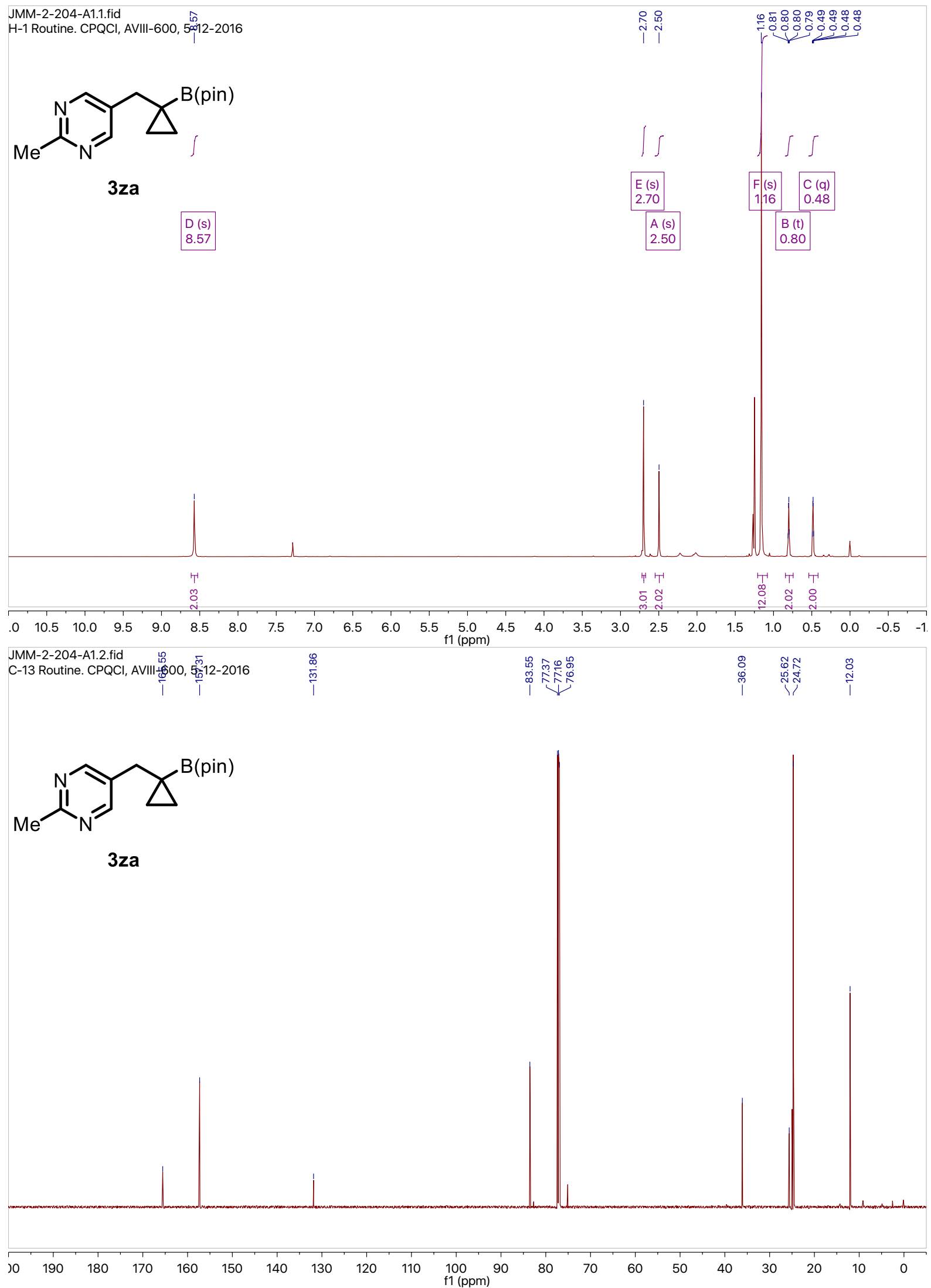



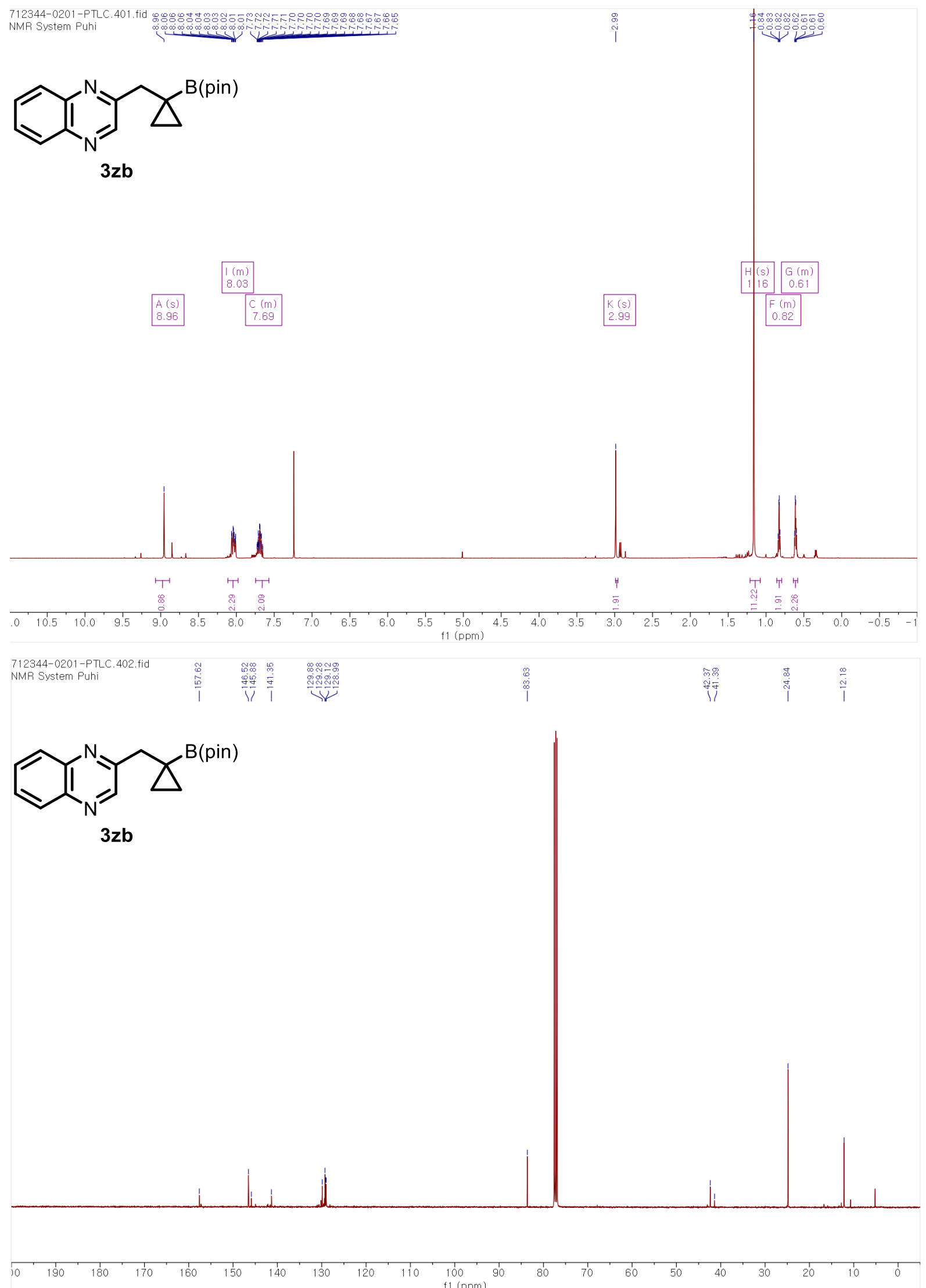


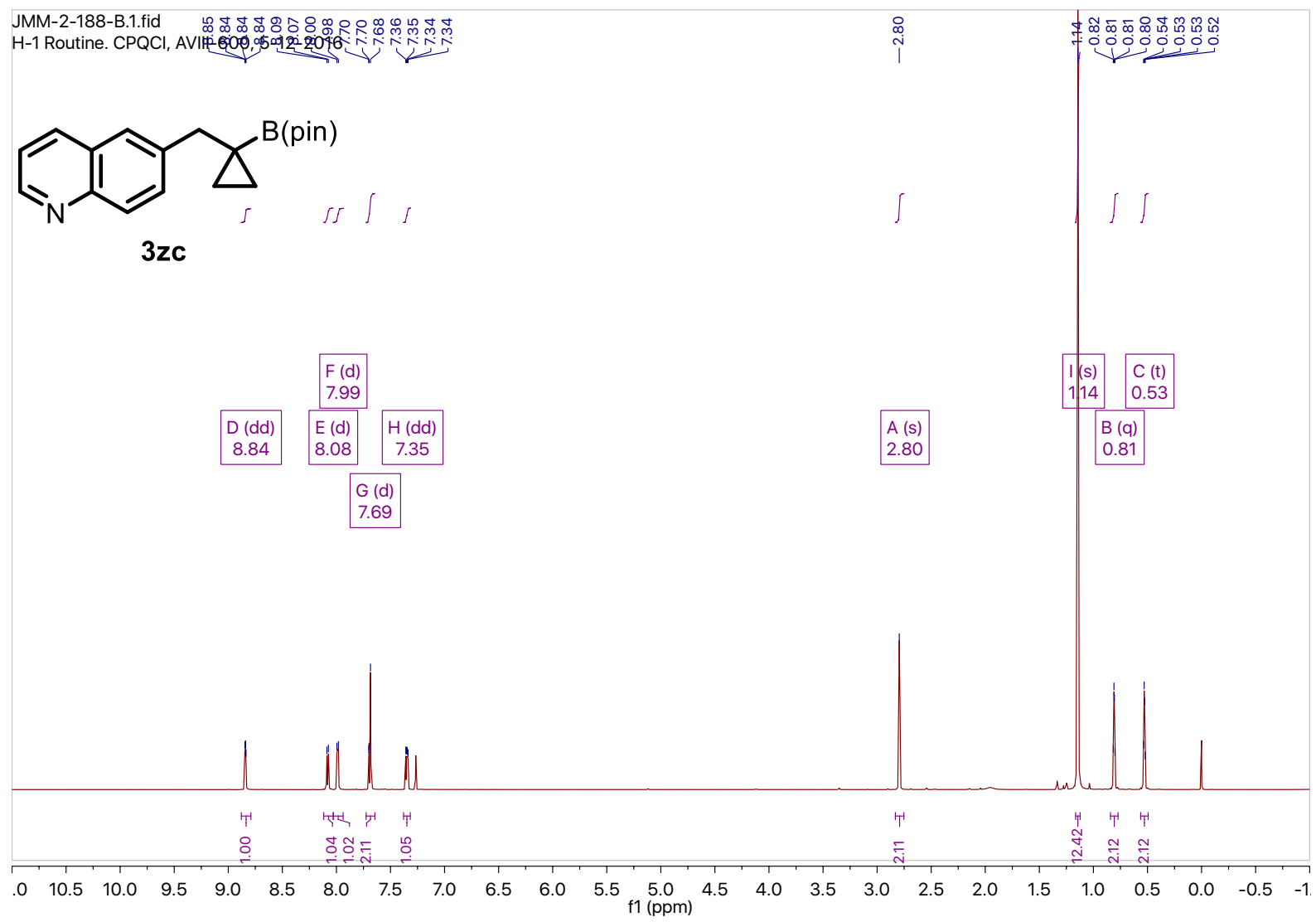

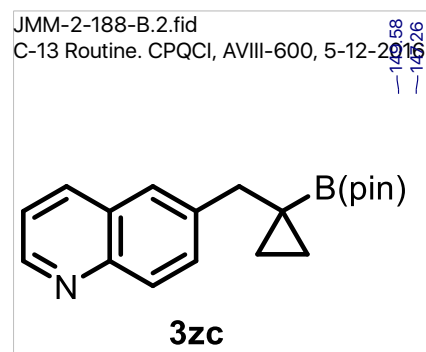

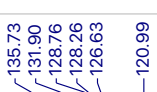

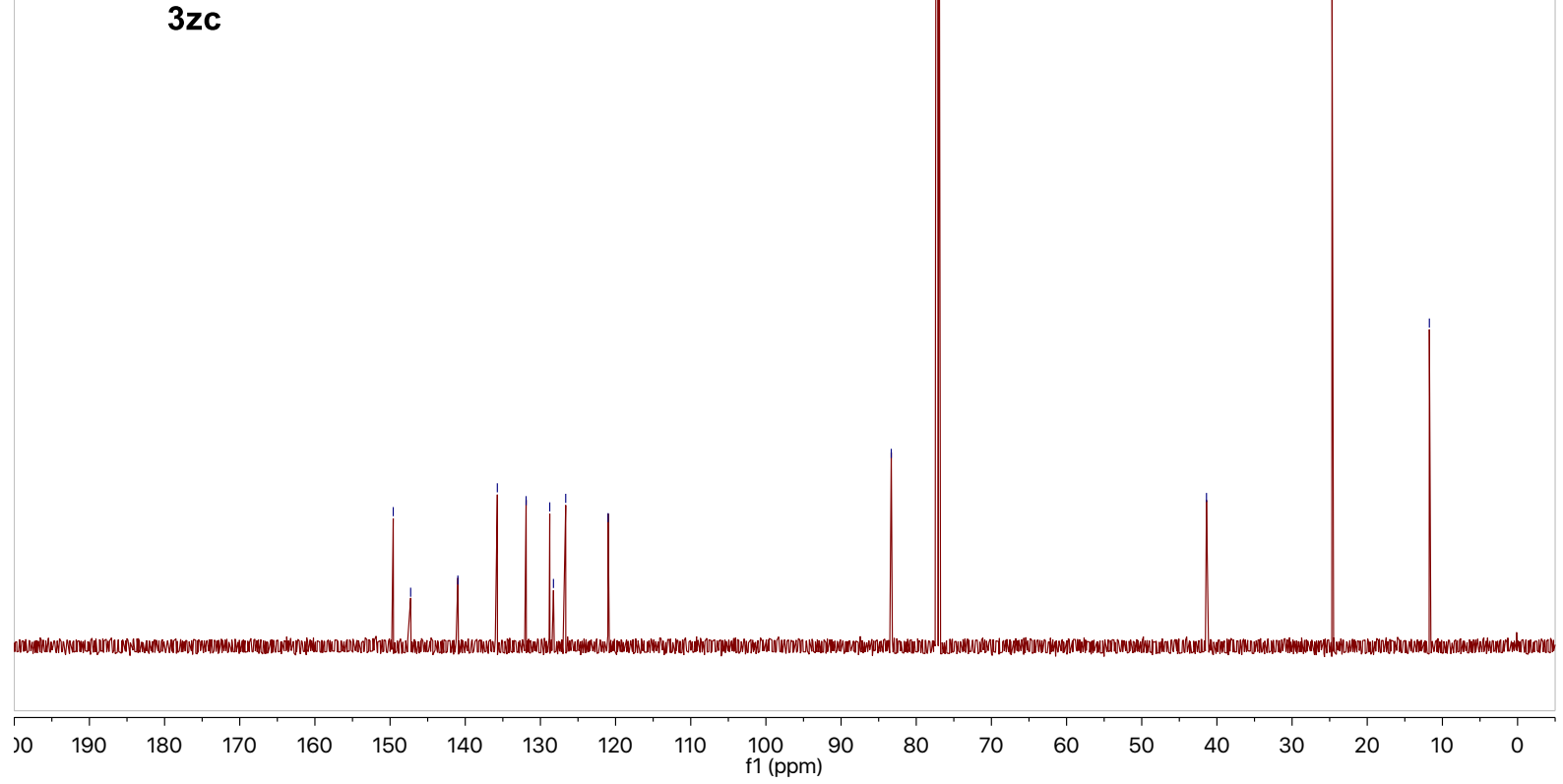




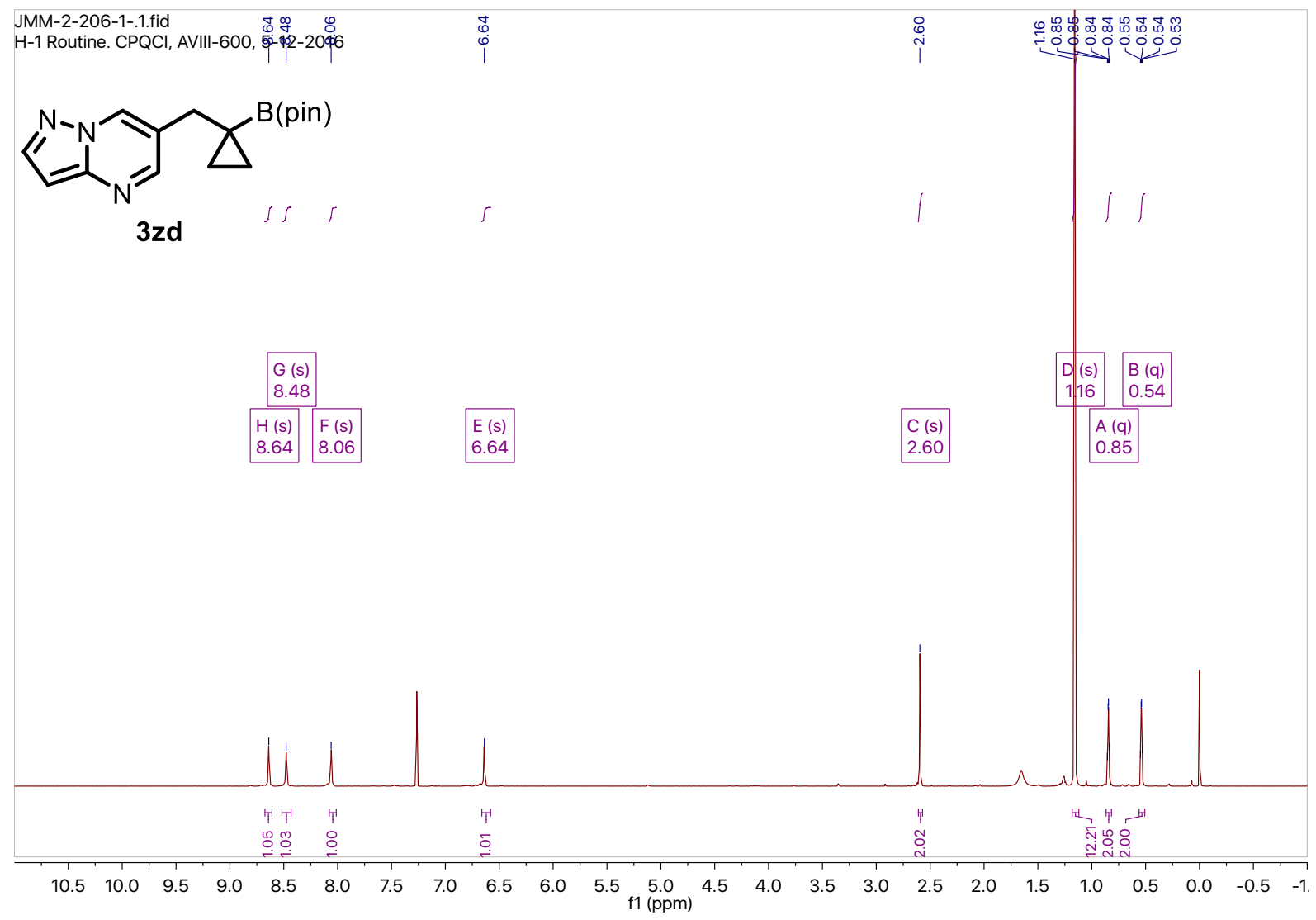

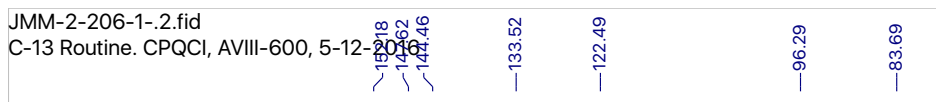
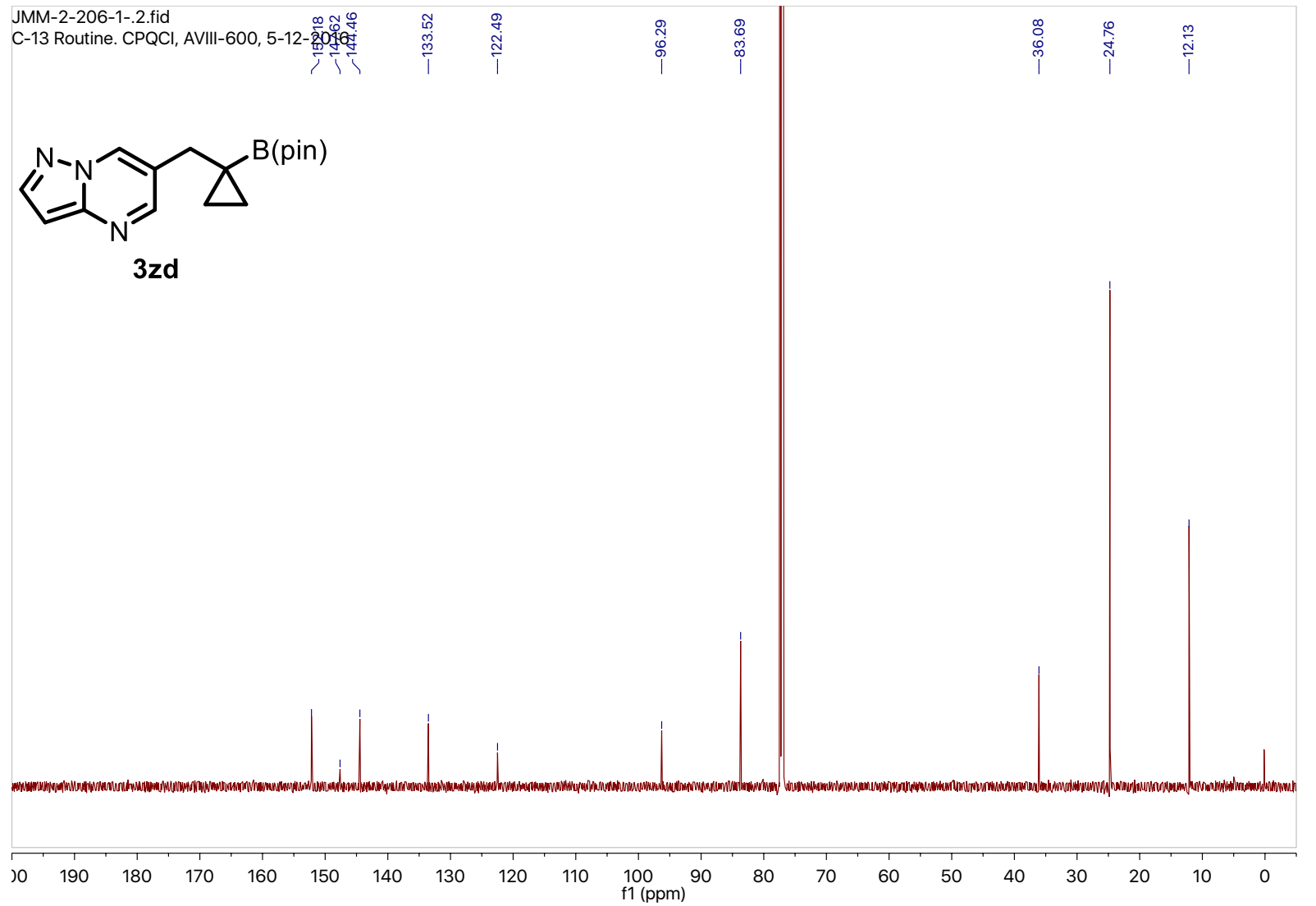

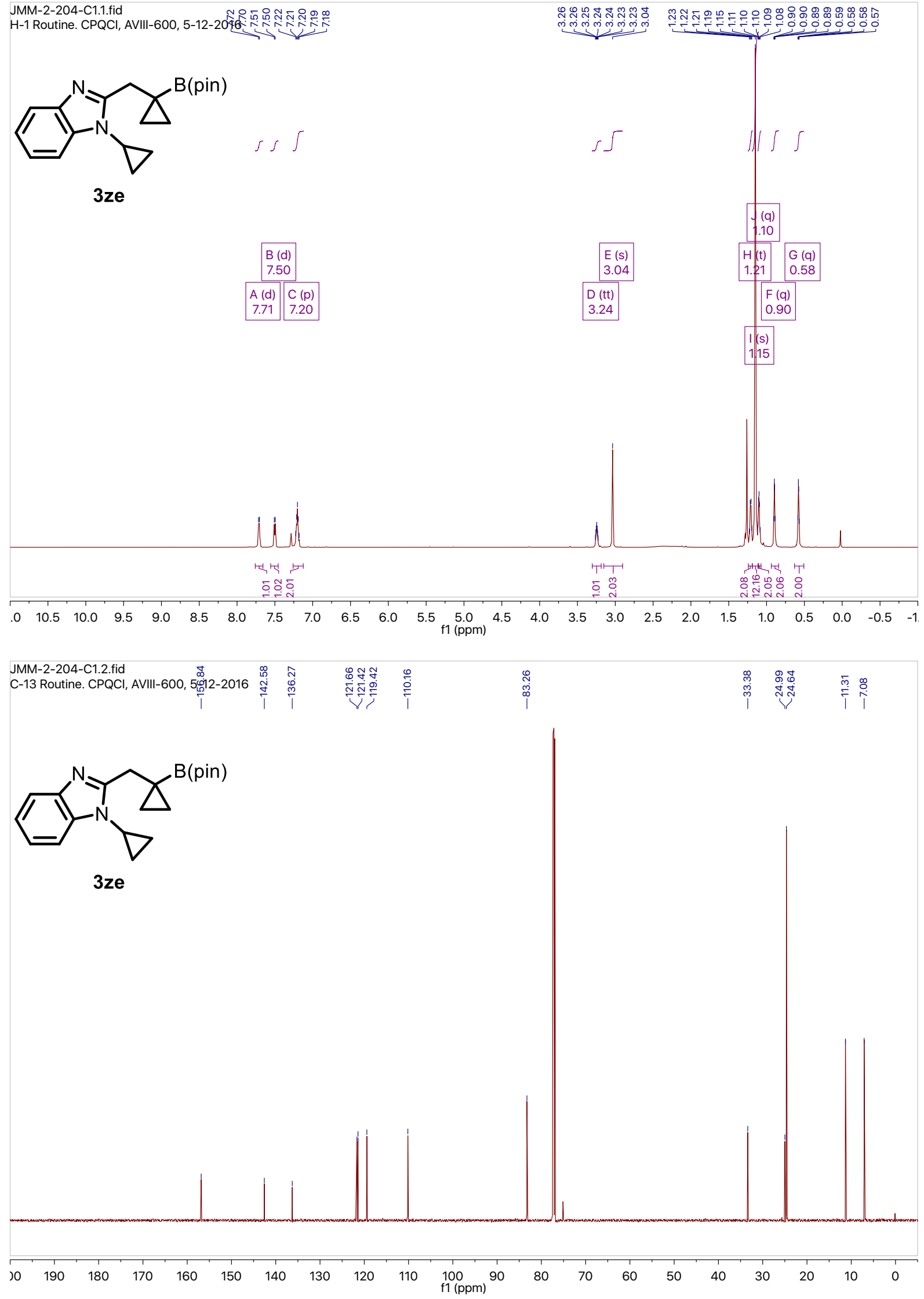


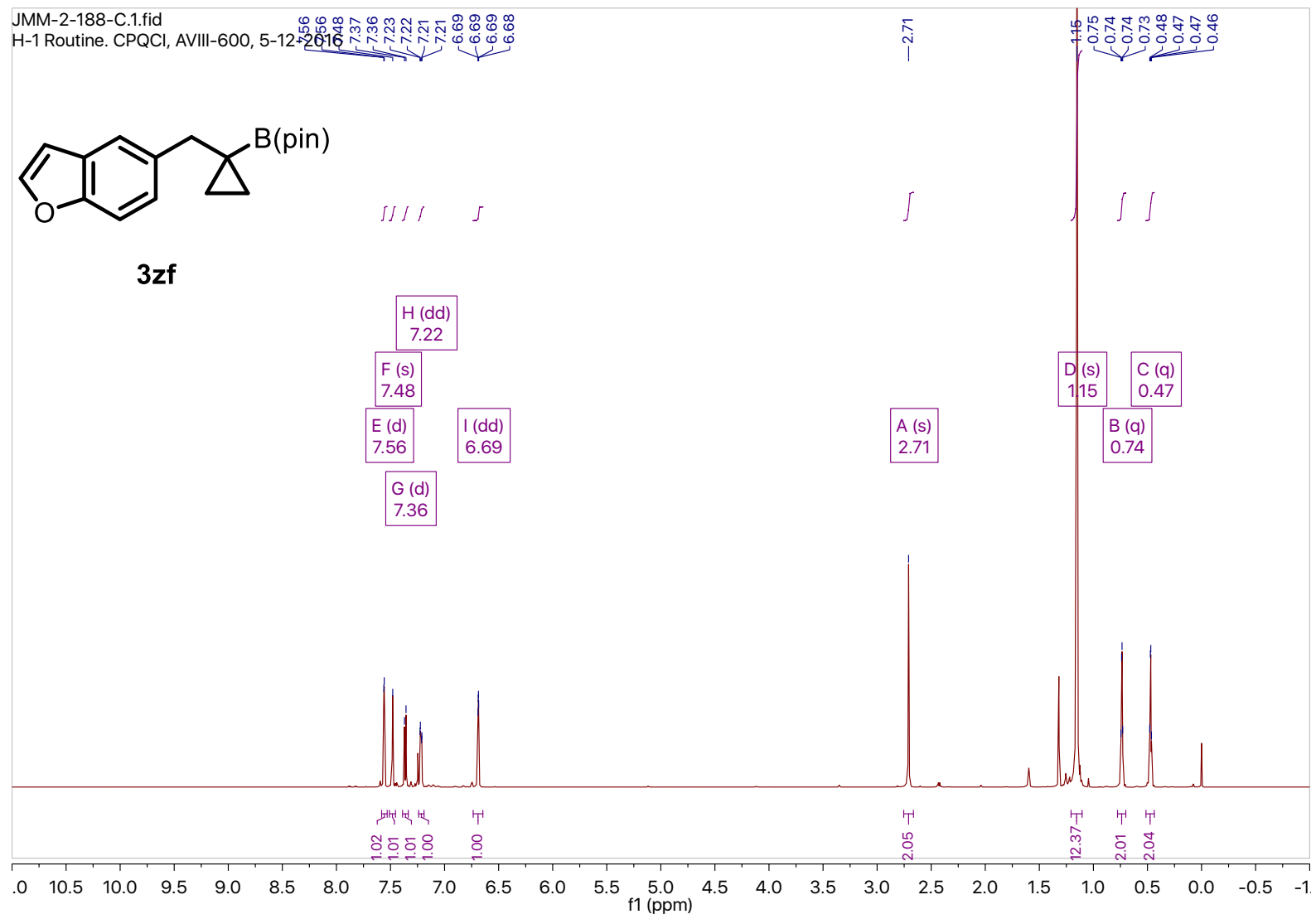

\begin{tabular}{|c|c|c|c|c|c|c|}
\hline 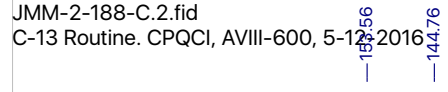 & $\begin{array}{l}\hat{i} \\
\stackrel{0}{0} \\
\stackrel{m}{i}\end{array}$ & 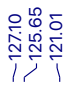 & 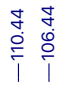 & 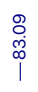 & $\begin{array}{l}\stackrel{\circ}{\circ} \\
\stackrel{+}{+} \\
1\end{array}$ & 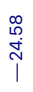 \\
\hline
\end{tabular}
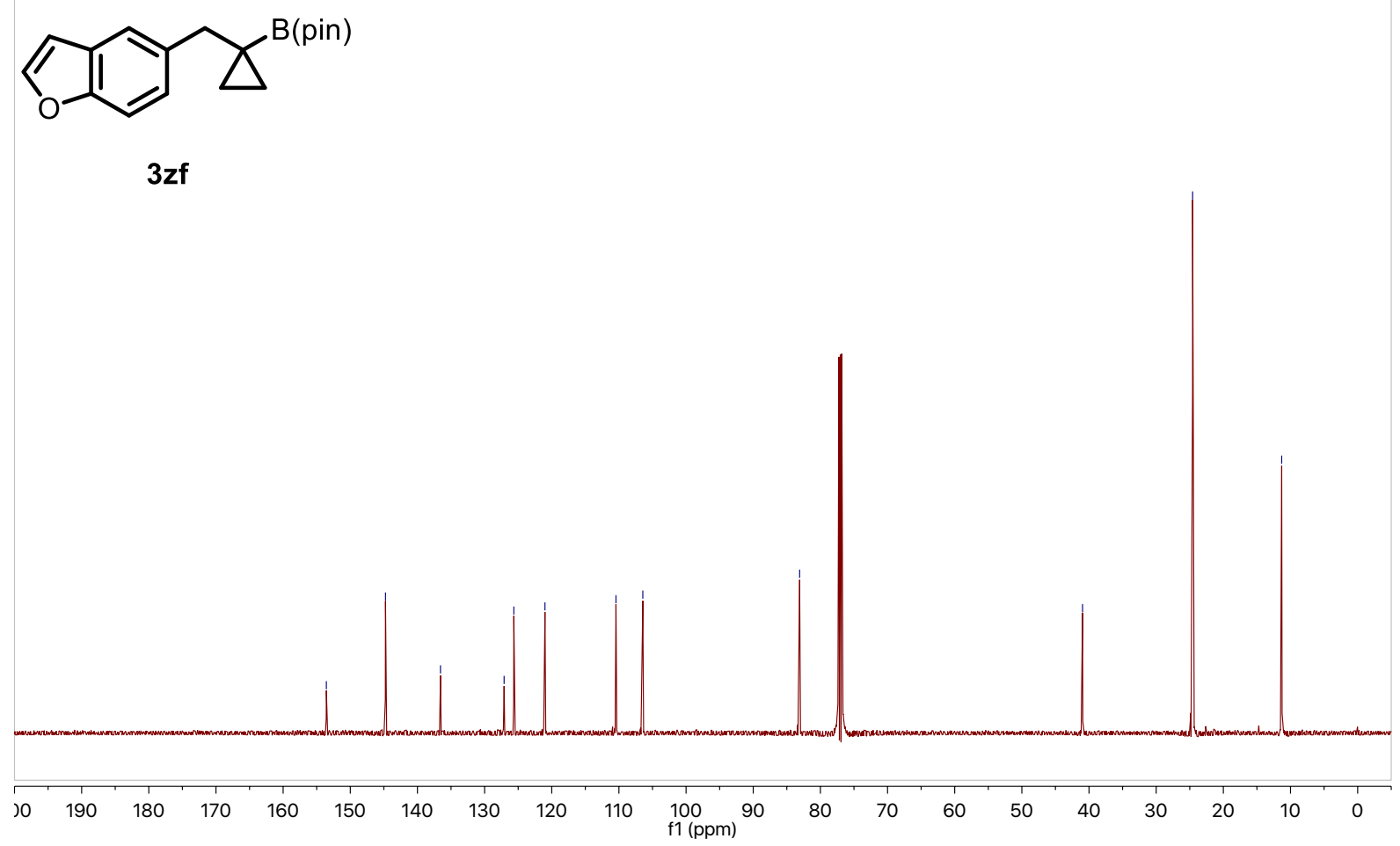
Medina et al.: BCP Hydroboration-Supporting Information - S174

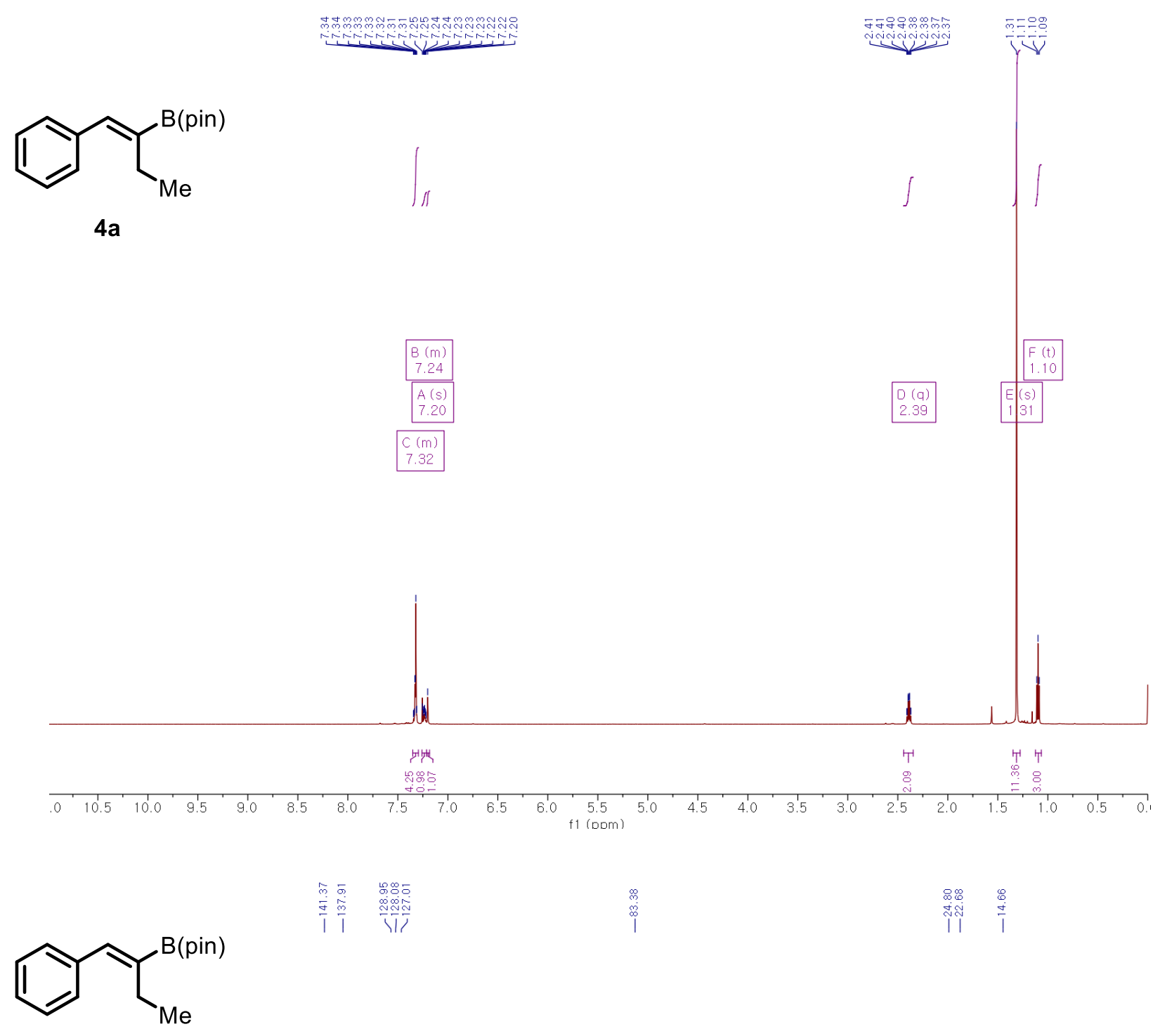

4a

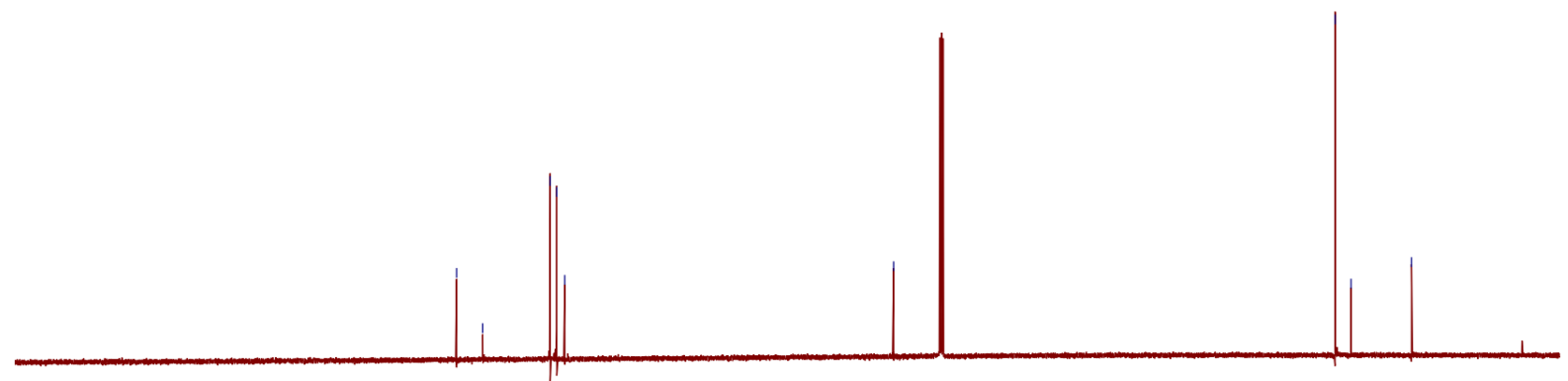

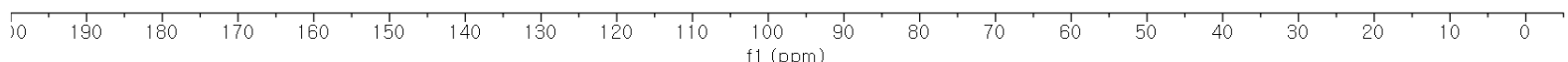




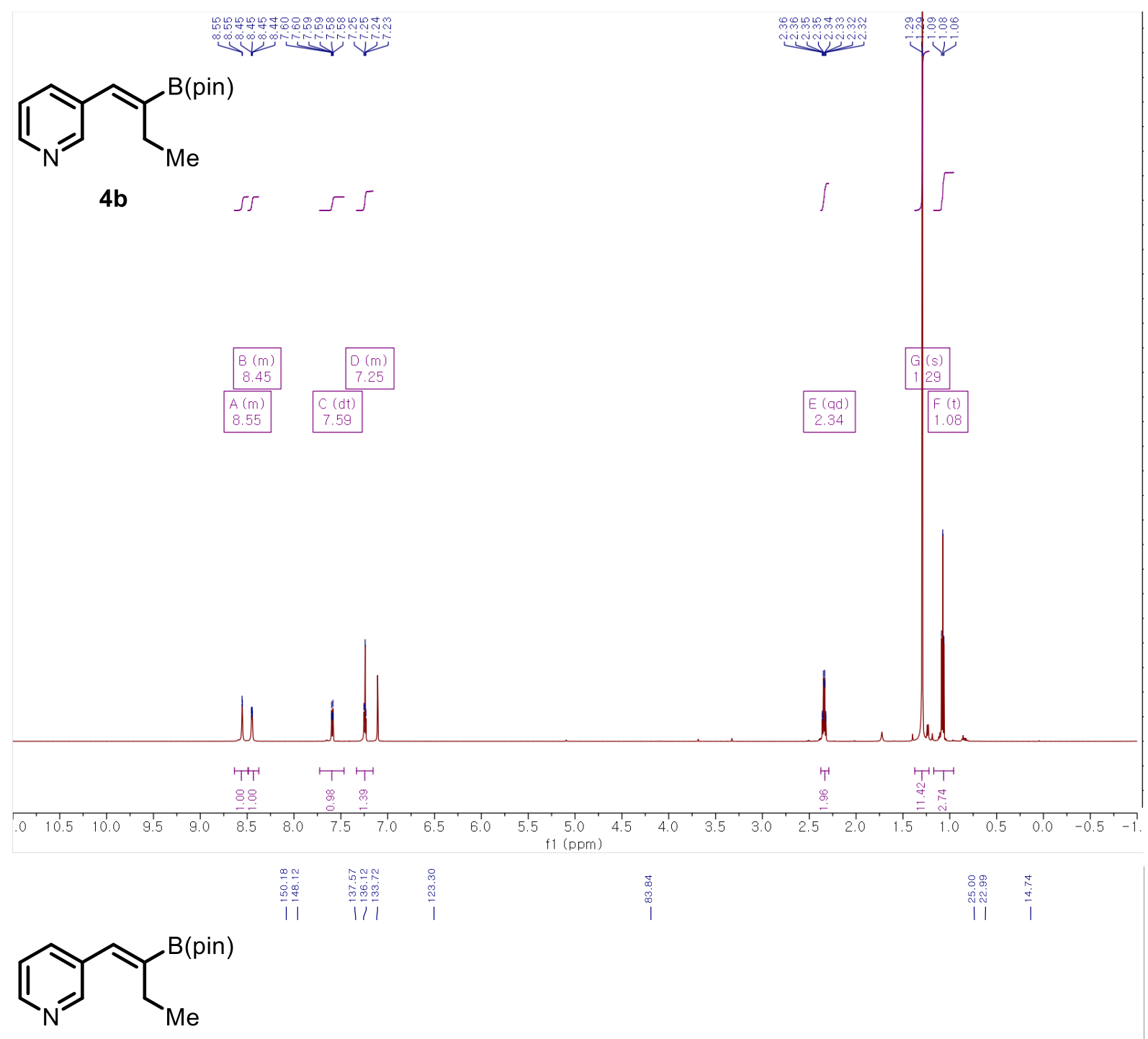

4b
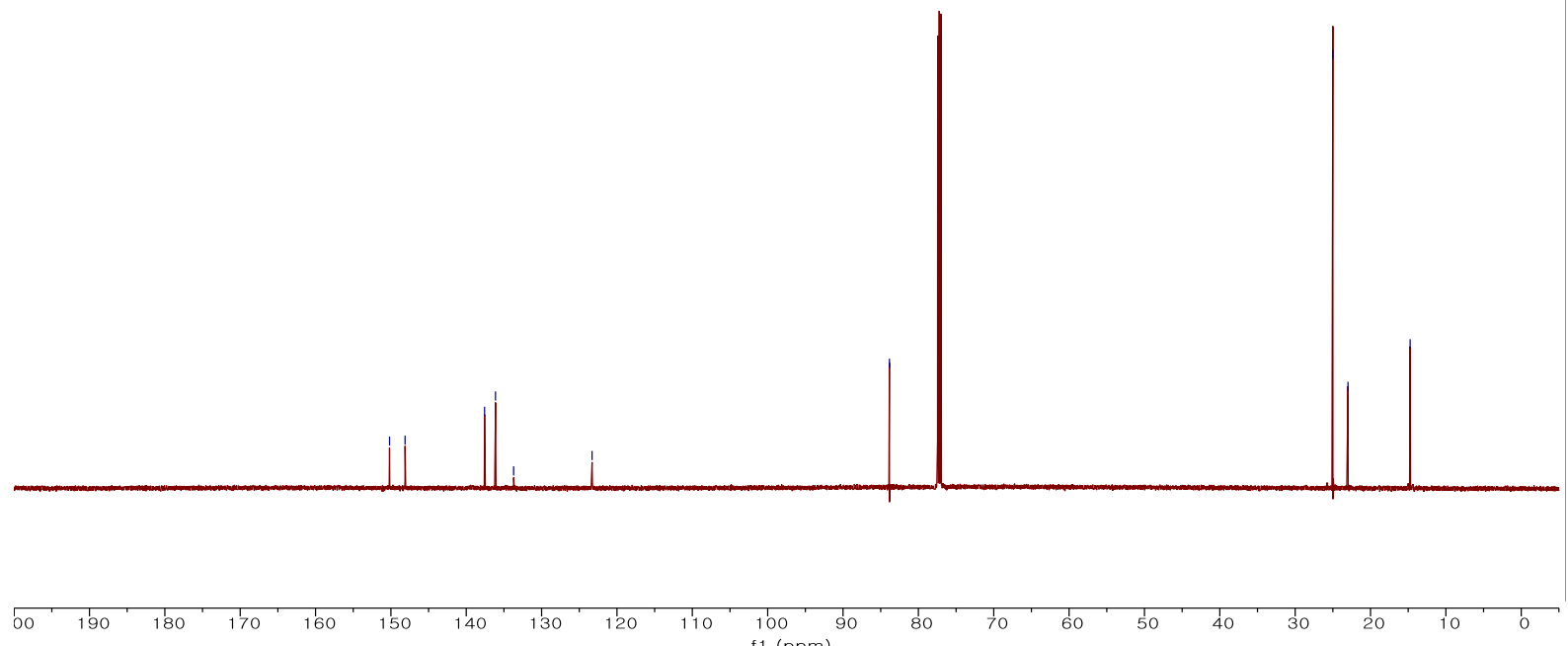

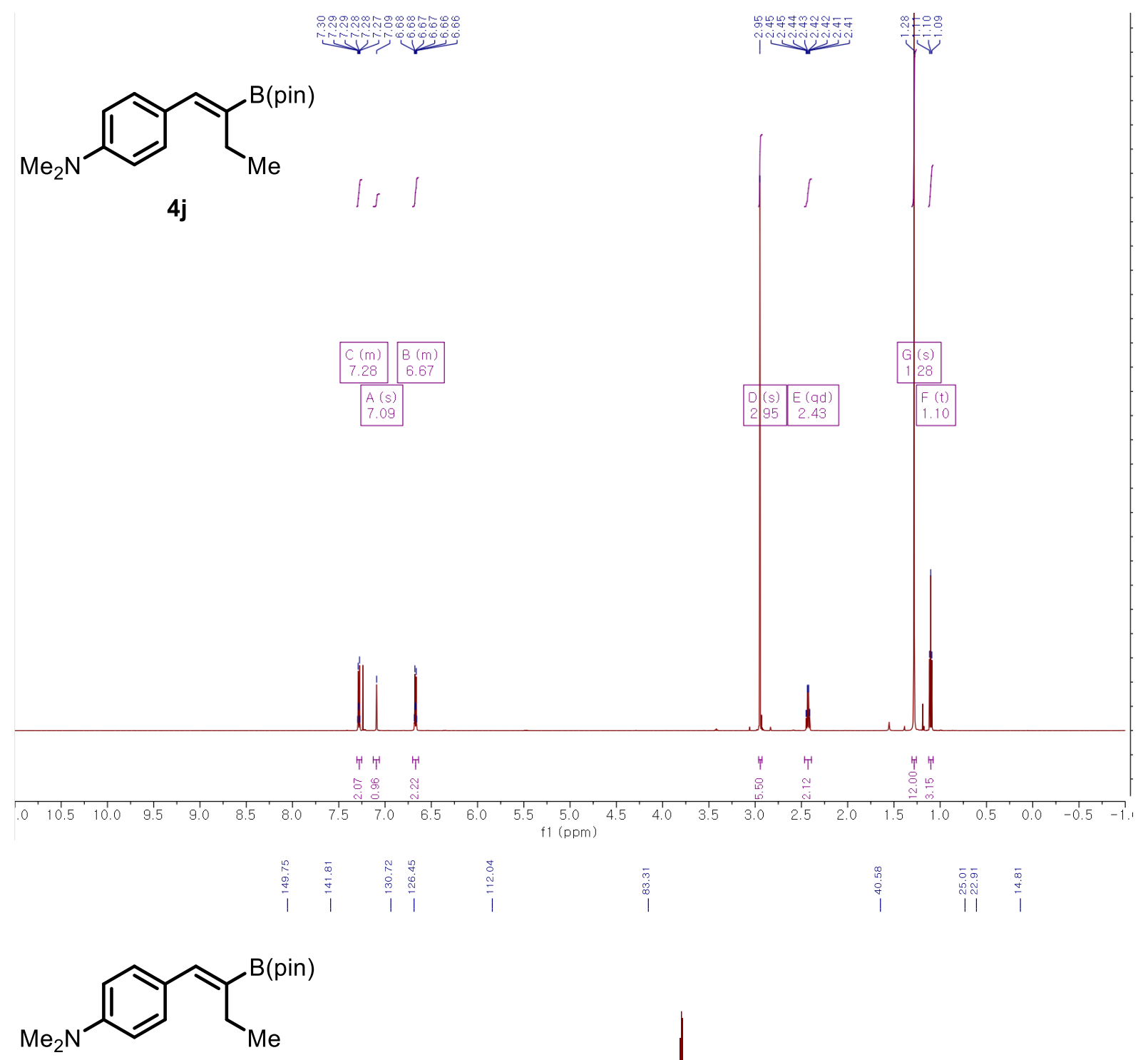

$4 \mathbf{j}$

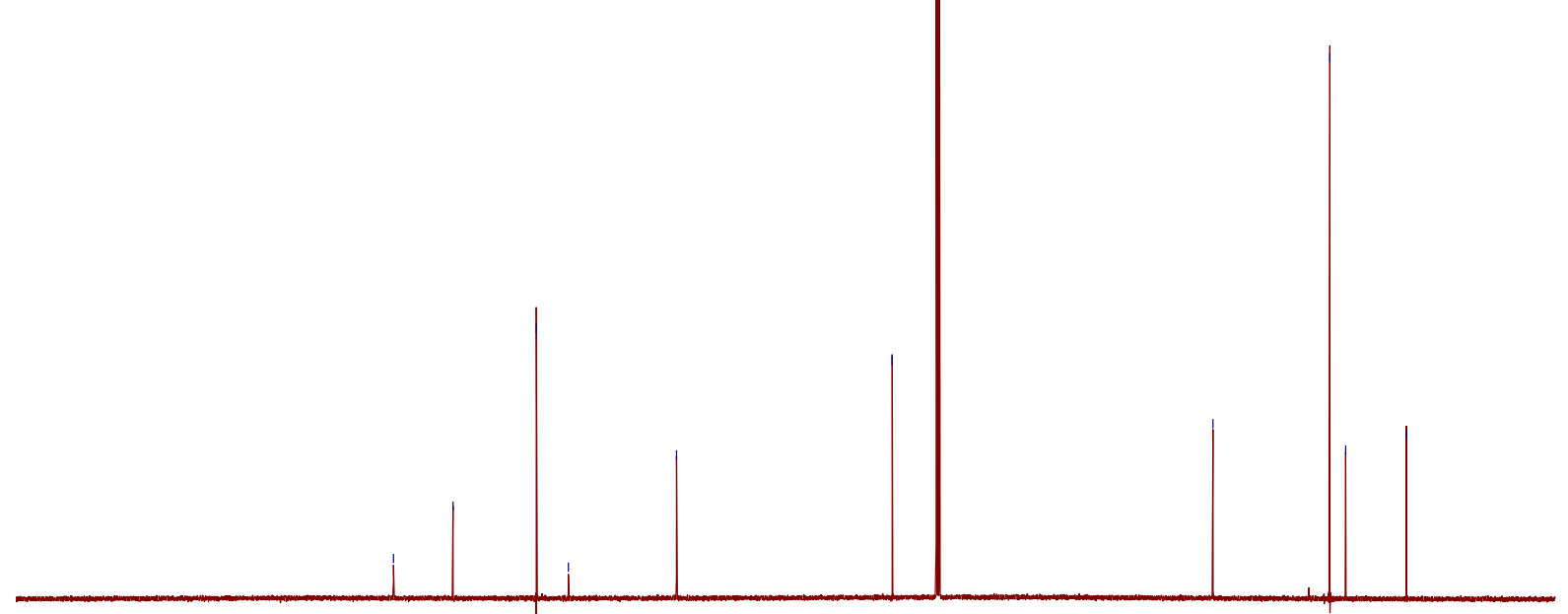

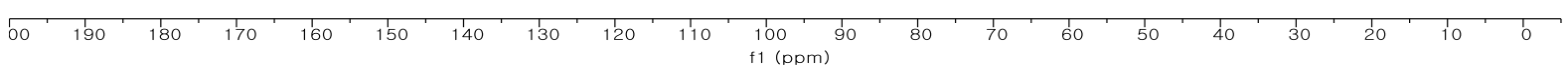



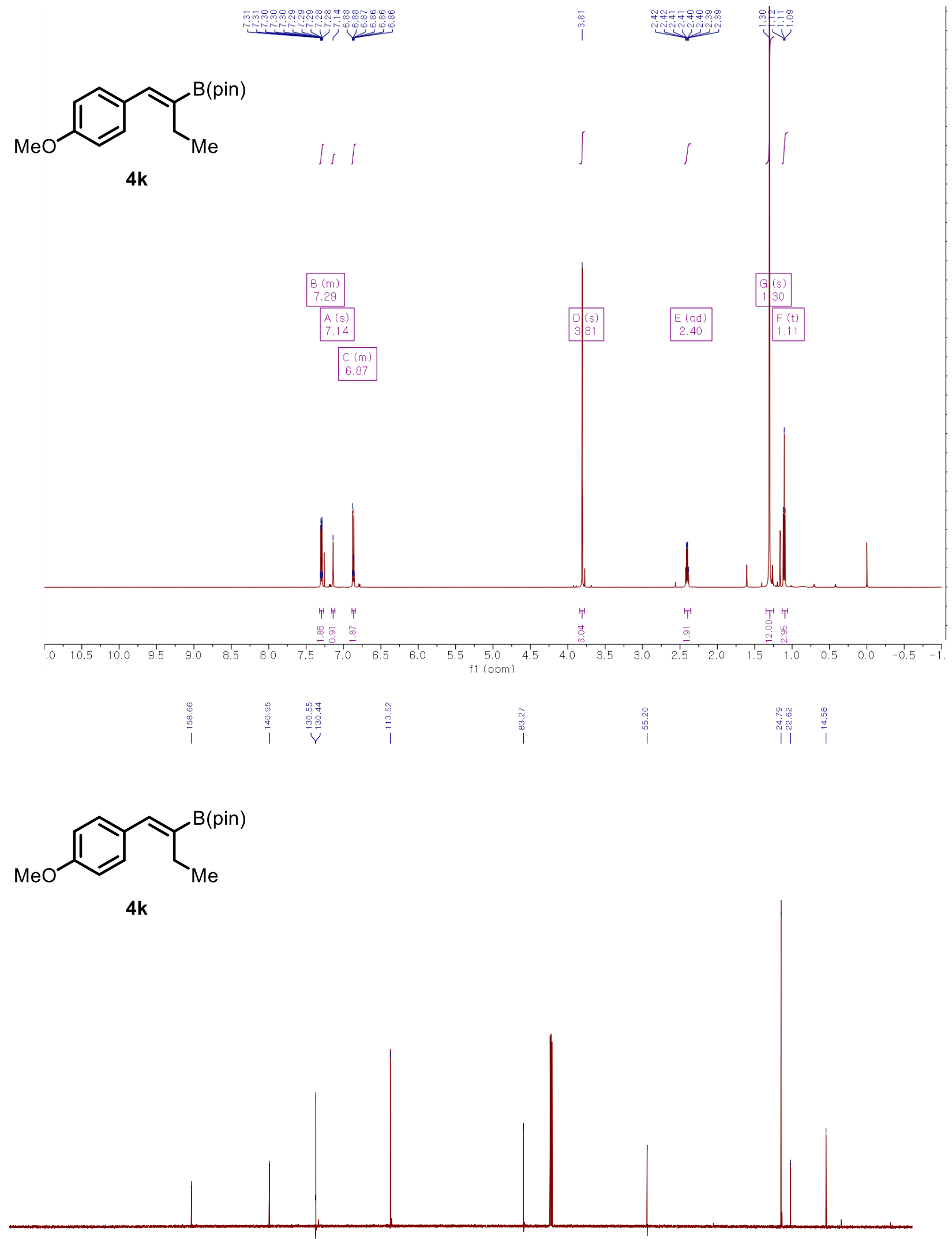
Medina et al.: BCP Hydroboration-Supporting Information - S178

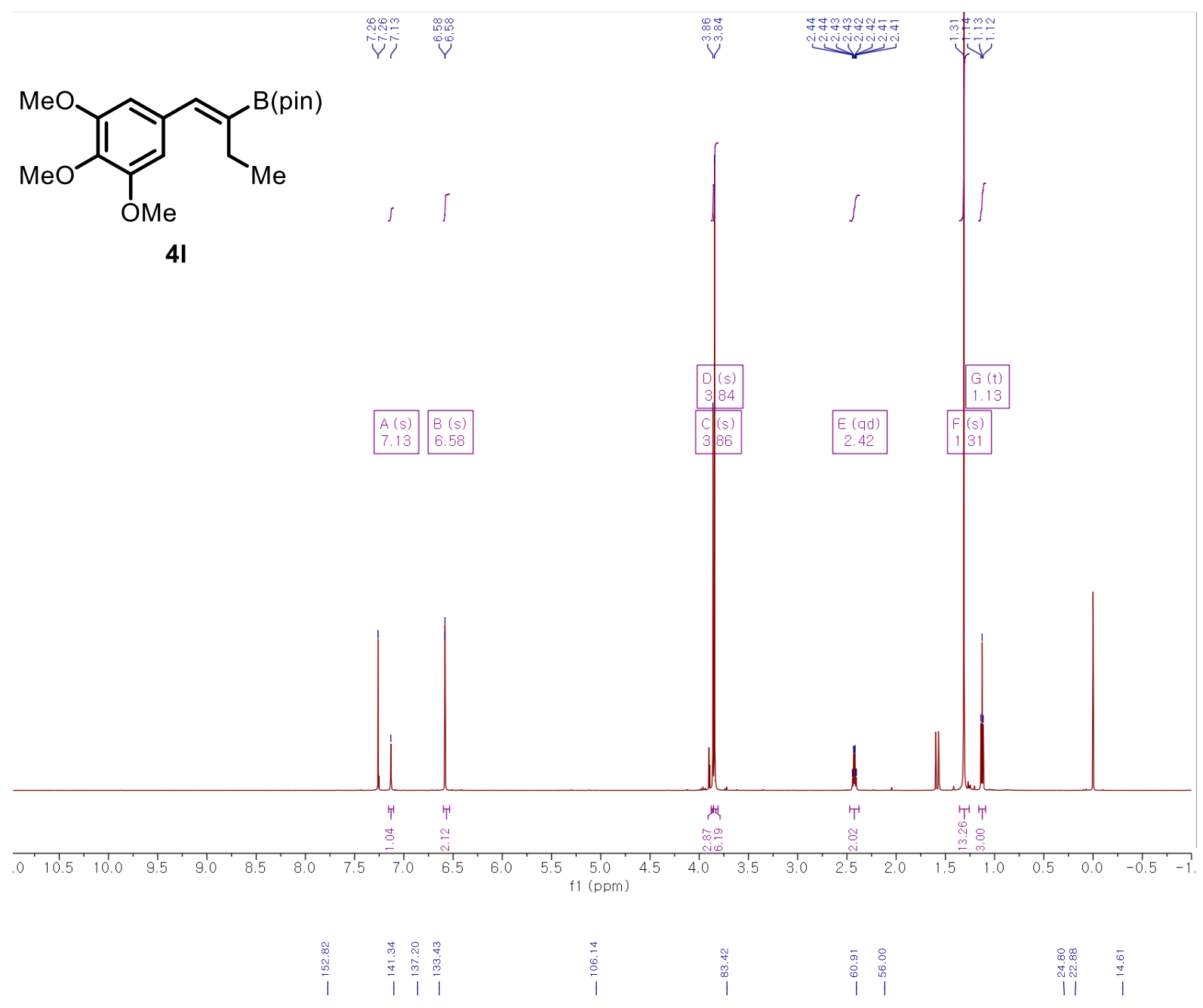

41
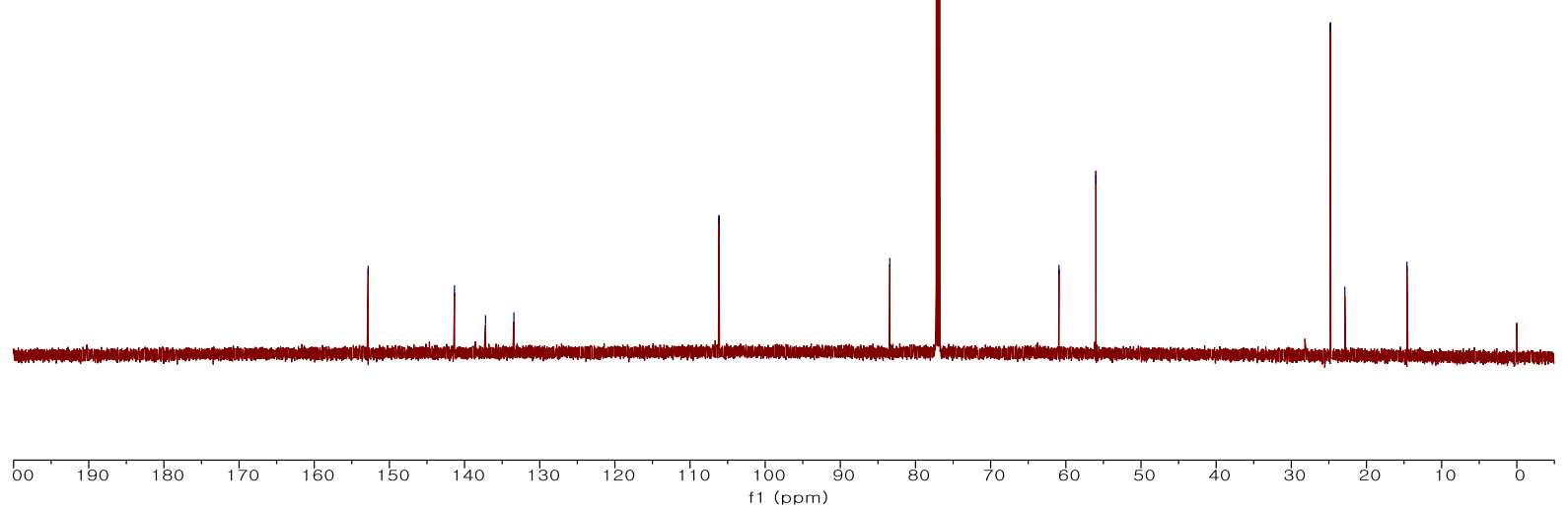

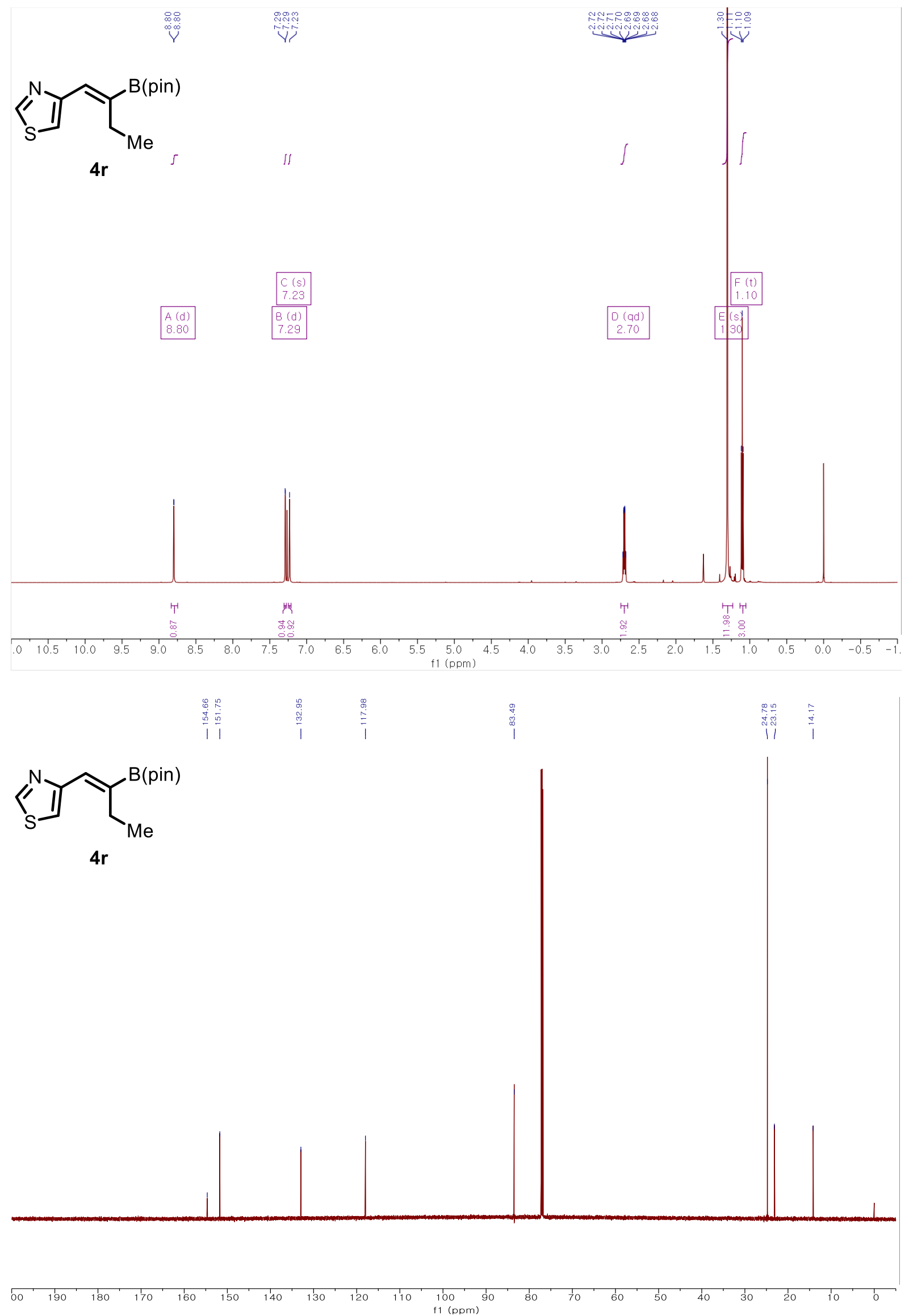

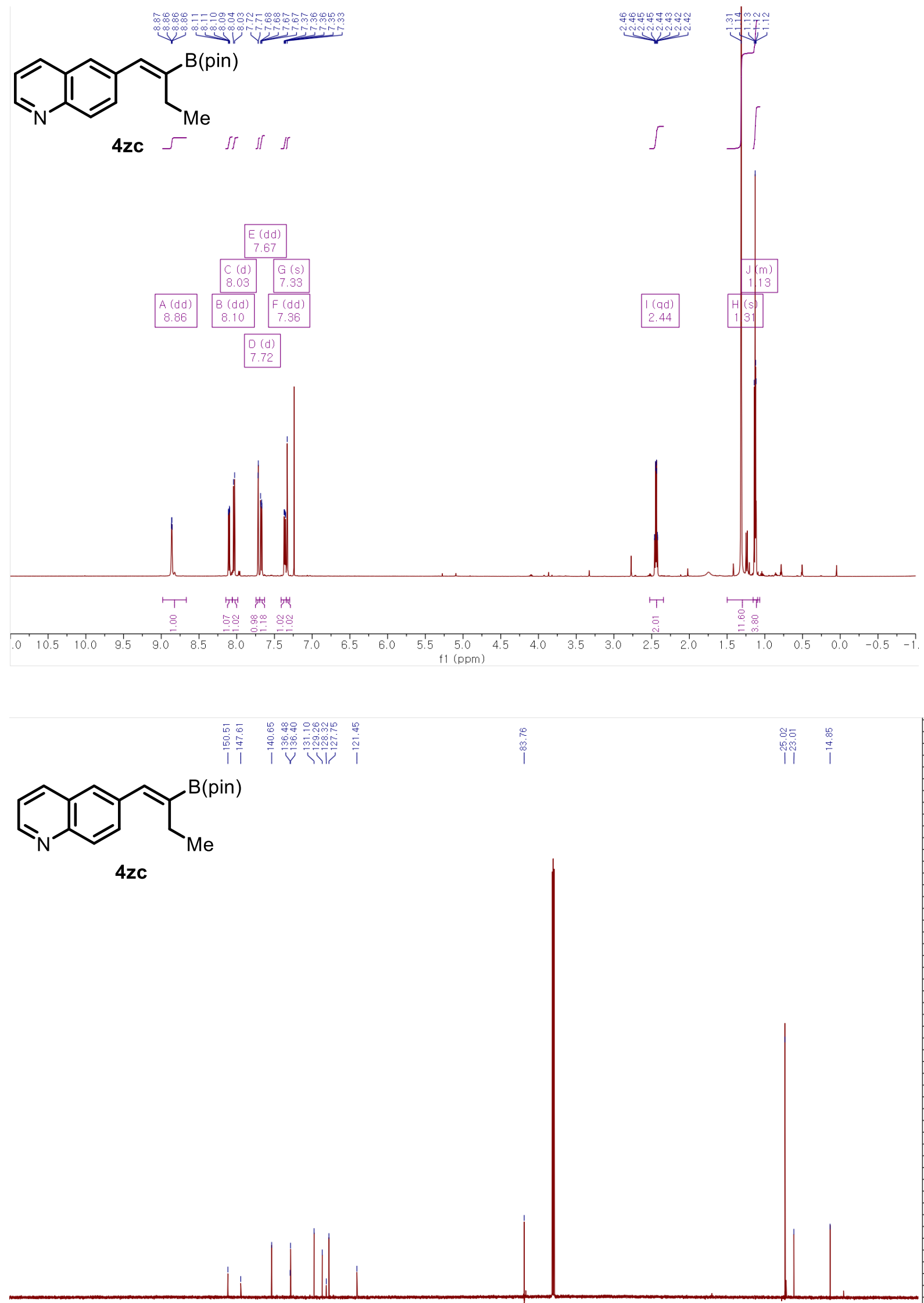

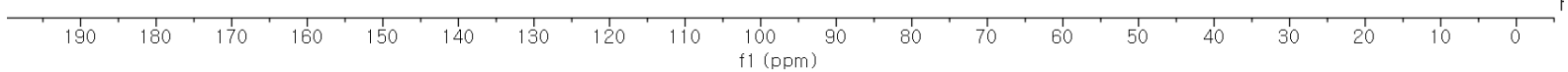



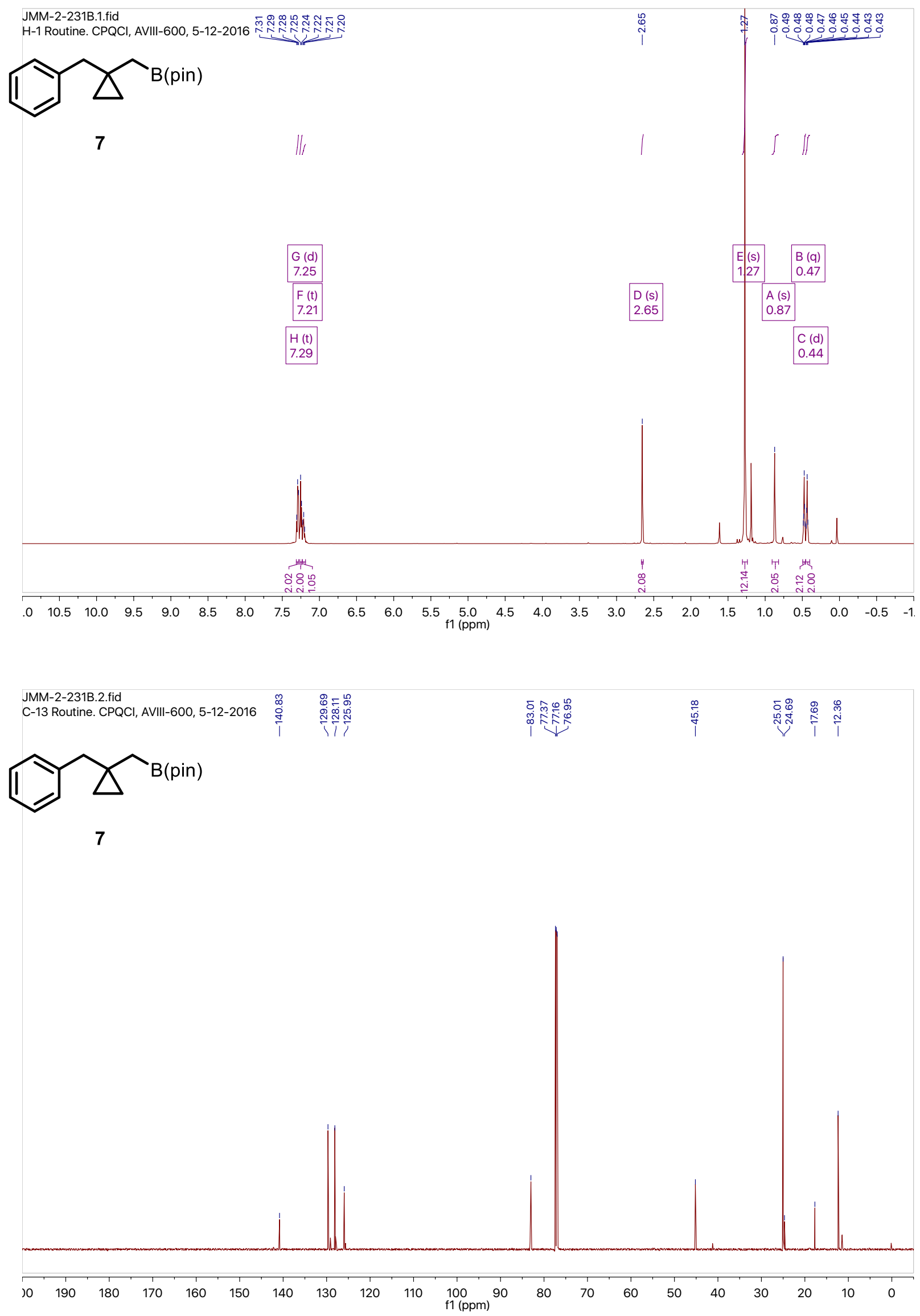


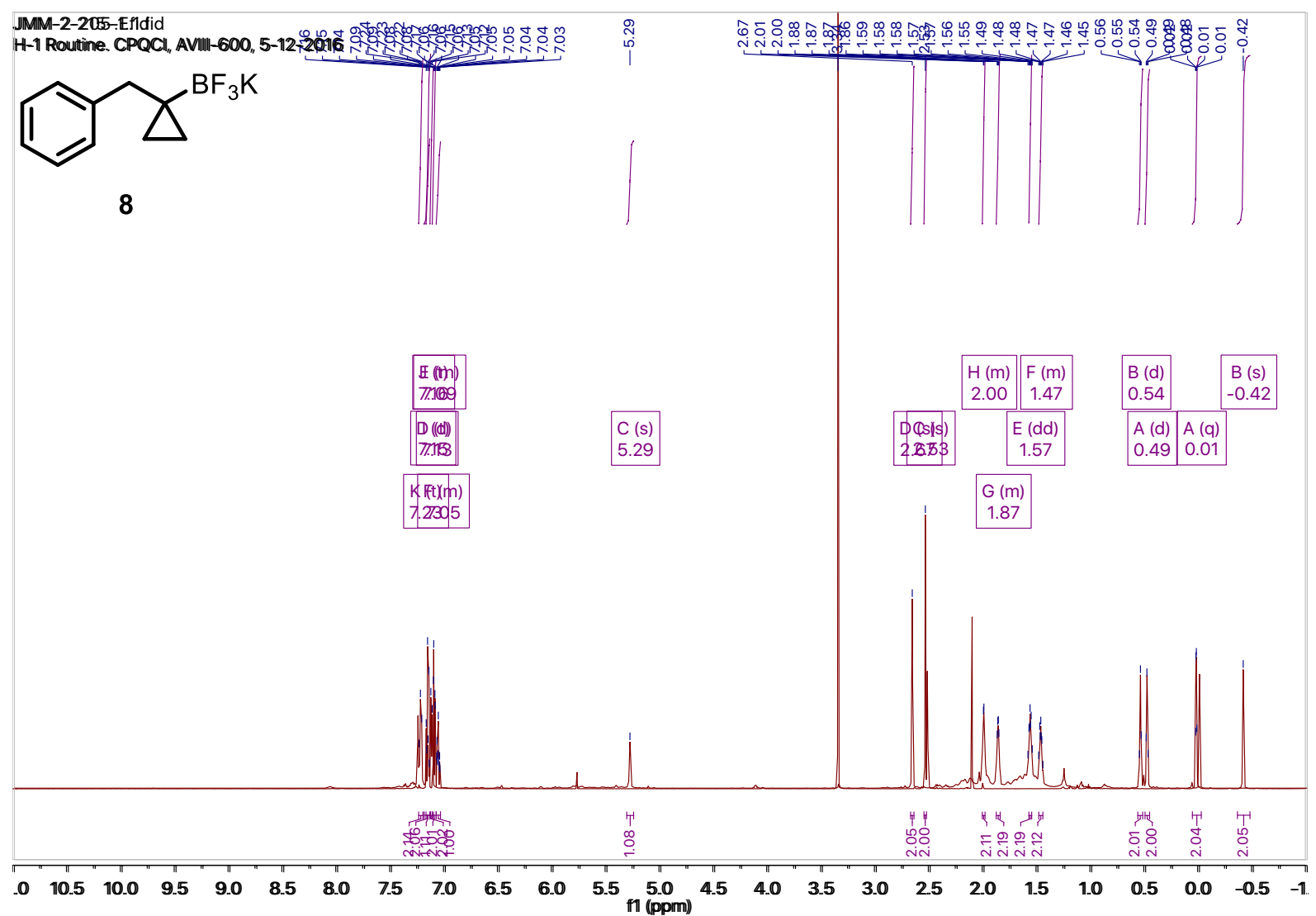

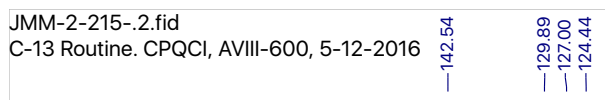<smiles>[TeH]</smiles>

8
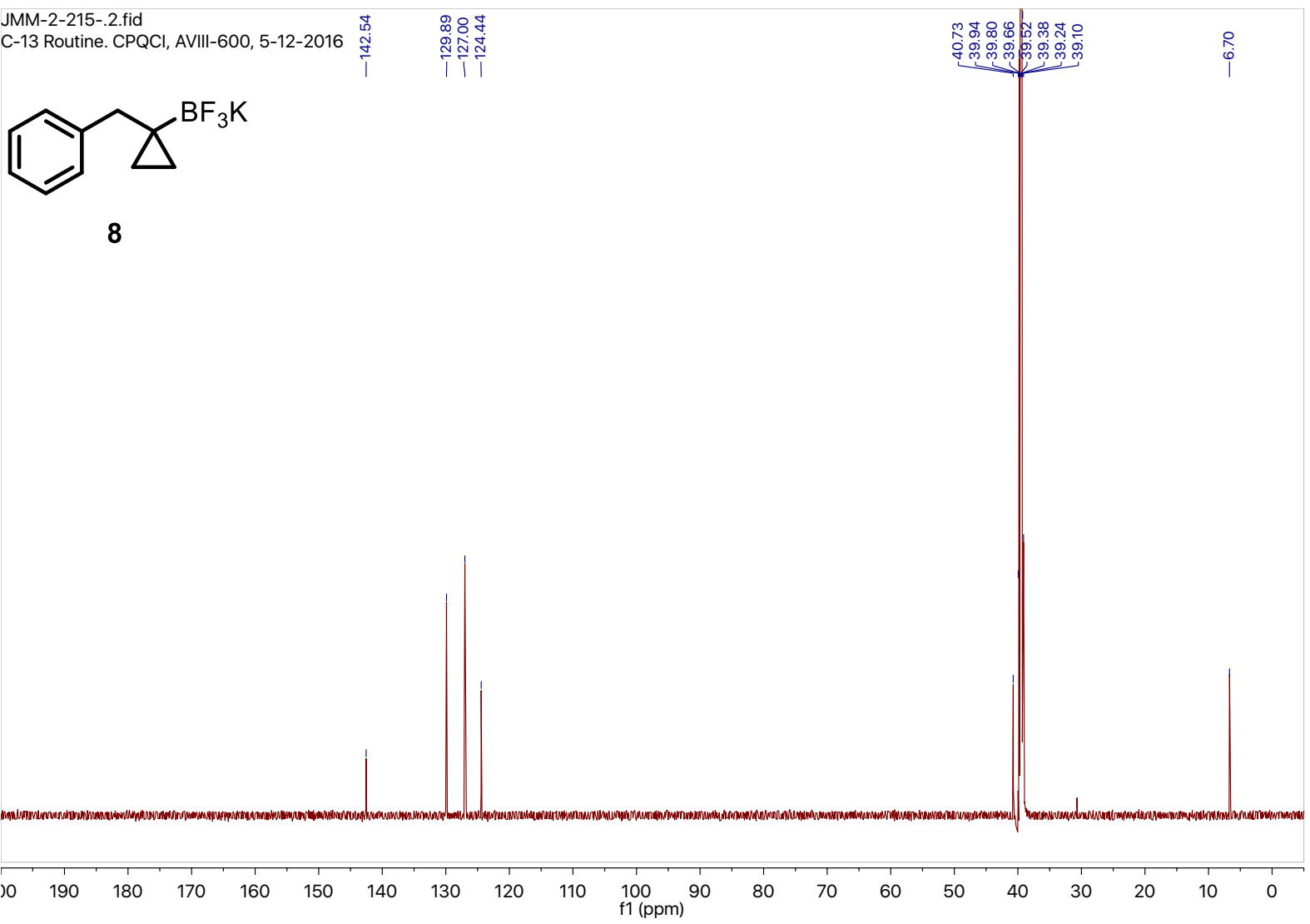

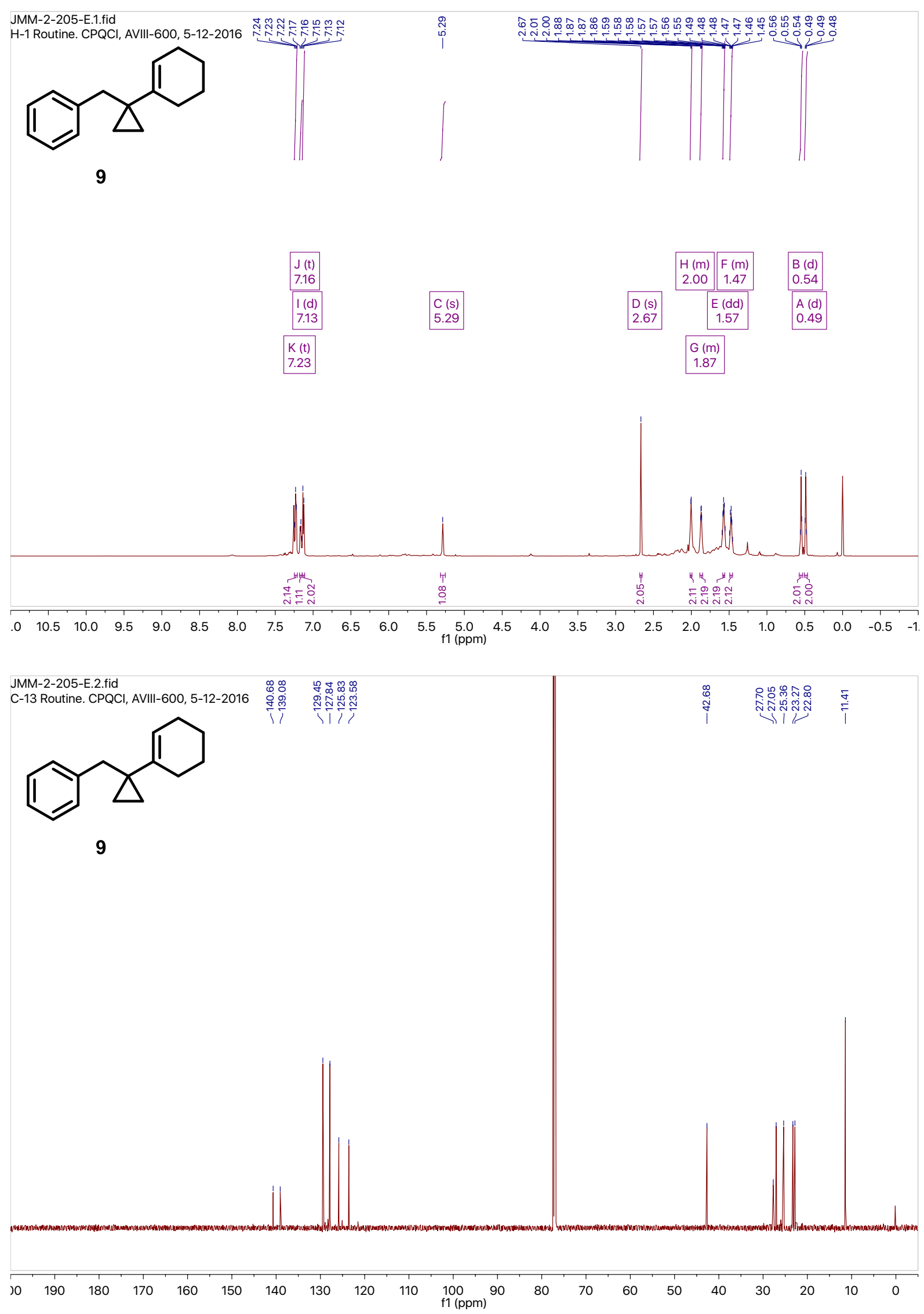

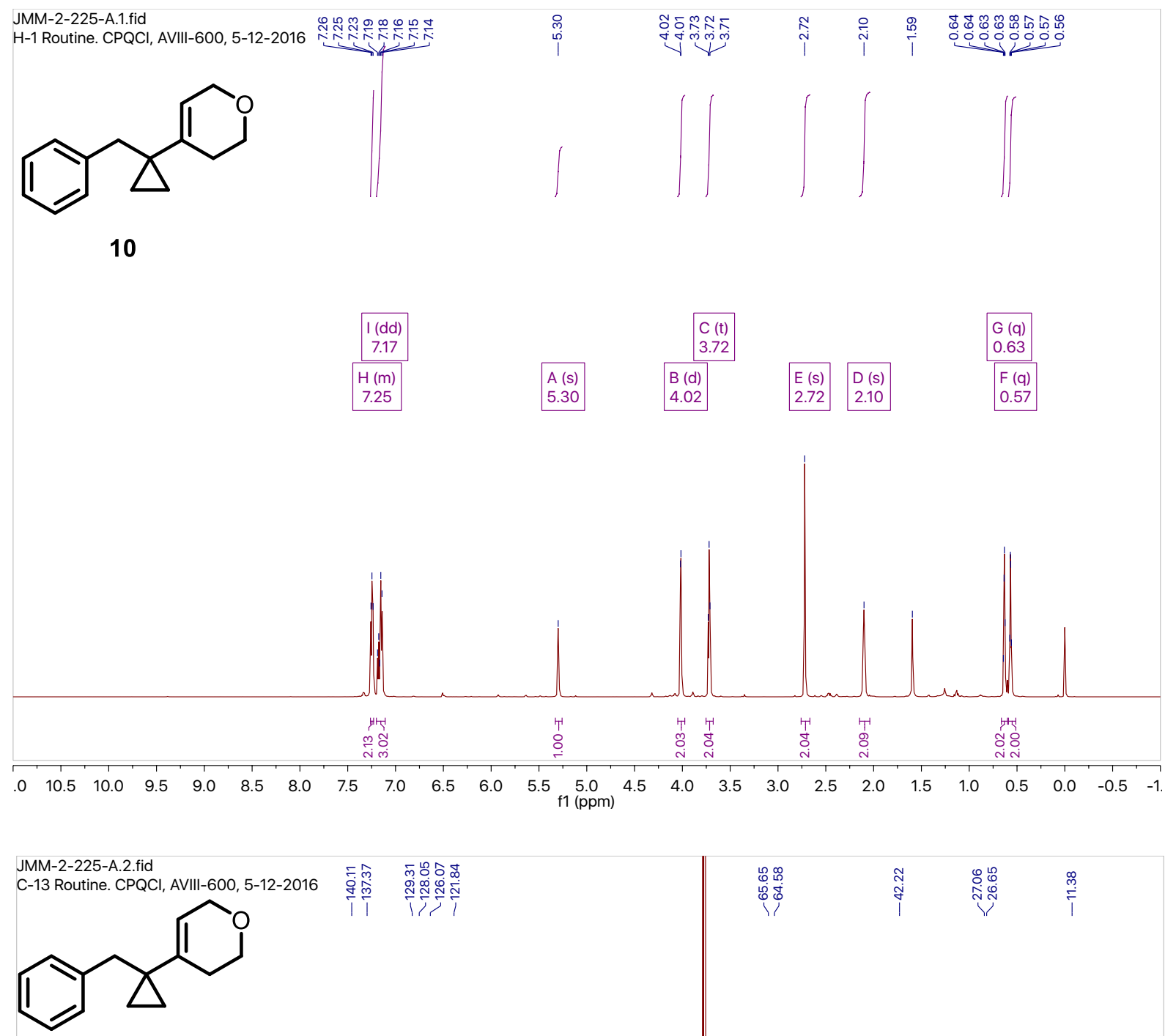

10

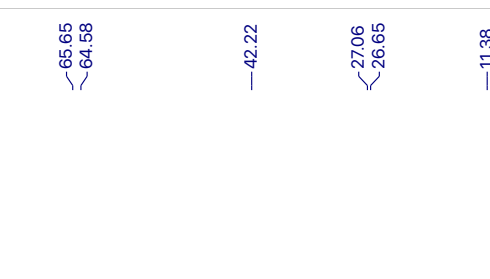



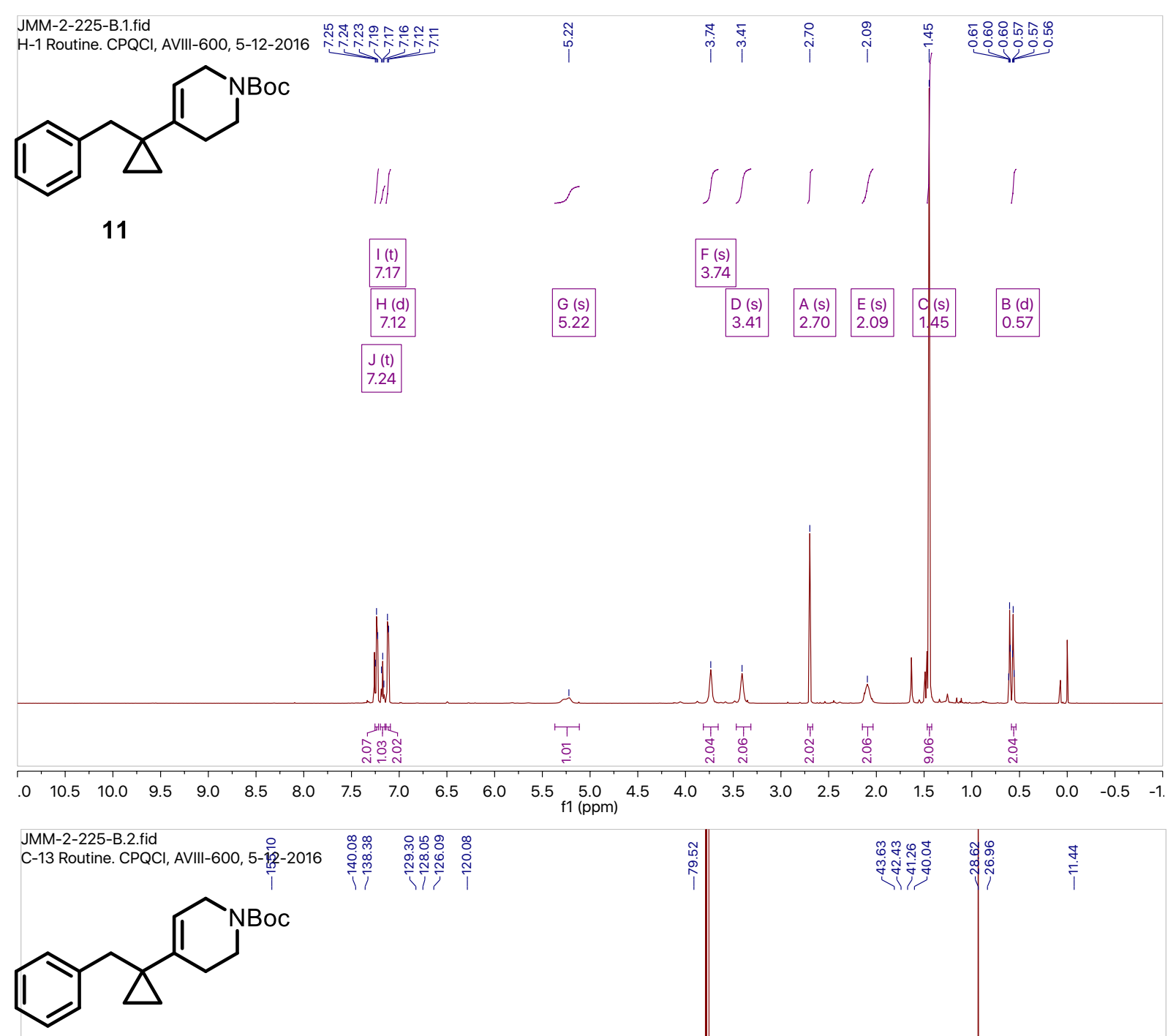

11
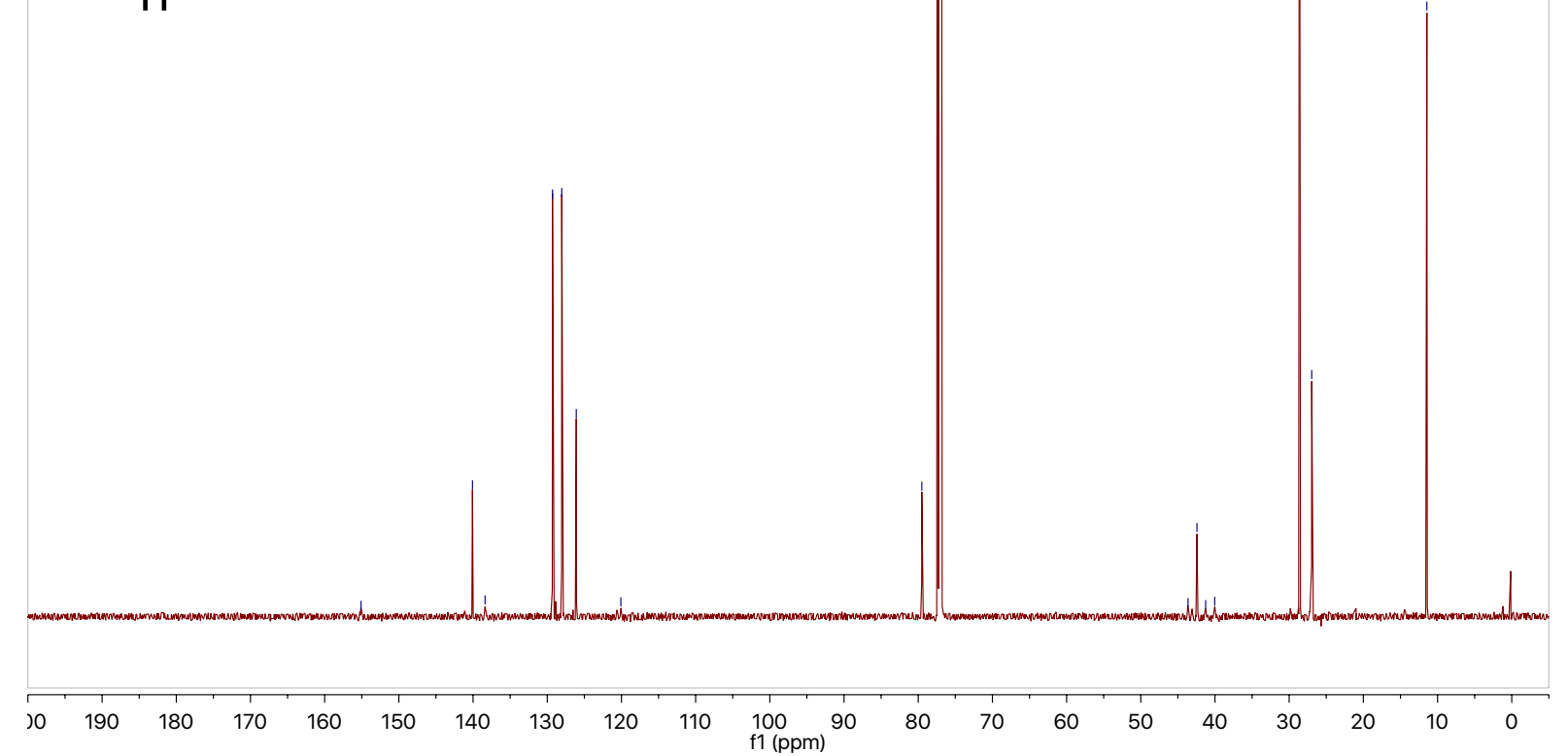

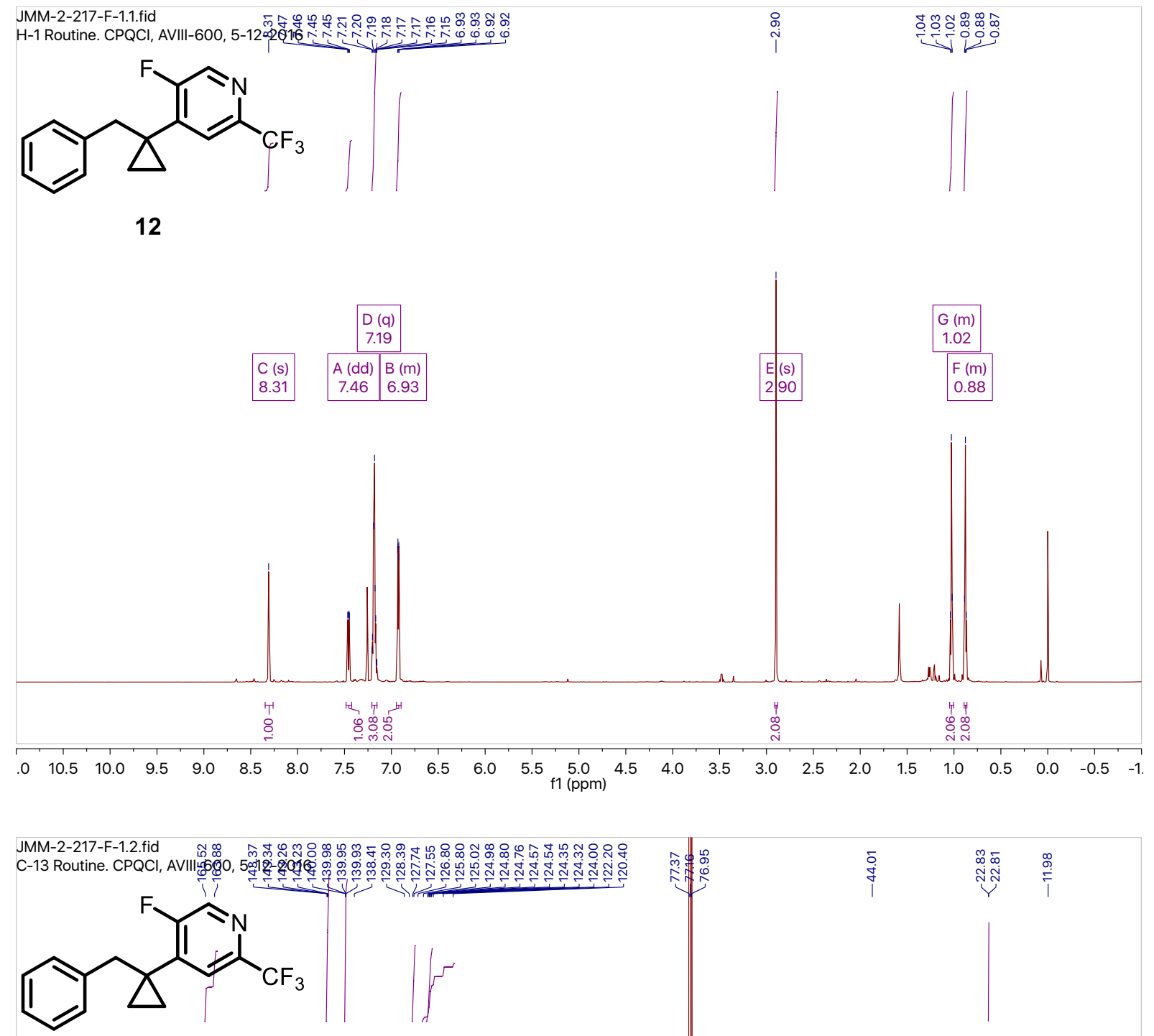

12

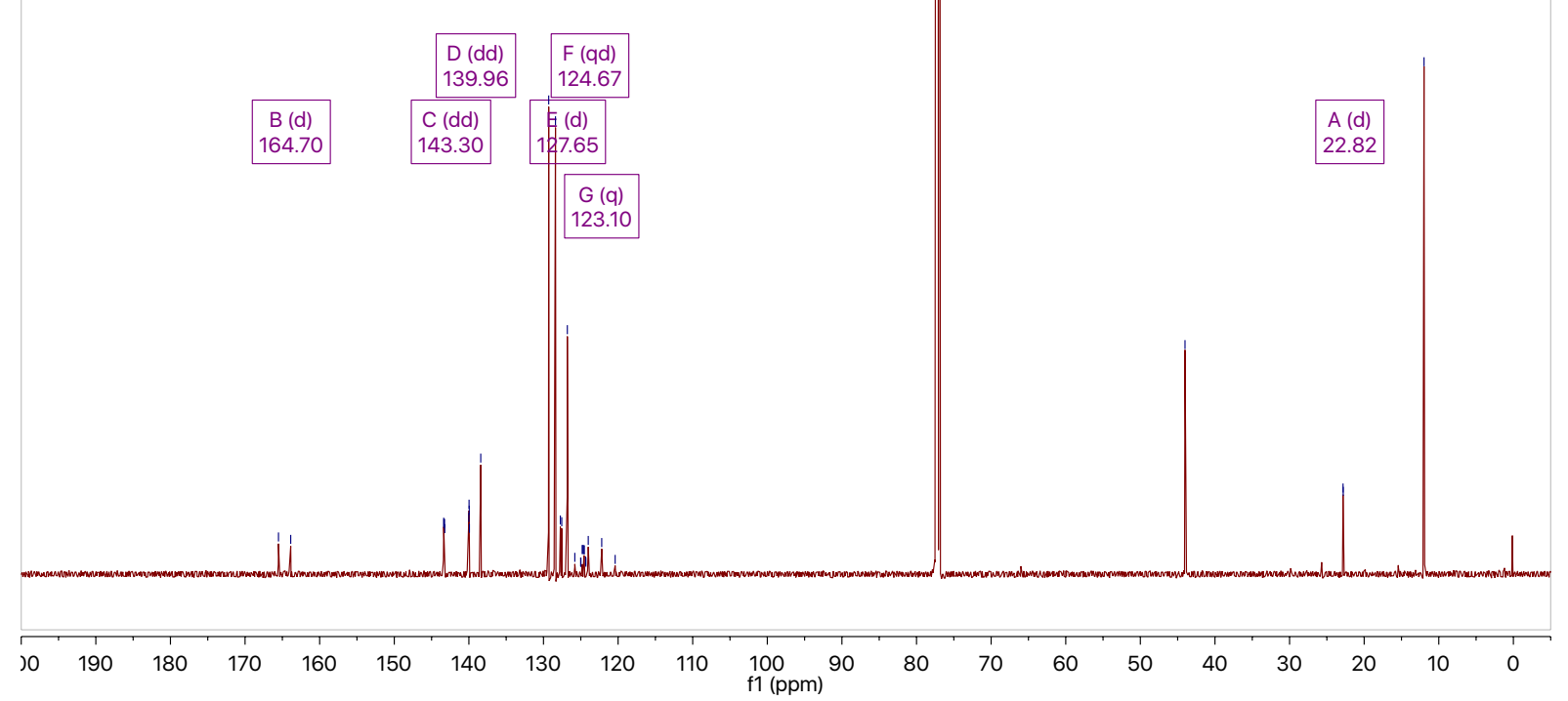



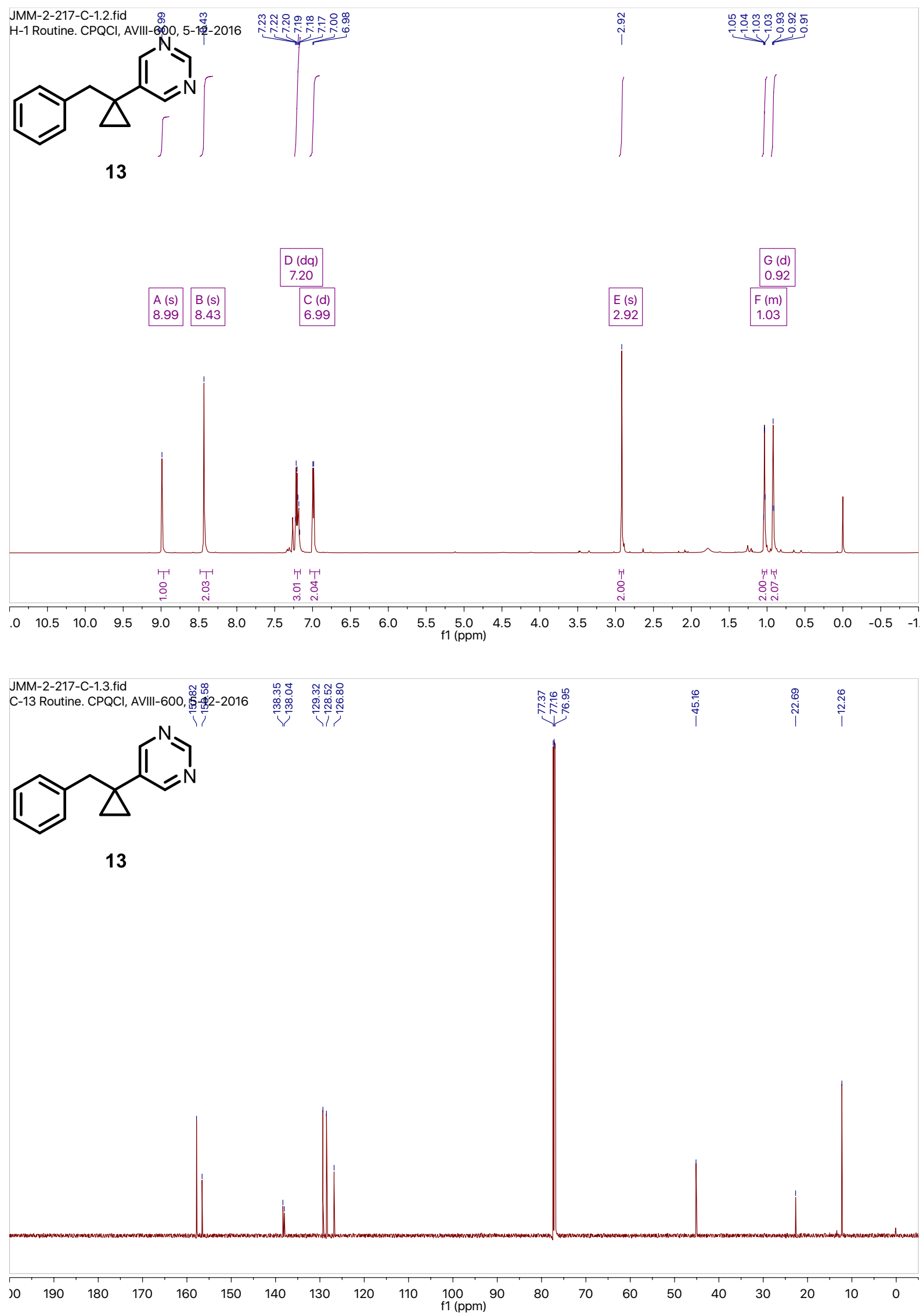

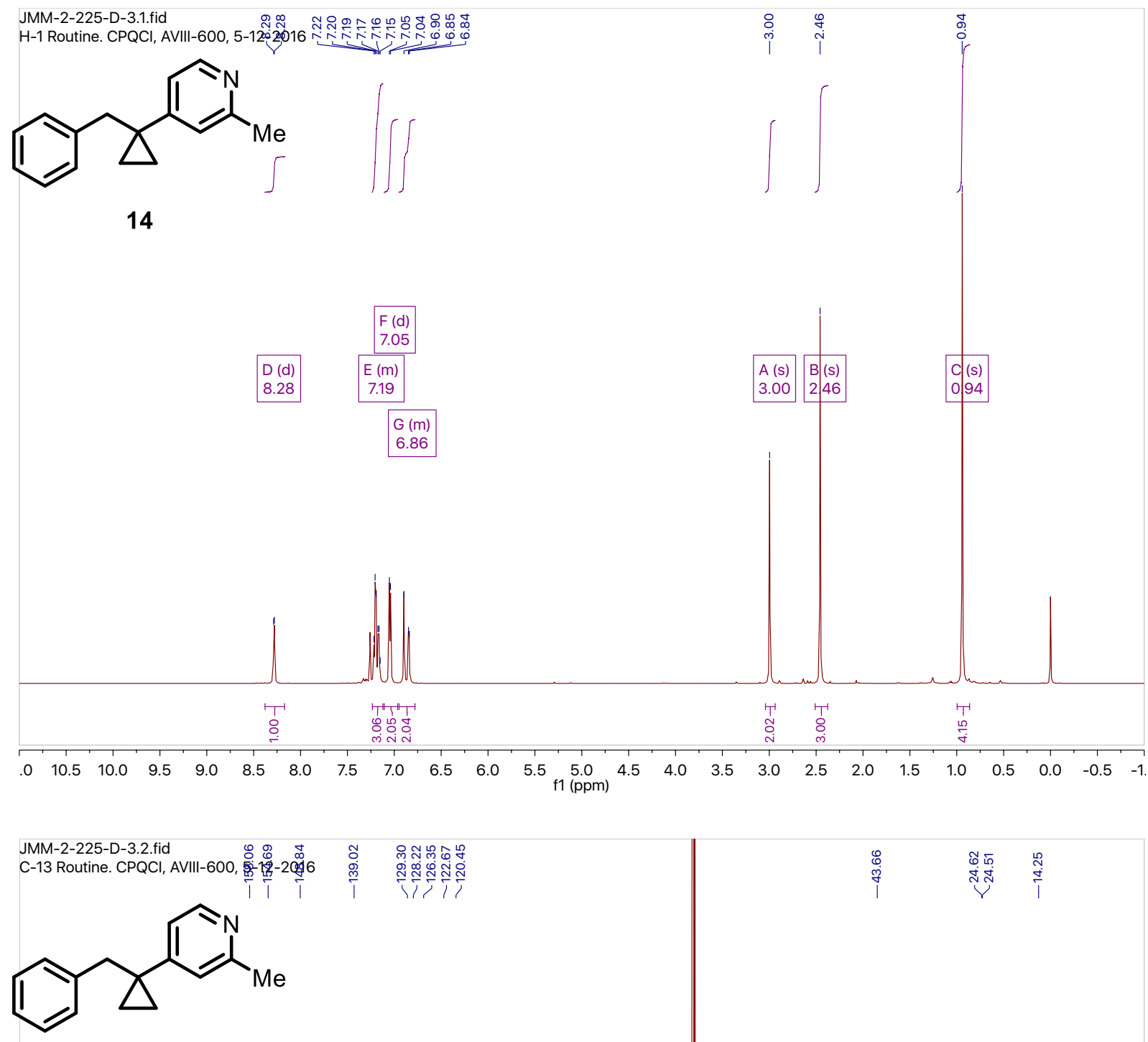

14

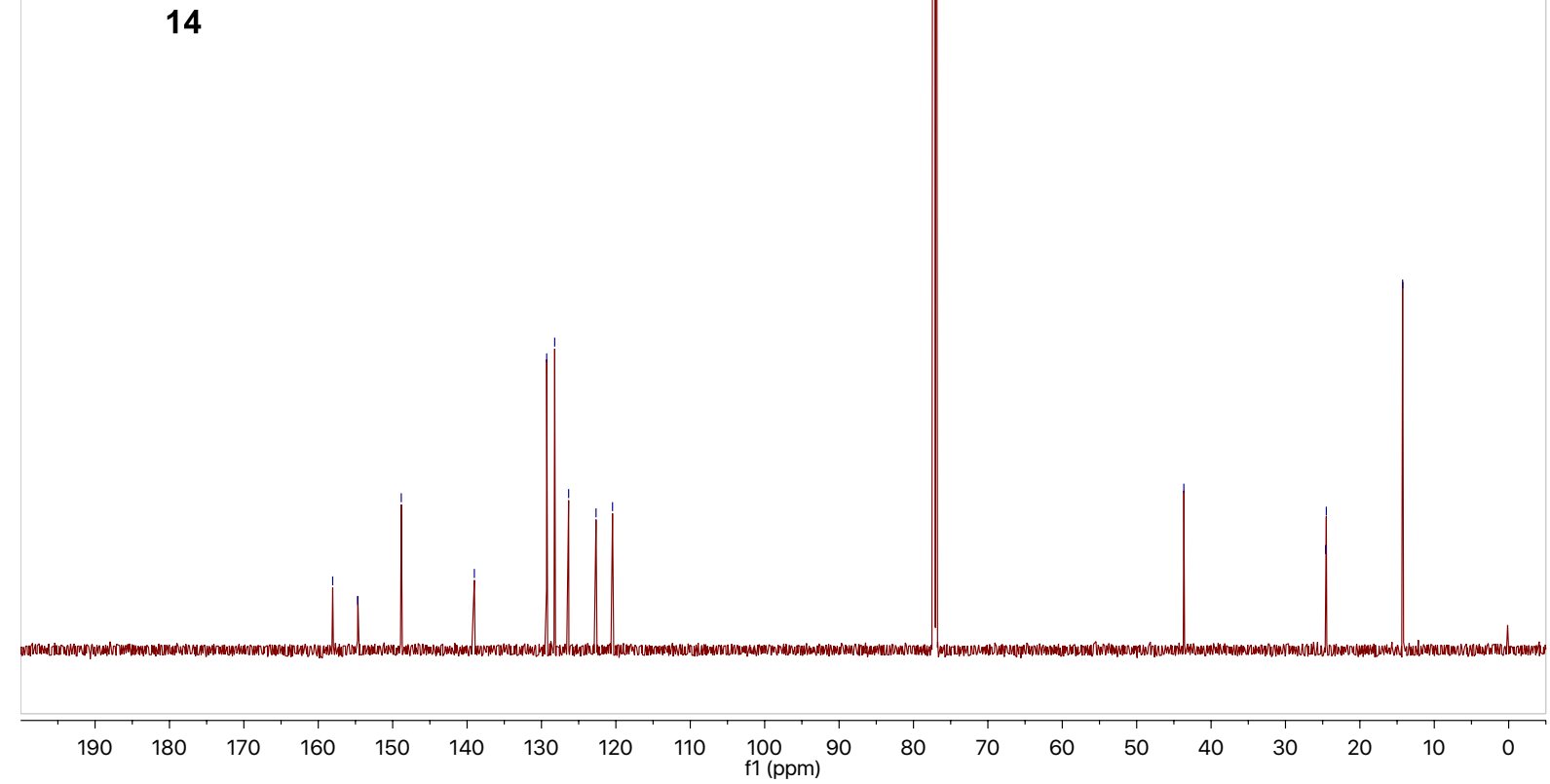




\section{References.}

${ }^{1}$ Wang, Y.; Muratore, M. E.; Rong, Z.; Echavarren, A. M. Formal (4+1) Cycloaddition of Methylenecyclopropanes with 7-Aryl-1,3,5-cycloheptatrienes by Triple Gold(I) Catalysis Angew. Chem., Int. Ed. 2014, 53, 14022-14026.

${ }^{2}$ Nordvik, T.; Brinker, U. H. Thermolysis of 1-(1-Aryl-1-bromomethyl)cyclopropyl Bromides: A Reinvestigation. J. Org. Chem. 2003, 68, 7092-7093.

${ }^{3}$ Farthing, C. N.; Marsden, S. P. Chiral Vinyl Dioxazaborocines in Synthesis: Asymmetric Cuprate Additions to $\beta$-boronyl Acrylates and Vinyl Sulfones. Tetrahedron Lett. 2000, 41, 42354238.

${ }^{4}$ Wu, P.; Jia, M.; Lin, W.; Ma, S. Matched Coupling of Propargylic Carbonates with Cyclopropanols. Org. Lett. 2018, 20, 554-557.

${ }^{7}$ Mlynarski, S. N.; Karns, A. S.; Morken, J. P. Direct Stereospecific Amination of Alkyl and Aryl Pinacol Boronates. J. Am. Chem. Soc. 2012, 134, 16449-16451.

${ }^{6}$ Bertus, P.; Szymoniak, J. New and Easy Route to Primary Cyclopropylamines from Nitriles.

Chem. Commun. 2001, 18, 1792-1793.

${ }^{7}$ Fujioka, Y.; Amii, H. Boron-Substituted Difluorocyclopropanes: New Building Blocks of gemDifluorocyclopropanes. Org. Lett. 2008, 10, 769-772.

${ }^{8}$ Fang, G.-H.; Yan, Z.-J.; Deng, M.-Z. Palladium-Catalyzed Cross-Coupling of Stereospecific Potassium Cyclopropyl Trifluoroborates with Aryl Bromides. Org. Lett. 2004, 6, 357-360.

9 Harris, M. R.; Li, Q.; Lian, Y.; Xiao, J.; Londregan, A. T. Construction of 1-Heteroaryl-3azabicyclo[3.1.0]hexanes by $\mathrm{sp}^{3}-\mathrm{sp}^{2}$ Suzuki-Miyaura and Chan-Evans-Lam Coupling Reactions of Tertiary Trifluoroborates. Org. Lett. 2017, 19, 2450-2453.

${ }^{10}$ Frisch, M. J.; Trucks, G. W.; Schlegel, H. B.; Scuseria, G. E.; Robb, M. A.; Cheeseman, J. R.; Scalmani, G.; Barone, V.; Petersson, G. A.; Nakatsuji, H.; Li, X.; Caricato, M.; Marenich, A. V.; Bloino, J.; Janesko, B. G.; Gomperts, R.; Mennucci, B.; Hratchian, H. P.; Ortiz, J. V.; Izmaylov, A. F.; Sonnenberg, J. L.; Williams; Ding, F.; Lipparini, F.; Egidi, F.; Goings, J.; Peng, B.; Petrone, A.; Henderson, T.; Ranasinghe, D.; Zakrzewski, V. G.; Gao, J.; Rega, N.; Zheng, G.; Liang, W.; Hada, M.; Ehara, M.; Toyota, K.; Fukuda, R.; Hasegawa, J.; Ishida, M.; Nakajima, T.; Honda, Y.; Kitao, O.; Nakai, H.; Vreven, T.; Throssell, K.; Montgomery Jr., J. A.; Peralta, J. E.; Ogliaro, F.; Bearpark, M. J.; Heyd, J. J.; Brothers, E. N.; Kudin, K. N.; Staroverov, V. N.; 
Keith, T. A.; Kobayashi, R.; Normand, J.; Raghavachari, K.; Rendell, A. P.; Burant, J. C.; Iyengar, S. S.; Tomasi, J.; Cossi, M.; Millam, J. M.; Klene, M.; Adamo, C.; Cammi, R.; Ochterski, J. W.; Martin, R. L.; Morokuma, K.; Farkas, O.; Foresman, J. B.; Fox, D. J. Gaussian 16 Rev. B.01, Wallingford, CT, 2016.

11 Zhao, Y.; Truhlar, D. G. The M06 suite of density functionals for main group thermochemistry, thermochemical kinetics, noncovalent interactions, excited states, and transition elements: two new functionals and systematic testing of four M06-class functionals and 12 other functionals. Theor. Chem. Acc. 2008, 120, 215-241.

${ }^{12}$ Marenich, A. V.; Cramer, C. J.; Truhlar, D. G. Universal Solvation Model Based on Solute Electron Density and on a Continuum Model of the Solvent Defined by the Bulk Dielectric Constant and Atomic Surface Tensions. J. Phys. Chem. B 2009, 113, 6378-6396.

13 Ribeiro, R. F.; Marenich, A. V.; Cramer, C. J.; Truhlar, D. G. Use of Solution-Phase Vibrational Frequencies in Continuum Models for the Free Energy of Solvation. J. Phys. Chem. $B$ 2011, 115, 14556-14562.

${ }^{14}$ Paton, R. F.-A., Ignacio GoodVibes v2.0.1, 2017.

${ }^{15}$ Legault, C. Y. CYLview, 1.0b, 2009. 
